\title{
Aerodynamic and Structural Design of Flow Conditioning, Flow Seeding and Testing Sections of a High-Speed Wind-Tunnel
}

\author{
by \\ Chanon C. Pretorius
}

A thesis submitted to the Faculty of Graduate and Postdoctoral Affairs in partial fulfillment of the requirements for the degree of

\section{Master of Applied Science \\ in \\ Aerospace Engineering}

\author{
Carleton University \\ Ottawa, Ontario
}

(C) 2020

Chanon C. Pretorius 


\begin{abstract}
This thesis presents the design of two test sections, one annular and one rectilinear, to facilitate the study of ducted flows at high subsonic Mach numbers on the Carleton high-speed wind-tunnel. The structural performance of the two designs was evaluated using finite element tools and were deemed compliant with provincial pressure-vessel regulatory requirements. Both designs benefit from lighter-weight materials and a greater degree of modularity than existing high-speed test sections. The aerodynamic performance of an annular-flow air-supply system was evaluated using a transient, one dimensional algorithm. The annular flow path is designed to provide a steady Mach number flow in the range of 0.3 to 0.8 at the test section discharge, with minimal pressure loss to achieve minimum run times of 25-30 seconds on a blow-down type wind-tunnel. The present study also evaluates the flow seeding performance of a seed material injection system on the annular-flow air-supply system for particle image velocimetry measurements. The study demonstrates the ability of computational fluid engineering tools to accurately evaluate the sensitivity of the seed injection system's performance to a range of geometric parameters, and velocity and pressure conditions specific to this design.
\end{abstract}




\section{Acknowledgements}

First and foremost I would like to acknowledged my thesis supervisor, Dr. Metin I. Yaras for providing me this opportunity to better myself and for his unwavering mentorship throughout this journey. I also wish to thank Pratt and Whitney Canada as well as the Natural Sciences and Engineering Research Council (NSERC) for their financial support. Without their involvement, this work would not have been possible.

My sincerest appreciation goes out to the technical and support staff of the university; Alex Proctor, Neil McFadyen, Kevin Sangster, and Stephan Biljan; whose expertise were relied on more times than I can count. Lastly but most certainly not least, I wish to thank my colleagues Andrei Bronipolsky, Andrew Copping, Riley Simpson, Shuhao Wu, Ted Gan, and Utku Caylan, who's collaboration and friendship made this journey very enjoyable. 


\section{Table of Contents}

Abstract $\quad$ ii

Acknowledgements

List of Tables viii

List of Figures $\quad$ xi

$\begin{array}{ll}\text { Nomenclature } & \text { xviii }\end{array}$

Chapter 1 Introduction 1

$\begin{array}{lll}\text { Chapter } 2 & \text { Literature Review } & 4\end{array}$

2.1 High Speed Wind-Tunnel Design . . . . . . . . . . . . . . 4

2.1.1 Air Compressor . . . . . . . . . . . . . . 4

2.1.2 Air Drying System . . . . . . . . . . . . . 5

2.1.3 Storage Tanks . . . . . . . . . . . . . . . 5

2.1.4 Control Valves .................. 6

2.1.5 Flow Conditioning . . . . . . . . . . . . . . . . 7

2.2 Laser-Based Flow Measurement Techniques . . . . . . . . . . . . . . . 10

2.2.1 Laser Doppler Anemometry (LDA) . . . . . . . . . . . . 10

2.2.2 Particle Image Velocimetry (PIV) . . . . . . . . . . . . . . . . 11

2.3 Flow Seeding Materials for Particle Image Velocimetry . . . . . . . . 15

2.3.1 Solid Particle Seeding . . . . . . . . . . . . . . . . . 15

2.3.2 Liquid Particle Seeding . . . . . . . . . . . . . . . . . 17

Chapter $3 \quad$ Experimental Facilities $\quad 23$

3.1 Low-Speed Open-Circuit Wind-Tunnel . . . . . . . . . . . . . . 23

3.2 High-Speed Blow-Down Wind-Tunnel . . . . . . . . . . . . . . 24

3.2.1 Aerodynamic Model of the Wind-Tunnel Flow Path . . . . . . 26 
Chapter 4 Design of an Air-Supply System for Annular-Flow Test Sections at Subsonic Mach Numbers 32

4.1 Introduction . . . . . . . . . . . . . . . . . . . . . 32

4.2 Pre-compression Rods . . . . . . . . . . . . . . . . . . . . . 34

$4.3 \quad$ V-band Clamps . . . . . . . . . . . . . . . . . . . 35

4.4 Upstream Flange . . . . . . . . . . . . . . . . . . . . . . . . . . 38

4.4 .1 Structural Analysis . . . . . . . . . . . . . . . . . . 40

4.5 Expansion Section . . . . . . . . . . . . . . . 46

4.5 .1 Structural Analysis . . . . . . . . . . . . . . . 47

4.6 Transition Section I . . . . . . . . . . . . . . . . . . . . . . . . . . 51

4.6 .1 Structural Analysis . . . . . . . . . . . . . . . 53

4.7 Honeycomb Flow Straightener . . . . . . . . . . . . . . . 57

4.7.1 Structural Analysis . . . . . . . . . . . . . . . . . 59

4.8 Turbulence-Reduction Screen Assembly . . . . . . . . . . . . . . 63

4.8 .1 Structural Analysis . . . . . . . . . . . . . . . . 65

4.9 Swirl Generator Assembly . . . . . . . . . . . . . . . . . . . . . . . . 69

4.9.1 Structural Analysis . . . . . . . . . . . . . . . . 71

4.10 Core-Pipe Assembly . . . . . . . . . . . . . . . . . . . 75

4.11 Seed Injection Assembly _ . . . . . . . . . . . . . . . 76

4.11 .1 Structural Analysis . . . . . . . . . . . . . . . . . . 79

4.12 Transition Section II _ . . . . . . . . . . . . . . . . . 83

4.12 .1 Structural Analysis . . . . . . . . . . . . . . . 85

4.13 Downstream Flange . . . . . . . . . . . . . . . . . . . . 89

4.13 .1 Structural Analysis . . . . . . . . . . . . . . . . 99 90

4.14 Air-Supply System Aerodynamic Performance Prediction . . . . . . . 94

4.15 Design Summary . . . . . . . . . . . . . . . . . . 97

Chapter 5 Design of a Rectilinear Test Section for Lobed-Mixer $\begin{array}{ll}\text { Studies at Subsonic Mach Numbers } & 98\end{array}$

5.1 Design . . . . . . . . . . . . . . . . . . . . . . . . . . . . . . 98 
$5.2 \quad$ Structural Analysis . . . . . . . . . . . . . . . . . . . 100

5.2 .1 Computational Models . . . . . . . . . . . . . . . . . . . 100

5.2 .2 Computational Results . . . . . . . . . . . . . . . . . . 103

5.3 Design Summary . . . . . . . . . . . . . . . . . . . . . 106

$\begin{array}{lll}\text { Chapter } 6 & \text { Seed Injection Port Design } & 107\end{array}$

6.1 Design Considerations . . . . . . . . . . . . . . . . . . 107

6.2 Computational Domain and Boundary Conditions . . . . . . . . 108

6.3 Computational Grid . . . . . . . . . . . . . . . . . . . . . . 112

6.4 Governing Equations . . . . . . . . . . . . . . . . . . 114

6.5 Discretization . . . . . . . . . . . . . . . . . . 117

6.6 Solution Method . . . . . . . . . . . . . . . . . . 117

6.7 Computational Results . . . . . . . . . . . . . . . . . . . . . 118

6.7.1 Low-speed Annular Inflow . . . . . . . . . . . . . . . 118

6.7.2 High-speed Annular Inflow _. . . . . . . . . . . . . 130

6.8 Validation of the Numerical Flow Model . . . . . . . . . . . 133

6.8.1 Computational Model . . . . . . . . . . . . . . . . . . . . 133

6.8.2 Computational Results . . . . . . . . . . . . . . . 136

6.9 Design Summary . . . . . . . . . . . . . . . . . . . . . . 140

$\begin{array}{lll}\text { Chapter } 7 & \text { Conclusions and Recommendations for Future Work } & 141\end{array}$

$\begin{array}{ll}\text { References } & 142\end{array}$

Appendix A Derivation of Aerodynamic Performance Equations Pertaining to the Wind-tunnel Aerodynamic Model 152

A.1 Stagnation Pressure Loss in Compressible Duct Flow . . . . . . . . 152

A.2 Storage Tank Expansion Process Equations . . . . . . . . . . . 153

Appendix B Technical Drawings for the Air-Supply System 155 
$\begin{array}{lll}\text { Appendix C Finite Element Analysis Benchmark Study } & 200\end{array}$

C.1 Pressurized Thick-Walled Cylinder _. . . . . . . . . . . . 200

C.1.1 Computational Model . . . . . . . . . . . . . . . . . . . 200

C.1.2 Results . . . . . . . . . . . . . . . . . . . . . . . . 202

C.2 Circular Beam in Bending . . . . . . . . . . . . . . . . 203

C.2.1 Computational Model . . . . . . . . . . . . . . . . . . . 203

C.2.2 Results . . . . . . . . . . . . . . . . . . . . . . 205

Appendix D Aerodynamic Performance Prediction Algorithm 206

Appendix E Technical Drawings for the Rectilinear Test Section 215

Appendix F Validation of the Numerical Grid Used to Resolve the Expansion/Compression Wave Pattern of an Under-expanded Jet 221

F.1 Computational Model . . . . . . . . . . . . . . . . . . . . . . . 221

F.2 Computational Results . . . . . . . . . . . . . . . . . . . 223

Appendix G Seed Injection Port Design - Additional Figures 226 


\section{List of Tables}

$2.1 \quad$ Physical properties of seed materials. . . . . . . . . . . 21

2.2 Health and safety hazard properties of seed materials (DeLapp,

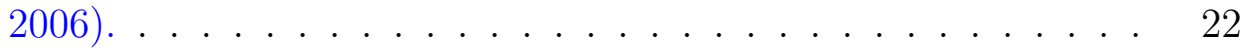

4.1 Main components of the air-supply system. . . . . . . . . . 34

4.2 Dimensions and installation torque of V-band clamps. . . . . . 36

4.3 Design parameters for the upstream flange. . . . . . . . . . 38

4.4 Parameters of the computational grid. . . . . . . . . . . . . . 42

4.5 Mechanical properties of the materials in the subassembly. . . . 43

4.6 Summary of the structural analysis results for the upstream flange subassembly. . . . . . . . . . . . . . . . . . . 45

4.7 Design parameters for the expansion section. . . . . . . . . 46

4.8 Parameters of the computational grid. . . . . . . . . . . 49

4.9 Mechanical properties of the materials in the subassembly. . . . 49

4.10 Summary of the structural analysis results for the sudden expansion subassembly. . . . . . . . . . . . . . 50

4.11 Design parameters for transition section I. . . . . . . . . . . 51

4.12 Parameters of the computational grid. . . . . . . . . . . 55

4.13 Summary of the structural analysis results for the transition section I subassembly. . . . . . . . . . . . . . . . 56

4.14 Design parameters for the flow straightener housing. . . . . . . 57

4.15 Parameters of the computational grid. . . . . . . . . . 61

4.16 Summary of the structural analysis results for the flow straightener subassembly. . . . . . . . . . . . . . 62

4.17 Design parameters for the turbulence screen housing. . . . . . . 63

4.18 Parameters of the computational grid. . . . . . . . . . 67

4.19 Summary of the structural analysis results for the turbulence screen subassembly. . . . . . . . . . . . . . . 68 
4.20 Design parameters for the swirl generator housing. . . . . . . . 69

4.21 Parameters of the computational grid. . . . . . . . . . . 73

4.22 Summary of the structural analysis results for the swirl generator housing subassembly. . . . . . . . . . . . . . . . . 74

4.23 Design parameters for the seed injection housing. . . . . . . . . 76

4.24 Parameters of the computational grid. . . . . . . . . . . . 81

4.25 Summary of the structural analysis results for the seed injection subassembly. . . . . . . . . . . . . . . . . . . 82

4.26 Design parameters for transition section II. . . . . . . . . . . 83

4.27 Parameters of the computational grid. . . . . . . . . . . . 87

4.28 Summary of the structural analysis results for the transition section II subassembly. . . . . . . . . . . . . . . . . . . . . . . . . 88

$4.29 \quad$ Design parameters for the downstream flange. . . . . . . . . . . 89

$4.30 \quad$ Parameters of the computational grid. . . . . . . . . . . . 92

4.31 Summary of the structural analysis results for the downstream flange subassembly. . . . . . . . . . . . . . . . . 93

$4.32 \quad$ Air-supply system empirical loss coefficients. . . . . . . . . . 95

5.1 Mechanical properties of the materials of the rectilinear lobedtail test section. . . . . . . . . . . . . . . . 101

$5.2 \quad$ Parameters of the computational grid. . . . . . . . . . 101

5.3 Summary of results for the aligned inflow condition. . . . . . . 105

$5.4 \quad$ Summary of results for the misaligned inflow condition. . . . . 105

$6.1 \quad$ Parameters of the CFD test matrix. . . . . . . . . . 110

6.2 Initial values of the normalized flow-field parameters in the domain.111

$6.3 \quad$ Grid specifications. . . . . . . . . . . . . 113

$6.4 \quad$ Nomenclature for the parameters in Eqns. 6.1 to 6.4 . . . . . . 116

6.5 Initial values of the normalized flow-field parameters in the domain.135

C.1 Parameters of the computational grid. . . . . . . . . . 201 
C.2 Geometric and mechanical properties of the thick-walled cylinder.201

C.3 Parameters of the computational grid. . . . . . . . . . . . 204

C.4 Geometric and mechanical properties of the circular beam. . . . 204

F.1 Initial values of the normalized flow-field parameters in the domain.222

F.2 Comparison of computational results to empirical and analytical

predictions. . . . . . . . . . . . . . . . . 225 


\section{List of Figures}

2.1 Variable-contour nozzle in the Lockheed Martin high-speed windtunnel (Lockheed Martin, 2002) . . . . . . . . . . . . . 7

2.2 Pressure loss across wire-mesh screens as a function of $R e_{d}$ (adapted from Groth and Johansson, 1988). . . . . . . . . . 10

$2.3 \quad$ Operating principle of planar PIV . . . . . . . . . . . . 13

3.1 Rendered model of the Carleton low-speed turbomachinery windtunnel. ........................... 24

3.2 Rendered model of the Carleton high-speed wind-tunnel. . . . 25

3.3 Simplified model of the wind-tunnel flow path. . . . . . . . . 26

3.4 Time variation of (a) $P_{\text {oTank }}$ and (b) valve open area ratio, $A_{2} / A_{3}$, for several Mach numbers at station 4. . . . . . . . 30

3.5 Time variation of (a) $P_{o T a n k}$ and (b) valve open area ratio, $A_{2} / A_{3}$, for several values of flow area $\left(A_{4}\right)$ with $M_{4}=0.6$. . . 31

4.1 Assembled air-supply system with a lobed-mixer test section. .

4.2 Exploded schematic of the air-supply system; pre-compression rods and V-band clamps omitted for clarity. . . . . . . . . . 33 V-band clamp. . . . . . . . . . . . . . . . . . 35

4.4 Cross-sectional view of the V-band clamp. . . . . . . . . 36

4.5 V-band clamp installation. . . . . . . . . . . 36

4.6 V-band clamp and joint deformation without tongue-and-groove under (a) dominantly axial load, and (b) dominantly radial load. 37

4.7 V-band clamp and joint deformation with tongue-and-groove geometry under combined axial and radial load. . . . . . . . . 37

$4.8 \quad$ Upstream flange. . . . . . . . . . . . . . . . . . . . 39

4.9 Upstream flange installation. . . . . . . . . . . . . . 39

4.10 FEA computational model of the upstream flange as seen from the (a) downstream end; (b) upstream end. . . . . . . . . . . 41 
4.11 Schematic showing the loading of the V-band clamp and the location of the spring used to model the pre-compression rods. 42

4.12 Von Mises stress distribution in the upstream flange subassembly. 44

$4.13 \quad$ Expansion section. . . . . . . . . . . . . 46

4.14 Expansion section installation. . . . . . . . . . . 47

4.15 FEA computational model of expansion section as seen from the (a) downstream end; (b) upstream end. . . . . . . . . . 48

4.16 Schematic showing the loading of the V-band clamp and the location of the spring used to model the pre-compression rods.

4.17 Von Mises stress distribution in the sudden expansion subassembly. . . . . . . . . . . . . . . . 5 50

$4.18 \quad$ Transition section I. . . . . . . . . . . . . . . . . . 52

$4.19 \quad$ Transition section I installation. . . . . . . . . . . . . 52

4.20 FEA computational model of transition section I as seen from the (a) downstream end; (b) upstream end. . . . . . . . . . . 54

4.21 Schematic showing the loading of the V-band clamp and the location of the spring used to model the pre-compression rods.

4.22 Von mises stress distribution in the transition section I subassembly. . . . . . . . . . . . . . . . 5 56

$4.23 \quad$ Honeycomb flow straightener assembly. . . . . . . . . 58

4.24 Honeycomb flow straightener assembly with steel insert replacing the honeycomb insert. . . . . . . . . . . . 58

$4.25 \quad$ Honeycomb flow straightener installation. . . . . . . . 59

4.26 FEA computational model of the flow straightener subassembly as seen from the (a) downstream end; (b) upstream end. . . . 60

4.27 Schematic showing the loading of the V-band clamp and the location of the spring used to model the pre-compression rods. 61

4.28 Von Mises stress distribution in the flow straightener subassembly. 62

4.29 Turbulence screen assembly. . . . . . . . . . . . . 64

4.30 Turbulence-screen-assembly installation. . . . . . . . . . 64 
4.31 FEA computational model of the turbulence screen subassembly as seen from the (a) downstream end; (b) upstream end. . 66

4.32 Schematic showing the loading of the V-band clamp and the location of the spring used to model the pre-compression rods.

4.33 Von Mises stress distribution in the turbulence screen subassembly. . . . . . . . . . . . . . . . . . 68

4.34 Swirl generator housing. . . . . . . . . . . . . 70

4.35 Swirl generator assembly installation. . . . . . . . . . . 70

4.36 FEA computational model of swirl generator housing assembly as seen from the (a) downstream end; (b) upstream end. . . . 72

4.37 Schematic showing the loading of the V-band clamp and the location of the spring used to model the pre-compression rods. 73

4.38 Von Mises stress distribution in the swirl generator housing subassembly. . . . . . . . . . . . . . . . . 74

$4.39 \quad$ Core pipe assembly. . . . . . . . . . . . . 75

$4.40 \quad$ Seed injection assembly. . . . . . . . . . . . . . 77

$4.41 \quad$ Seed injection assembly installation. . . . . . . . . . . 78

4.42 FEA computational model of the seed injection subassembly as seen from the (a) downstream end; (b) upstream end. . . . . 80

4.43 Schematic showing the loading of the V-band clamp and the location of the spring used to model the pre-compression rods. 81

4.44 Von mises stress distribution in the seed injection subassembly. 82

4.45 Transition section II. . . . . . . . . . . . . . . 84

4.46 Transition section II installation. . . . . . . . . . . . 85

4.47 FEA computational model of transition section II as seen from the (a) downstream end; (b) upstream end. . . . . . . . . . 86

4.48 Schematic showing the loading of the V-band clamp and the location of the spring used to model the pre-compression rods. 87

4.49 Von mises stress distribution in the transition section II subassembly. . . . . . . . . . . . . . . . . . 88 
$4.50 \quad$ Downstream flange. . . . . . . . . . . . . . . . 89

$4.51 \quad$ Downstream flange installation. . . . . . . . . . . . 90

4.52 FEA computational model of the downstream flange as seen from the (a) downstream end; (b) upstream end. . . . . . . . 91

4.53 Schematic showing the loading of the V-band clamp and the location of the spring used to model the pre-compression rods.

4.54 Von mises stress distribution in the downstream flange subassembly. . . . . . . . . . . . . . . . . 93

4.55 Predicted run time as a function of flow path exit-plane Mach number. . . . . . . . . . . . . . . . . . 96

5.1 Installation location for the rectilinear lobed-mixer test section. 99

$5.2 \quad$ Rectilinear lobed-mixer test section installation. . . . . . . 100

5.3 Loads and constraints for the scenario with the upstream inflow velocity aligned with the lobes (a) view from upstream end; (b) view from downstream end. . . . . . . . . . . . . . . 102

5.4 Loads and constraints for the scenario with misaligned inflow (a) view from upstream end; (b) front view of a single lobe; (c) view from downstream-left side; (c) view from downstreamright side. . . . . . . . . . . . . . . . . . 103

5.5 Von Mises stress distribution for the aligned inflow condition. 104

5.6 Von Mises stress distribution for the misaligned inflow condition.105

6.1 Computational domain and boundary conditions. . . . . . 108

6.2 Inflow radial profile of (a) time-averaged axial velocity, and (b) turbulence kinetic energy $(\mathrm{x}=-3 \Delta \mathrm{R}) . \ldots \ldots \ldots$

6.3 Inflow radial profile of (a) time-averaged axial velocity, and (b) stagnation temperature. . . . . . . . . . . . . 111

$6.4 \quad$ Grid-cell nomenclature. . . . . . . . . . . . . . . . 113 
6.5 Seed-particle density distribution in the $x=13.5 \Delta R$ plane for $\alpha_{j}=30$ deg. as a function of $D_{j} / \Delta R$ and $P_{o_{j}} / P_{o_{r e f}}$. Note the three times larger $\rho_{P}$ range used for the results with $D_{j} / \Delta R=0.077 .120$

6.6 Axial velocity distribution in the $x=13.5 \Delta R$ plane for $\alpha_{j}=30$ deg. as a function of $D_{j} / \Delta R$ and $P_{o_{j}} / P_{o_{r e f}}$. . . . . . . . . . 121

6.7 Stagnation pressure distribution in the $x=13.5 \Delta R$ plane for $\alpha_{j}=$ 30deg. as a function of $D_{j} / \Delta R$ and $P_{o_{j}} / P_{o_{\text {ref }}}$. . . . . . . 122

6.8 Streamwise seed-particle density distributions as a function of $P_{o_{j}} / P_{o_{\text {ref }}}$ for $D_{j} / \Delta R=0.077$ and $\alpha_{j}=30 \mathrm{deg}$. . . . . . . . . .

6.9 Numerical Schlieren image showing the density gradient magnitude of the under-expanded jet for $D_{j} / \Delta R=0.077$ and $P_{o_{j}} / P_{o_{r e f}}=3.93 .126$

6.10 Seed-particle density, axial velocity, and stagnation pressure distributions in the $x=13.5 \Delta R$ plane for $D_{j} / \Delta R=0.077$ and $\alpha_{j}=30 \mathrm{deg}$., and injection stagnation pressures of $P_{o_{j}} / P_{o_{r e f}}=1.65$

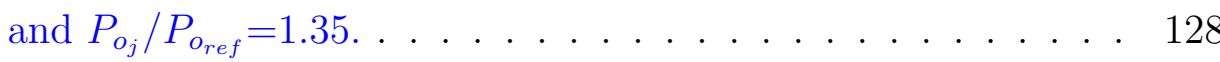

6.11 Streamwise seed-particle density distributions for $D_{j} / \Delta R=0.077$ and $\alpha_{j}=30 \mathrm{deg}$., and injection stagnation pressures of $P_{o_{j}} / P_{o_{r e f}}=1.65$

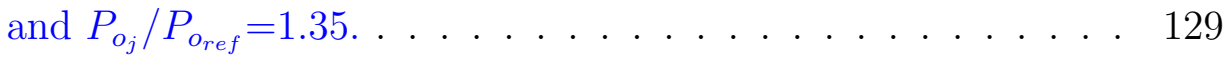

6.12 Seed-particle density and axial velocity distributions at $x / \Delta R=13.5$ for low and high annular flow speeds with $D_{j} / \Delta R=0.077$. . . 131

6.13 Comparison of the streamwise seed-particle density distributions for the low and high annular flow speeds with $D_{j} / \Delta R=0.077 .132$

6.14 Computational domain and boundary conditions. . . . . . 133

6.15 Inflow radial profile of (a) time-averaged axial velocity, and (b) turbulence kinetic energy at the annular-inflow plane (Strzelecki at al., 2009). . . . . . . . . . . . . . . . 134

6.16 Inflow cross-stream profile of time-averaged velocity at the jet inflow (Strzelecki et al., 2009). . . . . . . . . . . . 135 
6.17 Time-averaged and RMS seed-particle density distributions in the near region of a jet in crossflow as (a) measured by Strzelecki et al., 2009 (adapted), and (b) predicted by the simulation.137

6.18 Comparison of (a) the jet trajectory based on the maximum seed-particle density, and (b) the seed-particle density along the jet trajectory. . . . . . . . . . . . . . . . . 138

6.19 Comparison of the shape of the seed-particle density profile at (a) $x^{\prime} / J D_{j}=0.5$, and (b) $x^{\prime} / J D_{j}=2.5$; (c) profile of the jet envelope and depiction of the parameters used to normalize the quantities in the figures. . . . . . . . . . . . . . . . . 139

C.1 Computational model of the pressurized thick-walled cylinder. 201

C.2 Comparison of radial distribution of hoop stress between the ANSYS solution and the reference solution. . . . . . . . . 202

C.3 Comparison of radial distribution of radial stress between the ANSYS solution and the reference solution. . . . . . . . 203

C.4 Computational model of the circular beam. . . . . . . . . 204

C.5 Comparison of moment-rotation curves between ANSYS and reference solution. . . . . . . . . . . . . . . . . 205

F.1 Computational domain and boundary conditions. . . . . . . 222

F.2 Streamwise cross-section of the computational grid. . . . . . 223

F.3 (a) Density gradient-magnitude in the computed under-expanded jet showing the expansion/compression wave pattern as compared to an experimental Schlieren image (Xiao et al., 2019) and (b) Measurements of the expansion angle $(\omega)$ and Mach disk location $\left(X_{m} / D_{j}\right)$ in the computed under-expanded jet. . 224

G.1 Seed-particle density distribution in the $x=13.5 \Delta R$ plane as a function of $D_{j} / \Delta R$ and $P_{o_{j}} / P_{o_{r e f}}$ for $\alpha_{j}=45 \mathrm{deg} \ldots \ldots \ldots 227$ 
G.2 Axial velocity distribution in the $x=13.5 \Delta R$ plane as a function of $D_{j} / \Delta R$ and $P_{o_{j}} / P_{o_{r e f}}$ for $\alpha_{j}=45 \mathrm{deg}$. . . . . . . . . 228

G.3 Stagnation pressure distribution in the $x=13.5 \Delta R$ plane as a function of $D_{j} / \Delta R$ and $P_{o_{j}} / P_{o_{r e f}}$ for $\alpha_{j}=45 \mathrm{deg}$. . . . . . . . 229

G.4 Seed-particle density distribution in the $x=13.5 \Delta R$ plane as a function of $D_{j} / \Delta R$ and $P_{o_{j}} / P_{o_{\text {ref }}}$ for $\alpha_{j}=0 \mathrm{deg}$. . . . . . . 230

G.5 Axial velocity distribution in the $x=13.5 \Delta R$ plane as a function of $D_{j} / \Delta R$ and $P_{o_{j}} / P_{o_{r e f}}$, and $\alpha_{j}=0 \mathrm{deg}$. . . . . . . . . 231

G.6 Stagnation pressure distribution in the $x=13.5 \Delta R$ plane as a function of $D_{j} / \Delta R$ and $P_{o_{j}} / P_{o_{r e f}}$ for $\alpha_{j}=0 \mathrm{deg}$. . . . . . . 232

G.7 Streamwise seed-particle density distributions in the $y=0$ plane as a function of $P_{o_{j}} / P_{o_{r e f}}$ for $D_{j} / \Delta R=0.077$ and $\alpha_{j}=45$ deg. . . . . . . . . . . . . . . . . 233

G.8 Streamwise seed-particle density distributions as a function of $P_{o_{j}} / P_{o_{\text {ref }}}$ for $D_{j} / \Delta R=0.077$ and $\alpha_{j}=0 \mathrm{deg}$. . . . . . . . . . .

G.9 Streamwise seed-particle density distributions as a function of $P_{o_{j}} / P_{o_{\text {ref }}}$ for $D_{j} / \Delta R=0.036$ and $\alpha_{j}=45 \mathrm{deg}$. . . . . . . . . 235

G.10 Streamwise seed-particle density distributions as a function of $P_{o_{j}} / P_{o_{\text {ref }}}$ for $D_{j} / \Delta R=0.036$ and $\alpha_{j}=30 \mathrm{deg}$. . . . . . . . . 236

G.11 Streamwise seed-particle density distributions as a function of $P_{o_{j}} / P_{o_{r e f}}$ for $D_{j} / \Delta R=0.036$ and $\alpha_{j}=0 \mathrm{deg} . . . . . . .$.

G.12 Streamwise seed-particle density distributions as a function of $P_{o_{j}} / P_{o_{\text {ref }}}$ for $D_{j} / \Delta R=0.018$ and $\alpha_{j}=45 \mathrm{deg}$. . . . . . . . . . 238

G.13 Streamwise seed-particle density distributions as a function of $P_{o_{j}} / P_{o_{r e f}}$ for $D_{j} / \Delta R=0.018$ and $\alpha_{j}=30 \mathrm{deg}$. . . . . . . . . . 239

G.14 Streamwise seed-particle density distributions as a function of $P_{o_{j}} / P_{o_{r e f}}$ for $D_{j} / \Delta R=0.018$ and $\alpha_{j}=0 \mathrm{deg} . . . . . . . . .240$ 


\section{Nomenclature}

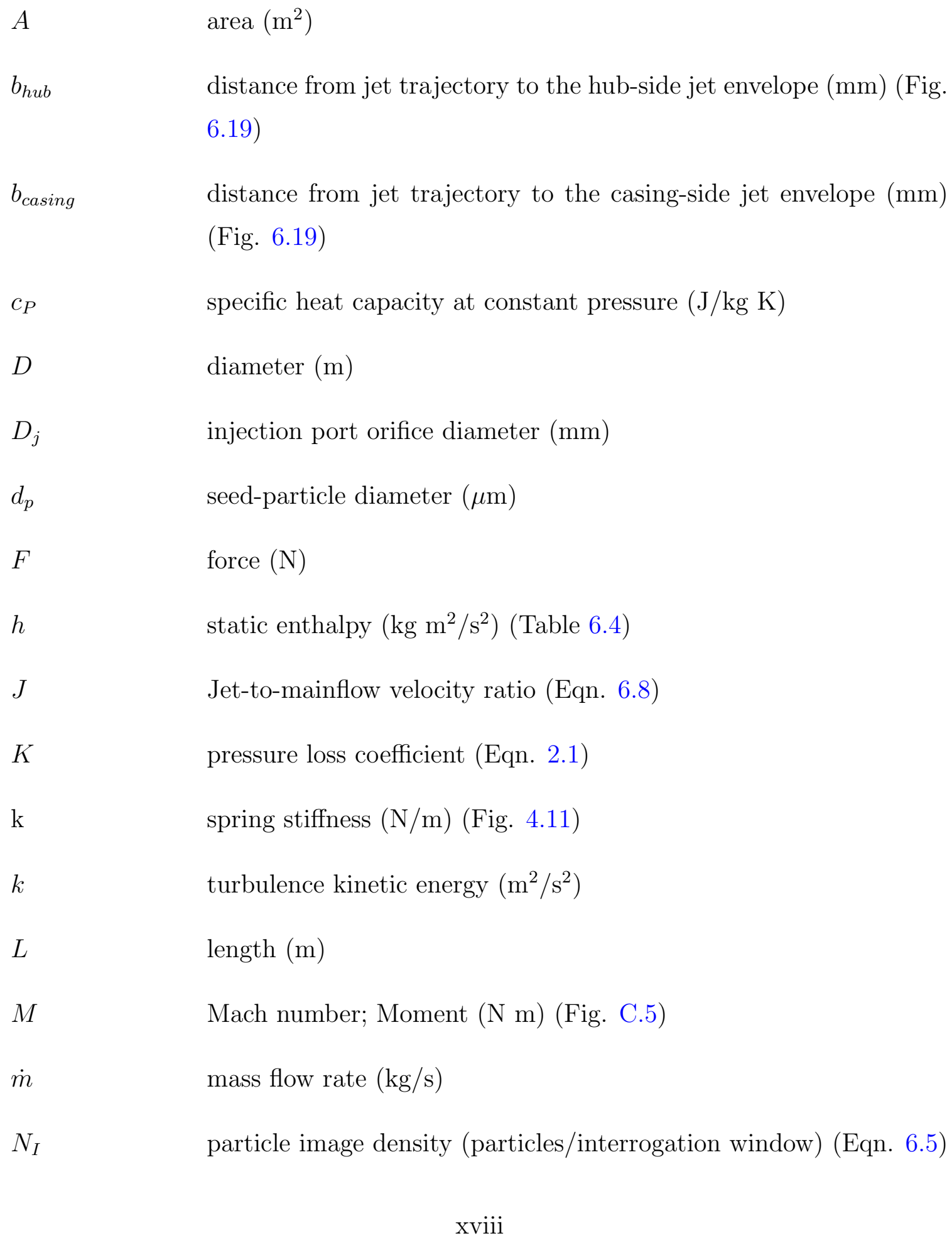




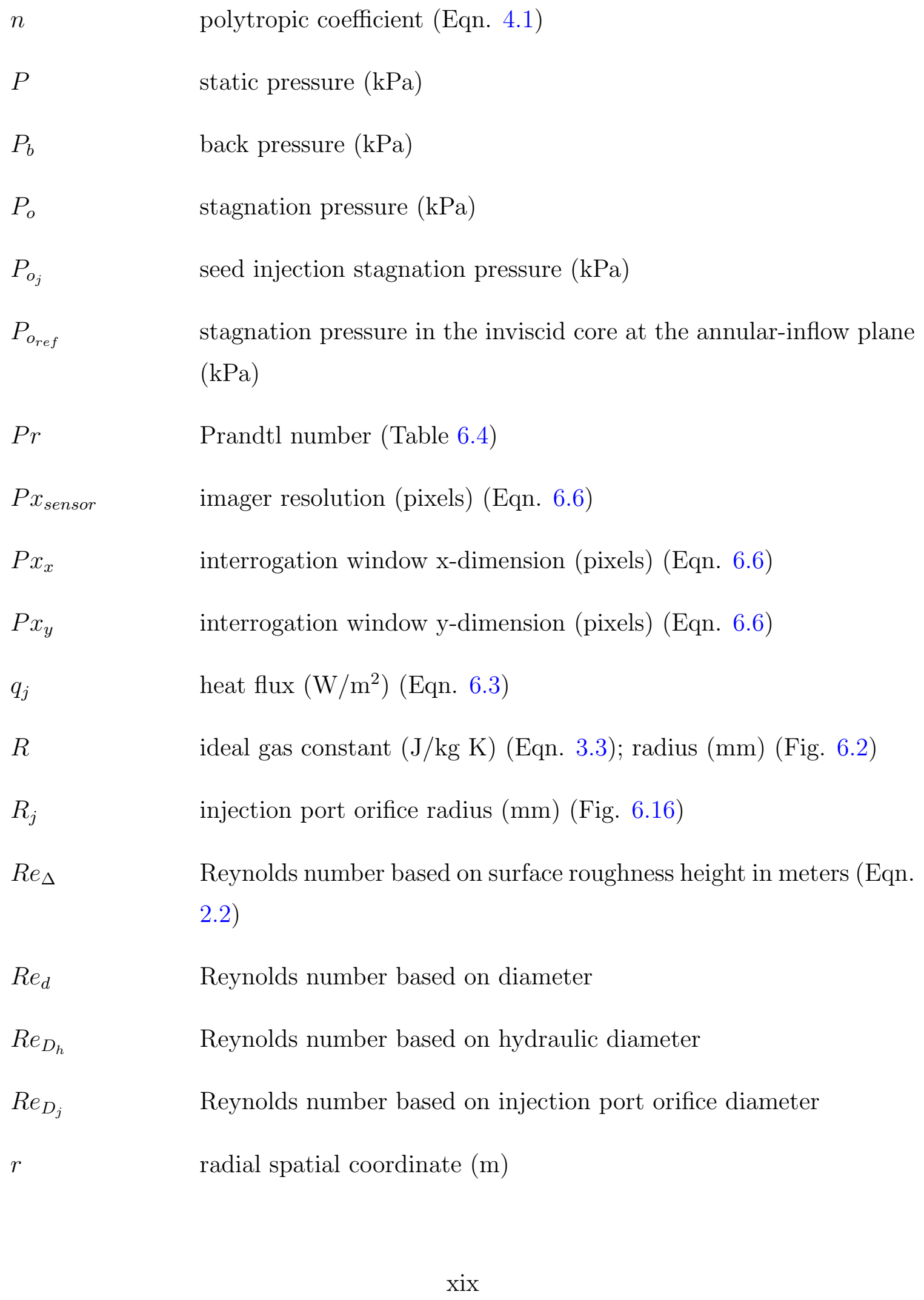




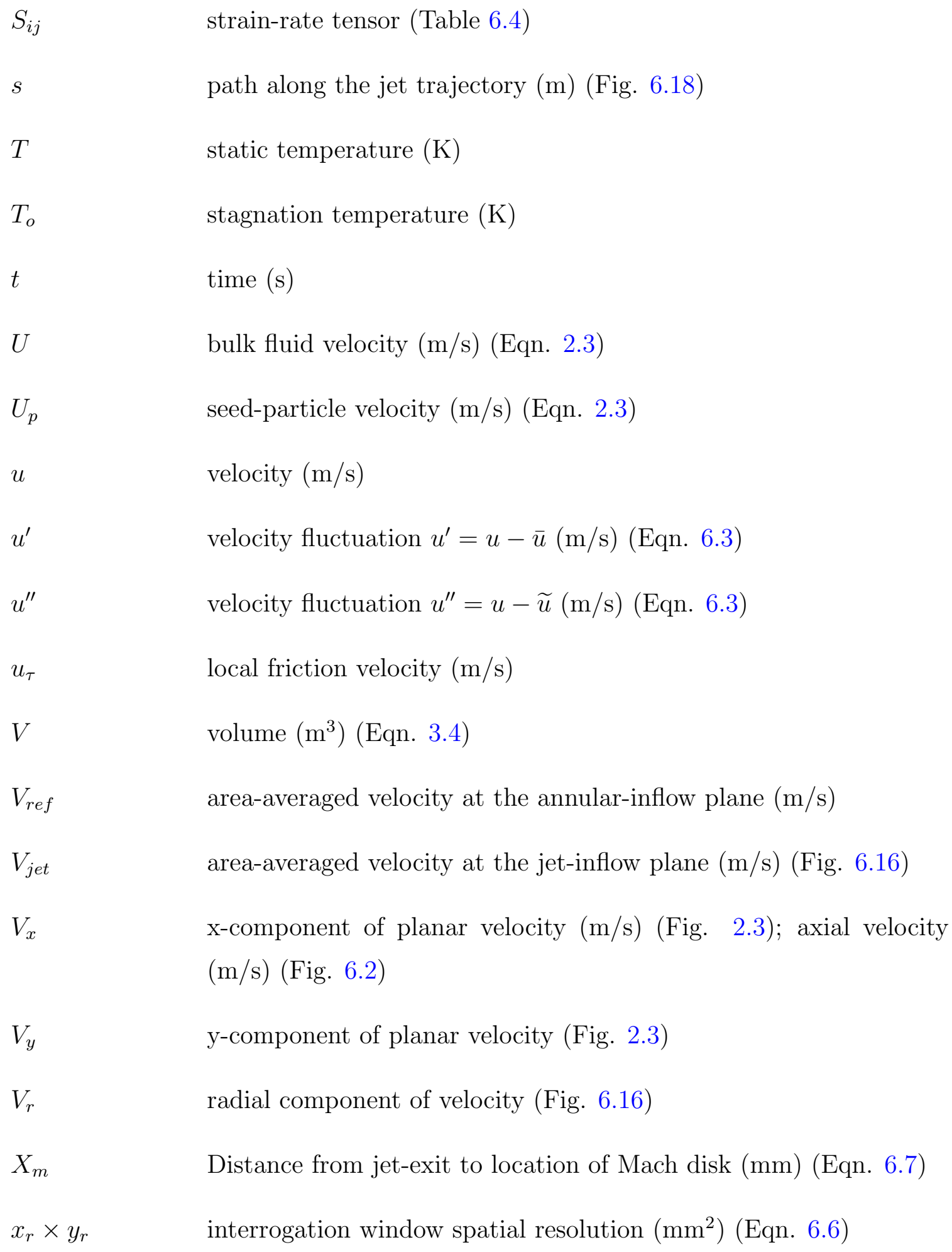


$x$

$x^{\prime}$

$y_{1}^{+}$

y

$y^{\prime}$

$z$

\section{Greek Symbols}

$\alpha_{j}$

$\gamma$

$\Delta$

$\Delta t$

$\Delta R$

$\Delta x$

$\Delta y$

$\Delta z$

$\delta_{i j}$

$\Delta \theta$

$\theta$

$\lambda$

$\mu$ streamwise spatial coordinate $(\mathrm{m})$

alternate streamwise spatial coordinate (mm) (Fig. 6.14)

normalized distance for the first grid node off the wall

wall-normal spatial coordinate $(\mathrm{m})$

alternate wall-normal spatial coordinate (mm) (Fig. 6.14)

spanwise spatial coordinate $(\mathrm{m})$

seed injection angle (deg.)

ratio of specific heats

surface roughness height (m) (Eqn. 2.2)

exposure time delay ( $\mu$ s) (Fig. 2.3); time increment (Eqn. 3.5)

annulus height (mm) (Fig. 6.1)

x-axis particle displacement ( $\mu \mathrm{m})$ (Fig. 2.3)

y-axis particle displacement ( $\mu \mathrm{m})$ (Fig. 2.3)

light sheet thickness (mm) (Eqn. 6.5)

Kronecker delta (Eqn. 6.2)

circumferential extent (deg.) (Fig. 6.12)

circumferential spatial coordinate (deg.) (Fig. 6.1)

fluid thermal conductivity (Table. 6.4)

friction coefficient; fluid dynamic viscosity $(\mathrm{kg} / \mathrm{m} \mathrm{s})$ (Table 6.4) 


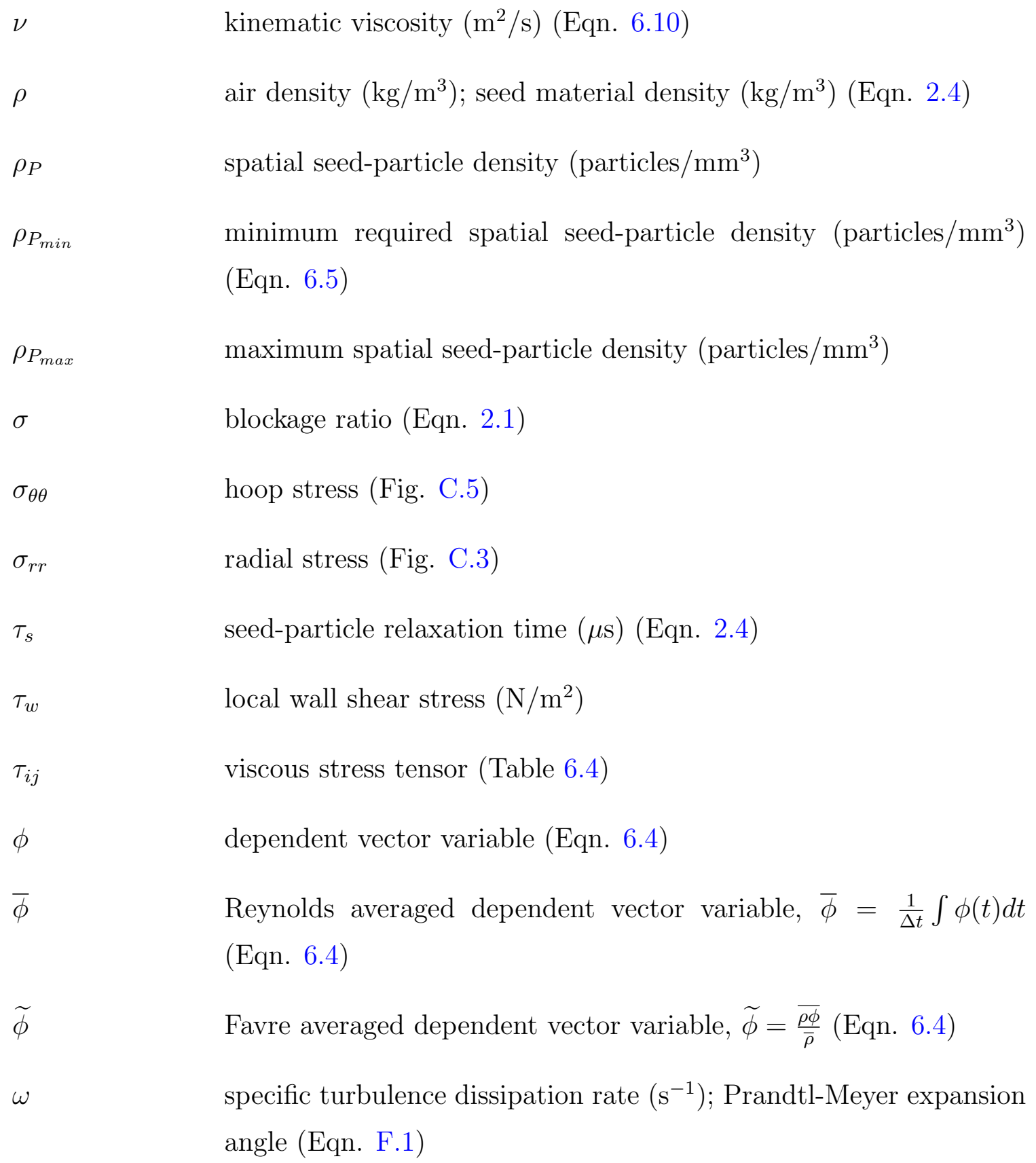

\section{Subscripts}

ambient ambient air conditions

in inflow value 


$\begin{array}{ll}i, j, k & \text { indices for the } \mathrm{x}, \mathrm{y} \text { and } \mathrm{z} \text { coordinate directions } \\ \text { jet } & \text { jet flow value } \\ \text { out } & \text { outflow value } \\ \text { ref } & \text { reference value } \\ \text { tank } & \text { storage tank air conditions } \\ \text { valve } & \text { control valve value } \\ x & \text { vector component in } \mathrm{x} \text {-direction } \\ y & \text { vector component in y-direction } \\ z & \text { vector component in } \mathrm{z} \text {-direction }\end{array}$




\section{Abbreviations}

$A B S \quad$ acrylonitrile butadiene styrene

ASTM American Society for Testing and Materials

ASME American Society of Mechanical Engineers

ANSI American National Standards Institute

CFD Computational Fluid Dynamics

CSA Canadian Standards Association

DEHS Di-Ethyl-Hexyl-Sebacate

FANS Favre-averaged Navier-Stokes

$F E A \quad$ finite element analysis

FOV field-of-view

HDPE high-density polyethylene

HSWT high-speed wind-tunnel

HFSB helium filled soap bubbles

LDA laser doppler anemometry

LEL lower explosive limit

PIV particle image velocimetry

PTV particle tracking velocimetry

PSL $\quad$ polystyrene latex

PVC poly-vinyl chloride 
RANS Reynolds-averaged Navier-Stokes

SST shear-stress transport

TSSA Technical Standards and Safety Authority

$V A P C O N \quad$ vaporization-condensation 


\section{Chapter 1}

\section{Introduction}

The flow instabilities and turbulence that prevail within the mixing regions of flow fields has long been an area of engineering interest. One area of application pertaining to this interest is the effect of these flow phenomena on the aerodynamic performance of gas-turbine engine components, including lobed mixers and inter-turbine and inter-compressor ducts. The majority of the experimental research endeavors related to the aerodynamic performance of these particular gas-turbine engine components have been conducted at incompressible flow Mach numbers, among them the work conducted by the author's own research group (Brinkerhoff et al., 2013; Brinkerhoff et al., 2014a). The interest of the academic community and industry to experimentally study the effects of flow compressiblity on the mixing processes and turbulence in lobed mixers and inter-turbine ducts has stimulated the present work.

The work presented in this thesis contains the design and performance analyses of flow-conditioning and test sections that will be used in future experimental studies of free shear layers and ducted annular flows at high subsonic Mach numbers. The designs uniquely emphasize light weight and modularity to enable rapid and safe experiment-specific reconfiguration of the setups. Furthermore, the designs strike a satisfactory balance between larger scales of flow that promote spatial resolution of measurements, and smaller scales of flow that enable longer durations of flow at the desired flow Mach number. These design features improve upon the capabilities of existing test sections in the transonic-speed blow-down wind tunnel at Carleton University. The first design effort involves the design of an annular-flow air-supply system. The work includes a one dimensional aerodynamic model of the high-speed wind-tunnel and the air-supply system. The operating range of the air-supply system

in terms of flow Mach number was selected to be comparable to that of the core-flow of a mid-sized high-bypass turbofan engine. The flow path was scaled to maximize the 
available run time of the blow-down type wind-tunnel, while maintaining a sufficiently large scale to ensure the aerodynamic phenomena of interest are of sufficient spatial scale to be accurately resolved by the available measurement techniques. The flow conditioning devices were sized in order to minimize pressure losses in the system in the interest of extending wind-tunnel run times while also facilitating a steady Mach number flow at the test section discharge. The modularity of the design and the use of polymer materials extends the design's versatility as well as its safety and ease of use. In addition to the aerodynamic design, exhaustive finite element analyses were performed to confirm the system's structural integrity under the expected operating conditions and to ensure it's compliance with the relevant provincial regulations.

The second design effort pertains to the design of a rectilinear test section that makes use of the existing infrastructure and flow conditioning devices in the Carleton high-speed wind-tunnel facility. The rectilinear test section provides an up-scaled version of the annular-flow air-supply system to more easily and reliably facilitate the measurement of the aerodynamic phenomena of interest. The rectilinear test section will serve as the primary apparatus for experimental investigations in the immediate future until the annular-flow air-supply system attains approval from the relevant provincial certification authorities.

Lastly, a third design effort involves the optimization of a seed injection system for delivering a seed material (atomized olive oil in the present work) into the air stream of the annular-flow air-supply system for the purpose of performing particle image velocimetry (PIV) measurements. Several parameters deemed to have appreciable effect on the dispersal of the seed material within the flow field were varied in parametric fashion using a computational fluid dynamics model. The parametric study was first performed under incompressible annular-inflow conditions to establish the most promising configuration in a low-speed wind-tunnel setting. A subset of the parametric study was repeated under high-speed annular-inflow conditions to determine the optimum configuration for the high-speed wind-tunnel. Finally, validation of the numerical flow model was performed by conducting a benchmark simulation based on experimental data sourced from the literature. 
To provide a context for the present design effort, existing design practices and related aerodynamic theory for high-speed aerodynamic testing facilities were reviewed in published literature. This review constitutes the first part of Chapter 2. A review of the PIV measurement technique, its underlying theory, and best practices pertaining to flow seeding constitutes the second half of Chapter 2.

Carleton's low- and high-speed wind-tunnel facilities as they relate to the present design efforts, and aerodynamic modeling of the high-speed facility is presented in Chapter 3.

The design and structural analysis of the high-speed air supply system are presented in Chapter 4. The design and structural analysis of a test section for testing of lobed mixers in a splitter-plate planar configuration are presented in Chapter 5 .

A CFD-based design optimization study for the seed injection port of the highspeed annular-flow supply system is documented in Chapter 6, followed by the conclusions of this thesis and recommended future work in Chapter 7.

The scope of this thesis is limited to the description of designs and analyses of their structural and aerodynamic performance. While actual experimentation using these setups is outside the scope of the present work, in part due to certification requirements that will take considerable time, the thesis contains information that will enable the execution of future experiments in a safe manner and with predictable accuracy. 


\section{Chapter 2}

\section{Literature Review}

\subsection{High Speed Wind-Tunnel Design}

The high-speed wind-tunnel (HSWT) at Carleton University is a blow-down tunnel that can generate air speeds in the high-speed subsonic and supersonic range for short durations. Wind-tunnels of this type consist of six main sub-assemblies; an air compressor, an air drying system, compressed air storage tanks, flow conditioning devices, air valves to control flow conditions during experimentation, and a test section.

\subsubsection{Air Compressor}

The compressor of a blow-down wind-tunnel is characterized by its flow rate and pressure rise. The rate at which the storage tanks are charged and the maximum charge pressure are dictated by these characteristics. The choice of compressor for a blow-down wind-tunnel is dictated by these two performance characteristics, the amount of electric power available to drive the compressor, and any space constraints in the wind-tunnel facility. The combination of flow rate and pressure rise together with a rotational speed dictate the type of compressor design. Reciprocating and rotary-screw compressors tend to be most suitable for the maximum pressure levels of typical blow-down wind-tunnels designed to achieve compressible air speeds (Pope and Goin, 1965).

Through the compression process, the air is heated to a temperature of about $100^{\circ} \mathrm{C}$. An aftercooler, or intercooler in the case of multi stage compressors, is typically used to cool down the air to approximately $25-30^{\circ} \mathrm{C}$ (Pope and Goin, 1965). This cooling process causes much of the air moisture to condense, which is collected by a moisture separator. Moisture removal at this level reduces the requirements of the air drying system which is discussed next. 


\subsubsection{Air Drying System}

During the course of a wind-tunnel run, the air expanding from the storage tanks, where it is at about room temperature, undergoes a drop in static temperature of about 20 to $60^{\circ} \mathrm{C}$, depending on the final Mach number achieved. Any air moisture that is still present will condense and possibly freeze onto the surfaces of the test section or test articles. As this is obviously undesirable, air dryers positioned between the compressor and the storage tanks extract any remaining moisture before the compressed air reaches the storage tanks. The air dryer capacity must be large enough to handle the flow rate coming from the compressor, and it must lower the dew point of the compressed air to a temperature below the lowest expected static temperature in the test section flow path. These requirements necessitate the use of a desiccant air dryer, which uses a desiccant material, usually silica gel beads or an activated alumina powder, to absorb the remaining moisture and lower the dew point of the compressed air to about $-40^{\circ} \mathrm{C}$ (Pope and Goin, 1965). Once the desiccant is saturated, about 15 to $20 \%$ of the dried air is diverted to flush the material and dry it in a process called "regeneration".

\subsubsection{Storage Tanks}

The volume and pressure rating of the storage tanks dictates the run times for a blowdown wind-tunnel, which varies depending on the scale and purpose of the tunnel or test section. TU Delft's TST-27 transonic wind-tunnel (TU Delft, 2019) has a test section roughly $25 \mathrm{~cm} \times 25 \mathrm{~cm}$ capable of achieving Mach numbers in the range of 0.5 to 4.2 for up to $300 \mathrm{~s}$, making it very similar in scale and operating range to the Carleton University HSWT. Hypervelocity tunnels such as NASA's Ames 16-inch Shock Tunnel (Tokarcik-Polsky et al., 1995) and the Institute of Saint-Louis's ISL Shock Tunnel (Havermann et al., 2002) operate at hypersonic Mach numbers (>5.0) yielding run times on the order of micro seconds. Facilities such as these require pressure retaining vessels that can withstand higher pressures (up to and beyond 500 atm). Such hypervelocity facilities generally also require a vacuum chamber, into 
which the tunnel exhausts rather than into the atmosphere.

For safety reasons, it is typically desirable to store compressed air at lower pressures. Lower charge pressures necessitate larger volume storage tanks, which may be limited in size by the available space of the facility. Furthermore, the cost of storing a given mass of air is the same regardless of the pressure, with higher pressures requiring smaller, but stronger vessels (ASME, 2010). The optimum storage tank design is therefore one where the largest mass of air is stored in vessels that utilize the available space most efficiently. All pressure vessels must be equipped with at least one pressure relief valve (ASME, 2010). Pressure relief, or safety relief valves, are normally a spring loaded blow-down design that activates if the internal vessel pressure exceeds $110 \%$ of the maximum allowable working pressure.

\subsubsection{Control Valves}

In blow-down wind-tunnels, a control valve regulates the rate of mass flow through the test section. Typical control valves on blow-down wind-tunnels are either ball valves (Hall, 2012; Dutton, 2008;) or butterfly valves (Nott et al., 2008). While either valve type can be made to work for the intended purpose, ball valves tend to be favored as they have negligible pressure drop when fully opened and are usually lighter, smaller, and less costly than an equivalent butterfly valve. Unlike butterfly valves, ball valves also provide an excellent seal with zero leakage when fully closed. High pressure regulators can be used in place of a control valve, although these tend to be applied on smaller scale wind-tunnels (Zheng et al., 2017).

A third method of flow control is through the use of variable-geometry nozzles. The TST-27 transonic blow-down wind-tunnel at TU Delft operates via continual adjustment of the throat area of a variable area nozzle (Mathijssen et al., 2009). A similar style control was used in Lockheed Martin's transonic blow-down windtunnel, where a variable-geometry converging-diverging nozzle is used to achieve Mach numbers in the range of 0.3 to 5.0 (Lockheed Martin, 2002). Fig. 2.1 shows a photograph of the variable-geometry nozzle in the Lockheed Martin high-speed windtunnel. The top and bottom surfaces of the nozzle are each connected to 28 jacks 
actuated by hydraulic pistons which adjust the nozzle geometry, and thereby control the Mach number in the test section.

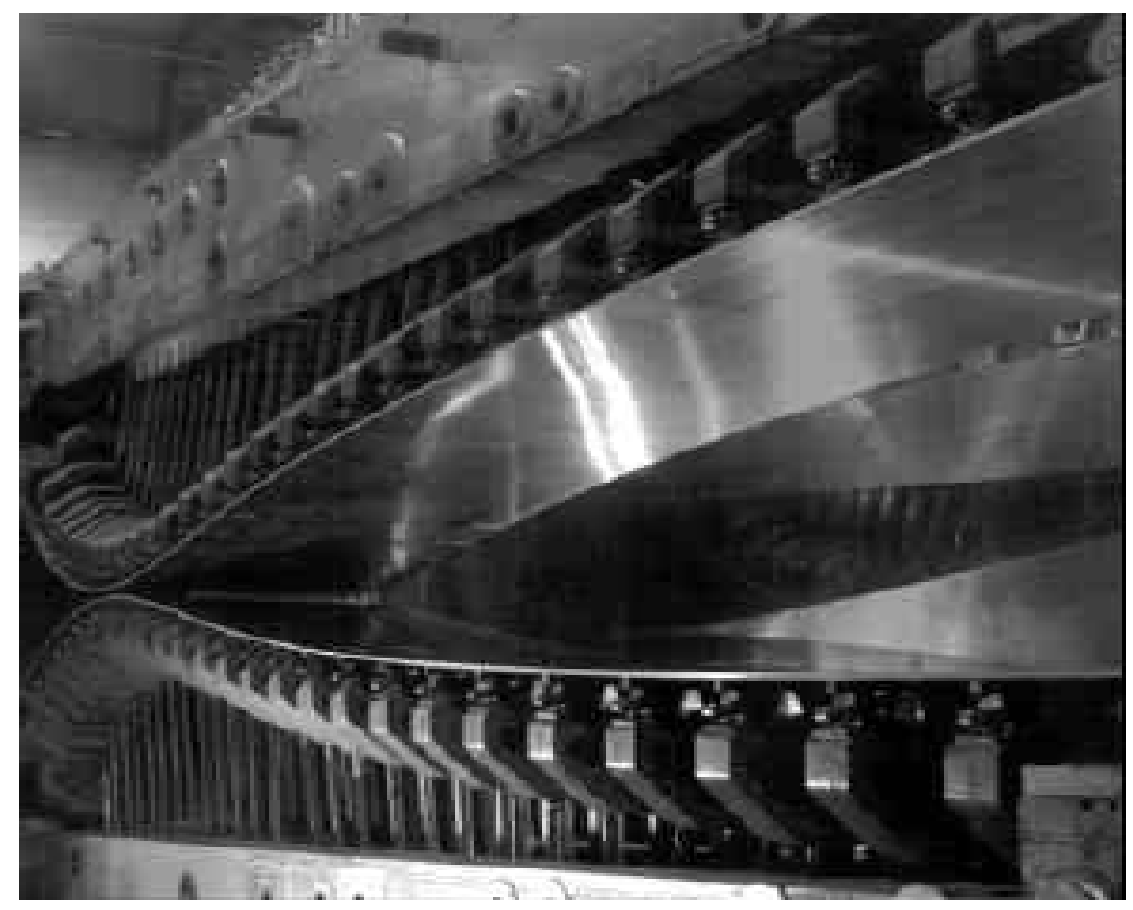

Figure 2.1: Variable-contour nozzle in the Lockheed Martin high-speed wind-tunnel (Lockheed Martin, 2002).

\subsubsection{Flow Conditioning}

\subsubsection{Honeycomb Flow Straighteners}

To re-establish a uniaxial flow downstream of any bends or orifices, a honeycomb flow straightener is typically installed in the flow path. In addition to removing the flow angularity, the honeycomb cells also serve to breakdown the turbulence eddies that are larger than the cell width, thereby contributing to the reduction of free-stream flow turbulence (Farell and Youssef, 1992). The axial length of the honeycomb is typically selected such that the length-to-width ratio of the honeycomb cell is no less than 8.0. Exceeding this limit would not sufficiently improve the flow straightening and turbulence reduction to justify the additional frictional pressure loss (Mehta 
and Bradshaw, 1979). The honeycomb pressure loss coefficient can be expressed as (Barlow et al., 1999):

$$
K=\lambda\left(\frac{L}{D}+3\right)\left(\frac{1}{1-\sigma}\right)^{2}+\left(\frac{1}{1-\sigma}-1\right)^{2}
$$

where

$$
\lambda=\left\{\begin{array}{cccc}
0.375\left(\frac{\Delta}{D}\right)^{0.4} & R e_{\Delta}^{-0.1} & \text { for } & R e_{\Delta} \leq 275 \\
0.214\left(\frac{\Delta}{D}\right)^{0.4} & \text { for } & R e_{\Delta} \geq 275
\end{array}\right\}
$$

In these equations, $L$ and $D$ are the honeycomb length and cell width, respectively; $\sigma$ is the cell solidity defined as the ratio of the honeycomb solid area to the flow path cross-sectional area; $\Delta$ is the surface roughness height of the honeycomb material, and $\operatorname{Re}_{\Delta}$ is the Reynolds number based on $\Delta$. If the honeycomb cells are too small, the flow through the cells may choke due to the vena contracta that forms at the discharge plane of the cells, resulting in a limit on the maximum Mach number achievable by the tunnel. Hence, both the pressure loss and the open-area characteristics of the honeycomb are critical design parameters.

\subsubsection{Turbulence Reduction Screens}

To further reduce the free-stream flow turbulence downstream of the honeycomb, one or more wire-mesh screens are usually installed in the flow path. The wire-mesh screens achieve this through the interaction of the small-scale turbulence prevailing in the wakes of the wires of the mesh with larger scale turbulence in the incoming flow (Mehta and Bradshaw, 1979). Three design parameters need to be considered; the wire diameter, $d$, the screen solidity, $\sigma$, defined as the ratio of the mesh solid area to the flow path cross-sectional area, and the stagnation pressure loss coefficient of the screen. A single wire-mesh screen with a pressure loss coefficient of about 2.0 (i.e. static pressure drop across the screen is twice the dynamic pressure of the flow immediately upstream of the screen) can eliminate nearly all longitudinal turbulence in the flow (Mehta and Bradshaw, 1979). This is achievable with a wire-mesh screen 
that has a subcritical Reynolds number based on the wire diameter of $\operatorname{Re}_{d} \leq 50$ (Groth and Johansson, 1988). A series of screens installed in tandem operating at $\operatorname{Re}_{d} \geq 50$, can achieve the same level of turbulence reduction with lower amount of pressure loss.

The screen solidity affects the pressure loss across the screen. Furthermore, if the solidity exceeds a value of about 0.43 , the flow passing through the screen may develop an instability (Baines and Peterson, 1951; Bradshaw, 1964). This instability, due to jet coalescence, results in an increase in turbulence intensity. Once $\mathrm{Re}_{d}$ exceeds a value of 600 , the pressure loss coefficient is independent of $\operatorname{Re}_{d}$ and becomes solely a function of the screen solidity (Mehta and Bradshaw, 1979). Fig. 2.2 presents the correlation between pressure loss as a function of the wire diameter Reynolds number. The streamwise spacing of multiple screens must be chosen such that flow turbulence downstream of the upstream conditioning device is mostly dissipated prior to the flow encountering the next conditioning device. This is true for the spacing between the first screen and the honeycomb as well, to allow for the wake regions of the multicell flow pattern to diffuse before the flow interacts with the first wire-mesh screen (Farell and Yousseff, 1992). Studies on screen combinations for turbulence reduction have shown that the turbulence generated in the wake of the wires interact with the incoming flow turbulence, dissipating the turbulence rapidly by viscous forces within 15 to 20 mesh-widths, defined as the spacing between adjacent wires (Groth and Johansson, 1988). 


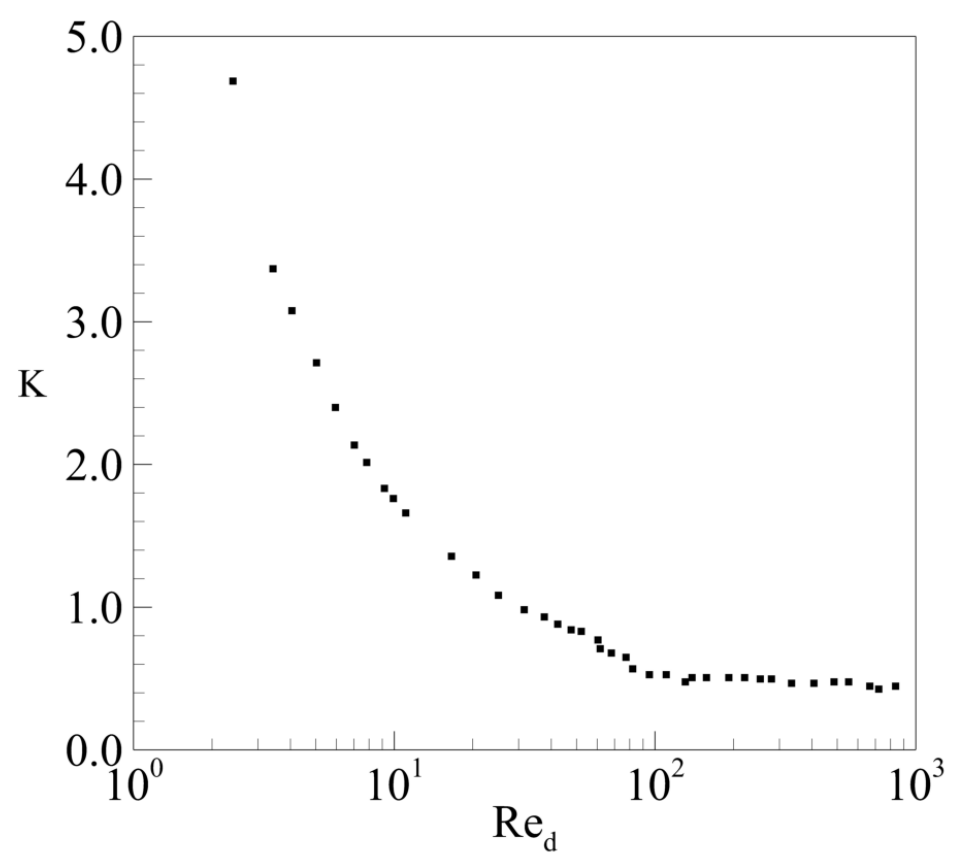

Figure 2.2: Pressure loss across wire-mesh screens as a function of $R e_{d}$ (adapted from Groth and Johansson, 1988).

\subsection{Laser-Based Flow Measurement Techniques}

There exists several laser-based flow measurement techniques for performing nonintrusive quantitative measurements in fluid flows. The present study focuses on the use of particle image velocimetry (PIV). While PIV is a proven flow measurement technique, to appreciate both its strengths and limitations it is necessary to assess both the precursors and variations of the PIV technique.

\subsubsection{Laser Doppler Anemometry (LDA)}

Laser Doppler anemometry (LDA), introduced in the early 1970s, was the first laserbased technique for measuring fluid flow velocity (Tropea, 1995). Two Gaussian laser beams are focused to a single point in the flow. Where the beams intersect, a region of constructive-destructive interference is generated. This region, which is ellipsoid in shape, is the measurement volume, with the bright fringes of the interference pattern within this volume aligned perpendicular to the velocity component that is 
to be measured. Like all laser based flow measurement techniques, the LDA method requires a seed particulate that will closely follow the local fluid motion. As these seed particles traverse the measurement volume, they reflect the laser light in the bright fringes of the interference pattern. This reflected light is also subject to a Doppler shift; the faster the particle, the higher the frequency of the reflected light. The reflected light is collected by a photo-detector and the magnitude of the Doppler shift is used to compute the flow velocity (Zhang, 2010).

While a single, two-laser LDA probe can only extract one component of the flow velocity, it can be combined with a four-laser LDA probe to expand the measurements to all three velocity components (Diasinos et al., 2013). With advances in fiber optics and silicon imagery, LDA probes shrunk in size, making LDA a preferred measurement technique when optical access is limited such as within the blade passages of an axial compressor stage (Faure et al., 2001), or inside a model of the human aorta (Haya, 2015). LDA is limited in is application by the size of the measurement volume. Typical LDA measurement volumes are on the order of $5.0 \times 10^{-3} \mathrm{~mm}^{3}$. While this provides excellent spatial resolution, study of a flow-field requires this measurement volume to be traversed throughout the region of interest in the flow.

\subsubsection{Particle Image Velocimetry (PIV)}

Particle image velocimetry is a laser-based flow-field measurement technique able to quantify the instantaneous velocity field in a fluid flow. PIV systems consist of three major components: a seed particulate that is injected into the flow; a pulsating laser light sheet about 1-3 mm in thickness that illuminates the seed particles; and, a camera or array of cameras that capture images of the illuminated seed articles. Section 2.3 addresses the considerations for choosing a seed particulate along with a review of the literature on the topic. The present section will address the evolution of PIV from planar PIV, to stereoscopic PIV, and finally to the most advanced, tomographic PIV. 


\subsubsection{Planar PIV}

The simplest execution of PIV is 2-component planar PIV. This technique makes use of a single laser light sheet and a single camera that is oriented perpendicular to the light sheet. This PIV arrangement is only capable of measuring a two-dimensional velocity field in an interrogation region in the plane of the light sheet. PIV recordings are typically acquired in a method known as double-frame double-exposure, whereby a single data point consists of two PIV images, captured at different times. The time difference between images is called the exposure time delay, a parameter selected by the user. The two images are then discretized into a matrix of interrogation windows, where each window is interrogated by a spatial cross-correlation algorithm that calculates the displacements of the particles (Adrian and Westerweel, 2011). The velocity vector in the interrogation window is then computed using the exposure time delay between the two images. The local $2 \mathrm{D}$ velocity vector in a given interrogation window is then determined statistically from a correlation plot in a process called peak detection. Fig. 2.3 illustrates the operating principle of planar PIV.

Compared to LDA, planar PIV is able to image a much larger area of the flowfield, with interrogation regions ranging in size from several millimeters squared to several hundreds of millimeters squared. With only a single camera, planar PIV is only suitable for two-dimensional flows, where the out-of-plane component of velocity (z-axis component in Fig. 2.3) is negligible. Planar PIV has been particularly useful in cases where optical access to the flow is limited and the space requirements for a two camera system are unfeasible. Dierksheide et al. (2002) used an 8 mm-diameter endoscopic attachment on both the laser and the camera to image the flow-field inside the cylinder of an internal combustion engine. Another example of successful use of planar PIV is the measurement of flows related to prosthetic heart valve applications (Marassi et al., 2004; Lim et al., 1994). 


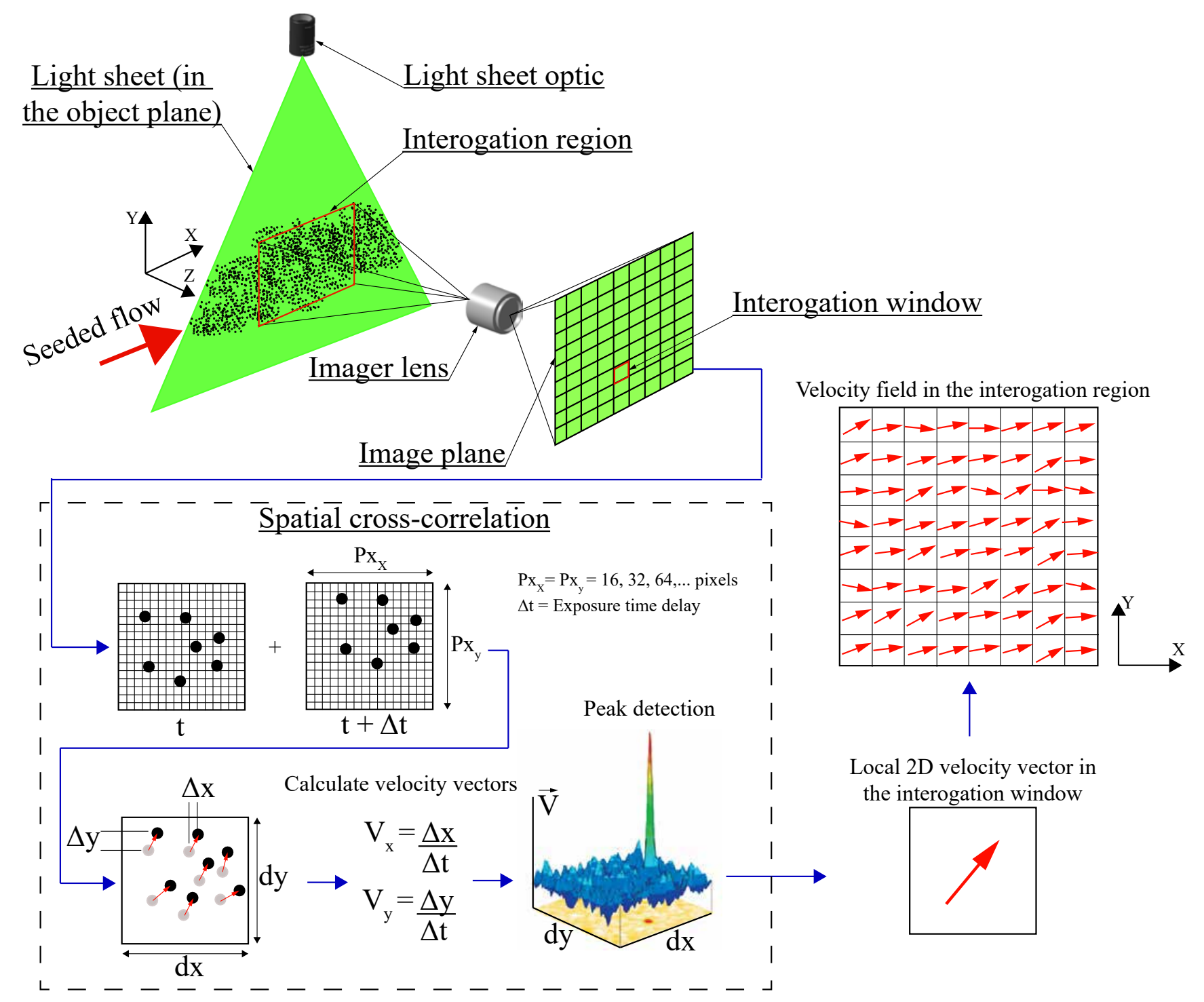

Figure 2.3: Operating principle of planar PIV. 


\subsubsection{Stereoscopic PIV}

Stereoscopic PIV is an extension of planar PIV. It is a 3-component measurement technique which is functionally the same as planar PIV, with the addition of a second camera which allows for stereoscopic viewing of the seed particles in the interrogation region, making it possible to resolve all three components of velocity. Stereoscopic PIV is the natural progression of planar PIV, as the technique becomes applicable to highly 3-dimensional flows. Reconstruction of a 3-dimensional velocity vector from pairs of $2 \mathrm{D}$ images is non-trivial, and its difficulty is exacerbated when the camera and light sheet are misaligned. Such misalignments can be corrected in-situ using the process of self-calibration, where actual measurements of the seeded flow-field are used to correct for imperfect alignments (Willert, 1997). This is a significant advantage over 3D LDA, where the LDA probes must be perfectly aligned for the measurement volumes to intersect at exactly the same point, and if they are not, the LDA measurements will not indicate the magnitude of this misalignment and it cannot be corrected for.

A variation of stereoscopic PIV is particle tracking velocimetry (PTV). PTV is usually only employed when the seed-particle density in the flow is too low to perform the statistical cross-correlation calculations accurately. Rather than compute the velocity vectors based on a statistical analysis of particle motions (peak detection), in PTV each individual particle in the interrogation region is tracked independently (Adrian and Westerweel, 2011). The operating principle of PTV gives it the advantage of having the ability to track the Lagrangian paths of the particles in the flow, which has been shown to be useful in understanding the nature of turbulence in a jet flow (Kim et al., 2016). Combining the statistical cross-correlation analysis of stereoscopic PIV with the particle tracking of PTV results in a method known as super-resolution PIV. The super-resolution method uses the result of the statistical cross-correlation to guide the measurement of the motion of individual particles (Keane et al., 1995). Keane et al. (1995) showed that the velocity field in a turbulent pipe flow could be computed to substantially higher levels of accuracy through 
super-resolution PIV compared to stereoscopic PIV alone. Because of the increased computing requirements, super-resolution PIV is most effective when applied only to regions of the imaged flow-field that contain especially high velocity gradients.

\subsubsection{Tomographic PIV}

The most recent advancement in PIV measurement technology is tomographic PIV. Tomographic PIV operates on the same working principle as stereoscopic PIV with two major modifications. First is the addition of more cameras (typically a total of four), and second is the expanding of the thickness of the light sheet to about 10-25 mm or approximately one quarter of the camera field-of-view (Scrano, 2013). The result of this is a $3 \mathrm{D}$ measurement volume used to resolve the $3 \mathrm{D}$ velocity field. Tomographic PIV makes use of a much larger measurement volume than stereoscpic PIV, facilitating a significant reduction in measurement uncertainty (Liu et al., 2018). The widespread use of tomographic PIV seems to be limited mostly by the cost of the additional cameras needed and the higher laser power required to achieve a thicker light sheet.

\subsection{Flow Seeding Materials for Particle Image Velocimetry}

The following review of seeding materials and techniques for PIV focuses on highspeed subsonic flows given the focus of the present research on such flows. Seed material requirements for high-speed, subsonic air-flows are: (1) micron, to sub-micron particle size for high fidelity flow tracking; (2) high refractive index and particle shape uniformity for effective light scattering properties; and (3) mono-disperse particle size distribution. Of course, any choice of seed particles that meet these technical specifications must also meet the health and safety standards of the laboratory facility.

\subsubsection{Solid Particle Seeding}

Solid metal powders such as titanium di-oxide $\left(\mathrm{TiO}_{2}\right)$, magnesium oxide $(\mathrm{MgO})$, and alumina powder $\left(\mathrm{Al}_{2} \mathrm{O}_{3}\right)$ have long been used for seeding flows for laser-based 
velocimetry (Crosswy, 1985). These solid powders are attractive for PIV for their high refractive index and sub-micron nominal particle sizes, which, in the case of titanium di-oxide powder, can be less than $0.1 \mu \mathrm{m}$. The high dynamic response with particle diameters of 0.3-0.4 $\mu \mathrm{m}$ has also been well documented for solid powders in supersonic flows (Havermann et al., 2002; Humble et al., 2007). Fang et al. (2017) demonstrated the flow tracking ability of $0.03 \mu \mathrm{m}$-diameter titanium di-oxide powder in supersonic air flow up to a Mach number of 4.0 to successfully minimize the "smearing" effect in measurements across shockwaves. However, delivery of these powders into the working fluid tends to be problematic. Electrostatic forces and even slight humidity may cause particles to agglomerate, resulting in highly poly-disperse seed clouds, with particle sizes varying by as much as an order of magnitude.

An alternative to the metallic powders is polystyrene latex. Solid polystyrene latex (PSL) particles are nearly perfectly shaped spherical particles. Their perfect spherical shape give PSL particles predictable flow tracking characteristics as well as ideal light scattering potential (Melling, 1997). Subsonic, transonic and supersonic air flows have all been successfully seeded with PSL particles (Seegmiller, 1985). Unlike alternative solid powders that have been used for air-flow seeding in PIV studies, PSL particles are stored in a liquid substrate or carrier fluid, which is typically a water-ethanol mixture. The PSL particle-substrate combination is injected into the working fluid through an atomizing nozzle. As the PSL particles travel, the carrier fluid evaporates, leaving only the mono-disperse PSL particles. PSL particles of 0.5 $\mu$ m-diameter have been shown to work well as a seed particulate in subsonic airflow with flow Mach numbers in the range of Mach 0.2 (Molezzi and Dutton, 1993) to 0.8 (Towers et al., 1991). In some supersonic flows, with low static temperatures and low static pressures, the carrier fluid has a tendency to re-condense on the PSL particles (Lepicovsky and Bruckner, 1996), resulting in poly-disperse particle sizes.

Solid particles and powders on the order of $1 \mu \mathrm{m}$-diameter are highly respirable and therefore pose a significant health hazard, requiring containment or filtration systems to maintain air quality in the laboratory. Recently, solidified $\mathrm{CO}_{2}$ has been proven to be an effective seed material for transonic and supersonic wind-tunnels (Love, 
2010). $\mathrm{CO}_{2}$ is an attractive seeding material because the solid particles sublimate into harmless $\mathrm{CO}_{2}$ gas, leaving no residue in the air or on laboratory equipment. Delapp (2006) modified a commercially available $\mathrm{CO}_{2}$ spray gun to produce solid $\mathrm{CO}_{2}$ seeds in the range of 5-12 $\mu$ m-diameter in Mach 2.9 flow. DeLapp found that particle size at the interrogation point is highly sensitive to the particle residence time; the longer the $\mathrm{CO}_{2}$ particle exists in the flow, the more sublimation will occur, reducing the size of the particle prior to reaching the interrogation region. $\mathrm{CO}_{2}$ particle sizes are also highly sensitive to the design of the injector probe, where larger diameter probes allow for greater particle agglomeration (McNiel, 2007). The geometry of the feedline connecting the probe and the liquid- $\mathrm{CO}_{2}$ dewar has been found to affect particle sizes to a similar extent (Love, 2010). All these factors lead to relatively large and poly-disperse particle sizes. Given the rapid sublimation rate of $\mathrm{CO}_{2}$, and the interdependency of injector probe location, injector probe geometry, feedline geometry, and flow velocity, $\mathrm{CO}_{2}$ has not yet become a common alternative to more conventional seeding methods.

\subsubsection{Liquid Particle Seeding}

Liquid seed materials must be atomized into an aerosol for use in PIV measurements of gas-flows. The most common and proven atomization method is the Laskin nozzle, where an aerosol is produced by injecting compressed air pressurized to 2-8 atm into a liquid reservoir (Melling, 1997). Atomized particles produced this way are typically on the order of $1 \mu \mathrm{m}$-diameter, but particle size is dependent on atomization pressure, with higher pressures typically producing particles with smaller mean diameters (Echols and Young, 1963). Many studies have had success using Di-Ethyl-HexylSebacate (DEHS), a non-toxic synthetic oil, as an effective seed material for flows in the transonic regime (Lang et al., 2002; Koike et al., 2007), and in the supersonic regime at flow Mach numbers from 1.95 (Mathijssen et al., 2009) to 3.0 (Scharnowski et al., 2018), with no apparent degradation in the flow tracking fidelity or light scatter of the tracers at the higher flow Mach numbers. DEHS is a popular choice for air flow seeding as the particles evaporate within a few hours, leaving no residue behind. 
Molezzi and Dutton (1993) produced an aerosol of $0.8 \mu \mathrm{m}$ mean diameter particles using a Laskin nozzle and silicone oil for PIV in a Mach 0.5 airflow, prompting the idea that for comparable air flows, any oily compound with similar viscosity and surface tension characteristics would be a suitable seed material. Legrand et al. (2016) designed an aerosol generator inspired by the classical Laskin nozzle (Kahler et al., 2002) using a propylene glycol and water solution. Their generator mixed the output, a propylene glycol-water aerosol, with compressed air to promote evaporation of the water, producing tracers with less than $1 \mu$ m-diameter. An atomized propylene glycol-ethanol solution was successfully used for PIV measurements inside a rotating transonic compressor stage, where the ethanol concentration was regulated to adjust the surface tension of the solution to improve atomization (Bhattacharya et al., 2016). Similar to florescent dye-doped PSL spheres (Petrosky et al., 2015), Bhatacharya et al. (2016) showed that the addition of a fluorescent dye to a propylene glycol-ethanol solution increased the signal-to-noise ratio of the PIV images due to the increased light intensity of the illuminated fluorescent particles.

Olive oil is a viable substitute for the synthetic oils, as $1 \mu$ m-diameter oil droplets are easily generated with a Laskin nozzle (Kompenhans and Raffel, 1993). In blowdown type wind-tunnels, the expanding air undergoes a large drop in static temperature. Successful use of atomized oil as seeding particulate at cryogenic temperatures would suggest no adverse effects of reduced static temperature on flow tracking fidelity or light scatter ability (Fey et al., 2010). While mean particle diameters of atomized liquids are larger than with solid seed materials, oils have a much lower density ratio with air, thus a $1 \mu$ m-diameter oil particle has the same dynamic response as a $0.45 \mu \mathrm{m}$-diameter titanium di-oxide particle (Melling, 1997). Atomized oils show a tendency to coalesce on the inside surfaces of the wind-tunnel. This can lead to collected oil bursting off, adding larger seed particles into the flow. If view ports are required for laser access, buildup of oil on view ports will obstruct a clean view of the interrogation region, requiring intermittent stoppage of the wind-tunnel for cleaning. 
An improvement on the classical Laskin nozzle is the vaporization-condensation seeder (VAPCON), commonly referred to as a fog generator. VAPCON devices use a Laskin nozzle to first generate an aerosol from a liquid oil, oil-water, or oil-alcohol solution. The aerosol is vaporized with a heating element and then allowed to recondense. The re-condensed aerosol particles are of a higher mono-dispersity than the original aerosol. Crosswy (1985) reported that a mixture of dioctyl phthalate oil (DOP) and ethanol passed through a VAPCON produced $0.4 \mu \mathrm{m}$-diameter seed particles with nearly perfect mono-dispersity, with the sizes of $99 \%$ of all particles produced within $\pm 0.3 \mu \mathrm{m}$. Mazumder et al. (1974) used a VAPCON aerosol generator to yield similar results as Crosswy using a DOP-alcohol mixture. They found that the particle size of the condensate could be controlled by altering the alcohol content of the mixture. Commercially available fog generators using a glycerin/glycol and water mixture have been used successfully for larger scale, low-speed PIV studies (Ikegaya et al., 2018).

Alternative to oils, alginic acid is a polysaccharide found in the cell walls of brown algae; combined with metals like sodium and calcium it forms salts, known as alginates, which are widely used in the biomedical industry as a drug delivery method (Sosnik, 2014; Lee and Mooney, 2012) and in the food industry as an emulsifying agent (Rehm, 2009). Erturk et al. (2013) used 15-30 $\mu$ m-diameter alginate gel beads impregnated with varying combinations of water, olive oil, and fluorescent dye to perform PIV in a closed-circuit wind-tunnel at subsonic air speeds. Submicron alginate microparticles on the order of $0.8 \mu \mathrm{m}$ are also readily achievable (Villaneuva et al., 2011, Rehm, 2009) by aerosol generators resembling a Laskin nozzle. The relative ease of generation of submicron particles and the fact that alginates are designed for inhalation make them an attractive potential alternative for PIV seeding in high-speed gas flows.

The body force arising from the inertia of even the smallest heavier-than-air particles can divert the tracer particles from the trajectories of the local fluid particles. Helium-filled soap bubbles (HFSB), which have a density comparable to air, can successfully seed the core of a vortex, where centripetal forces would radially displace 
seed particles that are not neutrally buoyant (Caridi et al., 2017). With a typical particle diameter on the order of 300-800 $\mu \mathrm{m}$, with maximum values reaching several millimeters, HFSB were initially considered as a seed particle for large scale PIV measurement volumes on the order of several cubic meters (Bosbach et al., 2009). Because of their size, HFSB do not have sufficient dynamic response for high-speed flows (Faleiros et al., 2019). For particles with densities notably higher than that of the working fluid, the particle velocity approaches the bulk fluid velocity in a manner that follows an exponential law given by:

$$
U_{p}(t)=U\left[1-\exp \left(-\frac{t}{\tau_{s}}\right)\right]
$$

where $U_{p}$ and $U$ are the velocity of the particles and the fluid, respectively, and $\tau_{s}$ is the relaxation time of the particle given by:

$$
\tau_{s}=d_{p}^{2}\left(\frac{\rho}{18 \mu}\right)
$$

where $d_{p}$ and $\rho$ are the diameter and density of the tracer particle, respectively, and $\mu$ is the dynamic viscosity of the working fluid. Table 2.1 provides a summary of the various seeding materials presented and their most relevant physical properties. It is evident that a reduction in particle relaxation time by one order of magnitude requires roughly a $60 \%$ reduction in particle diameter. Table 2.1 presents the relaxation times for particles of approximately 1.0-6.0 $\mu \mathrm{m}$-diameter, which have been shown suitable for use in high-speed flows (Adrian and Westerweel, 2011). Table 2.2 presents the most relevant health and safety hazard properties of the various seed materials (DeLapp, 2006). Some seed materials will form a combustive mixture in air at high enough concentrations. This property is often expressed as the material's lower explosive limit (LEL). 
Table 2.1: Physical properties of seed materials.

\begin{tabular}{|c|c|c|c|c|c|c|c|c|}
\hline Material & $\begin{array}{l}\text { Typical } \\
\text { Size } \\
\text { Range } \\
(\mu \mathrm{m})\end{array}$ & $\begin{array}{l}\text { Density } \\
\left(\mathrm{kg} / \mathrm{m}^{3}\right)\end{array}$ & $\begin{array}{c}\text { Density } \\
\text { Ratio } \\
\left(\rho / \rho_{\text {air }}\right)\end{array}$ & $\begin{array}{l}\text { Refractive } \\
\text { Index }\end{array}$ & $\begin{array}{c}\tau_{s} \\
(\mu \mathrm{s})\end{array}$ & $\begin{array}{c}d_{p} \\
(\mu \mathrm{m})\end{array}$ & $\begin{array}{l}\text { Operational } \\
\text { Temperature } \\
\text { Range }^{1} \\
\left({ }^{\circ} \mathrm{C}\right)\end{array}$ & $\begin{array}{c}\text { Operational } \\
\text { Velocity } \\
\text { Range }^{1} \\
\text { (Mach \#) }\end{array}$ \\
\hline $\mathrm{TiO}_{2}$ & $0.1-2.0$ & 3500 & 2800 & $2.6-2.7$ & $\begin{array}{r}0.1 \\
0.01\end{array}$ & $\begin{array}{l}3.0 \\
1.0\end{array}$ & $<+1830$ & $0.9-6.0+$ \\
\hline $\mathrm{MgO}$ & $0.1-1.5$ & 3580 & 2864 & 1.7 & $\begin{array}{r}0.1 \\
0.01 \\
\end{array}$ & $\begin{array}{l}3.0 \\
0.9 \\
\end{array}$ & $<+2800$ & $0.9-6.0+$ \\
\hline $\mathrm{Al}_{2} \mathrm{O}_{3}$ & $0.5-7.0$ & 3970 & 3176 & 1.7 & $\begin{array}{r}0.1 \\
0.01\end{array}$ & $\begin{array}{l}2.8 \\
0.9\end{array}$ & $<+1850$ & $0.9-6.0+$ \\
\hline PSL & $0.5-10$ & 1050 & 840 & 1.6 & $\begin{array}{r}0.1 \\
0.01\end{array}$ & $\begin{array}{l}5.6 \\
1.8\end{array}$ & $<+100$ & $0.01-1.0$ \\
\hline $\mathrm{CO}_{2}$ & $5.0-2.0$ & 1180 & 944 & 1.4 & $\begin{array}{r}0.1 \\
0.01\end{array}$ & $\begin{array}{l}5.3 \\
1.7\end{array}$ & $<+25$ & $0.3-3.0$ \\
\hline DEHS & $0.7-2.0$ & 912 & 729 & 1.3 & $\begin{array}{r}0.1 \\
0.01\end{array}$ & $\begin{array}{l}6.0 \\
1.9\end{array}$ & $-120-+25$ & $0.01-3.0$ \\
\hline $\begin{array}{l}\text { Propylene } \\
\text { Glycol }\end{array}$ & $0.4-2.0$ & 1040 & 832 & 1.4 & $\begin{array}{r}0.1 \\
0.01\end{array}$ & $\begin{array}{l}5.6 \\
1.8\end{array}$ & $-40-+25$ & $0.01-3.0$ \\
\hline Glycerin & $0.4-2.0$ & 1260 & 1008 & 1.4 & $\begin{array}{r}0.1 \\
0.01\end{array}$ & $\begin{array}{l}5.1 \\
1.6\end{array}$ & $-40-+25$ & $0.01-3.0$ \\
\hline Alginate & $0.8-40$ & Unknown & Unknown & $\approx 1.3-1.4$ & $\begin{array}{r}0.1 \\
0.01\end{array}$ & $\begin{array}{l}- \\
-\end{array}$ & Unknown & 0.01 - Unknown \\
\hline Olive Oil & $0.7-2.0$ & 918 & 734 & 1.3 & $\begin{array}{r}0.1 \\
0.01 \\
\end{array}$ & $\begin{array}{l}6.0 \\
1.9 \\
\end{array}$ & $-40-+25$ & $0.01-3.0$ \\
\hline HFSB & $300-800$ & $\approx 1.2$ & $\approx 1.0$ & 1.3 & $20-130$ & $300-800$ & $+15-+25$ & $0.01-0.1$ \\
\hline
\end{tabular}

${ }^{1}$ Based on physical properties of the material and evidence in the literature, where available 
Table 2.2: Health and safety hazard properties of seed materials (DeLapp, 2006).

\begin{tabular}{|c|c|c|c|c|}
\hline Material & 8-hour Exposure Limit & Health Effects $^{1}$ & LEL & Corrosiveness \\
\hline $\mathrm{TiO}_{2}$ & $50 \mathrm{ppm}$ & Nuisance & $\mathrm{N} / \mathrm{A}$ & $\mathrm{N} / \mathrm{A}$ \\
\hline $\mathrm{MgO}$ & $10 \mathrm{ppm}$ & Irritant & $\mathrm{N} / \mathrm{A}$ & $\mathrm{N} / \mathrm{A}$ \\
\hline $\mathrm{Al}_{2} \mathrm{O}_{3}$ & $50 \mathrm{ppm}$ & Nuisance, Carcinogenic & $\mathrm{N} / \mathrm{A}$ & $\mathrm{N} / \mathrm{A}$ \\
\hline PSL & $50 \mathrm{ppm}$ & $\begin{array}{c}\text { Nuisance, Carcinogenic, } \\
\text { Anesthetic, Irritant }\end{array}$ & $1.10 \%$ & N/A \\
\hline $\mathrm{CO}_{2}$ & 10000 ppm & Asphyxiant & $\mathrm{N} / \mathrm{A}$ & $\mathrm{N} / \mathrm{A}$ \\
\hline Propylene Glycol & $\mathrm{N} / \mathrm{A}$ & Nuisance & $2.6 \%$ & N/A \\
\hline Glycerin & $\mathrm{N} / \mathrm{A}$ & Nuisance & $0.9 \%$ & $\mathrm{~N} / \mathrm{A}$ \\
\hline Alginate & $\mathrm{N} / \mathrm{A}$ & $\mathrm{N} / \mathrm{A}$ & $\mathrm{N} / \mathrm{A}$ & N/A \\
\hline DEHS & $\mathrm{N} / \mathrm{A}$ & Nuisance & Unknown & $\mathrm{N} / \mathrm{A}$ \\
\hline Olive Oil & $\mathrm{N} / \mathrm{A}$ & Nuisance & Unknown & $\mathrm{N} / \mathrm{A}$ \\
\hline HFSB & $\mathrm{N} / \mathrm{A}$ & Asphyxiant & $\mathrm{N} / \mathrm{A}$ & $\mathrm{N} / \mathrm{A}$ \\
\hline
\end{tabular}

${ }^{1}$ Result of inhalation of atomized material 


\section{Chapter 3}

\section{Experimental Facilities}

\subsection{Low-Speed Open-Circuit Wind-Tunnel}

The low-speed wind-tunnel is illustrated in Fig. 3.1. The facility consists of a variablespeed centrifugal fan that draws ambient air and passes it through a settling chamber containing a honeycomb flow straightener and three turbulence-reducing screens. Downstream of the settling chamber, the air passes through two contractions with 9:1 and 4:1 area ratio, respectively. At the exit of the 4:1 contraction, a swirl generator section is installed, which also facilitates the transition of the flow path from a circular cross-section to an annular one. A third 2.5:1 contraction scales the flow path diameter to match that of the high-speed wind-tunnel air-supply system described in Chapter 4. 


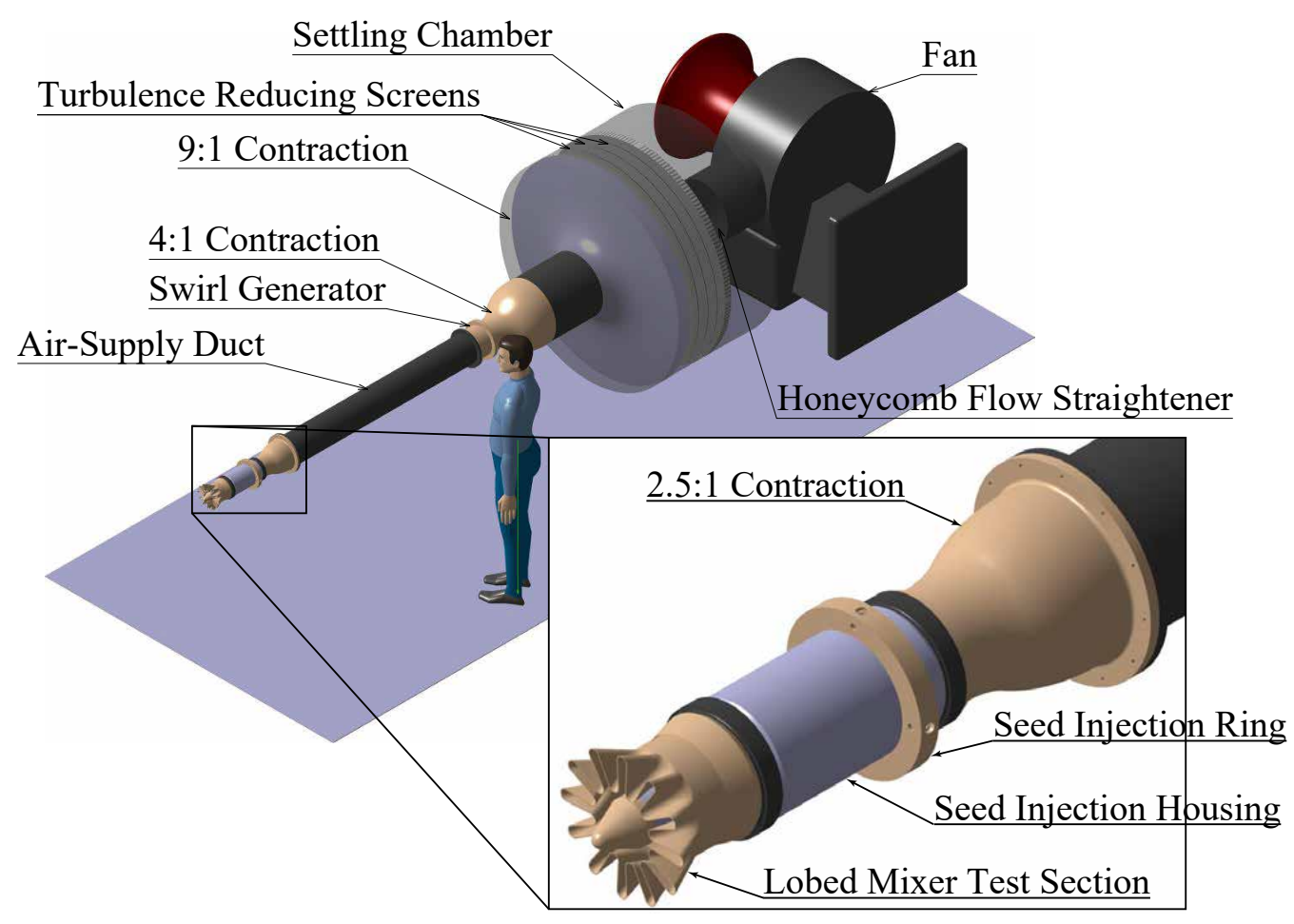

Figure 3.1: Rendered model of the Carleton low-speed turbomachinery wind-tunnel.

\subsection{High-Speed Blow-Down Wind-Tunnel}

The high-speed wind-tunnel is illustrated in Fig. 3.2. The facility consists of four compressed-air storage tanks, a flow control valve, and an air-supply duct, the end of which has a flange that facilitates the attachment of an annular-flow air-supply system developed in the present work. The air-supply duct feeds airflow discharging from the storage tanks to a test section through the flow-control valve that regulates the mass flow rate through the system with the goal of maintaining a constant Mach number at the inlet to the test section over a finite duration. A cascade test section is also part of the facility, the discharge duct of which facilitates attachment of an additional test section that is described in Chapter 5 . 


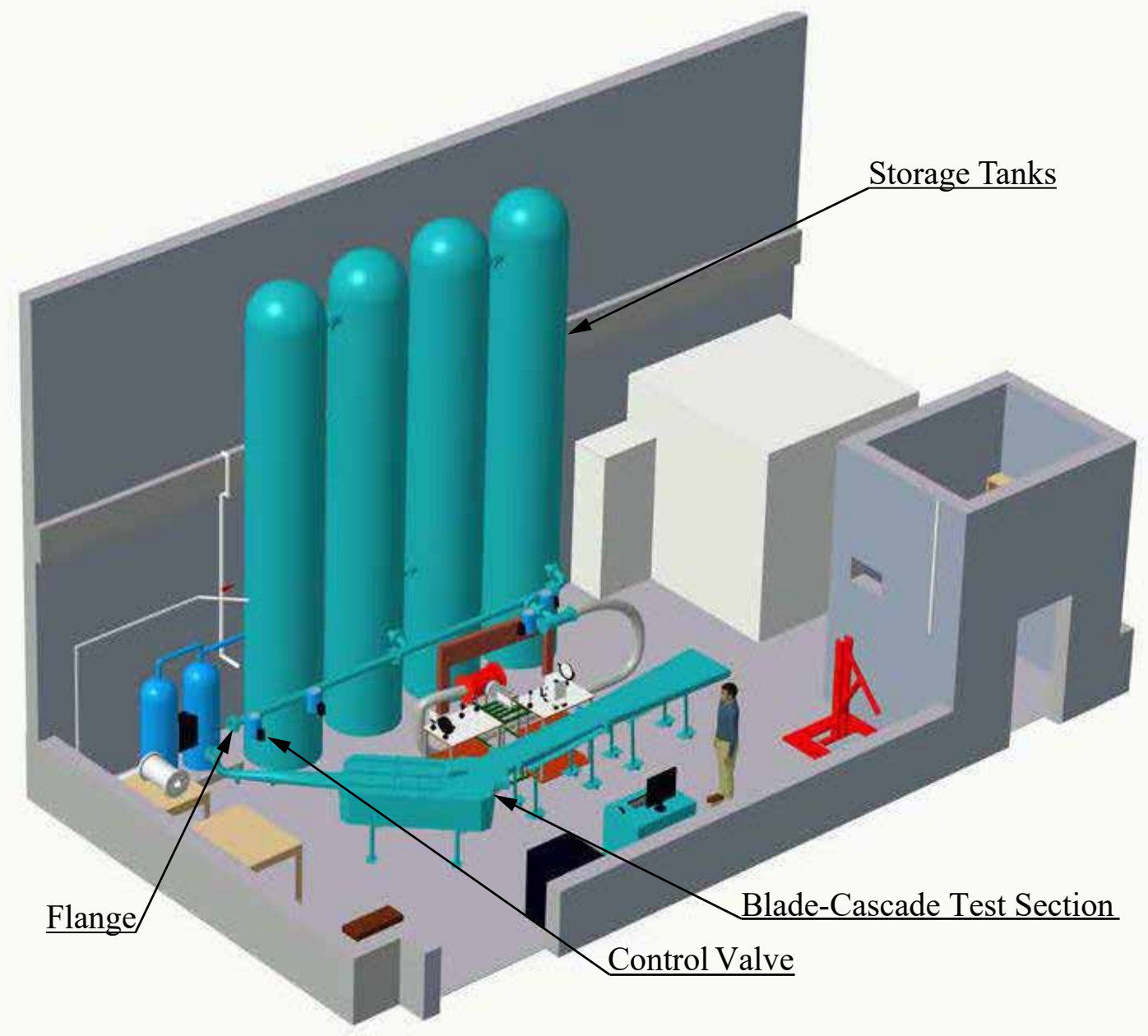

Figure 3.2: Rendered model of the Carleton high-speed wind-tunnel. 


\subsubsection{Aerodynamic Model of the Wind-Tunnel Flow Path}

The test-section mass flow rate and the run time for a single charge of the storage tanks are the two primary operational parameters of the blow-down wind-tunnel facility. The geometry and pressure-loss characteristics of the test section and the ducting connecting the test section to the flange labeled in Fig. 3.2 dictate the values of these parameters, hence constitute the focus of the aerodynamic design optimization study presented in the following sections. The one-dimensional aerodynamic model used for this design effort is best illustrated in the context of a simplified system shown in Fig. 3.3.

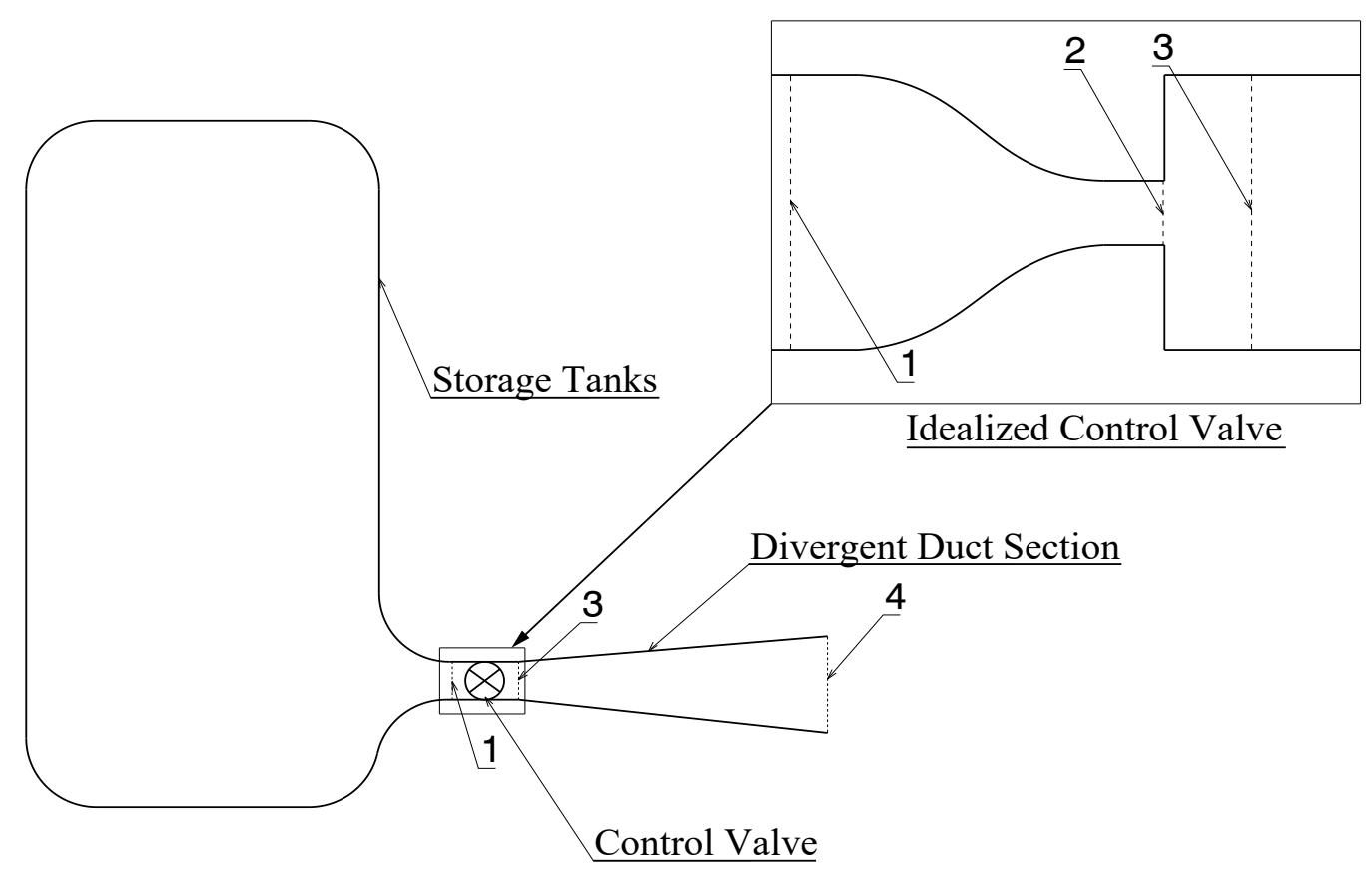

Figure 3.3: Simplified model of the wind-tunnel flow path.

In this idealized model, the control valve of the facility is represented by a converging nozzle that is terminated by a sudden expansion as shown in the inset of Fig. 3.3. 
The only frictional loss in this idealized flow path occurs as a result of this sudden expansion. As part of the one-dimensional modeling approach, at each of the labeled four stations, all flow and fluid parameters are uniformly distributed within the crosssectional area.

For the scenario where the flow is choked at the throat (station 2), the flow expands immediately downstream of the throat while experiencing stagnation pressure loss through turbulent mixing and a shock-wave system to yield subsonic flow conditions at station 3. The flow then decelerates via the area increase between stations 3 and 4 , reaching a static pressure value at station 4 that matches the pressure, $P_{b}$, of the surroundings into which the flow discharges. The subsonic Mach number at station 4 is related to the local stagnation pressure $\left(P_{o 4}\right)$ and local static pressure $\left(P_{4}=P_{b}\right)$ through:

$$
\frac{P_{o 4}}{P_{4}}=\left[1+\frac{\gamma-1}{2} M_{4}^{2}\right]^{\frac{\gamma}{\gamma-1}}
$$

where $\gamma=1.4$ is the ratio of specific heats. Accordingly, for a desired operational Mach number at station $4, M_{4}$, the local stagnation pressure is fixed by this relation. Assuming frictionless flow between stations 3 and 4, this then fixes the stagnation pressure at station $3\left(P_{o 3}=P_{o 4}\right)$. To realize this stagnation pressure at station 3 , the sudden expansion between 2 and 3 and the associated pressure losses need to be such that the stagnation pressure in the storage tanks $\left(P_{\text {oTank }}\right)$ is reduced to $P_{o 3}$ along this section of the flow path. For choked flow conditions at station $2\left(M_{2}=1.0\right)$, and representing the losses due to the sudden expansion by $K_{\text {valve }}, P_{o 2}\left(=P_{o T a n k}\right)$ and $P_{o 3}$ are related to each other through:

$$
P_{o 3}=P_{o_{T a n k}}\left(1-\left[K_{\text {valve }}\left(0.5 \gamma M_{2}^{2}\left[1+\frac{\gamma-1}{2} M_{2}^{2}\right]^{\frac{1-\gamma}{\gamma}}\right)\right]\right)
$$

The derivation for Eqn. 3.2 is presented in Appendix A. The mass flow rate, which remains constant along the length of the flow path under the intended steady conditions, can be determined at the throat (station 2) based on the stagnation pressure 
$P_{o 2}\left(=P_{o T a n k}\right)$ and temperature $T_{o 2}\left(=T_{o T a n k}\right)$ as:

$$
\dot{m}=A_{2} P_{o 2} \sqrt{\frac{2 \gamma}{R T_{o 2}(\gamma-1)}\left[\left(\frac{P_{b}}{P_{o 2}}\right)^{\frac{2}{\gamma}}-\left(\frac{P_{b}}{P_{o 2}}\right)^{\frac{\gamma+1}{\gamma}}\right]}
$$

where $R=287 \mathrm{~J} / \mathrm{kg} \mathrm{K}$ is the ideal-gas constant for air. As a result of this mass flow rate, the pressure in the storage tanks decreases over time during an experimental run. This expansion in the storage tanks takes place within the bounds of two extreme scenarios of isothermal expansion and adiabatic expansion. The isothermal case assumes instant heat transfer through the tank walls to maintain $T_{\text {oTank }}=T_{\text {ambient }}$, which would be a suitable approximation for a very slow expansion process. The adiabatic scenario assumes zero heat transfer through the tank walls, which would be a suitable approximation for a very fast expansion in the tanks. As per Eqn. 3.3, to maintain a time-independent mass flow rate at station 2 , the temporal decrease in $P_{o 2}\left(=P_{o T a n k}\right)$ must be compensated for by an increase of the flow area at station 2 . The time rate of change of $P_{\text {oTank }}$ for adiabatic expansion in the tanks can be expressed as:

$$
\frac{d P_{o T a n k}}{d t}=-\dot{m} \frac{R T_{o T a n k}}{V}
$$

where $V$ is the storage tank volume. The derivation for Eqn. 3.4 is presented in Appendix A. Substituting for the mass flow rate in this equation using the expression in Eqn. 3.3, and rearranging yields an expression for $A_{2}\left(=A_{\text {throat }}\right)$ as a function of $P_{o 2}\left(=P_{o T a n k}\right)$ and $T_{o 2}\left(=T_{o T a n k}\right)$ :

$$
A_{2}=\frac{-\Delta P_{o 2}}{\Delta t} \frac{V}{R T_{o 2} P_{o 2}}\left(\frac{2 \gamma}{R T_{o 2}(\gamma-1)}\left[\left(\frac{P_{b}}{P_{o 2}}\right)^{\frac{2}{\gamma}}-\left(\frac{P_{b}}{P_{o 2}}\right)^{\frac{\gamma+1}{\gamma}}\right]\right)^{-0.5}
$$

where $\Delta t$ is a user selected time increment for varying the throat area over time incrementally, and $\Delta P_{o 2}\left(=\Delta P_{\text {oTank }}\right)$ represents the change (with a negative value indicating a pressure decrease) in storage tank pressure during this time increment.

The time variation of tank pressure and the corresponding variation in the open area of the flow control valve that needs to be realized to maintain steady conditions in 
the test-section inlet (station 4) are presented in Fig. 3.4. The predictions are based on the assumption of adiabatic expansion in the storage tanks, which is deemed more suitable than isothermal expansion given the relatively short run times. Sensitivity of the run time (i.e. time available for experimentation) to the flow area in the test section is illustrated in Fig. 3.5. While a larger flow area in the test section is desirable for improved spatial resolution of the flow measurements, Fig. 3.5 illustrates that this comes at the cost of shorter run times. This trade off is an important factor in the design of the flow path that is described in the next chapter. It is important to note that the aerodynamic model that was used for predicting the aerodynamic performance of the air-supply system in this design exercise was not as simple as the one illustrated in Fig. 3.3. The performance predictions during the design iterations accounted for the frictional losses, flow area variations, and other loss elements along the flow path of the air-supply system. 


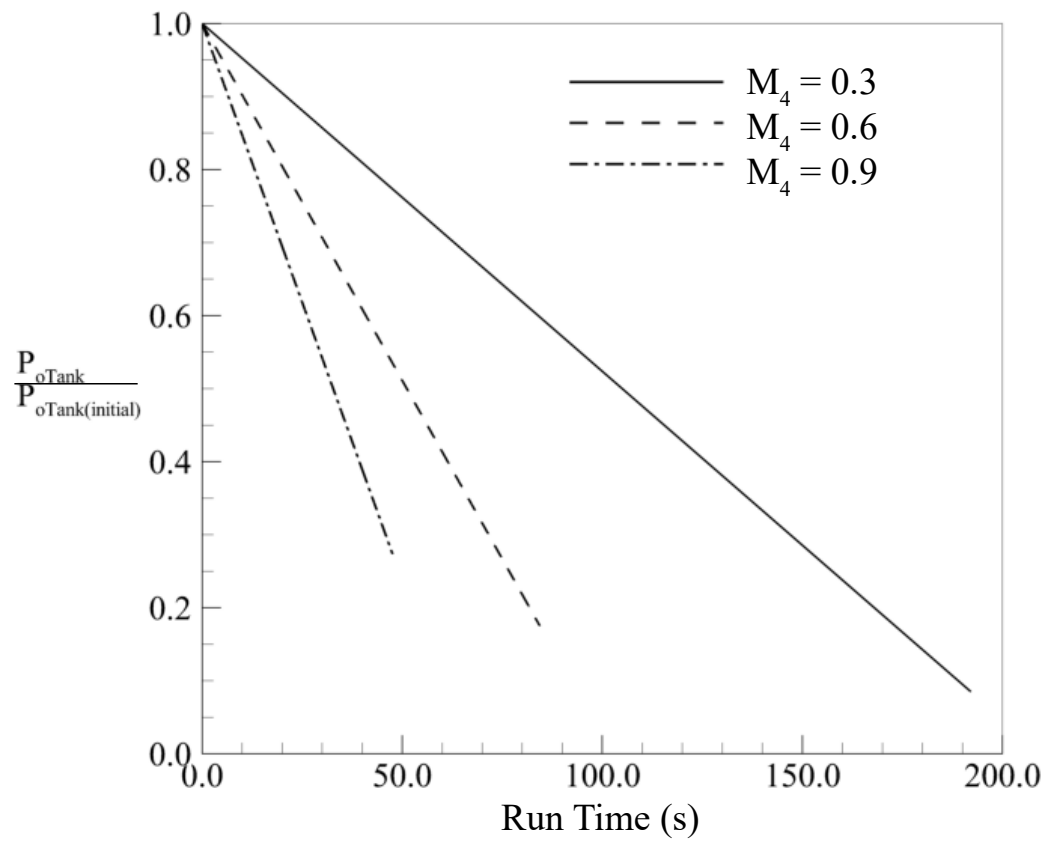

(a)

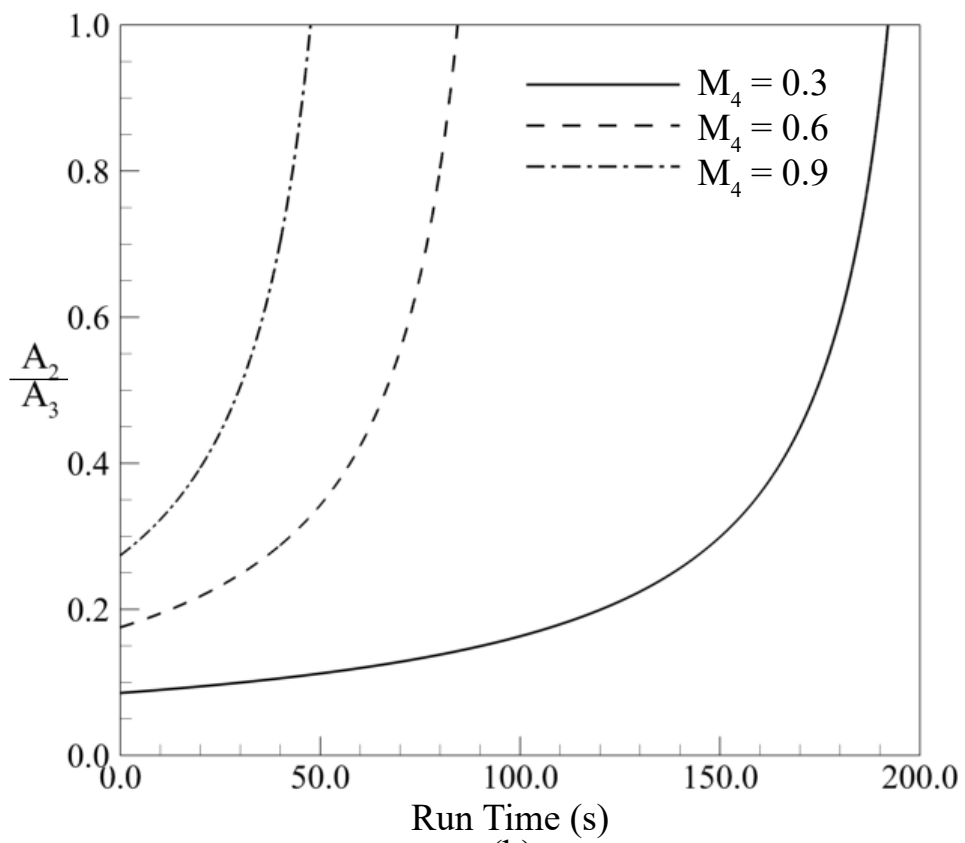

(b)

Figure 3.4: Time variation of (a) $P_{\text {oTank }}$ and (b) valve open area ratio, $A_{2} / A_{3}$, for several Mach numbers at station 4 . 


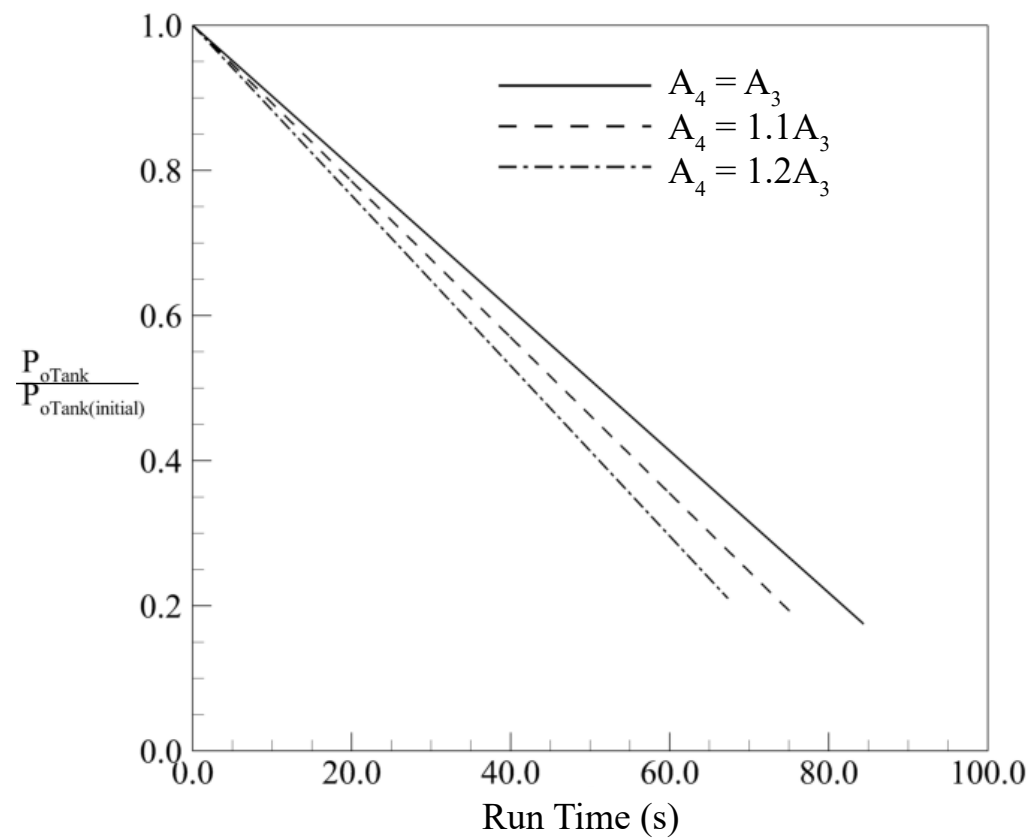

(a)

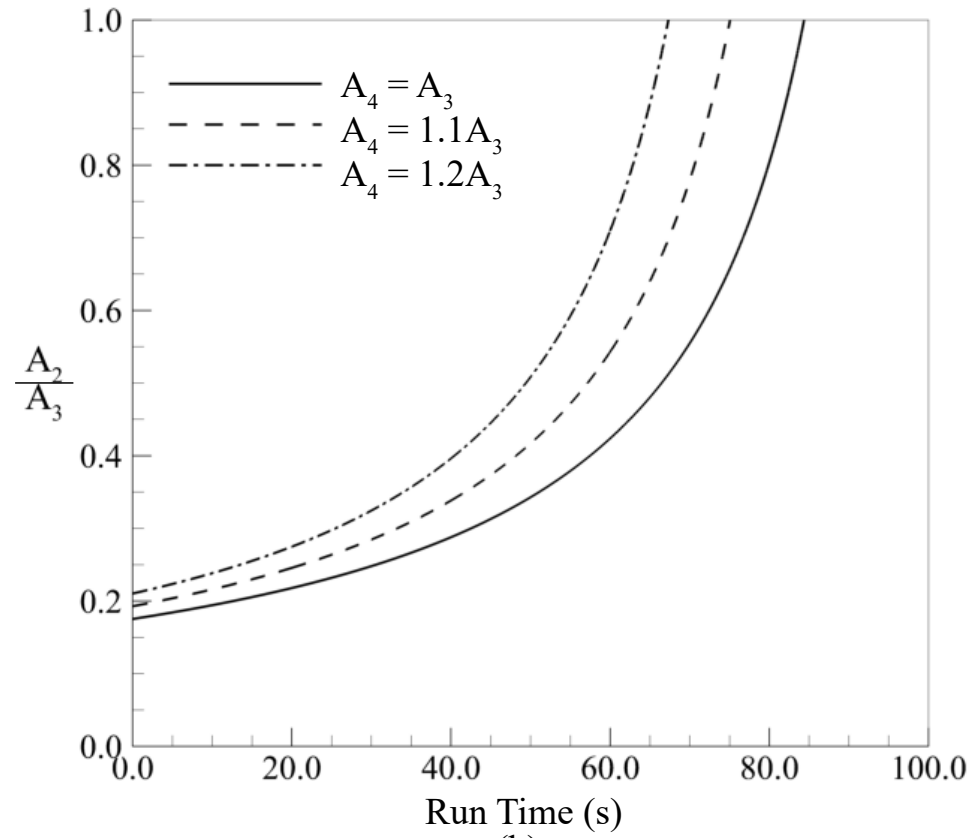

(b)

Figure 3.5: Time variation of (a) $P_{\text {oTank }}$ and (b) valve open area ratio, $A_{2} / A_{3}$, for several values of flow area $\left(A_{4}\right)$ with $M_{4}=0.6$. 


\section{Chapter 4}

\section{Design of an Air-Supply System for Annular-Flow Test Sections at Subsonic Mach Numbers}

\subsection{Introduction}

The philosophy maintained throughout the design process of the high-pressure airsupply system for the intended wind-tunnel investigations of ducted flows was that the design be light-weight, versatile, and easy to manufacture. The expected range of flow conditions consists of a flow Reynolds number range from $1.5 \times 10^{6}$ to $4.5 \times 10^{6}$ based on the flow-path hydraulic diameter and time-averaged velocity at the exit of the air-supply system, and a discharge flow Mach number range of 0.3 to 0.8. Fig. 4.1 presents a schematic of the assembled air-supply system. The supply air available on the high-speed wind-tunnel is at ambient stagnation temperature and a maximum stagnation pressure of $7 \mathrm{~atm}$. Accordingly, the air-supply system is designed for a peak pressure loading of $7 \mathrm{~atm}$. The system consists of multiple sections along its length, which are held together with the aid of V-band clamps and four pre-compression rods. The majority of the components that make up the flow path are made of high-density poly-ethylene (HDPE) to achieve a light-weight design. Alternative polymer-based piping materials such as ABS and PVC lack the toughness required for the present application. Fig. 4.2 shows an exploded schematic of the design, with the various parts labeled in this schematic identified in Table 4.1. In the following sections, the design of each component of the system is described. Technical drawings for all components are provided in Appendix B. 


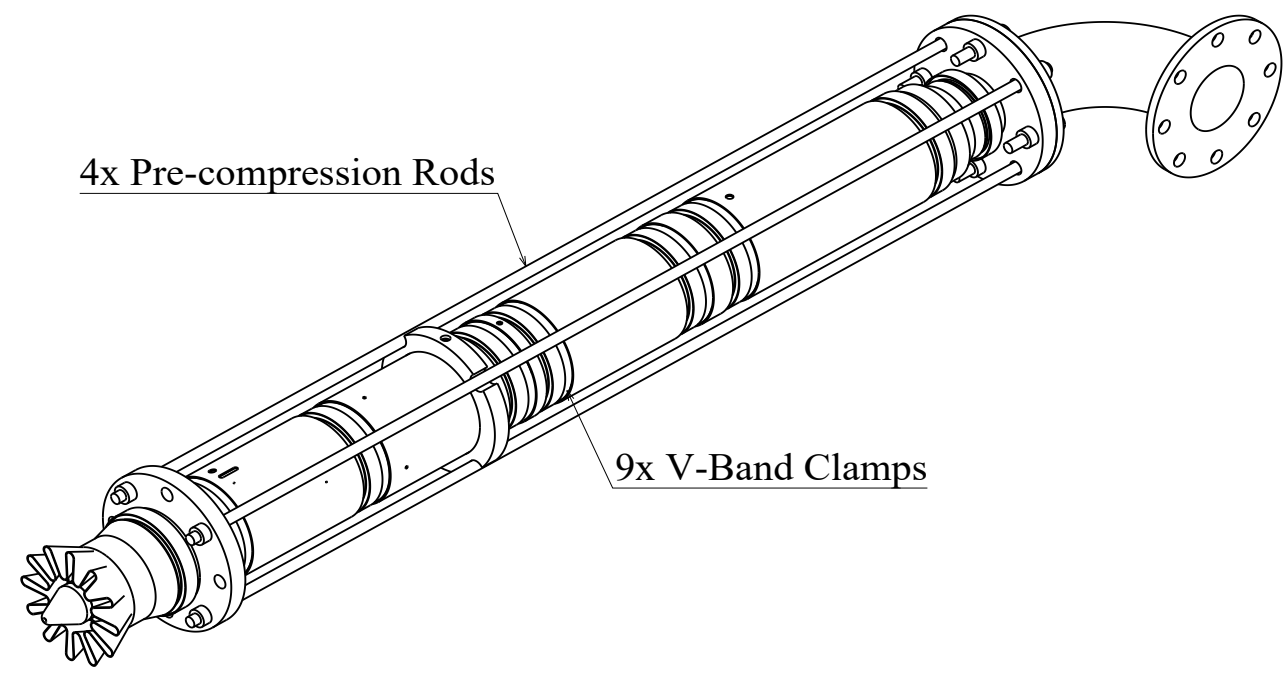

Figure 4.1: Assembled air-supply system with a lobed-mixer test section.

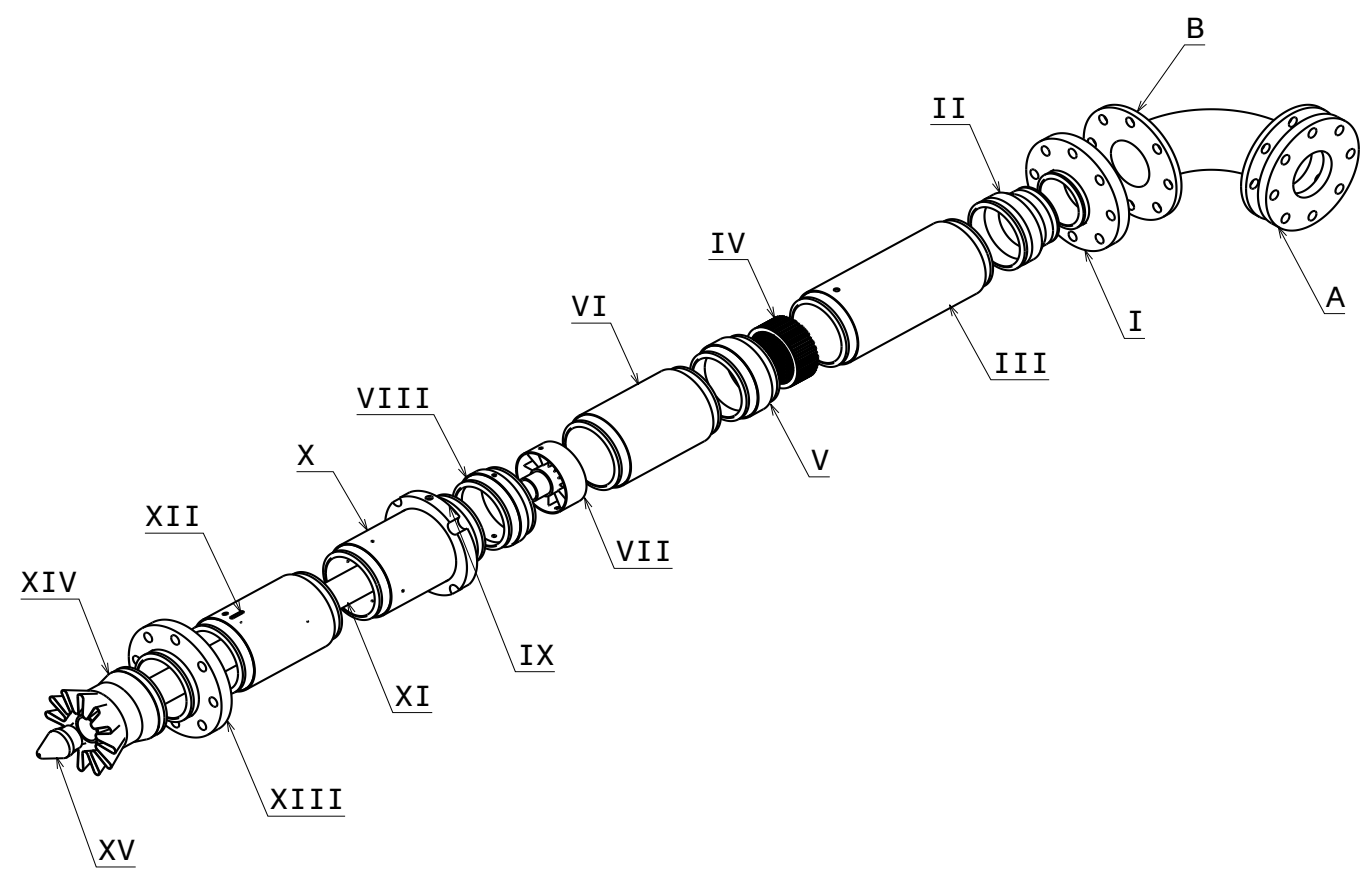

Figure 4.2: Exploded schematic of the air-supply system; pre-compression rods and V-band clamps omitted for clarity. 
Table 4.1: Main components of the air-supply system.

\begin{tabular}{lllc}
\hline Section & Designation & Material & Mass (kg) \\
A & Existing HSWT Flange & Steel & - \\
B & 90-deg. Bend & Steel & 16.0 \\
I & Upstream Flange & Steel & 7.3 \\
II & Expansion Section & Steel & 3.0 \\
III & Transition Section I & HDPE & 3.9 \\
IV & Honeycomb Insert & Aluminum & 0.2 \\
V & Flow Straightener Housing & HDPE & 3.1 \\
VI & Turbulence Screen Housing & HDPE & 2.6 \\
- & Turbulence Screen Inserts & Steel & 0.3 \\
VII & Swirl Generator Insert & Steel & 1.2 \\
VIII & Swirl Generator Housing & HDPE & 1.8 \\
IX & Seed Injection Ring & Steel & 2.6 \\
X & Seed Injection Housing & HDPE & 2.3 \\
XI & Core Pipe & Sch. 80 PVC & 0.7 \\
XII & Transition Section II & HDPE & 2.5 \\
XIII & Downstream Flange & Steel & 7.4 \\
XIV & Test Section (e.g. Lobed Mixer) & Steel & 1.6 \\
XV & End Plug & Steel & 0.3 \\
\hline
\end{tabular}

The downstream section of the flow path extending from the swirl generator to the test section (a lobed mixer in the illustrated configuration) is designed to be geometrically similar to the setup used in earlier low-speed lobed-mixer studies at Carleton University (Brinkerhoff et al., 2013) to facilitate comparison of the experimental results acquired in the two facilities.

\subsection{Pre-compression Rods}

Four steel pre-compression rods of $15.5 \mathrm{~mm}$-diameter installed between two ASME Class 150 steel flanges serve to pre-compress the system axially to yield safe levels of structural stresses when the system is pressurized. Each pre-compression rod has a longitudinal stiffness of $2.13 \times 10^{7} \mathrm{~N} / \mathrm{m}$ and is installed with a bolt torque of $1.4 \mathrm{Nm}$ to produce an axial pre-compression load of $375 \mathrm{~N}$ per rod. This choice of precompression load was determined through iterations of finite element analysis (FEA) 
calculations until the stresses and deformations in the air-supply system met the desired stress safety factors and geometric tolerances.

\subsection{V-band Clamps}

Each section of the air-supply system is connected to its upstream and downstream mating sections via a commercially available V-band clamp, depicted in Fig. 4.3 (Norma Group, 2016). These clamps were chosen based on their relatively low cost, simplicity of installation, and circumferential uniformity of the radial and axial loads imposed by the clamp on the mating parts.

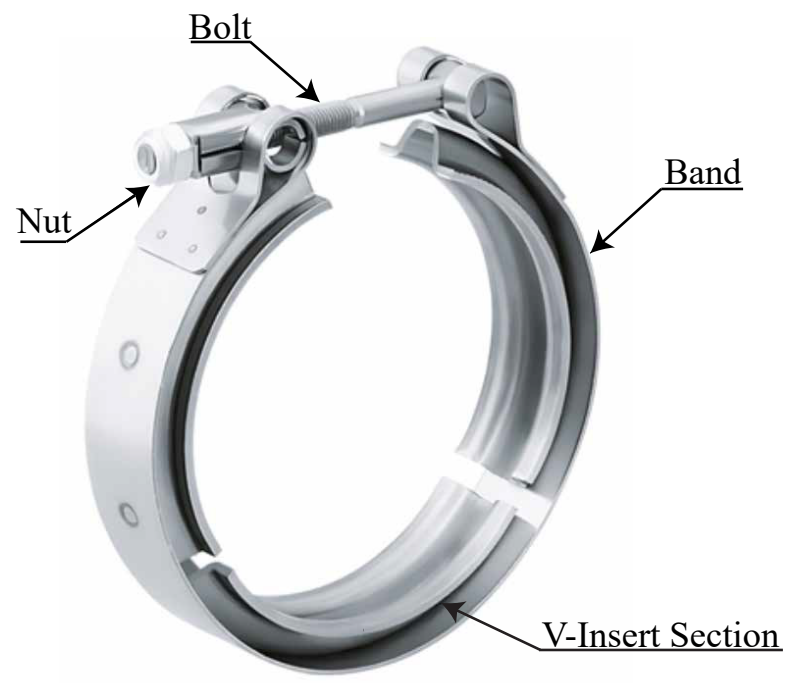

Figure 4.3: V-band clamp.

The stainless-steel clamp consists of a circular flat ring with an approximately V-shaped ring section welded along the inner surface of the flat ring. The clamp is installed and tightened around the mating sections using a single threaded bolt and hex nut of $6.35 \mathrm{~mm}$-diameter. Along the length of the air-supply system, two different diameters of this clamp are used with slightly different dimensions for the V-shaped section. The major dimensions and manufacturer-specified installation torque for each clamp size are presented in Fig. 4.4 and Table 4.2. 


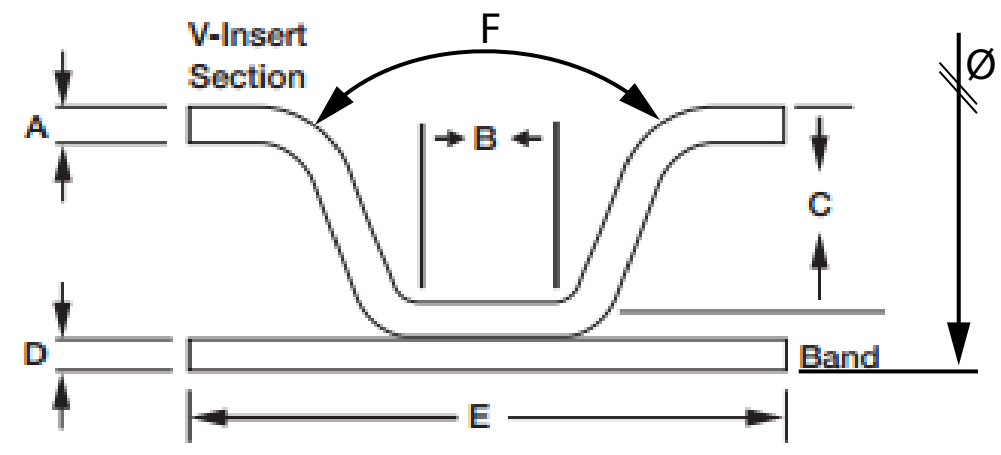

Figure 4.4: Cross-sectional view of the V-band clamp.

Table 4.2: Dimensions and installation torque of V-band clamps.

\begin{tabular}{ccccccccc}
\hline$\phi$ & $\mathrm{A}$ & $\mathrm{B}$ & $\mathrm{C}$ & $\mathrm{D}$ & $\mathrm{E}$ & $\mathrm{F}$ \\
$(\mathrm{mm})$ & $(\mathrm{mm})$ & $(\mathrm{mm})$ & $(\mathrm{mm})$ & $(\mathrm{mm})$ & $\begin{array}{c}\text { Ins. Torque } \\
(\mathrm{mm})\end{array}$ & $\begin{array}{c}\text { Post-installation } \\
(\mathrm{deg})\end{array}$ & $\begin{array}{c}\mathrm{N} \mathrm{m}) \\
\text { Radial Force }(\mathrm{N})\end{array}$ \\
125.5 & 1.52 & 4.6 & 7.14 & 0.89 & 19.05 & 40 & 5.7 & 4488 \\
149.4 & 1.27 & 10.16 & 7.14 & 1.02 & 22.23 & 40 & 8.5 & 6693 \\
\hline
\end{tabular}

Fig. 4.5 shows approximately how a V-band clamp would appear when installed on two mating parts of the air-supply system.

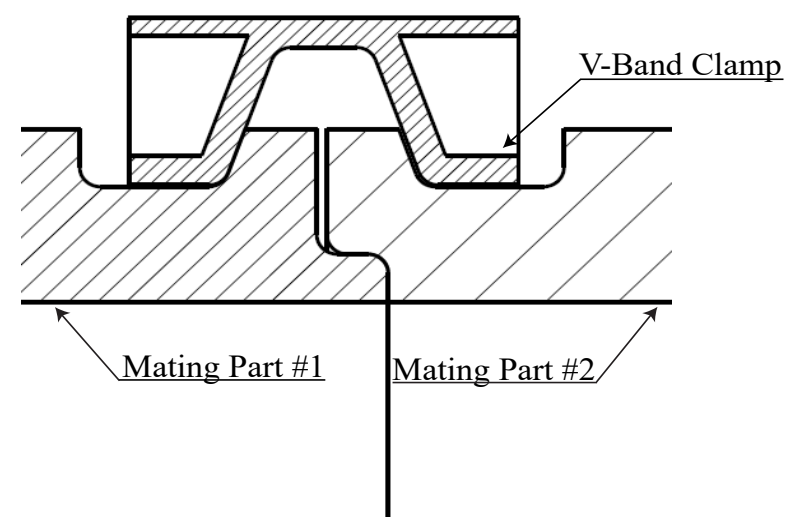

Figure 4.5: V-band clamp installation. 
The stress field in and the resultant deformation of the V-clamp under load are affected by the axial and radial components of the load applied to the clamp on its surfaces that are in contact with the parts being mated. This is illustrated schematically in Fig. 4.6. Furthermore, the load experienced by the clamp is not symmetric with respect to the mating plane if the mating parts are not symmetric at this plane due to asymmetric design features such as a tongue-and-groove interface, as depicted in Fig. 4.7.

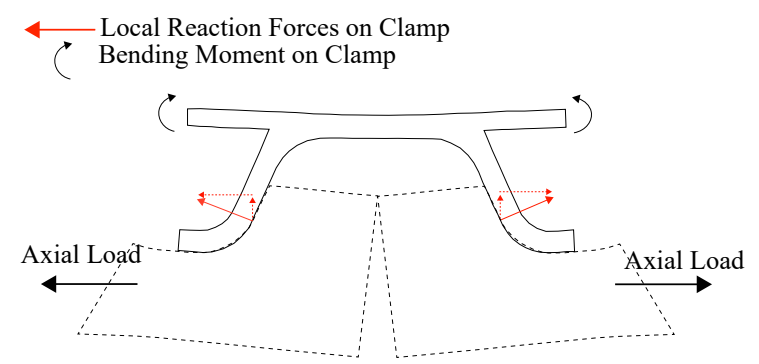

(a)

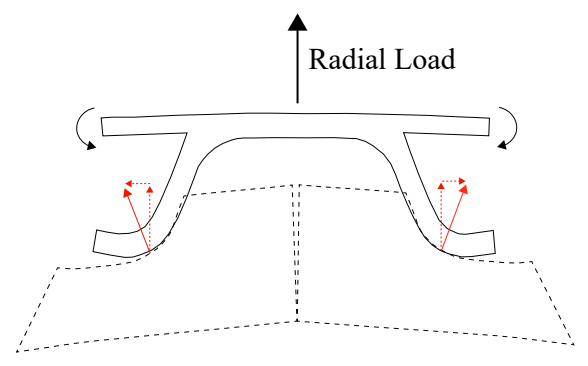

(b)

Figure 4.6: V-band clamp and joint deformation without tongue-and-groove under (a) dominantly axial load, and (b) dominantly radial load.

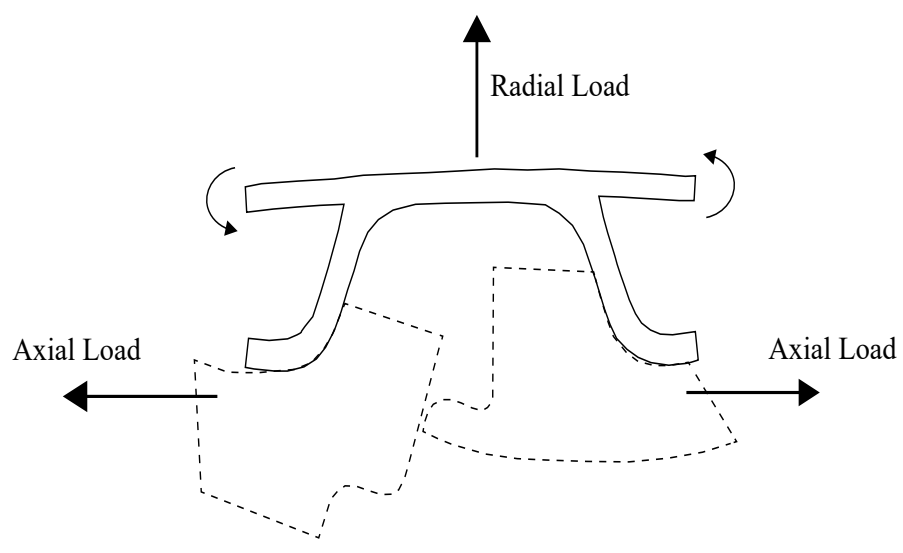

Figure 4.7: V-band clamp and joint deformation with tongue-and-groove geometry under combined axial and radial load. 


\subsection{Upstream Flange}

The upstream flange, shown in Fig. 4.8, is the first component of the air-supply system. Table 4.3 presents the design parameters of the flange. The flange is made of mild steel and is bolted to the existing steel pipe network of the facility. The flange has an inner diameter of $96 \mathrm{~mm}$ and a thickness of $25.4 \mathrm{~mm}$ based on ASME/ANSI code B16.5, which stipulates a minimum thickness of $22.3 \mathrm{~mm}$ for a Class 150 flange of this diameter (ASME, 2009). Eight holes of $20 \mathrm{~mm}$ diameter are evenly placed along the flange circumference. Every other hole in sequence is used for bolting the flange onto the above-noted existing flange of the wind-tunnel. The remaining holes are used with the pre-compression rods described in Section 4.2. A reusable fullface ANSI/ASME Class 150 rubber gasket of $1.5 \mathrm{~mm}$ thickness is installed between the flange and the existing flange. Fig. 4.9 shows the components involved in the assembly of the stated flange on the air-supply system.

Table 4.3: Design parameters for the upstream flange.

\begin{tabular}{cccc}
\hline Design Code & Press. Rating & Temp. Rating & ASTM Material Specifications \\
ASME B16.5/CSA B51 & $7 \mathrm{~atm}$ & $25^{\circ} \mathrm{C}$ & Steel (Table 4.5) \\
\hline
\end{tabular}



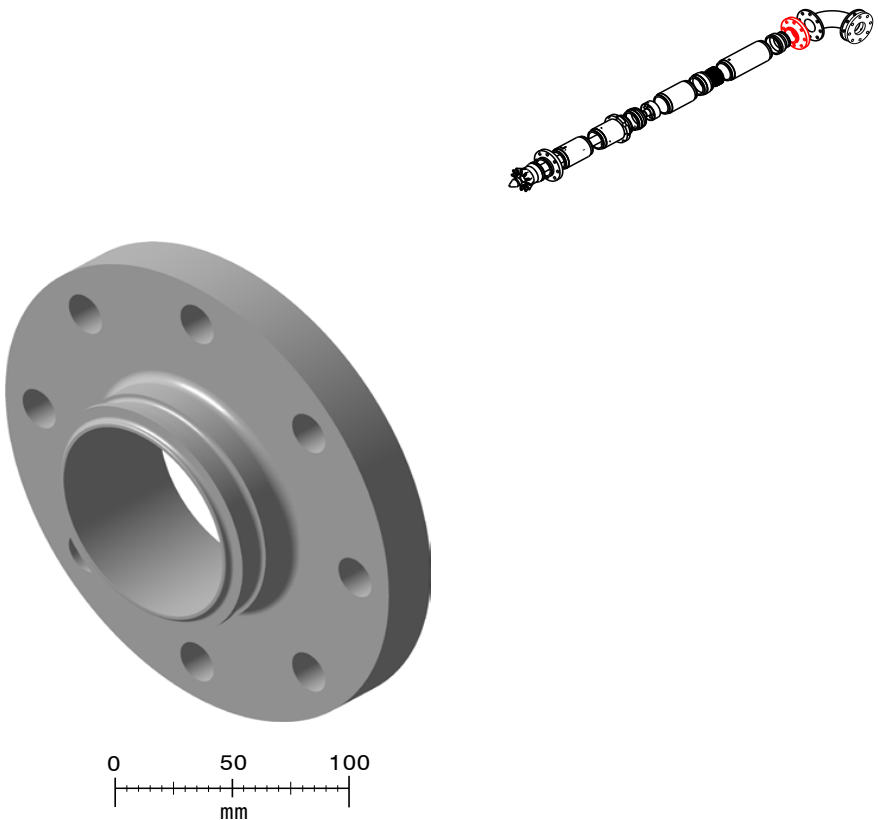

Figure 4.8: Upstream flange.

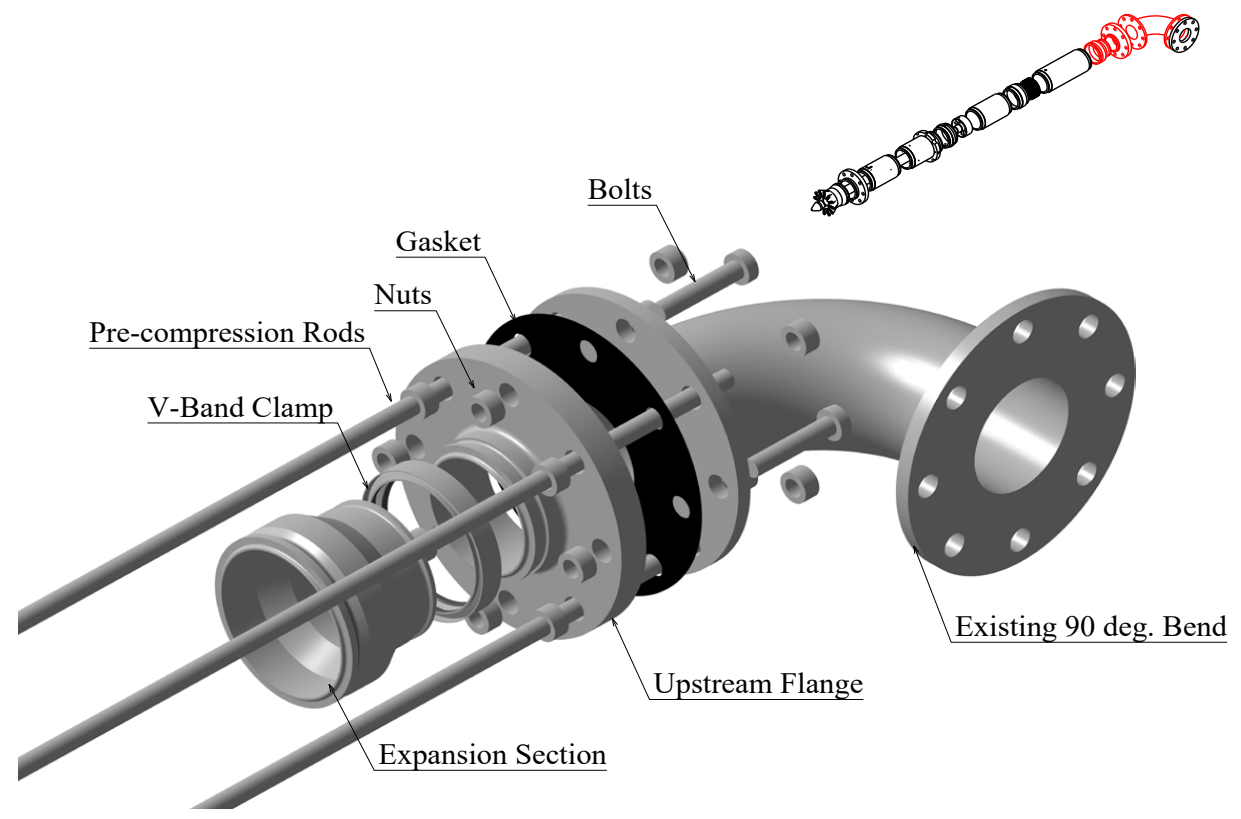

Figure 4.9: Upstream flange installation. 


\subsubsection{Structural Analysis}

\subsubsection{Computational Model}

To verify the structural integrity of the upstream flange, a static structural finite element analysis (FEA) is performed using ANSYS Mechanical APDL (version 19.2). Figs. 4.10 and 4.11 present the computational model for the structural analysis of the upstream flange. Table 4.4 presents the specifics of the computational grid. The grid consists of approximately 0.8 million tetrahedral elements, with approximately 0.3 million elements in the upstream flange. The elements are concentrated at the interfaces between the mating pipe segments and the V-band clamps to ensure adequate resolution of the stress gradients at the contact interfaces. To correctly capture the structural interaction of the upstream flange with its upstream and downstream mating parts, the computational model contains all three parts as a subassembly. The expansion section is replaced with a cylindrical segment, made of steel, terminated by a solid wall at its downstream end to simulate the extreme loading scenario of full blockage of the flow path that would pressurize the system to the level of the supply stagnation pressure. The contacts between the various parts are treated as frictional contacts with a friction coefficient of $\mu=0.2$. The results of the structural analysis showed very little sensitivity to the value of the friction coefficient so long as the value was nominally above zero to ensure that the numerical model was sufficiently constrained. 


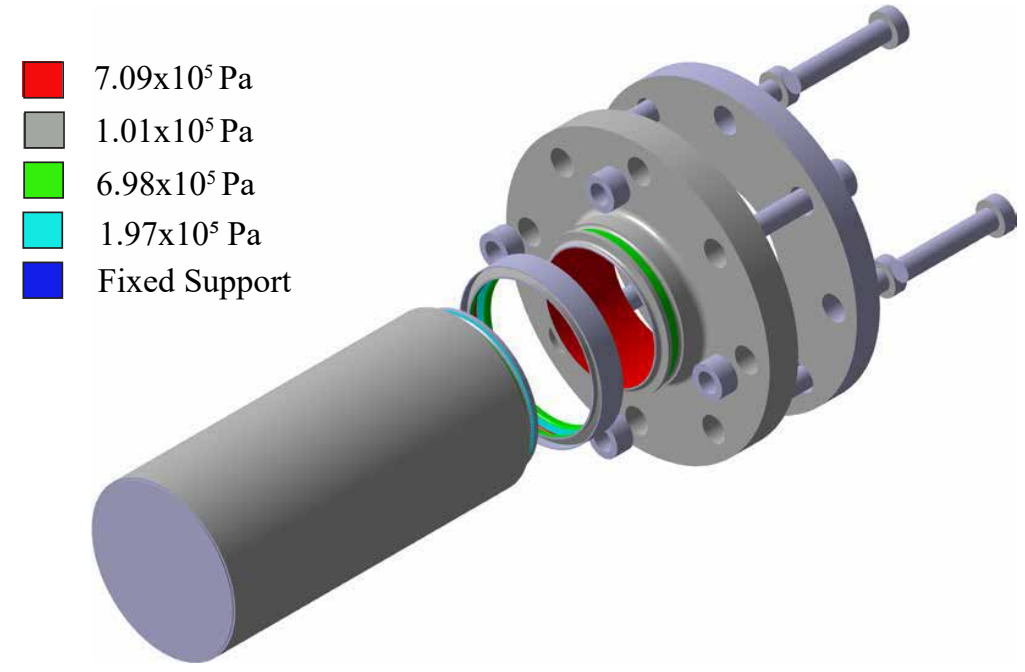

(a)

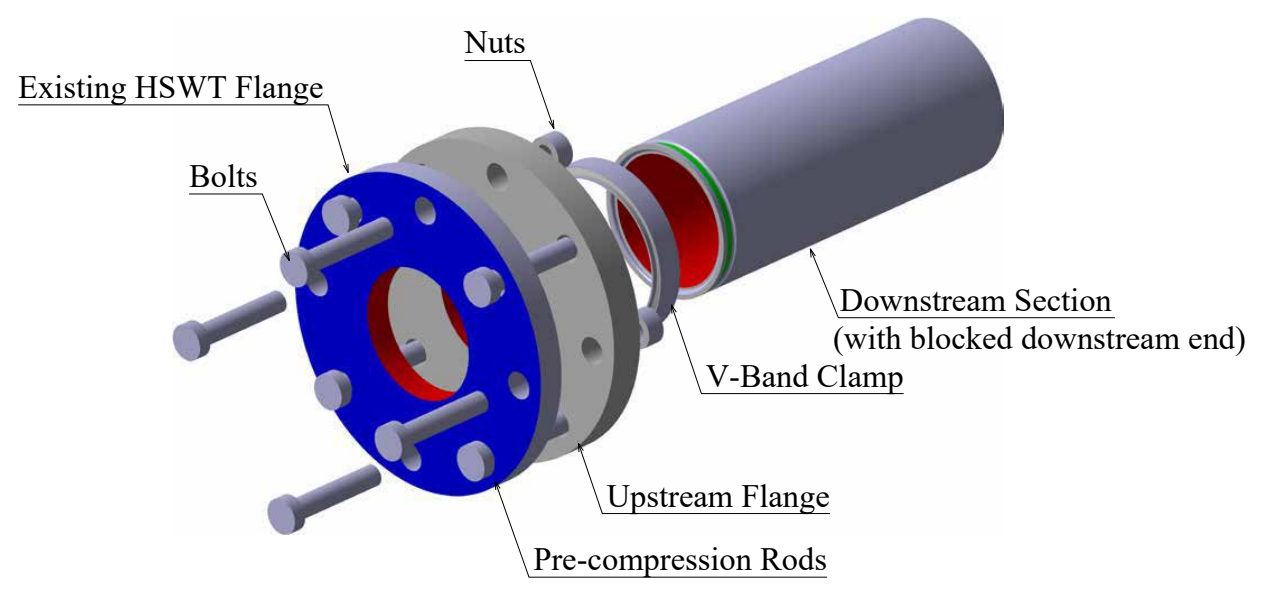

(b)

Figure 4.10: FEA computational model of the upstream flange as seen from the (a) downstream end; (b) upstream end. 

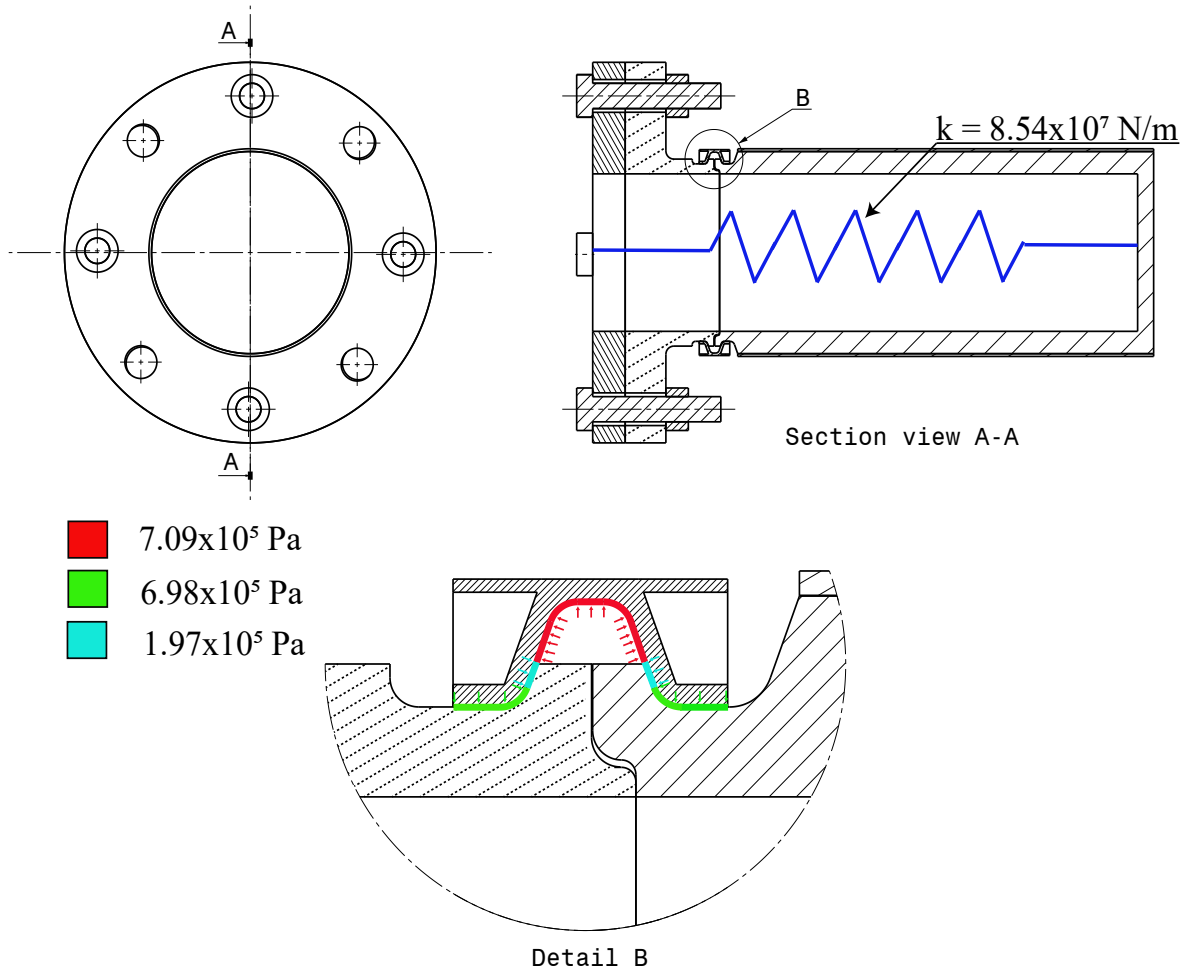

Figure 4.11: Schematic showing the loading of the V-band clamp and the location of the spring used to model the pre-compression rods.

Table 4.4: Parameters of the computational grid.

\begin{tabular}{lcc}
\hline & $\begin{array}{c}\text { Element size }(\mathrm{mm}) \\
\min / \max \end{array}$ & $\begin{array}{c}\text { Spatial growth rate } \\
\min / \max \end{array}$ \\
\hline Upstream Flange & $1.5 / 5.0$ & $0 \% / 15 \%$ \\
V-band Clamp & $1.0 / 1.0$ & $0 \%$ \\
\hline
\end{tabular}

The most upstream face of the assembly is constrained with a fixed support, which prevents all nodal deformation, translation, and rotation at this face, which is deemed to closely approximate the conditions that would prevail in the actual setup. The external faces of the parts are exposed to $1 \mathrm{~atm}$ and the internal faces are pressurized to the maximum supply stagnation pressure of 7 atm. Fig. 4.11 illustrates the loading of the V-band clamp. The installation clamping force is derived from the 
values listed in Table 4.2 and is applied appropriately where the clamp is in contact with the mating segments. The internal space of the $\mathrm{V}$-section is pressurized to 7 atm to simulate a leakage at the tongue-and-groove joint. The four bolts are installed with a bolt torque of $75 \mathrm{~N} \mathrm{~m}$, in accordance with typical ASME Class 150 gaskets. This load is applied in the computational model via a pre-tension force of $24,000 \mathrm{~N}$ on each bolt in the numerical model. A single spring with a longitudinal stiffness of $8.54 \times 10^{7} \mathrm{~N} / \mathrm{m}$ connects the upstream ends of the pre-compression rods to the downstream section to account for the stiffness of the four rods as shown in Fig. 4.11. The spring is initially pre-tensioned with a $1500 \mathrm{~N}$ axial load (corresponding to $375 \mathrm{~N}$ per pre-compression rod) which serves to pre-compress the subassembly. This choice of pre-compression load was determined through iterations until the stress and deformation levels in the components met the desired stress safety factors and deformation levels. Table 4.5 presents the mechanical properties of the materials used in the structural analysis.

Table 4.5: Mechanical properties of the materials in the subassembly.

\begin{tabular}{lc}
\hline & Steel \\
Density $\left(\mathrm{kg} / \mathrm{m}^{3}\right)$ & 8000 \\
Tensile yield strength (MPa) & 250 \\
Compressive yield strength (MPa) & 250 \\
Young's modulus (GPa) & 200 \\
Poisson's ratio & 0.29 \\
\hline
\end{tabular}

\subsubsection{Computational Results}

Fig. 4.12 presents the Von Mises stress distribution in the upstream flange and the V-band clamps. Stresses and deformations in the other components of the subassembly are not presented as these parts exist only to properly constrain the components of interest in this analysis. Table 4.6 presents a summary of the structural analysis results. Accuracy of the FEA results was established through a benchmark study presented in Appendix C. The simulation was repeated with zero pre-compression load to establish the effect of the pre-compression load on the stresses and deformations of the parts. The magnitude of the pre-compression load was found to be inconsequential. 
However, the longitudinal stiffness that is added to the system by the pre-compression rods is vital to limiting the axial deformation, and therefore stresses, in the parts. The stresses and deformations in the upstream flange and the V-band clamps confirm the structural integrity of the parts under the prescribed loading conditions.

In the subsequent sections, a similar structural analysis to the one presented here is performed for each component of the air-supply system. Unless otherwise specified, these subsequent analyses use identical computational setups as the present component in terms of grid element distributions, friction coefficients, material properties, geometric constraints, pre-compression loads, and internal and external surface pressures.
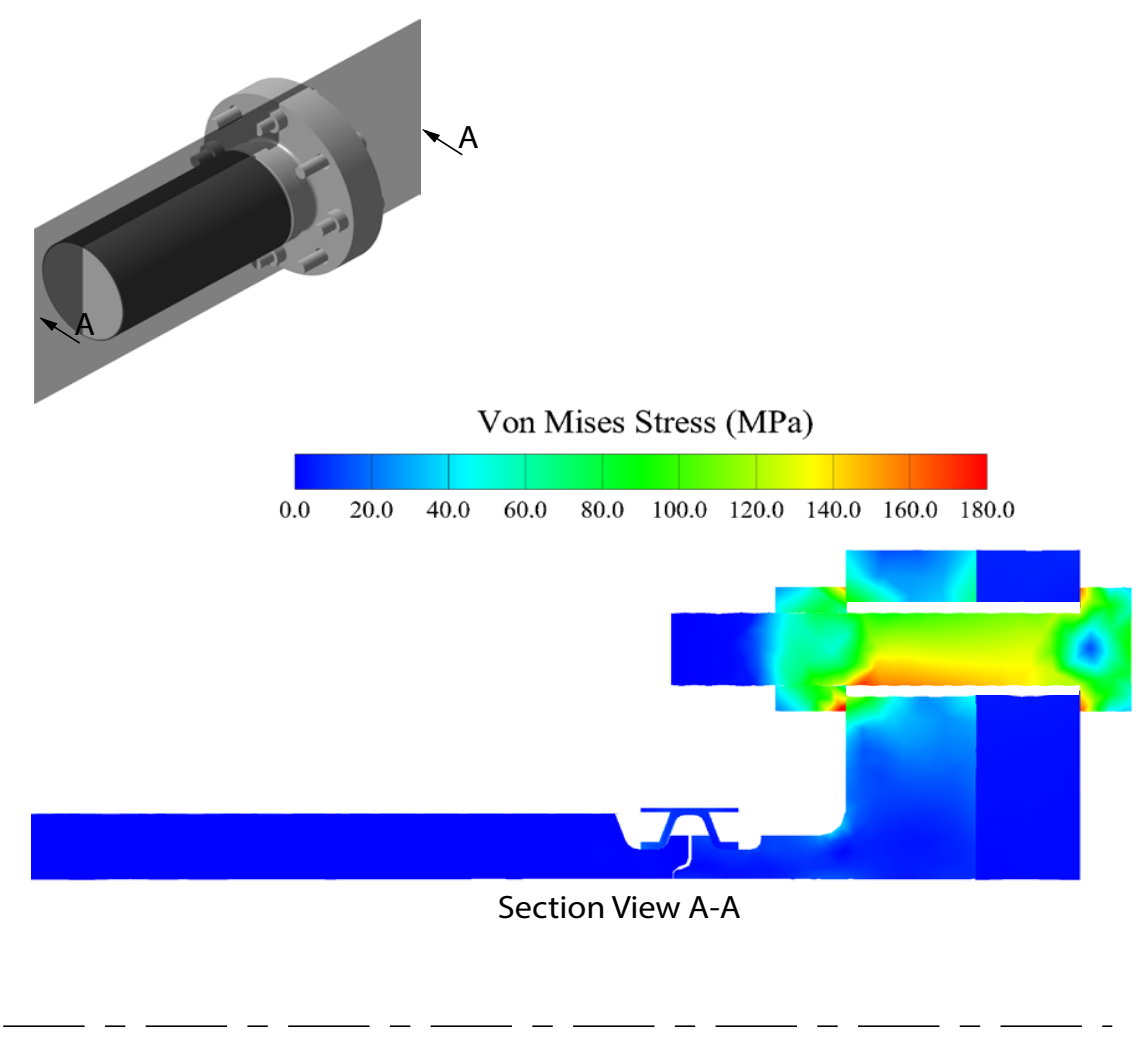

Figure 4.12: Von Mises stress distribution in the upstream flange subassembly. 
Table 4.6: Summary of the structural analysis results for the upstream flange subassembly.

\begin{tabular}{|c|c|c|c|c|c|}
\hline Component & $\begin{array}{l}\text { Pre-compression } \\
\text { Load }(\mathrm{N})\end{array}$ & $\begin{array}{c}\text { Max. } \\
\text { Deformation } \\
\left(\mathrm{x} 10^{-2} \mathrm{~mm}\right)\end{array}$ & $\begin{array}{c}\text { Max. Stress } \\
(\mathrm{MPa})\end{array}$ & $\begin{array}{c}\text { Min. Safety } \\
\text { Factor }\end{array}$ & $\begin{array}{l}\text { TSSA Required } \\
\text { Safety Factor }{ }^{1}\end{array}$ \\
\hline \multirow[t]{2}{*}{ Upstream Flange } & 1500 & $5.67 \pm 0.17$ & $62.2 \pm 1.9$ & $4.0 \pm 0.1$ & 4.0 \\
\hline & 0 & $5.67 \pm 0.17$ & $62.2 \pm 1.9$ & $4.0 \pm 0.1$ & 4.0 \\
\hline \multirow[t]{2}{*}{ V-band Clamp } & 1500 & $1.95 \pm 0.06$ & $24.5 \pm 0.7$ & $10.2 \pm 0.3$ & 4.0 \\
\hline & 0 & $1.95 \pm 0.06$ & $24.5 \pm 0.7$ & $10.2 \pm 0.3$ & 4.0 \\
\hline
\end{tabular}




\subsection{Expansion Section}

The test section must be large enough such that the aerodynamic phenomena of interest are of sufficient spatial scale for accurate resolution by the available measurement techniques. As such, an expansion section, depicted in Fig. 4.13, is incorporated into the flow path. Table 4.7 presents the design parameters of the expansion section. The section has a length of $100 \mathrm{~mm}$, an exit diameter of $122.2 \mathrm{~mm}$, and an exit-to-inlet area ratio of 1.62. Although a more gradual diffusion of the flow over a longer streamwise length would be aerodynamically more efficient, manufacturing limitations necessitated the use of this shorter, more abrupt expansion section. The section is made of mild steel with a wall thickness of $9.5 \mathrm{~mm}$ and mates with the upstream and downstream sections using V-band clamps, as illustrated in Fig. 4.14.

Table 4.7: Design parameters for the expansion section.

\begin{tabular}{cccc} 
Design Code & Press. Rating & Temp. Rating & ASTM Material Specifications \\
ASME B31.1/CSA B51 & $7 \mathrm{~atm}$ & $25^{\circ} \mathrm{C}$ & Steel (Table 4.9) \\
\hline
\end{tabular}

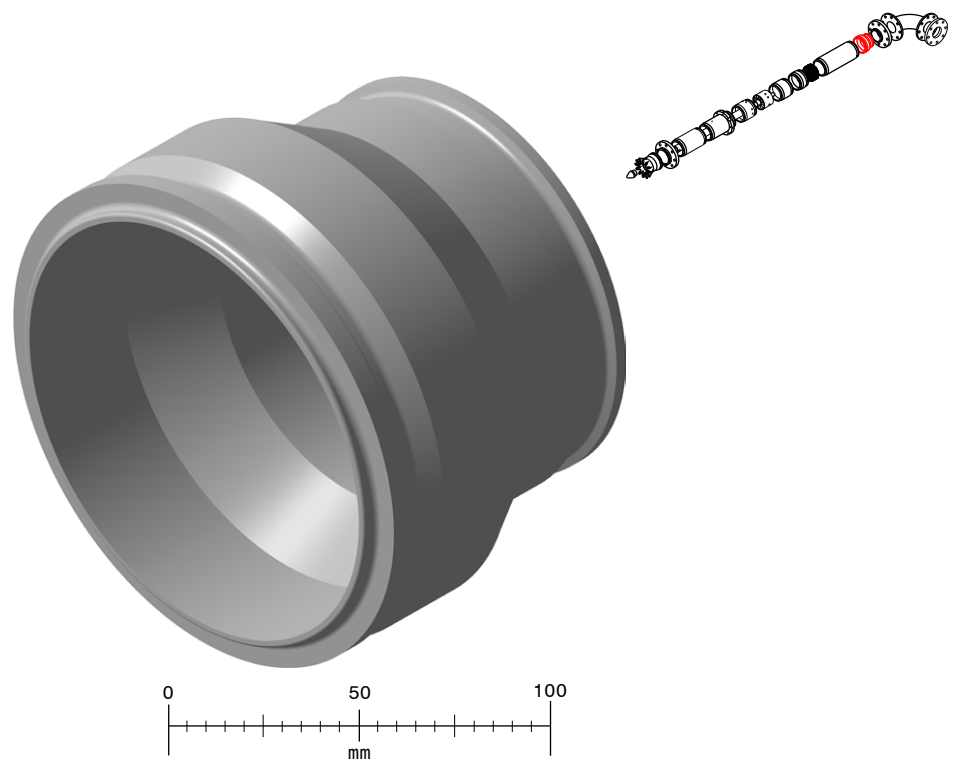

Figure 4.13: Expansion section. 


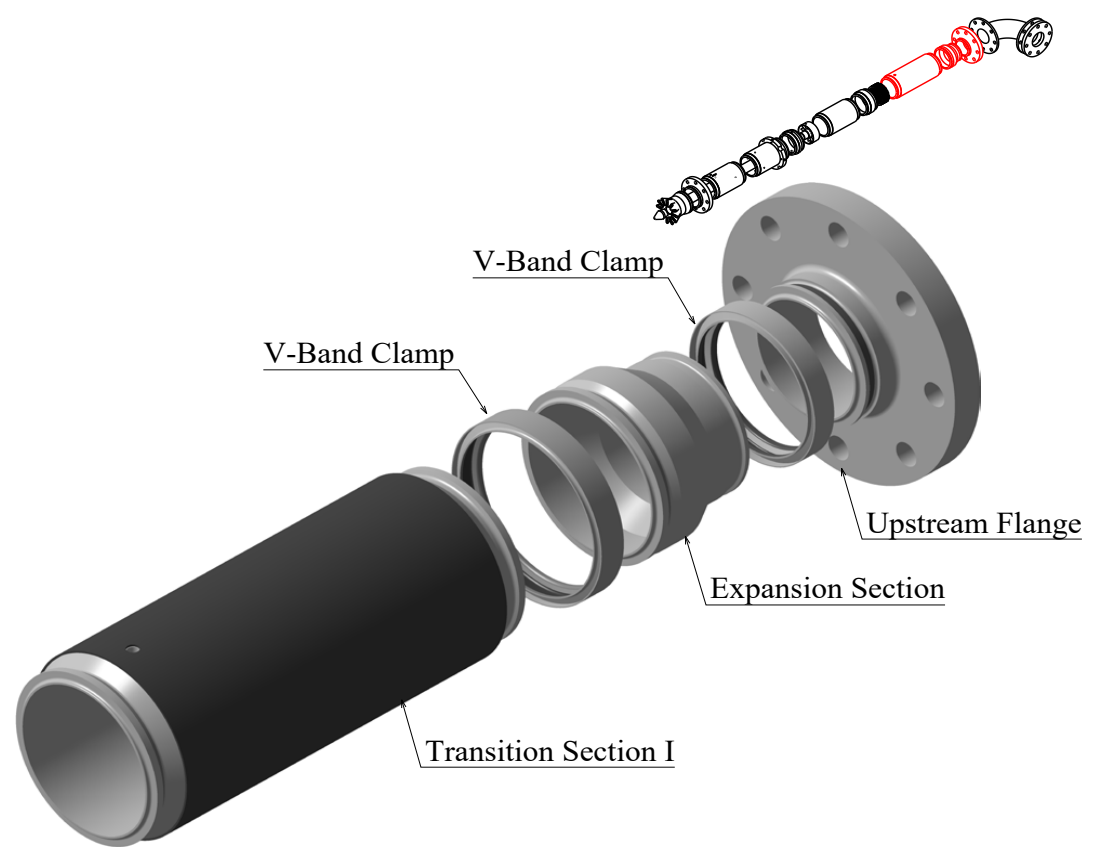

Figure 4.14: Expansion section installation.

\subsubsection{Structural Analysis}

\subsubsection{Computational Model and Results}

Figs. 4.15 and 4.16 present the computational model for the structural analysis of the expansion section. The downstream component (transition section I in this instance) is replaced with a cylindrical section made of the same material (HDPE and Steel) as the downstream section. Table 4.8 presents the specifics of the computational grid, which consists of approximately 1.0 million tetrahedral elements, with approximately 0.4 million elements in the expansion section. Fig. 4.17 presents the Von Mises stress distribution in the parts of the subassembly. Table 4.10 presents a summary of the structural analysis results. The stresses and deformations in the expansion section and the V-band clamps under the prescribed loading conditions confirm the structural integrity of the parts. 


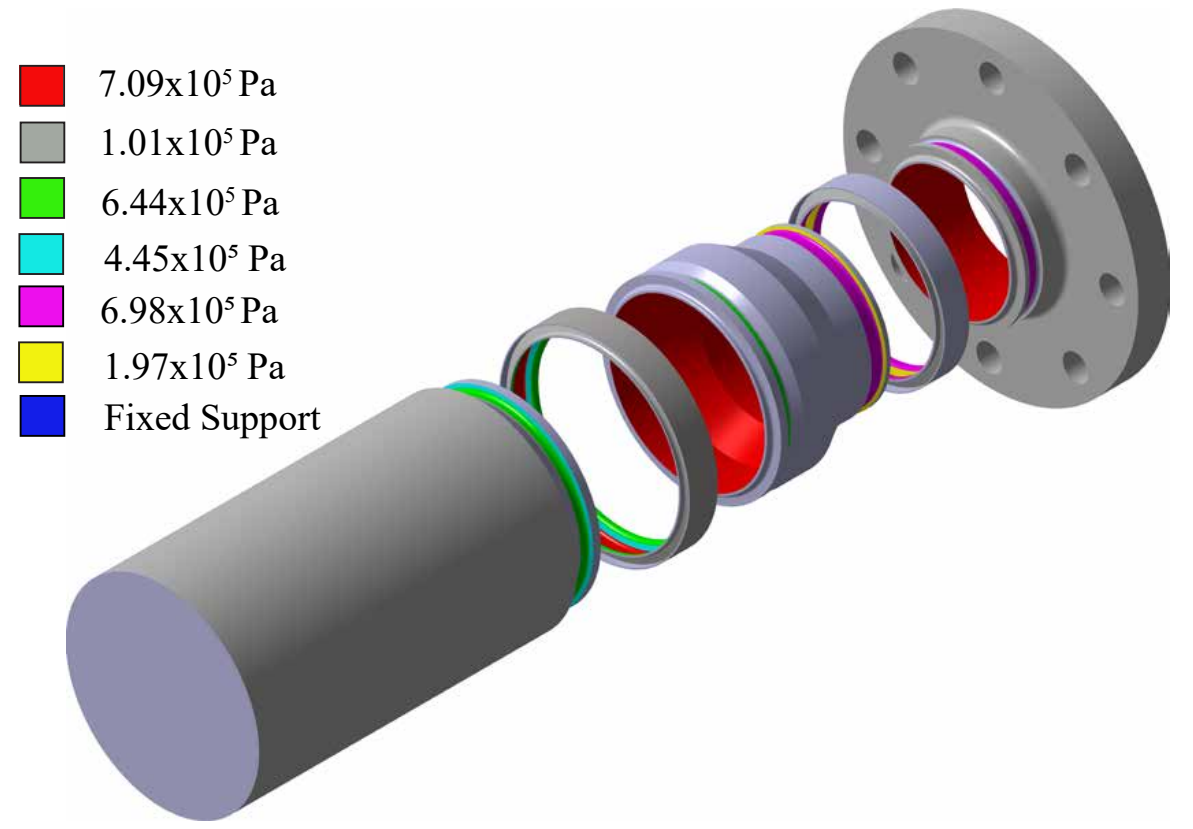

(a)

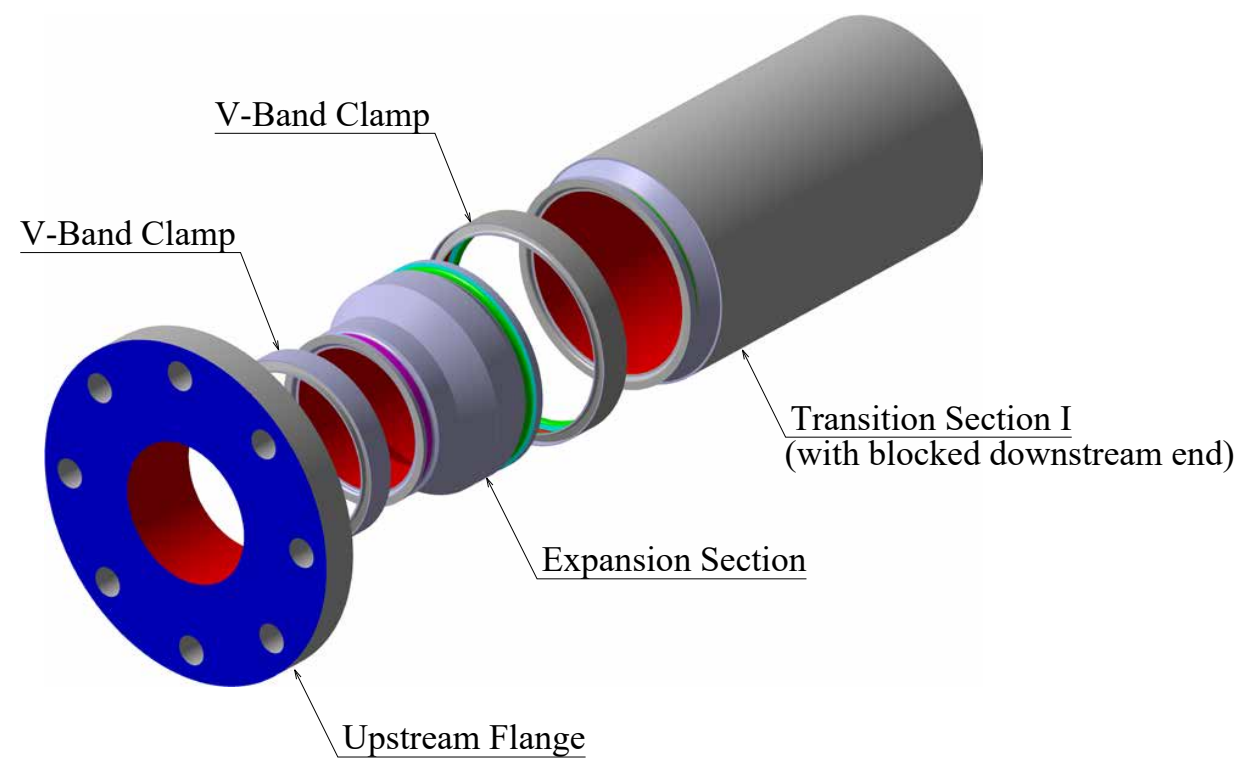

(b)

Figure 4.15: FEA computational model of expansion section as seen from the (a) downstream end; (b) upstream end. 

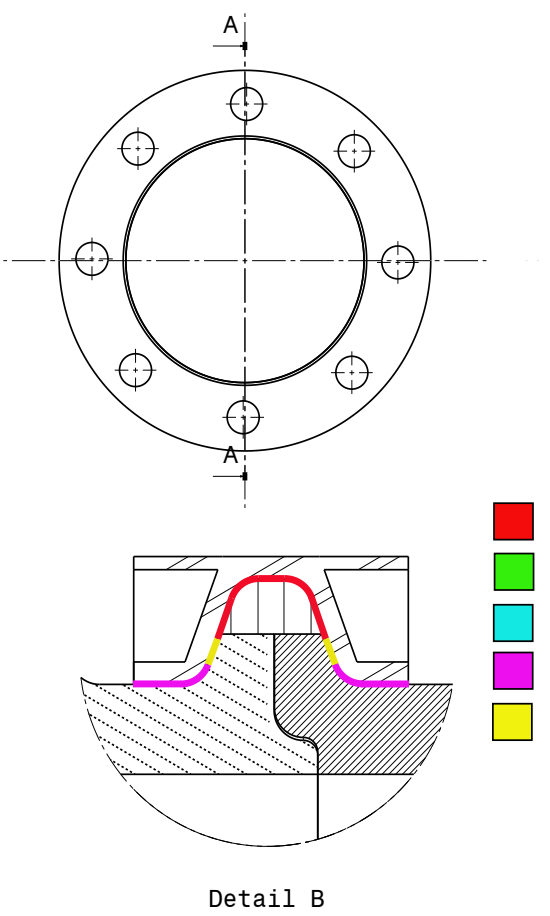

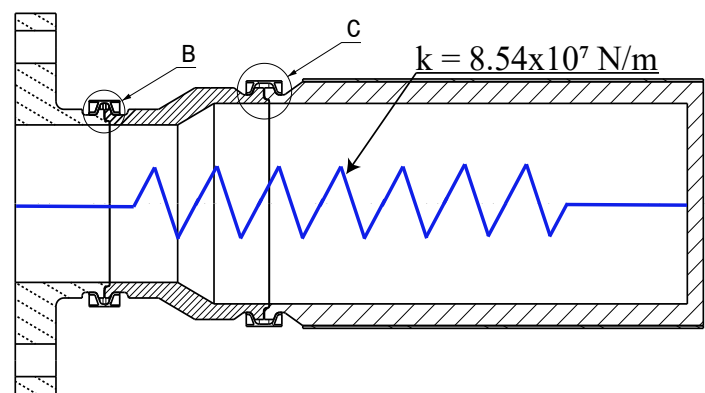

Section view A-A

$7.09 \times 10^{5} \mathrm{~Pa}$

$6.44 \times 10^{5} \mathrm{~Pa}$

$4.45 \times 10^{5} \mathrm{~Pa}$

$6.98 \times 10^{5} \mathrm{~Pa}$

$1.97 \times 10^{5} \mathrm{~Pa}$

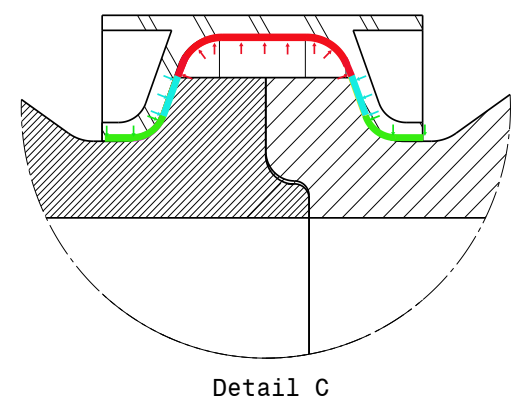

Figure 4.16: Schematic showing the loading of the V-band clamp and the location of the spring used to model the pre-compression rods.

Table 4.8: Parameters of the computational grid.

\begin{tabular}{lcc}
\hline & $\begin{array}{c}\text { Element size }(\mathrm{mm}) \\
\min / \max \end{array}$ & $\begin{array}{c}\text { Spatial Growth rate } \\
\min / \max \end{array}$ \\
\hline Expansion Section & $1.5 / 5.0$ & $0 \% / 15 \%$ \\
V-band Clamp & $1.0 / 1.0$ & $0 \%$ \\
\hline
\end{tabular}

Table 4.9: Mechanical properties of the materials in the subassembly.

\begin{tabular}{lcc}
\hline & HDPE & Steel \\
Density $\left(\mathrm{kg} / \mathrm{m}^{3}\right)$ & 958 & 8000 \\
Tensile yield strength (MPa) & 25.7 & 250 \\
Compressive yield strength (MPa) & 11.0 & 250 \\
Young's modulus (GPa) & 1.08 & 200 \\
Poisson's ratio & 0.42 & 0.29 \\
\hline
\end{tabular}




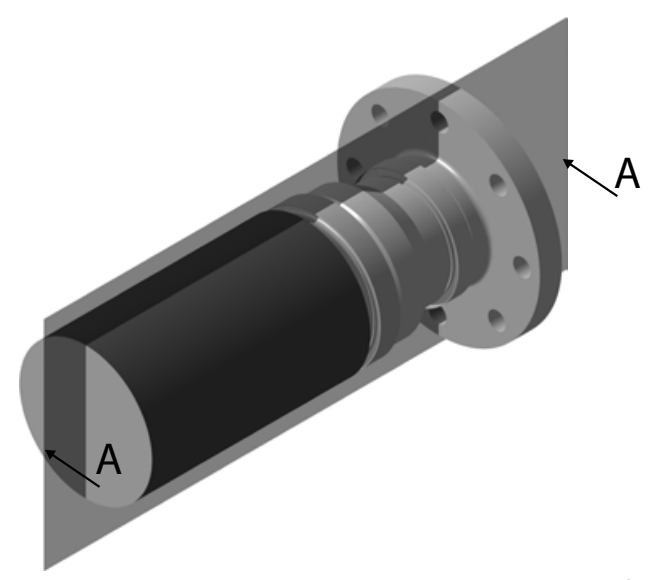

Von Mises Stress (MPa)
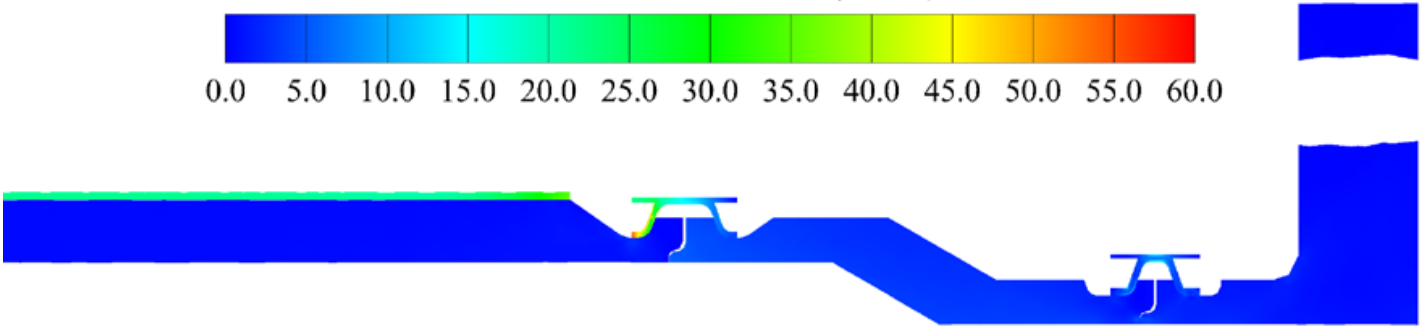

Section View A-A

$-$

Figure 4.17: Von Mises stress distribution in the sudden expansion subassembly.

Table 4.10: Summary of the structural analysis results for the sudden expansion subassembly.

\begin{tabular}{|c|c|c|c|c|c|}
\hline Component & $\begin{array}{l}\text { Pre-compression } \\
\text { Load }(\mathrm{N})\end{array}$ & $\begin{array}{c}\text { Max. } \\
\text { Deformation } \\
\left(\mathrm{x} 10^{-2} \mathrm{~mm}\right)\end{array}$ & $\begin{array}{l}\text { Max. Stress } \\
(\mathrm{MPa})\end{array}$ & $\begin{array}{l}\text { Min. Safety } \\
\text { Factor }\end{array}$ & $\begin{array}{l}\text { TSSA Required } \\
\text { Safety Factor }^{1}\end{array}$ \\
\hline Expansion & 1500 & $4.81 \pm 0.14$ & $9.7 \pm 0.3$ & $25.8 \pm 0.8$ & 4.0 \\
\hline Section & 0 & $4.81 \pm 0.14$ & $9.7 \pm 0.3$ & $25.8 \pm 0.8$ & 4.0 \\
\hline V-band Clamp & $\begin{array}{c}1500 \\
0\end{array}$ & $\begin{array}{l}4.81 \pm 0.14 \\
4.78 \pm 0.14\end{array}$ & $\begin{array}{l}59.0 \pm 1.8 \\
60.2 \pm 1.8\end{array}$ & $\begin{array}{l}4.2 \pm 0.1 \\
4.2 \pm 0.1\end{array}$ & $\begin{array}{l}4.0 \\
4.0\end{array}$ \\
\hline
\end{tabular}

${ }^{1}$ (TSSA, 2007) 


\subsection{Transition Section I}

Transition section I, shown in Fig. 4.18, is connected immediately downstream of the expansion section. Table 4.11 presents the design parameters of this section. The section has an inner diameter of $122.2 \mathrm{~mm}$ and a length of $367 \mathrm{~mm}$. The length of this section was chosen to allow for the flow to establish a more uniform flow profile over a length of three cross-section diameters (Miller, 1978). The section is made of HDPE with a wall thickness of $13.3 \mathrm{~mm}$ inserted in a steel sleeve of $1.65 \mathrm{~mm}$ wall thickness. Such a composite design was opted for to simultaneously achieve both the required strength and the desired light-weight. A $9.5 \mathrm{~mm}$-diameter port near the downstream end of the section allows for the installation of a flow measurement probe. The section mates with the upstream and downstream sections using V-band clamps, as illustrated in Fig. 4.19.

Table 4.11: Design parameters for transition section I.

\begin{tabular}{cccc}
\hline Design Code & Press. Rating & Temp. Rating & ASTM Material Specifications \\
ASME B31.1/CSA B51 & $7 \mathrm{~atm}$ & $25^{\circ} \mathrm{C}$ & HDPE, Steel (Table 4.9) \\
\hline
\end{tabular}




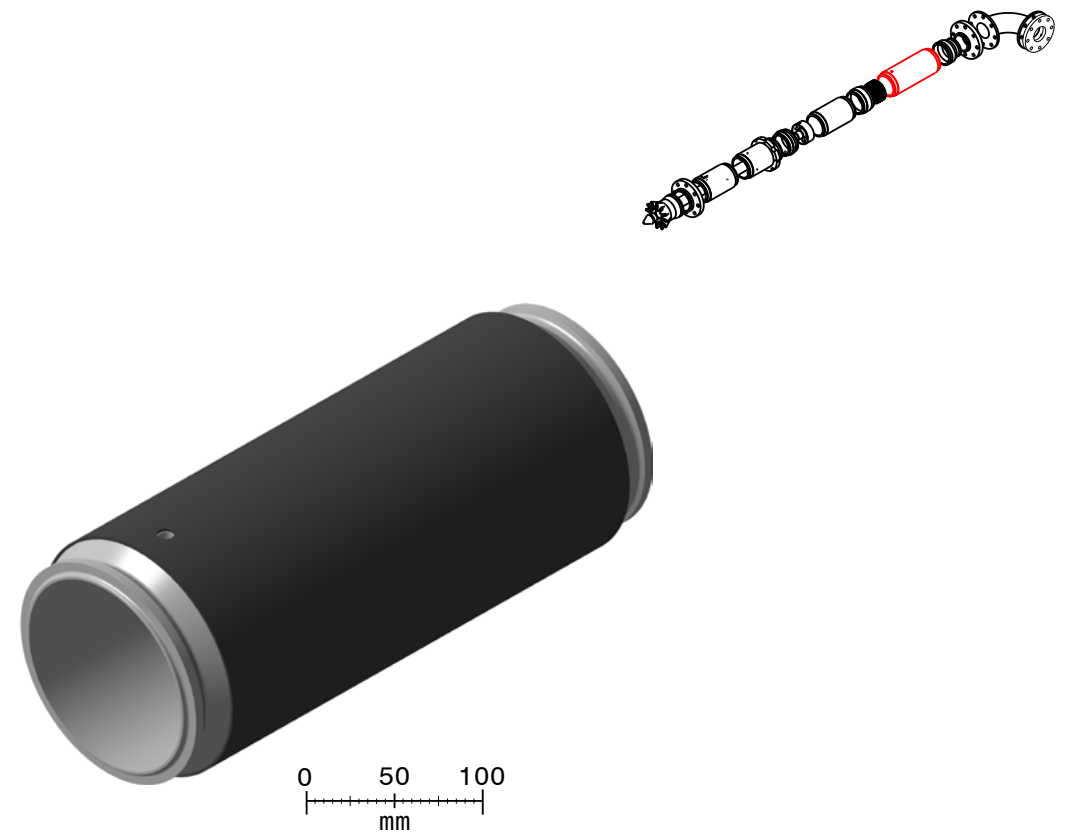

Figure 4.18: Transition section I.

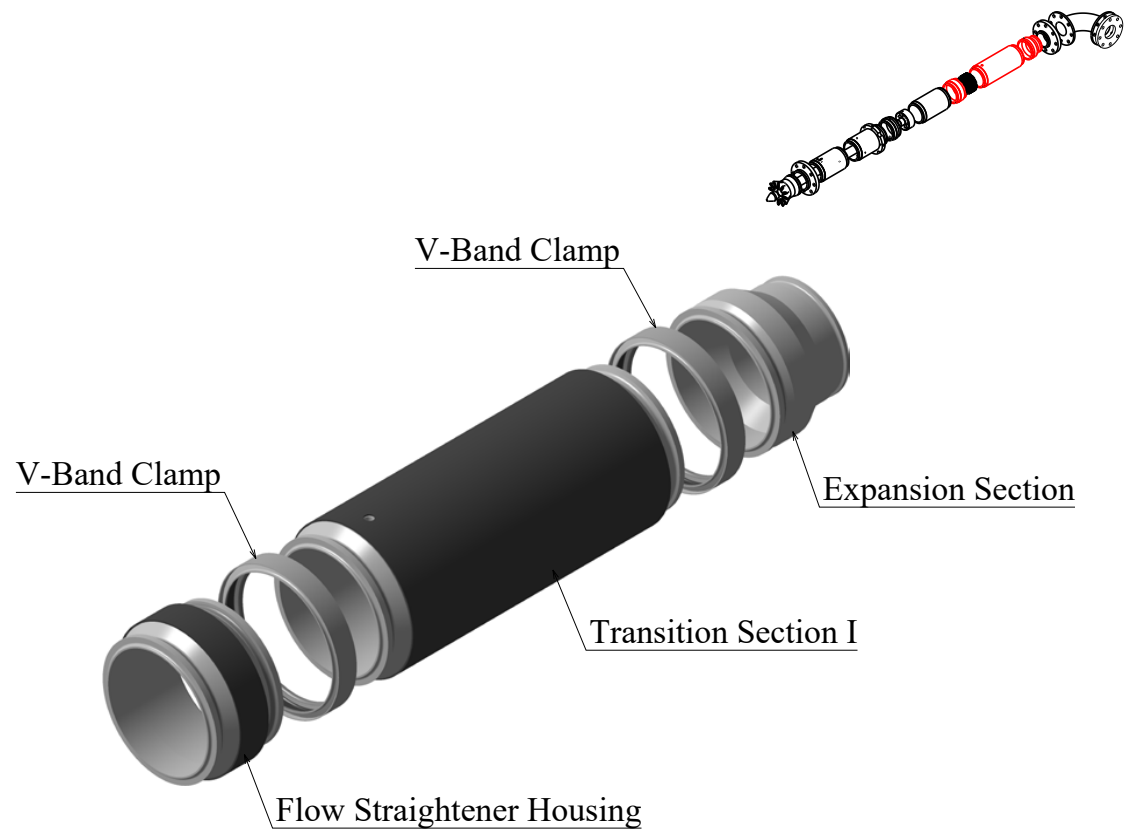

Figure 4.19: Transition section I installation. 


\subsubsection{Structural Analysis}

\subsubsection{Computational Model and Results}

Figs. 4.20 and 4.21 present the computational model for the structural analysis of transition section I. Table 4.12 presents the specifics of the computational grid. The grid consists of approximately 1.3 million tetrahedral elements, with approximately 0.2 million elements in the steel sleeve and 0.5 million elements in the HDPE housing. Fig. 4.22 presents the Von Mises stress distribution in the parts under the loading shown in Fig. 4.20. Table 4.13 presents a summary of the structural analysis results. The stresses and deformations in the transition section I components and the V-band

clamps confirm the structural integrity of the parts under the prescribed loading conditions. 


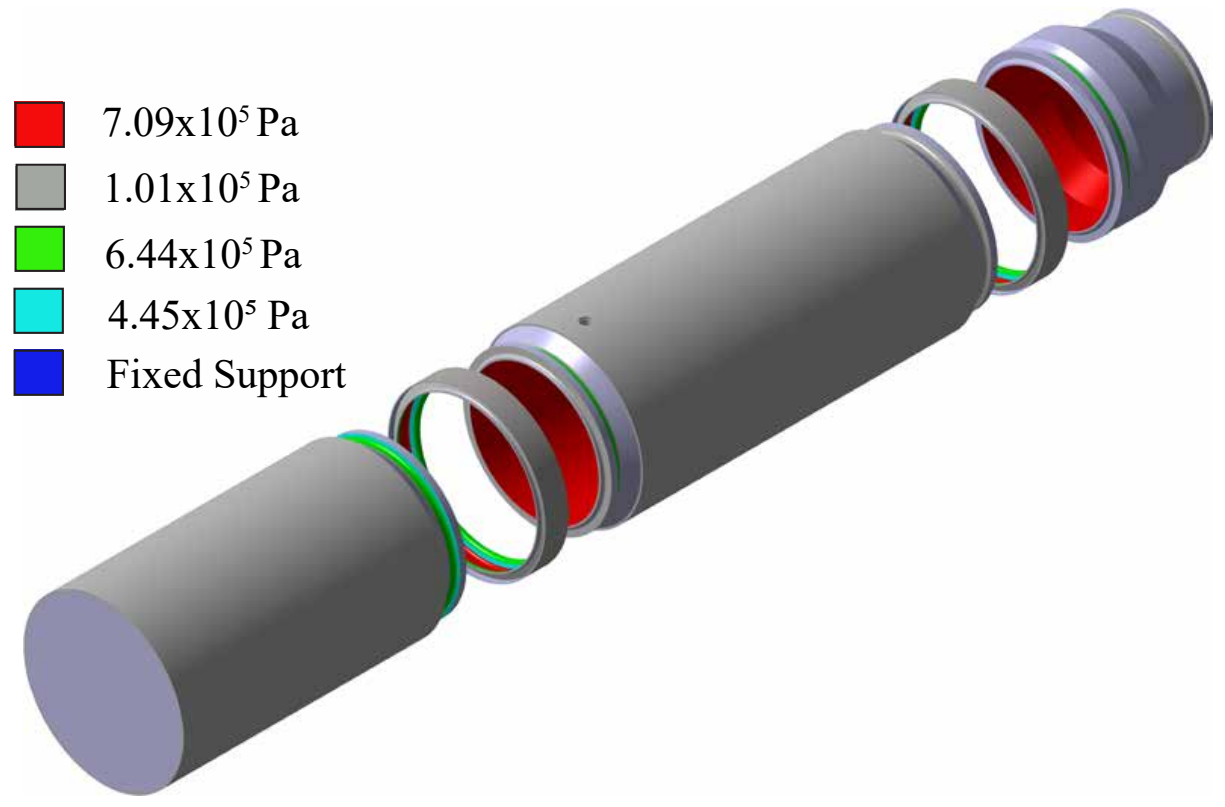

(a)

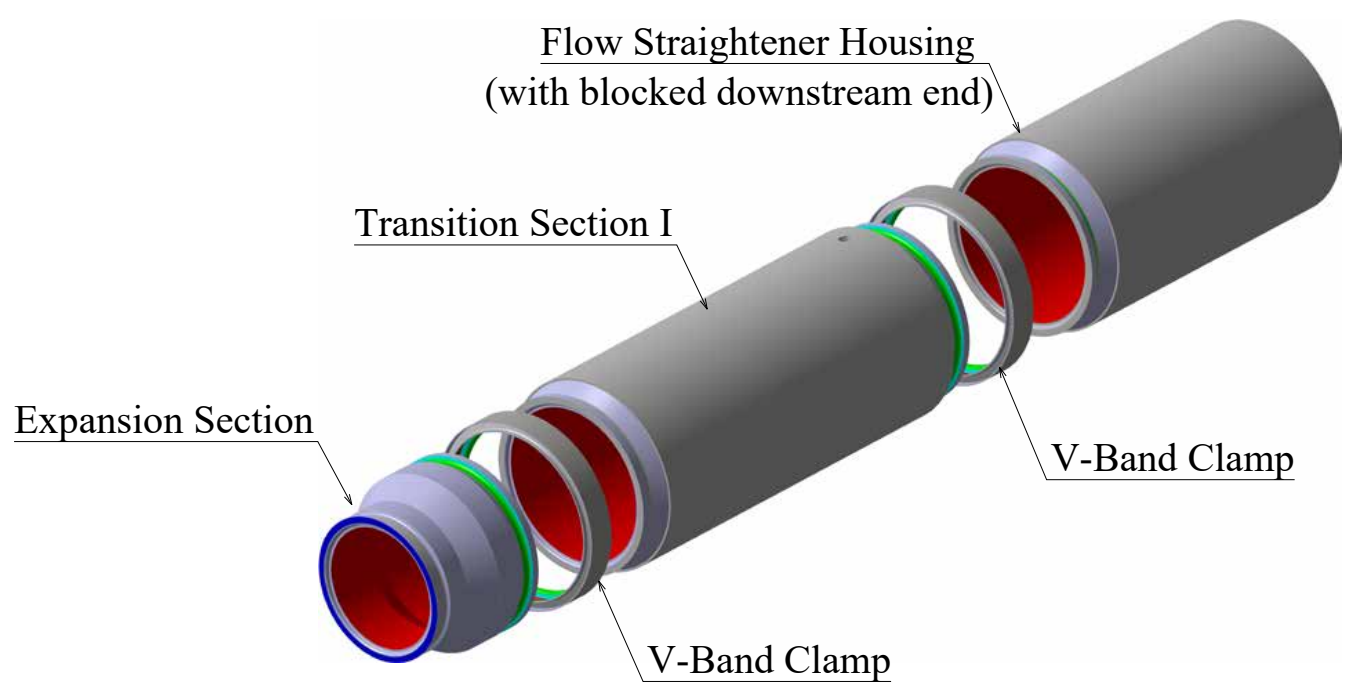

(b)

Figure 4.20: FEA computational model of transition section I as seen from the (a) downstream end; (b) upstream end. 

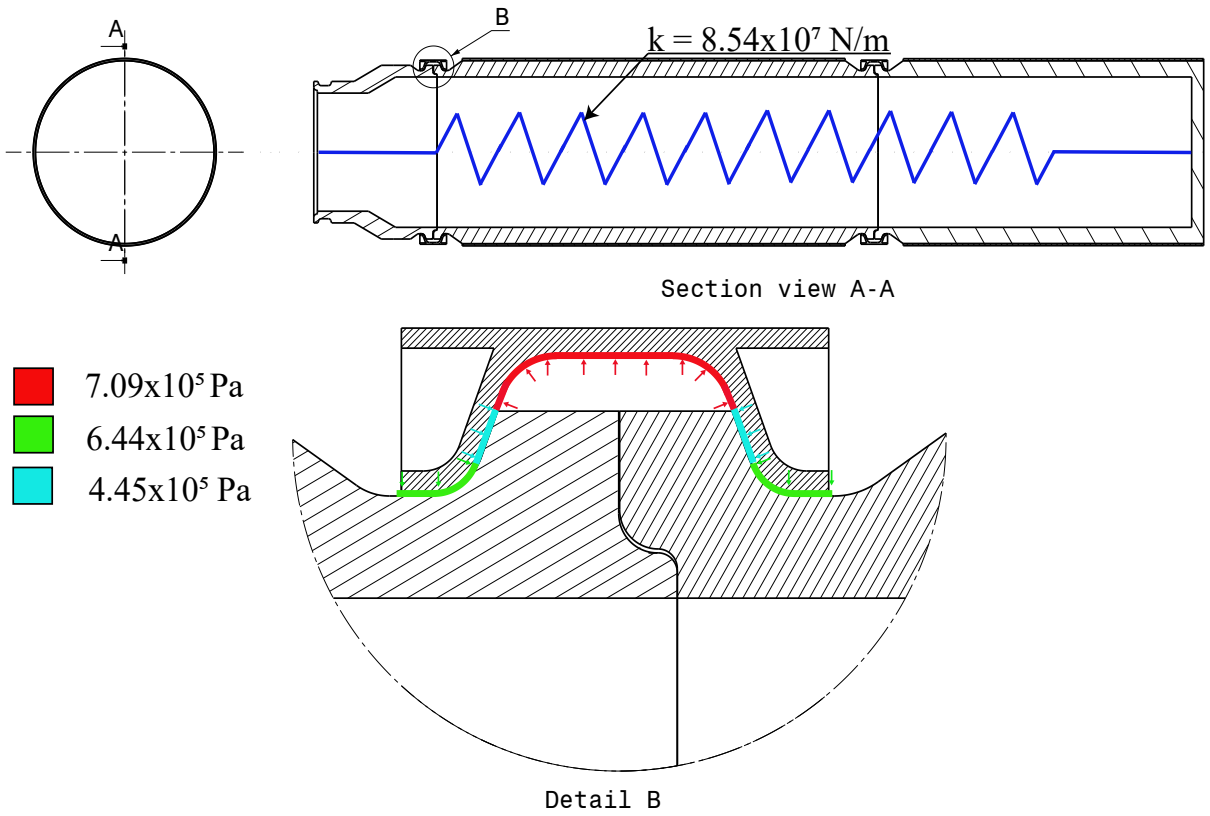

Figure 4.21: Schematic showing the loading of the V-band clamp and the location of the spring used to model the pre-compression rods.

Table 4.12: Parameters of the computational grid.

\begin{tabular}{lcc}
\hline & $\begin{array}{c}\text { Element size }(\mathrm{mm}) \\
\min / \max \end{array}$ & $\begin{array}{c}\text { Spatial Growth rate } \\
\min / \max \end{array}$ \\
\hline HDPE Pipe & $1.5 / 5.0$ & $0 \% / 15 \%$ \\
V-band Clamp & $1.0 / 1.0$ & $0 \%$ \\
Steel Sleeve & $0.5 / 0.5$ & $0 \%$ \\
\hline
\end{tabular}




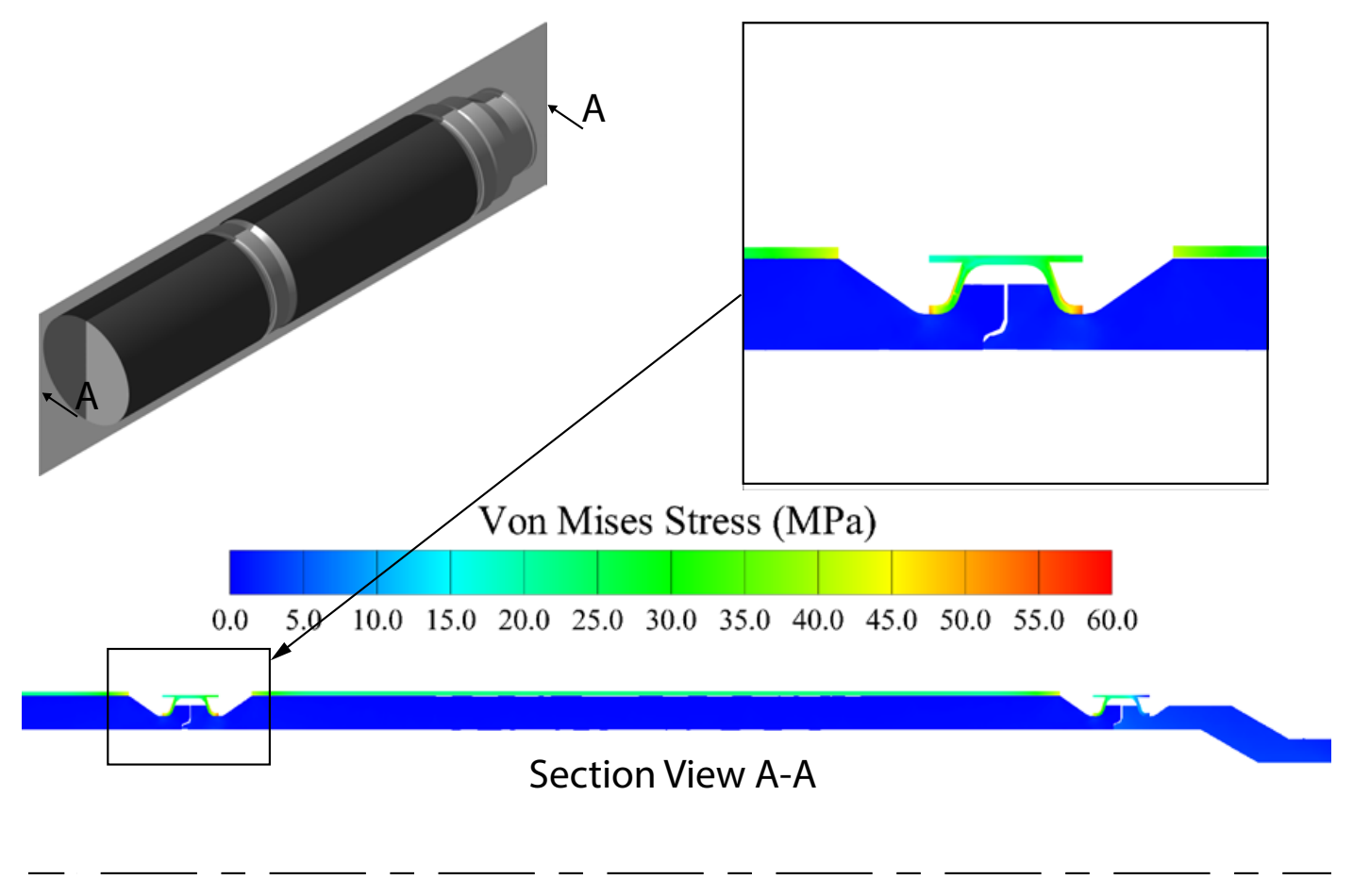

Figure 4.22: Von mises stress distribution in the transition section I subassembly.

Table 4.13: Summary of the structural analysis results for the transition section I subassembly.

\begin{tabular}{|c|c|c|c|c|c|}
\hline Component & $\begin{array}{l}\text { Pre-compression } \\
\text { Load }(\mathrm{N})\end{array}$ & $\begin{array}{c}\text { Max. } \\
\text { Deformation } \\
\left(\mathrm{x} 10^{-2} \mathrm{~mm}\right) \\
\end{array}$ & $\begin{array}{c}\text { Max. Stress } \\
\text { (MPa) }\end{array}$ & $\begin{array}{c}\text { Min. Safety } \\
\text { Factor }\end{array}$ & $\begin{array}{l}\text { TSSA Required } \\
\text { Safety Factor }^{1}\end{array}$ \\
\hline HDPE Pipe & $\begin{array}{c}1500 \\
0\end{array}$ & $\begin{array}{l}11.9 \pm 0.4 \\
12.9 \pm 0.4 \\
\end{array}$ & $\begin{array}{l}2.5 \pm 0.1 \\
2.5 \pm 0.1\end{array}$ & $\begin{array}{l}10.4 \pm 0.3 \\
10.3 \pm 0.3 \\
\end{array}$ & $\begin{array}{l}10.0 \\
10.0\end{array}$ \\
\hline V-band Clamp & $\begin{array}{c}1500 \\
0\end{array}$ & $\begin{array}{l}3.01 \pm 0.09 \\
3.97 \pm 0.12\end{array}$ & $\begin{array}{l}54.4 \pm 1.6 \\
55.6 \pm 1.7 \\
\end{array}$ & $\begin{array}{l}4.6 \pm 0.1 \\
4.5 \pm 0.1\end{array}$ & $\begin{array}{l}4.0 \\
4.0 \\
\end{array}$ \\
\hline Steel Sleeve & $\begin{array}{c}1500 \\
0\end{array}$ & $\begin{array}{l}3.36 \pm 0.10 \\
4.10 \pm 0.12\end{array}$ & $\begin{array}{l}45.0 \pm 1.4 \\
45.6 \pm 1.4\end{array}$ & $\begin{array}{l}5.6 \pm 0.2 \\
5.5 \pm 0.2\end{array}$ & $\begin{array}{l}4.0 \\
4.0\end{array}$ \\
\hline
\end{tabular}

${ }^{1}$ (TSSA, 2007) 


\subsection{Honeycomb Flow Straightener}

To remove any secondary flows downstream of the 90-deg. bend, a honeycomb flowstraightener is installed in the flow path. The cell width of the honeycomb insert is set to $6.35 \mathrm{~mm}$, which is approximately $7 \%$ of the local diameter of the duct. The length of the honeycomb is set to $50.8 \mathrm{~mm}$, which yields a cell length-to-width ratio of 8.0. The honeycomb, which has an estimated pressure loss coefficient of $K=0.3$, is made of commercially available $0.4 \mathrm{~mm}$-thick aluminum and is housed in a pipe of circular cross-section of $85 \mathrm{~mm}$ length, as shown in Fig. 4.23. The design parameters of the housing are presented in Table 4.14. The housing is made of HDPE with a wall thickness of $13.3 \mathrm{~mm}$ inserted in a steel sleeve of $1.65 \mathrm{~mm}$ wall thickness. To accommodate the honeycomb insert, the inner diameter of the housing is increased by $4 \mathrm{~mm}$ to an inner diameter of $126.2 \mathrm{~mm}$ over a length of $50.8 \mathrm{~mm}$ that matches the streamwise length of the honeycomb insert. During operation, the honeycomb is prevented from sliding in the streamwise direction as it rests against the downstream backward facing step of $2.0 \mathrm{~mm}$ height formed at the location where the housing inner diameter changes from $126.2 \mathrm{~mm}$ to $122.2 \mathrm{~mm}$. When the honeycomb insert is not in use, a circular steel insert is installed to maintain a smooth wall geometry for the flow. The installation of this insert is depicted in Fig. 4.24. The ends of the housing are shaped to allow for mating with the upstream and downstream components via V-band clamps, as illustrated in Fig. 4.25.

Table 4.14: Design parameters for the flow straightener housing.

\begin{tabular}{cccc}
\hline Design Code & Press. Rating & Temp. Rating & ASTM Material Specifications \\
ASME B31.1/CSA B51 & $7 \mathrm{~atm}$ & $25^{\circ} \mathrm{C}$ & HDPE, Steel (Table 4.9) \\
\hline
\end{tabular}




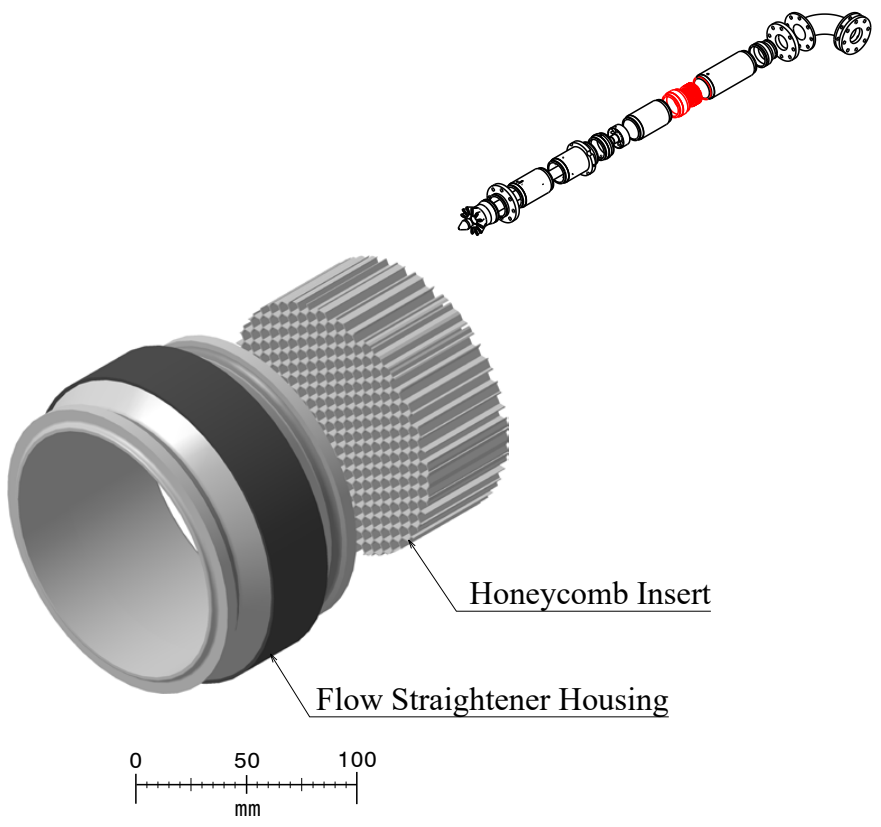

Figure 4.23: Honeycomb flow straightener assembly.

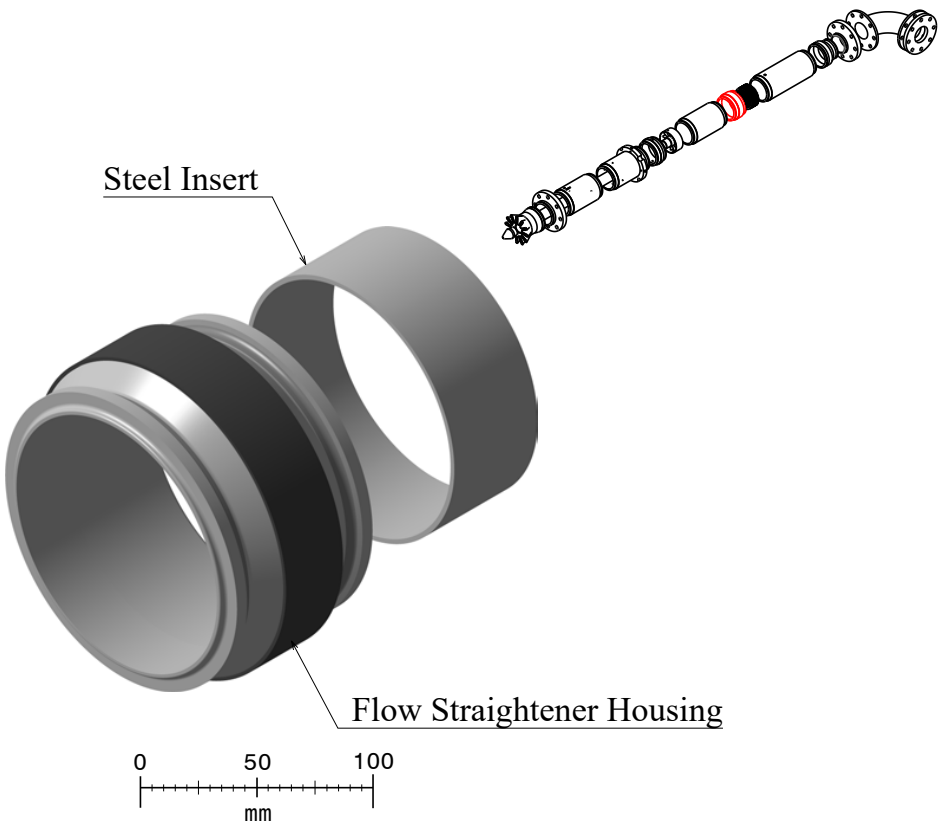

Figure 4.24: Honeycomb flow straightener assembly with steel insert replacing the honeycomb insert. 


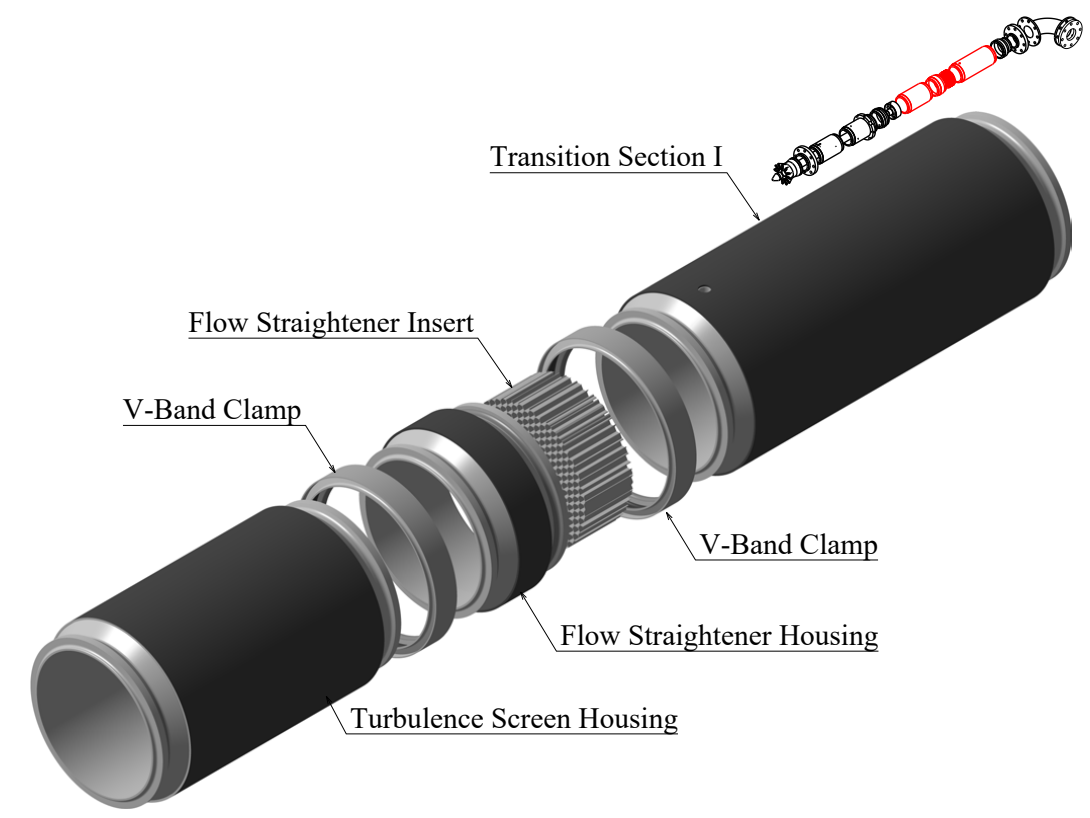

Figure 4.25: Honeycomb flow straightener installation.

\subsubsection{Structural Analysis}

\subsubsection{Computational Model and Results}

Figs. 4.26 and 4.27 present the computational model for the structural analysis of the flow straightener housing. In the structural analysis, the steel ring insert is used for the purposes of computational efficiency. Table 4.15 presents the specifics of the computational grid. The grid consists of approximately 1.1 million tetrahedral elements, with approximately 0.05 million elements in the steel sleeve and 0.3 million elements in the HDPE housing. Fig. 4.28 presents the Von Mises stress distribution in the parts of the subassembly. Table 4.16 presents a summary of the structural analysis results. The stresses and deformations in the flow straightener assembly components and the $\mathrm{V}$-band clamps confirm the structural integrity of the parts under the prescribed loading conditions. 


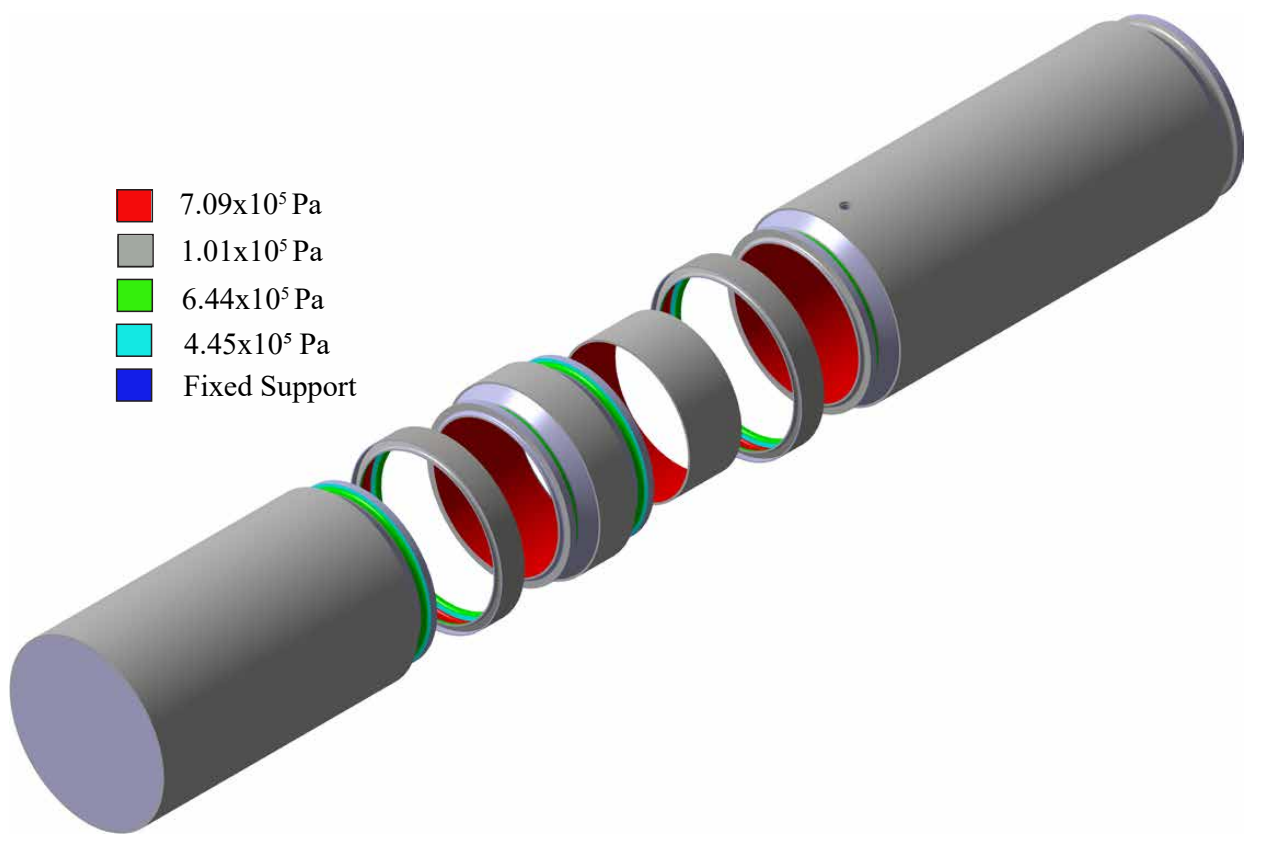

(a)

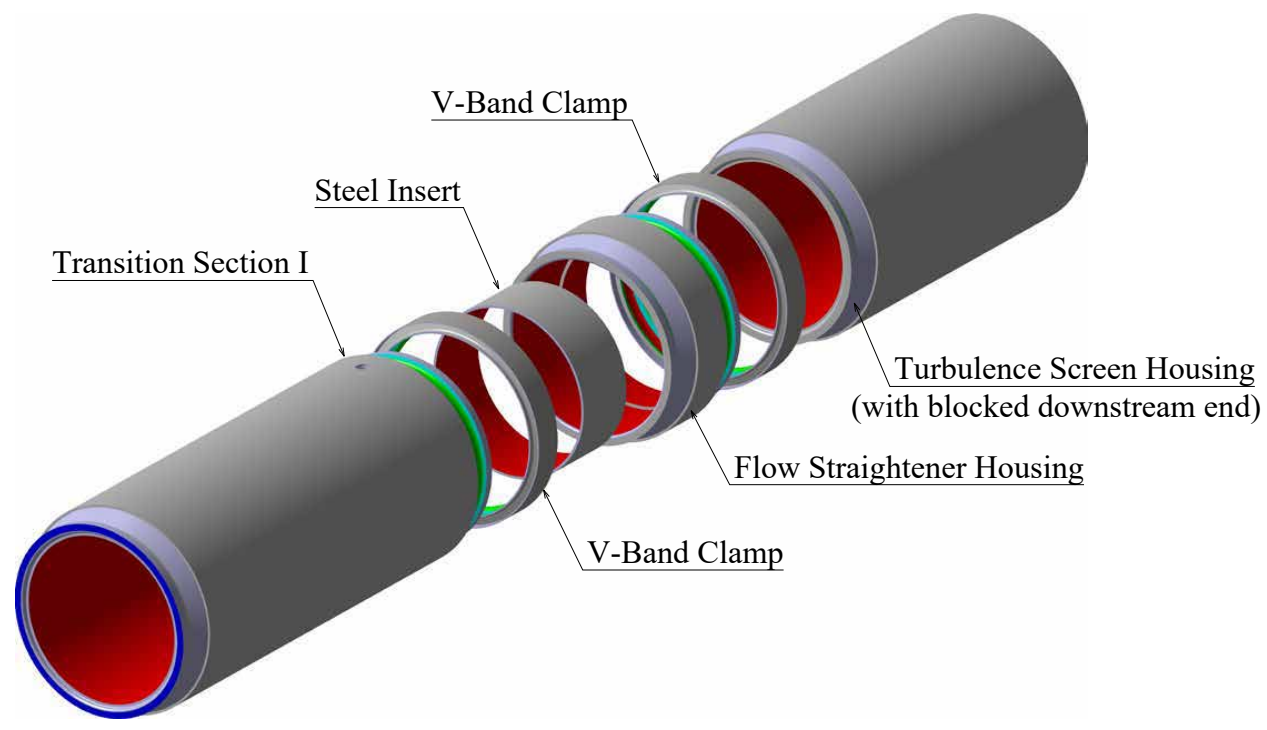

(b)

Figure 4.26: FEA computational model of the flow straightener subassembly as seen from the (a) downstream end; (b) upstream end. 

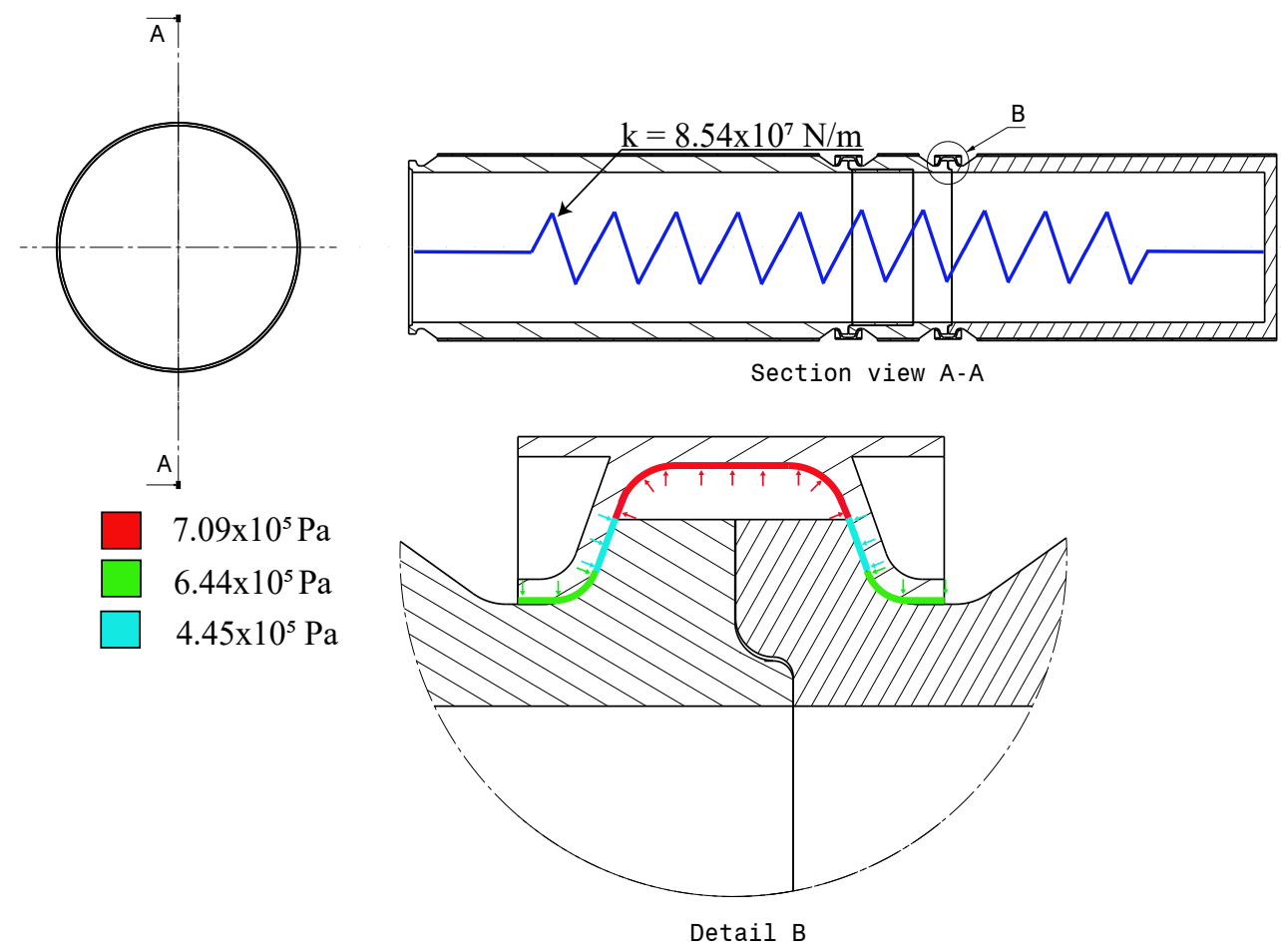

Figure 4.27: Schematic showing the loading of the V-band clamp and the location of the spring used to model the pre-compression rods.

Table 4.15: Parameters of the computational grid.

\begin{tabular}{lcc}
\hline & $\begin{array}{c}\text { Element size }(\mathrm{mm}) \\
\min / \max \end{array}$ & $\begin{array}{c}\text { Spatial Growth rate } \\
\min / \max \end{array}$ \\
\hline HDPE Pipe & $1.5 / 5.0$ & $0 \% / 15 \%$ \\
V-band Clamp & $1.0 / 1.0$ & $0 \%$ \\
Steel Sleeve & $0.5 / 0.5$ & $0 \%$ \\
Steel Insert & $0.5 / 0.5$ & $0 \%$ \\
\hline
\end{tabular}



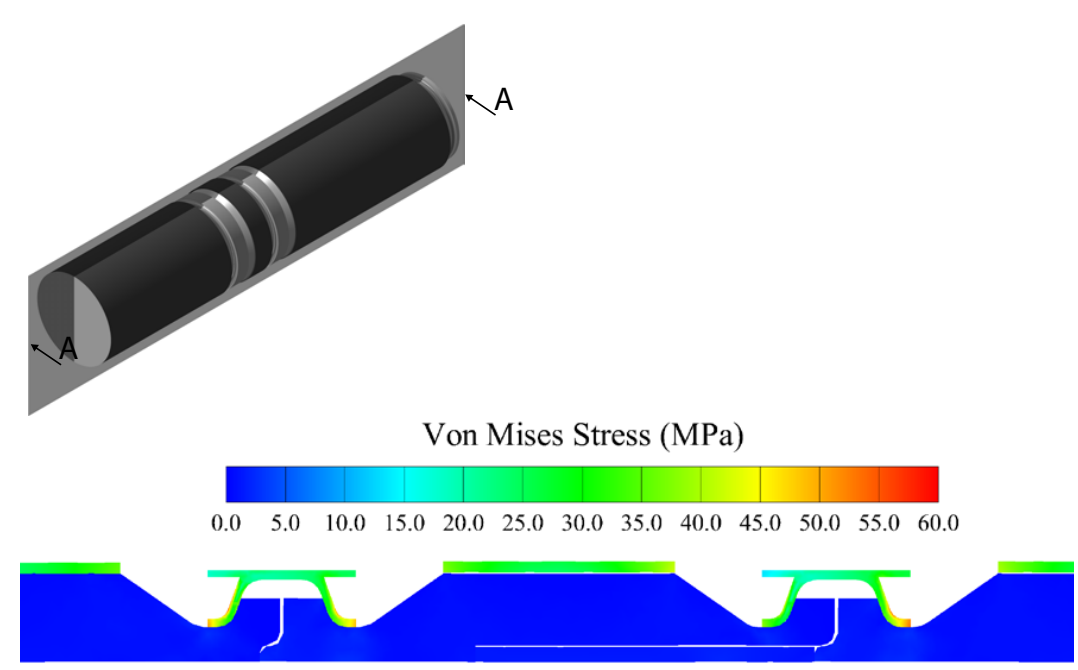

Section View A-A

Figure 4.28: Von Mises stress distribution in the flow straightener subassembly.

Table 4.16: Summary of the structural analysis results for the flow straightener subassembly.

\begin{tabular}{cccccc}
\hline \multirow{2}{*}{ Component } & $\begin{array}{c}\text { Pre-compression } \\
\text { Load (N) }\end{array}$ & $\begin{array}{c}\text { Max. } \\
\text { Deformation } \\
\left(\mathrm{x} 10^{-2} \mathrm{~mm}\right)\end{array}$ & $\begin{array}{c}\text { Max. Stress Min. Safety TSSA Required } \\
(\mathrm{MPa})\end{array}$ & $\begin{array}{c}\text { Factor } \\
\text { Safety Factor }\end{array}$ \\
\hline \multirow{2}{*}{ HDPE Pipe } & 1500 & $11.5 \pm 0.3$ & $2.5 \pm 0.1$ & $10.4 \pm 0.3$ & 10.0 \\
& 0 & $12.6 \pm 0.4$ & $2.6 \pm 0.1$ & $10.0 \pm 0.3$ & 10.0 \\
\hline \multirow{2}{*}{ V-band Clamp } & 1500 & $2.62 \pm 0.08$ & $49.0 \pm 1.47$ & $5.1 \pm 0.15$ & 4.0 \\
& 0 & $1.67 \pm 0.05$ & $48.6 \pm 1.46$ & $5.1 \pm 0.15$ & 4.0 \\
\hline \multirow{2}{*}{ Steel Sleeve } & 1500 & $1.49 \pm 0.04$ & $42.1 \pm 1.3$ & $5.9 \pm 0.2$ & 4.0 \\
& 0 & $2.22 \pm 0.07$ & $41.7 \pm 1.3$ & $6.0 \pm 0.2$ & 4.0 \\
\hline
\end{tabular}

${ }^{1}$ (TSSA, 2007) 


\subsection{Turbulence-Reduction Screen Assembly}

Two commercially available wire-mesh screens are installed downstream of the honeycomb flow straightener to reduce the level of flow turbulence. The first and second screens are chosen with wire diameters of $0.23 \mathrm{~mm}\left(\operatorname{Re}_{d}=1475\right)$ and $0.17 \mathrm{~mm}\left(\operatorname{Re}_{d}\right.$ $=1065)$ and percentage open areas of 0.7 and 0.62 , respectively. The noted screen Reynolds number values are the lowest values expected in the operating range of the air-supply system. Based on the correlation presented in Fig. 2.2, the pressure loss coefficients for these screens are estimated as 1.04 and 1.60, respectively.

The first screen is placed 5 honeycomb cell diameters downstream of the honeycomb to allow for the wake regions of the multi-cell flow pattern to diffuse before the flow interacts with the screen (Farell and Yousseff, 1992). The second screen is positioned $31.75 \mathrm{~mm}$ downstream of the first, which corresponds to 23 times the spacing of the wires of the first screen as recommended by the literature (Farell and Yousseff, 1992).

The relative positioning of the screens is realized via three steel spacers. Each spacer has a wall thickness of $2.5 \mathrm{~mm}$. Each screen is welded to its downstream spacer at a series of points along its perimeter. The housing is made of HDPE with a wall thickness of $13.3 \mathrm{~mm}$ inserted in a steel sleeve of $1.65 \mathrm{~mm}$ wall thickness. The design parameters of the turbulence screen housing are presented in Table 4.17. The ends

of this housing are shaped to allow for mating with the upstream and downstream components via V-band clamps, as illustrated in Fig. 4.30.

Table 4.17: Design parameters for the turbulence screen housing.

\begin{tabular}{cccc}
\hline Design Code & Press. Rating & Temp. Rating & ASTM Material Specifications \\
ASME B31.1/CSA B51 & $7 \mathrm{~atm}$ & $25^{\circ} \mathrm{C}$ & HDPE, Steel (Table 4.9) \\
\hline
\end{tabular}




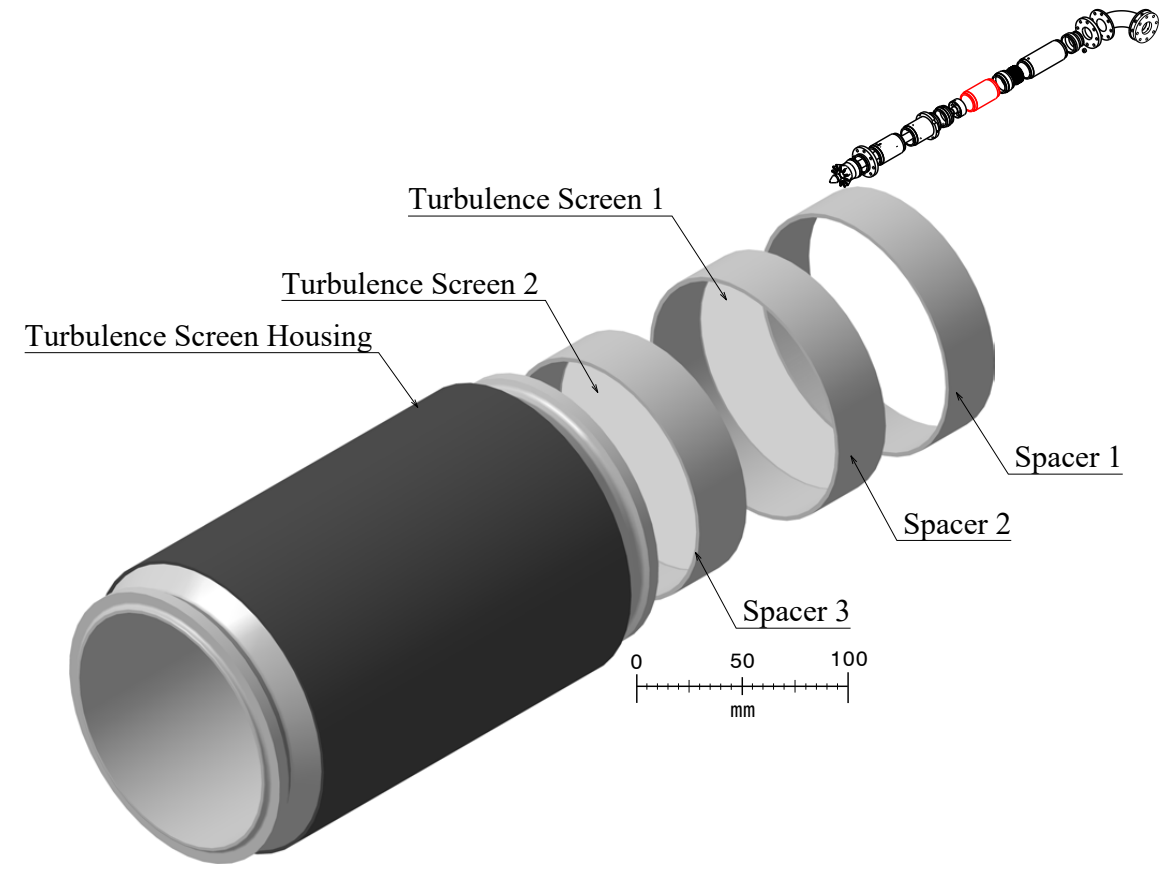

Figure 4.29: Turbulence screen assembly.

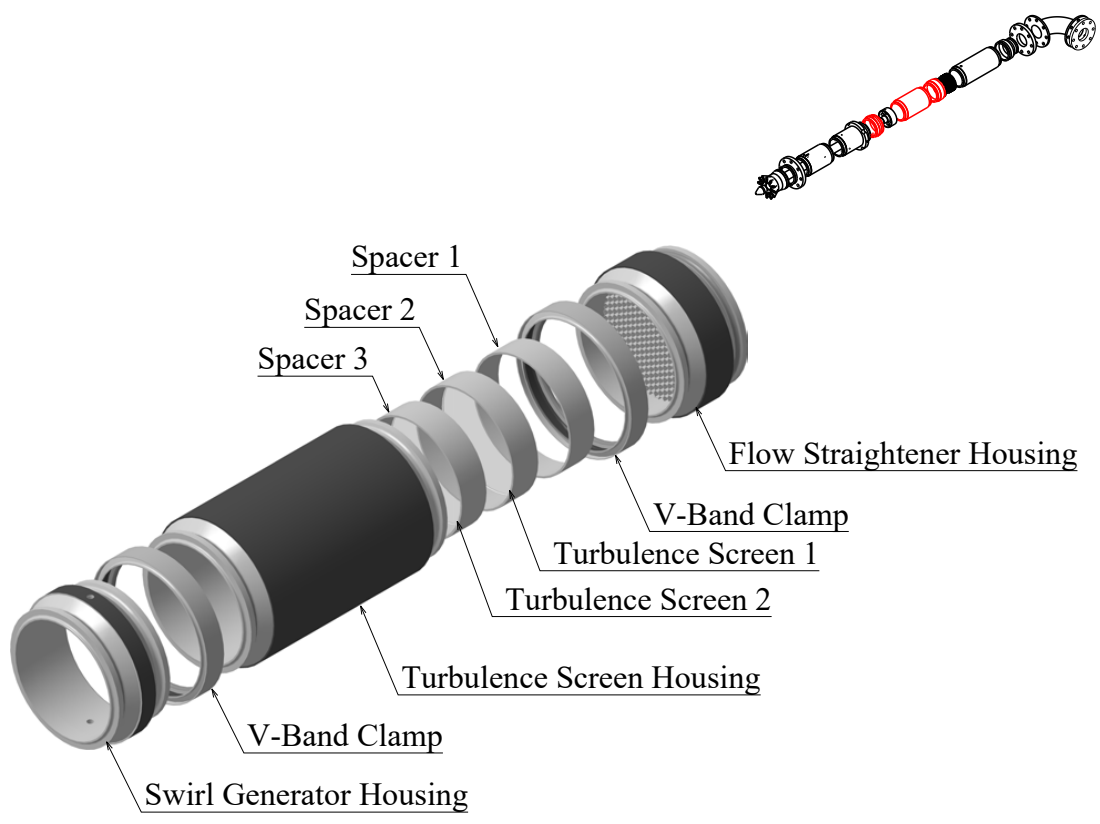

Figure 4.30: Turbulence-screen-assembly installation. 


\subsubsection{Structural Analysis}

\subsubsection{Computational Model and Results}

Figs. 4.31 and 4.32 present the computational model for the structural analysis of the turbulence screen assembly. Table 4.18 presents the specifics of the computational grid. The grid consists of approximately 1.2 million tetrahedral elements, with approximately 0.15 million elements in the steel sleeve and 0.4 million elements in the HDPE housing. Fig. 4.33 presents the Von Mises stress distribution in the parts of the subassembly. Table 4.19 presents a summary of the structural analysis results. The stresses and deformations in the turbulence screen housing components and the V-band clamps confirm the structural integrity of the parts under the prescribed loading conditions. 


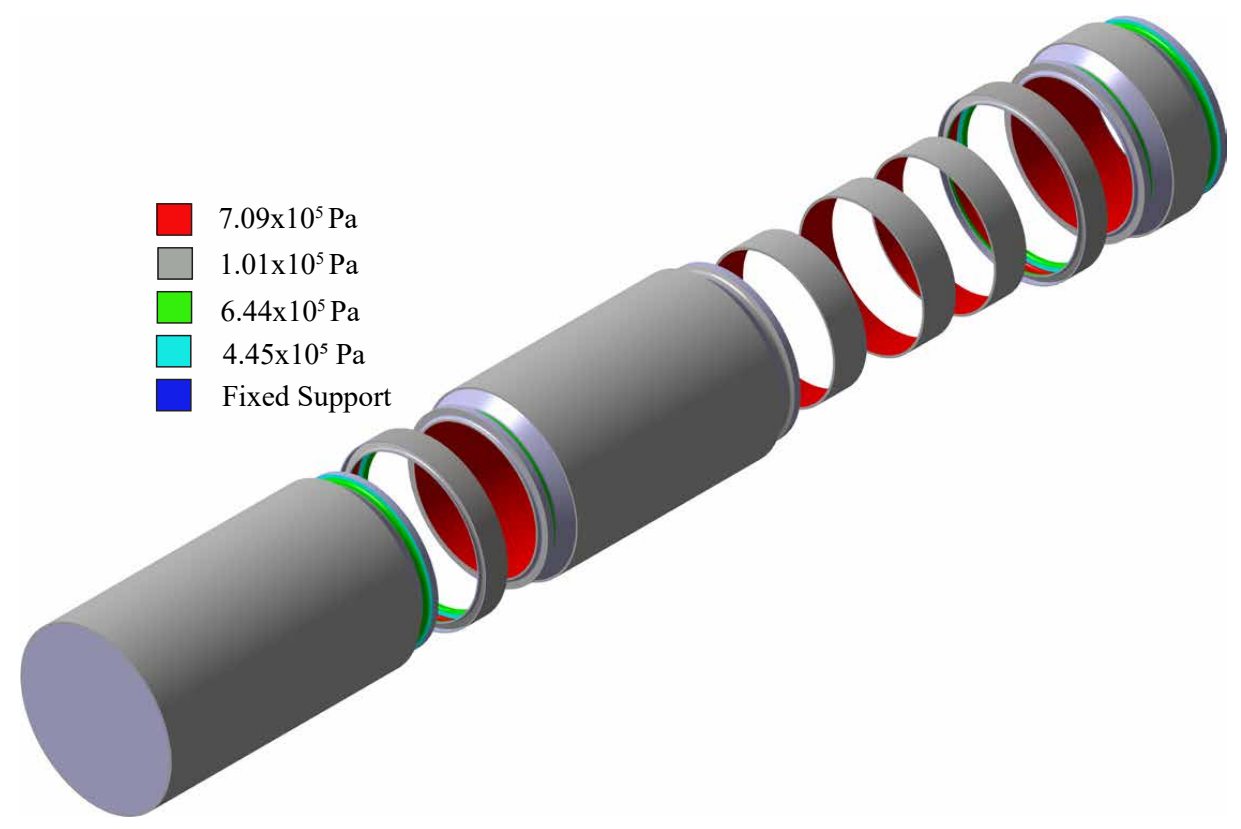

(a)

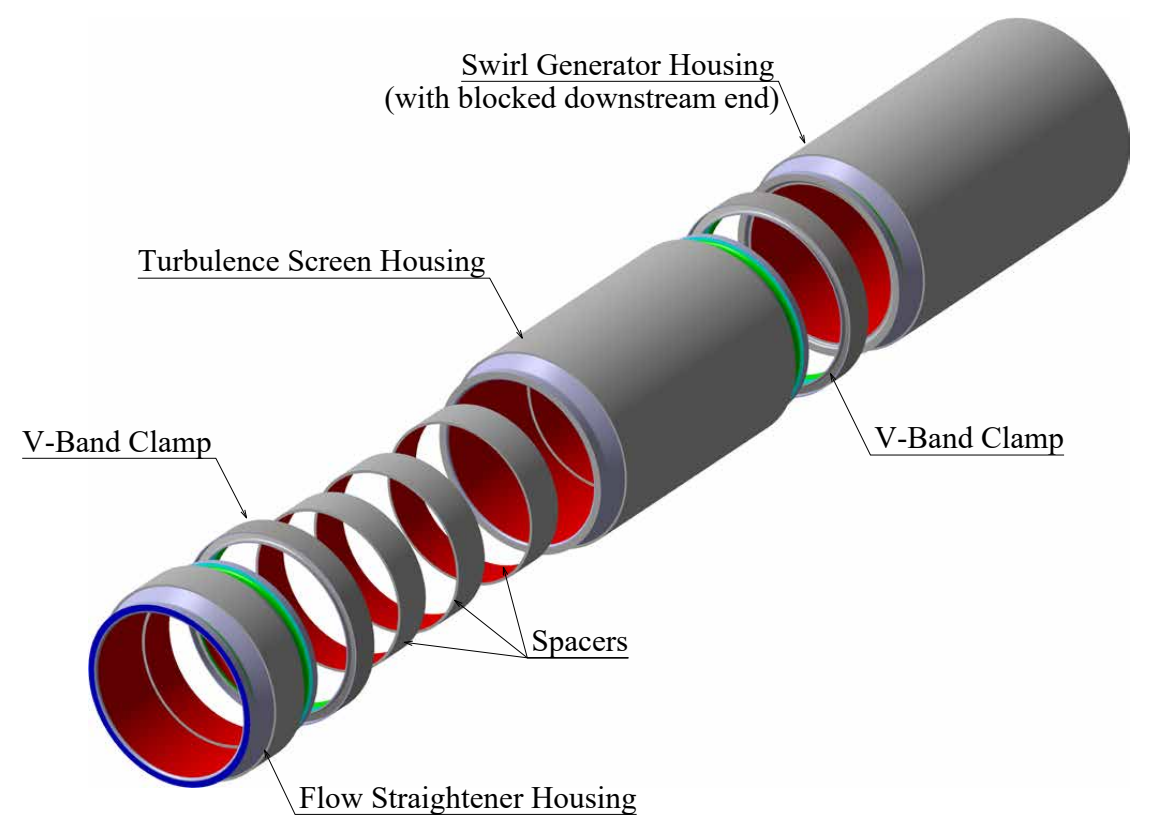

(b)

Figure 4.31: FEA computational model of the turbulence screen subassembly as seen from the (a) downstream end; (b) upstream end. 

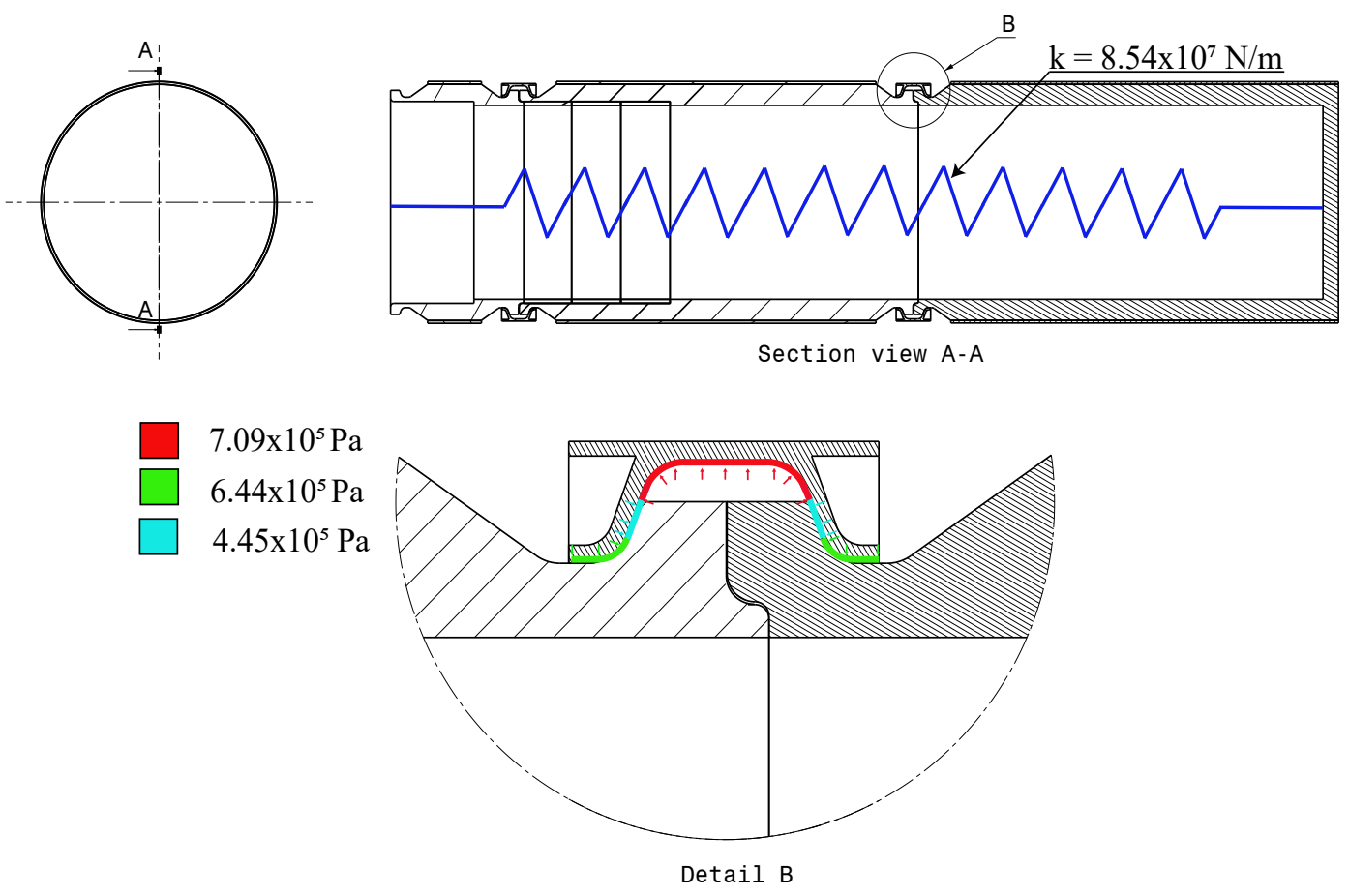

Figure 4.32: Schematic showing the loading of the V-band clamp and the location of the spring used to model the pre-compression rods.

Table 4.18: Parameters of the computational grid.

\begin{tabular}{lcc}
\hline & $\begin{array}{c}\text { Element size }(\mathrm{mm}) \\
\min / \max \end{array}$ & $\begin{array}{c}\text { Spatial Growth rate } \\
\min / \max \end{array}$ \\
\hline HDPE Pipe & $1.5 / 5.0$ & $0 \% / 15 \%$ \\
V-band Clamp & $1.0 / 1.0$ & $0 \%$ \\
Steel Sleeve & $0.5 / 0.5$ & $0 \%$ \\
Spacers & $0.5 / 0.5$ & $0 \%$ \\
\hline
\end{tabular}




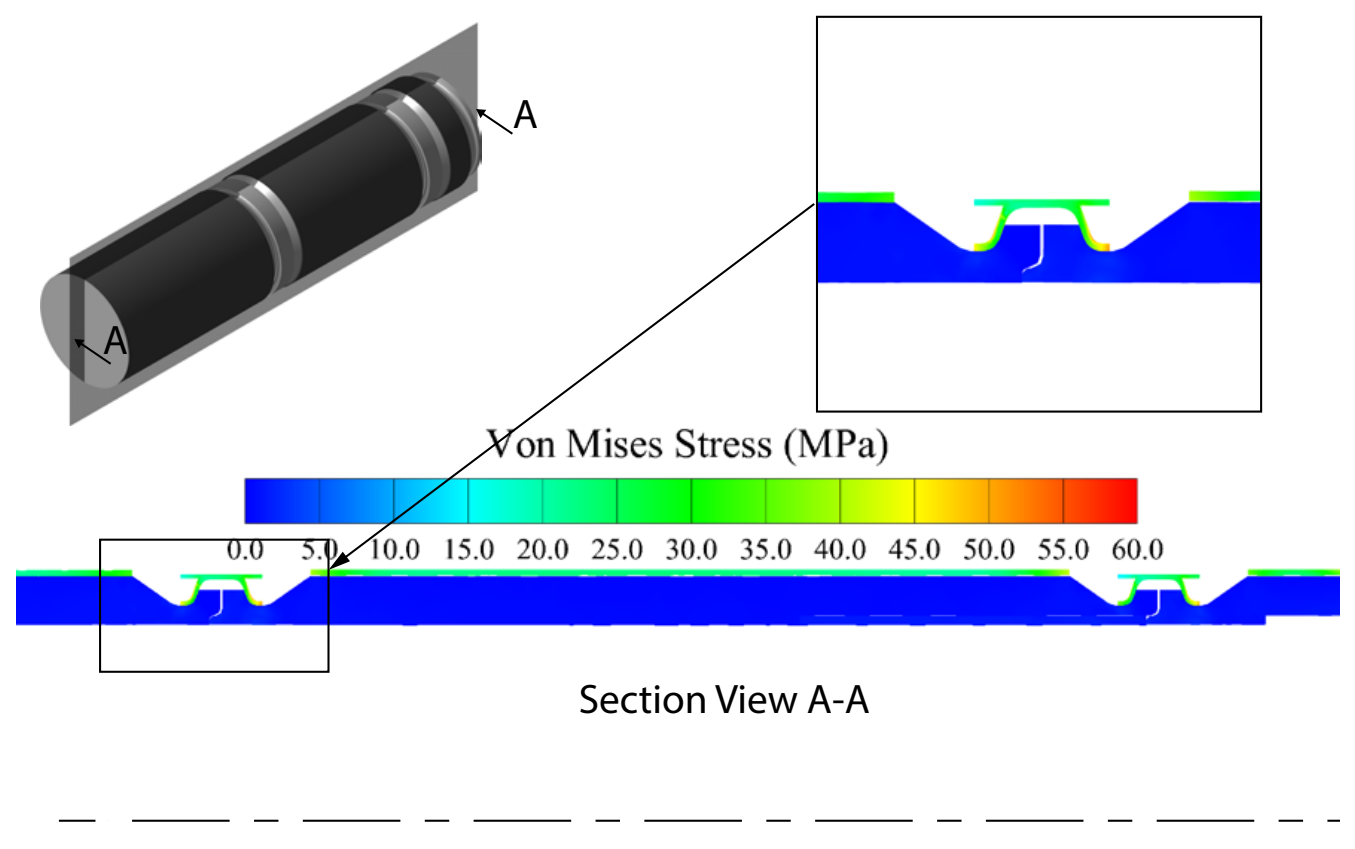

Figure 4.33: Von Mises stress distribution in the turbulence screen subassembly.

Table 4.19: Summary of the structural analysis results for the turbulence screen subassembly.

\begin{tabular}{|c|c|c|c|c|c|}
\hline Component & $\begin{array}{l}\text { Pre-compression } \\
\text { Load }(\mathrm{N})\end{array}$ & $\begin{array}{c}\text { Max. } \\
\text { Deformation } \\
\left(\mathrm{x} 10^{-2} \mathrm{~mm}\right)\end{array}$ & $\begin{array}{l}\text { Max. Stress } \\
(\mathrm{MPa})\end{array}$ & $\begin{array}{c}\text { Min. Safety } \\
\text { Factor }\end{array}$ & $\begin{array}{l}\text { TSSA Required } \\
\text { Safety Factor }{ }^{1}\end{array}$ \\
\hline \multirow[t]{2}{*}{ HDPE Pipe } & 1500 & $8.21 \pm 0.25$ & $2.5 \pm 0.1$ & $10.3 \pm 0.3$ & 10.0 \\
\hline & 0 & $9.28 \pm 0.28$ & $2.7 \pm 0.1$ & $9.6 \pm 0.3$ & 10.0 \\
\hline \multirow[t]{2}{*}{ V-band Clamp } & 1500 & $2.90 \pm 0.09$ & $27.7 \pm 0.8$ & $9.0 \pm 0.3$ & 4.0 \\
\hline & 0 & $2.69 \pm 0.08$ & $28.0 \pm 0.8$ & $8.9 \pm 0.3$ & 4.0 \\
\hline \multirow[t]{2}{*}{ Steel Sleeve } & 1500 & $2.49 \pm 0.07$ & $22.4 \pm 0.7$ & $11.2 \pm 0.3$ & 4.0 \\
\hline & 0 & $3.22 \pm 0.09$ & $22.4 \pm 0.7$ & $11.2 \pm 0.3$ & 4.0 \\
\hline
\end{tabular}




\subsection{Swirl Generator Assembly}

A set of guide vanes is used to impart angular momentum onto the air stream to facilitate swirl at the test section inlet. The swirl generator, shown in Fig. 4.34, consists of a housing made of $13.3 \mathrm{~mm}$-thick HDPE, and an insert containing the vanes of the swirl generator made of steel via additive manufacturing. The housing is inserted in a steel sleeve of $1.65 \mathrm{~mm}$ wall thickness. To accommodate the swirl generator insert, the inner diameter of the housing is increased by $4 \mathrm{~mm}$ to 49.5 $\mathrm{mm}$ over a length that matches the streamwise length of the insert. The housing is designed to facilitate mating with the upstream and downstream sections via V-band clamps, as illustrated in Fig. 4.35. The swirl generator insert consists of concentric hub and casing sections with 12 flow-turning vanes that span the annulus height. Each vane is based on a circular-arc camberline of $101.6 \mathrm{~mm}$ radius, and is configured to be aligned with the axis of the duct at its leading edge. The leading-to-trailing edge metal turning of the vane is 17.2-deg., which is expected to produce downstream swirl with an angle slightly less than this metal turning angle. The vane thickness is $2.4 \mathrm{~mm}$, which is uniform along the camberline. The leading and trailing edges are rounded to circular arcs. Table 4.20 presents the design parameters of the swirl generator housing. Two bolts of $6.35 \mathrm{~mm}$-diameter are installed through the insert and the housing to prevent rotation of the swirl generator insert within its housing. The suitability of these bolts to counter the torque generated by the flow passing through the turning vanes was confirmed though structural analysis based on the expected torque generated by a 15-deg. flow turning. The hub segment of the swirl generator also facilitates the transition from a circular flow-path geometry to an annular one. The annulus height of $33 \mathrm{~mm}$ at the swirl generator matches the desired annulus height at the discharge end of the air-supply system.

Table 4.20: Design parameters for the swirl generator housing.

\begin{tabular}{cccc} 
Design Code & Press. Rating & Temp. Rating & ASTM Material Specifications \\
ASME B31.1/CSA B51 & $7 \mathrm{~atm}$ & $25^{\circ} \mathrm{C}$ & HDPE, Steel (Table 4.9) \\
\hline
\end{tabular}



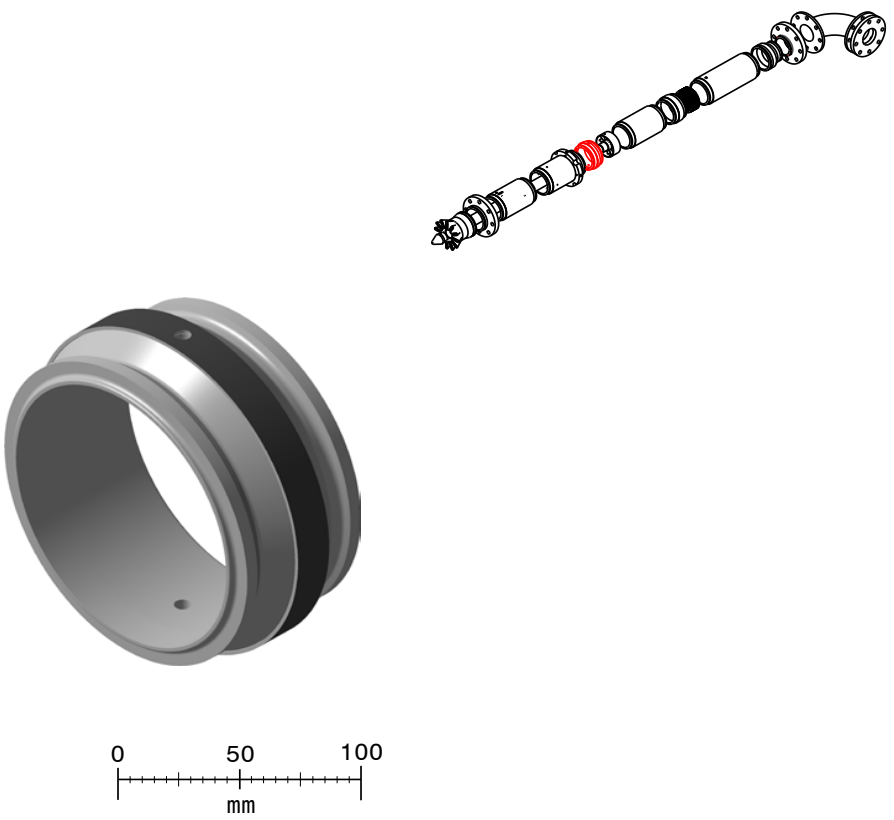

Figure 4.34: Swirl generator housing.

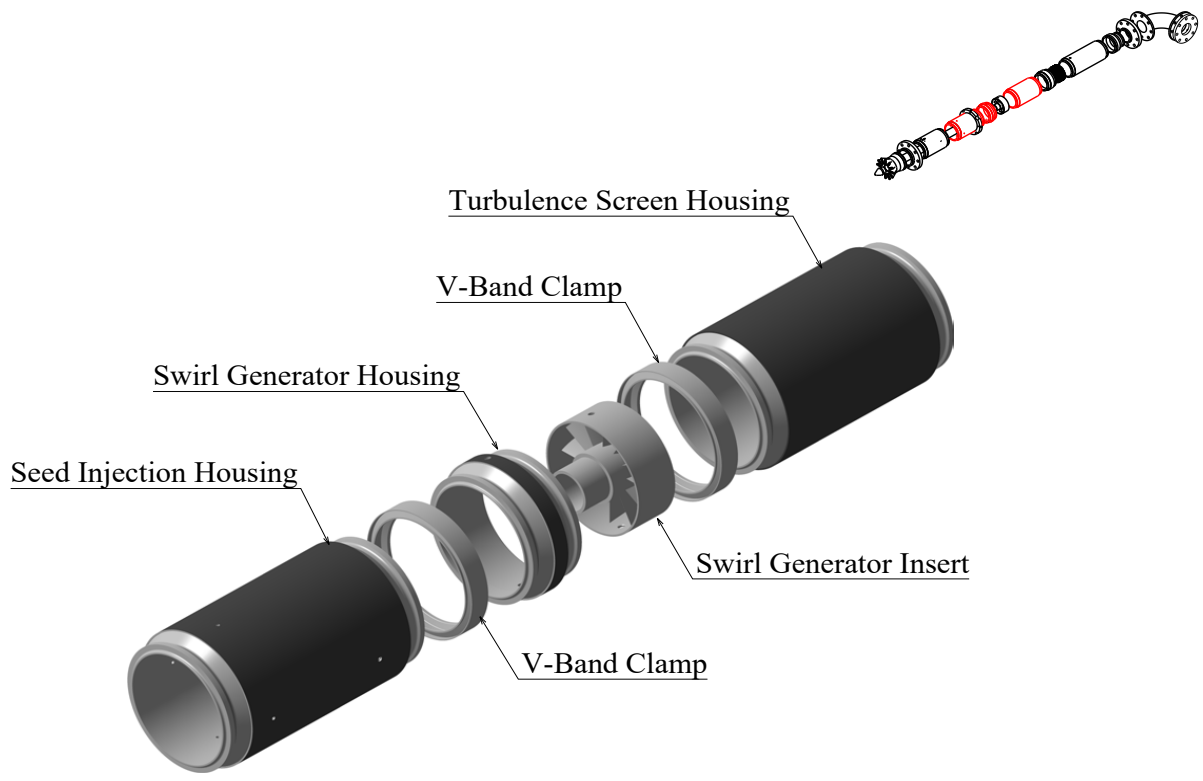

Figure 4.35: Swirl generator assembly installation. 


\subsubsection{Structural Analysis}

\subsubsection{Computational Model and Results}

Figs. 4.36 and 4.37 present the computational model for the structural analysis of the swirl generator housing assembly. For computational efficiency, the swirl generator insert is replaced with a cylindrical housing insert made of steel. Table 4.21 presents the specifics of the computational grid. The grid consists of approximately 1.4 million tetrahedral elements, with approximately 0.02 million elements in the steel sleeve and 0.2 million elements in the HDPE housing. Fig. 4.38 presents the Von Mises stress distribution in the parts of the subassembly. Table 4.22 presents a summary of the structural analysis results. The stresses and deformations in the swirl generator housing components and the V-band clamps confirm the structural integrity of the parts under the prescribed loading conditions. 


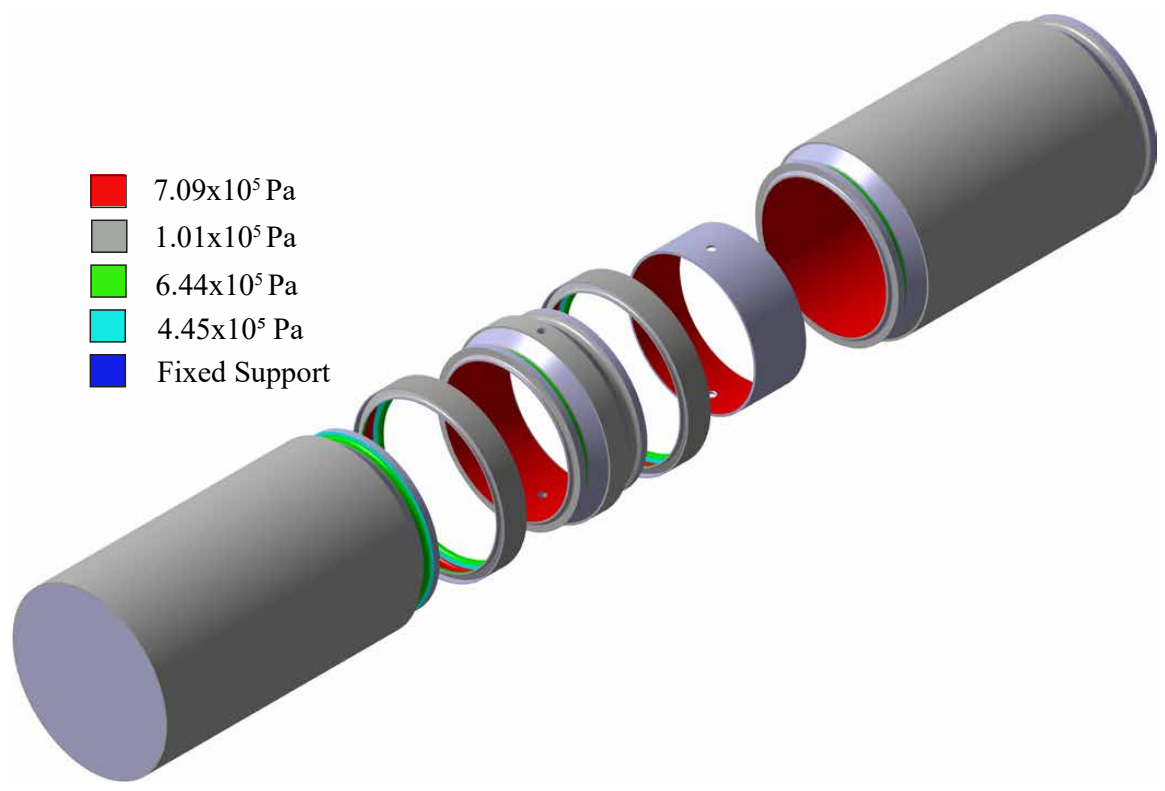

(a)

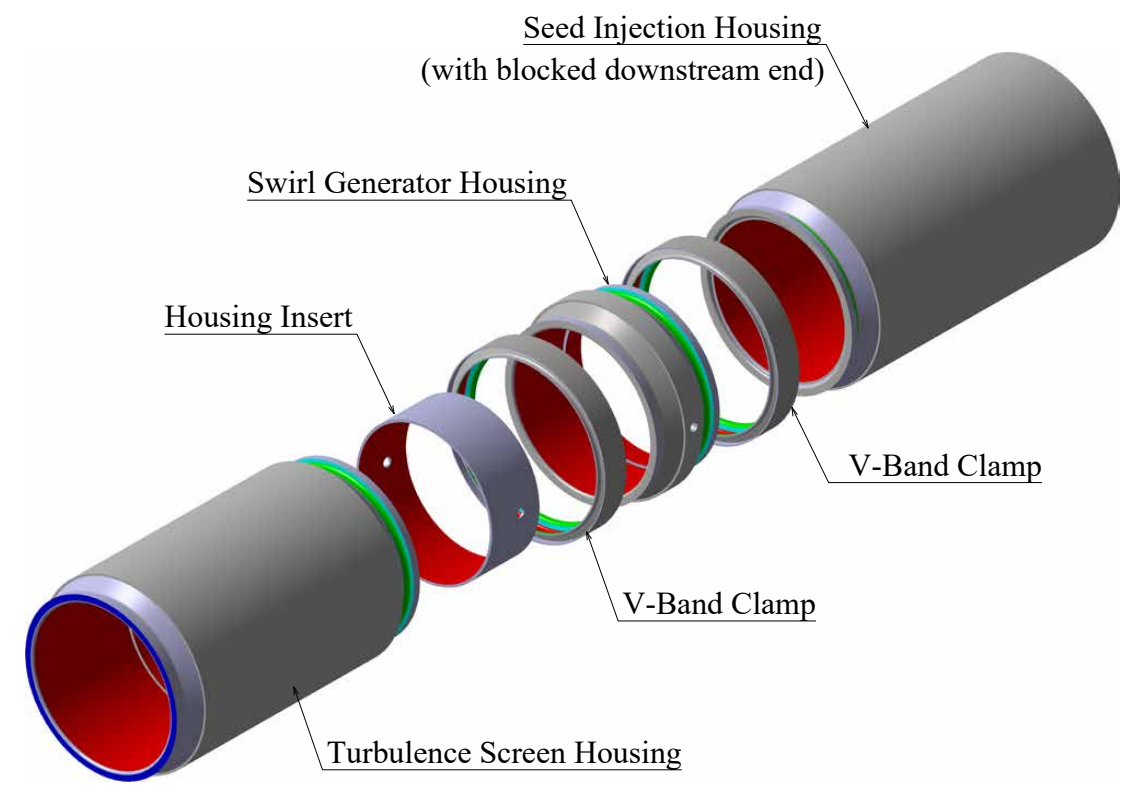

(b)

Figure 4.36: FEA computational model of swirl generator housing assembly as seen from the (a) downstream end; (b) upstream end. 

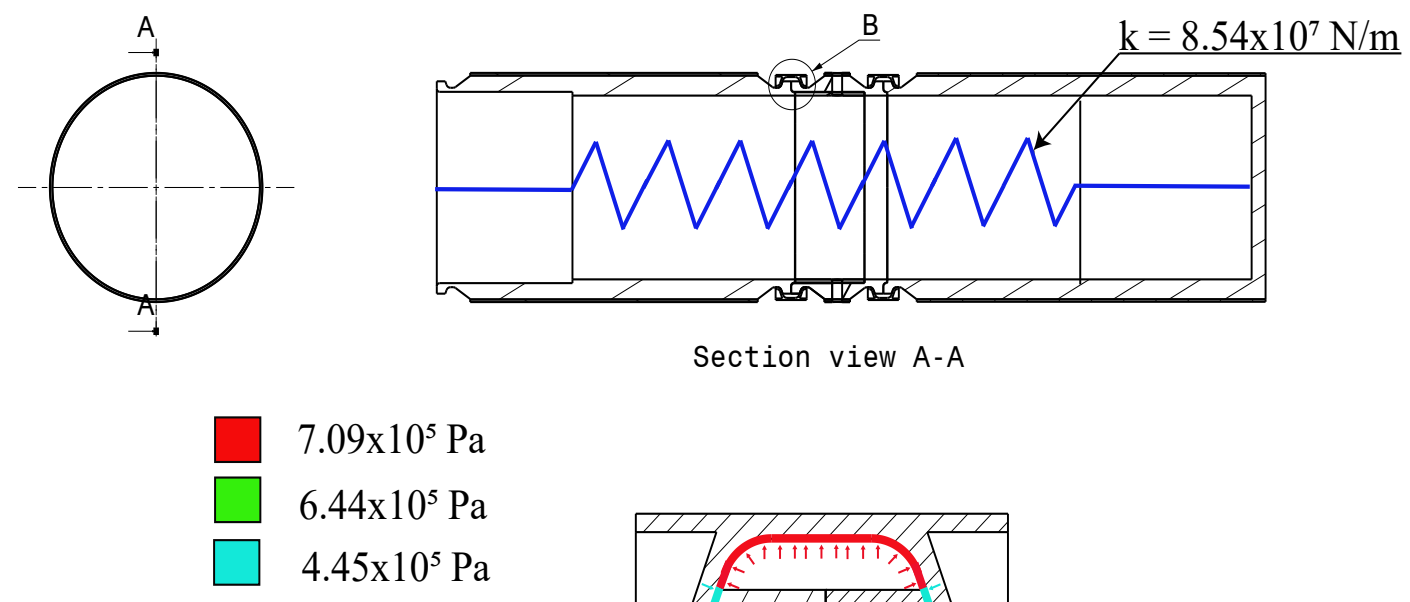

of the spring used to model the pre-compression rods.

Table 4.21: Parameters of the computational grid.

\begin{tabular}{lcc}
\hline & $\begin{array}{c}\text { Element size }(\mathrm{mm}) \\
\min / \max \end{array}$ & $\begin{array}{c}\text { Spatial Growth rate } \\
\min / \max \end{array}$ \\
\hline HDPE Pipe & $1.5 / 5.0$ & $0 \% / 15 \%$ \\
V-band Clamp & $1.0 / 1.0$ & $0 \%$ \\
Steel Sleeve & $0.5 / 0.5$ & $0 \%$ \\
Housing Insert & $0.5 / 0.5$ & $0 \%$ \\
\hline
\end{tabular}




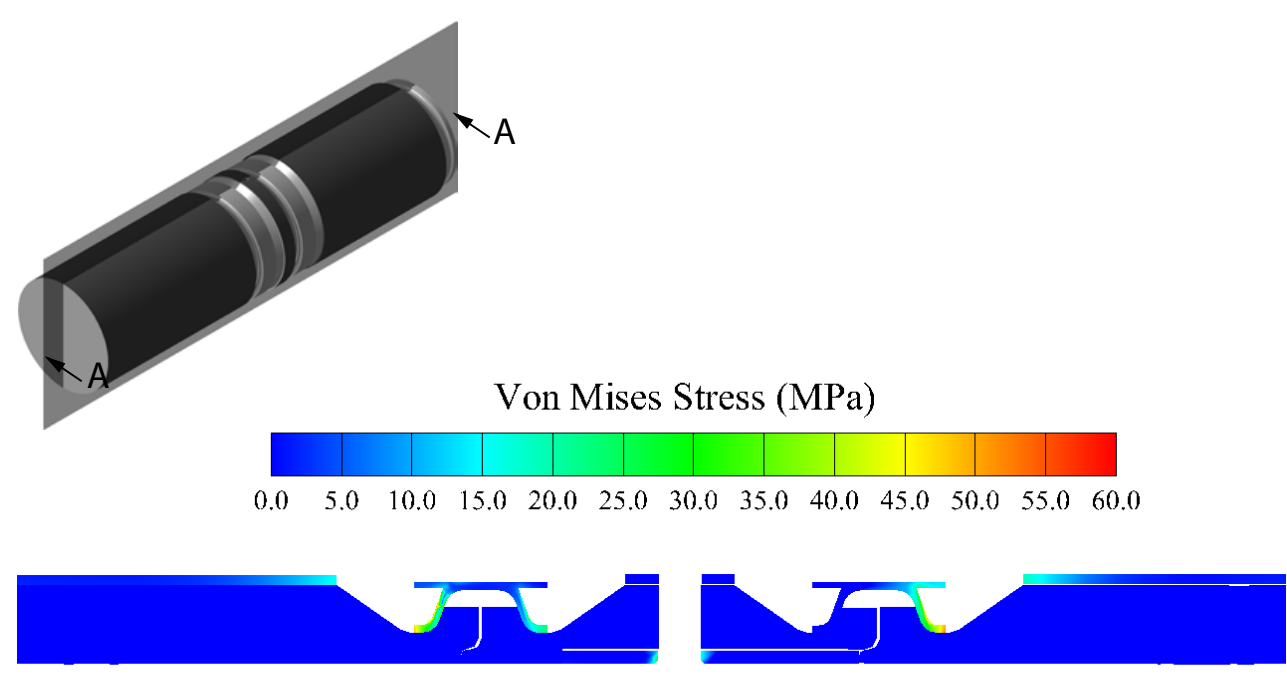

Section View A-A

Figure 4.38: Von Mises stress distribution in the swirl generator housing subassembly.

Table 4.22: Summary of the structural analysis results for the swirl generator housing subassembly.

\begin{tabular}{|c|c|c|c|c|c|}
\hline Component & $\begin{array}{c}\text { Pre-compression } \\
\text { Load }(\mathrm{N})\end{array}$ & $\begin{array}{c}\text { Max. } \\
\text { Deformation } \\
\left(\mathrm{x} 10^{-2} \mathrm{~mm}\right)\end{array}$ & $\begin{array}{c}\text { Max. Stress } \\
(\mathrm{MPa})\end{array}$ & $\begin{array}{c}\text { Min. Safety } \\
\text { Factor }\end{array}$ & $\begin{array}{l}\text { TSSA Required } \\
\text { Safety Factor }{ }^{1}\end{array}$ \\
\hline HDPE Pipe & $\begin{array}{c}1500 \\
0 \\
\end{array}$ & $\begin{array}{l}11.7 \pm 0.4 \\
12.9 \pm 0.4 \\
\end{array}$ & $\begin{array}{l}2.4 \pm 0.1 \\
2.6 \pm 0.1 \\
\end{array}$ & $\begin{array}{l}10.6 \pm 0.3 \\
10.0 \pm 0.3 \\
\end{array}$ & $\begin{array}{l}10.0 \\
10.0 \\
\end{array}$ \\
\hline V-band Clamp & $\begin{array}{c}1500 \\
0\end{array}$ & $\begin{array}{l}6.22 \pm 0.19 \\
7.38 \pm 0.22\end{array}$ & $\begin{array}{l}55.8 \pm 1.7 \\
61.5 \pm 1.8\end{array}$ & $\begin{array}{l}4.4 \pm 0.1 \\
4.1 \pm 0.1\end{array}$ & $\begin{array}{l}4.0 \\
4.0\end{array}$ \\
\hline Steel Sleeve & $\begin{array}{c}1500 \\
0\end{array}$ & $\begin{array}{l}7.81 \pm 0.23 \\
8.91 \pm 0.27\end{array}$ & $\begin{array}{l}42.2 \pm 1.3 \\
42.0 \pm 1.3\end{array}$ & $\begin{array}{l}5.9 \pm 0.2 \\
6.0 \pm 0.2\end{array}$ & $\begin{array}{l}4.0 \\
4.0\end{array}$ \\
\hline
\end{tabular}

${ }^{1}$ (TSSA, 2007) 


\subsection{Core-Pipe Assembly}

As described in Section 4.9, the hub of the swirl generator insert facilitates the transition of the flow path from a circular to annular cross-section. The core-pipe assembly, depicted in Fig. 4.39, consists of a core pipe made of Schedule 80 PVC, the swirl generator insert described in Section 4.9, the swirl generator nose cone and an end-plug both made of steel, an aluminum bushing, a steel center-line support rod, and four steel radial support rods.

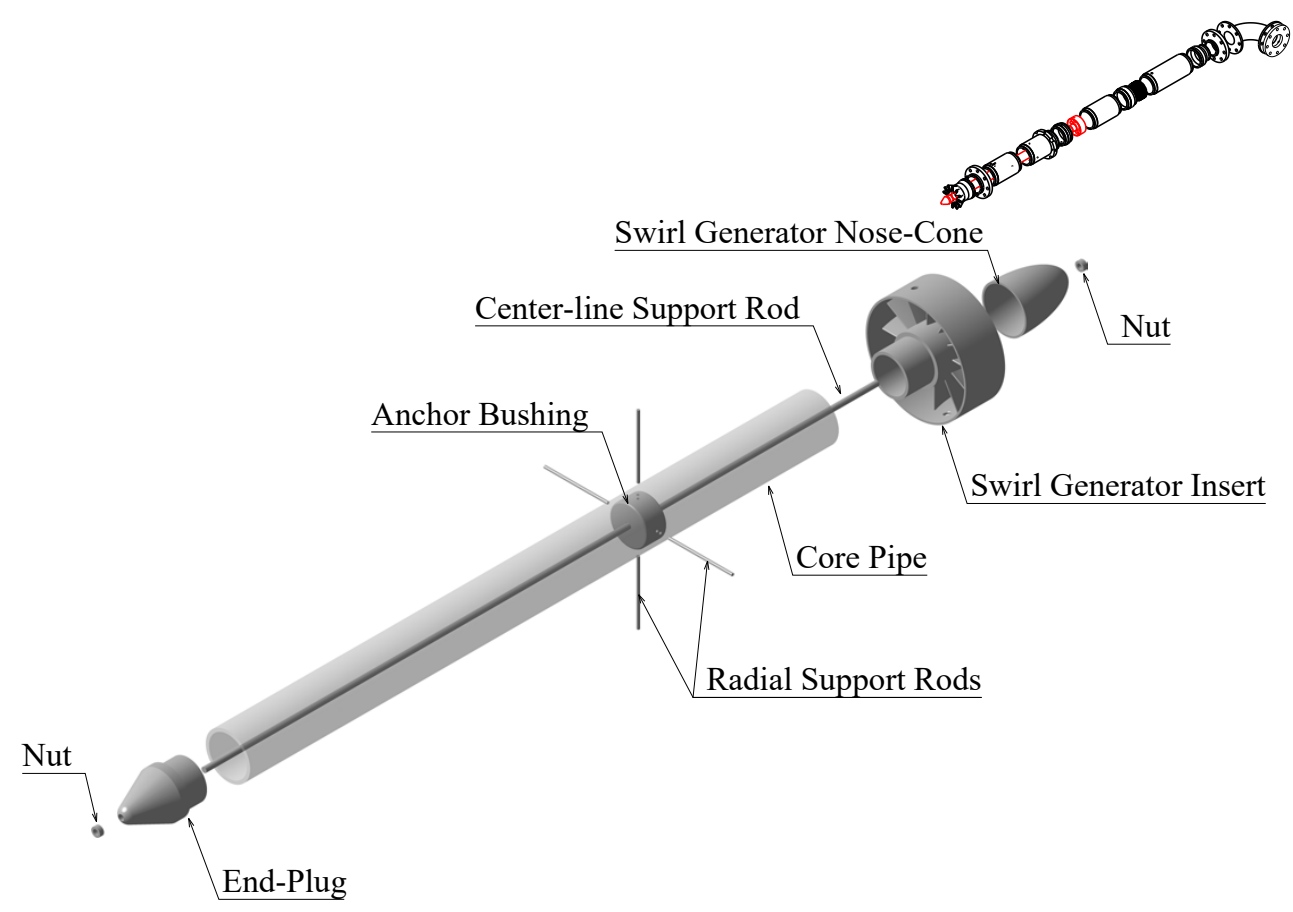

Figure 4.39: Core pipe assembly.

The 6.35 mm-diameter center-line support rod, fixed in place by a nut on either end, prevents the assembly from shifting axially. The four radial support rods of $3.2 \mathrm{~mm}$-diameter thread into the aluminum anchor bushing that resides inside the core-pipe. These radial rods serve to keep the core-pipe assembly centered within the casing components and are attached to the outer surface of the seed injection housing (Section $\mathrm{X}$ in Fig. 4.2) using a nut and a rubber washer to seal the connection. The 
axial position of the radial support rods was chosen such that the core pipe would be sufficiently supported to prevent cantilevering at the connection point with the swirl generator insert, while providing sufficient streamwise distance for the wake signatures of the rods to dissipate prior to the flow entering the test section. The nose cone is of elliptic cross-section in the axial-radial plane with an axial-to-radial axis ratio of 2.0. The end plug on the downstream end is presently set to a conical shape, which will likely be tailored for each test section depending on the specifics of the flow path being investigated in the test section.

\subsection{Seed Injection Assembly}

For the purpose of performing particle image velocimetry (PIV) measurements of the flow in the test section, a seed injection assembly is used to introduce a seed particulate into the flow. Table 4.23 presents the design parameters of the seed injection housing. The assembly, shown in Fig. 4.40 consists of a housing made of HDPE with a wall thickness of $13.3 \mathrm{~mm}$, and a seed injection ring made of steel. The housing is inserted in a steel sleeve of $1.65 \mathrm{~mm}$ wall thickness. Four $3.5 \mathrm{~mm}$-diameter holes placed at 90-deg. increments at the downstream end of the housing accommodate the radial support rods described in Section 4.10. The housing mates with its upstream and downstream sections via V-band clamps as illustrated in Fig. 4.41.

Table 4.23: Design parameters for the seed injection housing.

\begin{tabular}{cccc}
\hline Design Code & Press. Rating & Temp. Rating & ASTM Material Specifications \\
ASME B31.1/CSA B51 & $7 \mathrm{~atm}$ & $25^{\circ} \mathrm{C}$ & HDPE, Steel (Table 4.9) \\
\hline
\end{tabular}




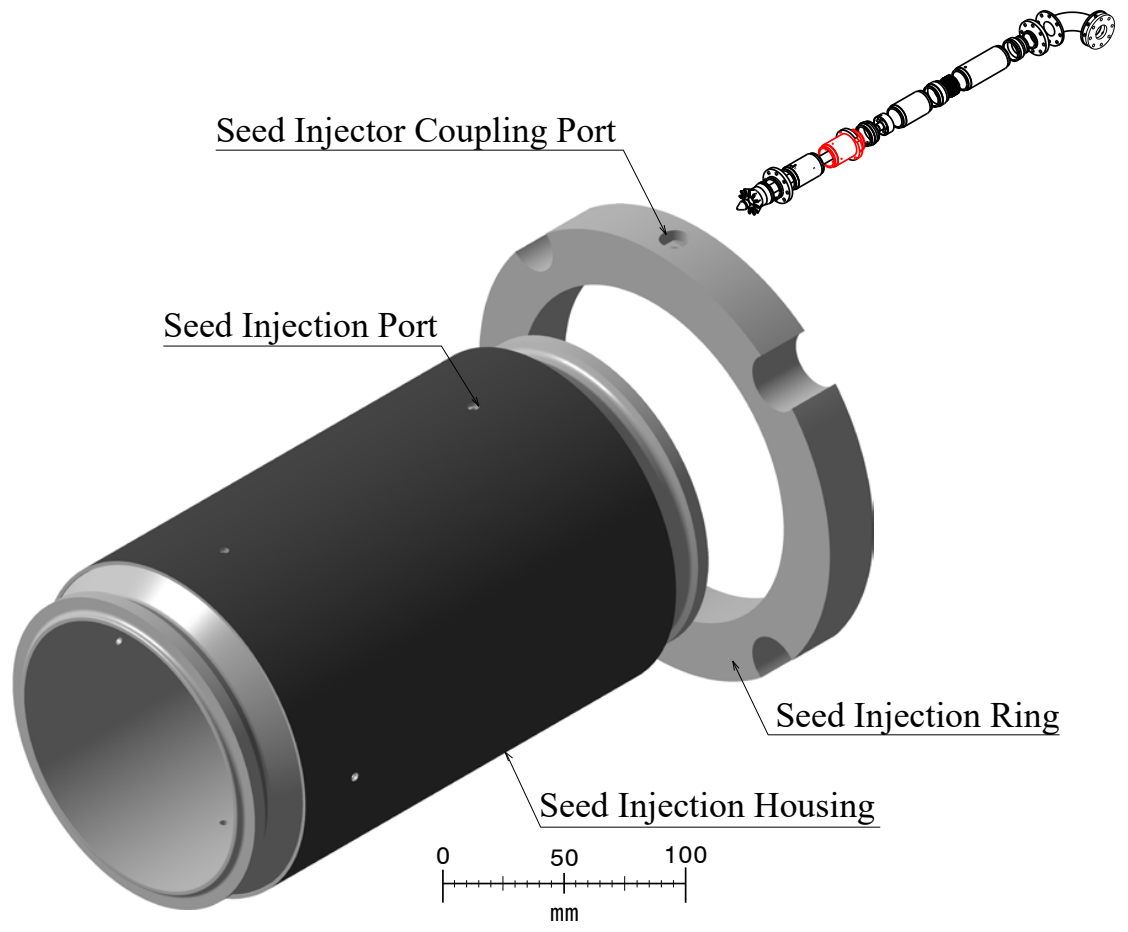

Figure 4.40: Seed injection assembly. 


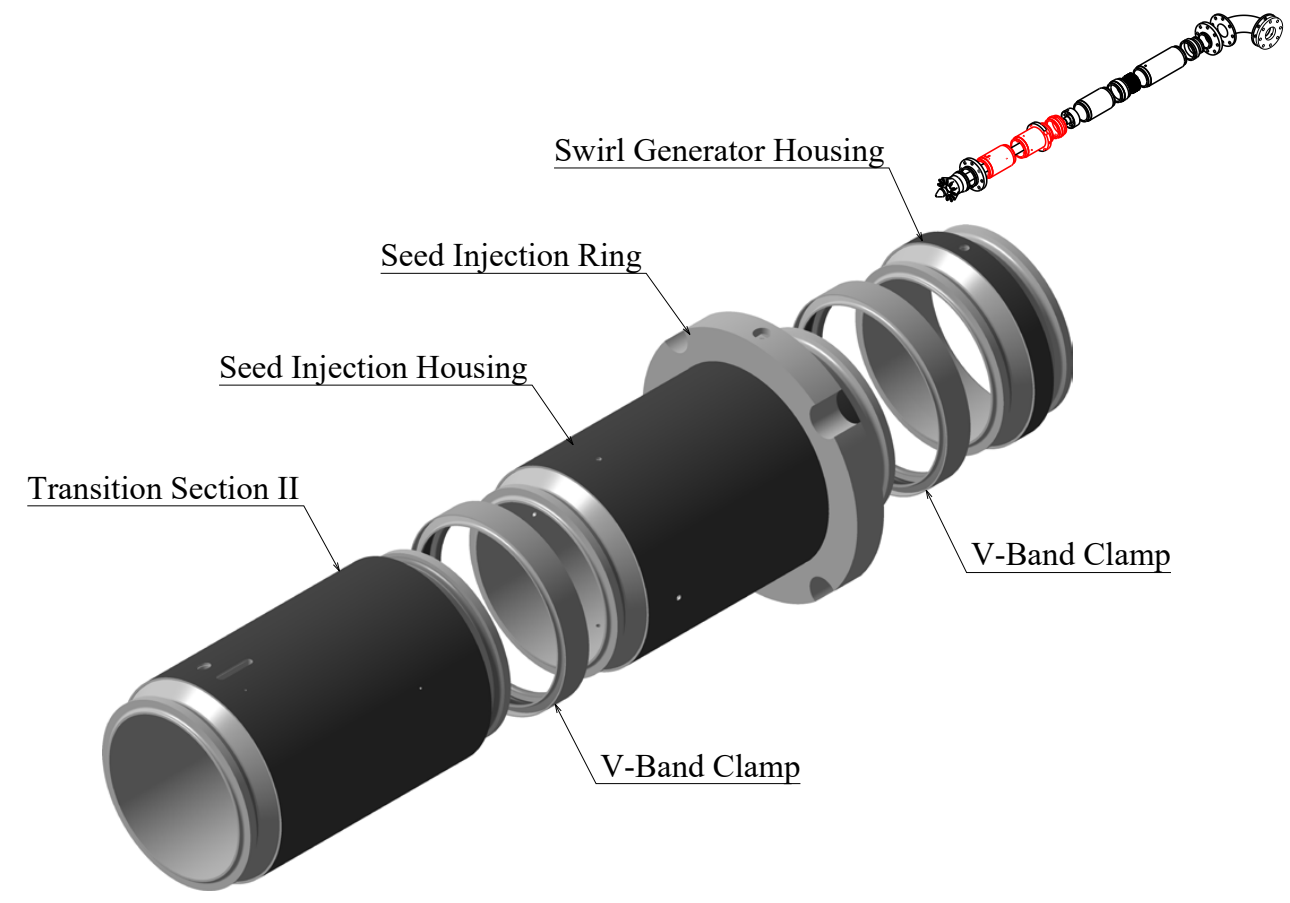

Figure 4.41: Seed injection assembly installation.

The seed injection ring facilitates the attachment of a feedline carrying atomized oil produced by a Laskin nozzle. When installed, the ring fits snug on the seed injection housing, and the injector coupling port on the ring aligns with the seed injection port on the housing. An o-ring seated on the inner diameter of the ring forms a leakage-free seal between the ring and the seed injection housing. Four circular cutouts spaced 90-deg. from each other on the outer circumference of the seed injection ring accommodate the pre-compression rods described in Section 4.2. The axial positioning of the injection port must be chosen to allow sufficient streamwise distance for the injected seed material to diffuse, resulting in a seed cloud at the region of interest that meets the density and size required for PIV measurements. As such, the injection port is placed immediately downstream of the swirl generator. More detailed design and analysis of the injection port is presented in Chapter 6. 


\subsubsection{Structural Analysis}

\subsubsection{Computational Model and Results}

Figs. 4.42 and 4.43 present the computational model for the structural analysis of the seed injection housing. Table 4.24 presents the specifics of the computational grid. The grid consists of approximately 1.0 million tetrahedral elements, with approximately 0.1 million elements in the steel sleeve and 0.4 million elements in the HDPE housing. Fig. 4.44 presents the Von Mises stress distribution in the parts under the loading shown in Fig. 4.42. Table 4.25 presents a summary of the structural analysis results. The stresses and deformations in the seed injection housing components and the V-band clamps confirm the structural integrity of the parts under the prescribed loading conditions. 


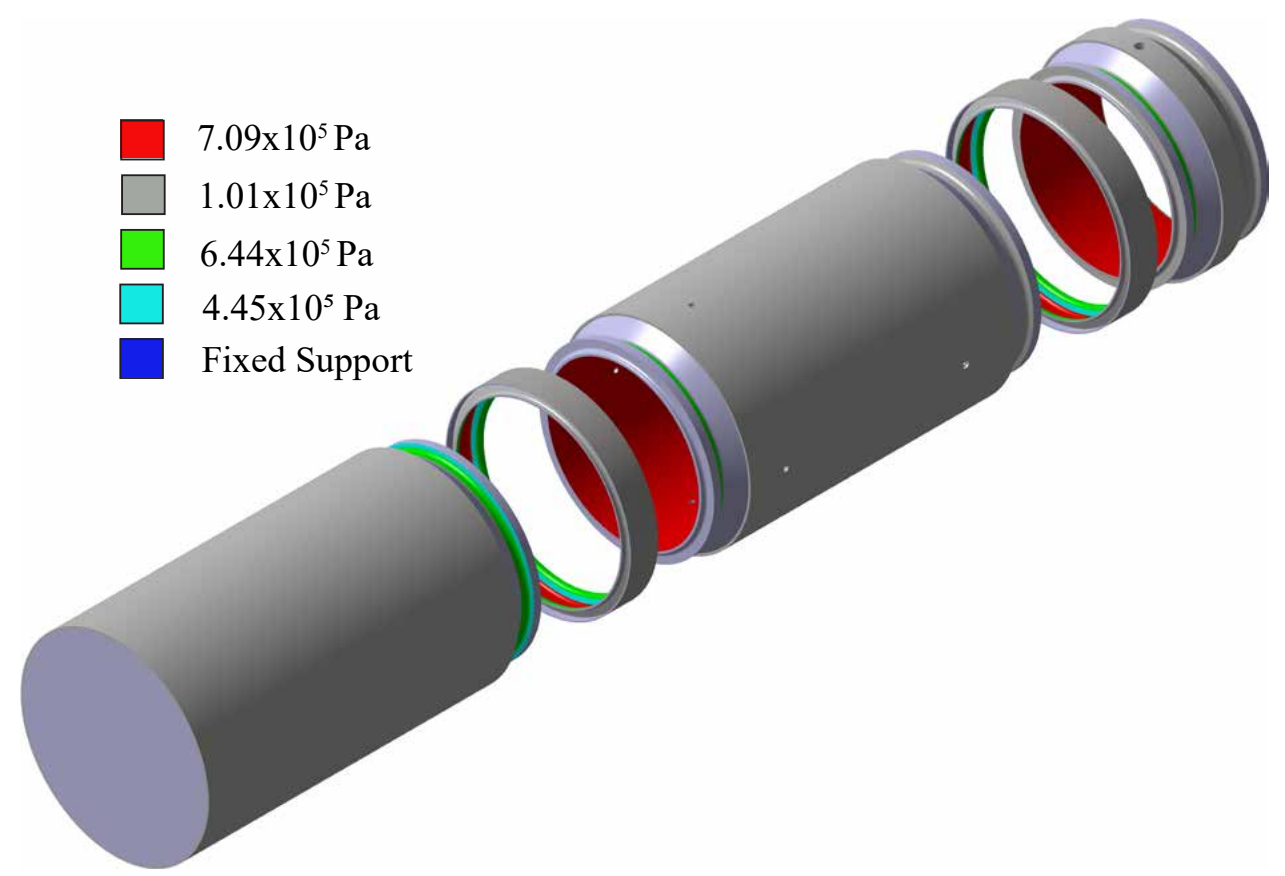

(a)

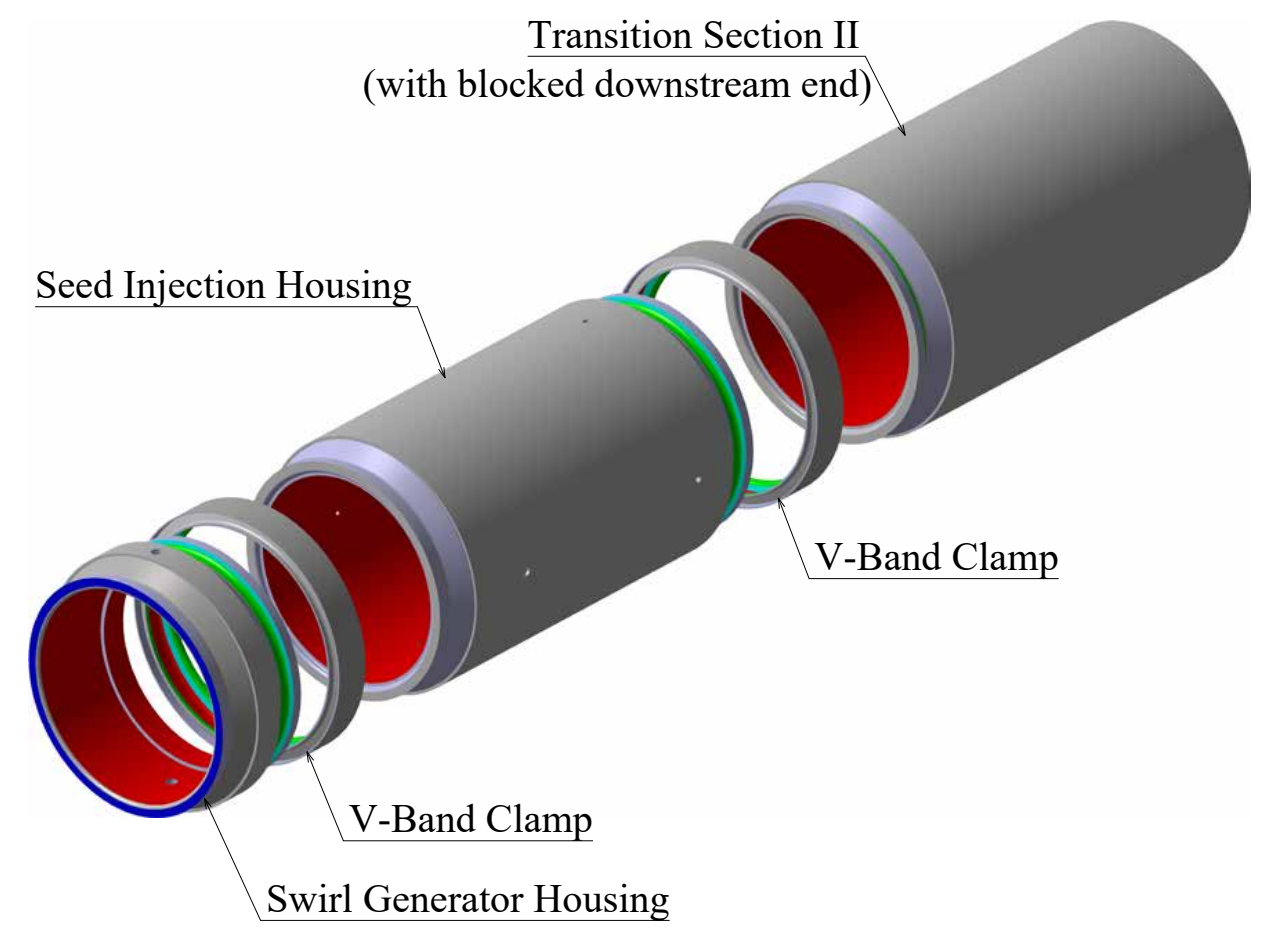

(b)

Figure 4.42: FEA computational model of the seed injection subassembly as seen from the (a) downstream end; (b) upstream end. 

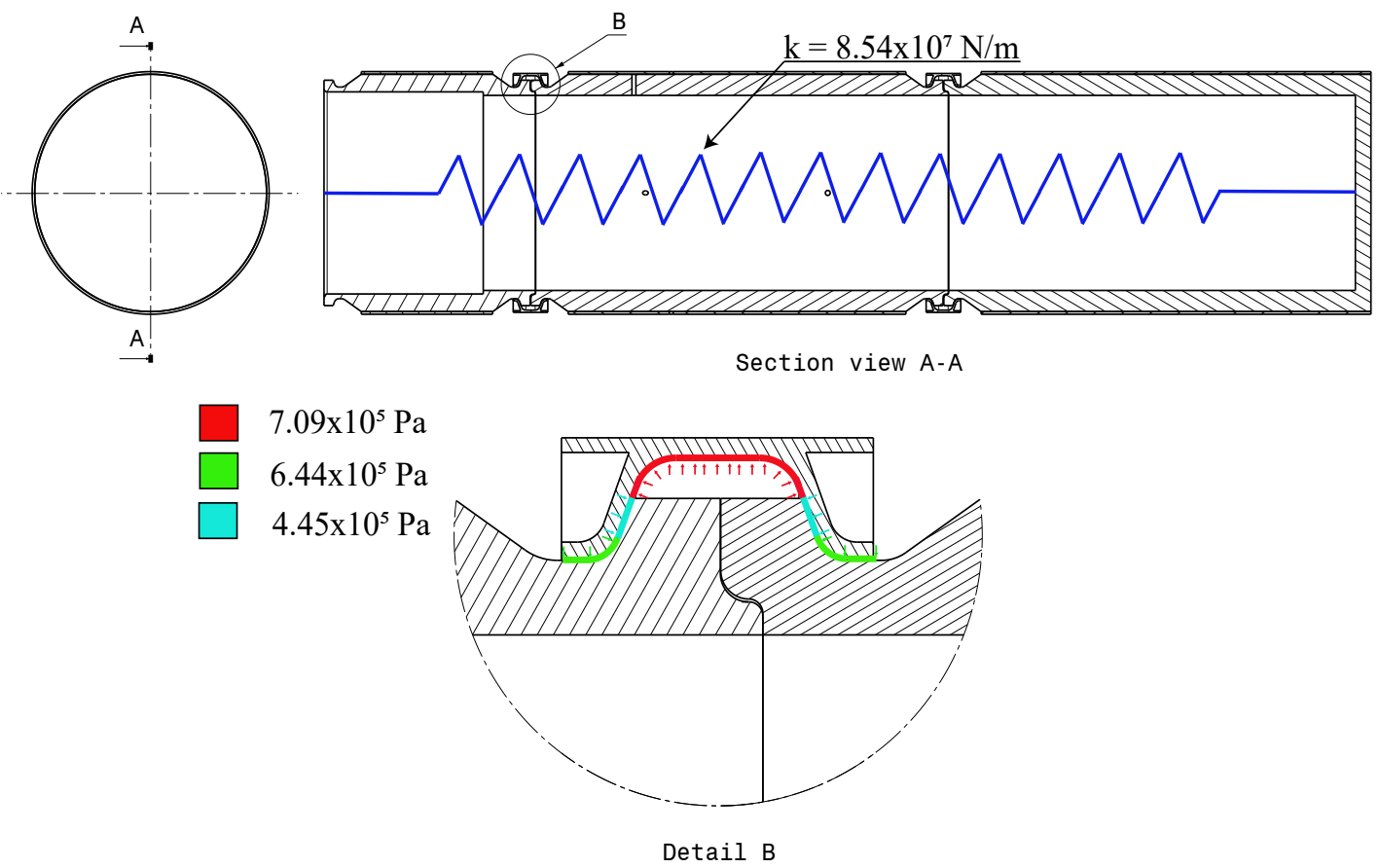

Figure 4.43: Schematic showing the loading of the V-band clamp and the location of the spring used to model the pre-compression rods.

Table 4.24: Parameters of the computational grid.

\begin{tabular}{lcc}
\hline & $\begin{array}{c}\text { Element size }(\mathrm{mm}) \\
\min / \max \end{array}$ & $\begin{array}{c}\text { Spatial Growth rate } \\
\min / \max \end{array}$ \\
\hline HDPE Pipe & $1.5 / 5.0$ & $0 \% / 15 \%$ \\
V-band Clamp & $1.0 / 1.0$ & $0 \%$ \\
Steel Sleeve & $0.5 / 0.5$ & $0 \%$ \\
\hline
\end{tabular}



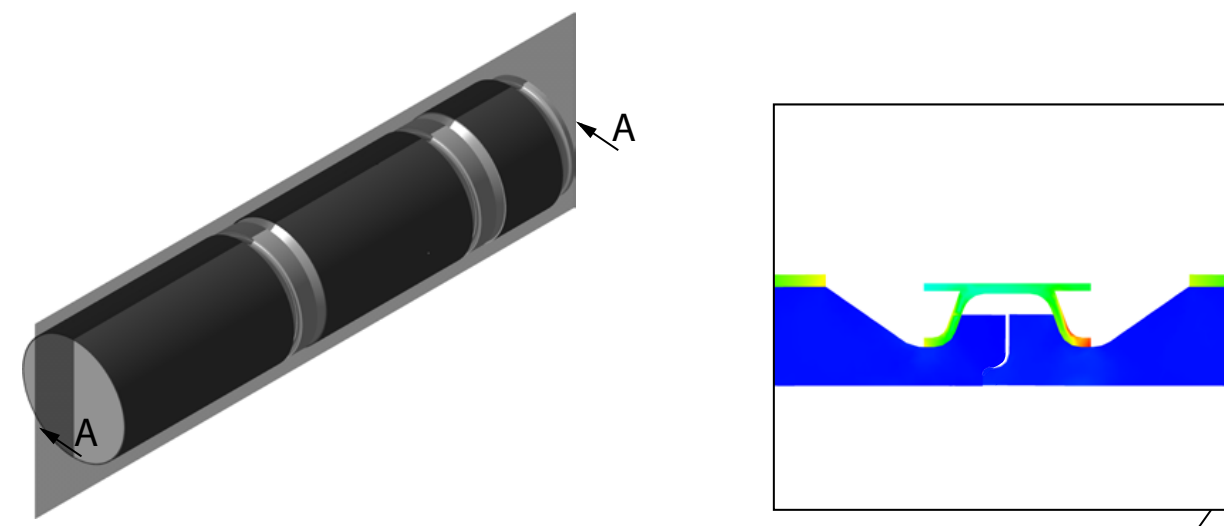

Von Mises Stress (MPa)

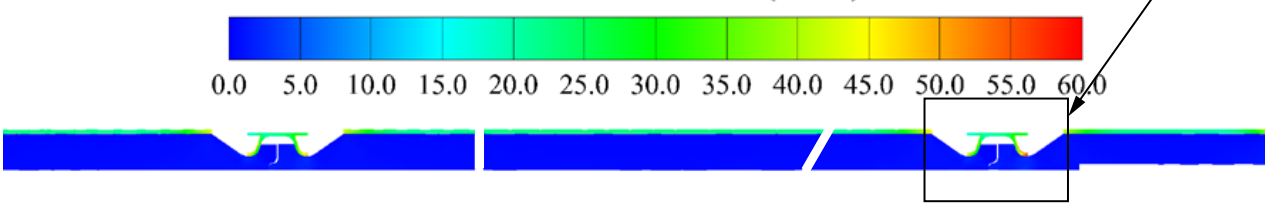

Section View A-A

Figure 4.44: Von mises stress distribution in the seed injection subassembly.

Table 4.25: Summary of the structural analysis results for the seed injection subassembly.

\begin{tabular}{|c|c|c|c|c|c|}
\hline Component & $\begin{array}{l}\text { Pre-compression } \\
\text { Load }(\mathrm{N})\end{array}$ & $\begin{array}{c}\text { Max. } \\
\text { Deformation } \\
\left(\mathrm{x} 10^{-2} \mathrm{~mm}\right) \\
\end{array}$ & $\begin{array}{c}\text { Max. Stress } \\
\text { (MPa) }\end{array}$ & $\begin{array}{c}\text { Min. Safety } \\
\text { Factor }\end{array}$ & $\begin{array}{l}\text { TSSA Required } \\
\text { Safety Factor }{ }^{1}\end{array}$ \\
\hline HDPE Pipe & $\begin{array}{c}1500 \\
0 \\
\end{array}$ & $\begin{array}{l}12.5 \pm 0.4 \\
13.4 \pm 0.4 \\
\end{array}$ & $\begin{array}{l}2.5 \pm 0.1 \\
2.7 \pm 0.1 \\
\end{array}$ & $\begin{array}{c}10.3 \pm 0.3 \\
9.6 \pm 0.3 \\
\end{array}$ & $\begin{array}{l}10.0 \\
10.0\end{array}$ \\
\hline V-band Clamp & $\begin{array}{c}1500 \\
0 \\
\end{array}$ & $\begin{array}{l}6.72 \pm 0.20 \\
7.26 \pm 0.22 \\
\end{array}$ & $\begin{array}{l}59.8 \pm 1.8 \\
60.0 \pm 1.8 \\
\end{array}$ & $\begin{array}{l}4.2 \pm 0.1 \\
4.2 \pm 0.1\end{array}$ & $\begin{array}{l}4.0 \\
4.0 \\
\end{array}$ \\
\hline Steel Sleeve & $\begin{array}{c}1500 \\
0\end{array}$ & $\begin{array}{l}5.85 \pm 0.17 \\
6.66 \pm 0.20\end{array}$ & $\begin{array}{l}46.2 \pm 1.4 \\
46.2 \pm 1.4\end{array}$ & $\begin{array}{l}5.4 \pm 0.2 \\
5.4 \pm 0.2\end{array}$ & $\begin{array}{l}4.0 \\
4.0\end{array}$ \\
\hline
\end{tabular}

${ }^{1}$ (TSSA, 2007) 


\subsection{Transition Section II}

Transition section II is shown in Fig. 4.45. Table 4.26 presents the design parameters of the section. The section has an inner diameter of $122.2 \mathrm{~mm}$ and a length of $247.8 \mathrm{~mm}$. The length of this section was chosen such such that the wake signatures of the swirl generator guide vanes and of the radial support rods would be sufficiently dissipated prior to the flow entering the test section. The chosen length is expected to be suitable for this purpose based on experience with a geometrically similar flow path used previously at Carleton University for lobed-mixer aerodynamic studies at incompressible Mach numbers (Brinkerhoff et al., 2013; Brinkerhoff et al., 2014a). The section is made of HDPE with a wall thickness of $13.3 \mathrm{~mm}$ inserted in a steel sleeve of $1.65 \mathrm{~mm}$ wall thickness. The section also has several ports to facilitate the installation of pressure taps and measurement probes. The installation of the section is illustrated in Fig. 4.46.

Table 4.26: Design parameters for transition section II.

\begin{tabular}{cccc} 
Design Code & Press. Rating & Temp. Rating & ASTM Material Specifications \\
ASME B31.1/CSA B51 & $7 \mathrm{~atm}$ & $25^{\circ} \mathrm{C}$ & HDPE, Steel (Table 4.9) \\
\hline
\end{tabular}




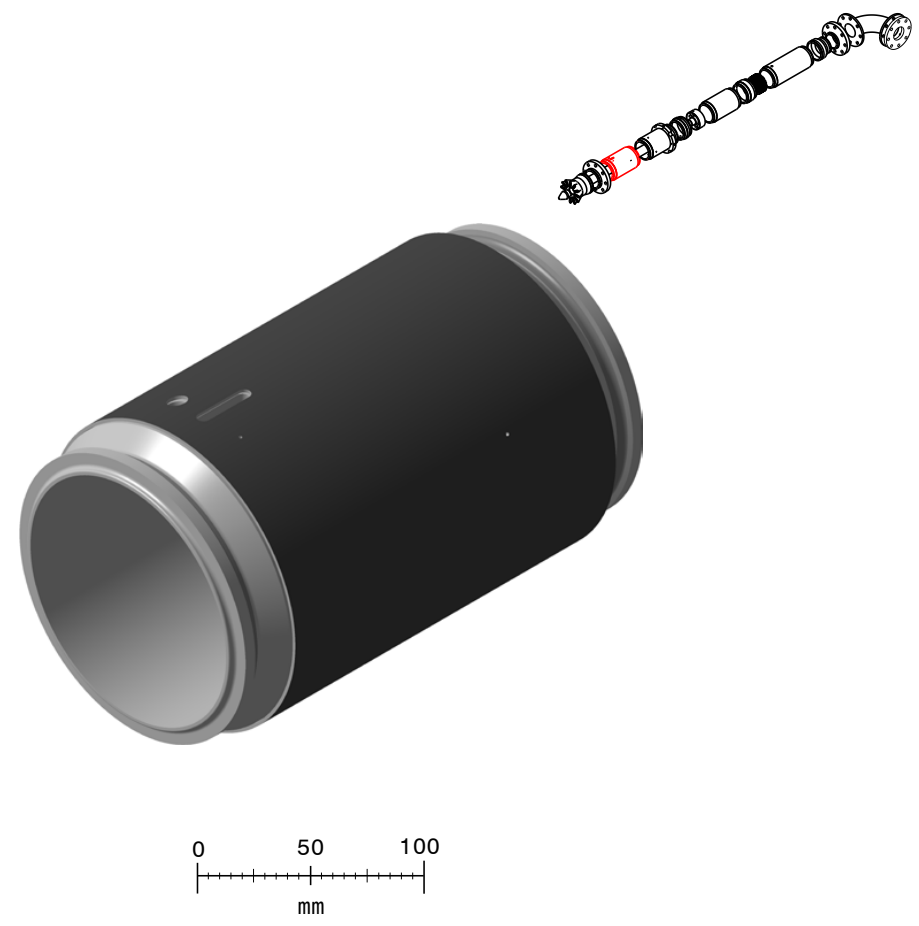

Figure 4.45: Transition section II. 


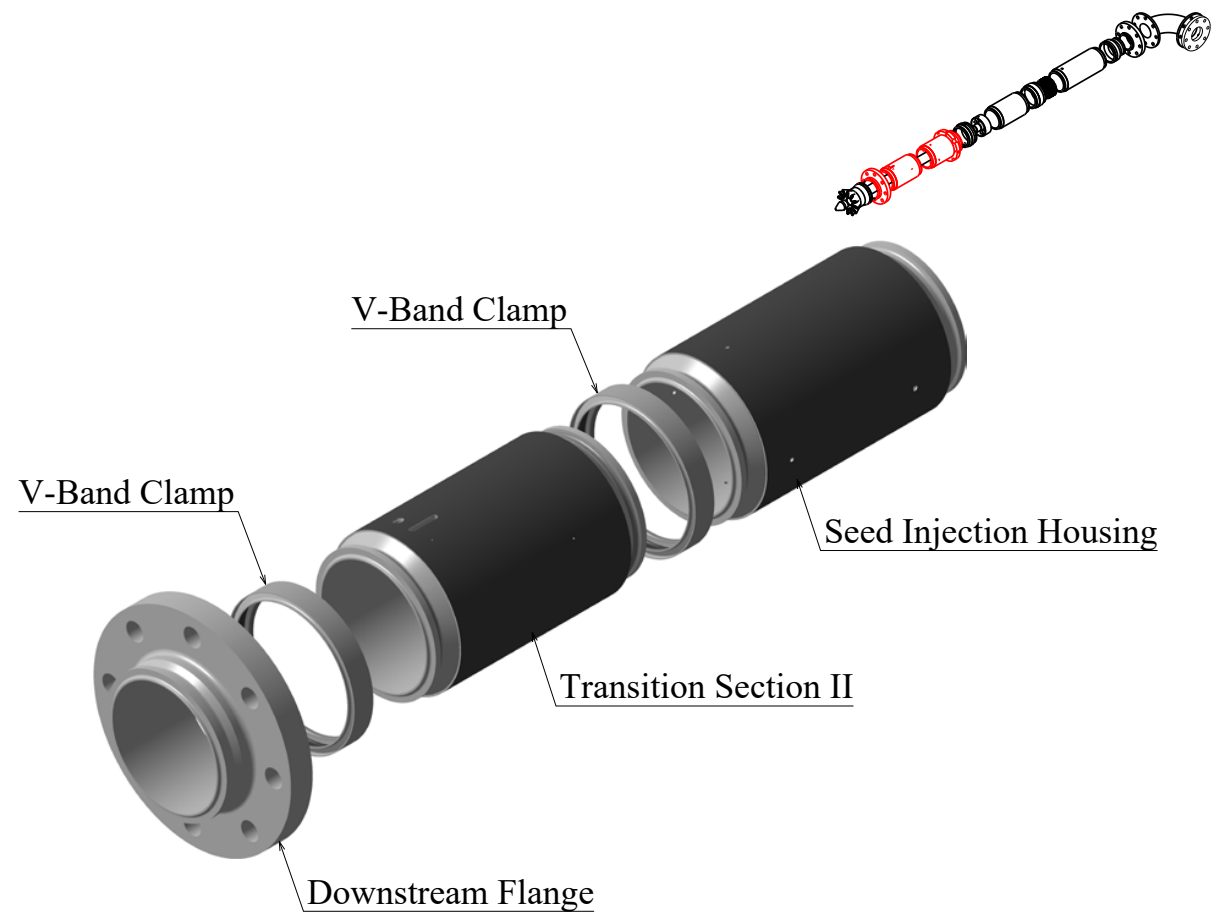

Figure 4.46: Transition section II installation.

\subsubsection{Structural Analysis}

\subsubsection{Computational Model and Results}

Figs. 4.47 and 4.48 present the computational model for the structural analysis of transition section II. Table 4.27 presents the specifics of the computational grid. The grid consists of approximately 1.3 million tetrahedral elements, with approximately 0.2 million elements in the steel sleeve and 0.5 million elements in the HDPE housing. Fig. 4.49 presents the Von Mises stress distribution in the parts of the subassembly. Table 4.13 presents a summary of the structural analysis results. The stresses and deformations in the section components and the V-band clamps confirm the structural integrity of the parts under the prescribed loading conditions. 


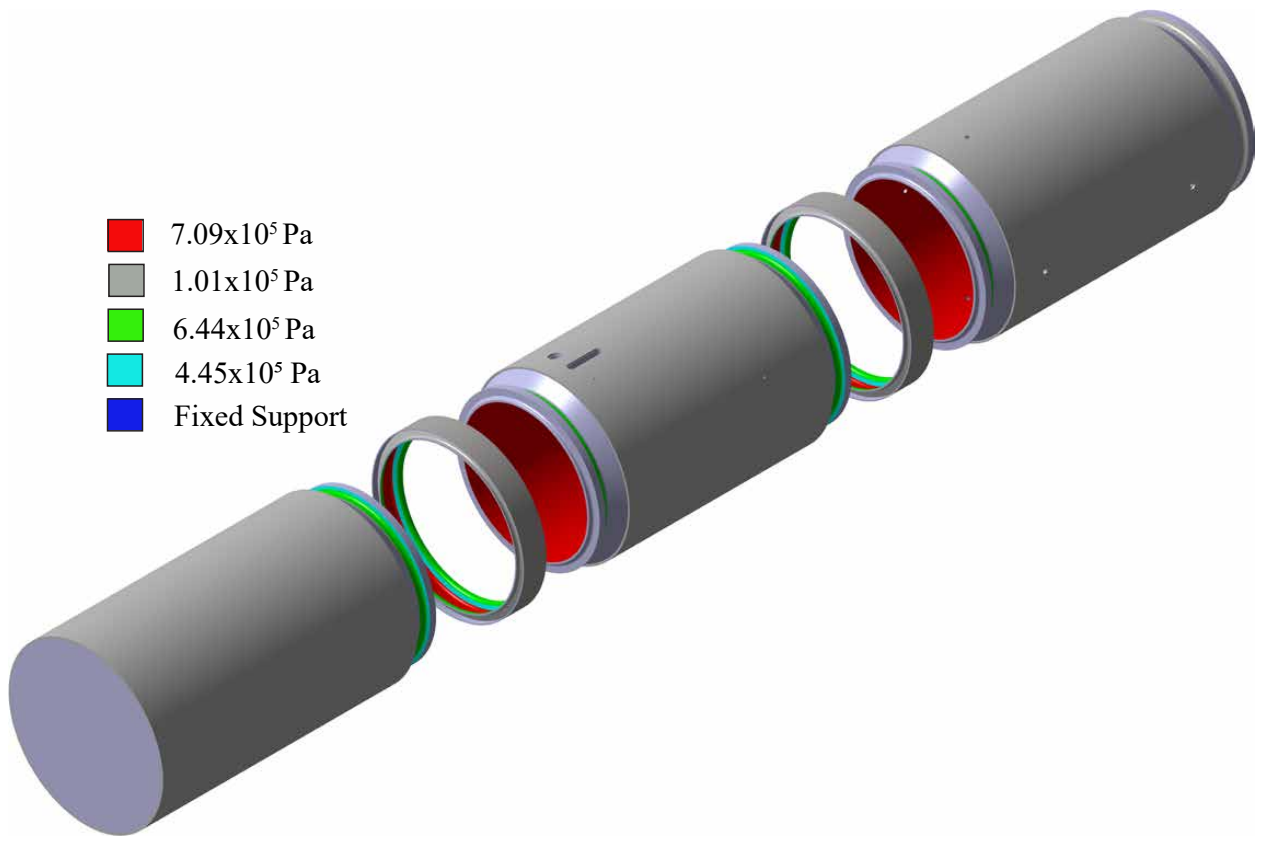

(a)

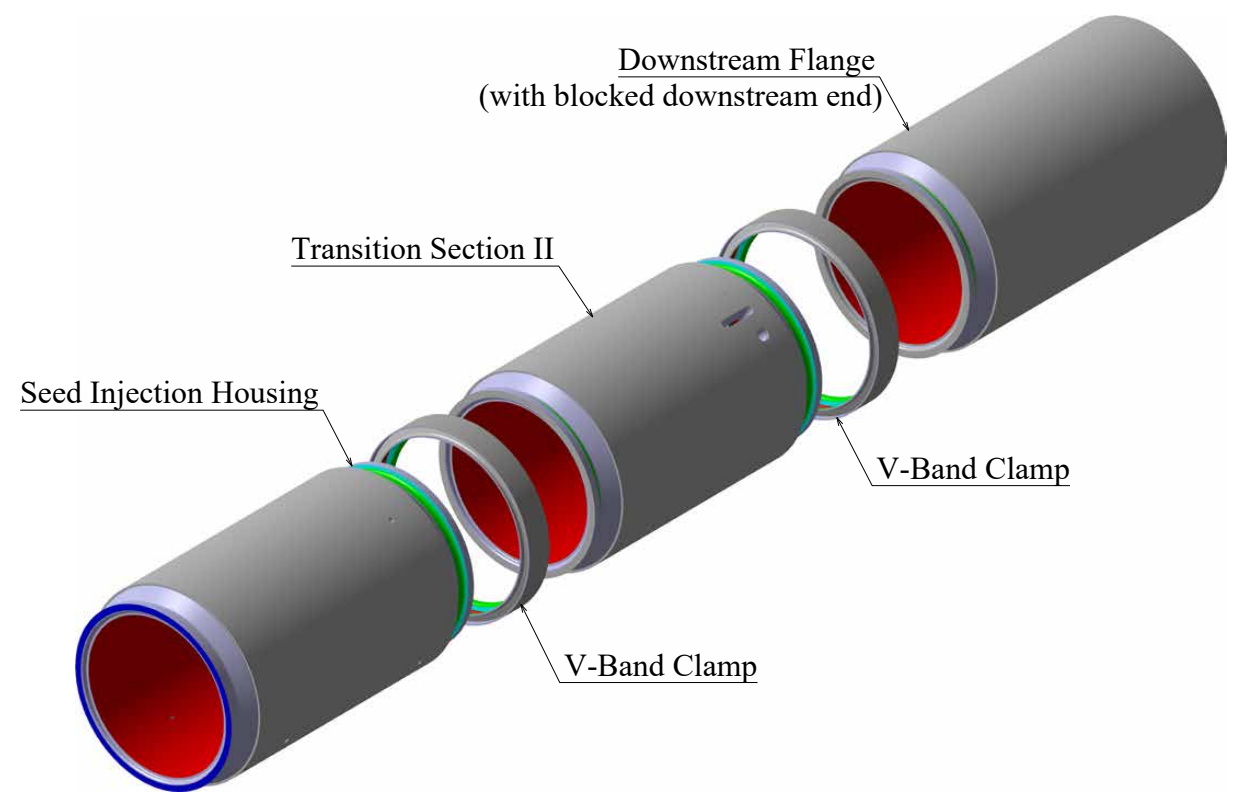

(b)

Figure 4.47: FEA computational model of transition section II as seen from the (a) downstream end; (b) upstream end. 

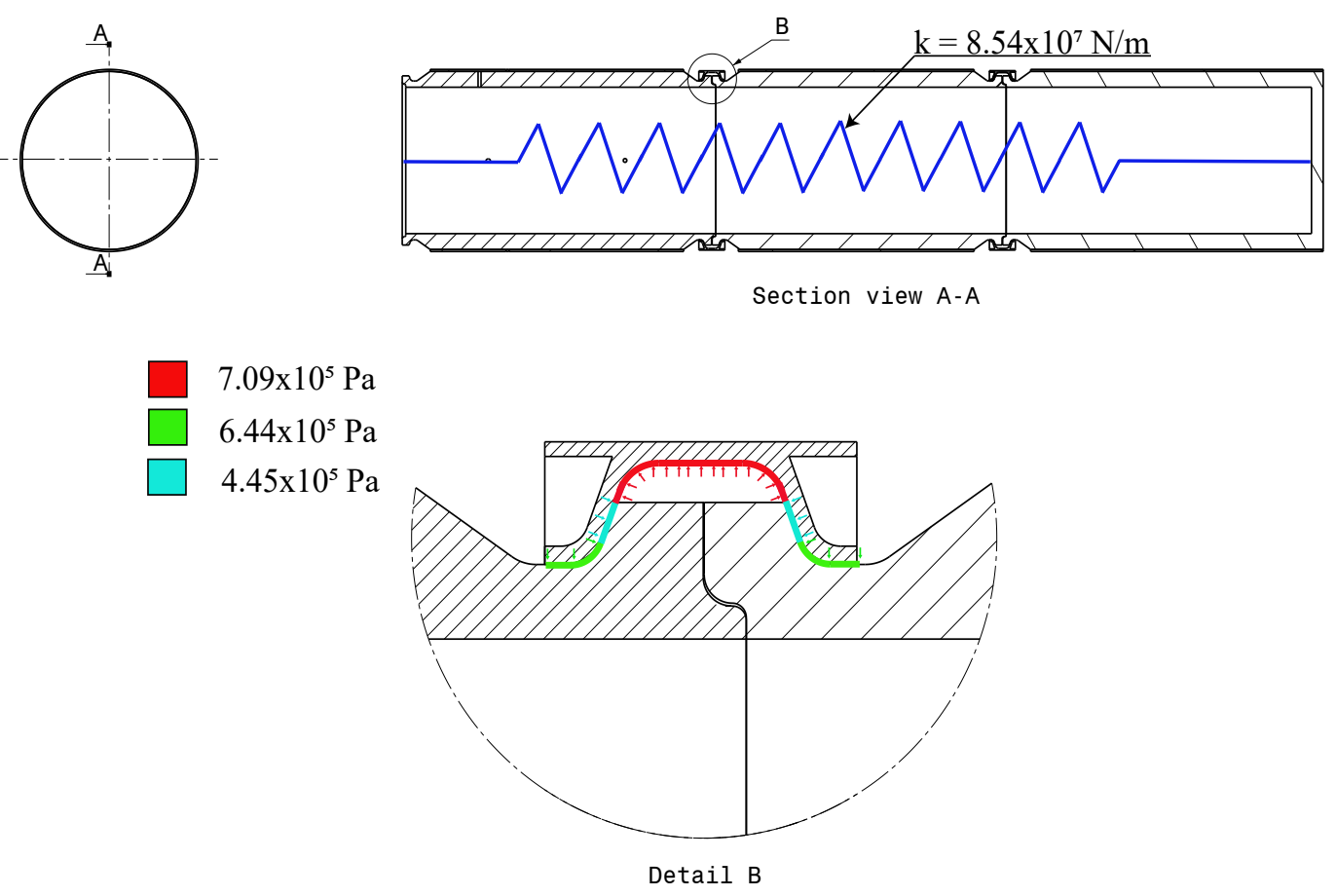

Figure 4.48: Schematic showing the loading of the V-band clamp and the location of the spring used to model the pre-compression rods.

Table 4.27: Parameters of the computational grid.

\begin{tabular}{lcc}
\hline & $\begin{array}{c}\text { Element size }(\mathrm{mm}) \\
\min / \max \end{array}$ & $\begin{array}{c}\text { Spatial Growth rate } \\
\min / \max \end{array}$ \\
\hline HDPE Pipe & $1.5 / 5.0$ & $0 \% / 15 \%$ \\
V-band Clamp & $1.0 / 1.0$ & $0 \%$ \\
Steel Sleeve & $0.5 / 0.5$ & $0 \%$ \\
\hline
\end{tabular}



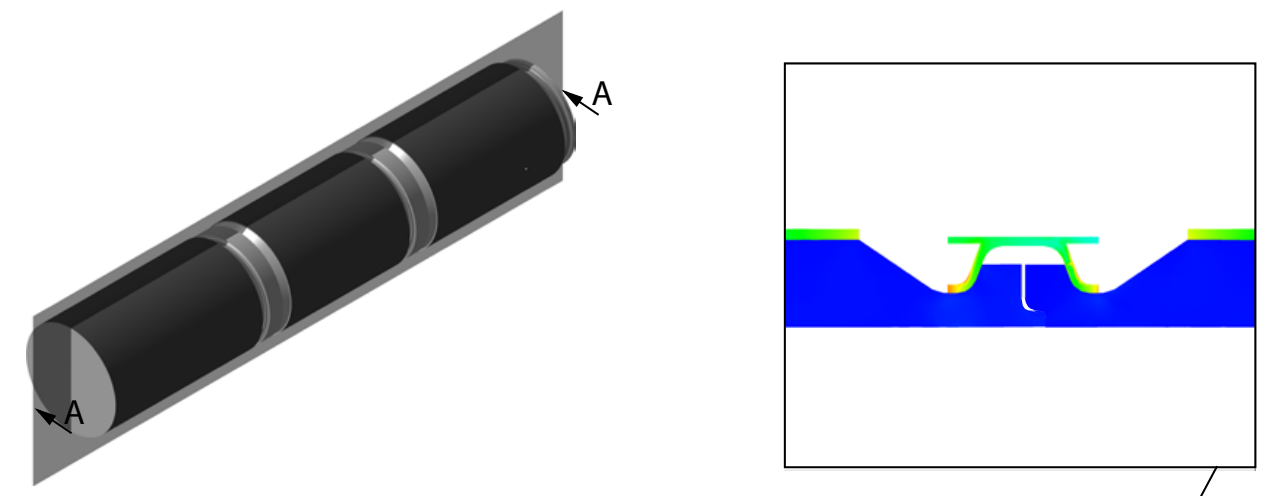

Von Mises Stress (MPa)

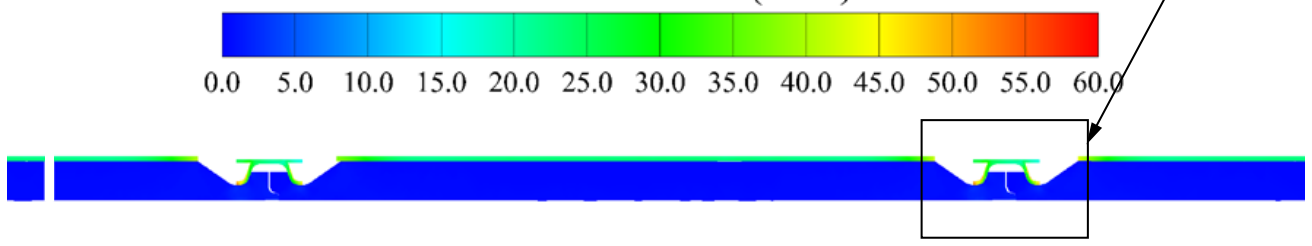

Section View A-A

Figure 4.49: Von mises stress distribution in the transition section II subassembly.

Table 4.28: Summary of the structural analysis results for the transition section II subassembly.

\begin{tabular}{|c|c|c|c|c|c|}
\hline Component & $\begin{array}{l}\text { Pre-compression } \\
\text { Load }(\mathrm{N})\end{array}$ & $\begin{array}{c}\text { Max. } \\
\text { Deformation } \\
\left(\mathrm{x} 10^{-2} \mathrm{~mm}\right)\end{array}$ & $\begin{array}{c}\text { Max. Stress } \\
\text { (MPa) }\end{array}$ & $\begin{array}{c}\text { Min. Safety } \\
\text { Factor }\end{array}$ & $\begin{array}{l}\text { TSSA Required } \\
\text { Safety Factor }{ }^{1}\end{array}$ \\
\hline HDPE Pipe & $\begin{array}{c}1500 \\
0 \\
\end{array}$ & $\begin{array}{l}12.9 \pm 0.4 \\
13.8 \pm 0.4\end{array}$ & $\begin{array}{l}2.5 \pm 0.1 \\
2.7 \pm 0.1\end{array}$ & $\begin{array}{c}10.3 \pm 0.3 \\
9.5 \pm 0.3\end{array}$ & $\begin{array}{l}10.0 \\
10.0\end{array}$ \\
\hline V-band Clamp & $\begin{array}{c}1500 \\
0 \\
\end{array}$ & $\begin{array}{l}5.53 \pm 0.17 \\
6.21 \pm 0.17 \\
\end{array}$ & $\begin{array}{l}61.1 \pm 1.8 \\
61.8 \pm 1.9 \\
\end{array}$ & $\begin{array}{l}4.1 \pm 0.1 \\
4.0 \pm 0.1\end{array}$ & $\begin{array}{l}4.0 \\
4.0 \\
\end{array}$ \\
\hline Steel Sleeve & $\begin{array}{c}1500 \\
0\end{array}$ & $\begin{array}{l}5.69 \pm 0.17 \\
6.54 \pm 0.19\end{array}$ & $\begin{array}{l}42.3 \pm 1.3 \\
42.2 \pm 1.3\end{array}$ & $\begin{array}{l}5.9 \pm 0.2 \\
5.9 \pm 0.2\end{array}$ & $\begin{array}{l}4.0 \\
4.0\end{array}$ \\
\hline
\end{tabular}

${ }^{1}$ (TSSA, 2007) 


\subsection{Downstream Flange}

The downstream flange is shown in Fig. 4.50. Table 4.3 presents the design parameters of the flange. It is made of mild steel and is fastened to the upstream and downstream mating pipe sections using V-band clamps as illustrated in Fig. 4.51. The flange has an inner diameter of $122.2 \mathrm{~mm}$ and a flange face thickness of 25.4 $\mathrm{mm}$. Eight holes of $20 \mathrm{~mm}$-diameter are evenly placed along the flange circumference. Four of these holes are needed to install the pre-compression rods described in Section 4.2, while the remaining holes provide attachment points for securing the air-supply system to anchor points in the laboratory.

Table 4.29: Design parameters for the downstream flange.

\begin{tabular}{cccc}
\hline Design Code & Press. Rating & Temp. Rating & ASTM Material Specifications \\
ASME B16.5/CSA B51 & $7 \mathrm{~atm}$ & $25^{\circ} \mathrm{C}$ & Steel (Table 4.5) \\
\hline
\end{tabular}

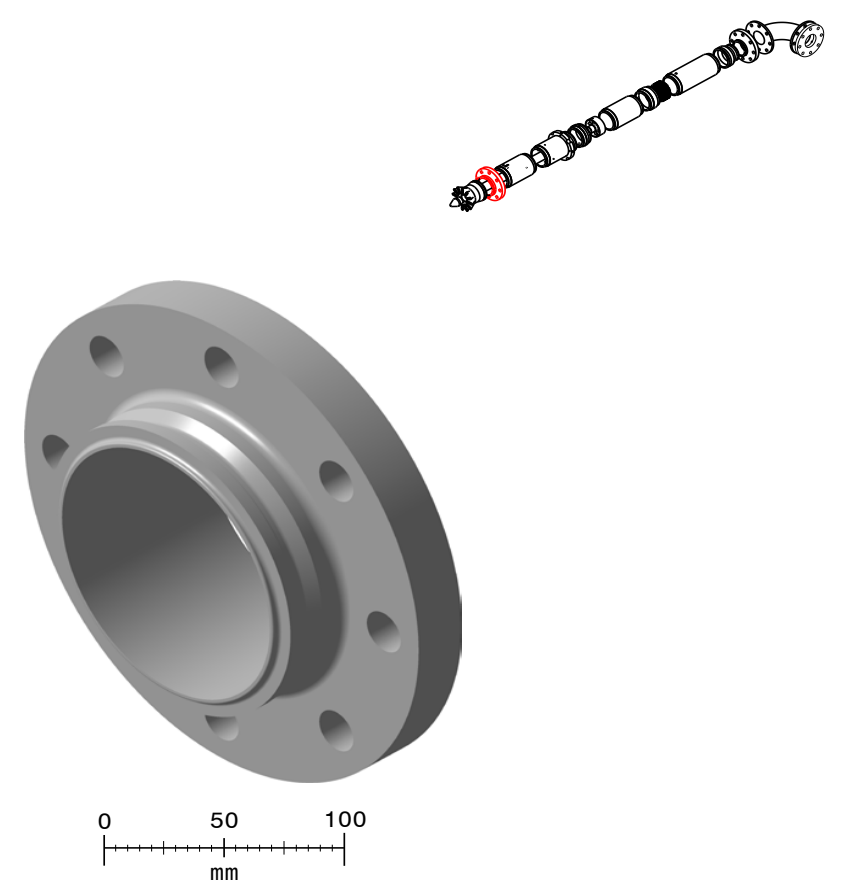

Figure 4.50: Downstream flange. 


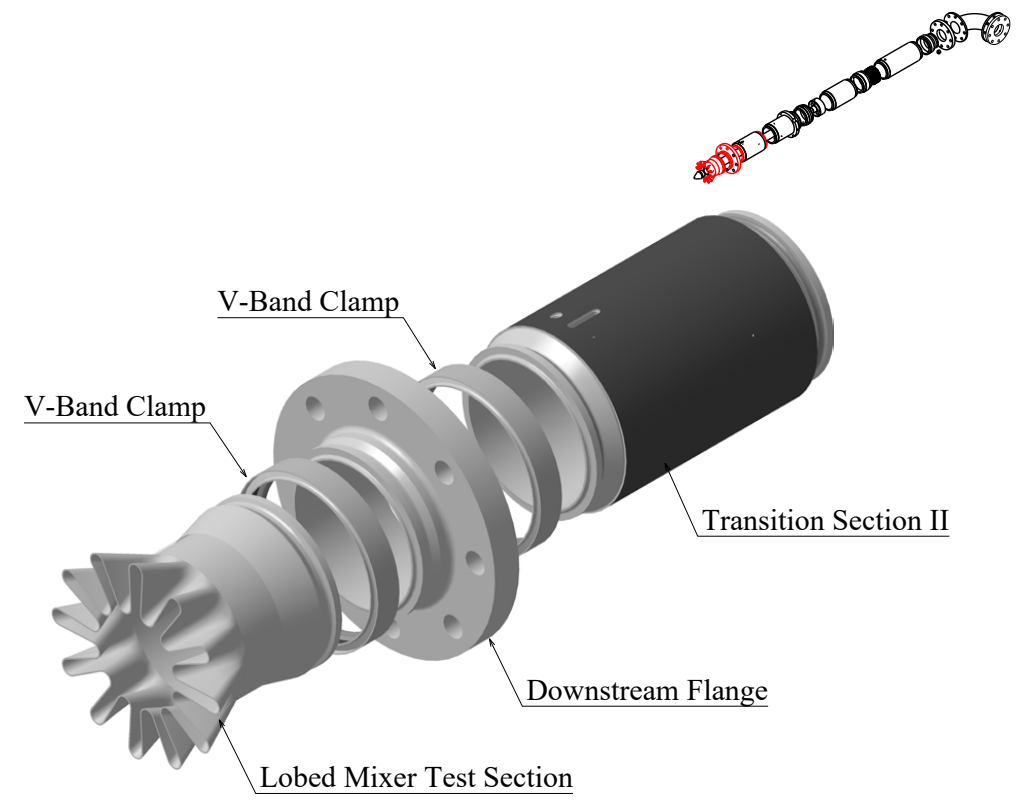

Figure 4.51: Downstream flange installation.

\subsubsection{Structural Analysis}

\subsubsection{Computational Model and Results}

Figs. 4.52 and 4.53 present the computational model for the structural analysis of the downstream flange. Table 4.30 presents the specifics of the computational grid. The grid consists of approximately 1.0 million tetrahedral elements, with approximately 0.4 million elements in the downstream flange. Fig. 4.54 presents the Von Mises stress distribution in the parts of the subassembly. Table 4.31 presents a summary of the structural analysis results which shows that the stresses and deformations in the downstream flange and the V-band clamps confirm the structural integrity of the parts under the prescribed loading conditions. 


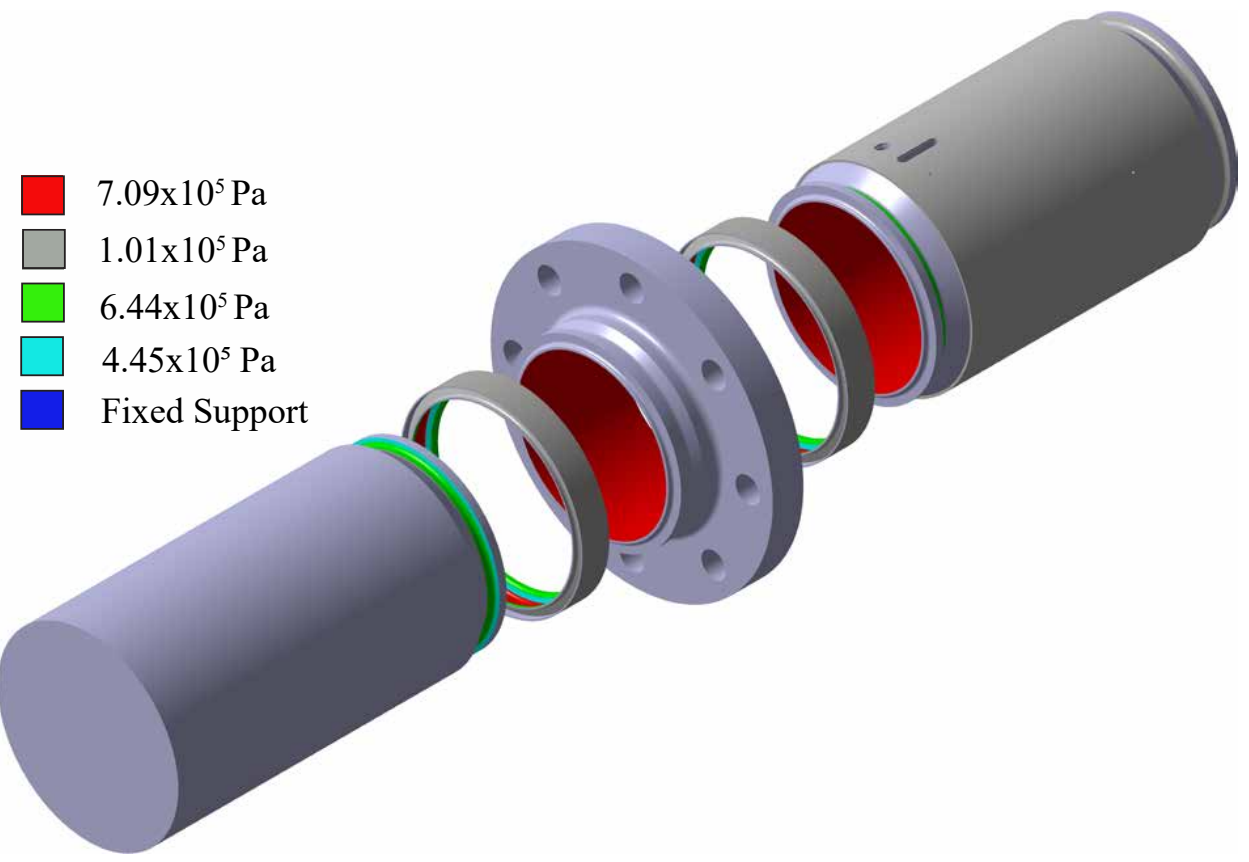

(a)

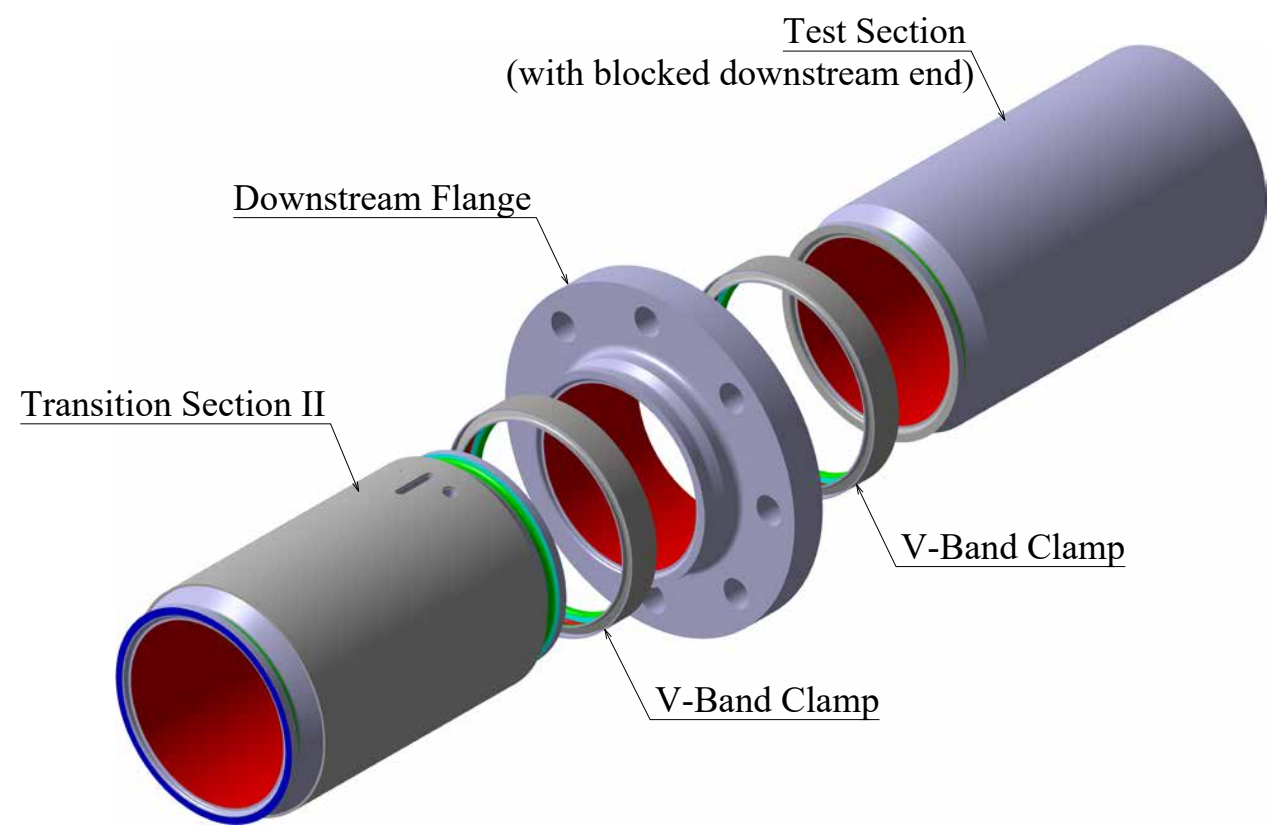

(b)

Figure 4.52: FEA computational model of the downstream flange as seen from the (a) downstream end; (b) upstream end. 


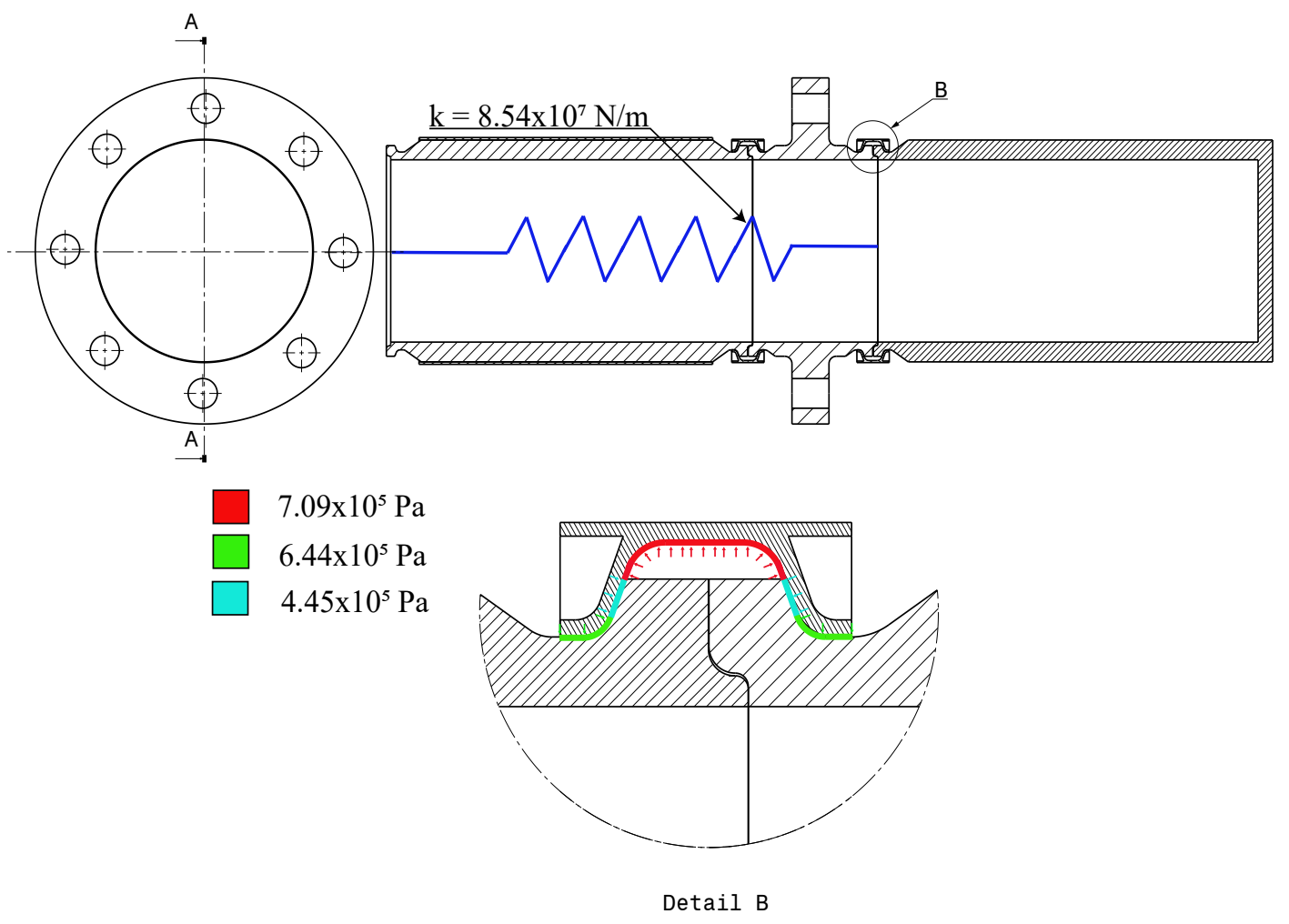

Figure 4.53: Schematic showing the loading of the V-band clamp and the location of the spring used to model the pre-compression rods.

Table 4.30: Parameters of the computational grid.

\begin{tabular}{lcc}
\hline & $\begin{array}{c}\text { Element size }(\mathrm{mm}) \\
\min / \max \end{array}$ & $\begin{array}{c}\text { Spatial Growth rate } \\
\min / \max \end{array}$ \\
\hline Downstream Flange & $1.5 / 5.0$ & $0 \% / 15 \%$ \\
V-band Clamp & $1.0 / 1.0$ & $0 \%$ \\
\hline
\end{tabular}




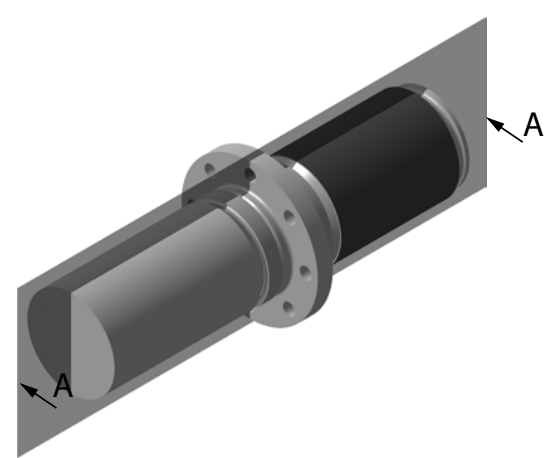

Von Mises Stress (MPa)

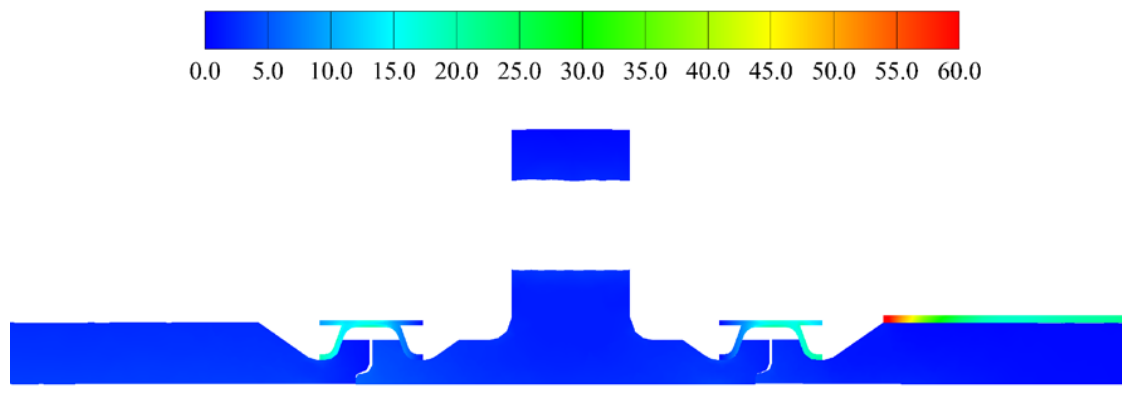

Section View A-A

Figure 4.54: Von mises stress distribution in the downstream flange subassembly.

Table 4.31: Summary of the structural analysis results for the downstream flange subassembly.

\begin{tabular}{|c|c|c|c|c|c|}
\hline Component & $\begin{array}{l}\text { Pre-compression } \\
\text { Load }(\mathrm{N})\end{array}$ & $\begin{array}{c}\text { Max. } \\
\text { Deformation } \\
\left(\mathrm{x} 10^{-2} \mathrm{~mm}\right)\end{array}$ & $\begin{array}{l}\text { Max. Stress } \\
(\mathrm{MPa})\end{array}$ & $\begin{array}{c}\text { Min. Safety } \\
\text { Factor }\end{array}$ & $\begin{array}{l}\text { TSSA Required } \\
\text { Safety Factor }^{1}\end{array}$ \\
\hline \multirow[t]{2}{*}{ Downstream Flange } & 1500 & $8.15 \pm 0.24$ & $5.8 \pm 0.2$ & $43.2 \pm 1.3$ & 4.0 \\
\hline & 0 & $8.72 \pm 0.26$ & $5.7 \pm 0.2$ & $43.8 \pm 1.3$ & 4.0 \\
\hline \multirow[t]{2}{*}{ V-band Clamp } & 1500 & $7.95 \pm 0.24$ & $23.1 \pm 0.7$ & $10.8 \pm 0.3$ & 4.0 \\
\hline & 0 & $7.97 \pm 0.24$ & $23.1 \pm 0.7$ & $10.8 \pm 0.3$ & 4.0 \\
\hline
\end{tabular}




\subsection{Air-Supply System Aerodynamic Performance Prediction}

An algorithm that predicts the aerodynamic performance of the air-supply system was developed using the methodology described in Section 3.2.1. The computer program, printed in Appendix D, computes the time it takes for the control valve to reach its fully opened position for a specified exit-plane Mach number. A fully open valve signifies the end of the time period over which the exit-plane Mach number can be maintained at the specified value. The calculation process is driven by the drop in stagantion pressure in the storage tanks which is modeled as:

$$
\frac{d P_{o T a n k}}{d t}=-\dot{m}\left(\frac{n R T_{o T a n k}}{V}\right)
$$

where $n=1.0$ for an isothermal process and $n=1.4$ for an isotropic process. The static pressure at the exit-plane of the flow path is assumed equal to the ambient pressure, restricting the algorithm to subsonic and uniaxial flow conditions at this plane. The flow path leading from the air-storage tanks to the exit plane is assumed to be adiabatic. The short duration of a typical experimental run on this setup will substantially limit the amount of heat transfer that can be realized, thus justifying this assumption. Accordingly, the stagnation temperature along the flow path is the same as the stagnation temperature in the storage tanks. However, as the air in the storage tank expands, the stagnation temperature drops over time which can be expressed as:

$$
\frac{d T_{o T a n k}}{d t}=-\dot{m}\left(\frac{(n-1) R T_{o T a n k}^{2}}{P_{o T a n k} V}\right)
$$

where $n=1.0$ for isothermal expansion in the tanks and $n=1.4$ for adiabatic expansion. The Mach number, static pressure and stagnation temperature at the exit plane yield values for the local mass flow rate and stagnation pressure. The calculated value of mass flow rate applies to all flow cross-sections of the flow path for the steady operating conditions realized through manipulation of the flow area at the control valve.

The stagnation pressure increases in the upstream direction along the flow path due to frictional pressure losses. The local values of stagnation pressure along the 
flow path are calculated based on estimated values of stagnation pressure losses along segments of constant-area flow using the Fanno compressible friction flow theory. Stagnation pressure losses across other geometries such as bends, sudden changes in flow cross-sectional area, screens, and honeycombs are calculated using empirical loss coefficients as summarized in Table 4.32.

Table 4.32: Air-supply system empirical loss coefficients.

\begin{tabular}{lr}
\hline Loss Device & Loss Coefficient $(K)$ \\
\hline Upstream of control valve (facility losses) & \\
\hline Sudden contraction (x4) & $0.5(\mathrm{x} 4)$ \\
Sudden expansion (x4) & $1.0(\mathrm{x} 4)$ \\
Shutoff valve & 0.1 \\
Supply duct & 0.5 \\
\hline Downstream of control valve (air-supply system losses) & 0.20 \\
\hline Existing 90 deg. bend & 1.0 \\
Expansion section & 1.30 \\
Honeycomb & $1.04,1.60$ \\
Wire mesh screens & 0.5 \\
Circular-to-annular transition & 0.5 \\
Lobed mixer test section & \\
\hline
\end{tabular}

The local stagnation pressure together with the local values of mass flow rate, stagnation temperature and cross-sectional area, yield the local Mach number, flow velocity and the static thermodynamic properties. Since the calculation of stagnation pressure loss requires values of local flow velocity and static density, the calculation process is iterative. The algorithm monitors possible flow choking along the flow path, which could potentially prevent the realization of the desired exit-plane Mach number for given stagnation conditions. In such occurrences, the algorithm prompts the user to repeat the calculations with a revised target Mach number and initial stagnation pressure and temperature values. Fig. 4.55 shows the predicted wind-tunnel run time as a function of exit-plane Mach number. Since the actual expansion process in the storage tanks will fall somewhere between an isothermal and an isentropic process, both models have been computed for completeness. The maximum exit-plane Mach number value of 0.8 considered in Fig. 4.55 corresponds to an inlet Mach number of 
about 0.5 for the lobed mixer test section considered in the present exercise (component XIV in Fig. 4.2). A run time of about 30 seconds is achieved for this exit-plane Mach number, which is sufficiently long for the existing measurement instrumentation in the lab facility. The curves in Fig. 4.55 labeled as ideal performance correspond to a frictionless flow path. Their comparison to the curves with frictional flow provide a sense for the effect of frictional losses on the run times.

The fundamental assumptions of the mathematical model used in this aerodynamic prediction algorithm were tested using experimental data in a undergraduate laboratory experiment at Carleton University involving the choked-flow discharge of a compressed-air tank through a converging nozzle. The results in this experiment were noted to be in very good agreement with the predictions of the model.

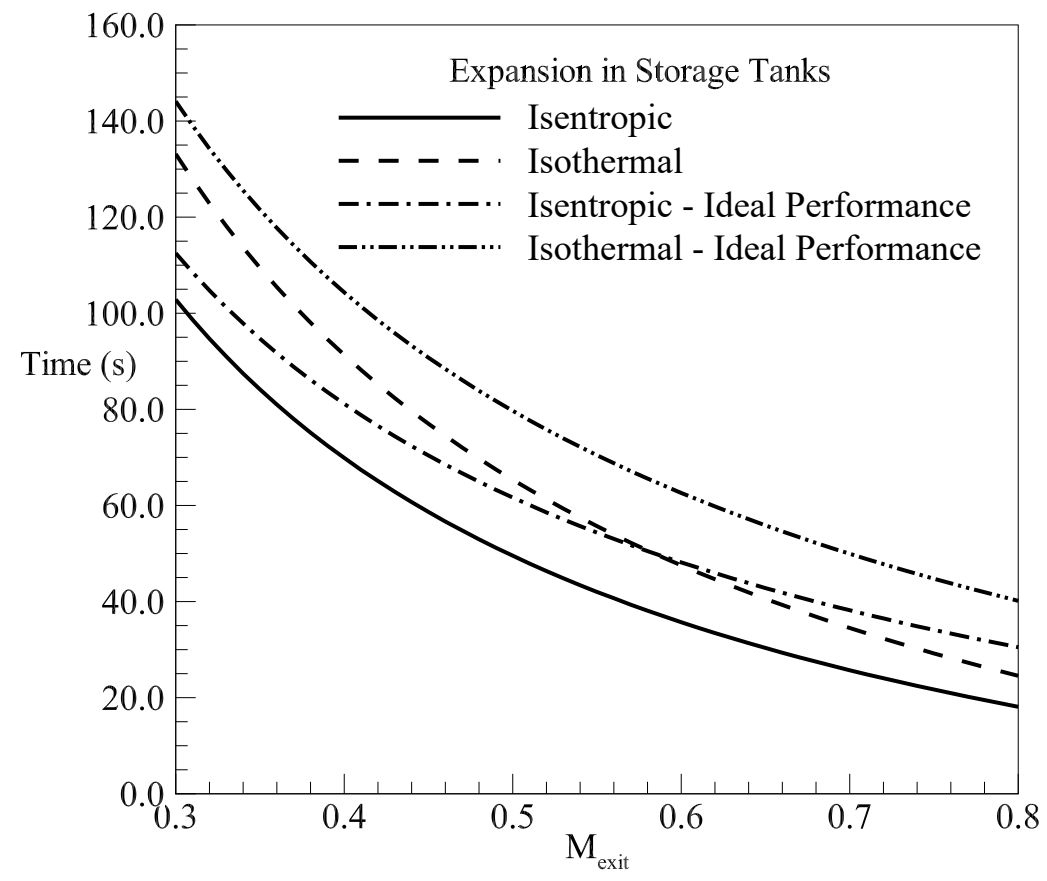

Figure 4.55: Predicted run time as a function of flow path exit-plane Mach number. 


\subsection{Design Summary}

The high-speed annular-flow air-supply system as described in this chapter is shown to produce the desired range of flow Mach numbers with satisfactory length of run times for each charge of the wind-tunnel. The design meets or exceeds the structural safety factors stipulated by the applicable provincial regulations. This functionality and structural integrity is complemented by modularity that will allow fast turn-around times for reconfiguration of the flow path to achieve the desired conditioning of the flow supplied to the test section in each instance. The total mass of the system, including all V-band clamps and fasteners is approximately $55 \mathrm{~kg}$. This compares very favorably to an alternative design that uses steel for all pressure boundaries and bolted flanges for all connections of the system yielding comparable safety factors for which the total mass would have been approximately $200 \mathrm{~kg}$.

Light-weight of the system will minimize the risk of accidents that are more likely to occur during handling of heavier items by relatively inexperienced students. As assembly/disassembly of the system during experiments is anticipated to occur frequently as part of the students' training, lightness of the system is particularly desirable. To contrast, the assembly/disassembly of the existing cascade test section and its upstream and downstream components identified in the facility image in Fig. 3.2 requires the use of a 2-ton shop crane and several hours of effort in each instance. Lastly, the design of the high-speed annular-flow air-supply system is deemed cost

effective, as the system utilizes parts that are either available off-the-shelf, or can be produced from relevant stock material with low amount of material removal using simple lathe and milling machine operations. 


\section{Chapter 5}

\section{Design of a Rectilinear Test Section for Lobed-Mixer Studies at Subsonic Mach Numbers}

\subsection{Design}

The scale of the annular-flow lobed-mixer test section compatible with the air-supply system described in the previous chapter is adequate for measurements of the mixing performance of the lobed mixer. To reliably study the detailed physics of the inviscid instability mechanisms dominating the mixing process, a somewhat larger lobed-mixer model is required. This was realized by developing a rectilinear test section that can be readily installed on the discharge flow path of an existing blade-cascade test section as shown in Fig. 5.1. The rectilinear test section consists of two aluminum wedges that are bolted to an existing flange as shown in Fig. 5.1. Air from the air-storage tanks discharges through a $447.7 \mathrm{~mm} \times 61.3 \mathrm{~mm}$ rectangular opening, and flows through the space between the two wedges (Fig. 5.2). At the wedge trailing edges, this high-speed air comes in contact with the ambient air that is dragged down the

outer slopes of the wedges by the jet-pump effect. The axial length of the wedges is $330.4 \mathrm{~mm}$, which was chosen to achieve a wedge angle of $15 \mathrm{deg}$. A relatively shallow wedge angle was deemed preferable to avoid significant redirection of the ambient air prior to its interaction with the high-speed core flow. Technical drawings for these components are provided in Appendix E. 


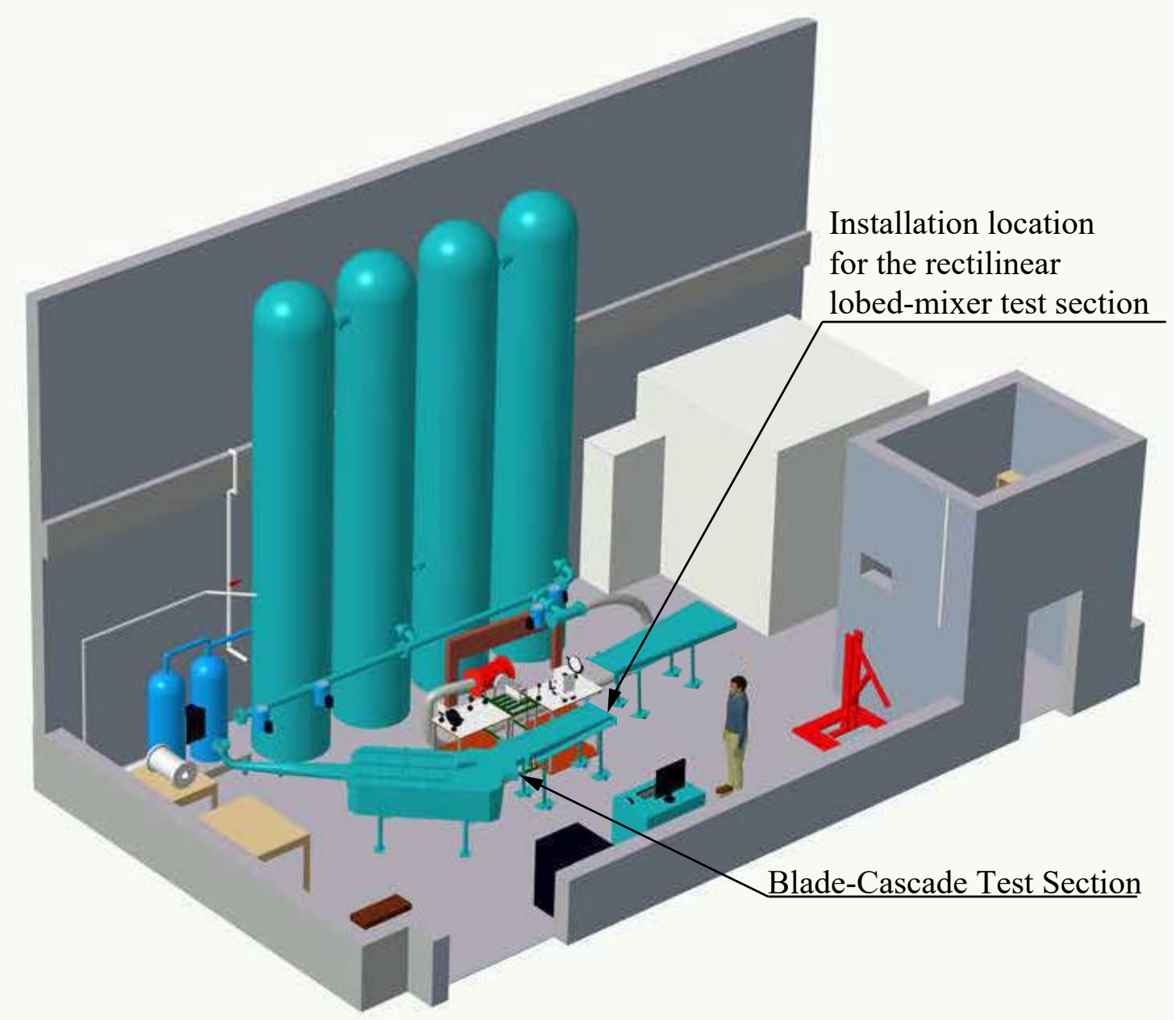

Figure 5.1: Installation location for the rectilinear lobed-mixer test section.

The lobed mixer configuration to be tested is mounted to the trailing edge of one of the wedges as illustrated in Fig. 5.2. The tongue-and-groove configuration for mounting the tail onto the wedge was chosen to promote precise alignment of the horizontal orientation of the tail surface with that of the wedge. Control of the streamwise pressure gradient on the high-speed side of the lobed mixer tail is achieved by mounting a "hub tail" onto the trailing edge of the second wedge. The streamwise pressure gradient can be affected by suitably profiling the hub tail in the streamwise direction, similar to the effect of the end-cone shaping on the hub pipe of the annular setup described in Chapter 4. The width of the setup allows for 7 to 10 lobes of 
proportions that are typical of lobed mixers. This lobe count is deemed sufficient to achieve spanwise periodic flow for the inner set of 4 to 5 lobes. The spanwise extent of the lobed-tail shown in Fig. 5.2 is dictated by the limitations of the metal additive manufacturing process available to the author's research group at this time.

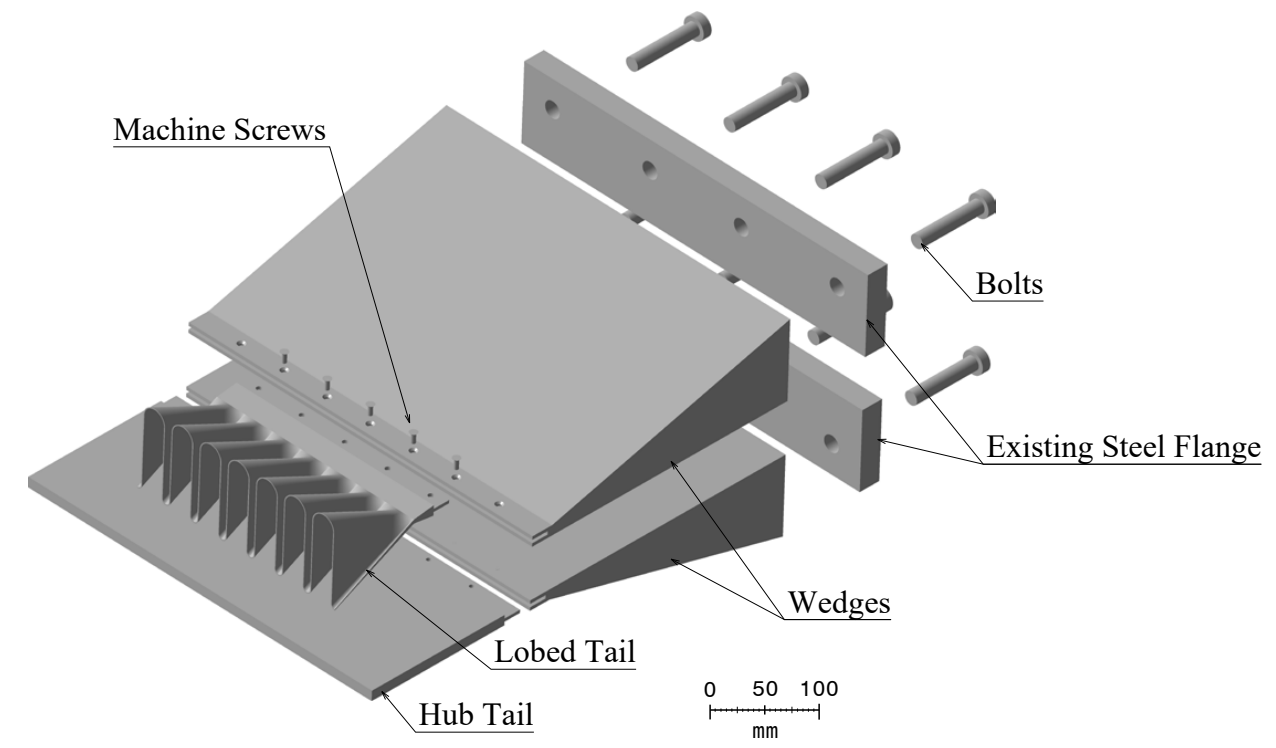

Figure 5.2: Rectilinear lobed-mixer test section installation.

\section{$5.2 \quad$ Structural Analysis}

\subsubsection{Computational Models}

Figs. 5.3 and 5.4 present the loads and constraints for the structural analysis of the rectilinear lobed-tail test section. Table 5.1 presents the mechanical properties of the materials used in the structural analysis. Two different loading scenarios are considered that correspond to the flow velocity upstream of the lobes that is either aligned with the lobes or is at a finite angle to the lobes. The latter scenario aims to approximate flow conditions that would be realized in an actual gas-turbine engine installation with swirling exhaust flow entering the lobed mixer. In the first scenario, the high-speed side surfaces are very conservatively pressurized to the level of the inflow stagnation pressure. To determine the structural limits of the test 
section, this surface pressure was incrementally increased over successive simulations until the stresses and deformations exceeded the allowable limits. In the second scenario, as shown in Fig. 5.4, the surface pressures on the high-speed side are set up asymmetrically to account for the misalignment of the upstream flow velocity with the lobes. In both loading scenarios, negligible friction is assumed between the wedges and the tails. This is a conservative assumption as it implies that the load is transferred between the wedges and the tails solely via the machine screws. Table 5.2 summarizes the specifics of the computational grid which contains about 2.1 million tetrahedral elements.

Table 5.1: Mechanical properties of the materials of the rectilinear lobed-tail test section.

Density $\left(\mathrm{kg} / \mathrm{m}^{3}\right)$

Tensile yield strength $(\mathrm{MPa})$

Compressive yield strength (MPa)

Young's modulus (GPa)

Poisson's ratio
6061 Aluminum

(Wedges and Hub tail) (Lobed tail) (Machine screws)

2700

145

68.9

0.33
7700

1025

-

200

0.31
8000

250

250

200

0.29

Table 5.2: Parameters of the computational grid.

\begin{tabular}{lccc}
\hline & $\begin{array}{c}\text { Element size }(\mathrm{mm}) \\
\min / \max \end{array}$ & $\begin{array}{c}\text { Spatial Growth rate } \\
\begin{array}{c}\text { Element Count } \\
\mathrm{x} 10^{6}\end{array}\end{array}$ \\
\hline Wedges & $0.5 / 10.0$ & $10 \%$ & 0.65 \\
Hub tail & $0.5 / 10.0$ & $10 \%$ & 0.3 \\
Lobed tail & $0.25 / 0.50$ & $10 \%$ & 0.5 \\
Machine screws & $0.20 / 0.20$ & $10 \%$ & 0.0001 \\
\hline
\end{tabular}


Surface Pressure 1 (matching inflow stagnation pressure) - High-Speed Side

$\square$ Ambient Pressure - $101 \mathrm{kPa}$

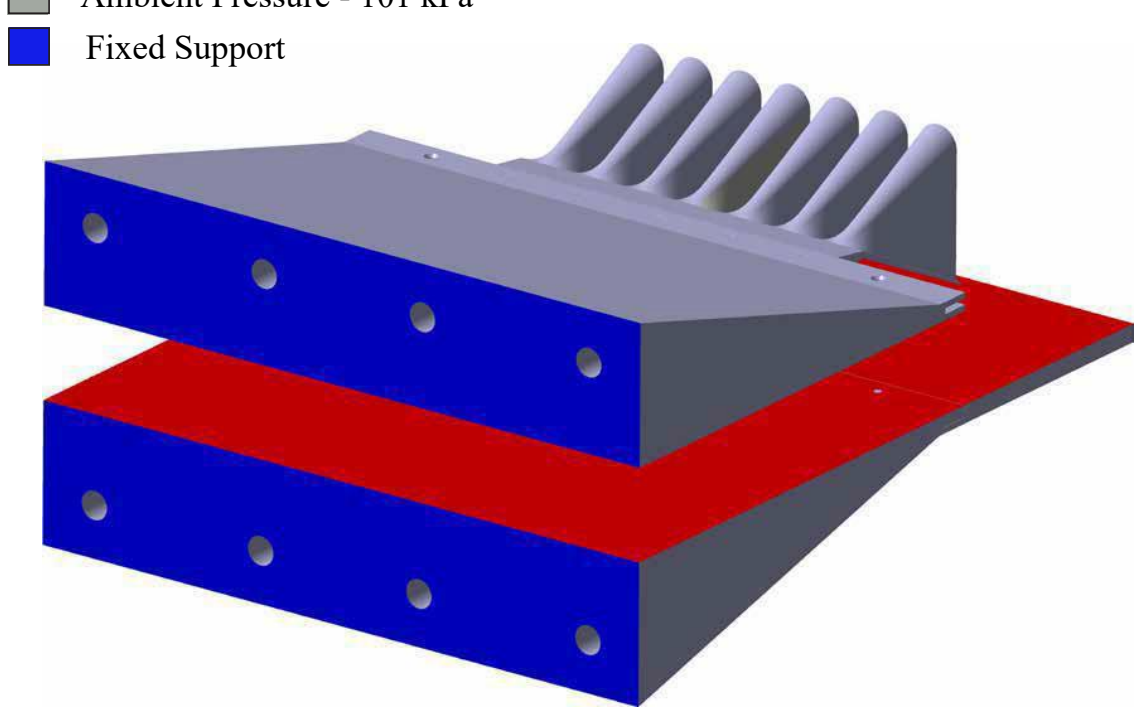

(a)

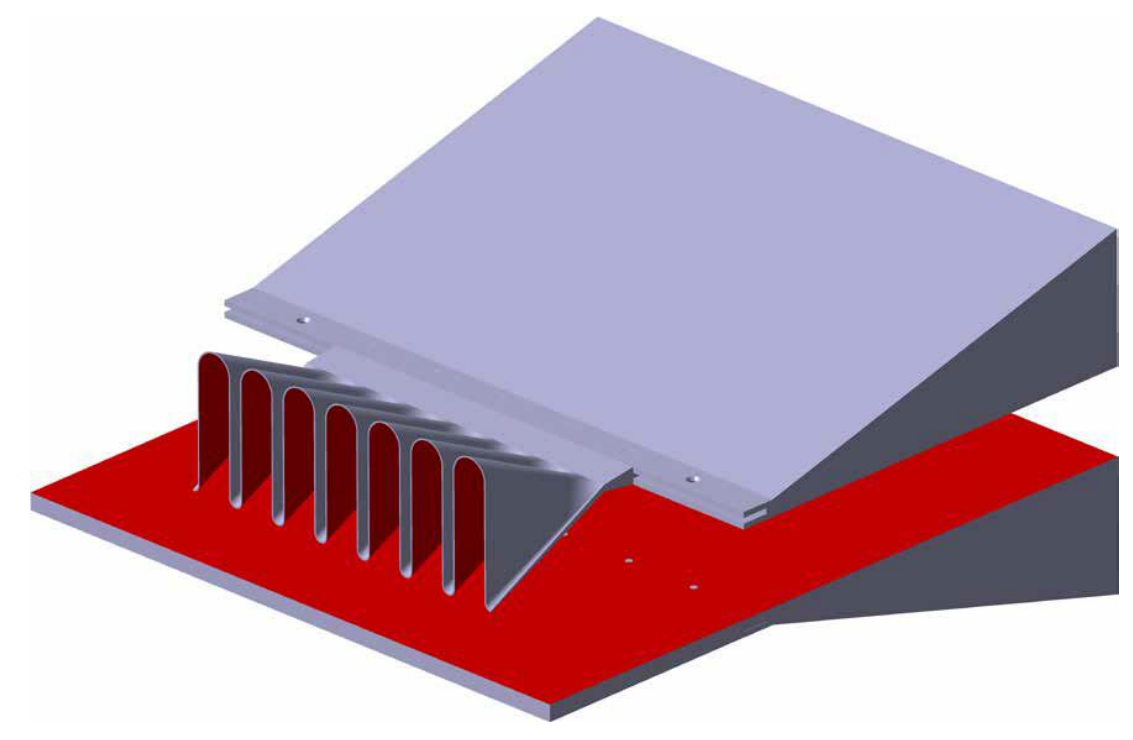

(b)

Figure 5.3: Loads and constraints for the scenario with the upstream inflow velocity aligned with the lobes (a) view from upstream end; (b) view from downstream end. 
Surface Pressure 1 (matching inflow stagnation pressure) - High-Speed Side

Ambient Pressure - $101 \mathrm{kPa}$

Surface Pressure 2 (varying linearly between the pressure levels of the two side walls of the lobe) - High-Speed Side

Fixed Support

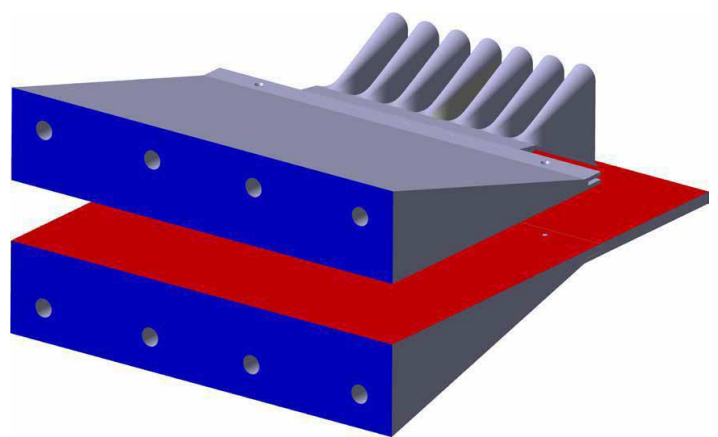

(a)

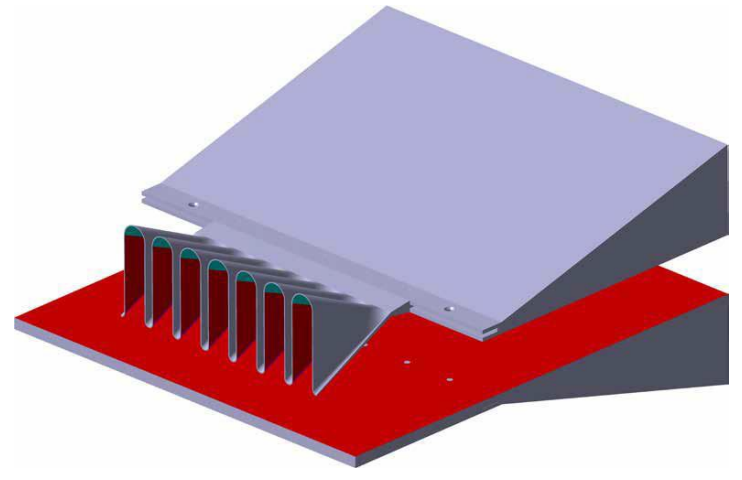

(c)

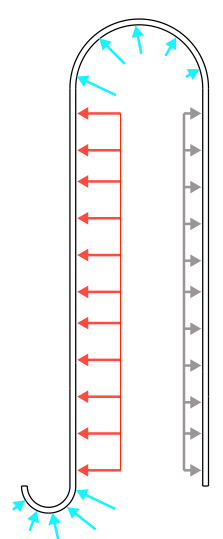

(b)

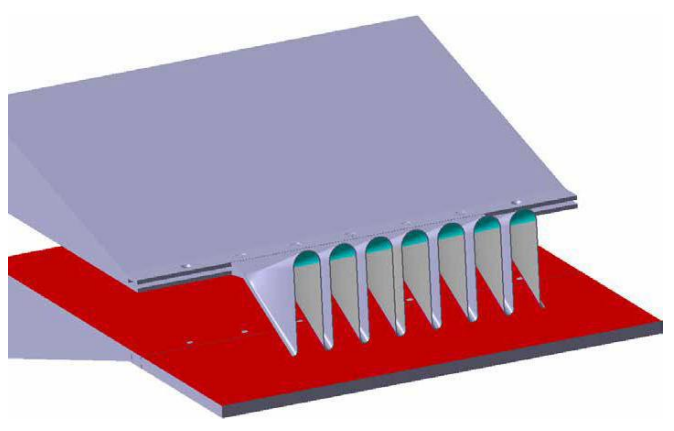

(d)

Figure 5.4: Loads and constraints for the scenario with misaligned inflow (a) view from upstream end; (b) front view of a single lobe; (c) view from downstream-left side; (c) view from downstream-right side.

\subsubsection{Computational Results}

Figs. 5.5 and 5.6 present the Von Mises stress distribution under the maximum allowable loading scenario for the aligned and misaligned inflow conditions, respectively. Table 5.3 and Table 5.4 summarize the analysis results. 
For the aligned inflow condition, the maximum allowable inflow stagnation pressure was determined to be $130 \mathrm{kPa}$, which corresponds to a dynamic pressure of 29 $\mathrm{kPa}$ assuming the inflow static pressure to be equal to the ambient pressure. This dynamic pressure corresponds to an inflow Mach number of 0.60 . Which is deemed sufficient for the planned lobed-mixer aerodynamics studies. The minimum safety factor under these conditions is 2.2 , dictated by the stresses in the machine screws that secure the lobed tail to the wedge. As already stated, the analysis is conservative given that the contact between the lobed tail and the wedge is treated as frictionless. Additionally, the test section is open to atmosphere at its spanwise ends, which will prevent the pressure levels on the surfaces exposed to the high-speed stream to reach the stagnation pressure of this stream.

In the misaligned inflow case, for an inflow total pressure of $130 \mathrm{kPa}$ that was established as the upper limit for the aligned inflow case, the maximum stresses yield suitably large safety factors.

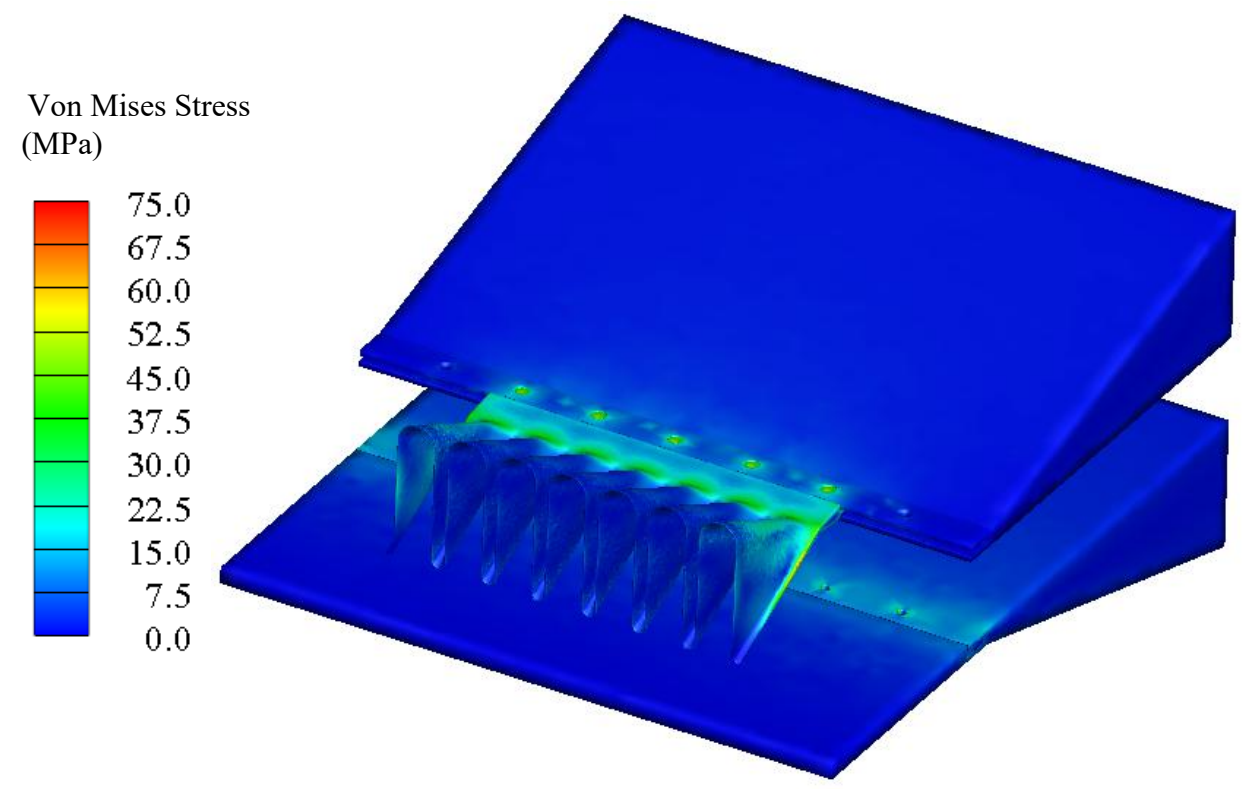

Figure 5.5: Von Mises stress distribution for the aligned inflow condition. 
Table 5.3: Summary of results for the aligned inflow condition.

\begin{tabular}{clccc}
\hline $\begin{array}{c}\text { Inflow } \\
\text { Stagntion Pressure } \\
(\mathrm{kPa})\end{array}$ & Component & \multicolumn{3}{c}{ Max. Deformation Max. Stress Min. Safety } \\
$(\mathrm{mm})$ & & & \\
& & MPa $)$ & Factor \\
\hline \multirow{4}{*}{130} & Wedge & $0.10 \pm 0.00$ & $47.1 \pm 1.4$ & $3.1 \pm 0.1$ \\
& Hub plate & $1.10 \pm 0.03$ & $36.7 \pm 1.1$ & $3.9 \pm 0.1$ \\
& Lobed tail & $0.89 \pm 0.03$ & $157.4 \pm 4.7$ & $6.5 \pm 0.2$ \\
& Machine screws & $0.17 \pm 0.01$ & $111.3 \pm 3.3$ & $2.2 \pm 0.1$ \\
\hline
\end{tabular}

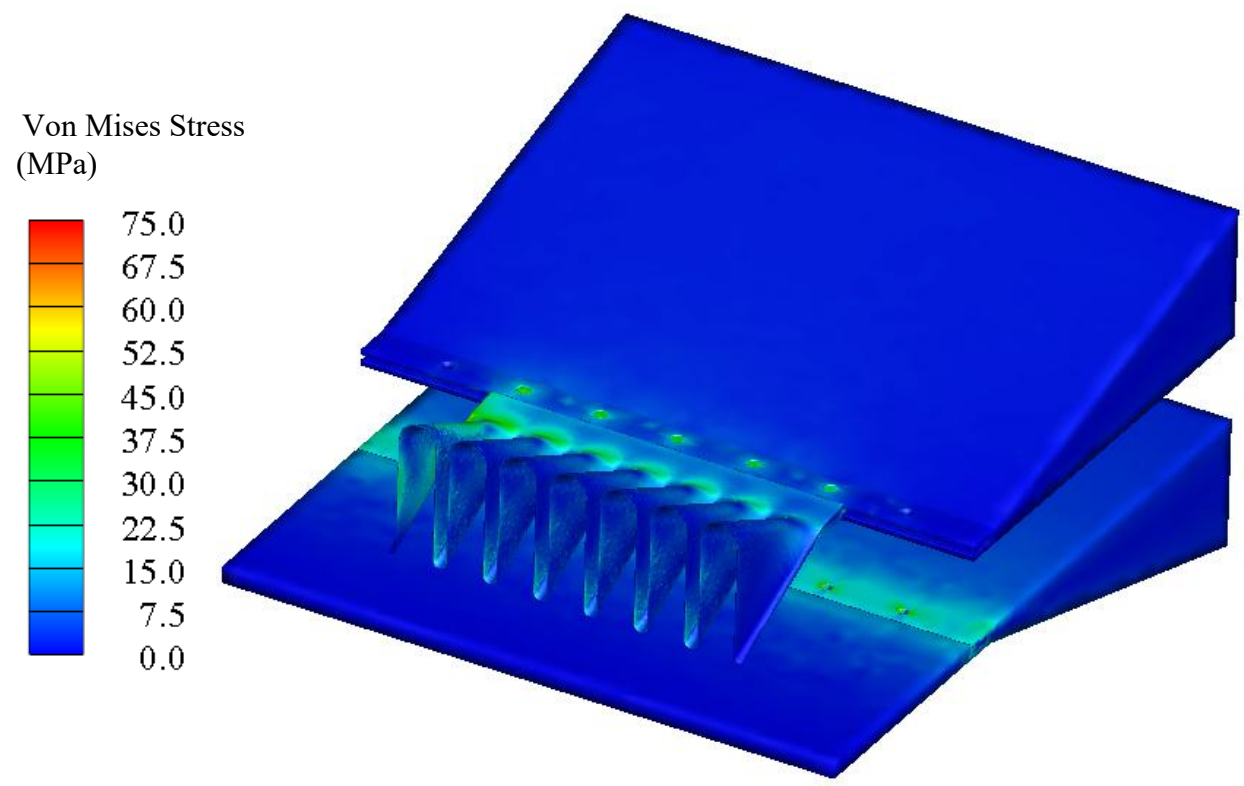

Figure 5.6: Von Mises stress distribution for the misaligned inflow condition.

Table 5.4: Summary of results for the misaligned inflow condition.

\begin{tabular}{clccc}
\hline $\begin{array}{c}\text { Inflow } \\
\begin{array}{c}\text { Stagnation Pressure } \\
(\mathrm{kPa})\end{array}\end{array}$ & Component & $\begin{array}{c}\text { Max. Deformation Max. Stress Min. Safety } \\
(\mathrm{mm})\end{array}$ & & \\
& & & & Factor \\
\hline & Wedge & $0.06 \pm 0.00$ & $27.5 \pm 0.8$ & $5.3 \pm 0.2$ \\
130 & Hub plate & $1.10 \pm 0.03$ & $36.7 \pm 1.1$ & $3.9 \pm 0.1$ \\
& Lobed tail & $0.69 \pm 0.02$ & $83.9 \pm 2.5$ & $12.2 \pm 0.4$ \\
& Machine screws & $0.05 \pm 0.00$ & $62.5 \pm 1.9$ & $4.0 \pm 0.1$ \\
\hline
\end{tabular}




\subsection{Design Summary}

The design of a high-speed rectilinear test section utilizing a lobed tail described in this chapter will allow larger scale study of the flow development downstream of the lobes for promising flow and geometry parameters that will be studied on the smaller scale annular-flow setup described in Chapter 4. The rectilinear design utilizes very few parts as it takes advantage of the flow conditioning realized in the flow path of the existing cascade test section. Apart from the lobed tail, which will require costly metal-based additive manufacturing, the few parts required for this test section can be produced on a conventional three-axis milling machine from aluminum stock without difficulty. 


\section{Chapter 6}

\section{Seed Injection Port Design}

\subsection{Design Considerations}

One of the components of the high-speed air-supply system described in Chapter 4 is a section where seeding material is injected into the air stream (component IX in the assembly as illustrated in Fig. 4.2). An ideal seed injection system should produce uniform distribution of seeding particles in the flow region of interest at a desired spatial seed-particle density with minimal disruption to the flow field. As described in Chapter 4, the seeding material is injected into the flow through a small port on the outer wall of the seed injection housing at 13.5 annulus heights upstream of the test section inflow plane. The diameter of this port, the orientation of its axis relative to the direction of the annular flow, and the supply pressure are the design parameters that can be varied to achieve a suitable injection configuration.

The present chapter contains a computational fluid dynamics (CFD) study of the injection-port design parameters. The high-speed air-supply system described in Chapter 4 will not be manufactured until it is registered with the Technical Standards and Safety Authority (TSSA) of Ontario. In the interim, experimental research will be conducted on a low-speed wind-tunnel with a test setup that closely approximates the flow path of the high-speed air-supply system (Section 3.1). As such, it is desirable to establish design-parameter values of the injection-port that are suitable for both lowspeed and high-speed flow conditions. Accordingly, the simulation results presented in this chapter with variations in injection-port geometry and seeding-fluid supply pressure were initially performed at low test-section flow speeds corresponding to incompressible flow conditions. Upon identification of the most promising injectionport configuration through these low-speed simulations, a final series of simulations

was conducted at compressible subsonic test-section flow conditions to determine the effect of the test-section flow Mach number on the performance of the seed injection 
system. The chapter concludes with a recommendation for the design of the seed injection port.

Review of relevant publications in the literature on seeding of flows for particle image velocimetry, as was presented in Section 2.3, suggests olive oil to have the most favorable combination of parameters for all Mach numbers in the subsonic range. Accordingly, the study presented in this chapter is based on this particular seeding material.

\subsection{Computational Domain and Boundary Conditions}

Fig. 6.1 presents the computational domain. The inflow boundary is placed $3 \Delta R$ upstream of the injection port, where $\Delta R=33 \mathrm{~mm}$ is the annulus height.

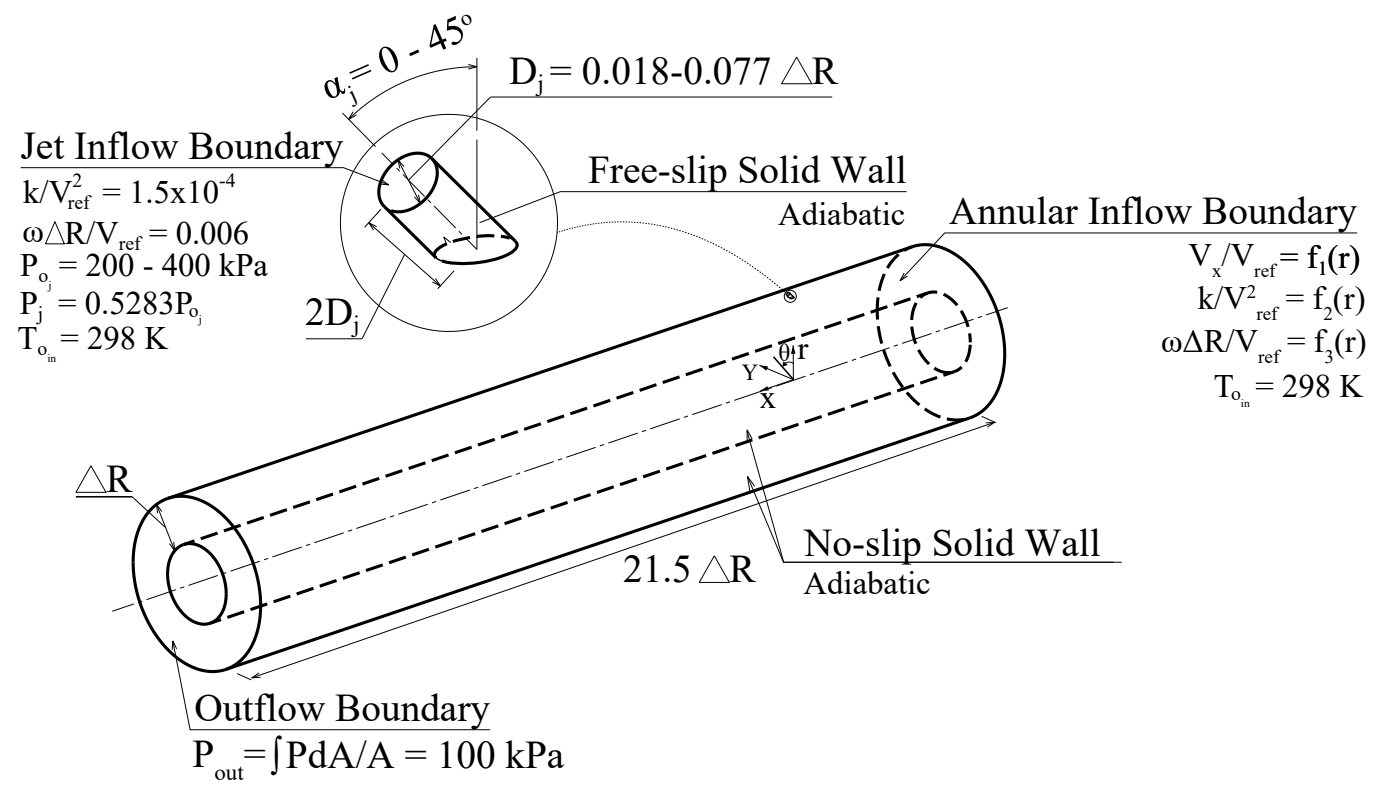

Figure 6.1: Computational domain and boundary conditions. 
The domain extends $21.5 \Delta R$ in the axial direction. The first $17.5 \Delta R$-long segment is the region of interest, for the axial location of the test-section inflow plane corresponds to the downstream end of this length. The outflow boundary is placed $4 \Delta R$ downstream of this region of interest to ensure any standing pressure waves that may be created by the constraint imposed on static pressure at the outflow boundary do not affect the flow field of interest. A uniaxial velocity profile is imposed at the annular inflow boundary. The radial profiles of velocity $\left(V_{x}\right)$ and turbulence kinetic energy $(k)$ are presented in Fig. 6.2. The turbulence energy dissipation rate, $\omega$, at this boundary is computed by the CFD software based on the turbulence kinetic energy profile and an integral length scale of $0.2 \Delta R$. The data points denoted with symbols in these profiles are measurements obtained on the low-speed wind-tunnel setup, with an area-averaged $V_{x}$ value of $V_{r e f}=15.8 \mathrm{~m} / \mathrm{s}$ and an area-averaged turbulence intensity of $3 \%$.

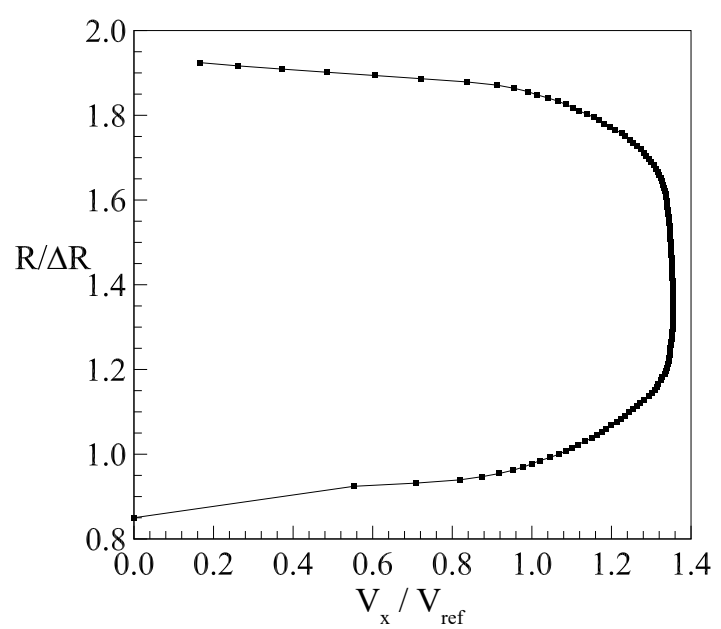

(a)

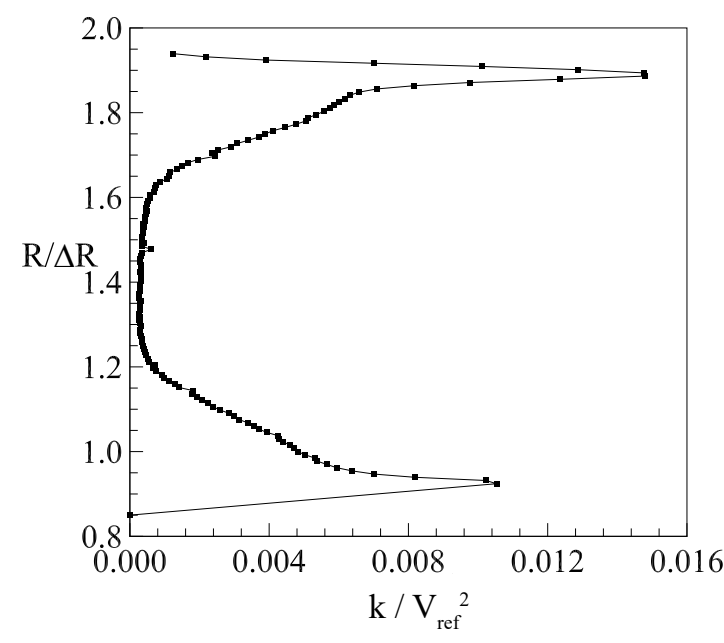

(b)

Figure 6.2: Inflow radial profile of (a) time-averaged axial velocity, and (b) turbulence kinetic energy $(\mathrm{x}=-3 \Delta \mathrm{R})$.

At the jet inflow boundary, the static pressure is set to $P_{j}=0.5283 P_{o_{j}}$, which corresponds to choked air flow $\left(M_{j}=1.0\right)$ conditions. With the outflow boundary of the annular flow set to a spatially-averaged static pressure value of $100 \mathrm{kPa}$, for 
the contemplated jet-feed stagnation pressure range of 200 to $400 \mathrm{kPa}$, the jet pipe is expected to remain choked $\left(M_{j}=1.0\right)$. Accordingly, the static pressure at the jet pipe inlet is fixed at $0.5283 P_{o_{j}}$ as part of the boundary conditions, which corresponds to $M_{j}=1.0$. As the jet pipe is set to free-slip, adiabatic walls, the jet flow conditions imposed at its inflow plane apply through the two diameters length of the pipe. Imposition of the jet inflow conditions some distance upstream of the discharge plane of the jet was preferred to allow for the flow at the jet discharge plane to develop in a manner that is not artificially constrained. At the jet inflow, the values of $k$ and $\omega$, normalized by the reference velocity and the annulus height, are set to $1.5 \times 10^{-4}$ and 0.006 , respectively, which correspond to a turbulence intensity of $1.0 \%$. The jet is a mixture of $99.99 \%$ air and $0.01 \%$ oil aerosol by mass. This is based on the manufacturer specifications of the aerosol generator that will be used in pending experimental research with these setups (LaVision, 2015). To determine the most promising seed injection configuration, three parameters were varied: injection port orifice diameter $\left(D_{j}\right)$; injection angle in the x-r plane $\left(\alpha_{j}\right)$; and, injection stagnation pressure $\left(P_{o_{j}}\right)$. Table 6.1 summarizes the test matrix for the parametric study covering a total of 27 combinations of the stated parameter values. In the table, $P_{o_{r e f}}$ is the stagnation pressure at the inviscid core at the annular inflow.

Table 6.1: Parameters of the CFD test matrix.

\begin{tabular}{|c|c|cc|}
\hline$D_{j} / \Delta R$ & $\alpha_{j}$ (deg.) & $P_{o_{j}}(\mathrm{kPa})$ & $P_{o_{j}} / P_{o_{\text {ref }}}$ \\
\cline { 2 - 3 } 0.018 & 0.0 & 200 & 1.96 \\
0.036 & 30.0 & 300 & 2.95 \\
0.077 & 45.0 & 400 & 3.93 \\
\hline
\end{tabular}

Based on the most promising configuration determined from this test matrix, an additional high-speed test case with an inviscid core Mach number of 0.8 for the annular flow is simulated. Fig. 6.3 presents the radial distribution of the time-averaged axial inflow velocity normalized by the area-averaged velocity at this plane, $V_{r e f}=157.0 \mathrm{~m} / \mathrm{s}$, for the high-speed case. Also presented in the figure is the radial distribution of the stagnation temperature normalized by $T_{o_{i n}}=298 \mathrm{~K}$. These profiles were obtained in a 
separate simulation by allowing a turbulent boundary layer to develop in the annular domain over an entrance length of $80 \Delta R$ starting from a radially uniform velocity profile. The inflow values of $k$ and $\omega$, normalized by $V_{\text {ref }}$ and $\Delta R$ are set to $1.35 \times 10^{-3}$ and 2.04, respectively, which correspond to $3 \%$ turbulence intensity and an integral turbulence length scale of $0.2 \Delta R$. All other boundary conditions are the same as in the low-speed cases. The flow parameters used to initialize the computations are summarized in Table 6.2.

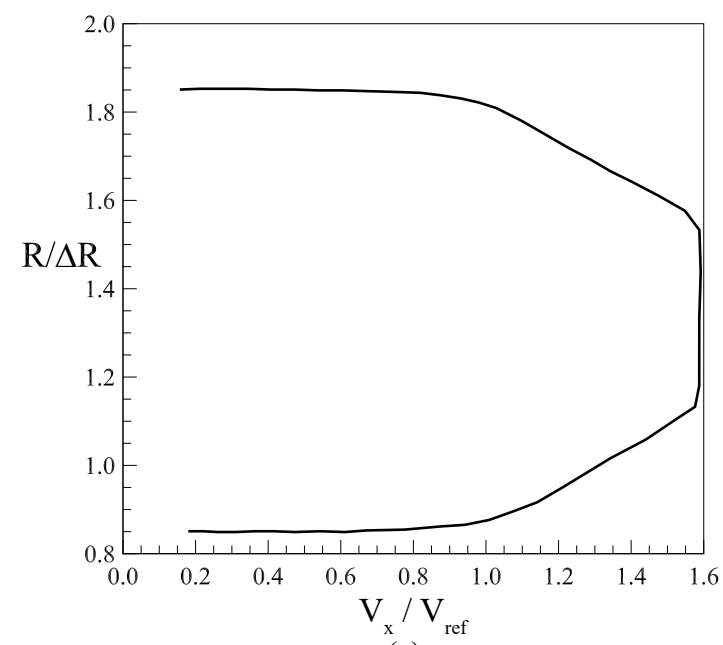

(a)

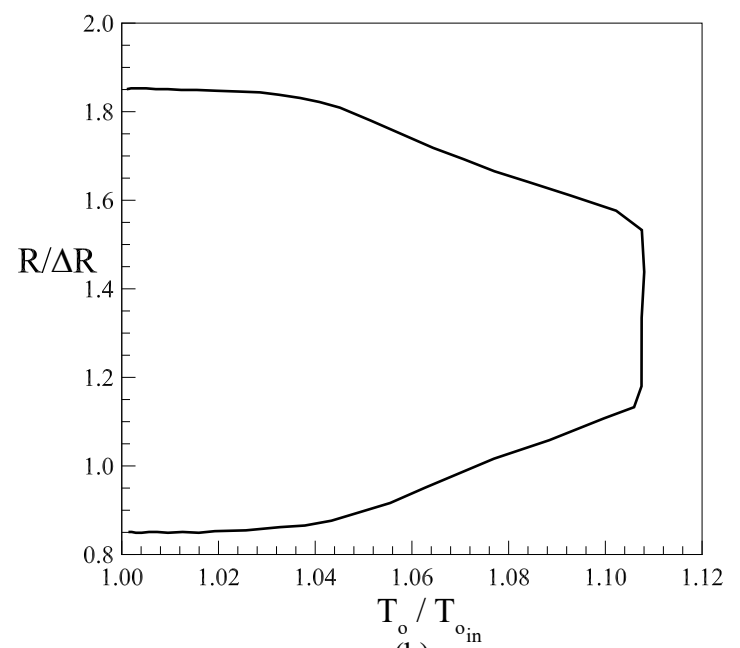

(b)

Figure 6.3: Inflow radial profile of (a) time-averaged axial velocity, and (b) stagnation temperature.

Table 6.2: Initial values of the normalized flow-field parameters in the domain.

\begin{tabular}{lc}
\hline$V_{x} / V_{\text {ref }}$ & 1.0 \\
$V_{y} / V_{\text {ref }}$ & 0.0 \\
$V_{r} / V_{\text {ref }}$ & 0.0 \\
$T_{o} / T_{\text {oin }}$ & 1.0 \\
$P / P_{\text {out }}$ & 1.0 \\
$k / V_{\text {ref }}^{2}$ & $1.35 \times 10^{-3}$ \\
$\omega \Delta R / V_{\text {ref }}$ & 2.04 \\
\hline
\end{tabular}




\subsection{Computational Grid}

The spatial grid consists of 36.2 million elements and is of the unstructured type. The grid is constructed with tetrahedral elements, which are replaced by prism elements along solid boundaries. Boundary layers are resolved with about 60 layers of prism elements across the boundary-layer thickness. The relevant parameters of the spatial grid are summarized in Table 6.3 with the corresponding nomenclature described in Fig. 6.4. The aspect ratio and skewness of the grid cells should be kept within acceptable bounds to prevent excessive build-up of round-off errors that may hinder the rate of convergence of the iterative solution algorithm. Furthermore, the skewness and spatial rate of change of the grid-cells should be kept low to minimize the discretization error in the converged solution. The grid-cell geometric specifications presented in Table 6.3 are consistent with the descriptions of good-quality spatial grids in published literature (e.g. Moukalled et. al, 2016). The $y_{1}^{+}$value of 1.2 places the grid node adjacent to the solid wall within the viscous sub-layer of the boundary layer as required by the low-Reynolds-number turbulence model used in the present simulations. The + superscript denotes normalization with the viscous length scale, $\nu / u_{\tau}$, where $\nu$ is the local kinematic viscosity, $u_{\tau}=\sqrt{\left(\tau_{w} / \rho\right)}$ is the so-called friction velocity at the wall, $\rho$ is the local fluid density, and $\tau_{w}$ is the local wall shear stress. The wall-normal extent of the flow region covered by the prism layers was confirmed to include the full thickness of the local boundary layer. The cell count of the grid was established through a grid sensitivity study, with the computed flow-field deemed grid independent when the maximum change in local velocity vector magnitude and seed-particle density at the downstream end of the flow region of interest $(\mathrm{x}=13.5 \Delta R$ from the location of the seeding jet) remained below $2 \%$ upon uniform refinement of the spatial grid to achieve twice as high a spatial resolution.

Given the injection stagnation pressure of the air stream carrying the seed particles, the jet exhaust will be under-expanded, resulting in a complex series of shock and expansion waves. These flow features are expected to affect the dispersion of the 
seed particles and therefore have to be adequately resolved by the spatial grid. Appendix $\mathrm{F}$ presents the validation of the spatial grid for resolving the said compression and expansion waves in a benchmark under-expanded jet.

Table 6.3: Grid specifications.

\begin{tabular}{lc}
\hline$y_{1}^{+}$ & $<1.2$ \\
Number of layers of prism cells along each solid boundary & 60 \\
Prism cell max. aspect ratio & 320 \\
Tetrahedral cell max. aspect ratio & 10 \\
Max. spatial rate of change of cell size & $15 \%$ \\
Cell skewness range & $0-0.5$ \\
Total number of tetrahedral elements & $11.1 \times 10^{6}$ \\
Total number of prism elements & $25.5 \times 10^{6}$ \\
\hline
\end{tabular}

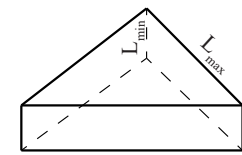

Prism cell

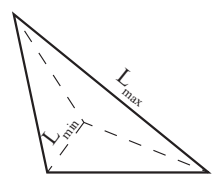

Tetrahedral cell

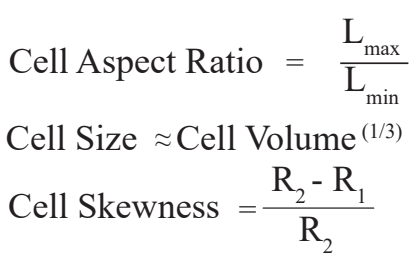

$\mathrm{R}_{1}=$ circumradius of the actual cell $\mathrm{R}_{2}=$ circumradius of the equilateral cell

Figure 6.4: Grid-cell nomenclature. 


\subsection{Governing Equations}

The Favre-averaged Navier-Stokes (FANS) equations at steady state were solved using ANSYS CFX (version 19.0), a commercial computational fluid dynamics software suite. Since the purpose of these simulations is to predict the seed-particle density distribution in the flow-field, a single-phase, multi-component simulation was performed with air for the annulus flow and oil aerosol for the jet flow, where both components are treated as calorically-perfect ideal gases. The primary assumption in the multicomponent model is that the two components of the aerosol mixture are intermixed at the molecular level, thus they share the same velocity, pressure, and temperature fields. The thermodynamic properties of the mixture are calculated at each spatial location in the flow field based on the relative mass proportions of the two components in the aerosol. The use of such a model for the aerosol implicitly assumes that the oil droplets are homogeneously distributed in the aerosol stream within the injection port, and each droplet follows the air stream perfectly as the aerosol mixes with the main flow. As the present design optimization study aims to identify the most effective injection port configuration to achieve the desired seed droplet distribution in the flow region of interest, the chosen aerosol modelling approach is deemed most suitable, for this approach decouples the physics of convection/diffusion of the seed material as part of the air stream from the matter of the extent to which the seed droplets are able to follow the local air motion. In the actual seed injector, the aerosol that is to be fed into the main flow through the injection port will be produced with a commercial Laskin nozzle designed to produce an aerosol with homogeneous distribution of droplets of a size range that has been well established to suitably follow the air motion. Accordingly, the only remaining possibility of the present simulations to inaccurately represent the actual mixing process is the coagulation of droplets as they convect through the flow field, driven by a phenomenon known as Brownian motion. In the present flow path design, the seed injection port is streamwise positioned such that the seed droplets convect to the measurement location in the test section within no longer than $20 \mathrm{~ms}$. This injection positioning was strategic, aiming at avoiding, 
or at least minimizing, coagulation of the oil droplets, while creating a design challenge of achieving seeding over a sufficiently large volume in the test section and of avoiding the disturbance of the test-section flow field by the process of seed injection relatively close to the test section inlet. In a study on the coagulation of olive-oil droplets in air injected into a closed box and allowed to recirculate for the duration of measurements, Hamdi et al. (2014) observed an increase in the mean size of the oil droplets from about $1 \mu \mathrm{m}$ to $4 \mu \mathrm{m}$ over a period of four minutes. Based on this data, coagulation is deemed very unlikely in the present flow path design.

In the presentation of simulation results later in this chapter, the spatial distribution of the seeding oil is presented as seed-particle density distributions rather than local mass fraction in the air-oil mixture as it is modeled. The seed-particle density is calculated by converting the computed local concentrations of the oil into local seed (droplet) counts per unit volume of mixture using an oil droplet diameter of $1 \mu \mathrm{m}$ as is expected to be produced in the experiments with the use of a Laskin nozzle.

The Favre-averaged compressible mass, momentum, and energy conservation equations for steady flow conditions can be expressed as:

$$
\begin{gathered}
\frac{\partial}{\partial x_{i}}\left(\bar{\rho} \widetilde{u_{i}}\right)=0 \\
\frac{\partial}{\partial x_{j}}\left(\bar{\rho} \widetilde{u_{i}} \widetilde{u_{j}}+\bar{P} \delta_{i j}+\overline{\rho u_{i}^{\prime \prime} u_{j}^{\prime \prime}}-\overline{\tau_{i j}}\right)=0 \\
\frac{\partial}{\partial x_{j}}\left[\bar{\rho} \widetilde{u_{j}}\left(\widetilde{h}+\frac{1}{2} \widetilde{u_{i}} \widetilde{u_{i}}\right)+\frac{\widetilde{u_{j}}}{2} \overline{\rho u_{i}^{\prime \prime} u_{i}^{\prime \prime}}-\left(\overline{\tau_{i j}}-\left(\overline{\rho u_{i}^{\prime \prime} u_{j}^{\prime \prime}}\right) \widetilde{u_{i}}-\overline{q_{j}}\right)\right]- \\
\frac{\partial}{\partial x_{j}}\left[-\overline{\rho u_{j}^{\prime \prime} h^{\prime \prime}}-\frac{1}{2} \overline{\rho u_{i}^{\prime \prime} u_{j}^{\prime \prime} u_{i}^{\prime \prime}}+\overline{\rho u_{i}^{\prime \prime} \tau_{i j}}\right]=0
\end{gathered}
$$

where the overbar indicates time averaging. The tilde indicates Favre averaging (density weighted time averaging) quantities, which is defined for a generic vector variable, $\phi$, as:

$$
\widetilde{\phi}=\frac{\overline{\rho \phi}}{\bar{\rho}}
$$


The various parameters appearing in the conservation equations are summarized in Table 6.4.

Table 6.4: Nomenclature for the parameters in Eqns. 6.1 to 6.4.

\begin{tabular}{|c|c|}
\hline Parameter & Definition \\
\hline$\phi^{\prime}=\phi-\bar{\phi}$ & $\begin{array}{l}\text { Fluctuating component of parameter } \phi \\
\text { about the time-averaged mean value }\end{array}$ \\
\hline$\phi^{\prime \prime}=\phi-\tilde{\phi}$ & $\begin{array}{l}\text { Fluctuating component of parameter } \phi \\
\text { about the density weighted time-averaged mean value }\end{array}$ \\
\hline$u$ & Fluid velocity component \\
\hline$\rho=\frac{P}{R T}$ & Fluid static density (equation of state) \\
\hline$P$ & Fluid static pressure \\
\hline$R$ & Ideal gas constant \\
\hline$\tau_{i j}=2 \mu S_{i j}-\frac{2}{3} \mu \frac{\partial u_{k}}{\partial x_{k}} \delta_{i j}$ & Viscous stress tensor \\
\hline$h=c_{p} T$ & Fluid static enthalpy \\
\hline$S_{i j}=\left(\frac{\partial u_{i}}{\partial x_{j}}+\frac{\partial u_{j}}{\partial x_{i}}\right)$ & Strain-rate tensor \\
\hline$q_{j}=-\lambda \frac{\partial T}{\partial x_{j}}$ & Heat flux \\
\hline$T$ & Fluid static temperature \\
\hline$c_{p}$ & Fluid specific heat capacity at constant pressure \\
\hline$\lambda$ & Fluid thermal conductivity \\
\hline$\mu$ & Fluid dynamic viscosity \\
\hline$\delta_{i j}$ & Kronecker delta \\
\hline
\end{tabular}


Turbulence closure is achieved via the Boussinesq hypothesis and the Reynolds-

stress term, $\overline{\rho u_{i}^{\prime \prime} u_{j}^{\prime \prime}}$, is modeled using the well established two-equation eddy-viscositybased shear-stress transport (SST) turbulence model of Menter (Menter, 1994) with its standard empirical coefficients (Menter and Esch, 2001).

\subsection{Discretization}

In the solution algorithms utilized by ANSYS CFX, the mass conservation equation is modified by expressing the velocity appearing in this equation in terms of pressure via the momentum conservation equation, thereby yielding Poisson's equation governing the pressure distribution in the flow field. The spatial discretization is based on the vertex-centered finite volume technique, with a collocated grid arrangement so that all variable values are stored at the grid nodes. Pressure-velocity coupling is achieved with the scheme of Rhie and Chow (1983), whereby the velocities at the surfaces of the control volumes interpolated from the surrounding nodal values are adjusted by a redistribution of pressure gradients. The non-linear convection terms in the momentum and energy equations are linearized via Picard iteration, which uses the method of successive approximations by computing the momentum components using the velocity from the current iteration and the mass flux from the previous iteration (Ferziger and Peric, 2002). The spatial interpolations of the flow parameters from grid nodes to the control volume surfaces are realized in manners that are equivalent to second-order centered differencing of all spatial derivatives except for those in the convection terms of the transport equations, which are approximated using a blend of first-order upwind and second-order centered differencing.

\subsection{Solution Method}

The linear system of discretized equations is solved using an Incomplete Lower-Upper (ILU) factorization method (ANSYS, 2006). The convergence of the solution is accelerated using an W-type Algebraic-Multigrid technique called Additive Correction that removes long-wavelength errors in the domain by solving the governing equations 
on a series of successively coarser grids. The multigrid algorithm utilizes 8 grid levels. After each mapping (restriction) of the solution to a coarser grid, a single smoothing iteration is performed on the restricted solution using the ILU factorization method. As the "corrections" to the fine-grid solution are successively mapped (prolongated) back from the coarsest grid to the finest grid, on each grid level two solution sweeps are performed using the ILU factorization technique to smooth the corrections. This cycle involves solution of linear algebraic equations, with the linearization affected by freezing the coefficients of the coefficient matrix at their values prevailing at the start of the solution cycle as described in Section 6.5. Upon completion of the last prolongation operation, the coefficient matrix is updated. The solution is under-relaxed between coefficient updates for the purposes of numerical stability. The solution is declared converged when the root-mean-square residuals of the governing equations are reduced to less than $1 \times 10^{-6}$, which has been found by the author's research group to be a suitable convergence threshold for accurate RANS solutions (e.q. Brinkerhoff et al., 2013, Brinkerhoff et al., 2014a).

\subsection{Computational Results}

\subsubsection{Low-speed Annular Inflow}

27 simulations were performed to cover the test matrix summarized in Table 6.1, of which the most promising results are presented here. The corresponding results for the remaining cases are included in Appendix G. For a successful PIV measurement, the seed-particle density in the flow field must meet certain requirements. The optimum injection-port configuration should produce a seed cloud over the region of interest with a normalized seed-particle density, $\rho_{P} / \rho_{P_{\min }}$, of at least 1.0 , where minimum seed-particle density, $\rho_{P_{\min }}$, is defined as:

$$
\rho_{P_{\text {min }}}=\frac{N_{I}}{x_{r} \times y_{r} \Delta z}
$$

where $N_{I}$ is the particle image density, which refers to the number of particle images in the PIV interrogation window. As a general rule, $N_{I}$ must have a value of at 
least 15-30 for accurate statistical cross-correlation (Adrian and Westerweel, 2011). In the present study, a seed-particle density of $\rho_{p_{\min }}=19$ particles $/ \mathrm{mm}^{3}$ is aimed for, which corresponds to a particle image density of $N_{I}=30$ for a light sheet thickness of $\Delta z=1.0 \mathrm{~mm}$ and a spatial resolution of $x_{r} \times y_{r}=1.25 \times 1.25 \mathrm{~mm}^{2}$. The spatial resolution, $x_{r} \times y_{r}$, of the PIV images is determined by:

$$
x_{r} \times y_{r}=F O V \frac{P x_{x} \times P x_{y}}{P x_{\text {sensor }}}
$$

where $P x_{x} \times P x_{y}=32 \times 32$ is the selected number of pixels in an interrogation window (see Fig. 2.3), and $P x_{\text {sensor }}=2560 \times 2160$ is the number of pixels in the camera intended to be used. A field-of-view (FOV) of $100 \times 84.4 \mathrm{~mm}^{2}$ is selected. While it is vital that injection system produce the desired seed density and uniformity for accurate PIV, it is of even greater importance that the desired seeding conditions be achieved without significant perturbation of the annular flow-field by the penetrating air jet carrying the seed particles. Of the tested injection angles, $\alpha_{j}=30 \mathrm{deg}$. produced the least disturbance in the annular flow. Fig. 6.5, Fig. 6.6, and Fig. 6.7 present the seed-particle density, axial velocity, and total pressure distributions, respectively, at the test section inflow plane $(x=13.5 \Delta R)$ for a fixed injection angle of $\alpha_{j}=30$ deg. and three values each of $D_{j} / \Delta R$ and $P_{o_{j}} / P_{o_{r e f}}$. In these figures, $V_{r e f}$ and $P_{o_{r e f}}$ are respectively the area-averaged velocity at the annular inflow, and the stagnation pressure in the inviscid core at the annular inflow. 

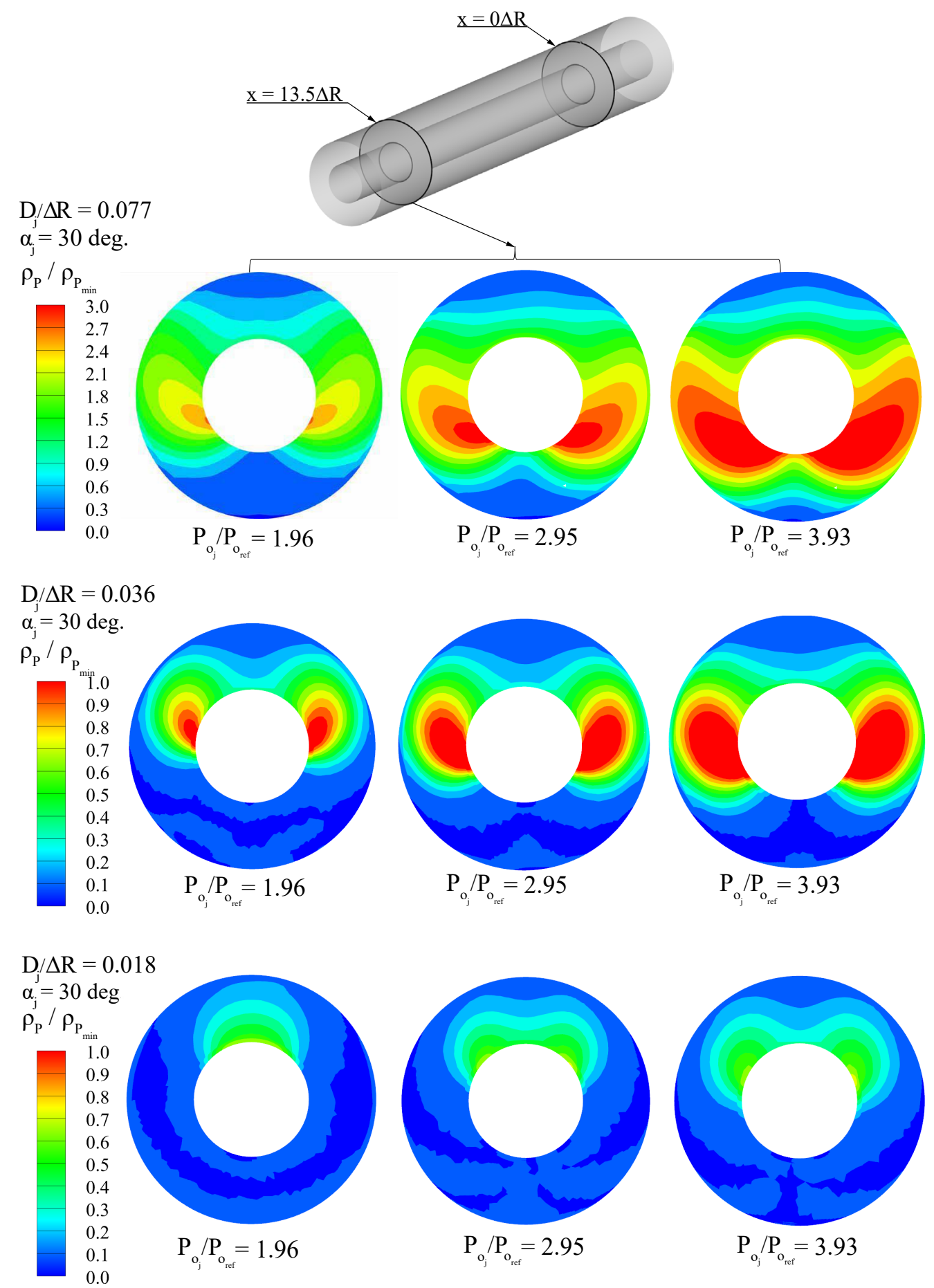

Figure 6.5: Seed-particle density distribution in the $x=13.5 \Delta R$ plane for $\alpha_{j}=30 \mathrm{deg}$. as a function of $D_{j} / \Delta R$ and $P_{o_{j}} / P_{o_{r e f}}$. Note the three times larger $\rho_{P}$ range used for the results with $D_{j} / \Delta R=0.077$. 

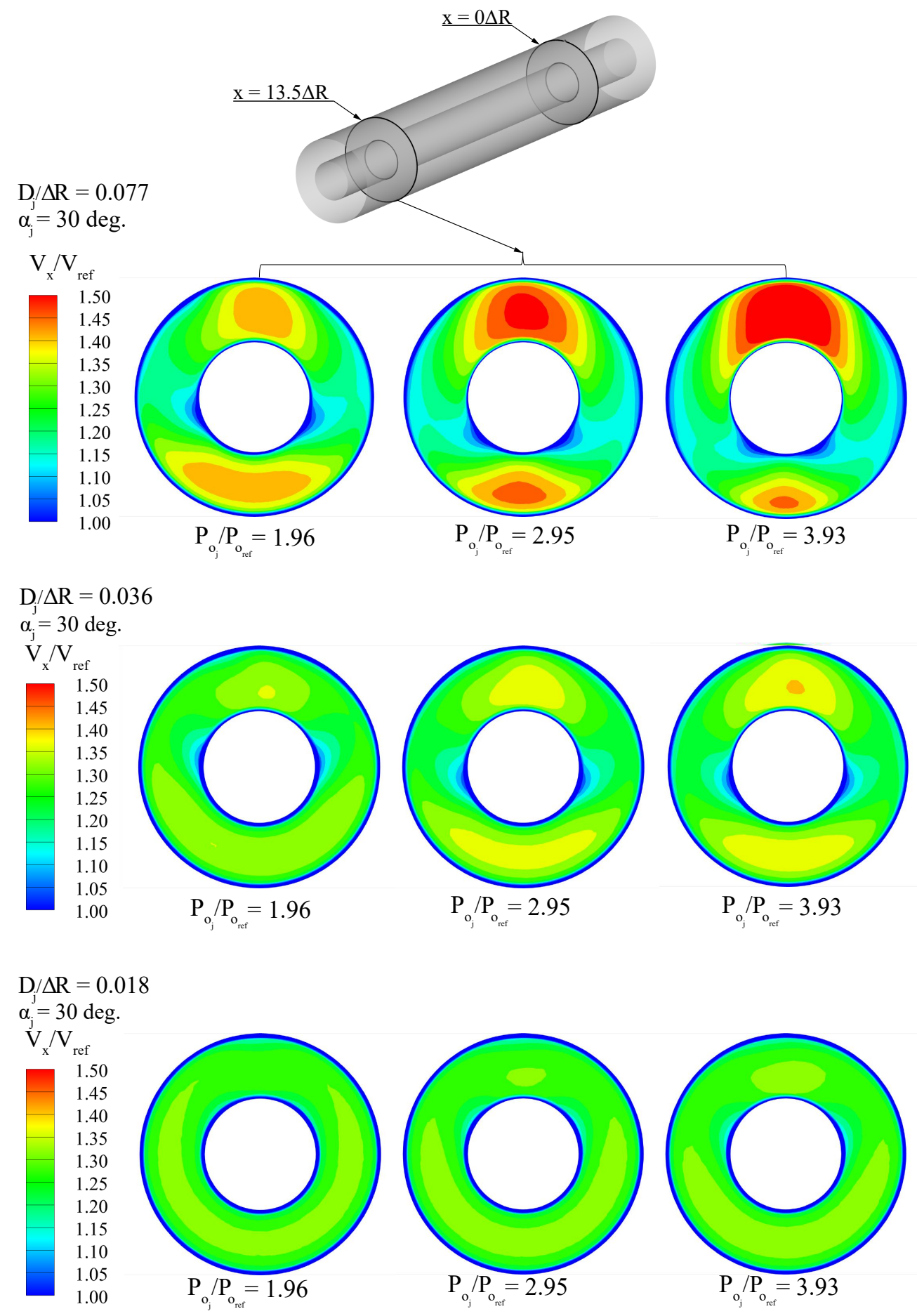

Figure 6.6: Axial velocity distribution in the $x=13.5 \Delta R$ plane for $\alpha_{j}=30$ deg. as a function of $D_{j} / \Delta R$ and $P_{o_{j}} / P_{o_{r e f}}$. 

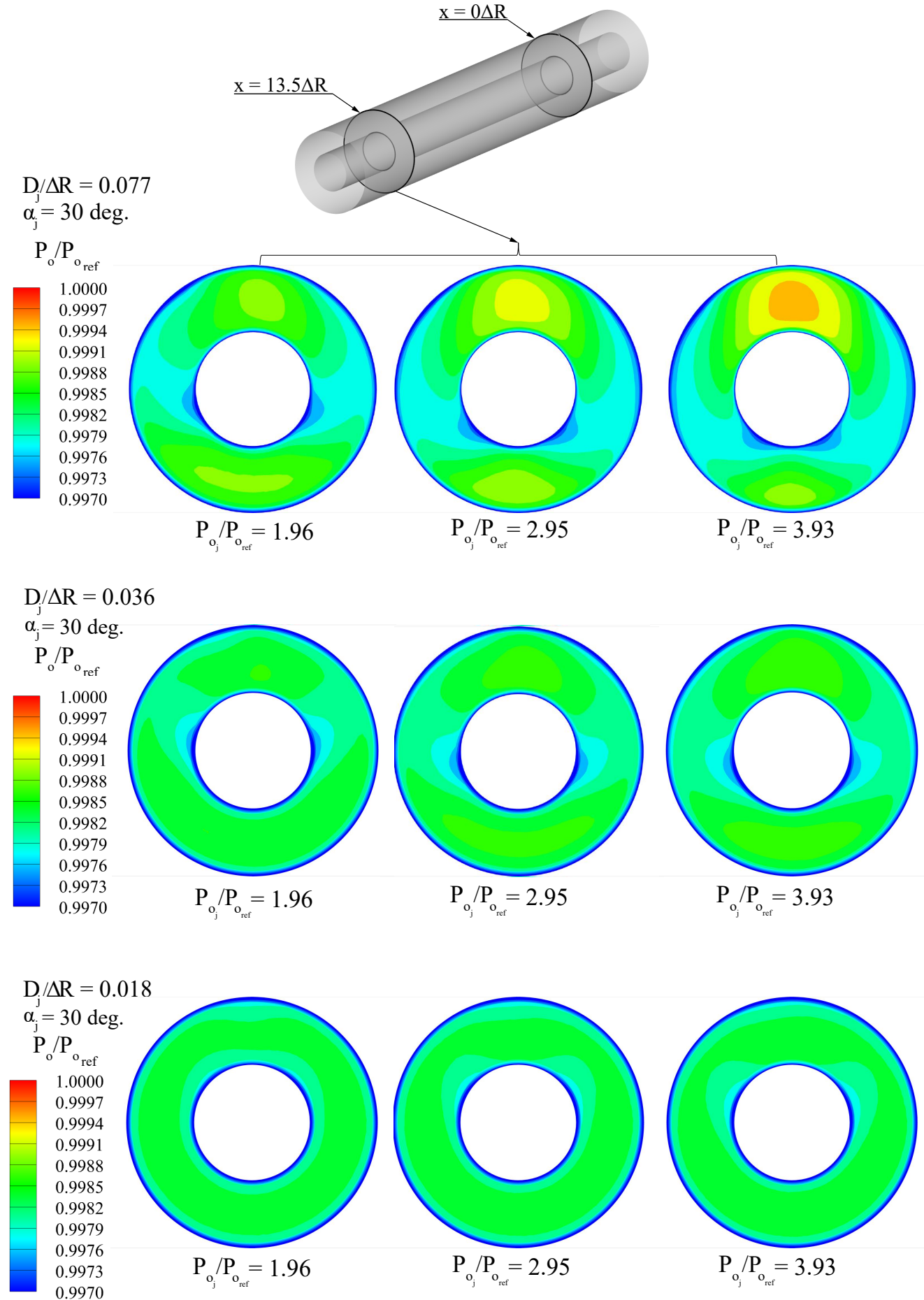

Figure 6.7: Stagnation pressure distribution in the $x=13.5 \Delta R$ plane for $\alpha_{j}=30$ deg. as a function of $D_{j} / \Delta R$ and $P_{o_{j}} / P_{o_{r e f}}$. 
The desired level of seed density is achieved only for the largest of the three jet discharge diameters, $D_{j} / \Delta R=0.077$, illustrated in the first row of Fig. 6.5. The reader is cautioned that the density distribution scale for this row is three times larger than that for the remaining two rows corresponding to smaller jet discharge diameters. Even for $D_{j} / \Delta R=0.077$, the seed density distribution is quite nonuniform, both radially and circumferentially, with the desired seed density developing only over part of the annulus circumference. The axial velocity and stagnation pressure fields presented in Figs. 6.6 and 6.7 shed further light on this seed density distribution. In Fig. 6.6, the axial velocity is observed to be relatively higher in the circumferential region corresponding to the location of the seeding jet, and in the region located on the diametrically opposite side. This is particularly evident for the $D_{j} / \Delta R=0.077$ cases presented in the first row, and the two higher $P_{o_{j}}$ cases for $D_{j} / \Delta R=0.036$ presented in the second row. The seeding jet is injected into the annular flow at 30 deg. angle tilted towards the upstream direction, as such has a momentum component in the negative $\mathrm{x}$ direction, opposing the momentum of the local annular flow. Such an interaction of the jet with the local annular flow should create a region of fluid of relatively low axial momentum that is rich with seeding particles. This development is shown in Fig. 6.8 through the seed density distribution in a streamwise-radial plane circumferentially aligned with the seeding jet. For brevity, only the cases corresponding to $D_{j} / \Delta R=0.077$ are shown here. The results for all of the remaining test cases are included in Appendix $\mathrm{G}$ as is done with the data excluded from all of the figures pertaining to the simulation results in this chapter. 


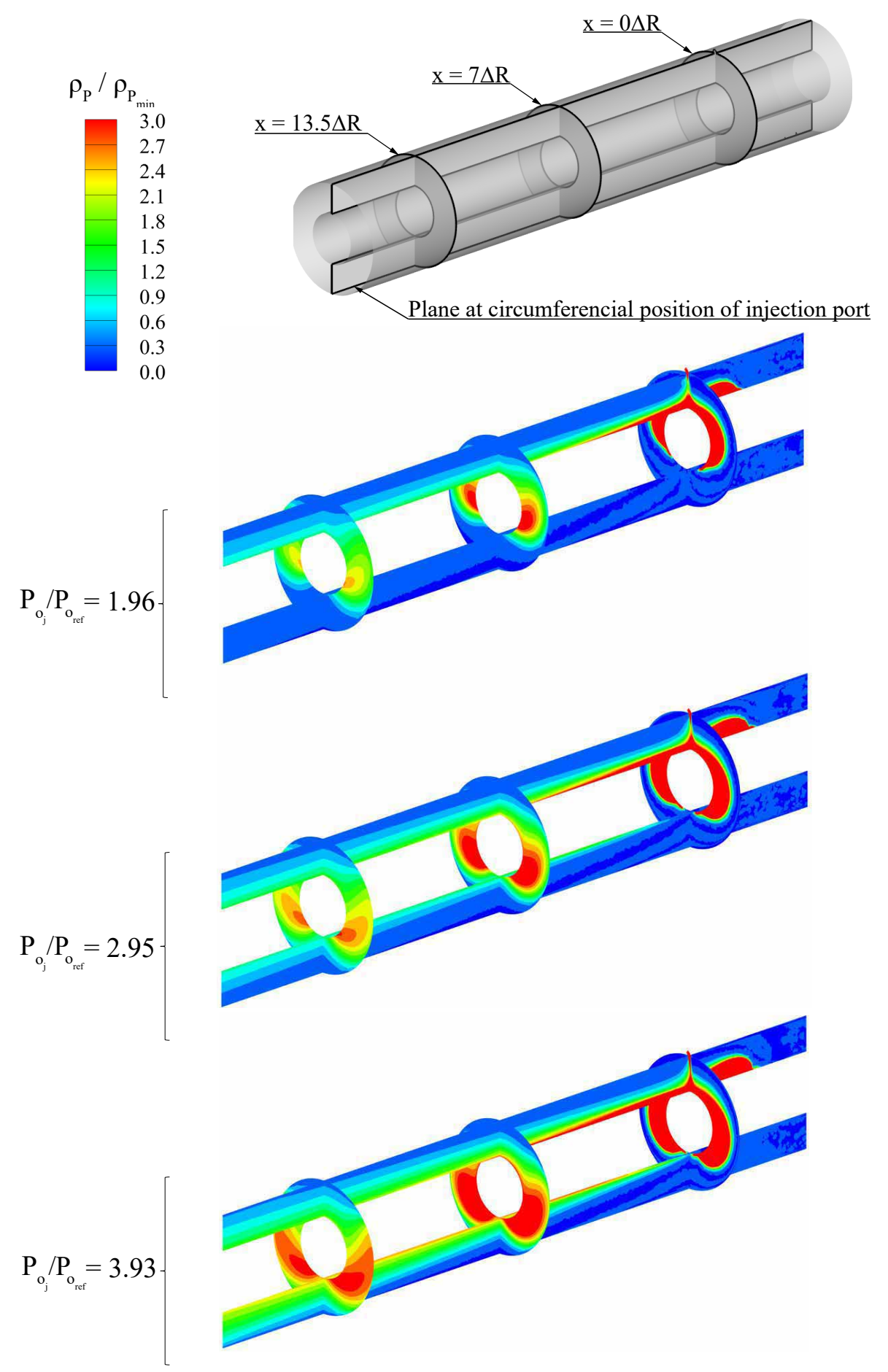

Figure 6.8: Streamwise seed-particle density distributions as a function of $P_{o_{j}} / P_{o_{\text {ref }}}$ for $D_{j} / \Delta R=0.077$ and $\alpha_{j}=30 \mathrm{deg}$. 
In Fig. 6.8, for all three $P_{o_{j}} / P_{o_{r e f}}$ values considered, the jet is observed to have sufficient momentum to span the annulus height and impinge upon the inner wall of the annulus. This is not surprising given the relatively high velocity of the jet compared to that of the approaching annular flow. This impingement is observed to create a local region of seed-rich fluid that extends some distance upstream of the impingement location. The streamwise extent of this accumulation zone is noted to increase when $P_{o_{j}} / P_{o_{r e f}}$ is increased from 1.96 to 2.95 , but negligible further variation is observed when the pressure increased to 3.93. These trends are dictated by the relative rates at which the seed particles are carried to the impingement zone by the jet and are then removed from this zone via convection and diffusion. As the seedloaded jet traverses across the annulus height, it is noted that a portion of it is mixed with the annulus flow and convects in the downstream direction. However, as per the corresponding cross-stream seed density distributions shown in the first row of Fig. 6.5 , the majority of the seeded fluid seems to first accumulate in the impingement zone and then spread around the inner wall of the annulus. While the seeded jet is of relatively high stagnation pressure, its impingement on the inner wall of the annulus where it is affected by the no-slip condition of the wall and the associated frictional losses and its mixing interaction with the prevailing annular-flow boundary layer along this wall may yield seed-rich fluid of relatively low stagnation pressure. This indeed turns out to be the case as illustrated in the first row of Fig. 6.7 by the relatively lower stagnation pressure in the circumferential regions that correspond to regions of higher seed density in Fig. 6.5. To compensate for the lower flow rate of these well-seeded circumferential zones, the annular flow should streamwise accelerate in the remaining circumferential zones to maintain mass conservation, which explains the higher axial velocities (Fig. 6.6) in the circumferential zone corresponding to the position of the injection port and the zone on the diametrically opposite side.

The rate at which the seeding jet is spreading as it travels across the annulus height, visualized through plots of seed-particle density distribution in Fig. 6.8, is affected by a fairly complex set of compression and expansion waves given the choked 
state of the injection port in these cases. Upon the seeded air jet reaching the discharge plane of the injection port at a Mach number of 1.0 and a static pressure that is higher than the back pressure prevailing downstream of the discharge port, the jet accelerates to supersonic speeds through a series of expansion waves that fan out from the edges of the discharge plane. Further downstream, these waves reflect off the perimeter of the jet as compression waves, the compression waves coalesce into an intercepting shock, and this shock converges towards the center of the jet to form a normal shock often referred to as a Mach disk. This flow development is summarized in Fig. 6.9 for $D_{j} / \Delta R=0.077$ and $P_{o_{j}} / P_{o_{r e f}}=3.93$.

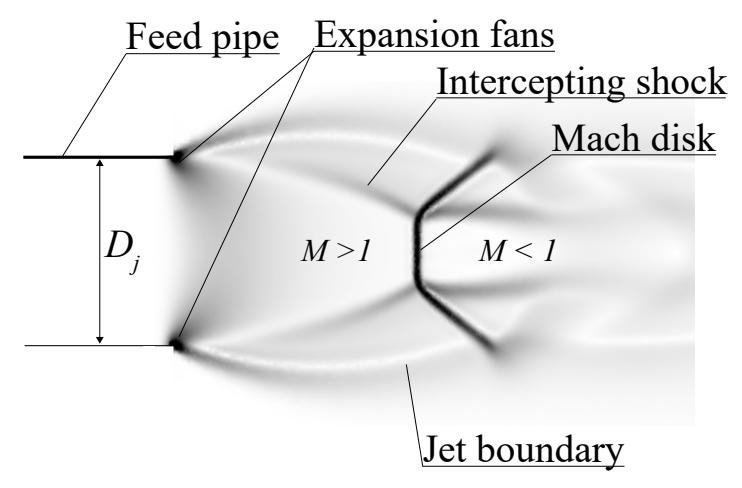

Figure 6.9: Numerical Schlieren image showing the density gradient magnitude of the under-expanded jet for $D_{j} / \Delta R=0.077$ and $P_{o_{j}} / P_{o_{r e f}}=3.93$. 
The distance of the Mach disk from the discharge plane is $1.28 D_{j}$ for $D_{j} / \Delta R=0.077$ and $P_{o_{j}} / P_{o_{r e f}}=3.93$. This value agrees favourably with the value of $1.28 D_{j}$ predicted with the empirical correlation put forward by Crist et al. in 1966:

$$
\frac{X_{m}}{D_{j}}=\sqrt{\frac{P_{o_{j}}}{2.4 P_{b}}}
$$

where $X_{m}$ is the distance of the Mach disk from the discharge plane and $P_{b}$ is the back pressure into which the jet discharges.

It is evident from the presented results that the desired seed density is achievable over part of the circumference of the annular flow path and with the least amount of disturbance of the annular flow only for the largest tested jet diameter $\left(D_{j} / \Delta R=0.077\right)$ and the lowest tested jet pressure $\left(P_{o_{j}} / P_{o_{r e f}}=1.96\right)$. Even in this configuration, the axisymmetry of the axial velocity of the annular flow is perturbed by as much as $40 \%$. This level of flow distortion would not be acceptable in test sections such as annular lobed mixers where the axisymmetry of the incoming flow is critical to the aerodynamic performance of the device.

Additional simulations were therefore performed for $D_{j} / \Delta R=0.077$ for lower values of $P_{o_{j}} / P_{o_{r e f}}=1.65$ and 1.35 . For these jet stagnation pressure values the injection port would be unchoked. The simulation results summarized in Fig. 6.10 and Fig. 6.11 show that the manner in which the jet mixes with the annular flow remains the same at these lower jet pressures as the cases discussed earlier for choked jet conditions. At the lowest jet stagnation pressure considered $\left(P_{o_{j}} / P_{o_{r e f}}=1.35\right)$, the desired seed density is noted to be achieved in the $x / \Delta R=13.5$ plane over part of the annulus circumference, with significantly less distortion in the axial velocity distribution than the choked scenario with $P_{o_{j}} / P_{o_{r e f}}=1.96$. These trends demonstrate that one may be able to achieve a satisfactory seed density in the flow region of interest without disturbing the annulus flow to a significant extent through adjustment of the stagnation pressure with $D_{j} / \Delta R=0.077$ and $\alpha_{j}=30 \mathrm{deg}$. 


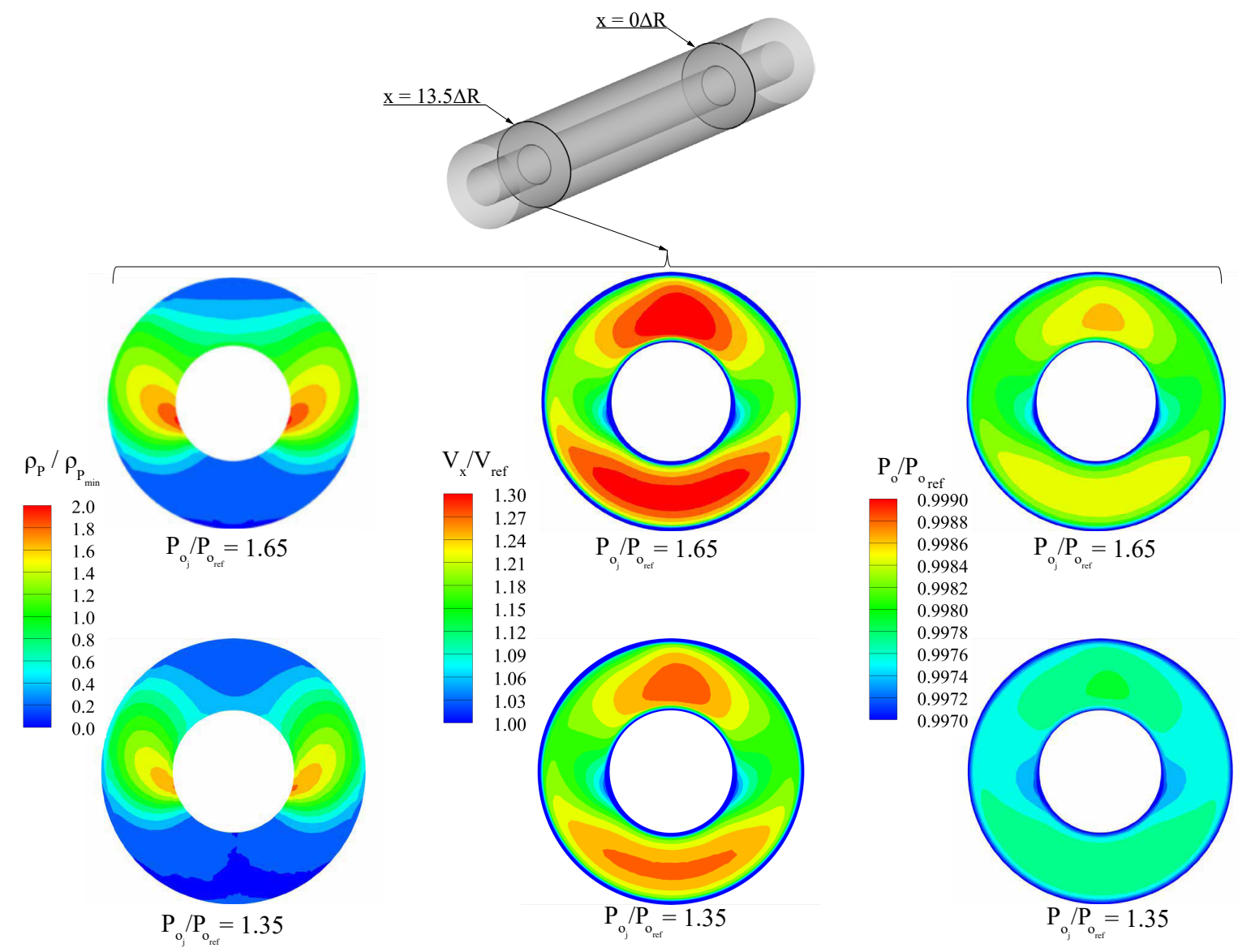

Figure 6.10: Seed-particle density, axial velocity, and stagnation pressure distributions in the $x=13.5 \Delta R$ plane for $D_{j} / \Delta R=0.077$ and $\alpha_{j}=30$ deg., and injection stagnation pressures of $P_{o_{j}} / P_{o_{r e f}}=1.65$ and $P_{o_{j}} / P_{o_{r e f}}=1.35$. 


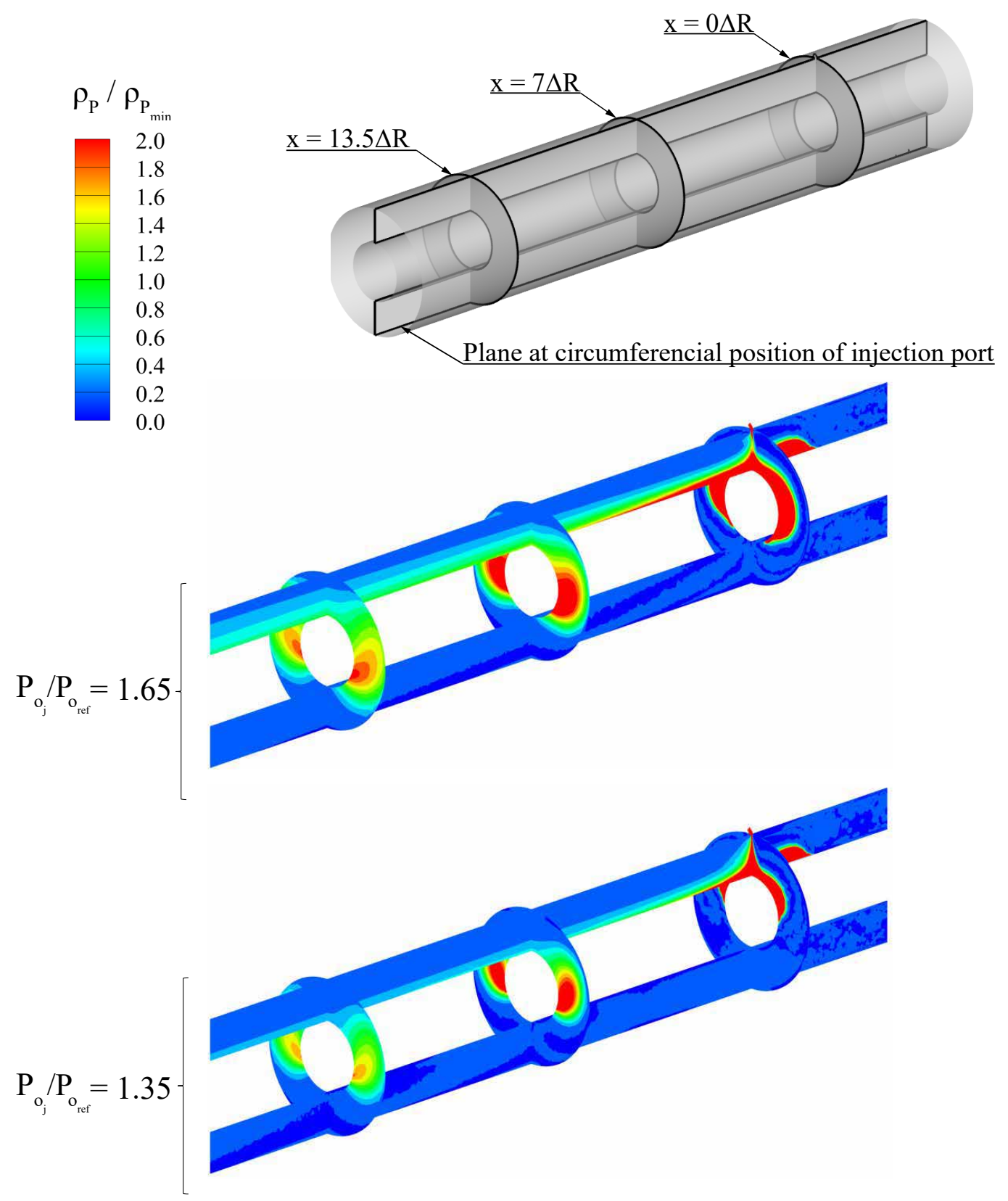

Figure 6.11: Streamwise seed-particle density distributions for $D_{j} / \Delta R=0.077$ and $\alpha_{j}=30$ deg., and injection stagnation pressures of $P_{o_{j}} / P_{o_{r e f}}=1.65$ and $P_{o_{j}} / P_{o_{r e f}}=1.35$. 


\subsubsection{High-speed Annular Inflow}

The seeding system being designed in the present study will be used in both the low-speed and high-speed wind-tunnels as discussed previously in this chapter. Accordingly, it is important to establish whether the seed-injection system performance trends for the low-speed annular flow presented in the previous section are also applicable to higher annular-flow speeds. Simulations with higher annular flow speeds indicated that for these conditions a jet injection angle of $45 \mathrm{deg}$. rather than $30 \mathrm{deg}$. provides more effective seeding. The seed density distribution and the perturbation of the annular flow by the seeding jet for this case are compared to the low-speed case in Figs. 6.12 and 6.13. This comparison illustrates that the flow physics of the interaction of the seeding jet with the annular flow is altered significantly by the tenfold increase of the annular flow velocity. Specifically, it is evident that the increased annular flow velocity results in the seeding jet not being able to traverse across the annulus height to reach the inner wall of the annulus. Instead, the comparable jet velocity and annular-flow velocity results in the jet mixing with the annular flow locally, shortly after it discharges from the injection port. This also results in the disturbance of the annular flow by the jet to remain localized as shown by the axial velocity and stagnation pressure distributions. The level of local disturbance of the annulus velocity field is sufficiently small enough, and the seed density is sufficiently high and uniform in a reasonably wide circumferential portion of the annulus to declare this particular configuration $\left(D_{j} / \Delta R=0.077, P_{o_{j}} / P_{o_{r e f}}=2.05\right.$ and $\alpha_{j}=45$ deg. $)$ to be suitable for experimentation at annulus flow speeds of about $160 \mathrm{~m} / \mathrm{s}$. 


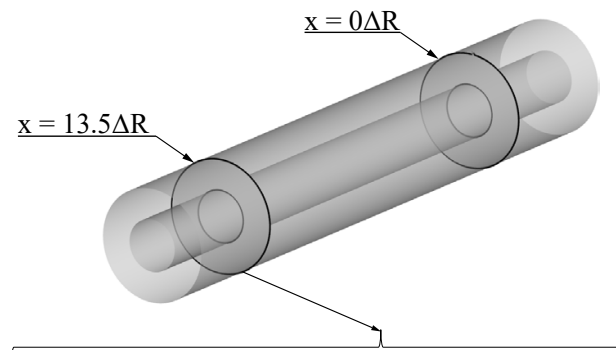

Low-speed Annular Inflow

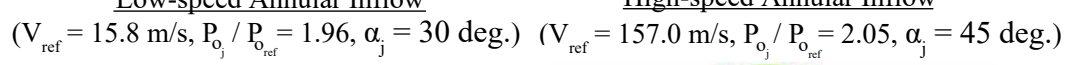

$\rho_{\mathrm{P}} / \rho_{\mathrm{P}_{\text {min }}}$
3.0
2.7
2.4
2.1
1.8
1.5
1.2
0.9
0.6
0.3
0.0
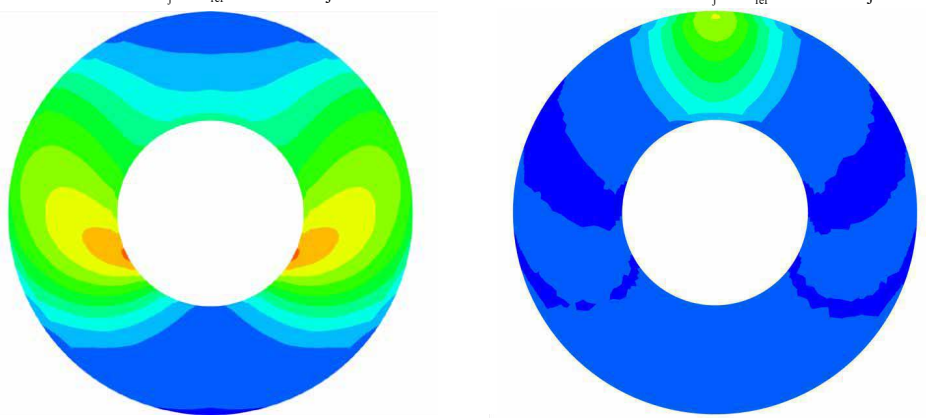

$\mathrm{V}_{\mathrm{x}} / \mathrm{V}_{\text {ref }}$
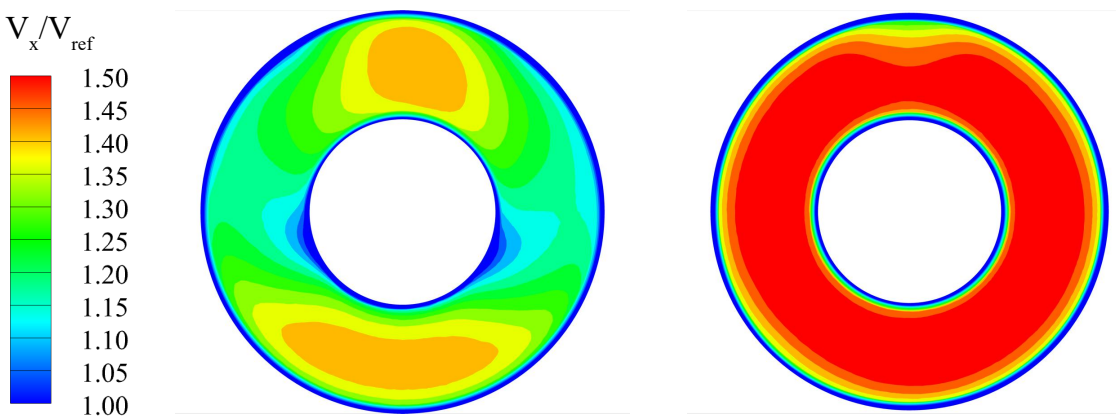

$\mathrm{P}_{\mathrm{o}} / \mathrm{P}_{\mathrm{o}_{\text {ref }}}$
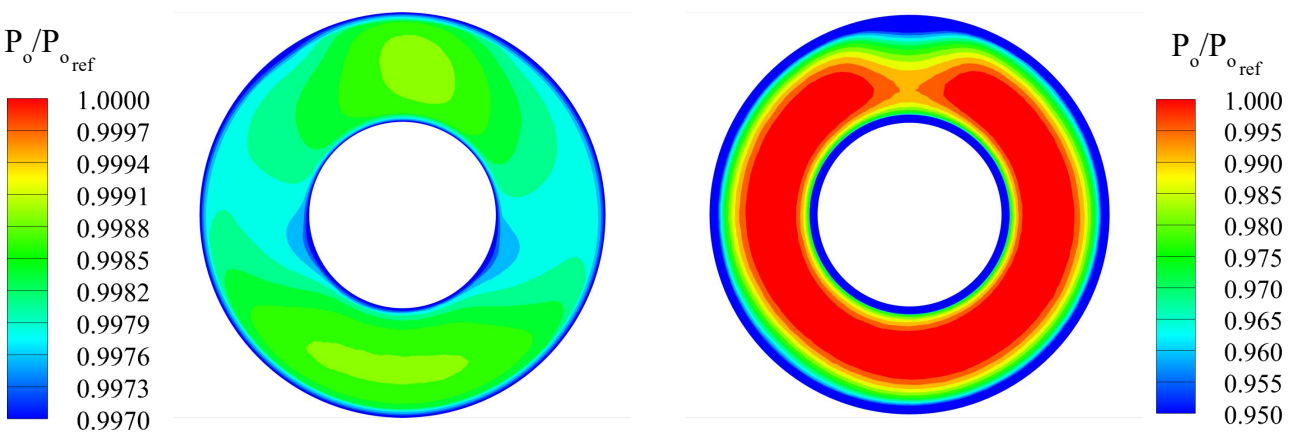

Figure 6.12: Seed-particle density and axial velocity distributions at $x / \Delta R=13.5$ for low and high annular flow speeds with $D_{j} / \Delta R=0.077$. 


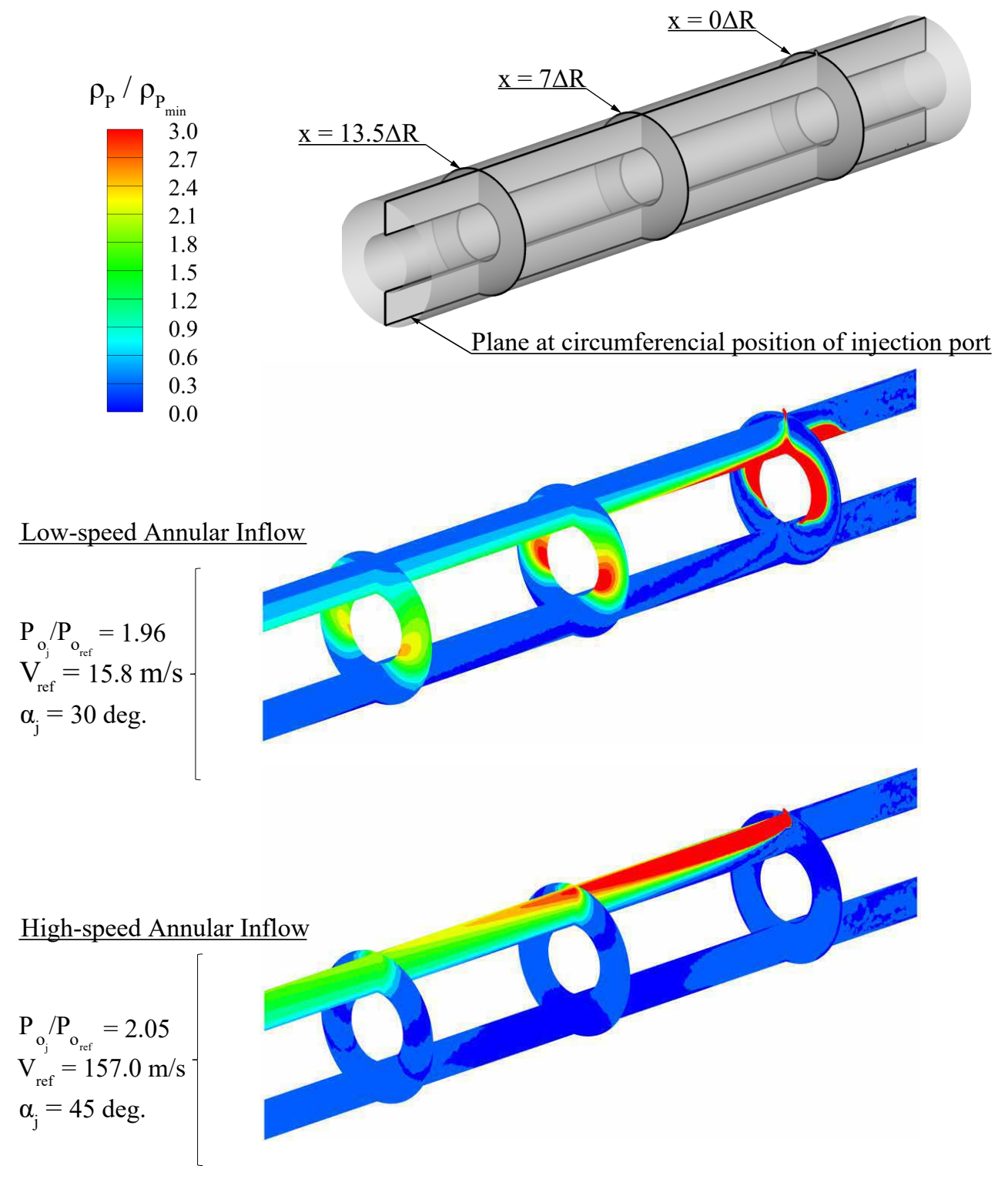

Figure 6.13: Comparison of the streamwise seed-particle density distributions for the low and high annular flow speeds with $D_{j} / \Delta R=0.077$. 


\subsection{Validation of the Numerical Flow Model}

\subsubsection{Computational Model}

Validation of the numerical flow model used to design the seed injection system was realized by performing a benchmark simulation based on the experimental study performed by Strzelecki et al. (2009) of a jet in crossflow. Fig. 6.14 presents the computational domain and boundary conditions used in this validation simulation.

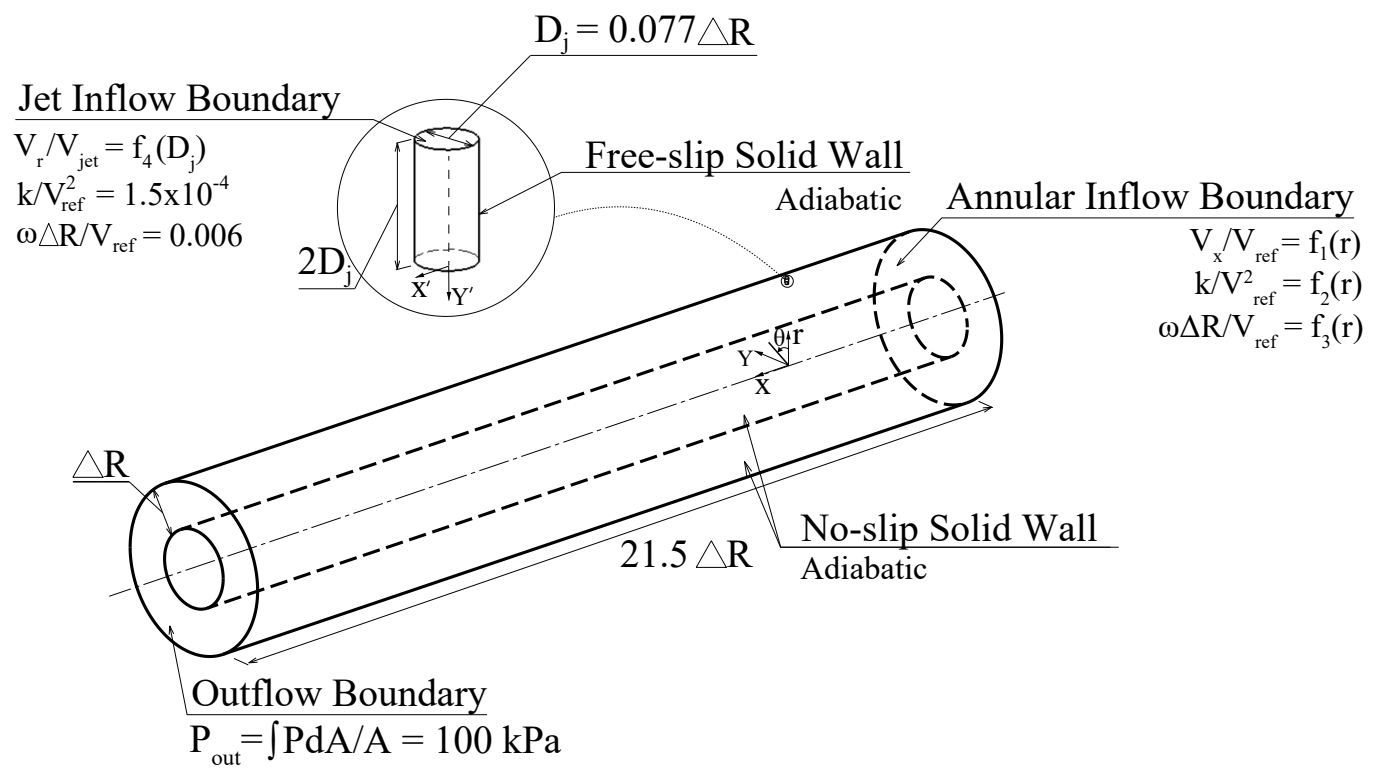

Figure 6.14: Computational domain and boundary conditions.

The injection port orifice diameter and injection angle are set to values of $D_{j} / \Delta R=0.077$ and $\alpha_{j}=0$ deg., respectively. The radial profiles of velocity and turbulence kinetic energy at the annular inflow were matched to those experimentally measured by Strzelecki et al. (2009), and are presented in Fig. 6.15. In these figures, $V_{r e f}=25 \mathrm{~m} / \mathrm{s}$ is the area-averaged axial velocity at the annular inflow. This corresponds to an annular flow Reynolds number based on the hydraulic diameter of about $R e_{D_{h}}=1.7 \times 10^{5}$, which which was matched in the simulation. 


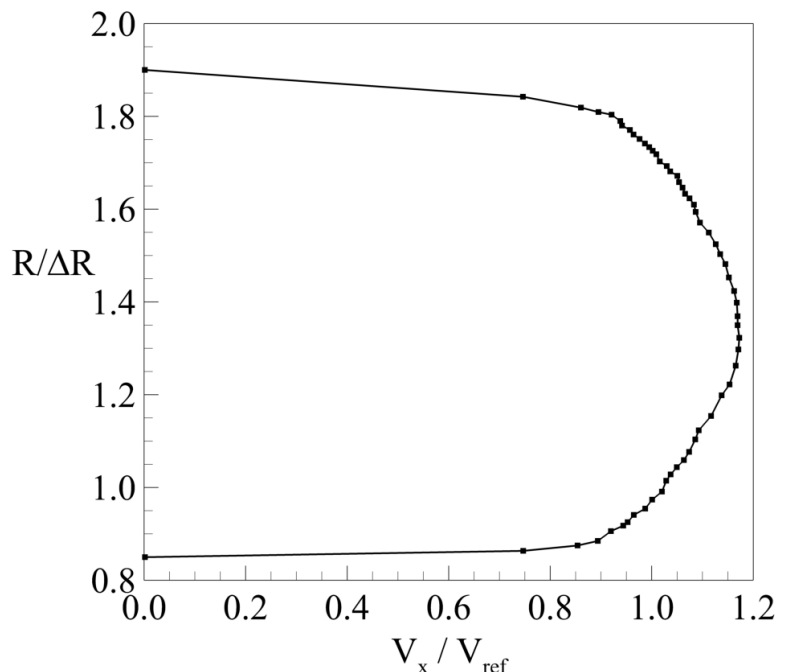

(a)

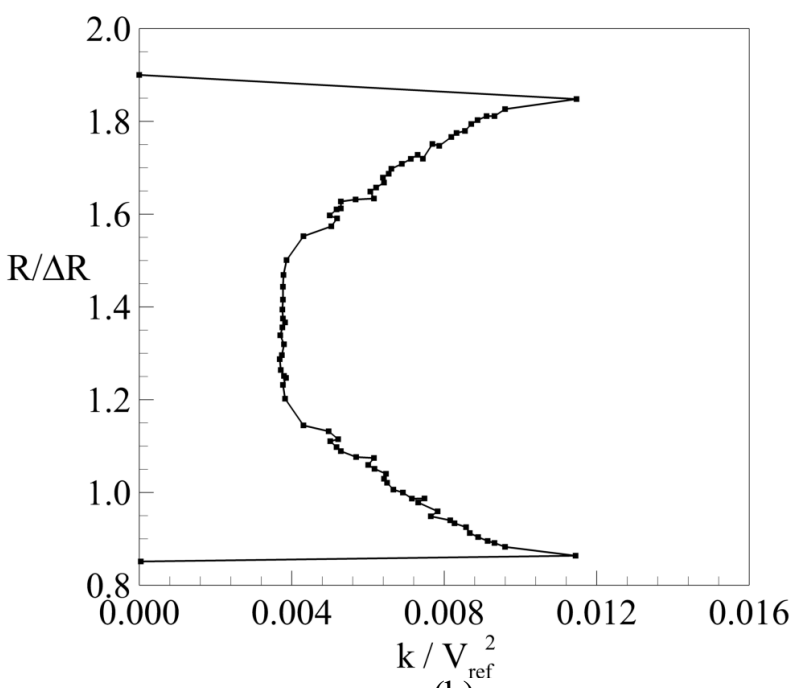

(b)

Figure 6.15: Inflow radial profile of (a) time-averaged axial velocity, and (b) turbulence kinetic energy at the annular-inflow plane (Strzelecki at al., 2009).

The jet inflow velocity profile is also set to match the one used in the experimental study and is presented in Fig. 6.16 where $V_{\text {jet }}=100 \mathrm{~m} / \mathrm{s}$, corresponding to a jet flow Reynolds number of about $R e_{D j}=3.3 \times 10^{4}$. The corresponding jet-to-annular flow velocity ratio is $J=4.0$, where $J$ is defined as:

$$
J=\frac{V_{\text {jet }}}{V_{\text {ref }}}
$$

At the jet inflow, the values of $k$ and $\omega$, normalized by the reference velocity, $V_{\text {ref }}=25 \mathrm{~m} / \mathrm{s}$, and the annulus height, $\Delta R=33 \mathrm{~mm}$, are set to $1.5 \times 10^{-4}$ and 0.006 , respectively, which corresponds to a turbulence intensity of $1.0 \%$. The flow parameter settings used to initialize the computation are summarized in Table 6.5. 


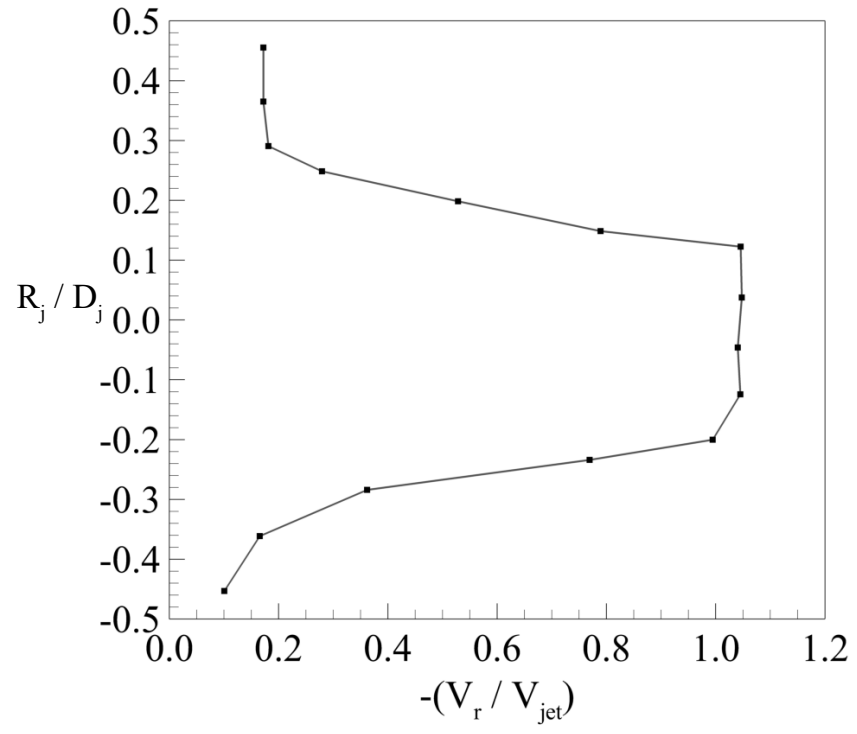

Figure 6.16: Inflow cross-stream profile of time-averaged velocity at the jet inflow (Strzelecki et al., 2009).

Table 6.5: Initial values of the normalized flow-field parameters in the domain.

\begin{tabular}{lc}
\hline$V_{x} / V_{\text {ref }}$ & 1.0 \\
$V_{y} / V_{\text {ref }}$ & 0.0 \\
$V_{r} / V_{\text {ref }}$ & 0.0 \\
$P / P_{\text {out }}$ & 1.0 \\
$k / V_{\text {ref }}^{2}$ & $1.35 \times 10^{-3}$ \\
$\omega \Delta R / V_{\text {ref }}$ & 2.04 \\
\hline
\end{tabular}

Consistent with the negligibly low Mach numbers in the benchmark study, the Reynoldsaveraged Navier Stokes (RANS) equations at steady state were solved using ANSYS CFX (version 19.0). The incompressible form of the RANS equations can be expressed as:

$$
\begin{gathered}
\frac{\partial}{\partial x_{i}} \overline{u_{i}}=0 \\
\frac{\partial}{\partial x_{j}}\left(\bar{u}_{i} \bar{u}_{j}+\frac{1}{\rho} \bar{P} \delta_{i j}+\overline{u_{i}^{\prime} u_{j}^{\prime}}-\nu\left[\frac{\partial \bar{u}_{i}}{\partial x_{j}}+\frac{\partial \bar{u}_{j}}{\partial x_{i}}\right]\right)=0
\end{gathered}
$$


where $\nu$ is the fluid kinematic viscosity. Turbulence closure, as before, is achieved via the Boussinesq hypothesis where the Reynolds-stress term, $\overline{u_{i}^{\prime} u_{j}^{\prime}}$, is modeled using the well established two-equation eddy-viscosity-based shear-stress transport (SST) turbulence model of Menter (Menter, 1994) with its standard empirical coefficients (Menter and Esch, 2001). All other aspects of the computational model are the same as those of the seed-system design optimization simulations described in previous sections of this chapter.

\subsubsection{Computational Results}

Fig. 6.17 presents a comparison of the simulated and experimental time-averaged and RMS seed-particle density, in the $x^{\prime}-y^{\prime}$ plane (see Fig. 6.14 for coordinate system) in the near region of the jet-exit. Both jets appear to penetrate into the mainflow to a similar extent within the same streamwise distance. The seed-particle density distribution of the two jets also appear to match quite favorably. 

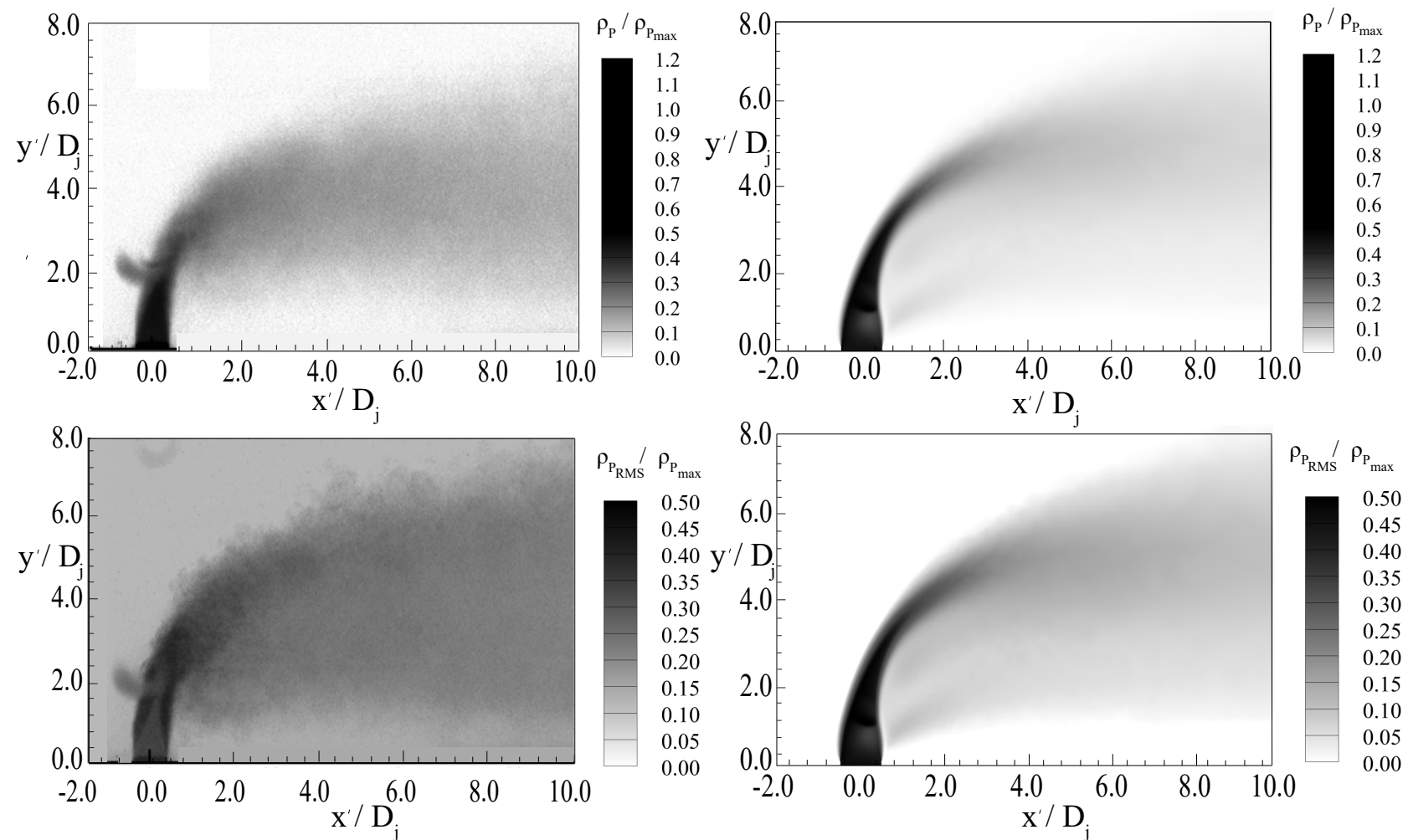

(a)

(b)

Figure 6.17: Time-averaged and RMS seed-particle density distributions in the near region of a jet in crossflow as (a) measured by Strzelecki et al., 2009 (adapted), and (b) predicted by the simulation.

This observation is supported by the comparisons in Fig. 6.18, which presents the jet trajectory as a function of streamwise distance and the seed-particle density along the jet trajectory as a function of $s / D_{j}$, where the jet trajectory is defined by the streamline of maximum seed-particle density, and $s$ is the curvilinear path along the jet trajectory. 


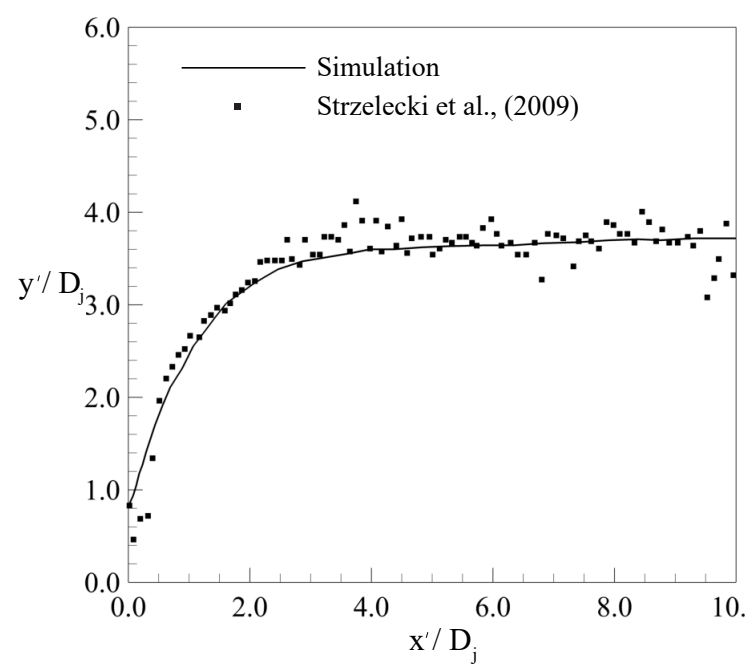

(a)

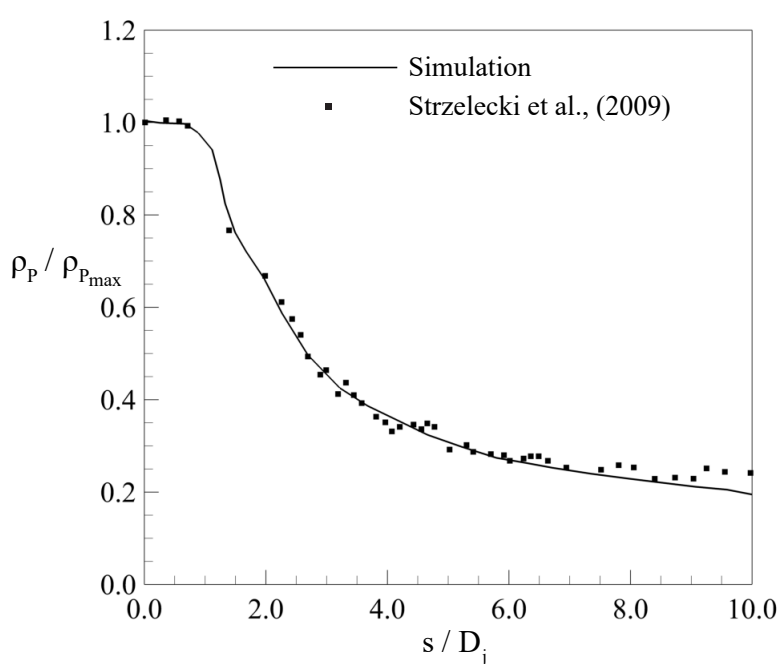

(b)

Figure 6.18: Comparison of (a) the jet trajectory based on the maximum seed-particle density, and (b) the seed-particle density along the jet trajectory.

The matching jet trajectory suggests that the jet-annular flow interaction is accurately captured by the numerical model. The favorable agreement in the seed-particle density along the jet trajectory indicates that the distribution of seed material is adequately captured by the model as well. The shape of the seed cloud emanating from the jet orifice is another worthy metric for comparison. Fig. 6.19 presents a comparison of the shape of the seed-particle density profile of the jets in two crossstream planes downstream of the jet port. The streamwise distance has been normalized by $D_{j}$ and $J$. In Fig. 6.19, the jet envelope is defined by two streamlines, one nearest the hub wall the other nearest the casing wall, where the seed-particle density is 0.5 times the maximum seed-particle density at that streamwise location. The favorable agreements presented in these figures further conclude that the numerical model does indeed adequately capture the flow physics that are essential to the present study. 


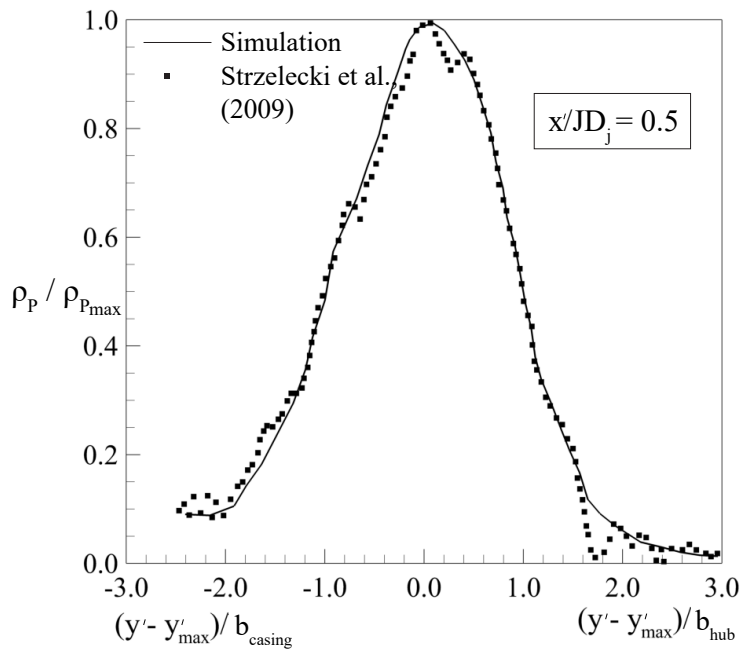

(a)

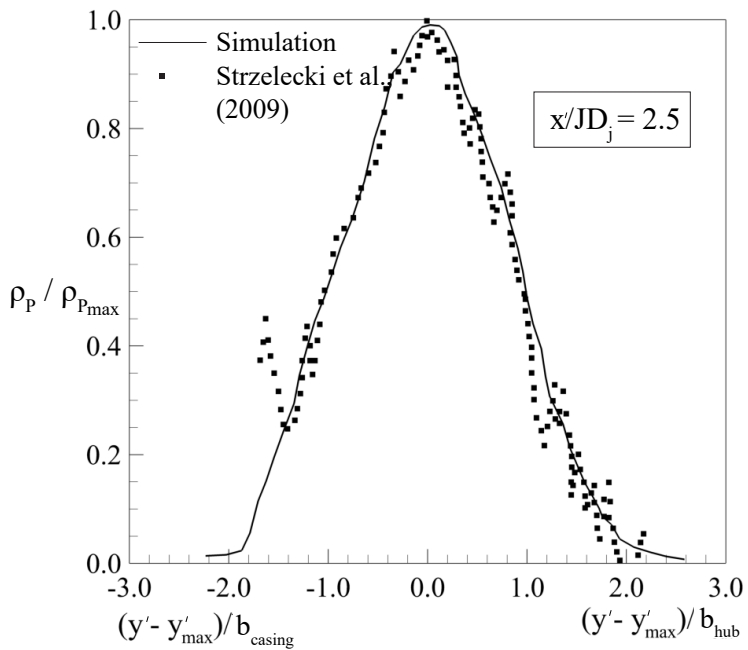

(b)

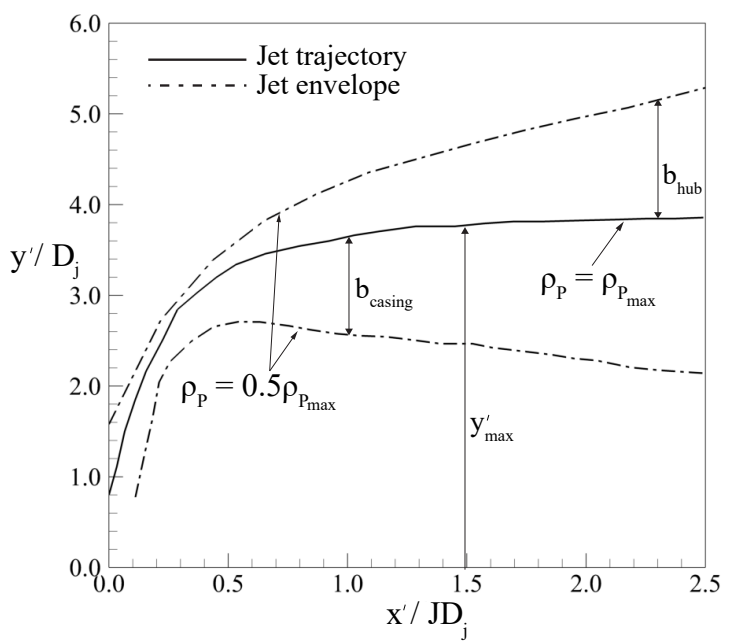

(c)

Figure 6.19: Comparison of the shape of the seed-particle density profile at (a) $x^{\prime} / J D_{j}=0.5$, and (b) $x^{\prime} / J D_{j}=2.5$; (c) profile of the jet envelope and depiction of the parameters used to normalize the quantities in the figures. 


\subsection{Design Summary}

The design iterations performed through CFD simulations as summarized in the preceding sections provide guidance on the choice of the injection port diameter, injection angle in the streamwise-radial plane, and injection stagnation pressure. Specifically, for the annular-flow geometry and speed range considered, a seeding jet discharge diameter of $D_{j} / \Delta R$ of about 0.077 is recommended. It is demonstrated that with this jet diameter, a sufficiently high seed-particle density can be achieved over part of the annulus circumference and that the extent of perturbation to the annular flow caused by the jet can be controlled with the stagnation pressure of the jet. The optimum value of the angle at which the jet is injected into the annular main stream is shown to be sensitive to the relative magnitudes of the jet and annular flow velocities. 


\section{Chapter 7}

\section{Conclusions and Recommendations for Future Work}

This thesis presents the aerodynamic and structural design of a high-speed annularflow air-supply system for the purpose of experimental investigations of ducted flows at high subsonic flow Mach numbers. The aerodynamic efficiency of the system is predicted to yield wind-tunnel run times on a blow-down type wind-tunnel of no less than 25-30 seconds at high subsonic Mach numbers. This performance is complemented with a cross-sectional discharge flow area that allows for test section sizes suitable for high-spatial-resolution measurements with existing measurement instrumentation. The design meets or exceeds the structural safety factors as stipulated by the applicable provincial regulations. The modular nature the air-supply system provides a highly versatile experimental apparatus for the testing of a wide range of ducted flows and allows for rapid turn-around times for reconfiguration of the flow path. The choice of a composite design with the use of durable polymer materials results in a light-weight design that minimizes the risks to students and other personnel when handling the system components without the need for external lifting devices such as cranes. Finally, the design is cost effective which is achieved by making use of off-the-shelf components and easily obtainable raw materials that require minimum stock removal and can be machined by standard milling and lathe operations. The next step in the development of the high-speed annular-flow air-supply system is certification of the design by the relevant provincial authority. This will be followed by manufacturing and commissioning of the setup.

The second design effort in this thesis pertains to the design of a rectilinear lobed mixer test section for experimentation at high subsonic Mach numbers. The test section is designed to interface with the discharge end of an existing experimental setup that has been used for blade cascade testing. This rectilinear test section will allow testing of geometries such as lobed mixers in a planar setting, complementing 
experiments that will be conducted in an annular configuration using the high-speed annular flow supply system. As with the annular-flow system, the structural integrity of the system under the anticipated aerodynamic loads have been confirmed through detailed structural analyses. The test section has since been manufactured and installed in the facility. Commissioning of the installation is the remaining step before experiments can commence with research specimens of lobed mixer geometries.

Experimentation using the annular and rectilinear test setups will utilize a broad range of measurement instrumentation. To date, all experiments conducted in Carleton's high-speed blow-down wind-tunnel have used pressure probes, surface pressure taps and thermal anemometry for flow measurements. Going forward, field measurements based on the particle image velocimetry technique will be used to complement point measurements with probes and surface pressure taps. In preparation for this, a comprehensive review of flow seeding techniques for high-speed flows was undertaken as part of this thesis. Additionally, a CFD-based design optimization study was performed for seeding an annular flow to establish a flow seeding configuration that yields satisfactory seed density over the desired flow region with minimal perturbation of the flow to be measured. Olive oil is demonstrated to have all of the characteristics desired for seeding particles in relatively high-speed air flows, and guidance is provided on the size, orientation and supply pressure of a seed injection port installed on the wall of an annular flow path.

The CFD-based design optimization study of a seeding system presented in this thesis could be expanded to include a broader range of values for the relevant design parameters, namely the size, inclination and supply pressure of the seed injection port. The trends presented in the thesis for 27 combinations of these parameters provide effective guidance for such a future study. 


\section{References}

Adrian, R., Westerweel, J. (2011). Particle Image Velocimetry. Cambridge University Press.

ASME (2010). Boiler and Pressure Vessel Code. Setion VIII, Div. 1.

ANSYS (2006). ANSYS CFX-Solver Theory Guide, 11ed., ANSYS, Inc., 275 Technology Drive, Canonsburg, PA.

Baines, W.D., Peterson, E.G. (1951). An investigation of Flow Through Screens.

Barlow, J.B., Rae, W.H., Pope, A. (1999). Low-Speed wind-tunnel Testing. John Wiley $\&$ Sons.

Bhattacharya, S., Charonko, J.J., Vlachos, P.P. (2017). Stereo-particle image velocimetry uncertainty quantification. Meas. Sci. Technol., 28.

Blevins, R.D. (1984). Applied Fluid Dynamics Handbook. Van Nostrad Reinhold Co.

Brinkerhoff, J., Oria, H., Yaras, M. (2013). Experimental and Computational Study of Mixing Mechanisms in an Axisymmetric Lobed Mixer. Journal of Propulsion and Power, 29(5), pp. 1017-1030.

Brinkerhoff, J., Yaras, M. (2014a). Computational and Experimental Study of the Effect of Inlet Swirl on Mixing Mechanisms in an Axisymmetric Lobed Mixer. ASME Turbo Expo 2014, GT2014-25691.

Brinkerhoff, J. (2014b). Numerical Investigations of Instability and Transition in Attached and Separated Shear Layers. Ph.D. thesis, Carleton University.

Bradshaw, P. (1964). Wind-Tunnel Screens: Flow Instability and its Effect on Aerofoil Boundary Layers. The Journal of the Royal Aeronautical Society, 68(639), pp. 198-198. 
Bosbach, J., Kuhn, M., Wagner, C. (2009). Large scale particle image velocimetry with helium filled soap bubbles. Exp. Fluids, 46, pp. 539-547.

Caridi, G., Sciacchitano, A., Scarano, F. (2017). Helium-filled soap bubbles for vortex core velocimetry. Exp. Fluids, 58(130), pp. 1-12.

Crosswy, F. (1985). Particle size distribution of several commonly used seeding aerosols. NASA Workshop, wind-tunnel Seeding Systems for Laser Velocimeters.

Crist, S., Sherman, P.M., F;ass, D.R. (1966). Study of the Highly Underexpanded Sonic Jet,. AIAA J., 4, pp. 68-71.

DeLapp, C. (2006). Particle Image Velocimetry Using Novel Non-intrusive Particle Seeding. M.A.Sc. thesis, Air Force Institute of Technology.

Diasinos, S., Beves, C., Barber, T. (2013). Meas. Sci. Technol., 24.

Dierksheide, U., Meyer, P., Hovestadt, T., Hentschel, W. (2002). Endoscopic 2D particle image velocimetry (PIV) flow-field measurements in IC engines. Experiments in Fluids, 33, pp. 794-800.

Dutton, J.C. (2008). Supersonic Blowdown wind-tunnel Control Using LabVIEW. 46th AIAA Aerospace Sciences Meeting and Exhibit.

Echols, W., Young, J. (1963). Studies of Portable Air-Operated Aerosol Generators. U.S. Naval Research Laboratory Report.

Erturk, N., Duzgun, A., Ferre, J., Varela, S., Vernet, A. (2013). Alginate flow seeding microparticles for use in Particle Image Velocimetry (PIV). 10th International Symposium on Particle Image Velocimetry.

Farell, C., Youssef, S. (1992). Experiments on Turbulence Management Using Screens and Honeycombs. National Science Foundation, Project Report No. 338.

Faleiros, D., Tuinstra, M., Sciacchitano, A., Scarano, F. (2019). Generation and control of helium-filled soap bubbles for PIV.Exp. Fluids, 60(40) 
Fang, C., Hong, L., Zifeng, Y., Hui, H. (2017). Tracking characteristics of tracer particles for PIV measurements in supersonic flows. Chinese Journal of Aeronautics, 30(2), pp. 527-585.

Faure, T.M., Michon, G.J., Miton, H., Vassilieff, N. (2001). Laser Doppler Anemometry Measurements in an Axial Compressor Stage. Journal of Propulsion and Power, 17(3), pp. 481-491.

Fey. U., Konrath, R., Kimse, T., Ahlefeldt, T. (2010). Advanced measurement techniques for high Reynolds number testing in cryogenic wind-tunnels. 48th AIAA Aerospace Sciences Meeting Including the New Horizons Forum and Aerospace Exposition.

Groth, J., Johansson, A.V. (1988). Turbulence reduction by screens. J. Fluid Mech., 197, pp. 139-155.

Hall, S. (2012). Investigation of the Compressibility on Profile Pressure Losses in Axial Turbine Cascades. M.A.Sc. thesis, Carleton University.

Haya., L.K. (2015). Measurement of Flow Through A Bileaflet Mechanical Heart Valve in an Anatomically Accurate Model of the Aorta. PhD. thesis, University of Ottawa.

Havermann, M., Haertig, J., Rey, C., and George, A. (2002). Application of particle image velocimetry to high-speed supersonic flows in a shock tunnel. 11th International Symposium on Application of Laser Techniques to Fluid Mechanics.

Hamdi, M., Havet, M., Rouaud, O. (2014). Comparison of different tracers for PIV measurements in EHD airflow. Exp. Fluids , 55:1702.

Humble, R., Scarano, F., van Oudheusden, B. (2007). Particle image velocimetry measurements of a shock wave/turbulent boundary layer interaction. Exp. Fluids, 43, pp. 173-183. 
Ikegaya, N., Hasegawa, S., Hagishima, A. (2018). Time-resolved particle image velocimetry for cross-ventilation flow of generic block sheltered urban-like block arrays. ELSEVIER: Building and Environment. vol 147, pp. 132-145.

Kahler, C., Sammler, B., Kompenhans, J. (2002) Generation and control of tracer particles for optical flow investigations in air. Experiments in Fluids, 33, pp. 736742.

Keane, R.D., Adrian, R.J., Zhang, Y. (1995). Super-resolution particle imaging velocimetry. Meas. Sci. Technol., 6, pp. 754-768.

Kim, J., Kim, D., Liberzon, A., Chamorro, L.P. (2016). Three-dimensional Particle Tracking Velocimetry for Turbulence Applications: Case of a Jet Flow. Journal of Visualized Experiments, 108.

Koike, S., Takahashi, H., Tanaka, K., Hirota, M. (2007). Correction Method for Particle Velocimetry Data Based on the Stokes Drag Law. AIAA Journal, 45(11), pp. 2770-2777.

LaVision (2015). Flow Master Product-Manual, 1105011-4.

Legrand, M., Noguiera, J., Rodriguez, P., Lecuona, A., Jimenez, R. (2016). Generation and droplet size distribution of tracer particles for PIV measurements in air, using propylene glycol/water solution. ELSEVIER: Experimental Thermal and Fluid Science, 81, pp. 1-8.

Lepicovsky, J., Bruckner, R.J. (1996). Seeding for laser velocimetry in confined supersonic flows with shocks. Eighth symposium on Applications of Laser Technology to Fluid Mechanics.

Lee, K., Mooney, D. (2012). Alginate: properties and biomedical applications. Prog Polym Sci. 37(1), pp. 106-126. 
Lim, W.L., Chew, Y.T., Chew, T.C., Low, H.T. (1994). Particle Image Velocimetry in the Investigation of Flow Past Artificial Heart Valves. Annals of Biomedical Engineering, 22, pp. 307-318.

Liu, N., Wu, Y., Ma, L. (2018). Quantification of tomographic PIV uncertainty using controlled experimental measurements. Applied Optics. 57(3), pp. 420-427.

Love, B. (2010). Particle Size Control for PIV Seeding using Dry Ice. M.A.Sc. thesis, Air Force Institute of Technology.

Lockheed Martin, (2002). High Speed wind-tunnel and Test Systems Design Handbook. Publication Number AER-EIR-13552-E.

Mazumder, M., Hoyle, B., Kirsch, K. (1974). Fluid dynamic studies on scattering aerosol and its generation for application as tracer particles in supersonic flow measurements utilizing laser Doppler velocimetry. NASA, Technical Report.

Mathijssen, T., Bannink, W.J., Scarano, F. (2009). Investigation of a sharp-edged delta wing in a supersonic flow using stereo PIV. 39th AIAA Fluid Dynamics Conference.

Marassi, M., Castellini, P., Pinotti, M., Scalise, L. (2004). Cardiac valve prosthesis flow performances measured by $2 \mathrm{D}$ and $3 \mathrm{D}$-stereo particle image velocimetry. Experiments in Fluids, 36, pp. 176-186.

McNiel, C. (2007). Demonstration of Clean Particle Seeding for Particle Image Velocimetry in a Closed Circuit Supersonic wind-tunnel. M.A.Sc. thesis, Air Force Institute of Technology.

Melling, A. (1997). Tracer Particles and Seeding for Particle Image Velocimetry. Meas. Sci. Technol., 8, pp. 1406-1416.

Menter, F.R. (1994). Two-Equation Eddy-Viscosity Turbulence Models for Engineering Applications. AIAA Journal, 32(8). 
Menter, F.R., Esch, T. (2001). Elements of industrial heat transfer predictions. 16th Brazilian Congress of Mechanical Engineering (COBEM), 109.

Mehta, R.D., Bradshaw, P. (1979). Design rules for small low speed wind-tunnels. The Aeronautical Journal of the Royal Aeronautical Society, 718, pp. 443-449.

Moukalled, F., Mangani, L., Darwish, M. (2016). The Finite Volume Method in Computational Fluid Dynamics. Springer.

NAFEMS (1989). National Agency for Finite Element Methods and Standards (U.K.): Test NL2, NAFEMS non-linear benchmarks, NNB, Rev. 1

NAFEMS (1993). National Agency for Finite Element Methods and Standards (U.K.): Test 3DNL-8, R0024.

Nott, C.R., Olcmen, S.M., Lewis, D.R., Williams, K. (2008). Supersonic, variablethroat, blow-down wind-tunnel control using genetic algorithms, neural networks, and gain scheduled PID. Applied Intelligence.

Norma Group (2016). https://www.normaamericasds.com/product-type/v-band-clamps

Olsen, M., Adrian, R. (2000). Brownian motion and correlation in particle image velocimetry, Optics and Laser Technology, pp. 621-627.

Petrosky, B., Maisto, P., Lowe, T., Andre, M., Bardet, P., Tiemsin, P., Whol, C., Danehy, P. (2015). Particle Image Velocimetry Applications Using Fluorescent Dye-doped Particles. AIAA SciTech.

Pope, A., Goin, K.L. (1965). High-Speed wind-tunnel Testing. Jon Wiley \& Sons.

Raw, M.J. (1985). A New Control-Volume Based Finite Element Procedure for the Numerical Solution of the Fluid Flow and Scalar Transport Equations. PhD. thesis, University of Waterloo.

Rehm, B. (2009). Alginates: Biology and Applications. Springer. 
Rhie, C.M., Chow, W.L. (1983). Numerical study of the turbulent flow past an airfoil with trailing edge separation. AIAA Journal, 21(11), pp. 1525-1532.

Scarano, F., (2013). Tomographic PIV: principles and practice. Meas. Sci Technol., 24.

Scarano, F., Poelma, C. (2009). Three-dimensional vorticity patterns of cylinder wakes. Exp. Fluids, 47, pp. 69-83.

Scharnowski, S., Bross, M., Kahler, C. (2018). Accurate turbulence level estimations using PIV/PTV. Experiments in Fluids, 60(1).

Seegmiller, H. (1985). Seeding Subsonic, Transonic and Supersonic Flows with 0.5 Micron Polystrene Spheres. NASA Workshop, wind-tunnel Seeding Systems for Laser Velocimeters.

Smith, S.H., Mungal, M.G. (1998). Mixing, structure and scaling of the jet in crossflow. Journal of Fluid Mechanics, 357, pp. 83-122.

Sosnik, A. (2014). Alginate Particles as Platform for Drug Delivery by Oral Route: State-of-the-Art, ISRN Pharmaceutics, 2014(926157), pp. 1-17.

Strzelecki, A., Gajan, P., Giquel, L., Michel, B. (2009). Experimental Investigation of the Jets in Crossflow: Nonswirling Flow Case. AIAA, 47(5), pp. 1079-1089.

Su, L.K., Mungal, M.G. (2004). Simultaneous Measurements of Scalar and Velocity Field Evolution in Turbulent Crossflowing Jets. Journal of Fluid Mechanics, 513, pp. 1-45.

Towers, C.E., Bryanston-Cross, P.J., Judge, T.R. (1991). Application of particle image velocimetry to large-scale transonic wind-tunnels. Optics and Laser Technology, 23, pp. 289-295.

Tokarcik-Polsky, S., Papadopoulos, P., Venkatapathy, E., Deiwert, G.S. (1995). The NASA Ames 16-Inch Shock Tunnel Nozzle Simulations and Experimental Comparison. International Aerospace Planes and Hypersonics Technologies. 
Tropea, C. (1995). Laser Doppler anemometry: recent developments and future challenges. Meas. Sci. Technol., 6, pp. 605-619.

TSI Incoporated (2019). TSI Particle Technology Particle Instruments. Product Catalog.

TSSA (2007). Guidelines for the Registration of Non-Nuclear Fittings in the Province of Ontario. Technical Standards and Safety Authority.

TU Delft (2019). TST-27 Transonic/Supersonic windtunnel, https://www.tudelft.nl/lr/organisatie/ wind-energy-flight-performance-and-propulsion/facilities/high-speed-wind-tunnels/tst27-transonicsupersonic-windtunnel/

Villanueva, D., Smyth, H., Ruiz, D., Sherbiny, I. (2011). A Novel Aerosol Method for the Production of Hydrogel Particles. J Nanomater.

Wieneke, B., Pfeiffer, K. (2010). Adaptive PIV with variable interrogation window size and shape. 15 International Symposium of Laser Techniques to Fluid Mechanics

Wilcox, D.C. (1994). Turbulence Modeling for CFD. DCW Industries.

Xiao, C., Fond, B., Beyaru, F., T'Joen, C., Henkes, R., Veenstra, P., van Wachem, B. (2019). Numerical Investigation and Experimental Comparison of the Gas Dynamics in a Highly Underexpanded Confined Real Gas Jet. Flow, Turbulence and Combustion, 103, pp. 141-173.

Yu, K., Xu, J., Tang, L., Mo, J. (2015) New adaptive method in particle image velocimetry. Meas. Sci. Technol., 26 (3).

Zheng, R., Li, M., Wang, Z., Zhang, Q. (2017). Control of Blow-Down wind-tunnel Using Combined Extended Kalman and Nonlinear Predictive Filters. Journal of Fluids Engineering, 139.

Zhang, Z. (2010). LDA Application Methods. Springer Heidelberg Dordrecht London New York. 
Zhang, Zh. (2005). Dual-Measurement-Method and its extension for accurately resolving the secondary flows in LDA (Laser Doppler Anemometry) applications. Flow Measurement and Instrumentation, 16, pp. 57-62. 


\section{Appendix A}

\section{Derivation of Aerodynamic Performance Equations Pertaining to the Wind-tunnel Aerodynamic Model}

\section{A.1 Stagnation Pressure Loss in Compressible Duct Flow}

For an incompressible flow, the total, or stagnation, pressure $\left(P_{o}\right)$ is defined as the sum of the fluid static pressure $(P)$ and dynamic pressure as:

$$
P_{o}=P+\frac{1}{2} \rho V^{2}
$$

where $\rho$ and $V$ are the density and velocity of the fluid flow, respectively. The change in total pressure in a duct flow is expressed as a dimensionless loss coefficient, $K$, as:

$$
K=\frac{P_{o 1}-P_{o 2}}{\frac{1}{2} \rho_{1} V_{1}^{2}}
$$

where subscripts 1 and 2 denote upstream and downstream stations, respectively. For incompressible flow the change in stagnation pressure is equal to the change in static pressure:

$$
P_{o 1}-P_{o 2}=P_{1}-P_{2}
$$

For compressible flow, the above relation does not apply as the fluid density is not constant. For compressible flow, the static and stagnation pressure are related through an expression in terms of the Mach number, $M$, as:

$$
\frac{P_{o}}{P}=\left[1+\frac{\gamma-1}{2} M^{2}\right]^{\gamma-1 / \gamma}
$$

where $\gamma=1.4$ is the ratio of specific heats. Eqn. A.2 can then be rearranged through the relation:

$$
\frac{1}{2} \rho_{1} V_{1}^{2}=\frac{1}{2} \gamma P_{1} M_{1}^{2}
$$


such that:

$$
K=\frac{P_{o 1}-P_{o 2}}{\frac{1}{2} \gamma P_{1} M_{1}^{2}}
$$

Combining Eqns. A.6 and A.4, the equation for the loss coefficient becomes:

$$
K=\frac{1-\frac{P_{o 2}}{P_{o 1}}}{\frac{1}{2} \gamma M_{1}^{2}\left[1+\frac{\gamma-1}{2} M_{1}^{2}\right]^{1-\gamma / \gamma}}
$$

\section{A.2 Storage Tank Expansion Process Equations}

As mass flow leaves the storage tanks, the stagnation pressure $\left(P_{o}\right)$ and stagnation temperature $\left(T_{o}\right)$ decrease through polytropic expansion defined as:

$$
\frac{T_{o 2}}{T_{o 1}}=\left(\frac{P_{o 2}}{P_{o 1}}\right)^{\frac{n-1}{n}}
$$

where the subscripts indicate the static conditions at two different instances in time. Written in differential form, Eqn. A.8 becomes:

$$
\ln \left(\frac{T_{o 2}}{T_{o 1}}\right)=\left(\frac{n-1}{n}\right) \ln \left(\frac{P_{o 2}}{P_{o 2}}\right)
$$

which is equivalent to:

$$
\int_{1}^{2} \frac{d T_{o}}{T_{o}}=\frac{n-1}{n} \int_{1}^{2} \frac{d P_{o}}{P_{o}}
$$

Therefore, in differential form:

$$
\frac{d T_{o}}{T_{o}}=\left(\frac{n-1}{n}\right) \frac{d P_{o}}{P_{o}}
$$

Now considering the equation of state:

$$
P=\frac{m R T}{V}
$$

where $m$ and $V$ are the mass and volume of air in the storage tanks, respectively. Differentiating Eqn. A.12 with respect to time gives:

$$
\frac{d P_{o}}{d t}=\frac{R T_{o}}{V}\left(\frac{d m}{d t}\right)+\frac{m R}{d V}\left(\frac{d T_{o}}{d t}\right)
$$


Rearranging Eqn. A.11 gives:

$$
\frac{d T_{o}}{d t}=\frac{n-1}{n} \frac{T_{o}}{P_{o}}
$$

And since;

$$
\frac{d T_{o}}{d t}=\frac{d T_{o}}{d P_{o}} \frac{d P_{o}}{d t}
$$

combining equations Eqn. A.14 and A.15,

$$
\frac{d T_{o}}{d t}=\frac{n-1}{n} \frac{T_{o}}{P_{o}} \frac{d P_{o}}{d t}
$$

Now combining Eqn. A.16 and A.13 gives:

$$
\frac{d P_{o}}{d t}=\frac{R T_{o}}{V} \frac{d m}{d t}+m\left(\frac{R T_{o}}{P_{o} V}\right) \frac{n-1}{n} \frac{d P_{o}}{d t}
$$

Reducing an rearranging gives:

$$
\frac{1}{n}\left(\frac{d P_{o}}{d t}\right)=\frac{R T_{o}}{V}\left(\frac{d m}{d t}\right)
$$

and since by definition,

$$
\dot{m}=\frac{d m}{d t}
$$

Substituting Eqn. A.19 into Eqn. A.18 and A.16 yields the following two equations governing the pressure and temperature change in the storage tanks:

$$
\frac{d P_{o}}{d t}=-\dot{m}\left(\frac{n R T_{o}}{V}\right)
$$

and

$$
\frac{d T_{o}}{d t}=-\dot{m}\left(\frac{(n-1) R T_{o}^{2}}{P V}\right)
$$

where the negative sign indicates mass flow leaving the storage tanks and the polytropic coefficient, $n$, is equal to 1.0 for isothermal expansion and 1.4 for isentropic expansion. Eqns. A.20 and A.21 describe the expansion process in the storage tanks and therefore $T_{o}=T_{o T a n k}$ and $P_{o}=P_{o T a n k}$. 


\section{Appendix B}

\section{Technical Drawings for the Air-Supply System}

This page left intentionally blank. 
口

0

$\infty$

$\varangle$

\begin{tabular}{|l|l|l|l|}
\hline Part & Designation & Material & Quantity \\
\hline A & Existing HSWT Flange & Steel & - \\
\hline B & Exiting HSWT 90 deg. Bend & Steel & 1 \\
\hline I & Upstream Flange & Steel & 1 \\
\hline II & Expansion Section & Steel & 1 \\
\hline II I & Transition Section I & HDPE & 1 \\
\hline IV & Honeycomb Insert & Aluminum & 1 \\
\hline V & Flow Straightener Housing & HDPE & 1 \\
\hline VI & Turbulence Screen Housing & HDPE & 1 \\
\hline- & Turbulence Screen Inserts & Steel & 3 \\
\hline VII & Swirl Generator Insert & Steel & 2 \\
\hline VIII & Swirl Generator Housing & HDPE & 1 \\
\hline IX & Seed Injection Ring & Steel & 1 \\
\hline X & Seed Injection Housing & HDPE & 1 \\
\hline XI & Core Pipe & Sch. 80 PVC & 1 \\
\hline XII & Transition Section II & HDPE & 1 \\
\hline XIII & Downstream Flange & Steel & 1 \\
\hline XIV & Test Section (eg. Lobed Mixer) & Steel & 1 \\
\hline XV & End Plug & Steel & 1 \\
\hline
\end{tabular}

$$
x \quad \text { VIII }
$$
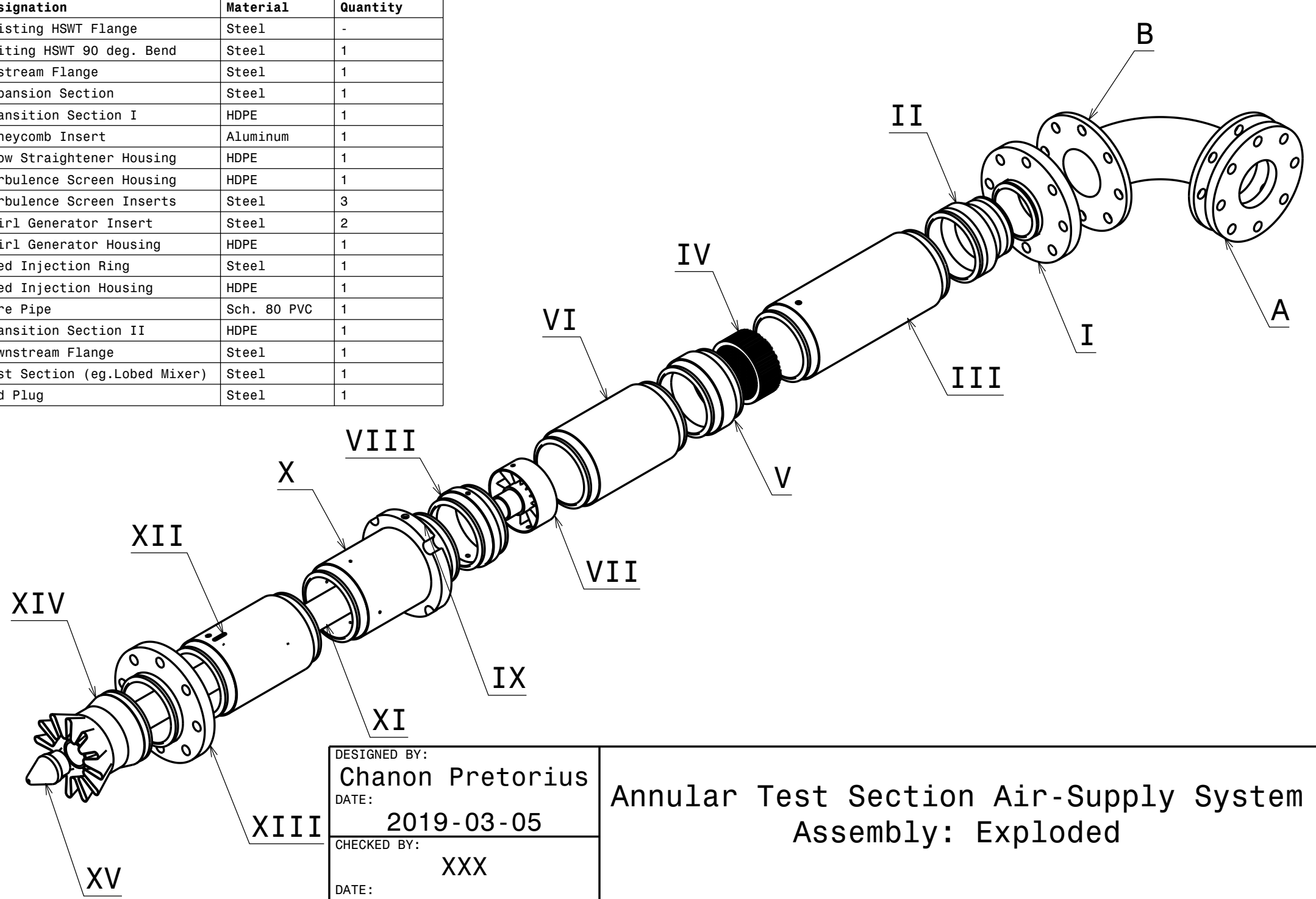

$$
X I
$$

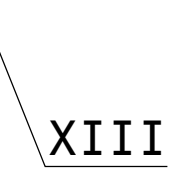

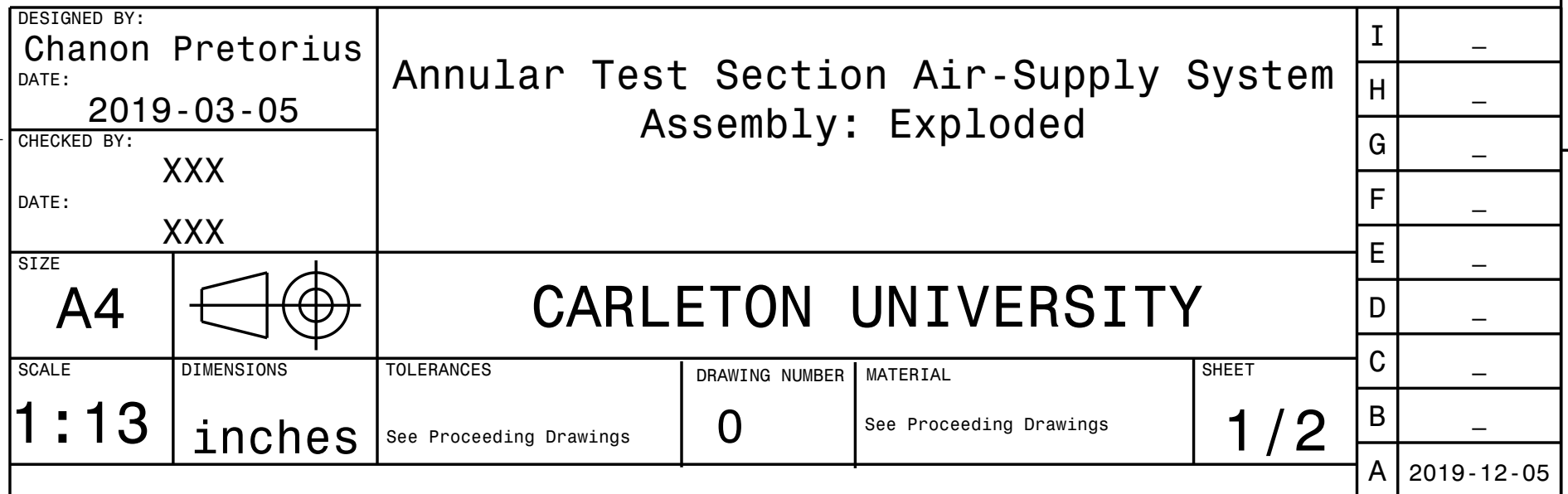




\begin{tabular}{|l|l|l|l|}
\hline Part & Designation & Material & Quantity \\
\hline 1 & 4.9 inch V-band Clamp & Steel & 1 \\
\hline 2 & 5.8 inch V-band Clamp & Steel & 8 \\
\hline 3 & Pre-compression Rod & Steel & 4 \\
\hline 4 & $5 / 8$ UNC Bolt & Steel & 4 \\
\hline 5 & $5 / 8$ UNC Hex Nut & Steel & 12 \\
\hline
\end{tabular}

$\underline{4}$

3

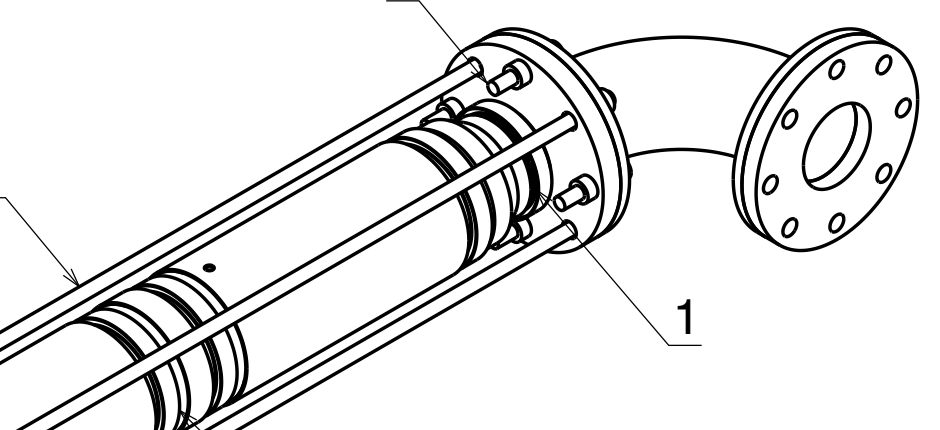

2

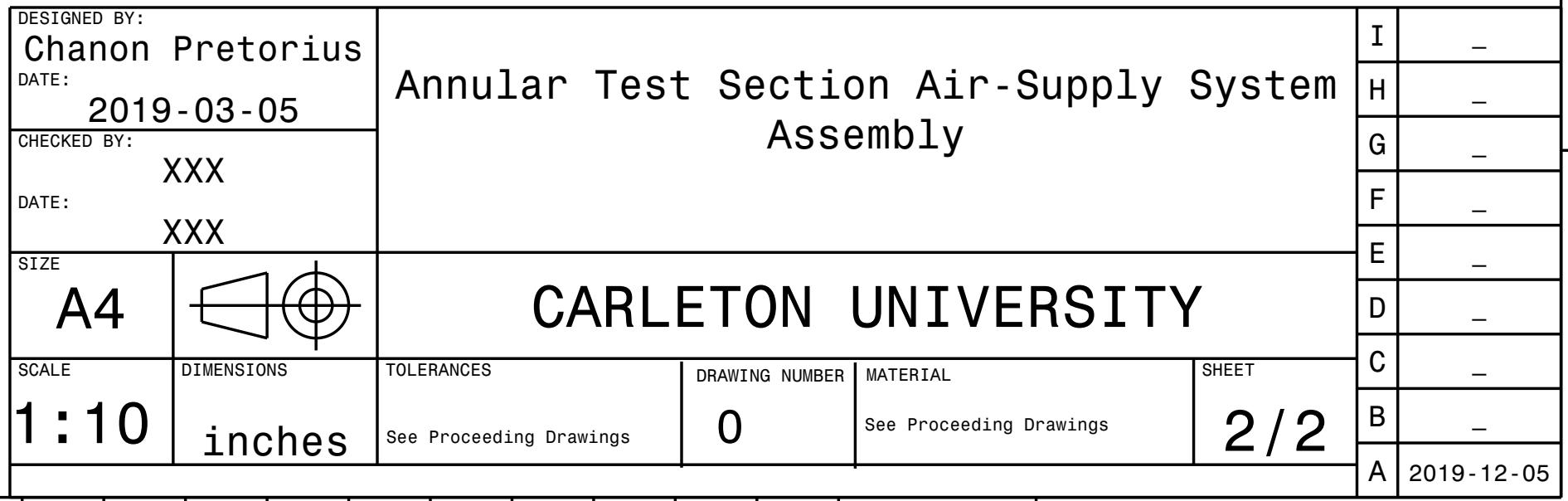


๑

0

$\infty$

$\varangle$

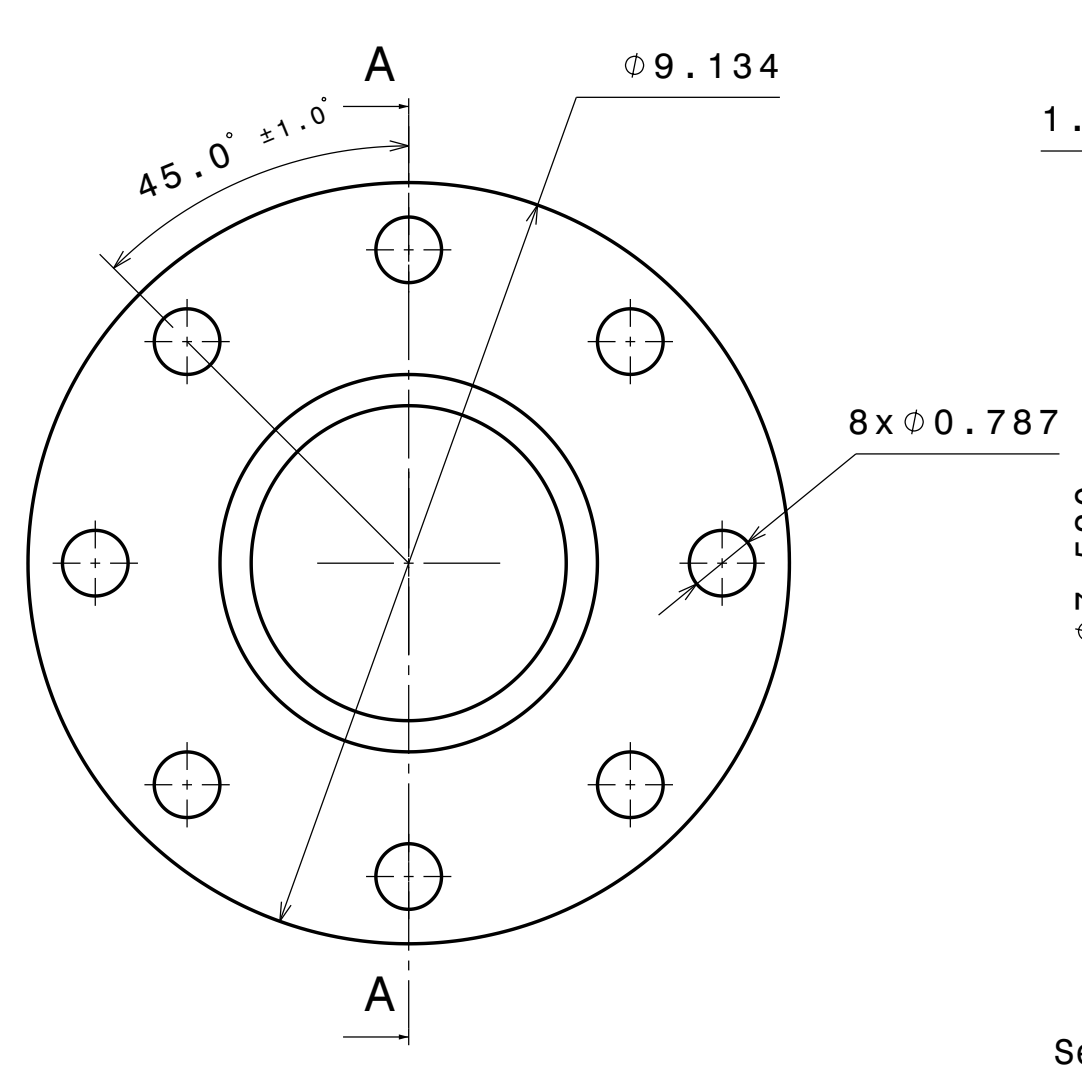

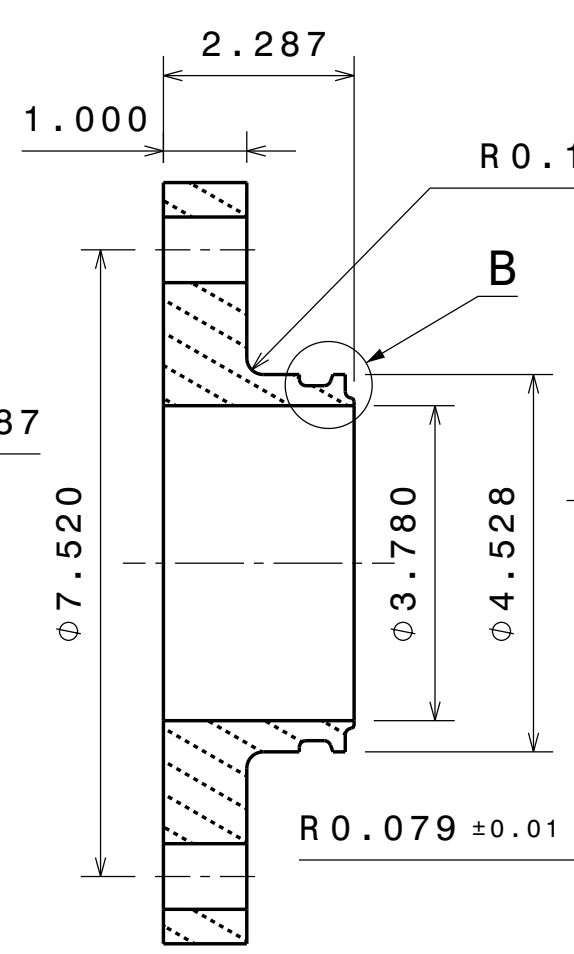

Section view A-A 0.197

R 0.079
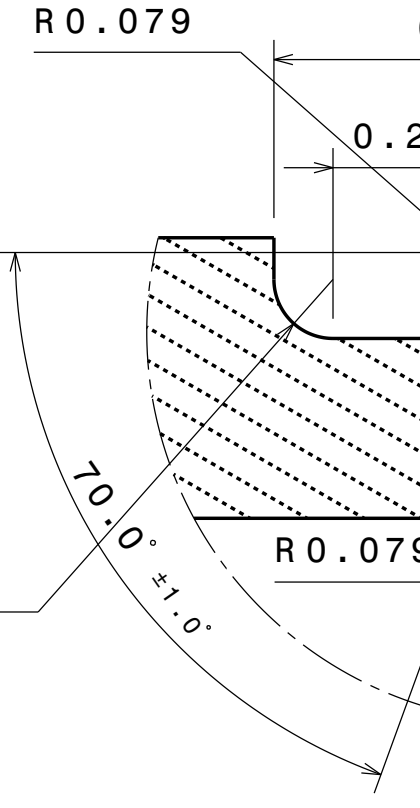

0.551

$0.148^{+0.005}$

0
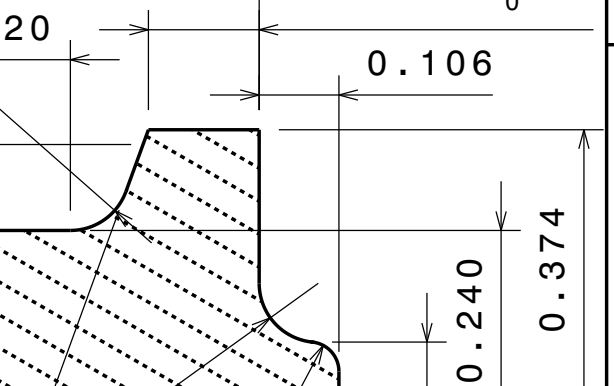

R 0.039

\begin{tabular}{|c|c|c|c|c|c|c|c|}
\hline \multirow{4}{*}{\multicolumn{2}{|c|}{$\begin{array}{l}\text { DESIGNED BY: } \\
\text { Chanon Pretorius } \\
\text { EMAIL: } \\
\text { chanonpretorius@email.carleton.ca } \\
\text { PHONE: } 613-462-5243 \\
\quad 613-46\end{array}$}} & \multirow{3}{*}{\multicolumn{4}{|c|}{ Upstream Flange }} & $\mathrm{H}$ & - \\
\hline & & & & & & $G$ & - \\
\hline & & & & & & $F$ & - \\
\hline & & \multirow{2}{*}{\multicolumn{4}{|c|}{ CARLETON UNIVERSITY }} & E & - \\
\hline A4 & 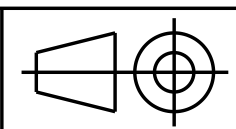 & & & & & $D$ & - \\
\hline SCALE & $\begin{array}{ll}\text { DIMENSIONS } & 1 \\
\end{array}$ & TOLERANCES & DRAWING NUMBER & MATERIAL & SHEET & C & $2019-08-02$ \\
\hline $1: 3$ & inches & $\begin{array}{l}+/-0.005 \text { unless otherwise } \\
\text { specified }\end{array}$ & 1 & Mild Steel & $1 / 1$ & $B$ & 2019-07-03 \\
\hline
\end{tabular}



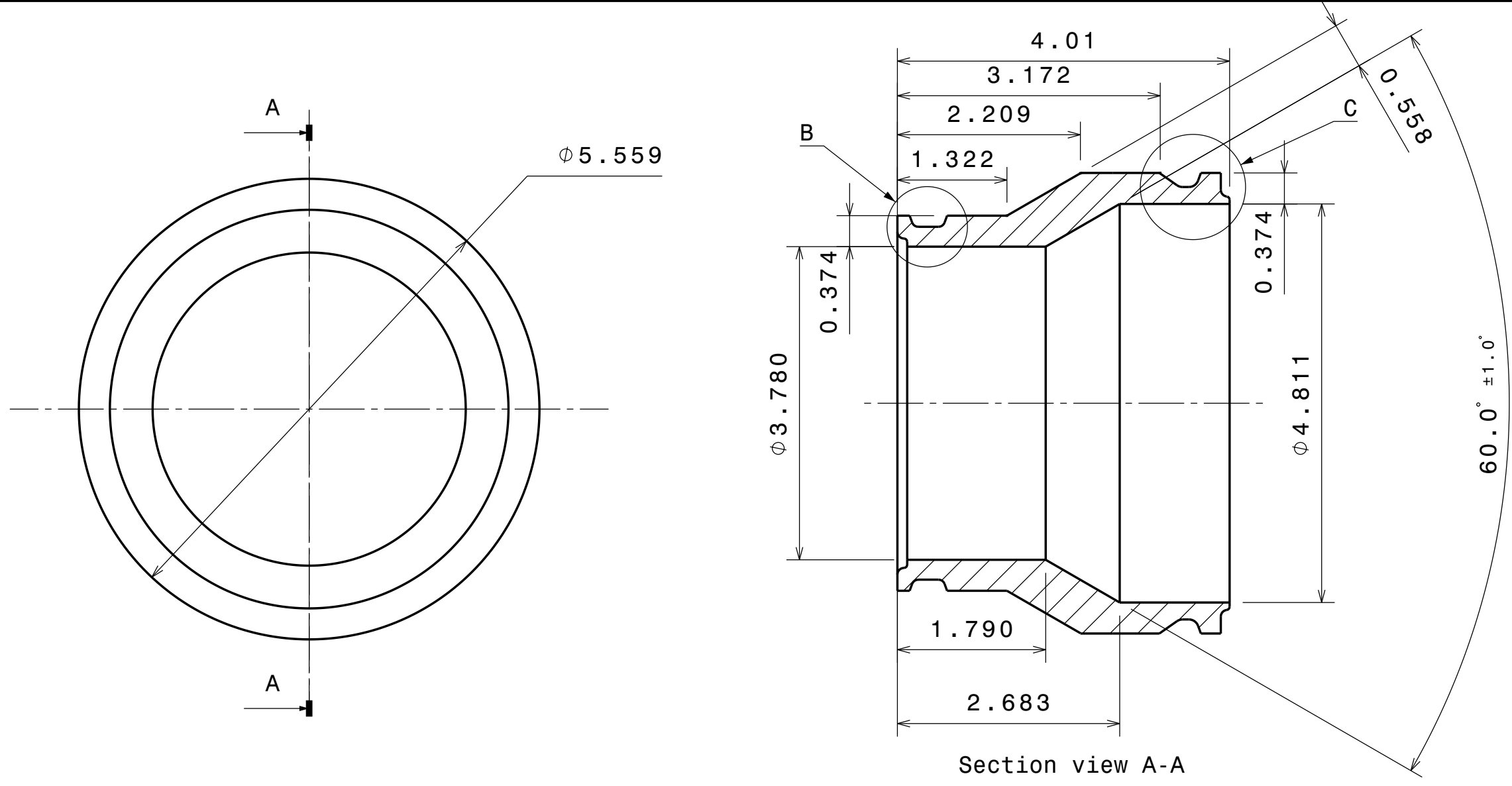

\begin{tabular}{|c|c|c|c|c|c|c|c|}
\hline DESIGNED BY: & & & & & & $\mathrm{H}$ & _ \\
\hline $\begin{array}{l}\text { Chanon } \\
\text { EmaLL: }\end{array}$ & Pretorius & & xpansic & n Section & & G & 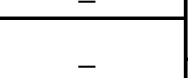 \\
\hline $\begin{array}{l}\text { Chanonppretor } \\
\text { PHowe: } 613\end{array}$ & secmaii.ccarleton.ca & & & & & $\mathrm{F}$ & - \\
\hline & & & & & & $E$ & - \\
\hline A4 & $\Leftarrow(\emptyset)$ & CA & ETON & UNIVERSI & & $\mathrm{D}$ & - \\
\hline $\begin{array}{ll}\text { SCALE } \\
\end{array}$ & $\begin{array}{l}\text { DIMENSIONS } \\
\end{array}$ & TOLERANGES & $\begin{array}{l}\text { DAAWING NUMBER } \\
\end{array}$ & MATERIAL & & c & - \\
\hline $1: 2$ & inches & $\begin{array}{l}+1-0.005 \text { in unless } \\
\text { otherwise specifified }\end{array}$ & 2 & Mild Steel & $1 / 2$ & $B$ & 2019-08-02 \\
\hline & 1 & 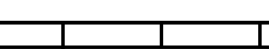 & 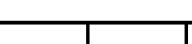 & & & & \\
\hline
\end{tabular}




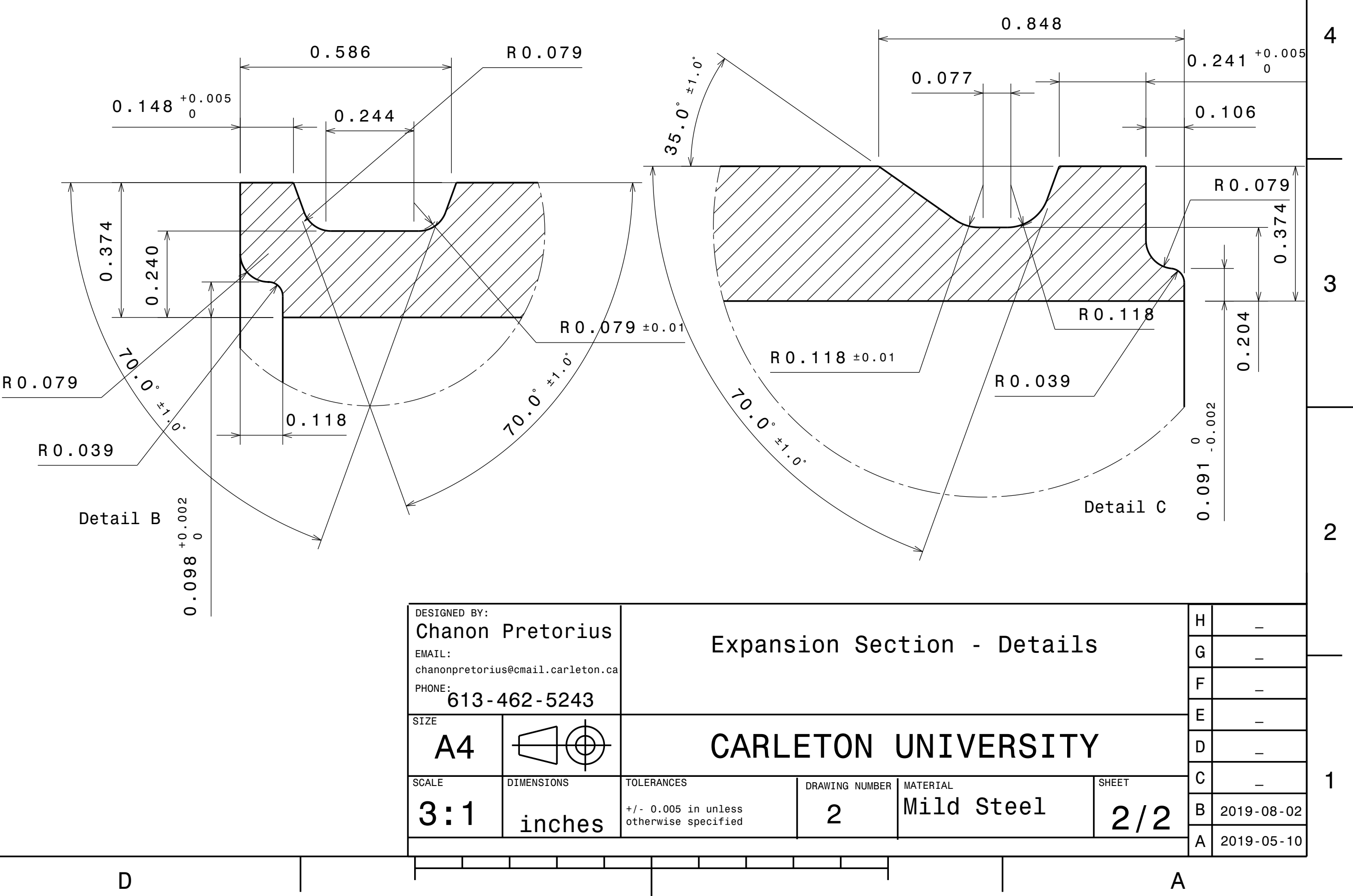


$\checkmark$

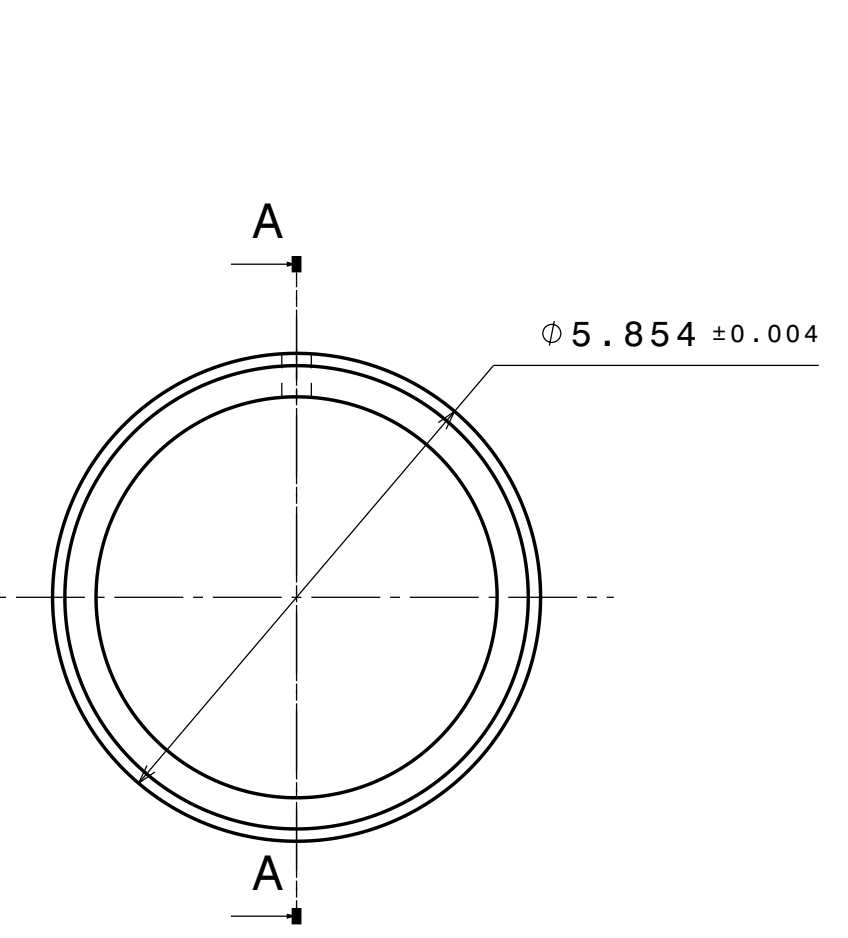

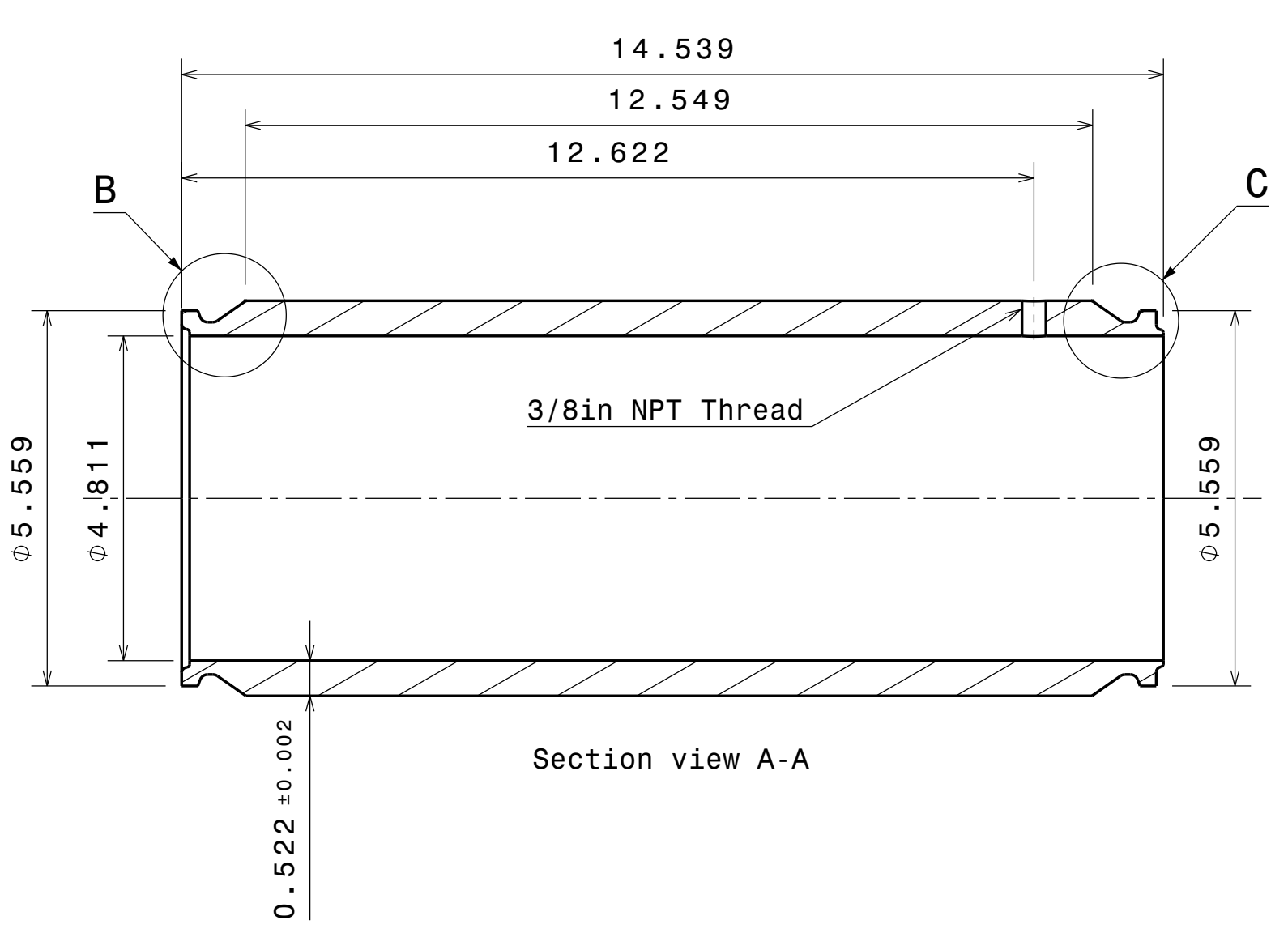

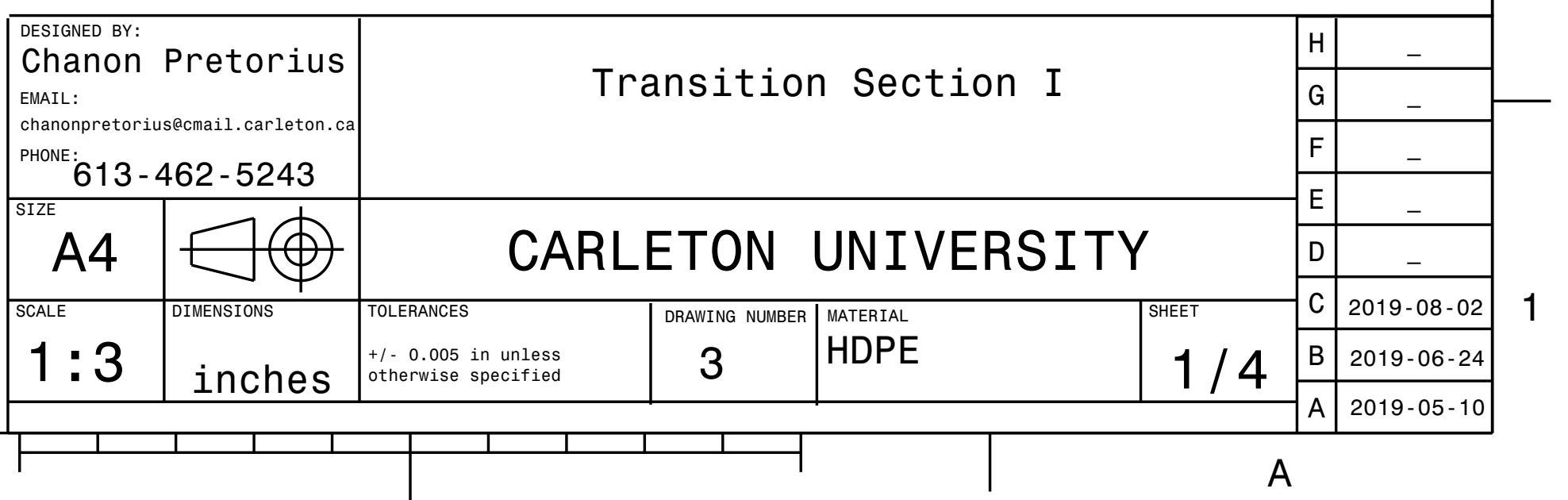




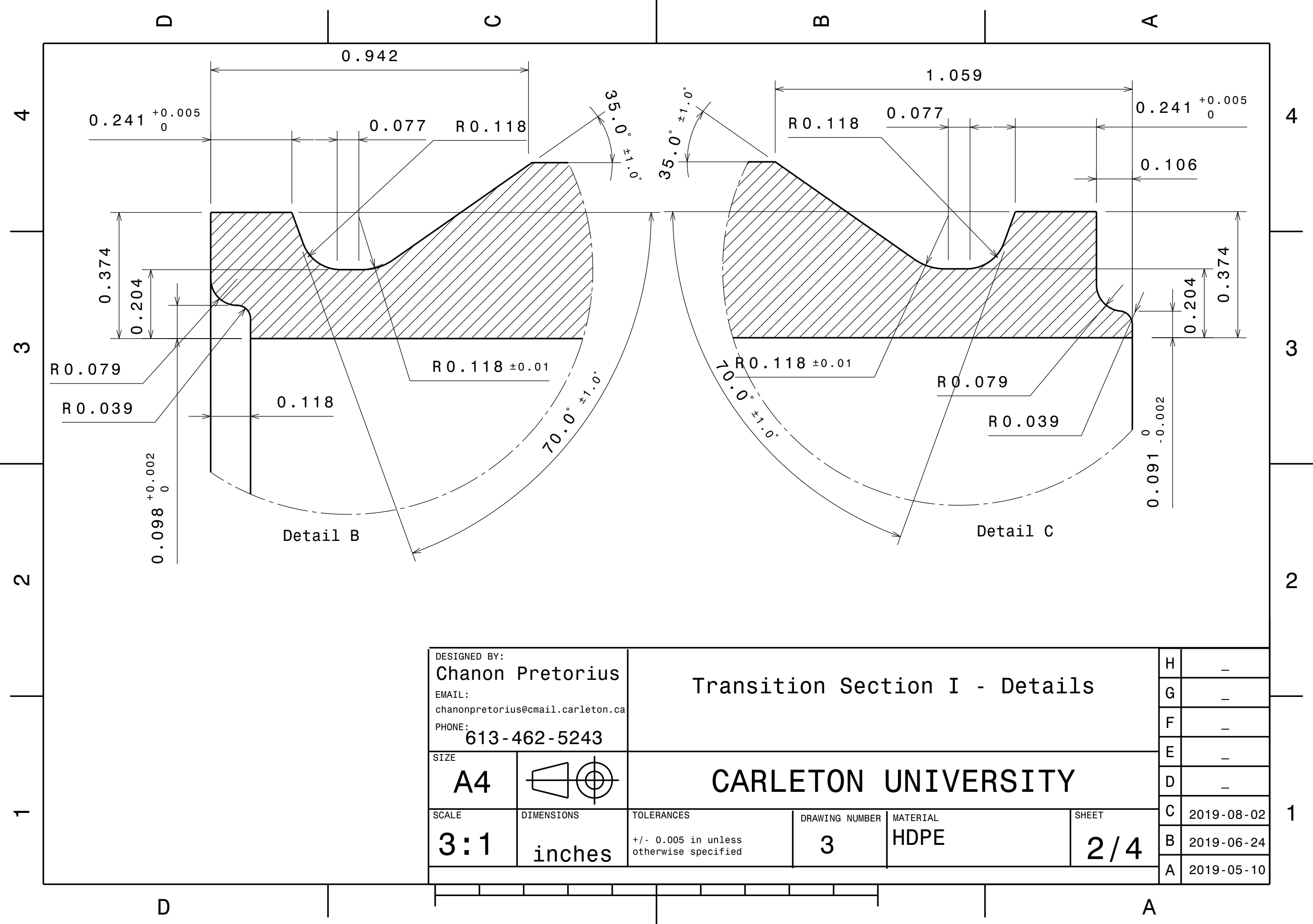



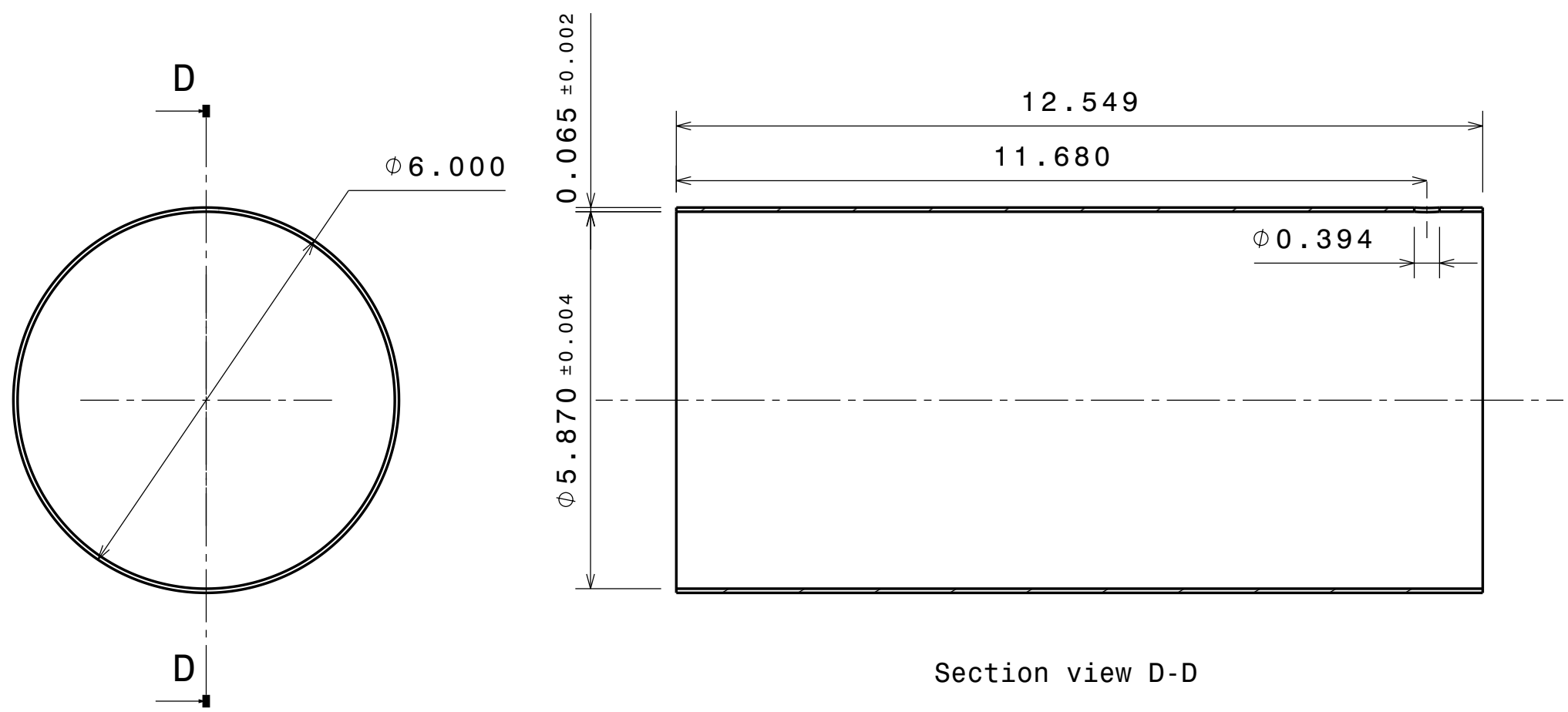

Section view D-D

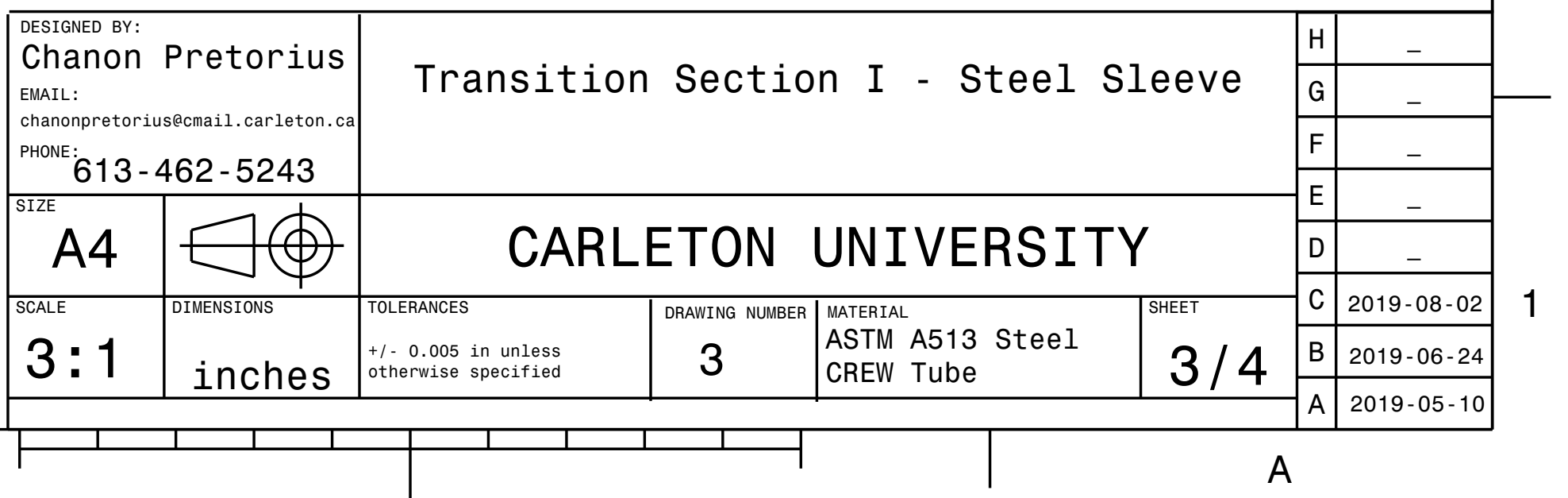




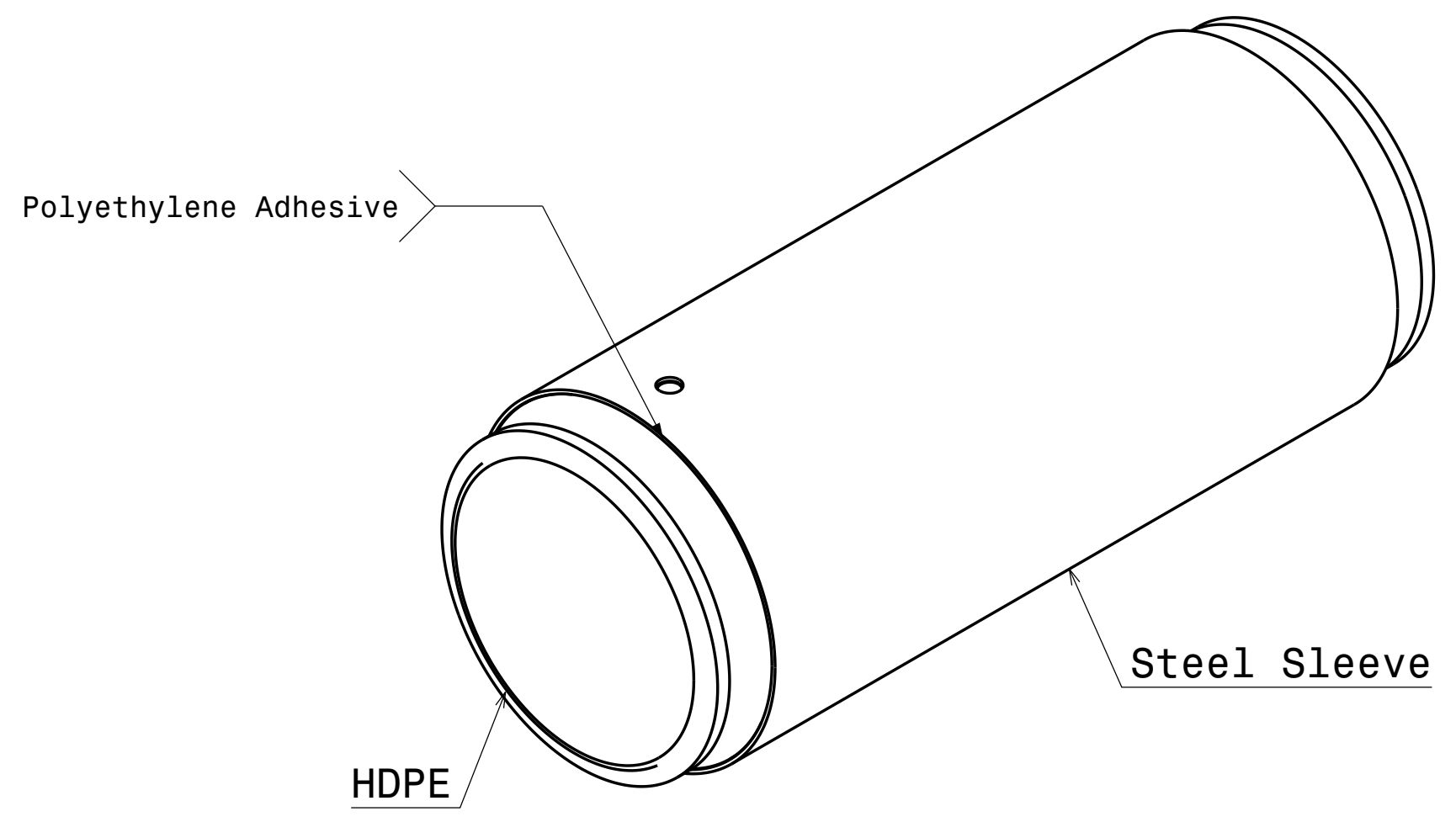

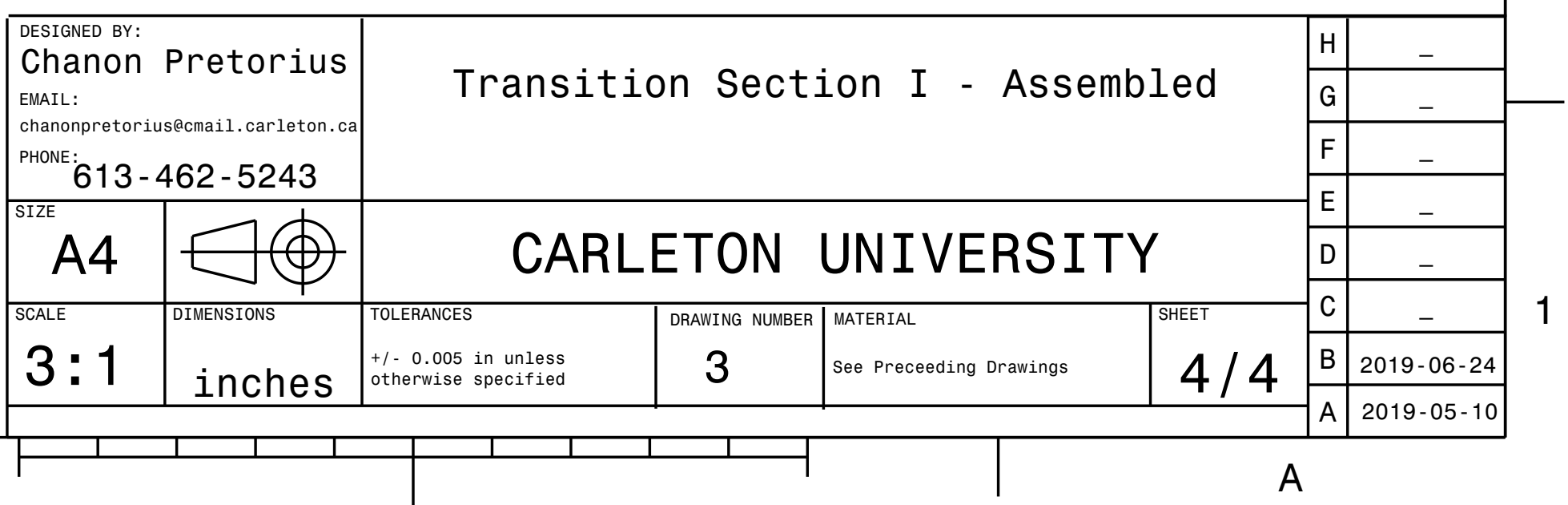


口

0

$\infty$ 

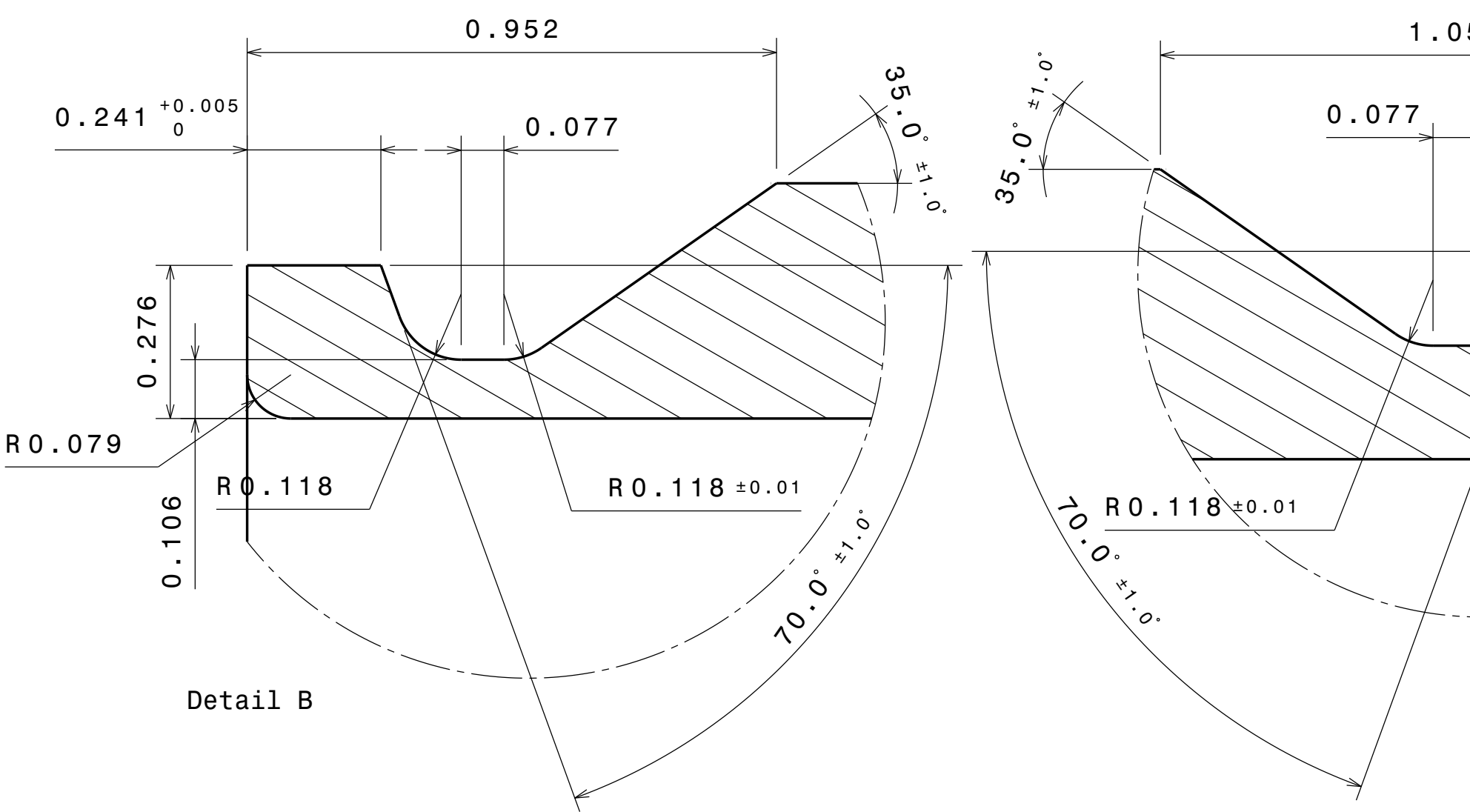

1. 059

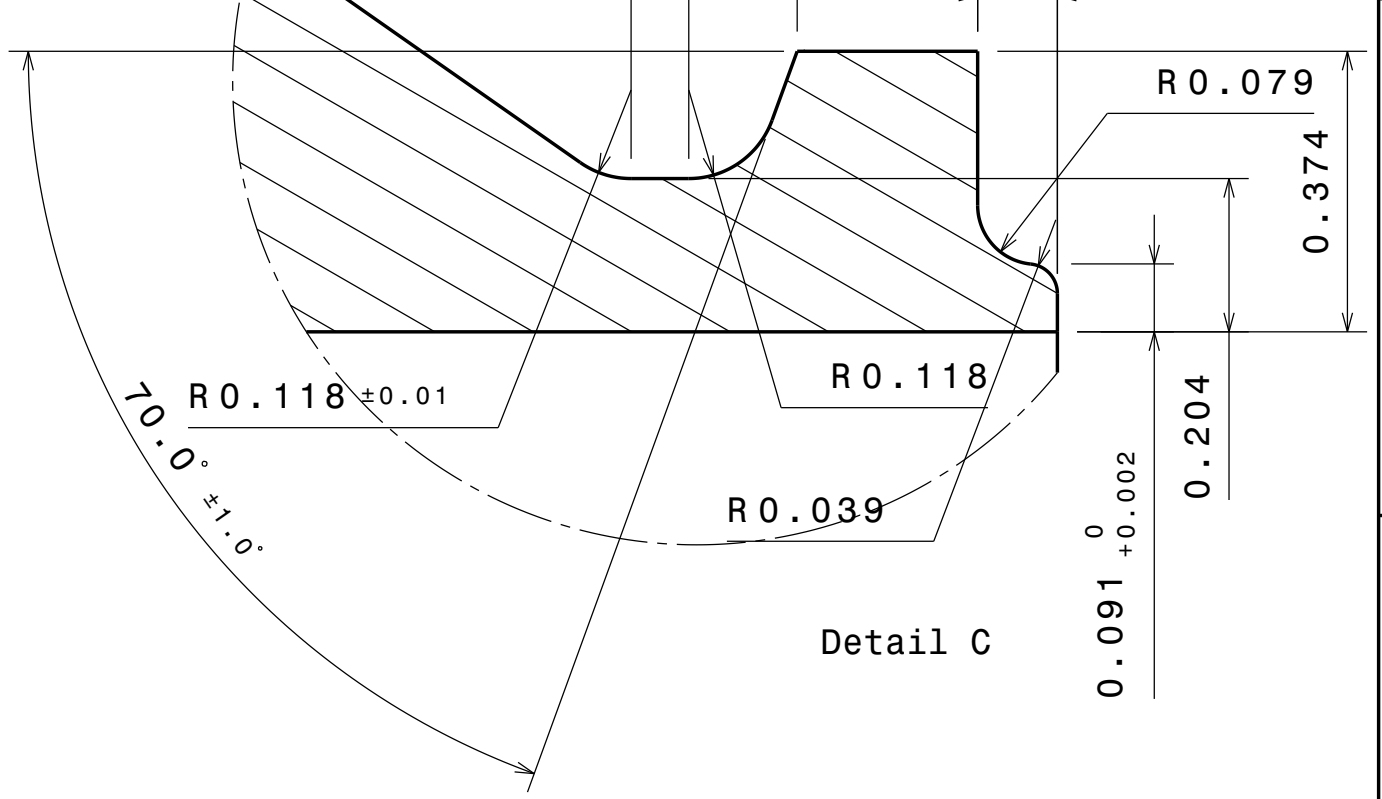

\begin{tabular}{|c|c|c|c|c|c|c|c|}
\hline \multirow{4}{*}{\multicolumn{2}{|c|}{$\begin{array}{l}\text { DESIGNED BY: } \\
\text { Chanon Pretorius } \\
\text { EMAIL: } \\
\text { chanonpretorius@email.carleton.ca } \\
\text { PHONE: } 613-462-5243 \\
\quad 613-462\end{array}$}} & \multirow{3}{*}{\multicolumn{4}{|c|}{ Flow Straightener Housing - Details }} & $\mathrm{H}$ & - \\
\hline & & & & & & G & - \\
\hline & & & & & & $\mathrm{F}$ & - \\
\hline & \multirow[b]{2}{*}{$E($} & \multirow{2}{*}{\multicolumn{4}{|c|}{ CARLETON UNIVERSITY }} & E & - \\
\hline A4 & & & & & & $\mathrm{D}$ & - \\
\hline \multirow{2}{*}{$3: 1$} & \multirow{2}{*}{$\begin{array}{l}\text { DIMENSIONS } \\
\text { inches }\end{array}$} & \multirow{2}{*}{$\begin{array}{l}\text { TOLERANCES } \\
+/-0.005 \text { in unless } \\
\text { otherwise specified } \\
\end{array}$} & DRAWING NUMBER & \multirow{2}{*}{ HATERIAL } & SHEET & C & 2019-08-02 \\
\hline & & & 4 & & $2 / 4$ & B & $2019-07-03$ \\
\hline \multicolumn{5}{|c|}{\begin{tabular}{l|l|l|l|l|l|l|l|l} 
& 1 & 1 & &
\end{tabular}} & & A & $2019-05-10$ \\
\hline
\end{tabular}



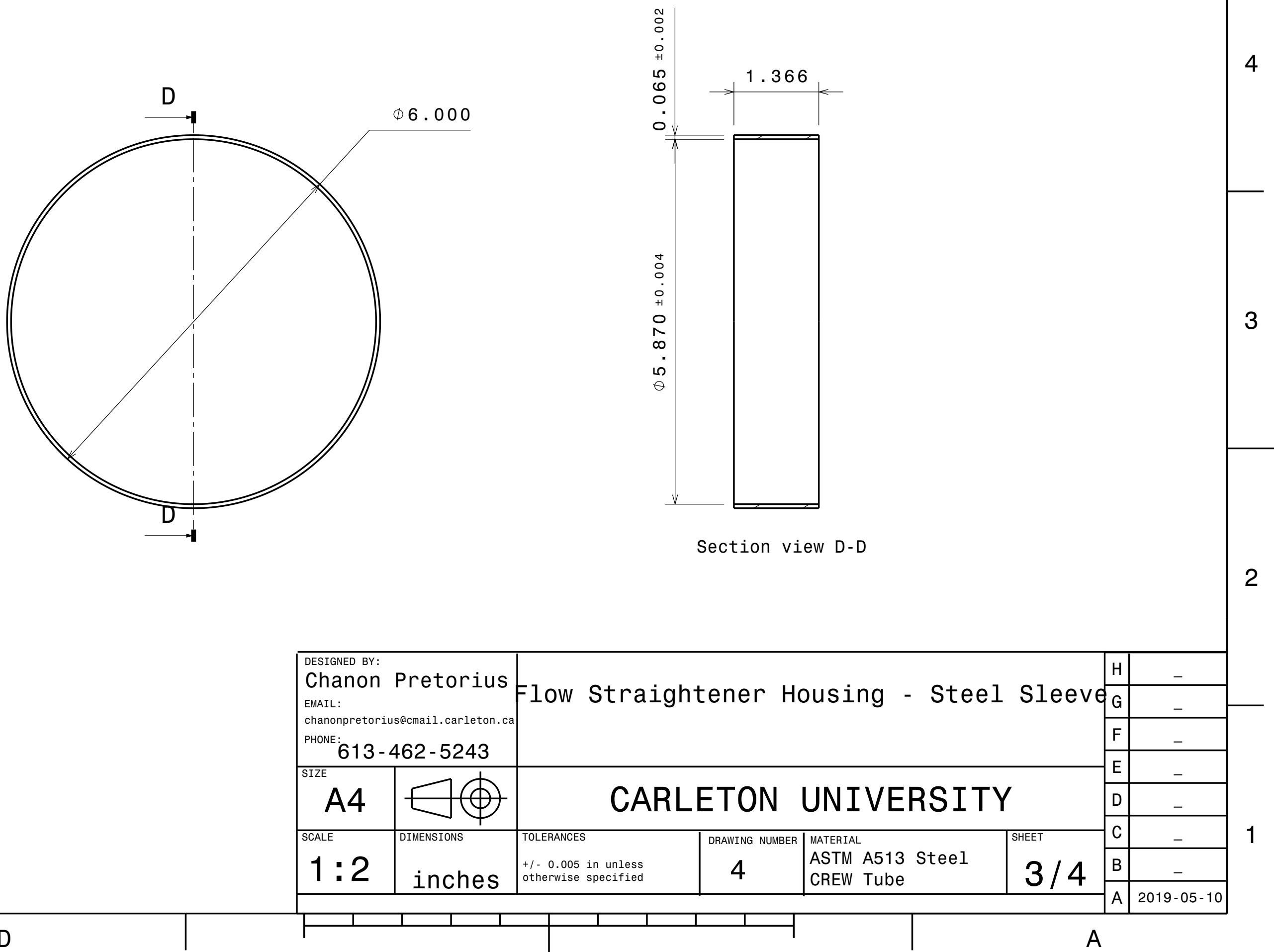
Polyethylene Adhesive

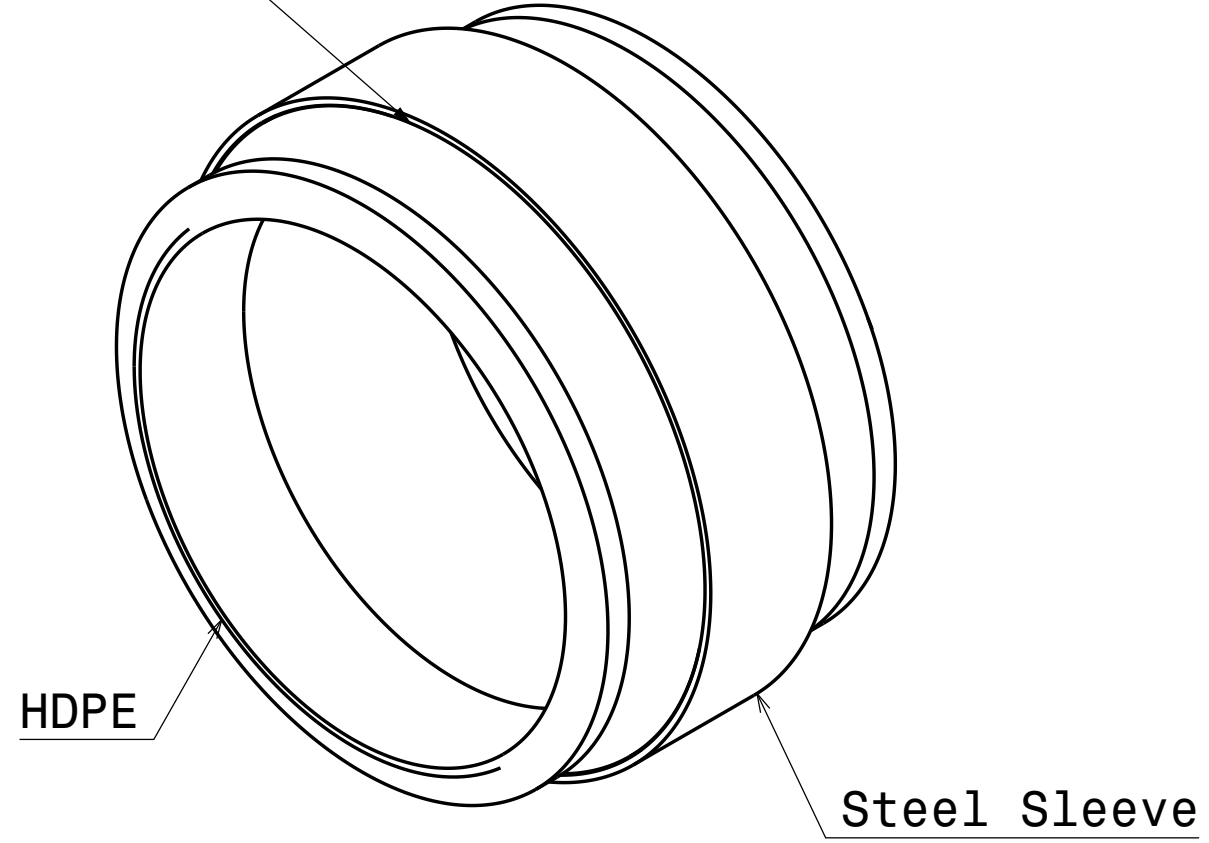

\begin{tabular}{|c|c|c|c|c|c|c|c|}
\hline \multirow{3}{*}{\multicolumn{2}{|c|}{\begin{tabular}{|l|} 
DESIGENED BY: \\
Chanon Pretorius \\
EMALL: \\
EManonpretoriusecmail.carleton.ca \\
PHONE: $613-462-5243$ \\
SIZE
\end{tabular}}} & \multirow{3}{*}{\multicolumn{4}{|c|}{ Flow Straightener Assembly }} & $\mathrm{H}$ & - \\
\hline & & & & & & G & - \\
\hline & & & & & & $\mathrm{F}$ & - \\
\hline A4 & $\square \phi$ & \multicolumn{4}{|c|}{ CARLETON UNIVERSITY } & \begin{tabular}{|l|l|}
$D$ \\
$D$
\end{tabular} & - \\
\hline \begin{tabular}{|l|l|l} 
SCALE \\
\end{tabular} & DIMENSIONS & TOLERANCES & $\begin{array}{l}\text { DRAWING NUMBER } \\
\end{array}$ & | MATERIAL & & C & - \\
\hline $1: 2$ & inches & $\begin{array}{l}+1-0.005 \text { in unless } \\
\text { otherwise specified }\end{array}$ & 4 & See Preceeding Drawings & $4 / 4$ & $B$ & - \\
\hline & & 1 & 7 & & & A & 2019-05-10 \\
\hline
\end{tabular}


口

0

$\infty$

$\varangle$ 


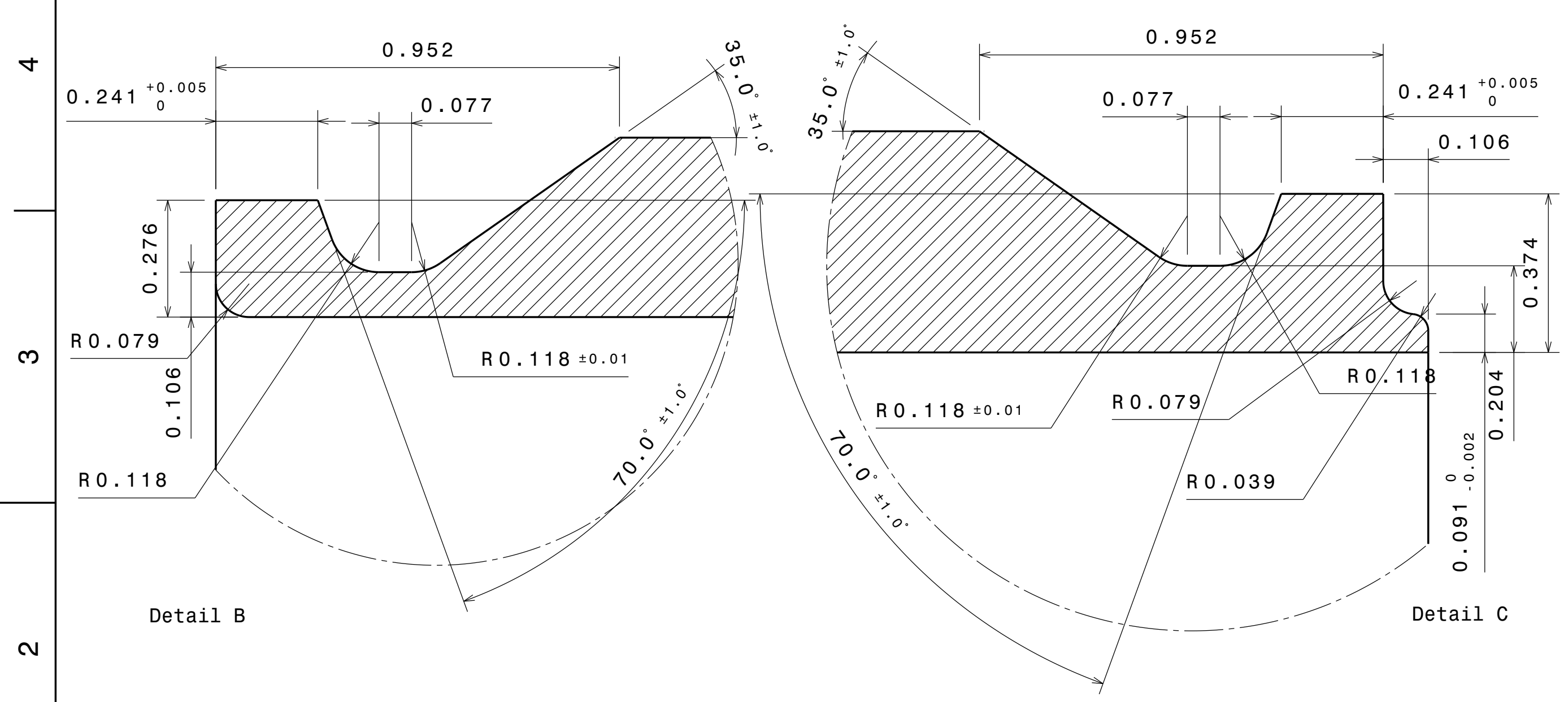



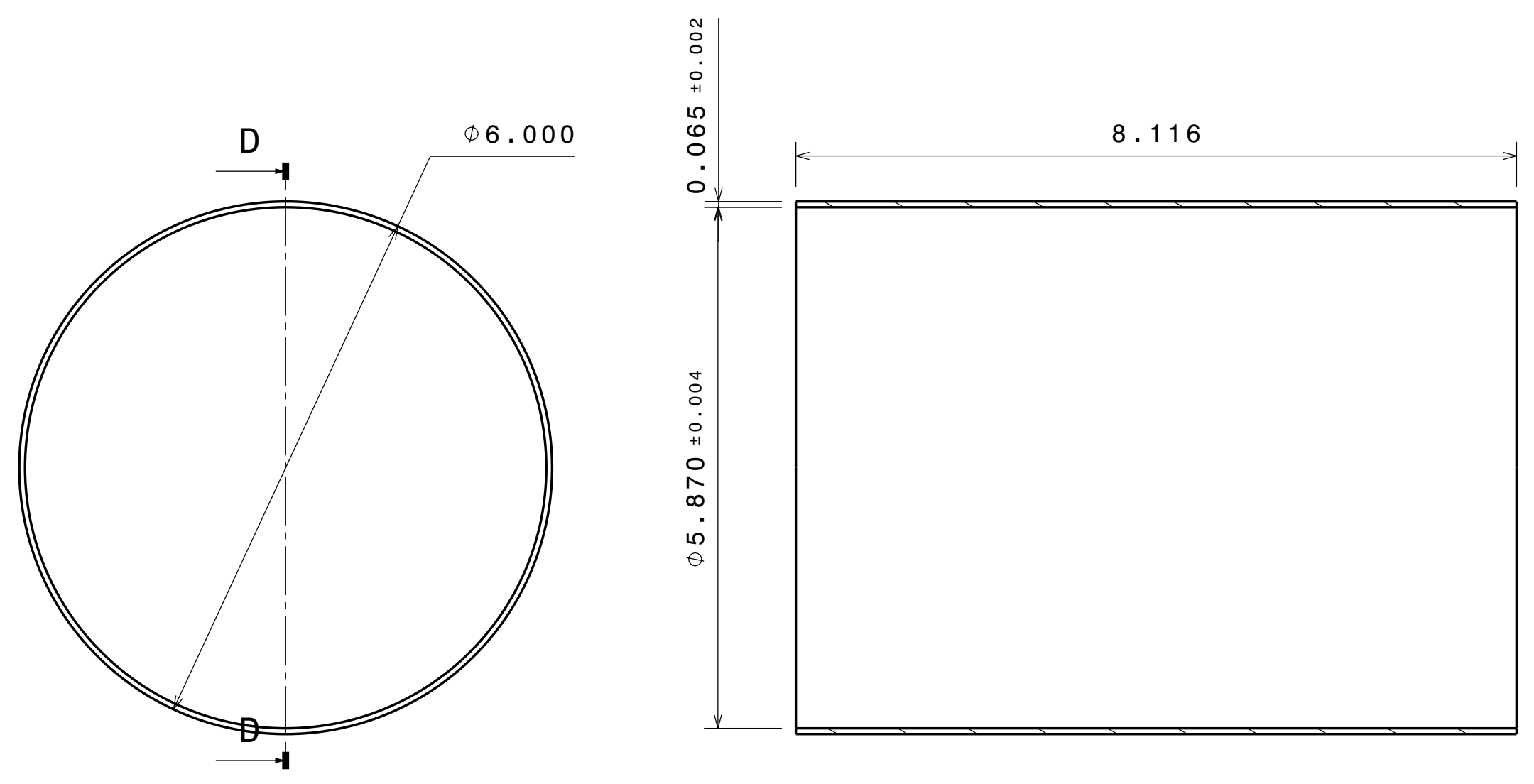

Section view D-D

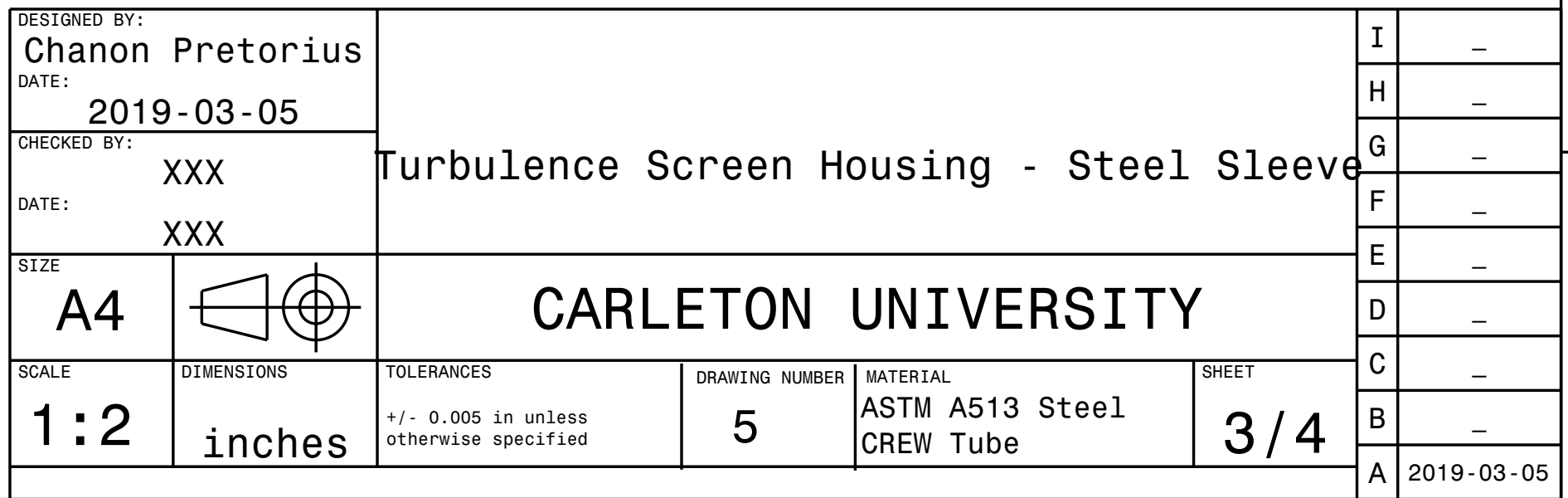




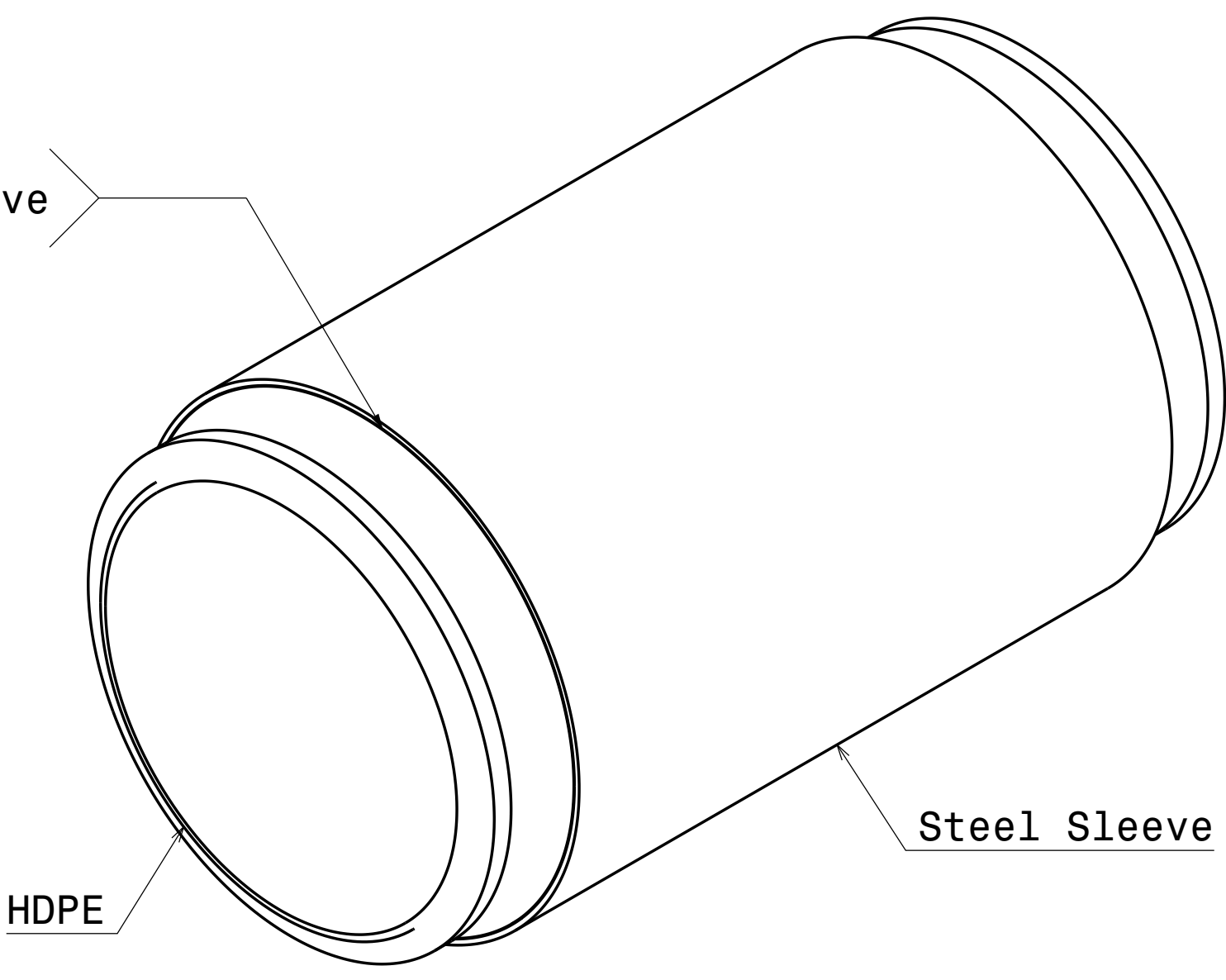

\begin{tabular}{|c|c|c|c|c|c|c|c|}
\hline $\begin{array}{l}\text { DESIGGNED BY: } \\
\text { Chanon }\end{array}$ & Pretorius & & & & & $\mathrm{H}$ & - \\
\hline $\begin{array}{l}\text { EMAIL: } \\
\text { chanonpretor }\end{array}$ & secmail.carleton.ca & Tur & ence Ho & using Assem & & $G$ & - \\
\hline PHONE: 613. & $62-5243$ & & & & & $\mathrm{~F}$ & - \\
\hline A4 & 7 (क) & CA & $=T 0 N$ & INTVFRS & & D & - \\
\hline$\overline{S C A L E}$ & DIMENSIONS & $\begin{array}{l}\text { TOLERANCES } \\
\end{array}$ & $\begin{array}{l}\text { DRAWING NUMBER } \\
\end{array}$ & |MATERTAL & SHEET & c & - \\
\hline $1: 2$ & inches & 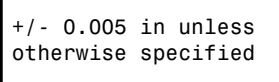 & 5 & See Preceeding Drawings & $4 / 4$ & B & - \\
\hline & & 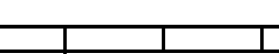 & $\bar{T}-1$ & & & A & 2019-05-10 \\
\hline
\end{tabular}



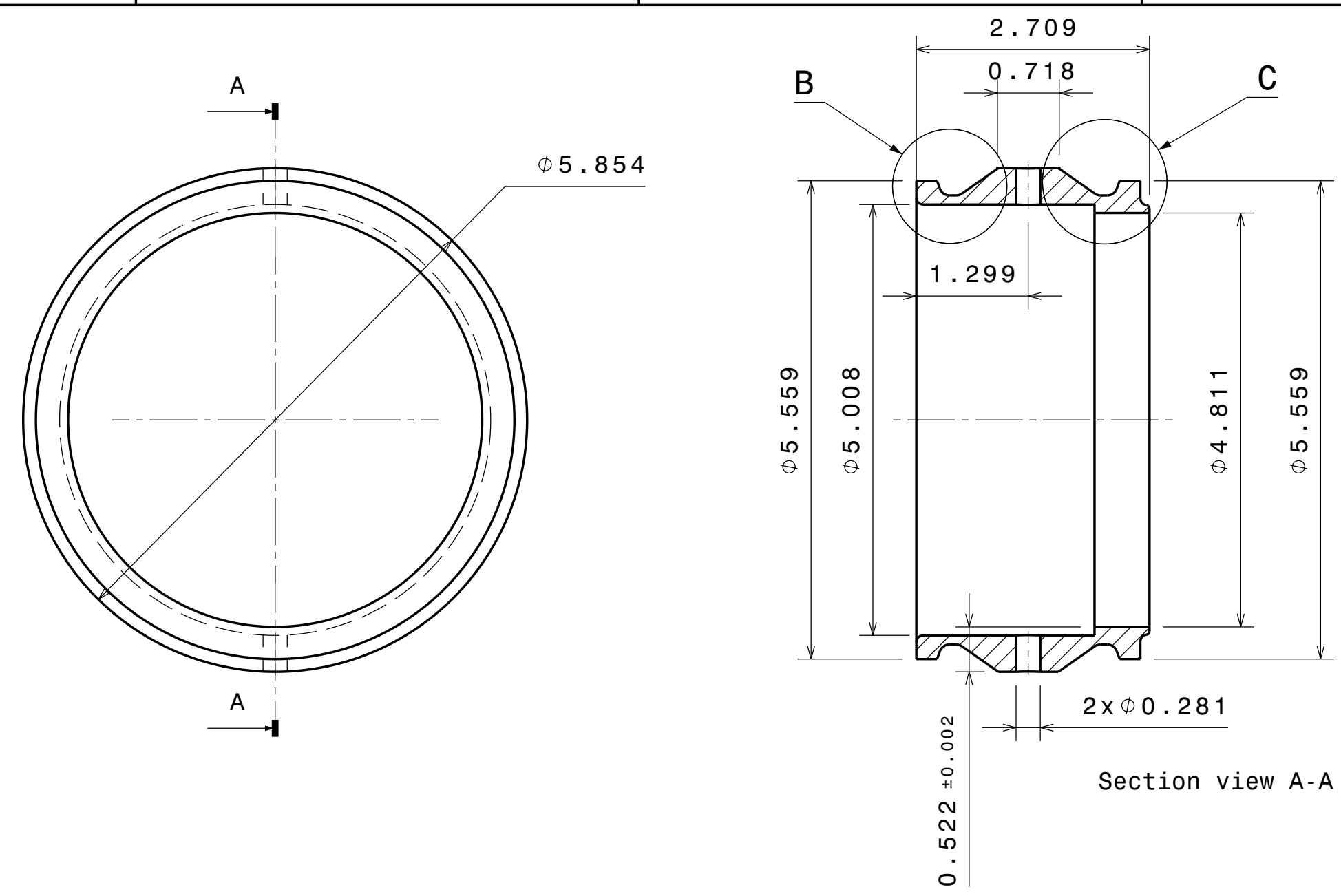

\begin{tabular}{|c|c|c|c|c|c|c|c|}
\hline \multirow{4}{*}{\multicolumn{2}{|c|}{$\begin{array}{l}\text { DESIGNED BY: } \\
\text { Chanon Pretorius } \\
\text { EMAIL: } \\
\text { chanonpretoriusecmail.carleton. ca } \\
\text { PHONEE } 613-462-5243 \\
\text { SIZE }\end{array}$}} & \multirow{4}{*}{\multicolumn{4}{|c|}{ Swirl Generator Housing }} & \multirow{3}{*}{\begin{tabular}{|l|}
$H$ \\
$G$ \\
$F$
\end{tabular}} & \multirow{2}{*}{ - } \\
\hline & & & & & & & \\
\hline & & & & & & & - \\
\hline & & & & & & $\mathrm{E}$ & - \\
\hline A4 & $\square(\emptyset)$ & CA & ETON & UNI & & $D$ & - \\
\hline$\overline{S C A L E}$ & DTweNS IONS & TOLERANCES & DRAWING NUMBER & 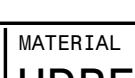 & & C & 2019-08-02 \\
\hline $1: 2$ & inches & $\begin{array}{l}+1,0.005 \text { in unless } \\
\text { otherwise specified }\end{array}$ & 6 & HDPE & $1 / 4$ & $B$ & $2019-05-29$ \\
\hline & $T$ & & 1 & & & $A$ & $2019-05-10$ \\
\hline
\end{tabular}




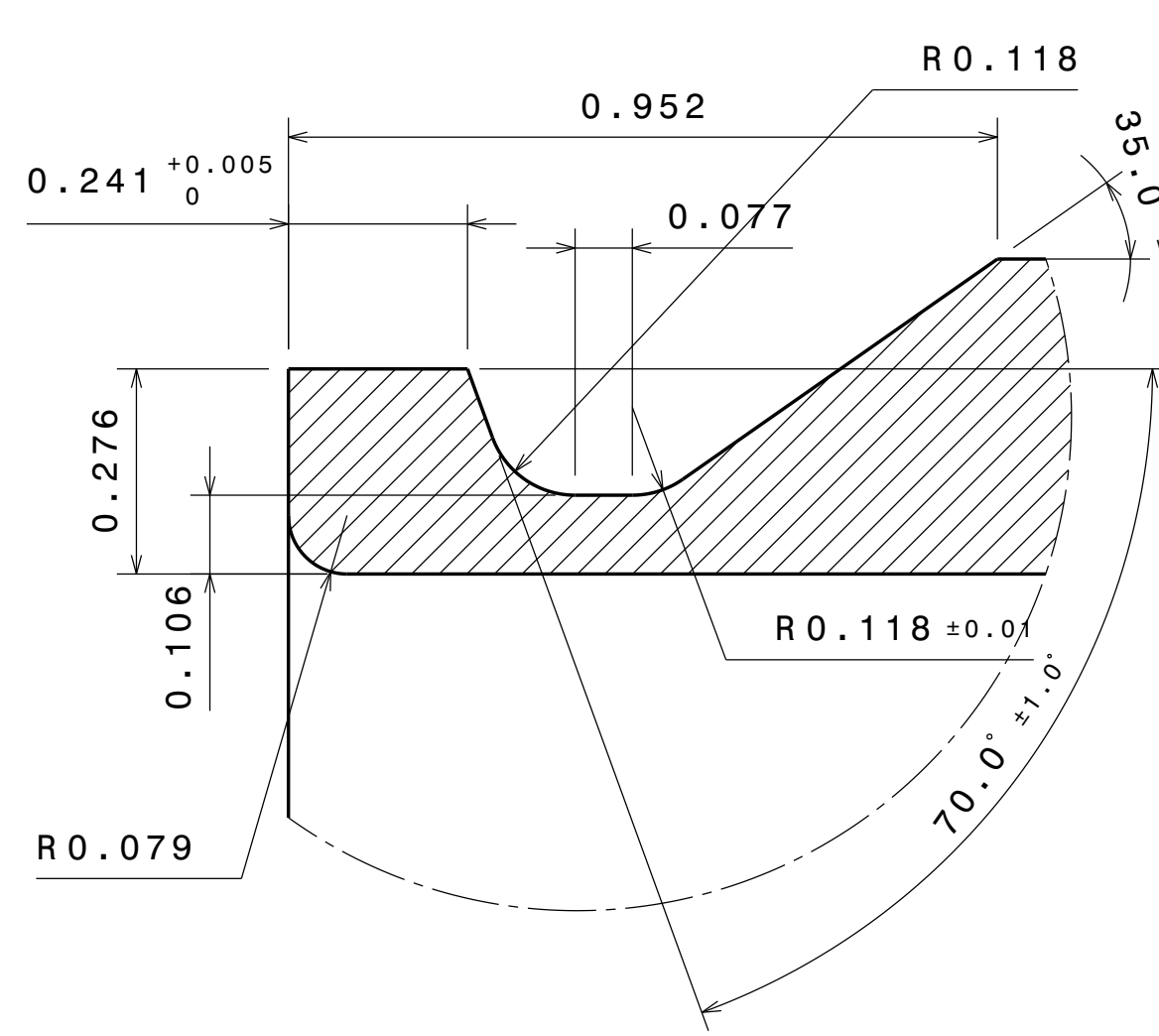

Detail B

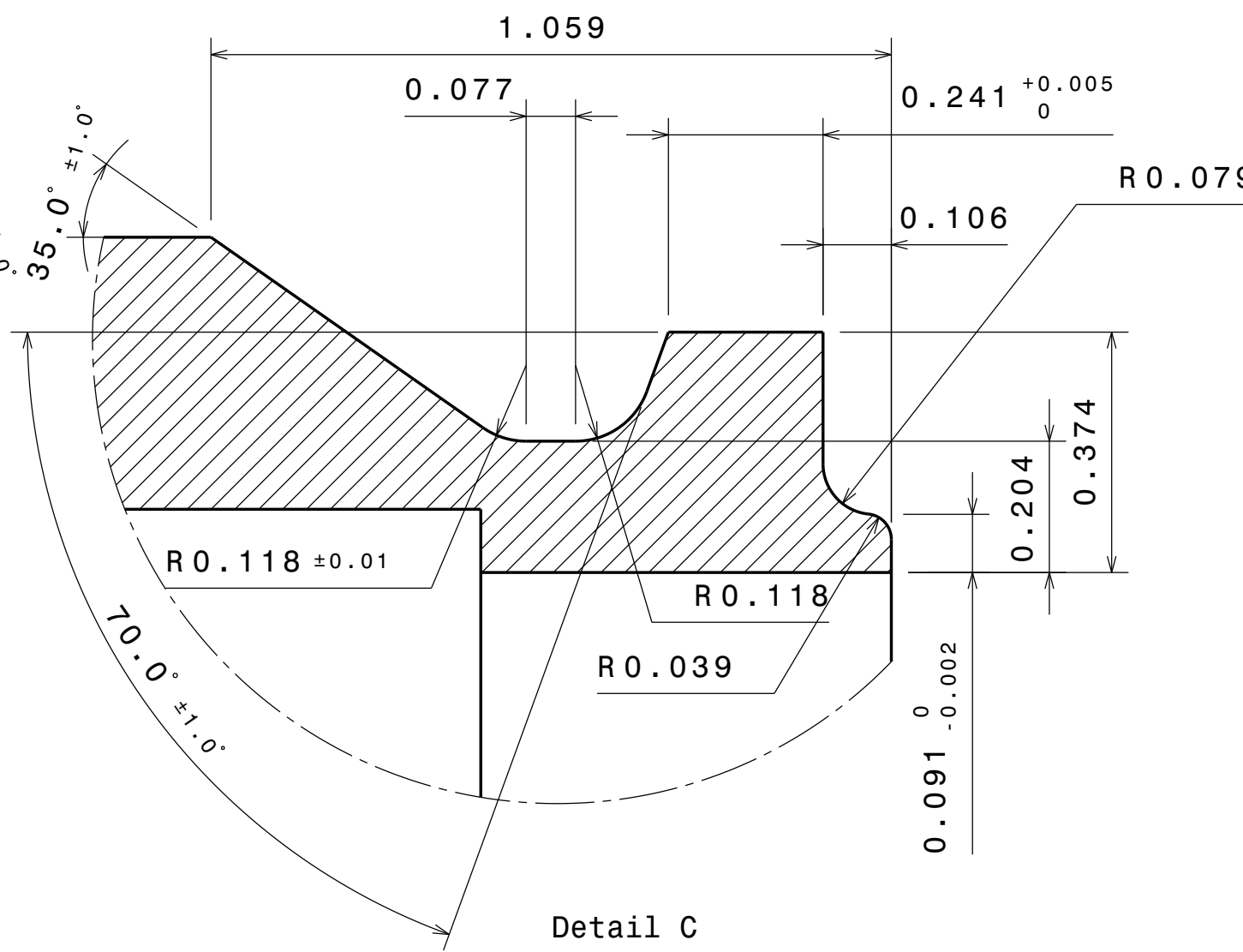

4

3

2

\begin{tabular}{|c|c|c|c|c|c|c|c|}
\hline \multirow{4}{*}{\multicolumn{2}{|c|}{$\begin{array}{l}\text { DESIGGED BY: } \\
\text { Chanon Pretorius } \\
\text { EMAIL: } \\
\text { chanonpretoriusecmail.carleton. ca } \\
\text { PHONE: } 613-462-5243 \\
\text { SIZE }\end{array}$}} & \multirow{4}{*}{\multicolumn{4}{|c|}{ Swirl Generator Housing - Details }} & $\mathrm{H}$ & - \\
\hline & & & & & & $G$ & - \\
\hline & & & & & & $F$ & - \\
\hline & & & & & & E & - \\
\hline A4 & $\Leftarrow(\oplus)$ & \multicolumn{2}{|c|}{ CARLETON } & \multicolumn{2}{|c|}{ UNIVERSITY } & $D$ & - \\
\hline$\overline{S C A L E}$ & DimENSIONS & TOLERANCES & $\begin{array}{l}\text { DRAWING NUMBER } \\
\end{array}$ & MATERIAL & & c & 2019-08-02 \\
\hline $3: 1$ & inches & $\begin{array}{l}+1-0.005 \text { in unless } \\
\text { otherwise specifified }\end{array}$ & 6 & HDPE & $2 / 4$ & $B$ & 2019-05-29 \\
\hline & \multicolumn{2}{|c|}{\begin{tabular}{l|l} 
A & $2019-05-10$ \\
\end{tabular}} \\
\hline
\end{tabular}


口

0

$\infty$

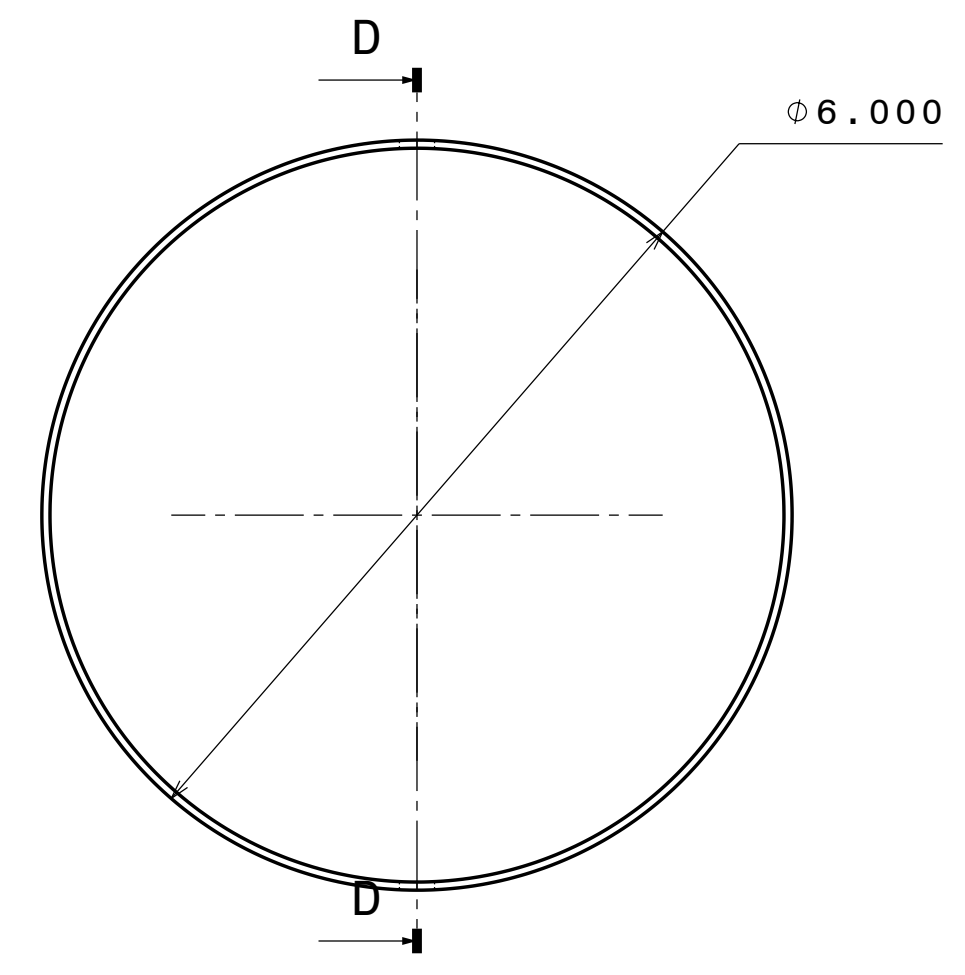

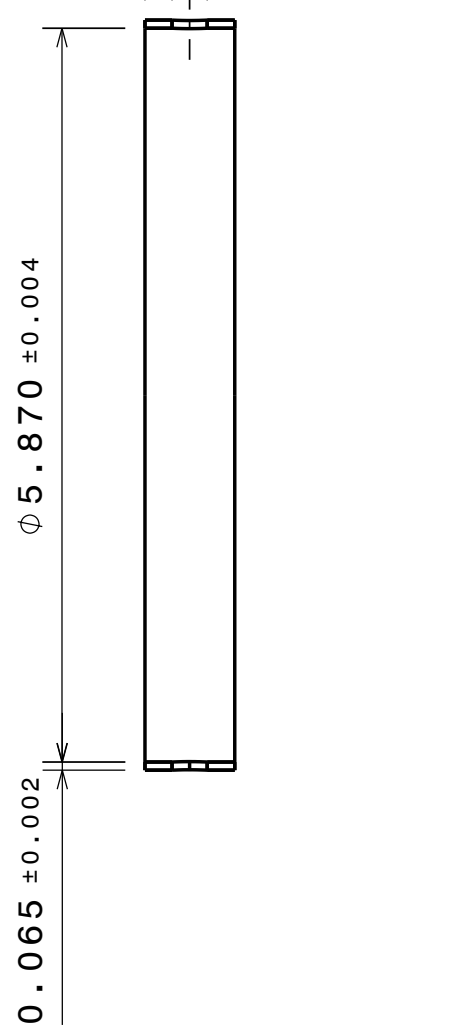

Section view D-D

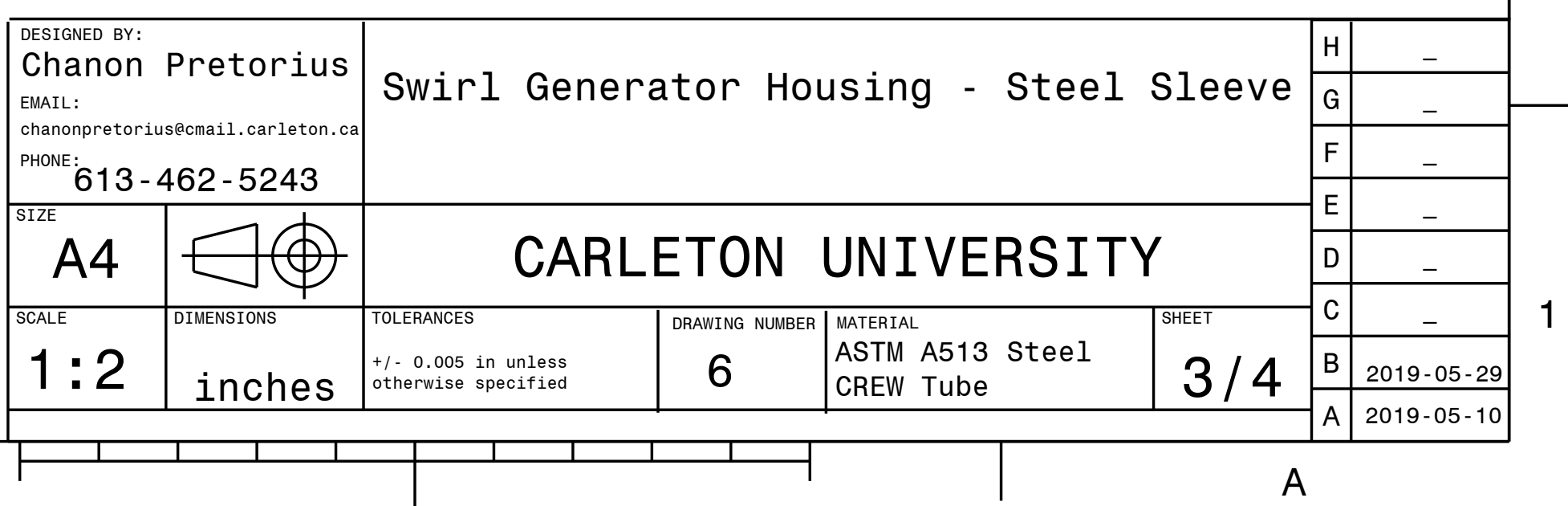


Polyethylene Adhesive

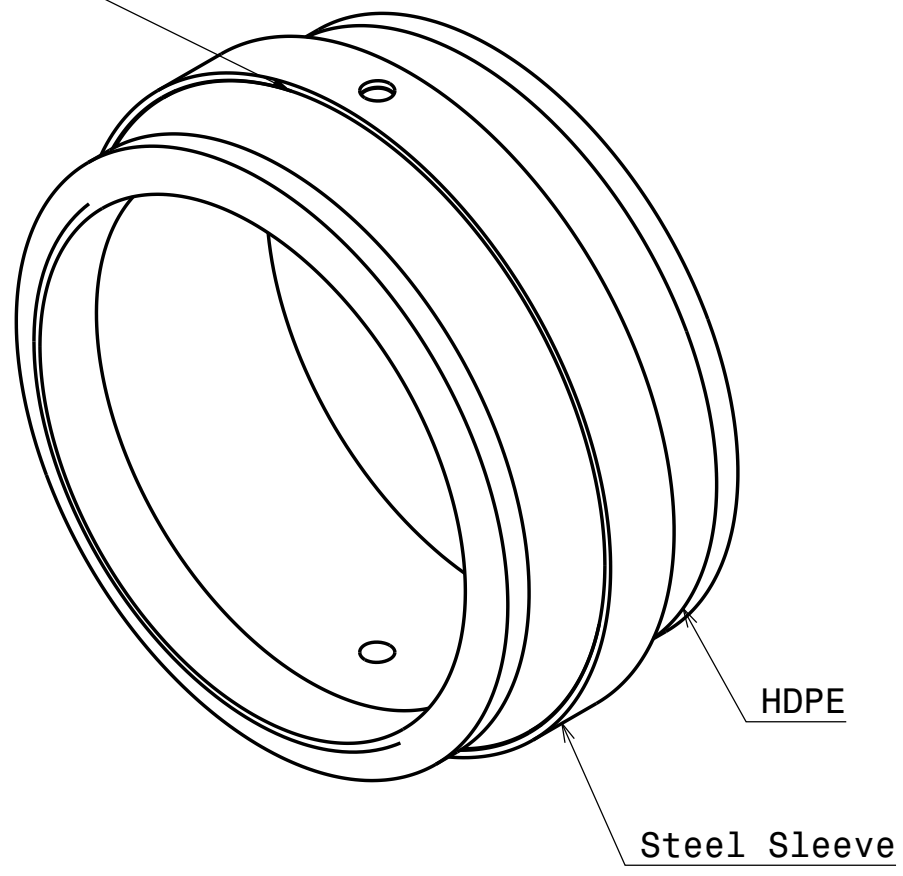

3

\begin{tabular}{|c|c|c|c|c|c|c|c|}
\hline \multirow{3}{*}{\multicolumn{2}{|c|}{$\begin{array}{l}\text { DESIGNED BY: } \\
\text { Chanon Pretorius } \\
\text { EMAIL: } \\
\text { chanonpretoriusecmail.carleton.ca } \\
\text { PHONE: } 613-462-5243 \\
\quad 613-46\end{array}$}} & \multirow{3}{*}{\multicolumn{4}{|c|}{ Swirl Generator Housing Assembly }} & $\mathrm{H}$ & - \\
\hline & & & & & & $\mathrm{G}$ & - \\
\hline & & & & & & $\mathrm{F}$ & - \\
\hline \multirow{2}{*}{$\mathrm{A} 4$} & \multirow[b]{2}{*}{ 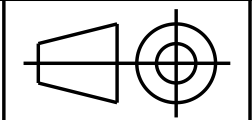 } & \multirow{2}{*}{\multicolumn{4}{|c|}{ CARLETON UNIVERSITY }} & $\mathrm{E}$ & - \\
\hline & & & & & & D & - \\
\hline \multirow{3}{*}{$1: 2$} & \multirow{3}{*}{$\begin{array}{l}\text { DIMENSIONS } \\
\text { inches }\end{array}$} & \multirow{2}{*}{$\begin{array}{l}\text { TOLERANCES } \\
+/-0.005 \text { in unless } \\
\text { otherwise specified } \\
\end{array}$} & DRAWING NUMBER & \multirow{3}{*}{\begin{tabular}{|l|} 
MATERIAL \\
See Preceding Drawings \\
\end{tabular}} & \multirow{3}{*}{$4 / 4$} & C & - \\
\hline & & & 6 & & & B & 2019-05-29 \\
\hline & & & & & & A & $2019-05-10$ \\
\hline
\end{tabular}


口

0 
口

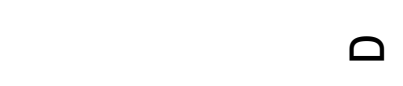

0

0.942

$\checkmark$

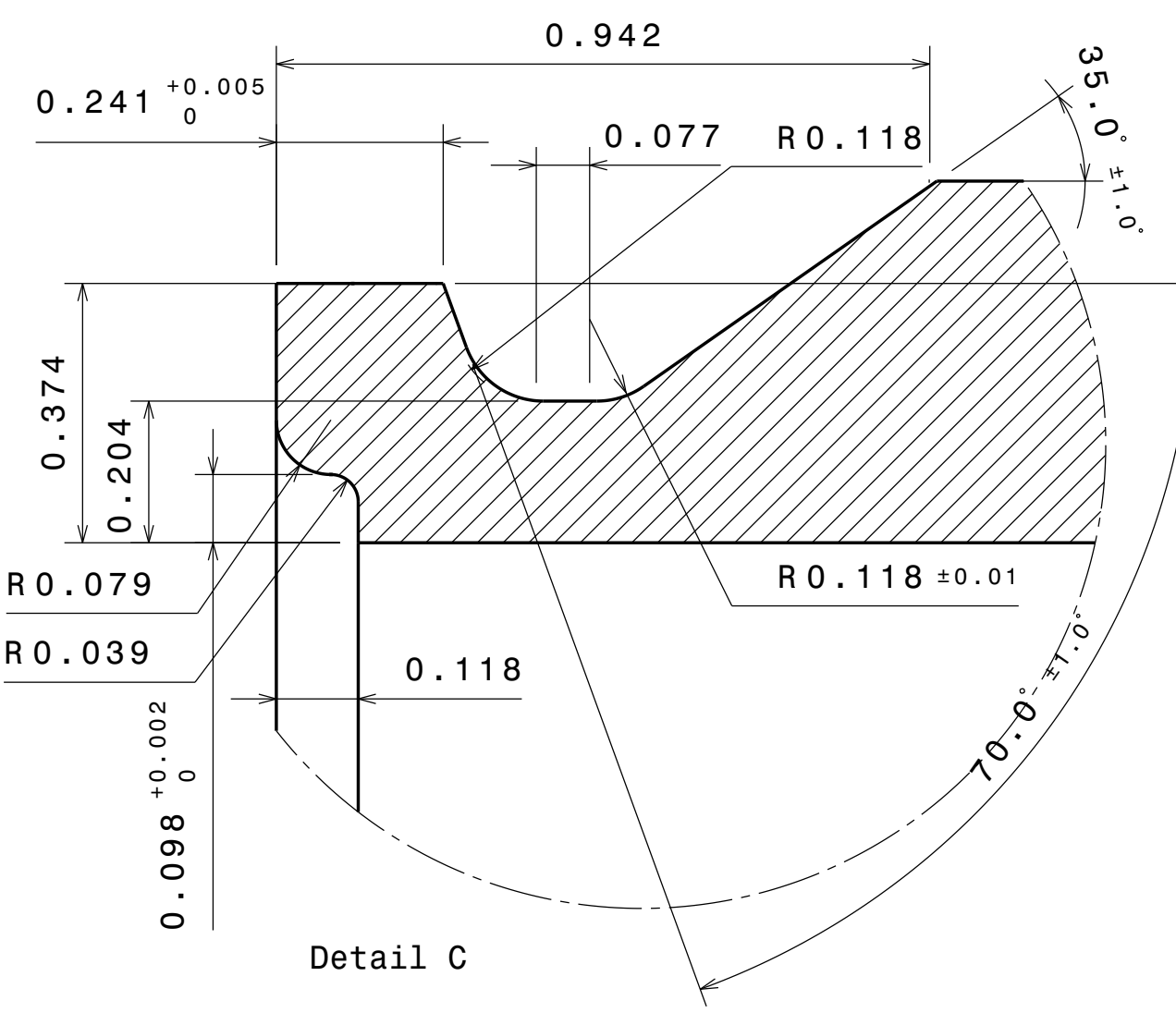

N

R 0.079 $+$ Detail C

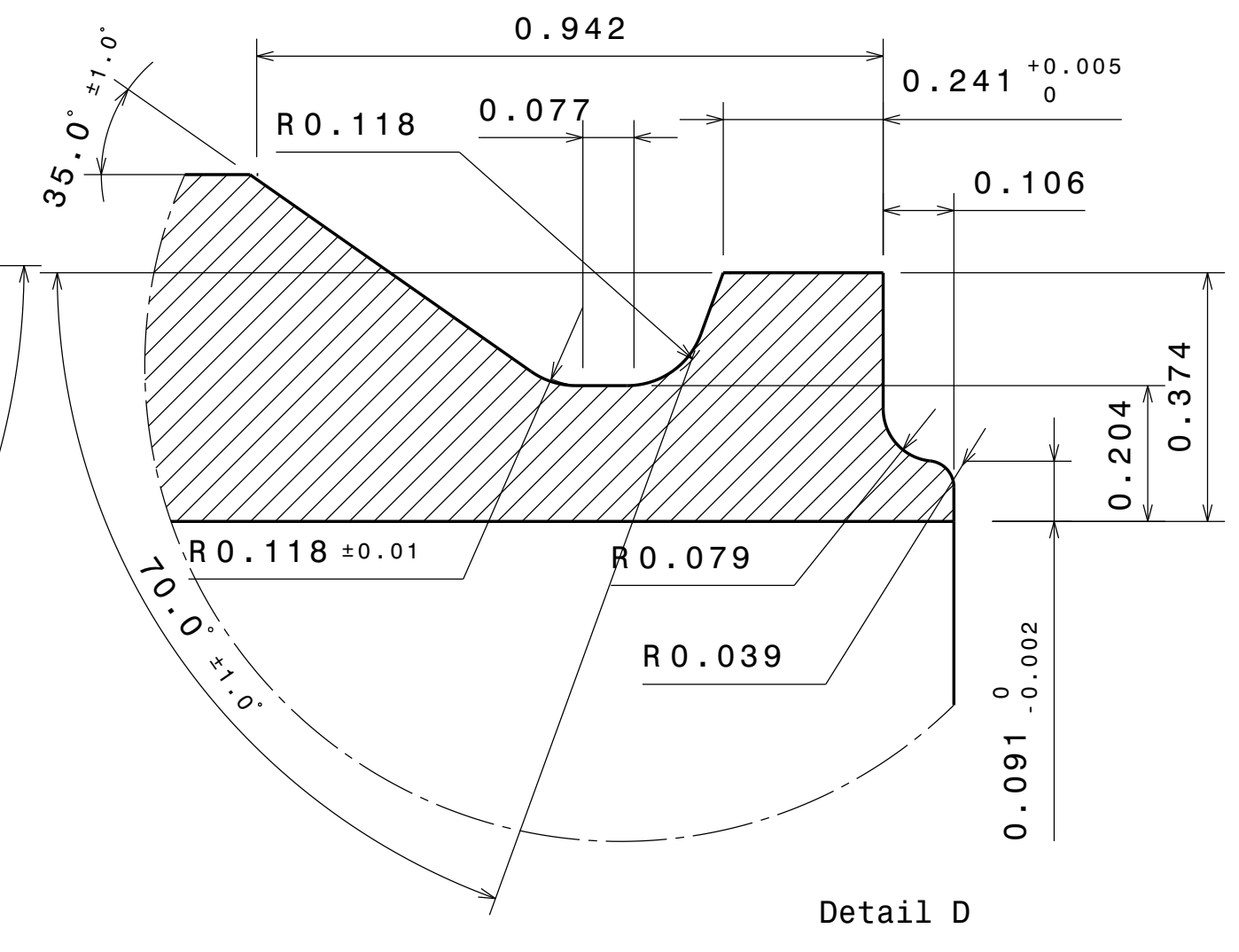

$m$

$\varangle$

2

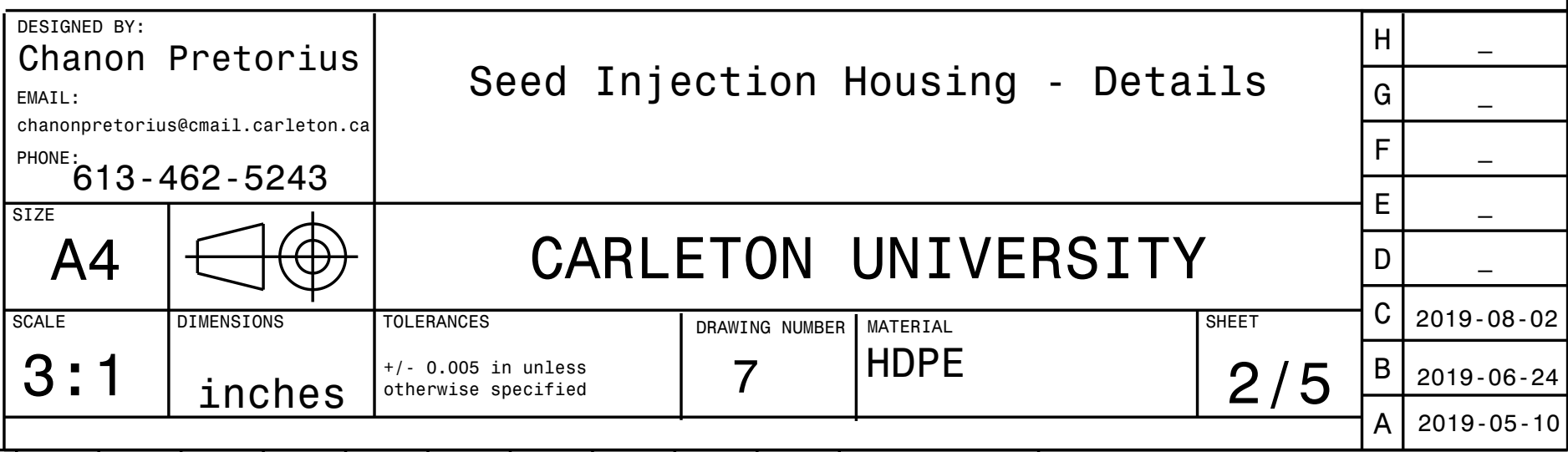



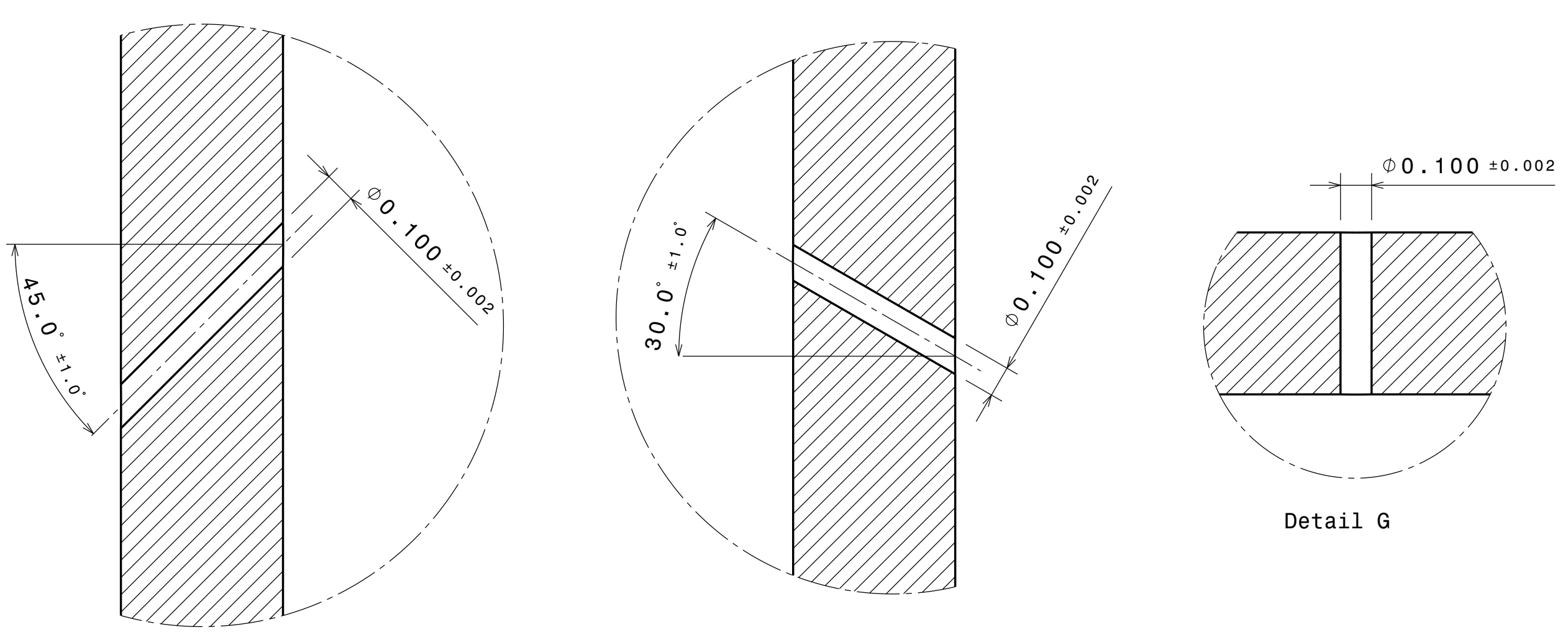

Detail G

Detail F

Detail E

\begin{tabular}{|c|c|c|c|c|c|c|c|}
\hline \multirow{3}{*}{\multicolumn{2}{|c|}{$\begin{array}{l}\text { DESIGNED BY: } \\
\text { Chanon Pretorius } \\
\text { EMAIL: } \\
\text { chanonpretorius@email.carleton.ca } \\
\text { PHONE: } 613-462-5243 \\
\quad 613-46\end{array}$}} & \multirow{3}{*}{\multicolumn{4}{|c|}{ Seed Injection Housing - Details 2}} & $\mathrm{H}$ & - \\
\hline & & & & & & G & - \\
\hline & & & & & & $\mathrm{F}$ & - \\
\hline \multirow{2}{*}{$\mathrm{A} 4$} & & \multirow{2}{*}{\multicolumn{4}{|c|}{ CARLETON UNIVERSITY }} & $\mathrm{E}$ & - \\
\hline & $\rightleftarrows(\emptyset)$ & & & & & D & - \\
\hline SCALE & \begin{tabular}{|l|l} 
DIMENSIONS \\
\end{tabular} & \multirow{2}{*}{$\begin{array}{l}\text { TOLERANCES } \\
+1-0.005 \text { in unless } \\
\text { otherwise specified }\end{array}$} & DRAWING NUMBER & \multirow{2}{*}{ 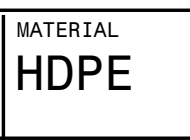 } & \multirow{2}{*}{\begin{tabular}{l|r} 
SHEET & $3 / 5$ \\
\end{tabular}} & $C$ & 2019-08-02 \\
\hline $2: 1$ & inches & & 7 & & & B & $2019-06-24$ \\
\hline \multicolumn{2}{|c|}{\begin{tabular}{|l|l|l|l|} 
& & & \\
\end{tabular}} & & 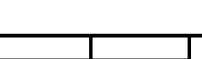 & & & A & $2019-05-10$ \\
\hline
\end{tabular}


口

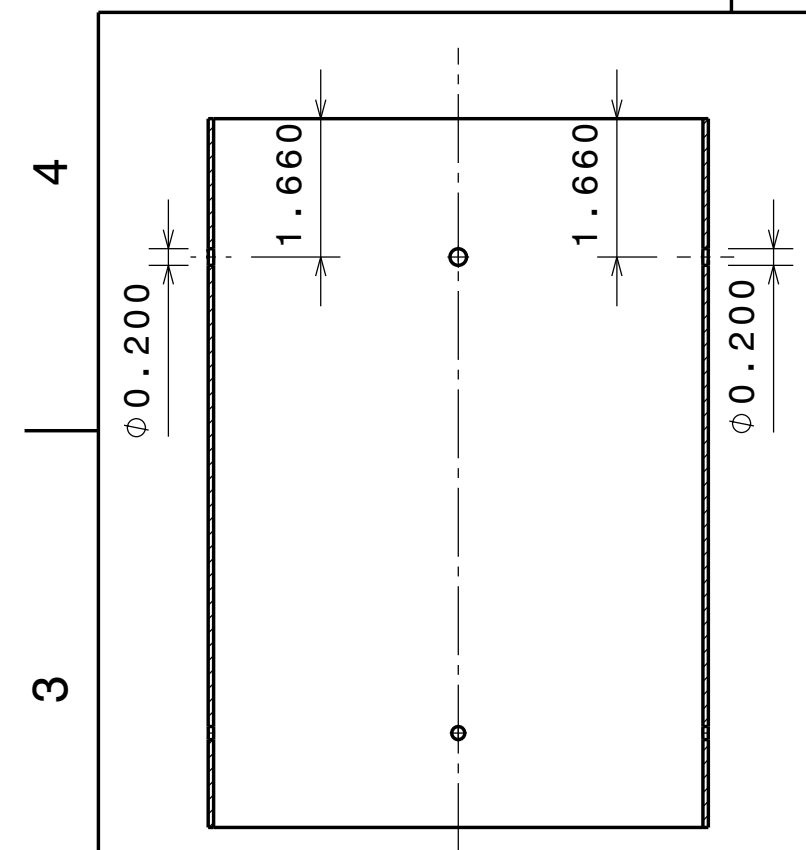

Section View J-J $\infty$

$\varangle$
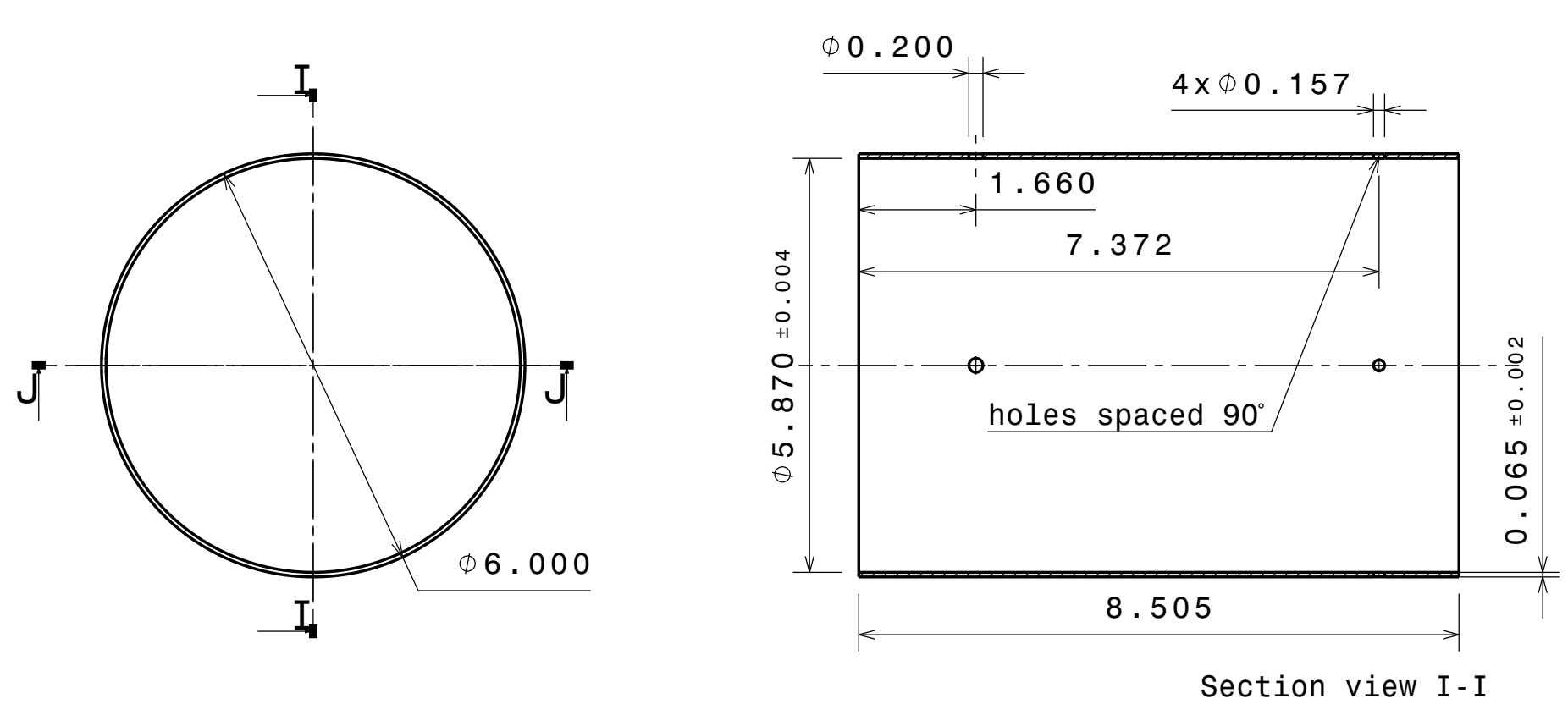

3

2

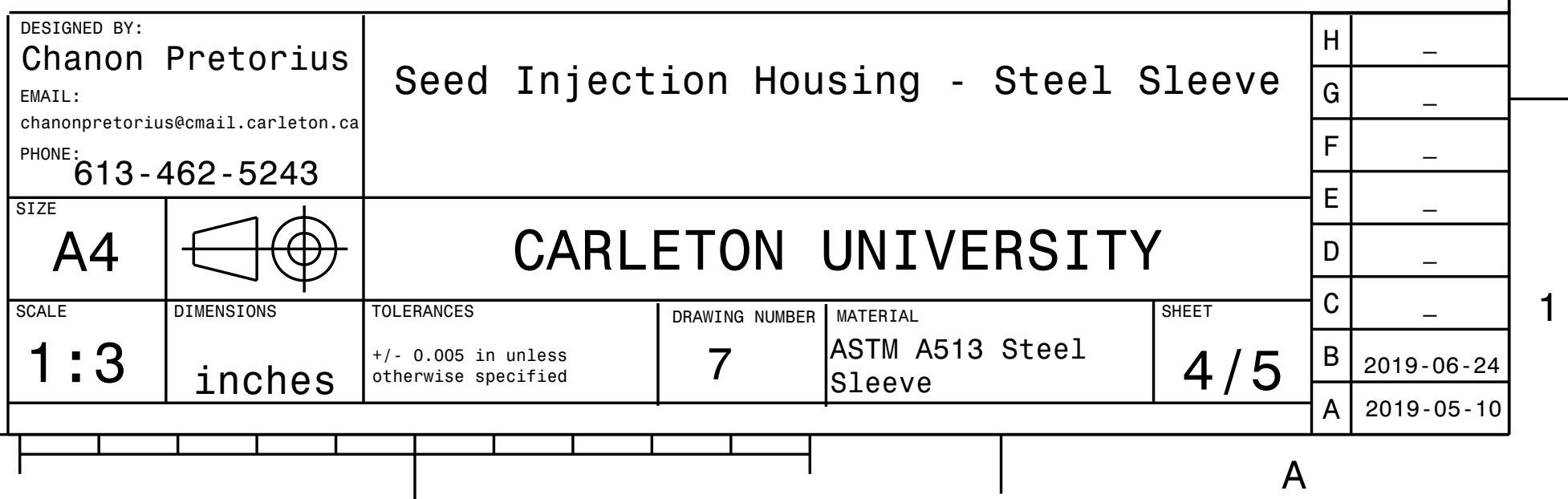



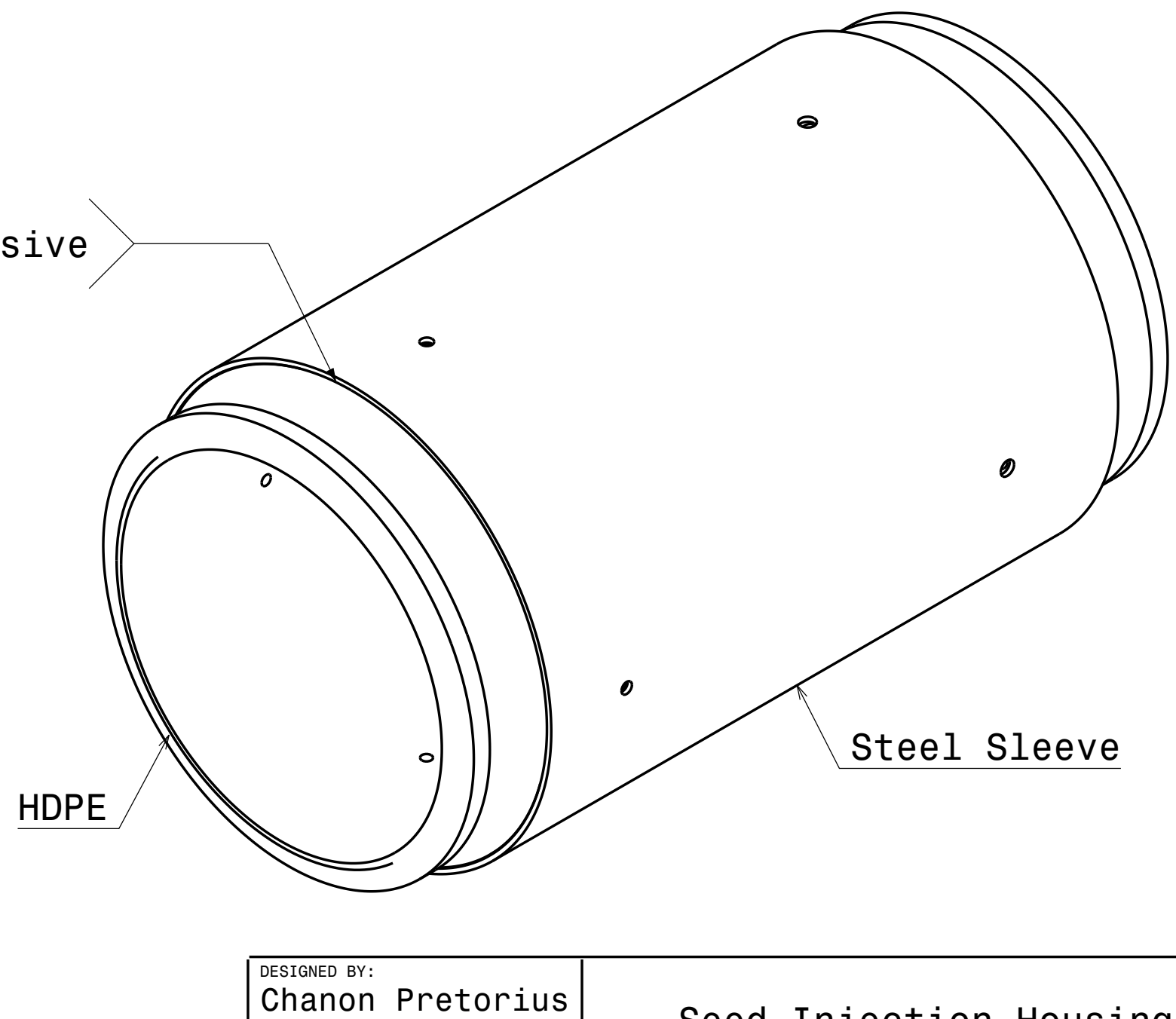

EMAIL:

Seed Injection Housing Assembly

chanonpretorius@cmail.carleton.ca

$\begin{array}{r}\text { PHONE: } 613-462-5243 \\ \hline\end{array}$

A4 $9 \bigoplus$ CARLETON UNIVERSITY

\begin{tabular}{|c|l|l|}
\hline SCALE & DIMENS IONS & $\begin{array}{l}\text { TOLERANCES } \\
+/-0.005 \\
\text { on unless } \\
\text { otherwise specified }\end{array}$ \\
\hline
\end{tabular}

\begin{tabular}{l|l}
7 & see Preceeding Drawings
\end{tabular} $5 / 5$ 


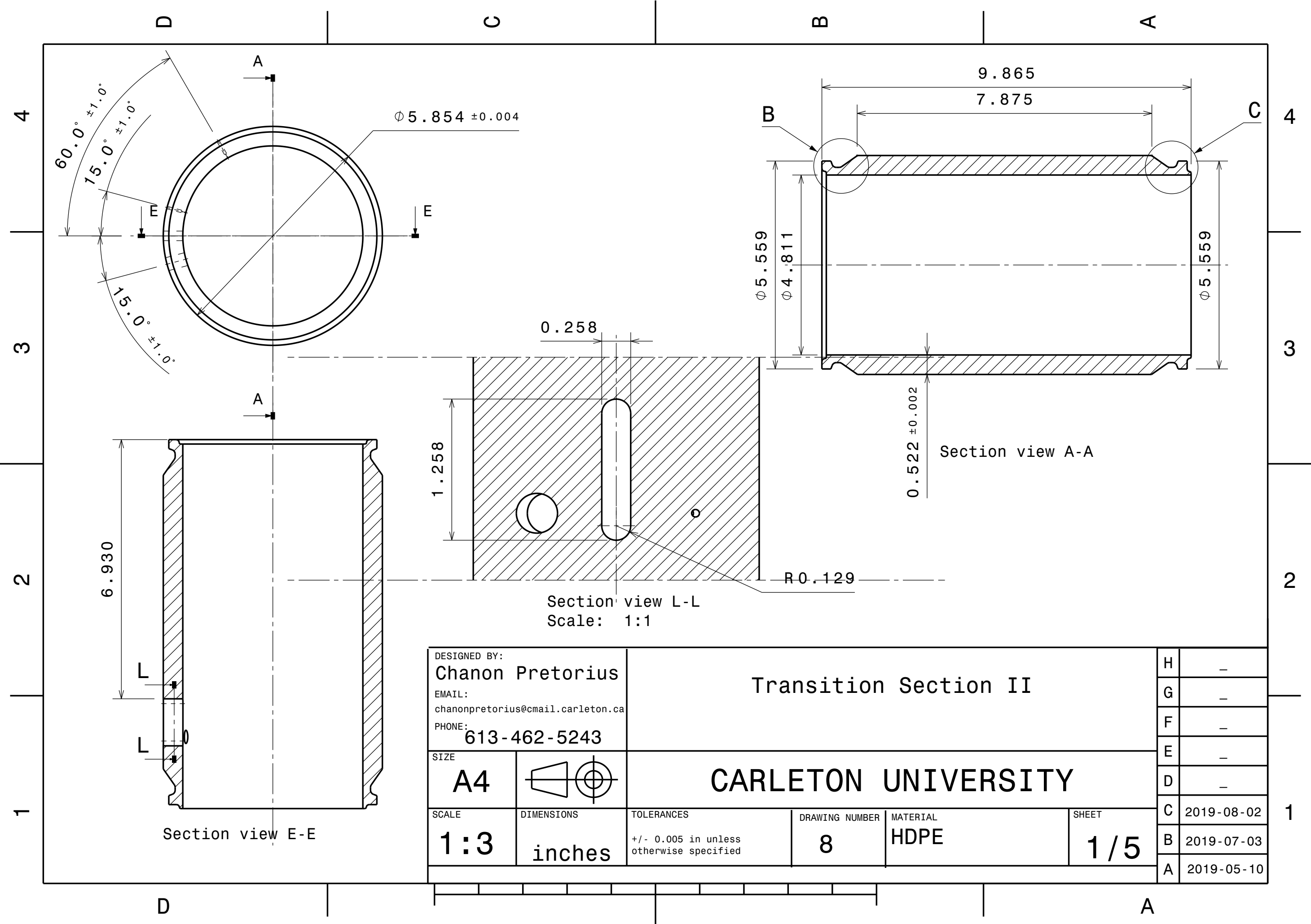



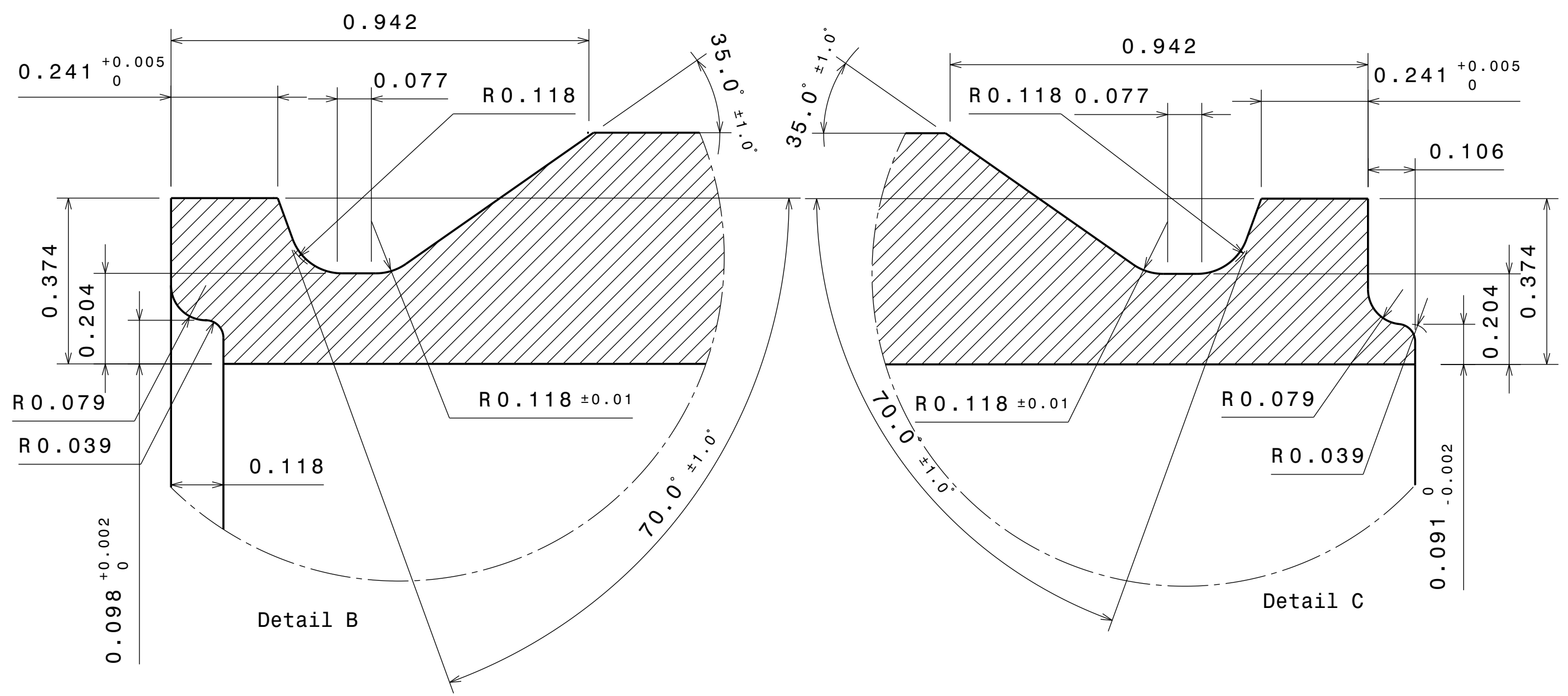

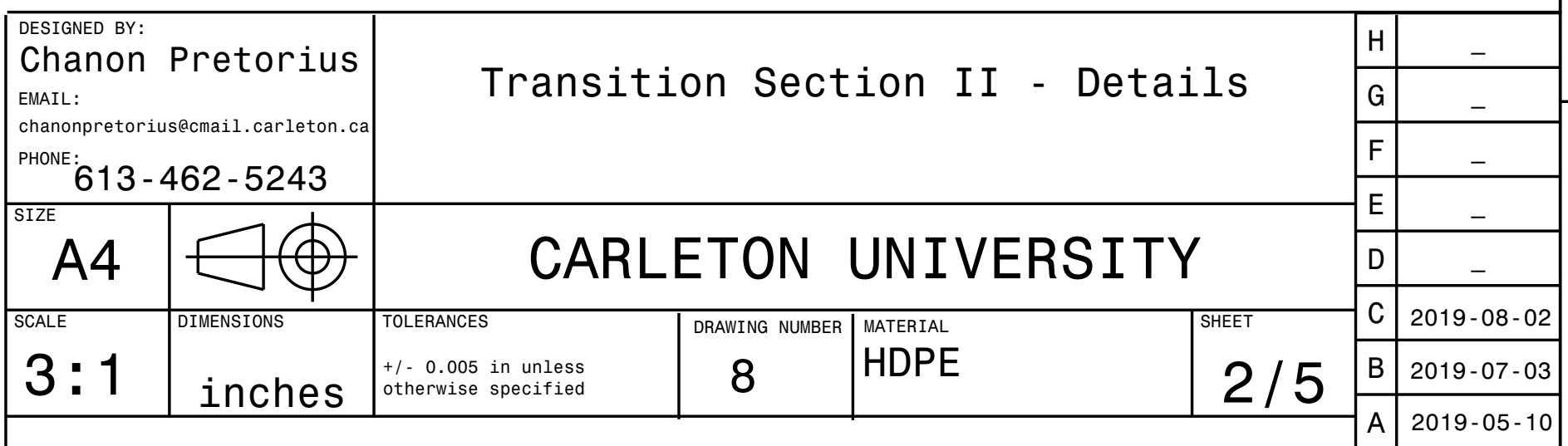


$\infty$

$\varangle$

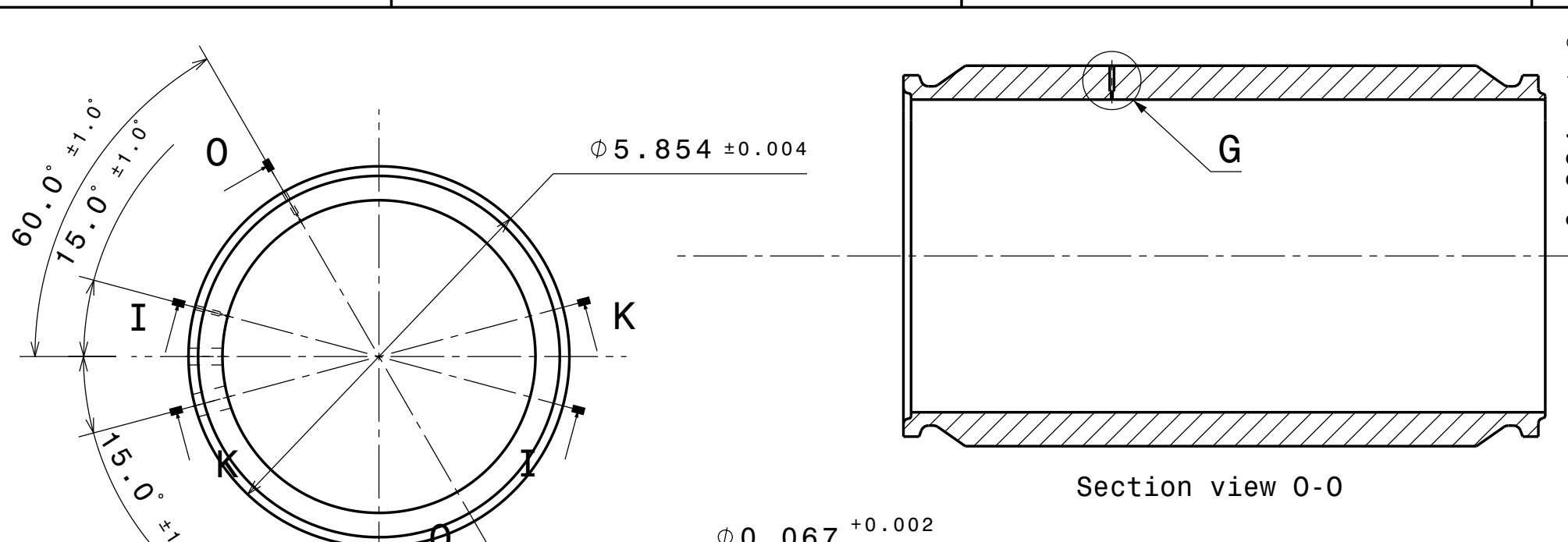

$\Phi 0.067_{-0.001}^{+0.002}$

$0.001+1, k$

$\Phi 5.854 \pm 0.004$

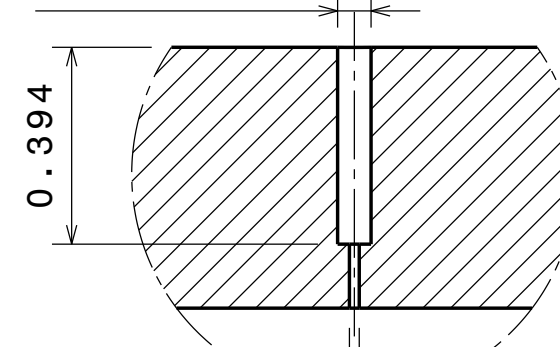

$\overline{\phi 0.020 \pm 0.002}$

Detail J

Scale: $2: 1$

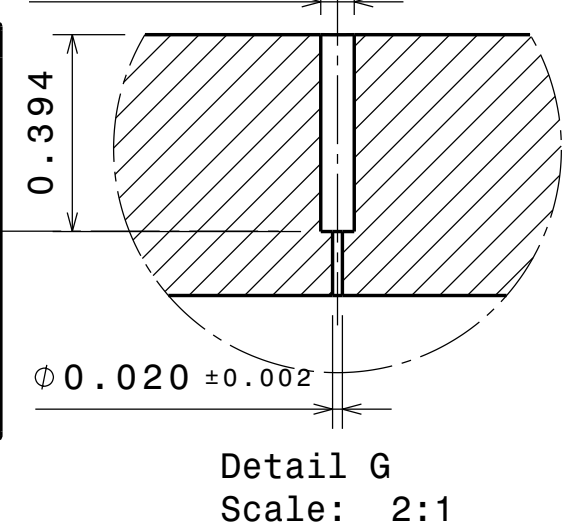

$\sim$

.7 .948

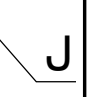

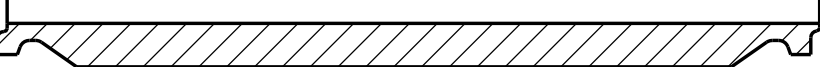

Section view I-I

\begin{tabular}{|c|c|c|c|c|c|}
\hline \multicolumn{2}{|c|}{$\begin{array}{l}\text { DESIGNED BY: } \\
\text { Chanon Pretorius } \\
\text { EMAIL: } \\
\text { chanonpretoriusecmail.carleton.caa } \\
\text { PHONEE } \\
613-462-5243\end{array}$} & \multicolumn{4}{|c|}{ Transition Section II - Details 2} \\
\hline A4 & $\square \oplus$ & \multicolumn{4}{|c|}{ CARLETON UNIVERSITY } \\
\hline $1: 3$ & & $\begin{array}{l}\text { TOLERANCES } \\
+1-0.005 \text { in unless } \\
\text { otherwise specified }\end{array}$ & \begin{tabular}{|l} 
MATERIAL \\
HDPE
\end{tabular} & & $3 / 5$ \\
\hline
\end{tabular}

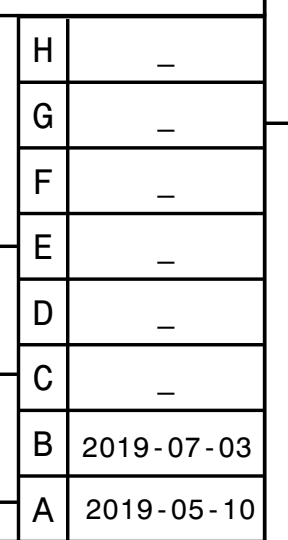




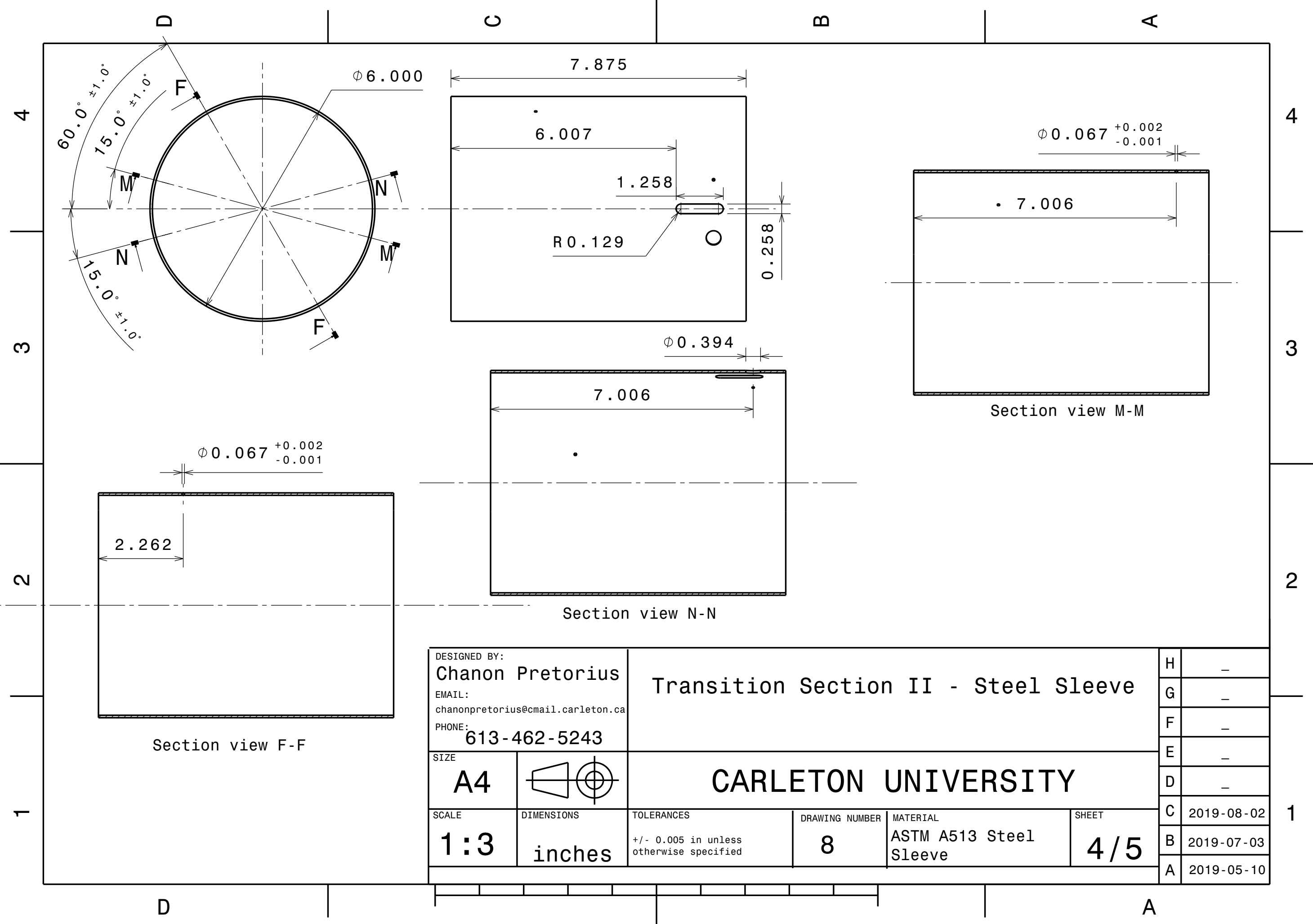


Polyethylene Adhesive

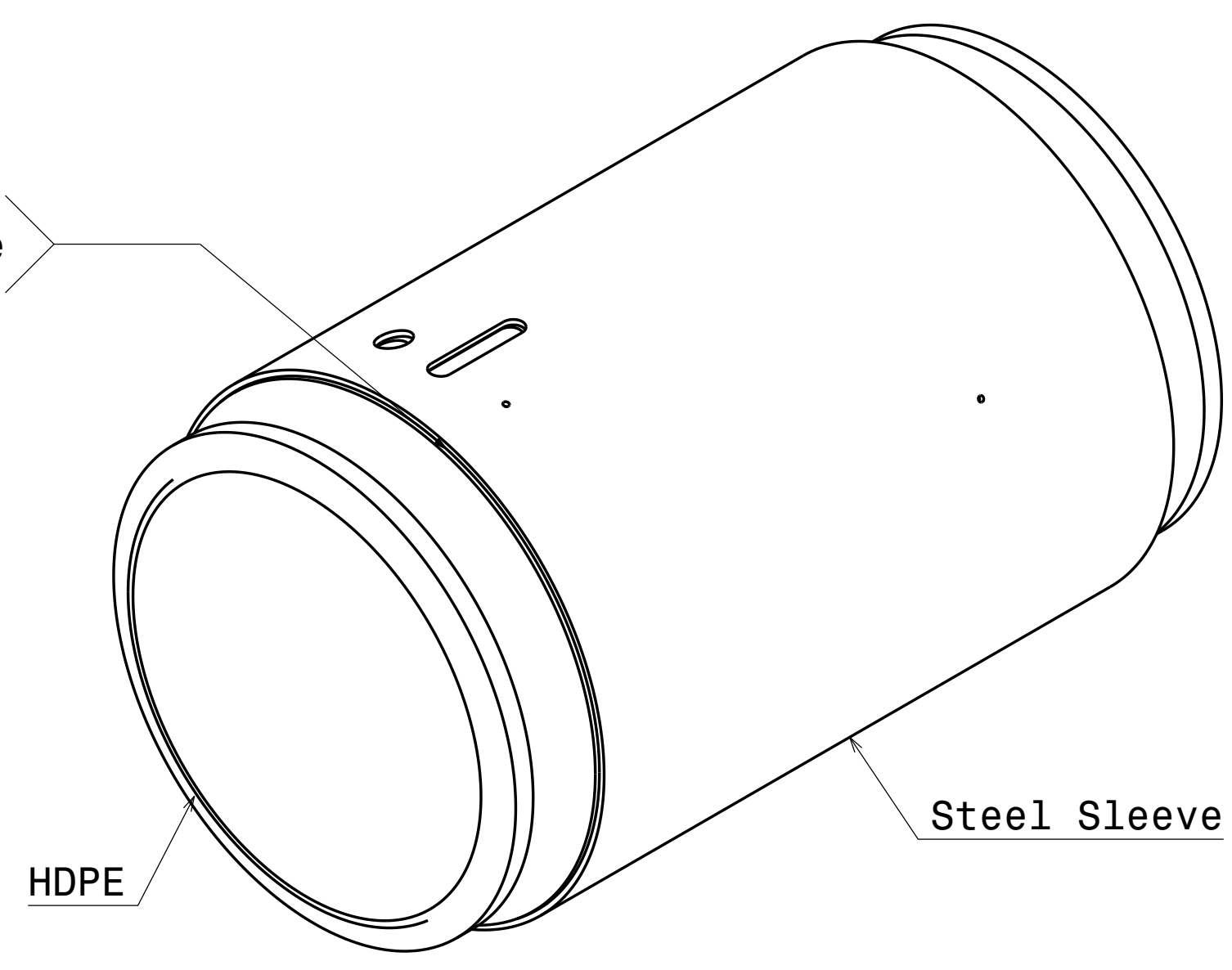

\begin{tabular}{|c|c|c|c|c|c|c|c|}
\hline \multirow{4}{*}{\multicolumn{2}{|c|}{$\begin{array}{l}\text { DESIGNED BY: } \\
\text { Chanon Pretorius } \\
\text { EMAIL: } \\
\text { chanonpretoriusecmail.carleton. ca } \\
\text { PHONE: } 613-462-5243 \\
\text { SIZE }\end{array}$}} & \multirow{4}{*}{\multicolumn{4}{|c|}{ Transition Section II - Assembly }} & $\mathrm{HH}$ & - \\
\hline & & & & & & G & - \\
\hline & & & & & & $\mathrm{F}$ & - \\
\hline & & & & & & E & - \\
\hline A4 & $\uplus(\emptyset)$ & \multicolumn{4}{|c|}{ CARLETON UNIVERSITY } & D & - \\
\hline$\overline{S C A L E}$ & DTwENSIONS & $\begin{array}{l}\text { TOLERANCES } \\
\end{array}$ & \begin{tabular}{|l|} 
DRAWING NUMBER \\
\end{tabular} & $\begin{array}{l}\text { MATERIAL } \\
\end{array}$ & & c & - \\
\hline $1: 2$ & inches & $\begin{array}{l}\text { l. } 0.005 \text { in unless } \\
\text { otherwise specifified }\end{array}$ & 8 & See Preceeding Drawings & $5 / 5$ & $B$ & 2019-07-03 \\
\hline
\end{tabular}



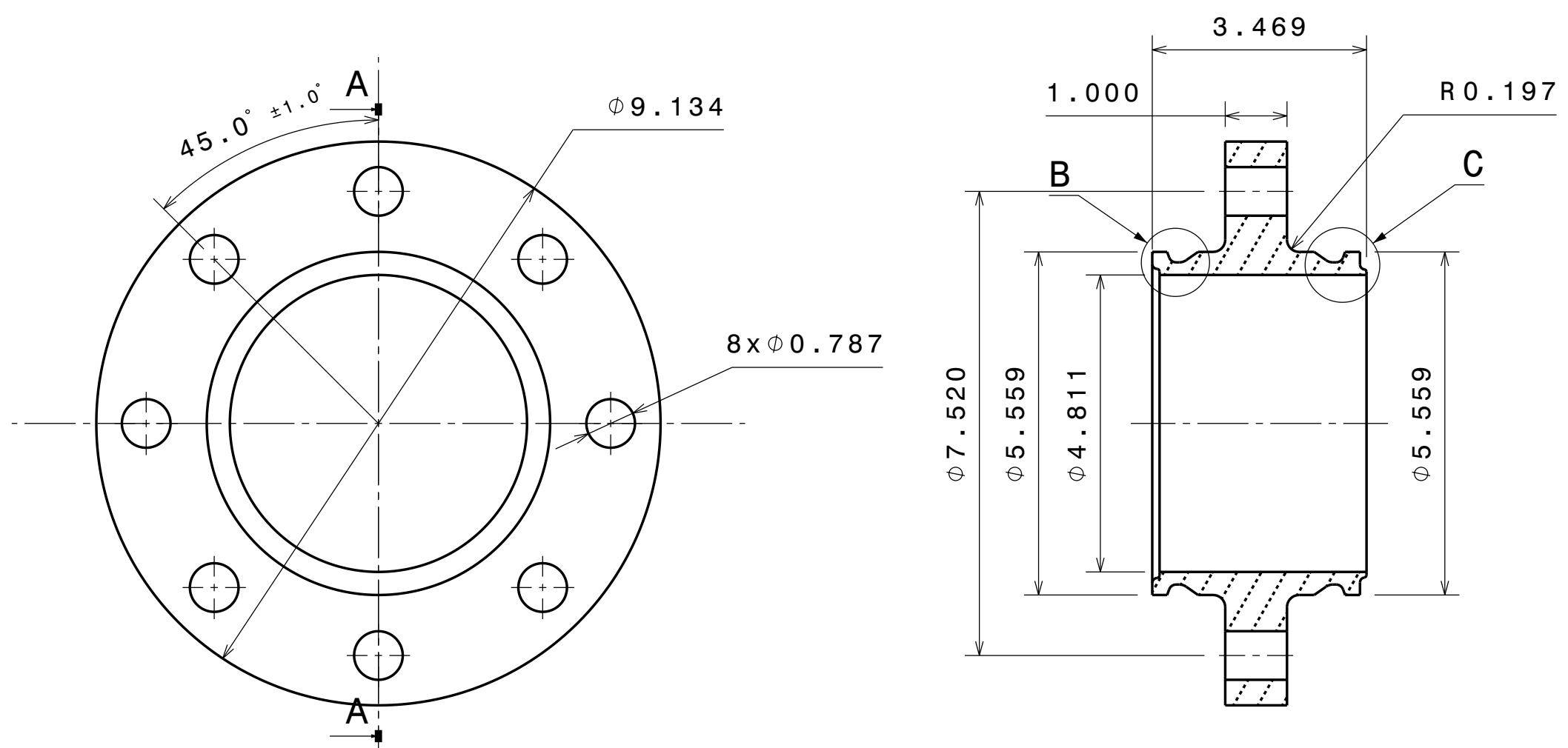

4

Section view A-A

\begin{tabular}{|c|c|c|c|c|c|c|c|}
\hline \multirow{4}{*}{\multicolumn{2}{|c|}{ 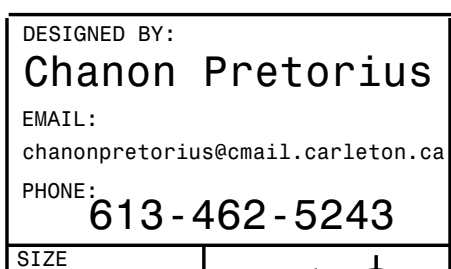 }} & \multirow{3}{*}{\multicolumn{4}{|c|}{ Downstream Flange }} & $\mathrm{H}$ & - \\
\hline & & & & & & $G$ & 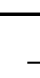 \\
\hline & & & & & & $\mathrm{F}$ & _- \\
\hline & & \multirow{2}{*}{\multicolumn{4}{|c|}{ CARLETON UNIVERSITY }} & $E$ & - \\
\hline A4 & $\notin(\phi$ & & & & & $\mathrm{D}$ & _- \\
\hline & & TOLERANCES & DRAWING NUMBER & & & $c$ & _- \\
\hline $1: 3$ & & $\begin{array}{l}+1-0.005 \text { in unless } \\
\text { othervise specifified }\end{array}$ & 9 & Mild Steel & $1 / 2$ & $B$ & - \\
\hline
\end{tabular}




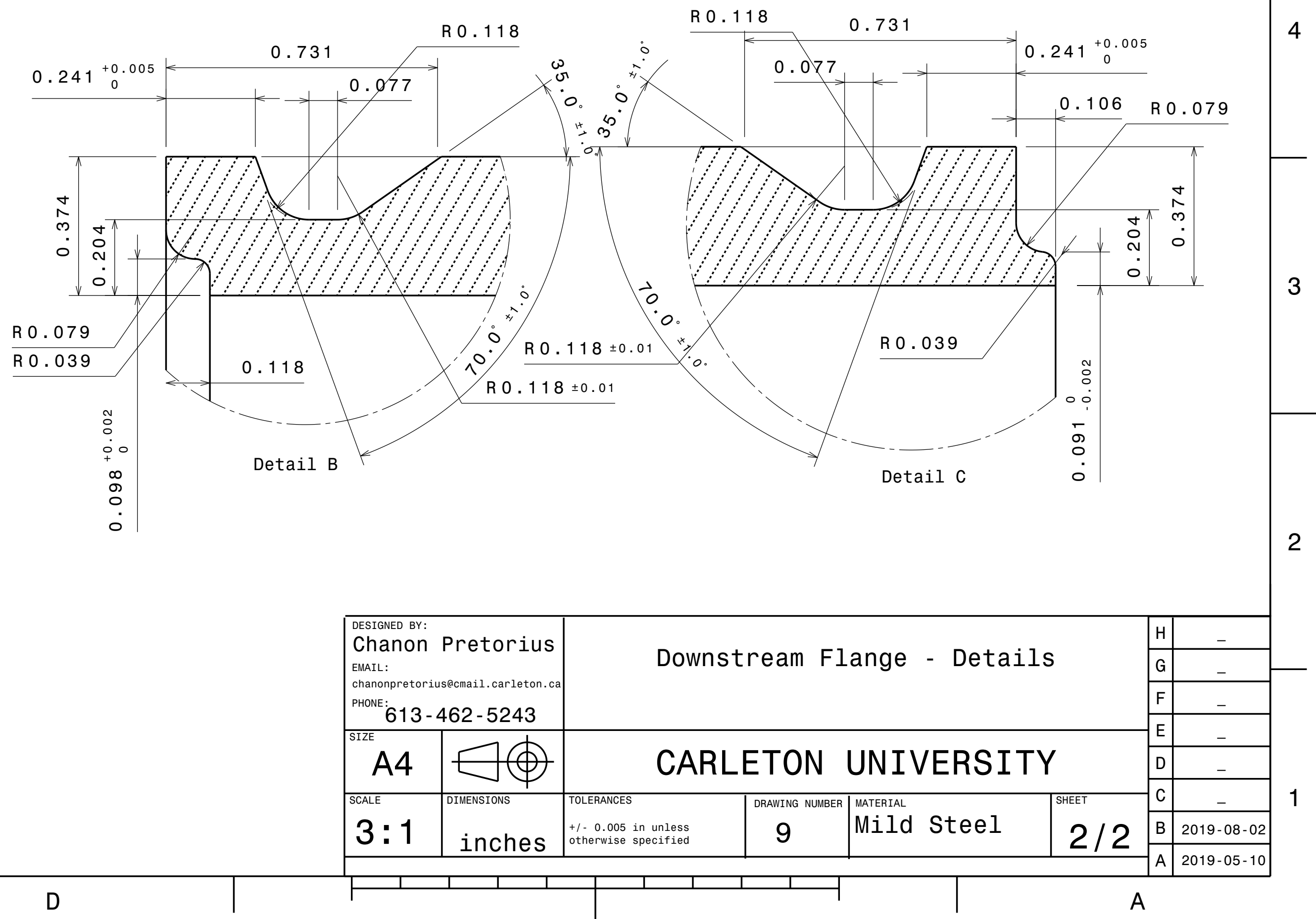


口

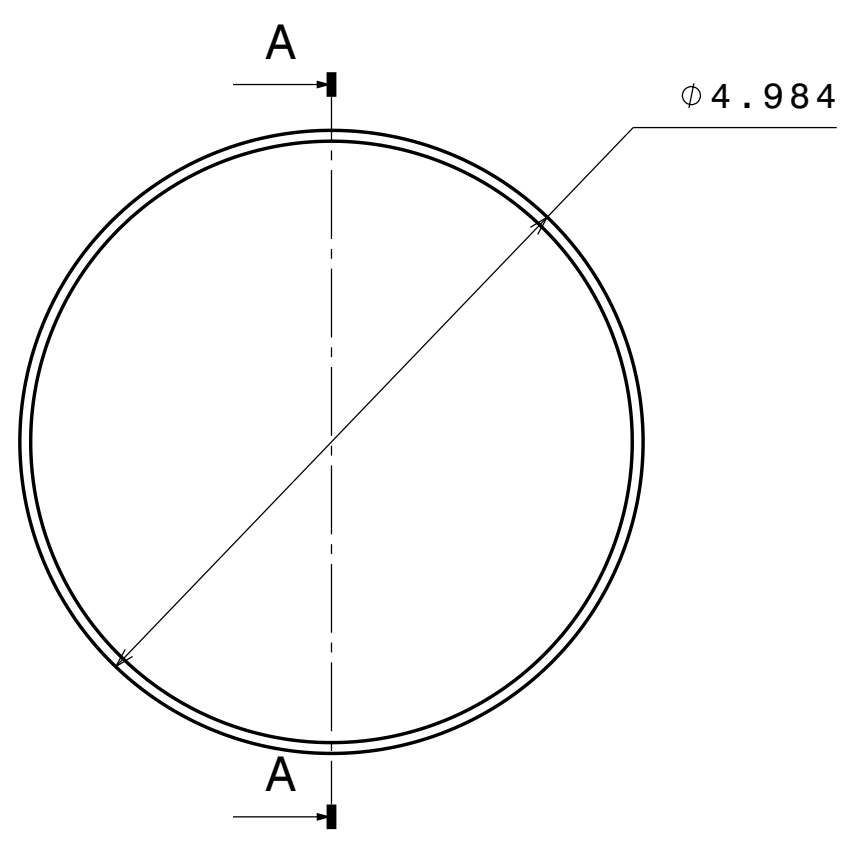

$\infty$

$\varangle$

Note: Quantity $=3$

\begin{tabular}{|c|c|c|c|c|c|c|c|}
\hline \multirow{4}{*}{\multicolumn{2}{|c|}{$\begin{array}{l}\text { DESIGNED BY: } \\
\text { Chanon Pretorius } \\
\text { EMAIL: } \\
\text { chanonpretor iusecmail.carleton.cal } \\
\text { PHONEE } \\
\quad 613-462-5243 \\
\text { SIZE }\end{array}$}} & \multirow{3}{*}{\multicolumn{4}{|c|}{ Turbulence Screen Spacer }} & $\mathrm{H}$ & _ \\
\hline & & & & & & \begin{tabular}{|l|l}
$G$ \\
\end{tabular} & - \\
\hline & & & & & & $\mathrm{F}$ & _- \\
\hline & & \multirow{2}{*}{\multicolumn{2}{|c|}{ CARLETON }} & \multirow{2}{*}{\multicolumn{2}{|c|}{ UNIVERSITY }} & $\mathrm{E}$ & - \\
\hline A4 & $\square(\emptyset)$ & & & & & $\mathrm{D}$ & _- \\
\hline$\overline{\text { SCALE }}$ & DIMENSIONS & TOLERANCES & DAAUING NUMBER & MAATERIAL & & c & - \\
\hline $1: 2$ & inches & $\begin{array}{l}+1-0.005 \text { in unless } \\
\text { otherwise specified }\end{array}$ & 10 & Steel & $1 / 3$ & $B$ & - \\
\hline & $T$ & & 1 & & & $A$ & $2019-06-16$ \\
\hline
\end{tabular}




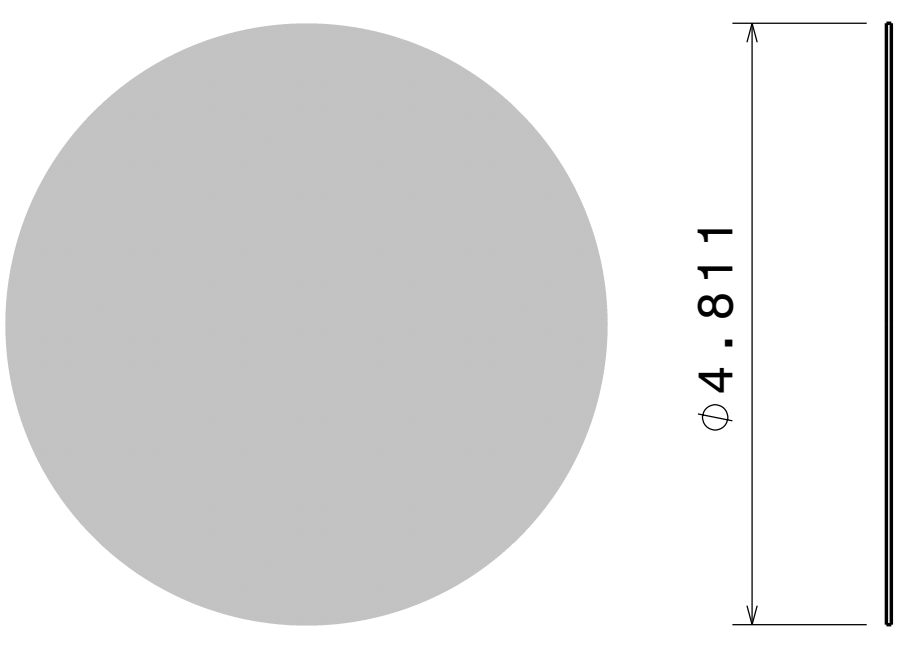

3

Note: Quantity $=2$

Note: To be cut from a larger rectangular sheet of steel wire mesh

\begin{tabular}{|c|c|c|c|c|c|c|}
\hline \multirow{5}{*}{\multicolumn{2}{|c|}{$\begin{array}{l}\text { DESIGNED BY: } \\
\text { Chanon Pretorius } \\
\text { EMAIL: } \\
\text { chanonpretoriusecmail.carleton.ca } \\
\text { PHONE: } 613-462-5243 \\
\end{array}$}} & \multirow{4}{*}{\multicolumn{3}{|c|}{$\begin{array}{c}\text { Turbulence Screen Spacer - Wire } \\
\text { Mesh Screen }\end{array}$}} & \multirow{3}{*}{$\mathrm{H}$} & \multirow{2}{*}{ - } \\
\hline & & & & & & \\
\hline & & & & & & \\
\hline & & & & & & - \\
\hline & & & & & E & - \\
\hline A4 & 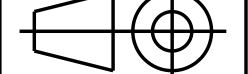 & CARLETON & 8 & & $\mathrm{D}$ & - \\
\hline $\begin{array}{l}\text { SCALE } \\
\end{array}$ & DIMENSIONS & DRAWING NUMBER & MATERIAL & $\begin{array}{l}\text { SHEET } \\
\end{array}$ & $\mathrm{C}$ & - \\
\hline $1: 2$ & inches & $\begin{array}{l}+-0.005 \text { in unless } \\
\text { otherwise specified }\end{array}$ & Steel & $2 / 3$ & $B$ & - \\
\hline & & & & & A & 2019-07-04 \\
\hline
\end{tabular}



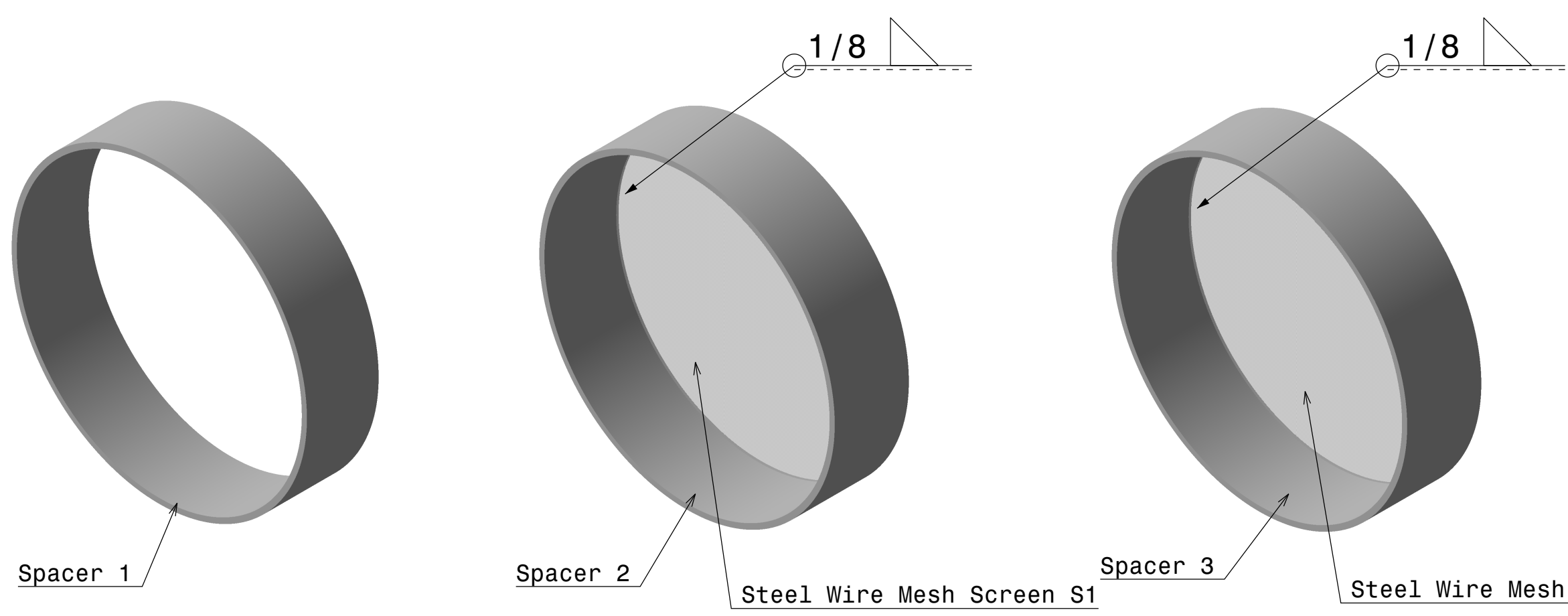

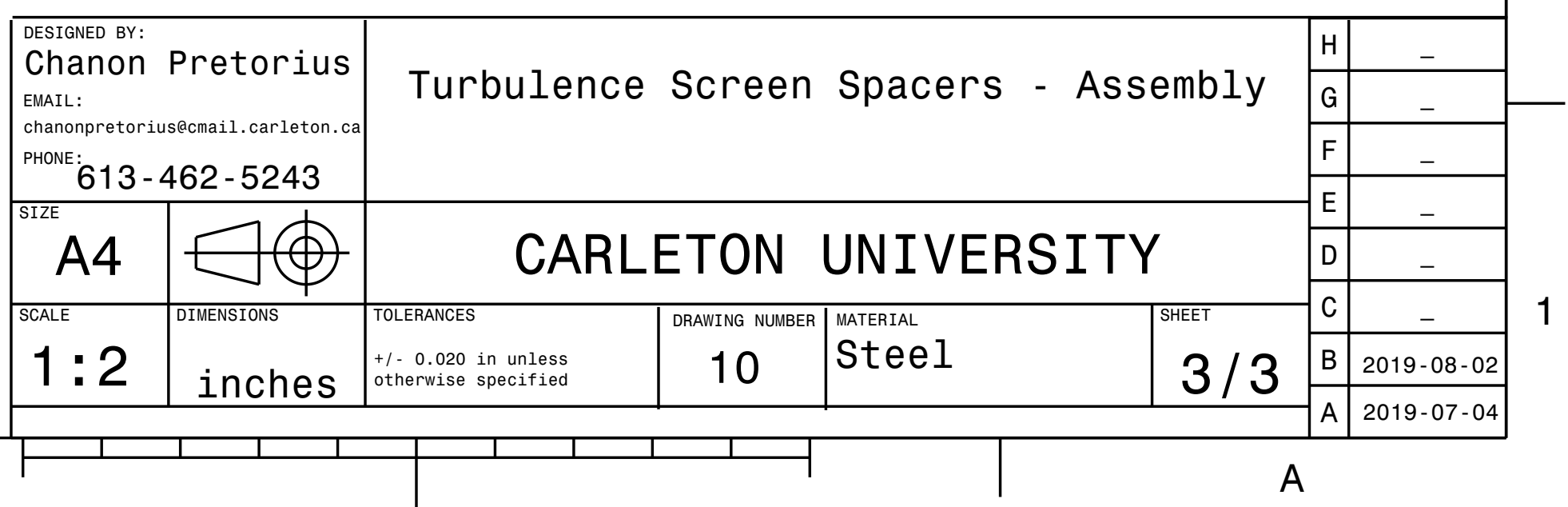



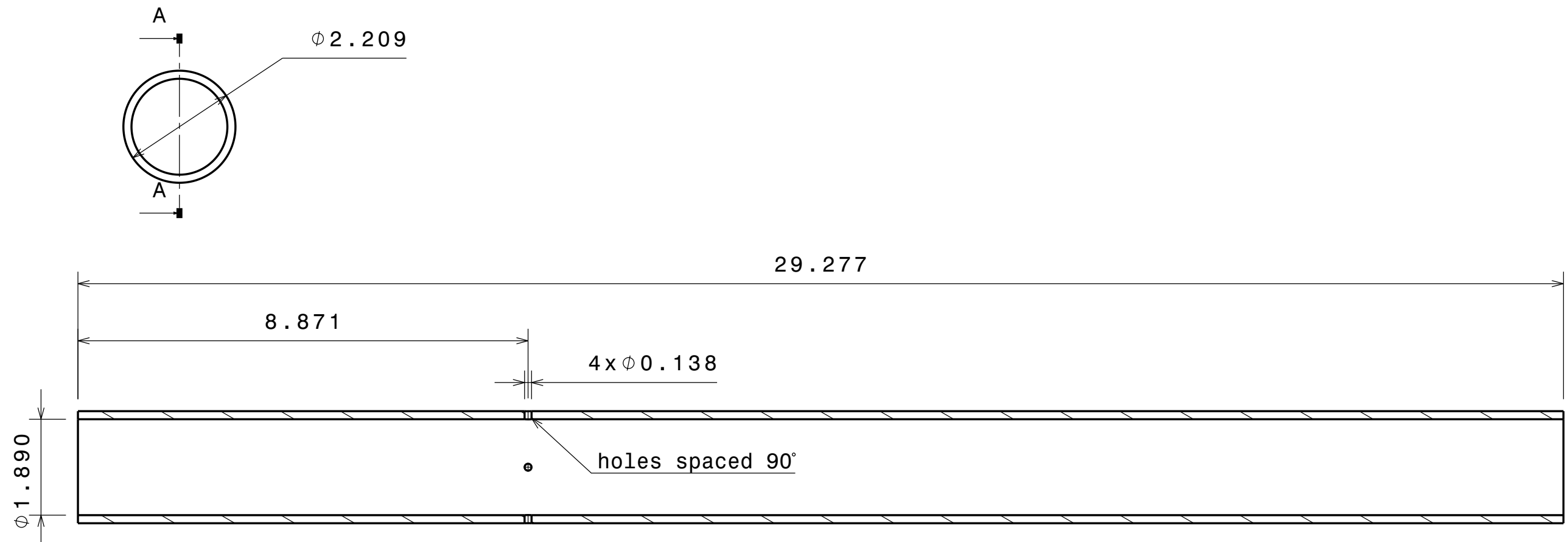

Section view A-A

\section{Chanon Pretorius}

EMAIL:

chanonpretorius@cmail.carleton.ca

PHONE: $613-462-5243$

\begin{tabular}{|c|c|c|}
\hline AIZE & \\
\hline SCALE & DIMENSIONS & $\substack{\text { TOLERANCES } \\
+-0.005 \\
\text { otherwise }}$ \\
\hline
\end{tabular}

CARLETON UNIVERSITY 
ص

0

$\infty$

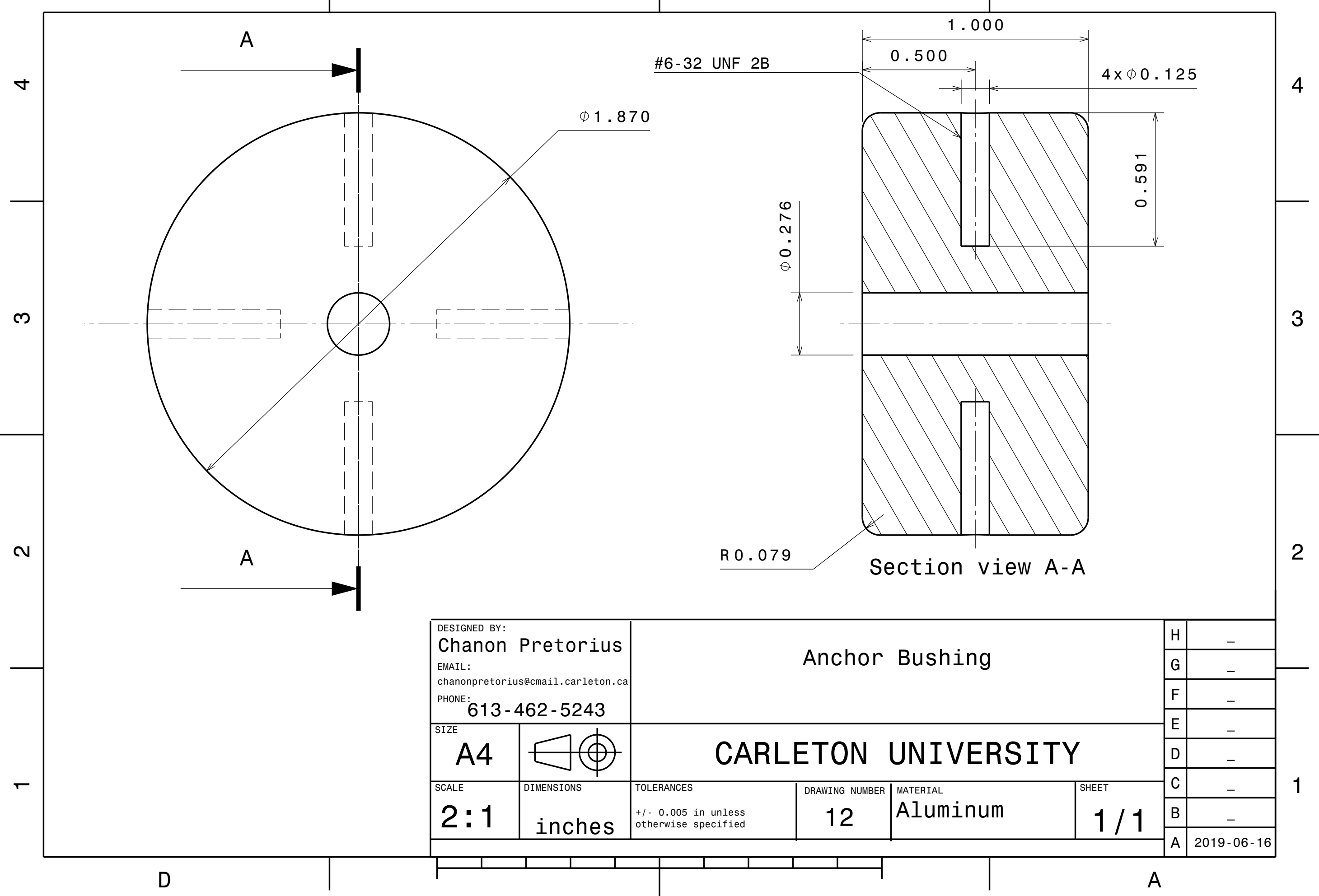



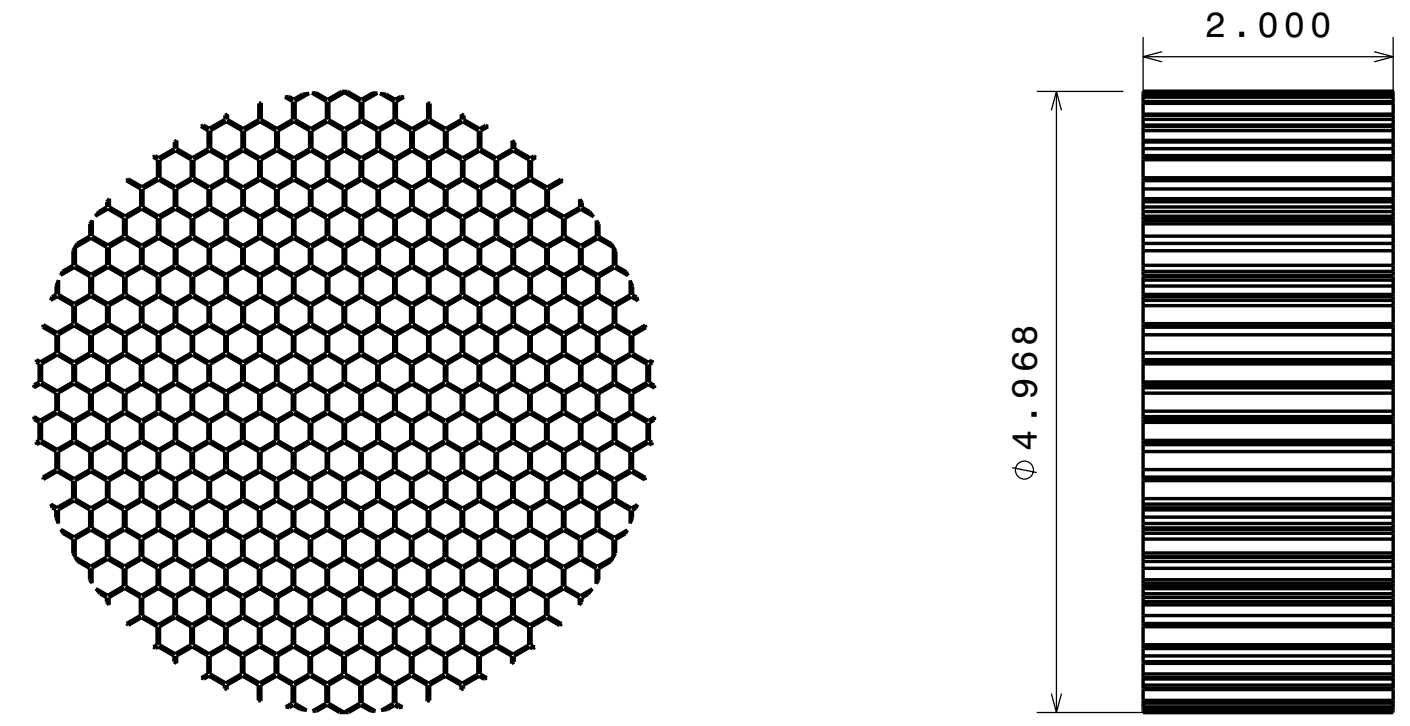

Note: Honeycomb cells must not become deformed during cutting.

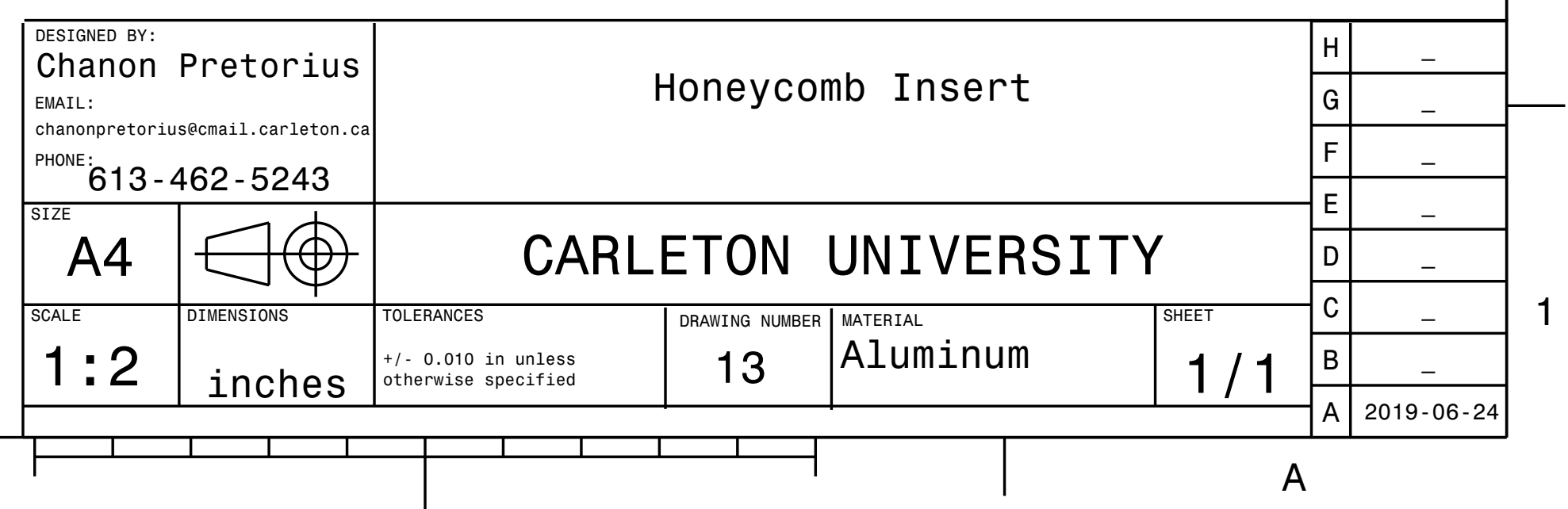


口

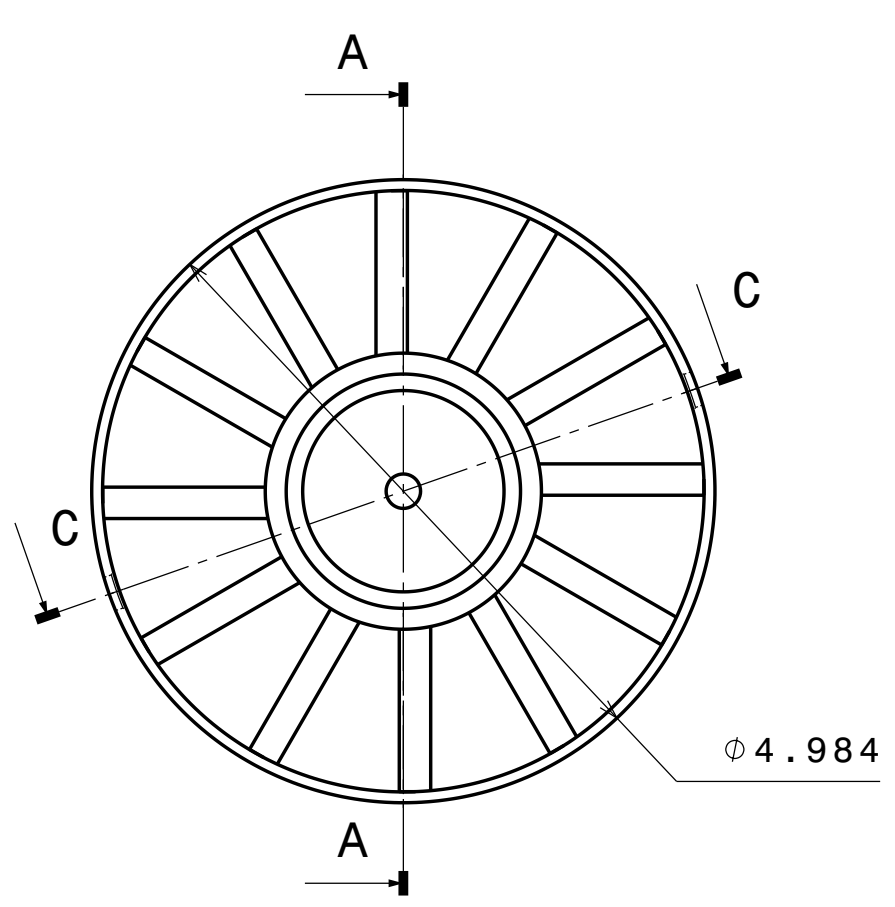

m

7
0
0
0
0
0
$1+$
0
0
0

$\infty$

$\varangle$

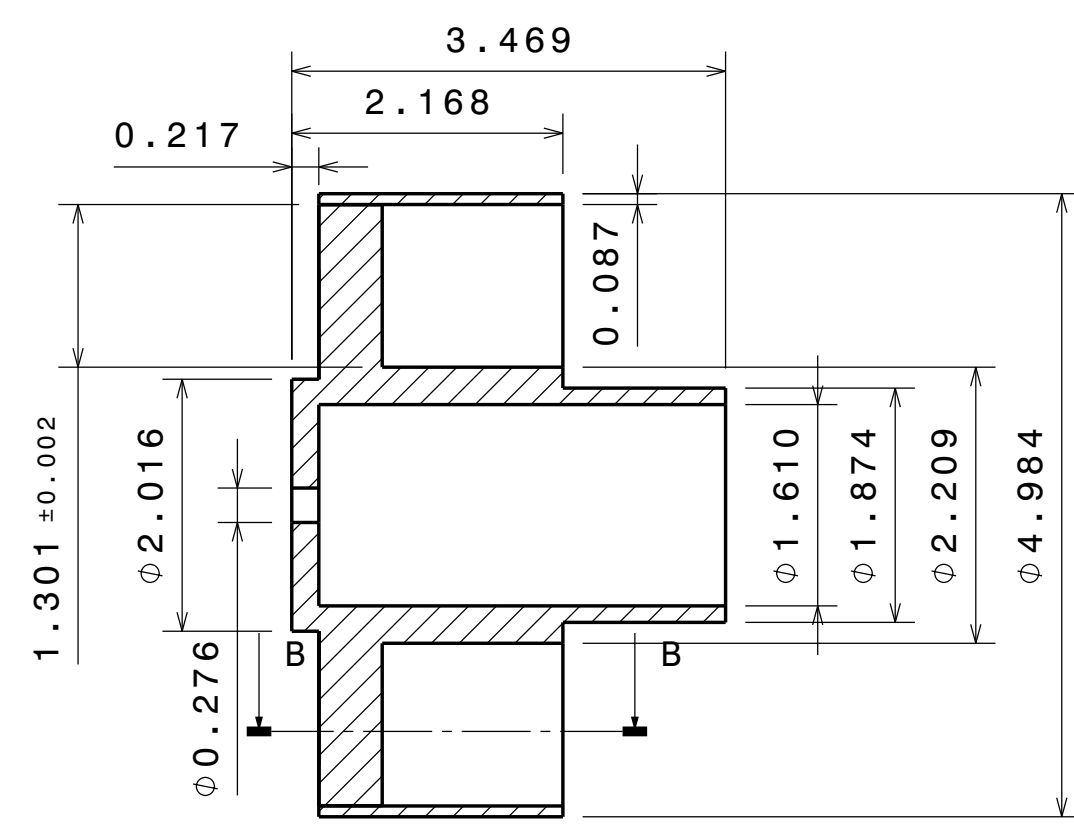

Section view A-A

2

R $0.032 \pm 0.02$

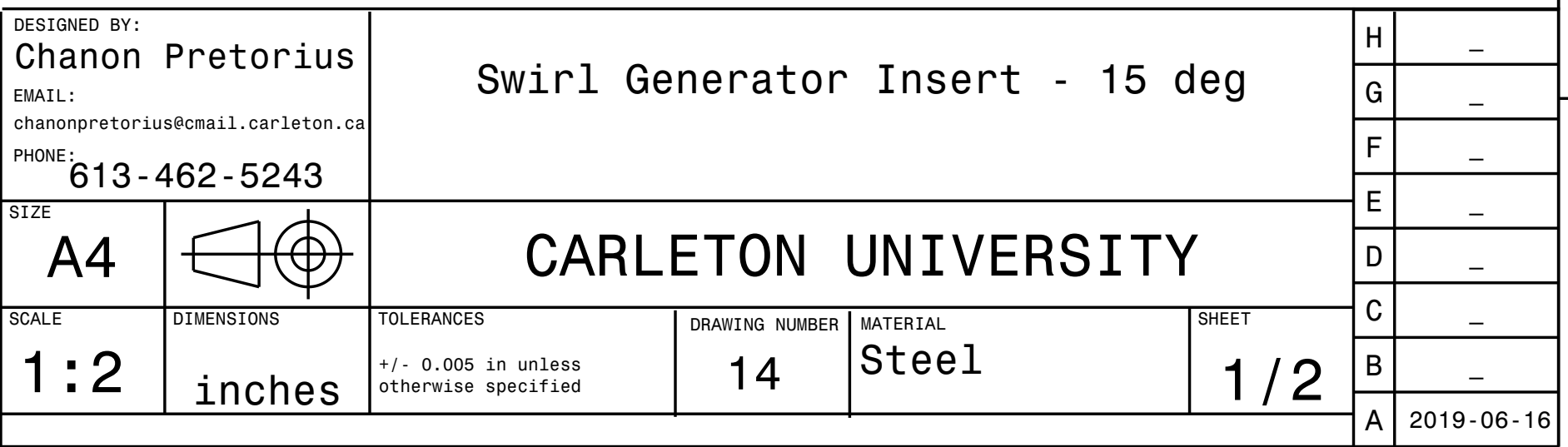

D

Section view B-B

Scale: 2:1

1

A 

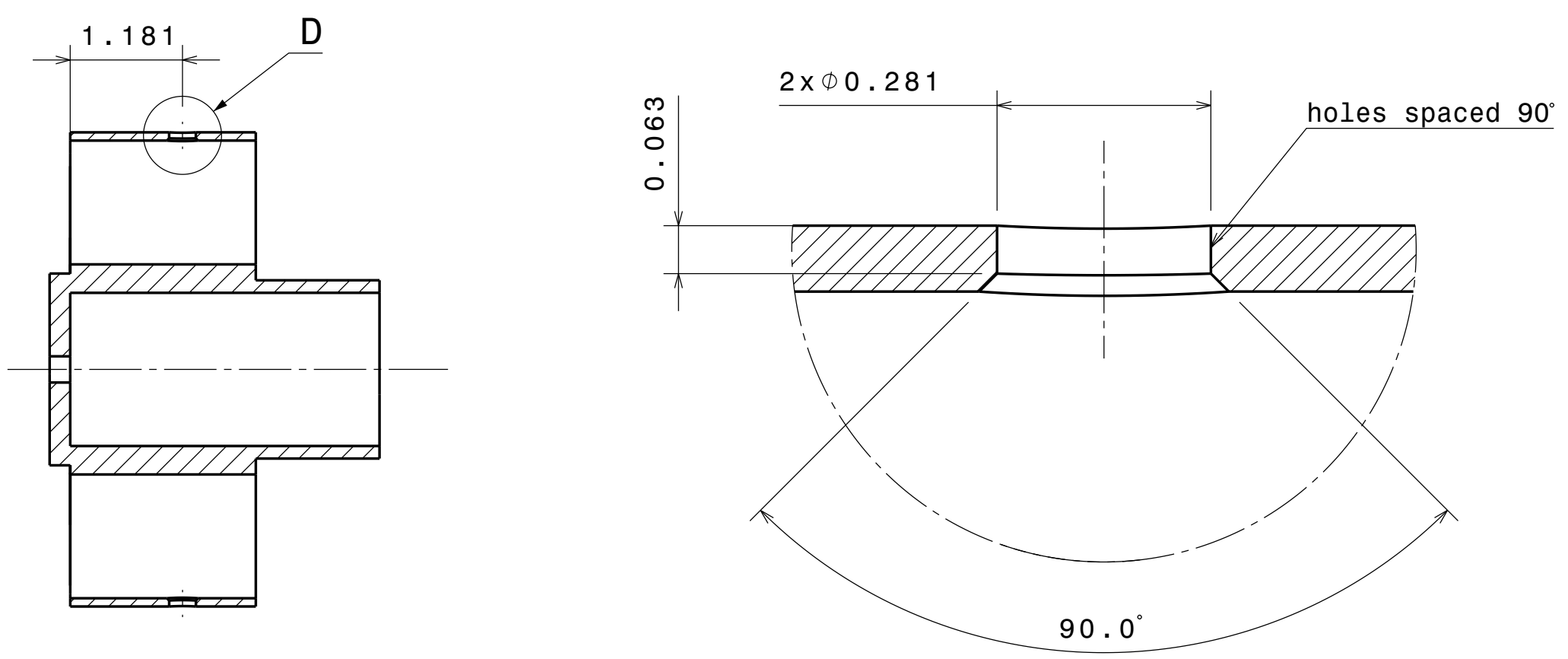

Section view C-C

Detail D

Scale: $4: 1$

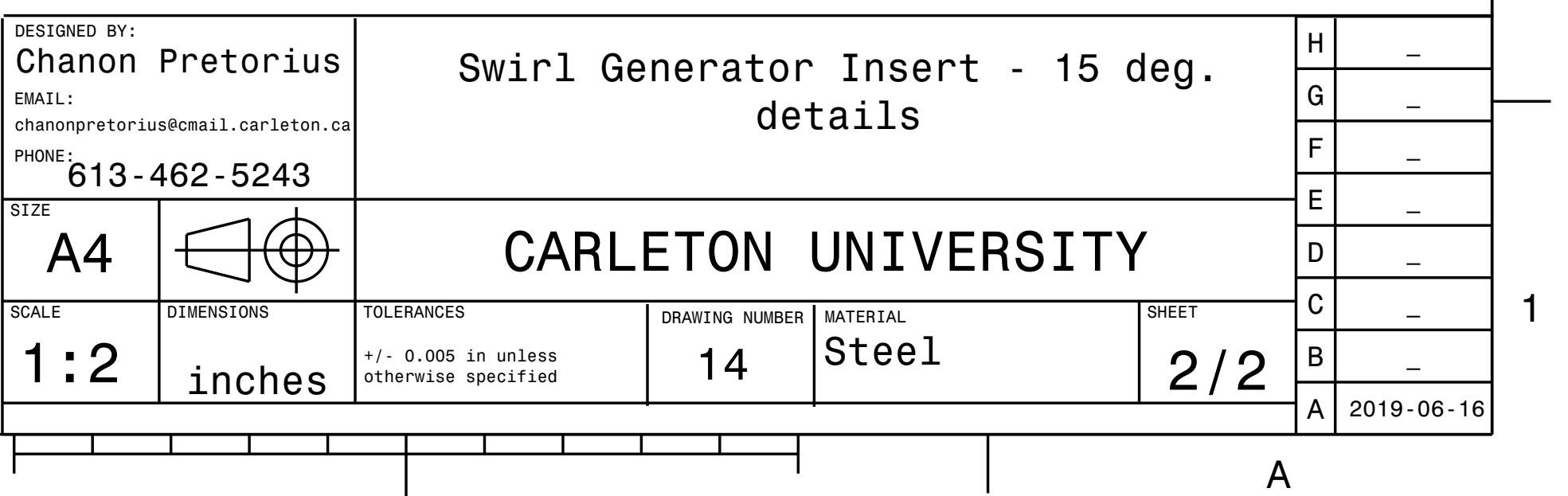




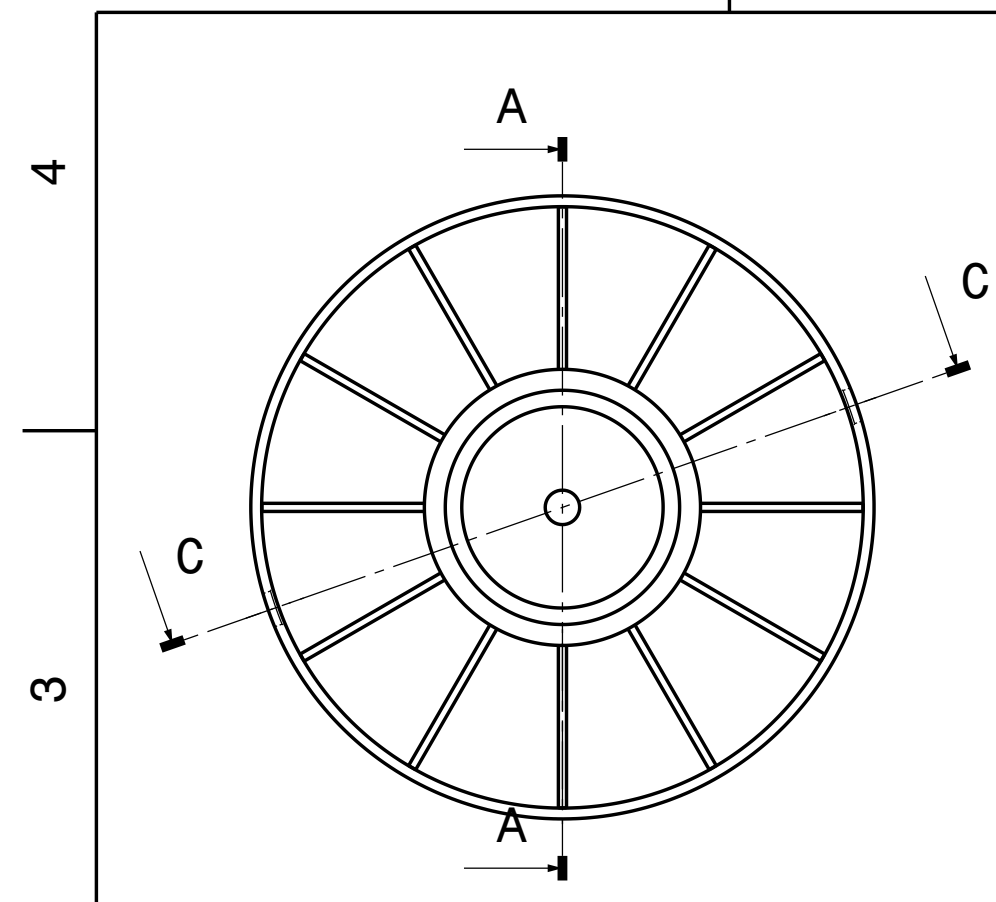

$1.228 \pm 0.02$

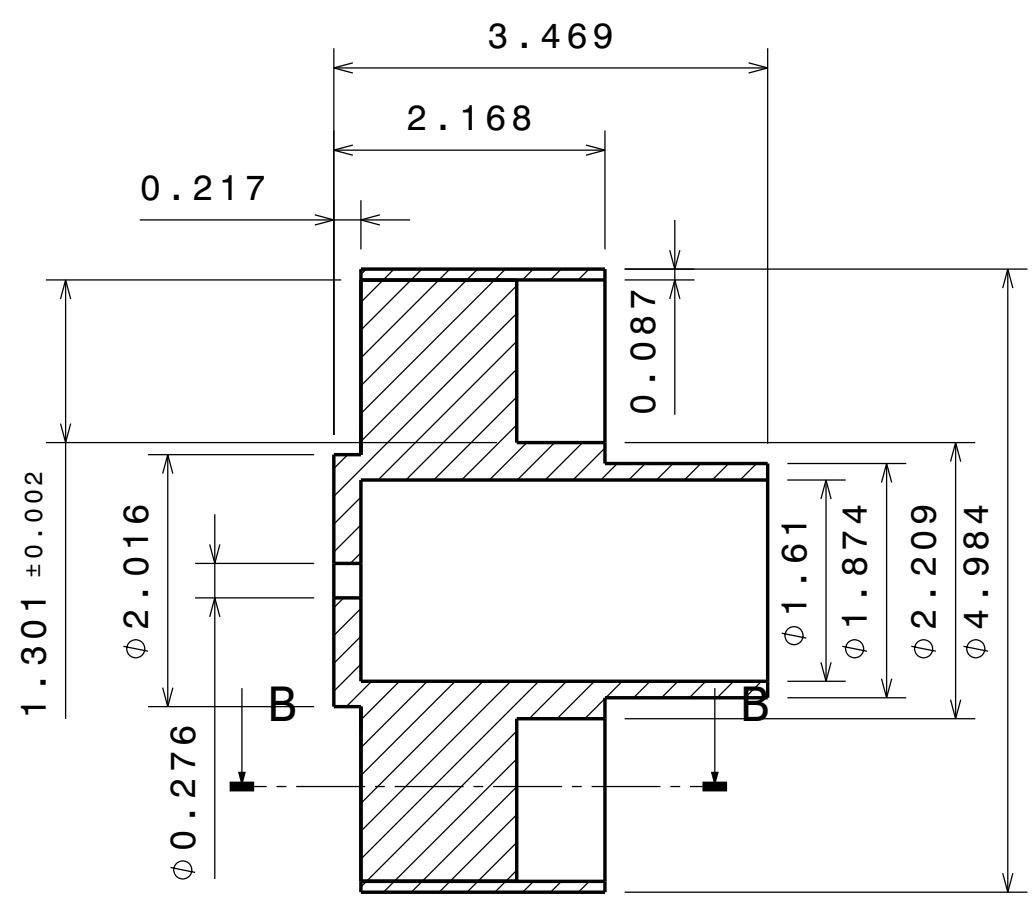

\section{3}

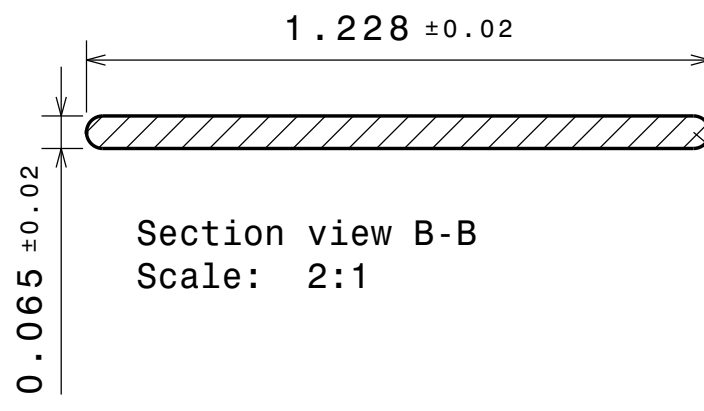

R $0.033 \pm 0.02$

Section view A-A

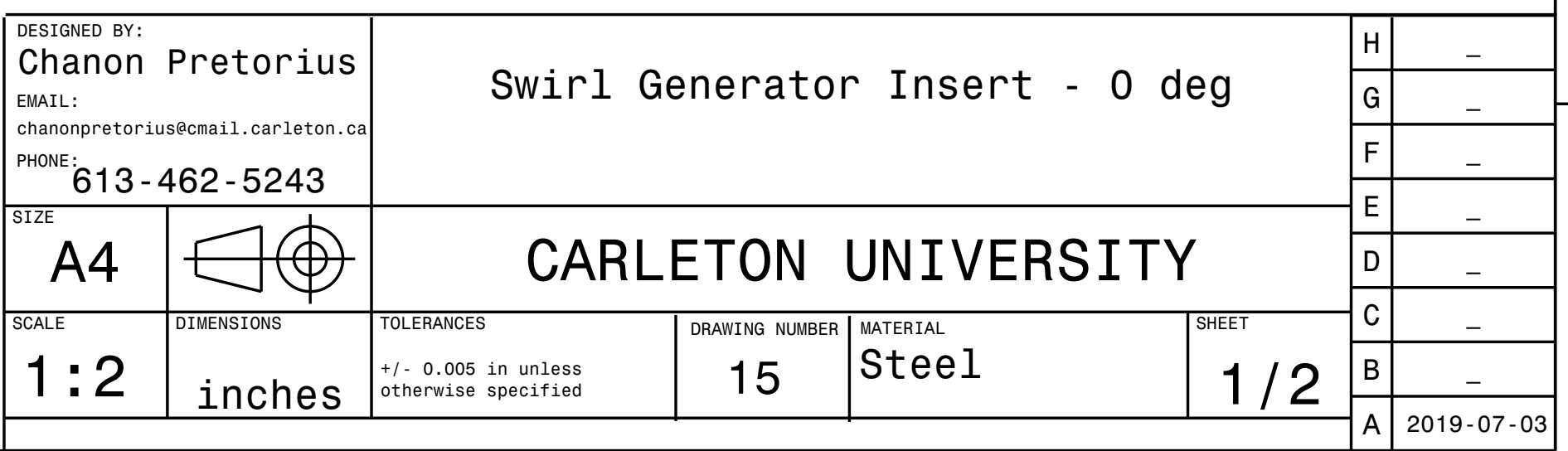



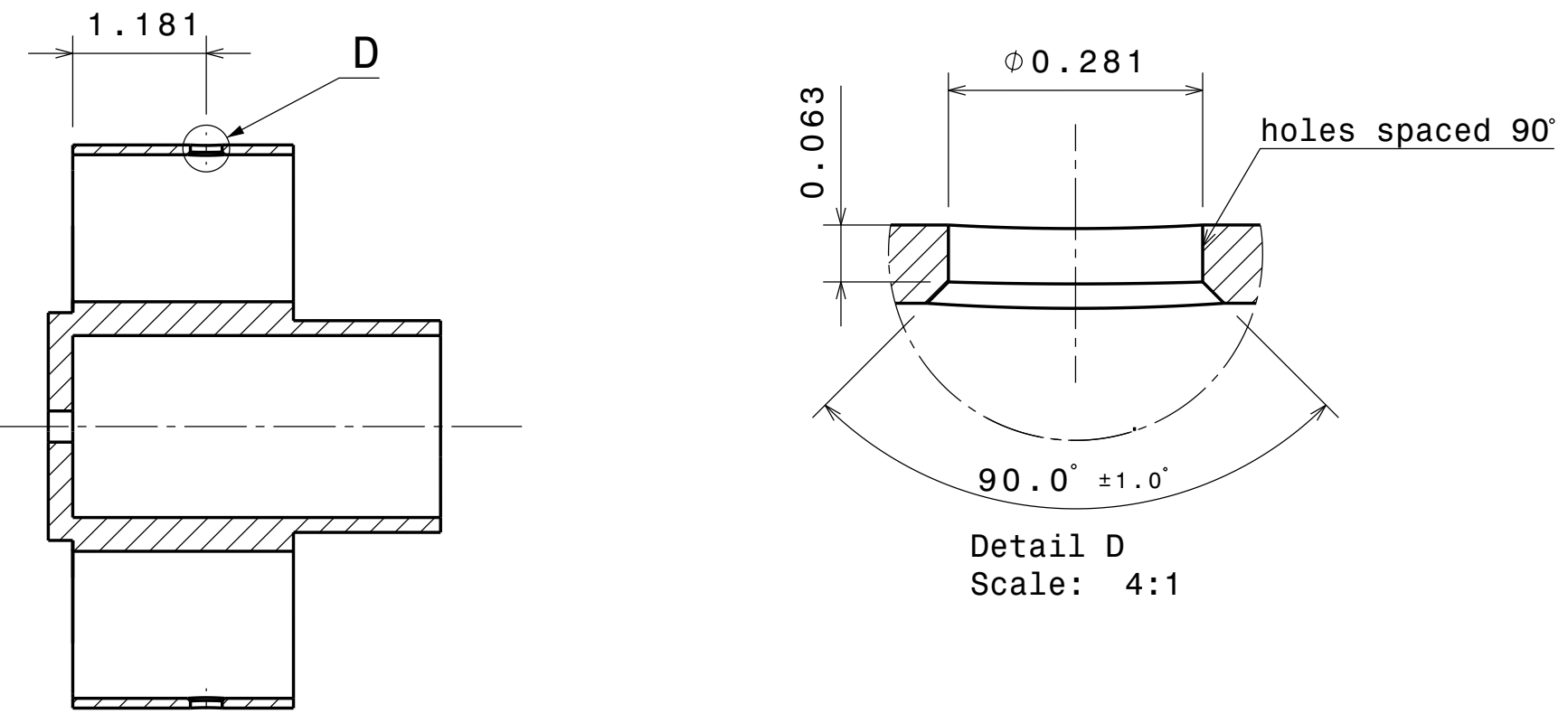

Section View C-C

Scale: 1:2

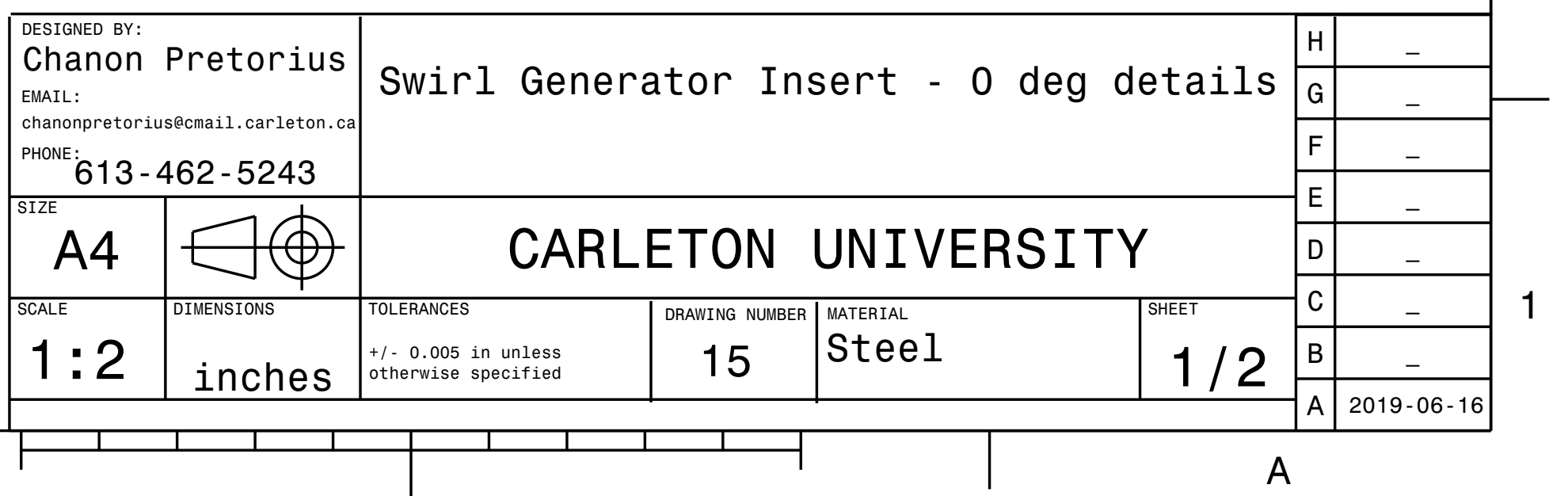




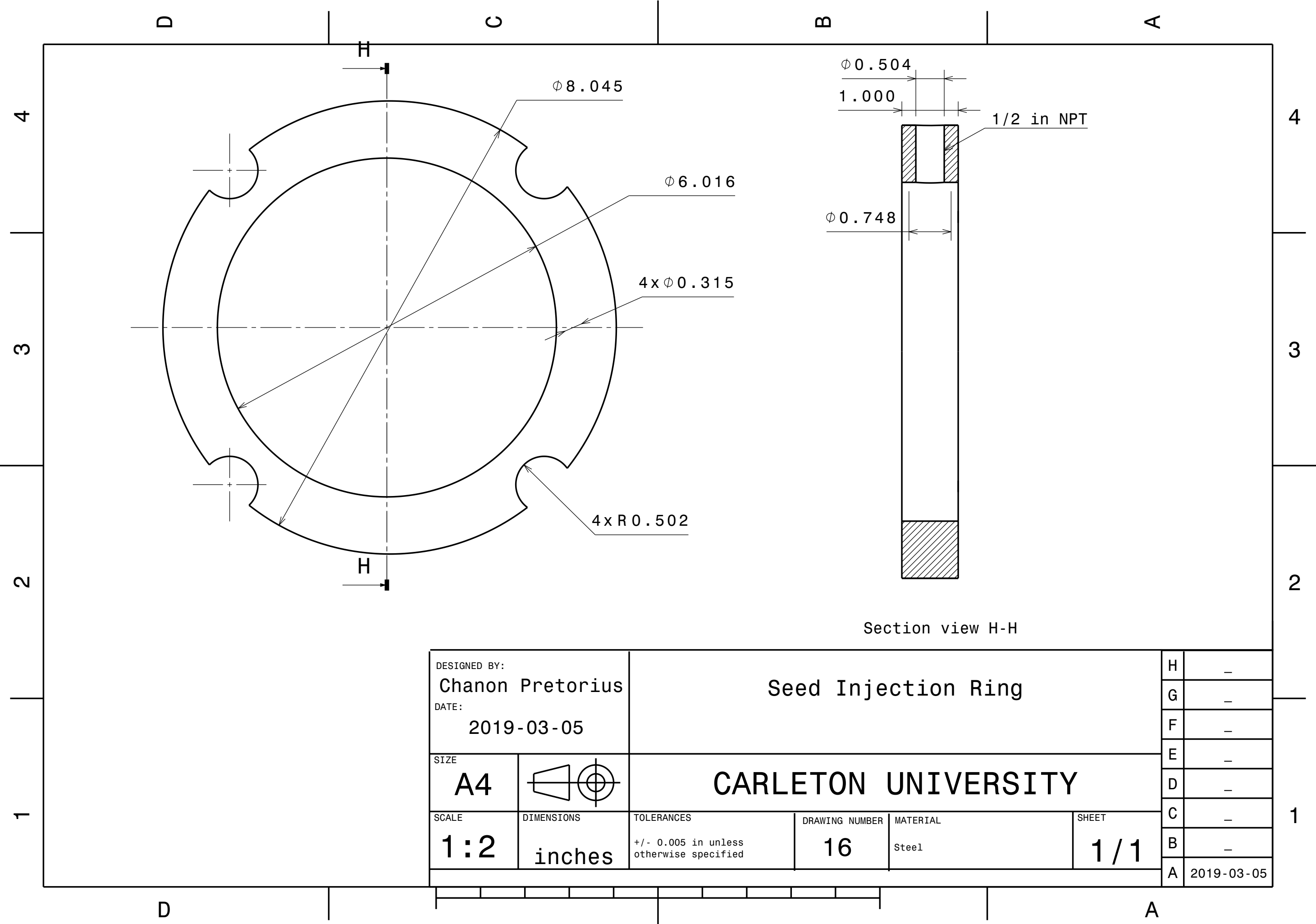




\section{Appendix C}

\section{Finite Element Analysis Benchmark Study}

This appendix presents the results of a benchmarking study used to validate the static structural finite element analysis (FEA) code in ANSYS Mechanical APDL (version 19.2).

\section{C.1 Pressurized Thick-Walled Cylinder}

\section{C.1.1 Computational Model}

Fig. C.1 presents the computational model used for the pressurized thick-walled cylinder benchmark. The geometric and mechanical properties of the cylinder are the same as those used by NAFEMS (1989) as a recommended test for finite element codes, and are listed in Table C.2. The internal pressure of the cylinder is increased from $80 \mathrm{MPa}$ to $120 \mathrm{MPa}$ in increments of $20 \mathrm{MPa}$. All the faces and edges of the cylinder are constrained from moving or deforming along the axial (x-axis) direction but are free to deform in the radial direction. The computational grid consists of approximately 50000 uniformly distributed tetrahedral elements, the details of which are presented in Table C.1 . 


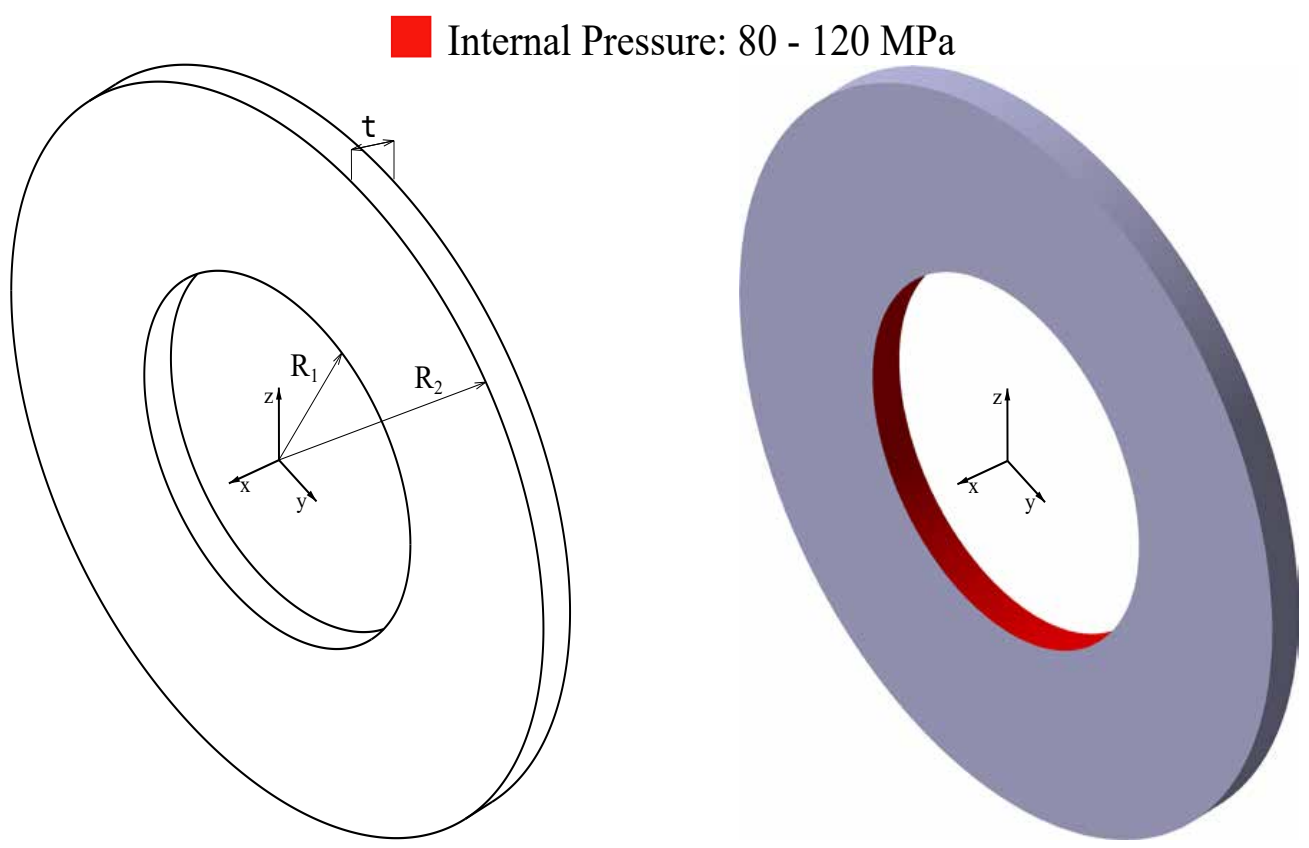

Figure C.1: Computational model of the pressurized thick-walled cylinder.

Table C.1: Parameters of the computational grid.

\begin{tabular}{lcc}
\hline & $\begin{array}{c}\text { Element size }(\mathrm{mm}) \\
\min / \max \end{array}$ & $\begin{array}{c}\text { Spatial growth rate } \\
\min / \max \end{array}$ \\
\hline Thick-walled Cylinder & $2.0 / 2.0$ & $0 \% / 0 \%$ \\
\hline
\end{tabular}

Table C.2: Geometric and mechanical properties of the thick-walled cylinder.

\begin{tabular}{lc}
\hline Inner radius, $R_{1}$ & $100 \mathrm{~mm}$ \\
Outer radius, $R_{2}$ & $200 \mathrm{~mm}$ \\
thickness, $t$ & $20 \mathrm{~mm}$ \\
Young's modulus & $207 \mathrm{GPa}$ \\
Poisson's ratio & 0.3 \\
Yield stress & $207.9 \mathrm{MPa}$ \\
\hline
\end{tabular}




\section{C.1.2 Results}

Fig. C.2 and Fig. C.3 present the radial distribution of the hoop stress, $\sigma_{\theta \theta}$, and radial stress, $\sigma_{r r}$, respectively. The ANSYS results agree favorably with those of the reference solution (NAFEMS, 1989). The maximum difference between the two solutions is about $3 \%$.

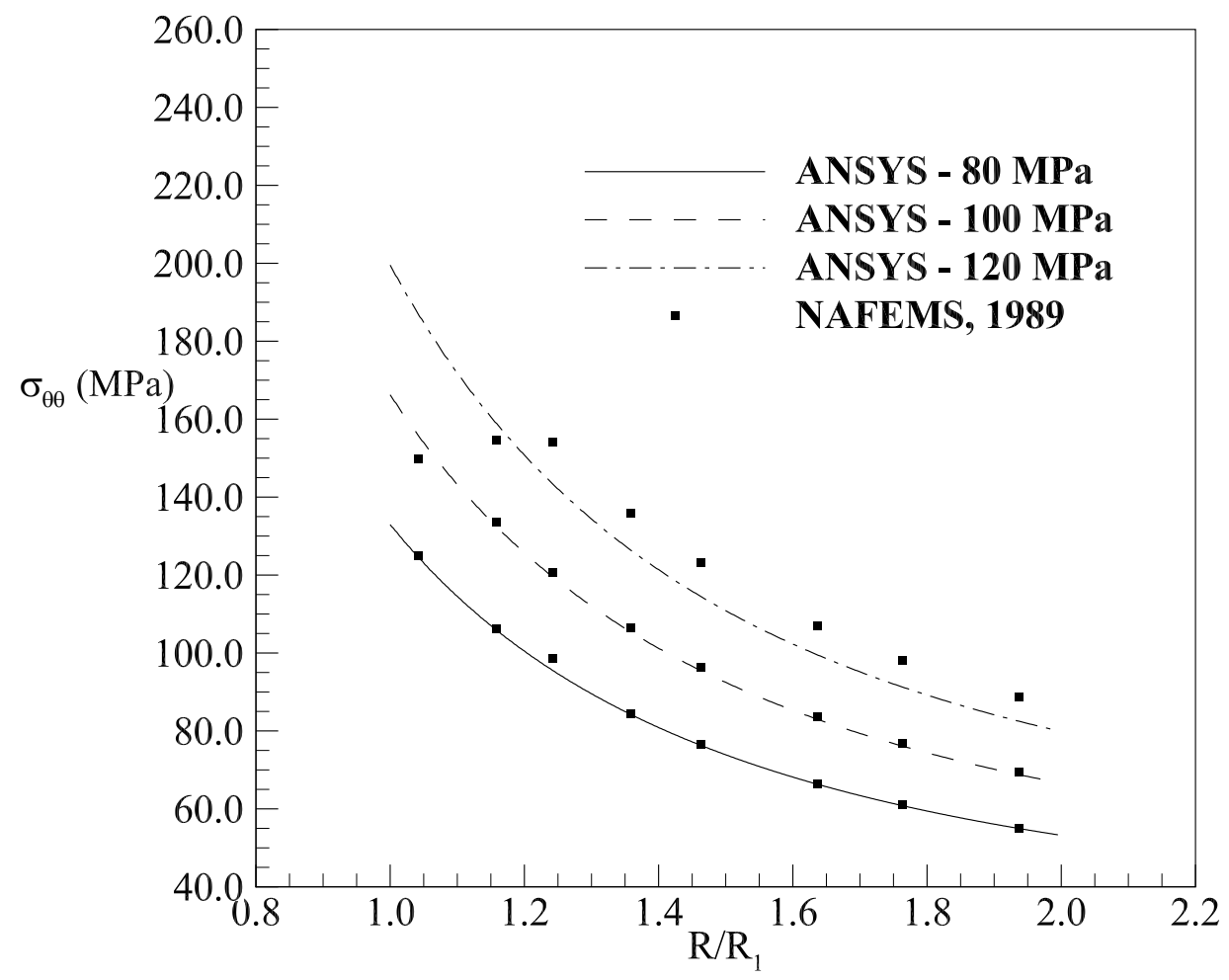

Figure C.2: Comparison of radial distribution of hoop stress between the ANSYS solution and the reference solution. 


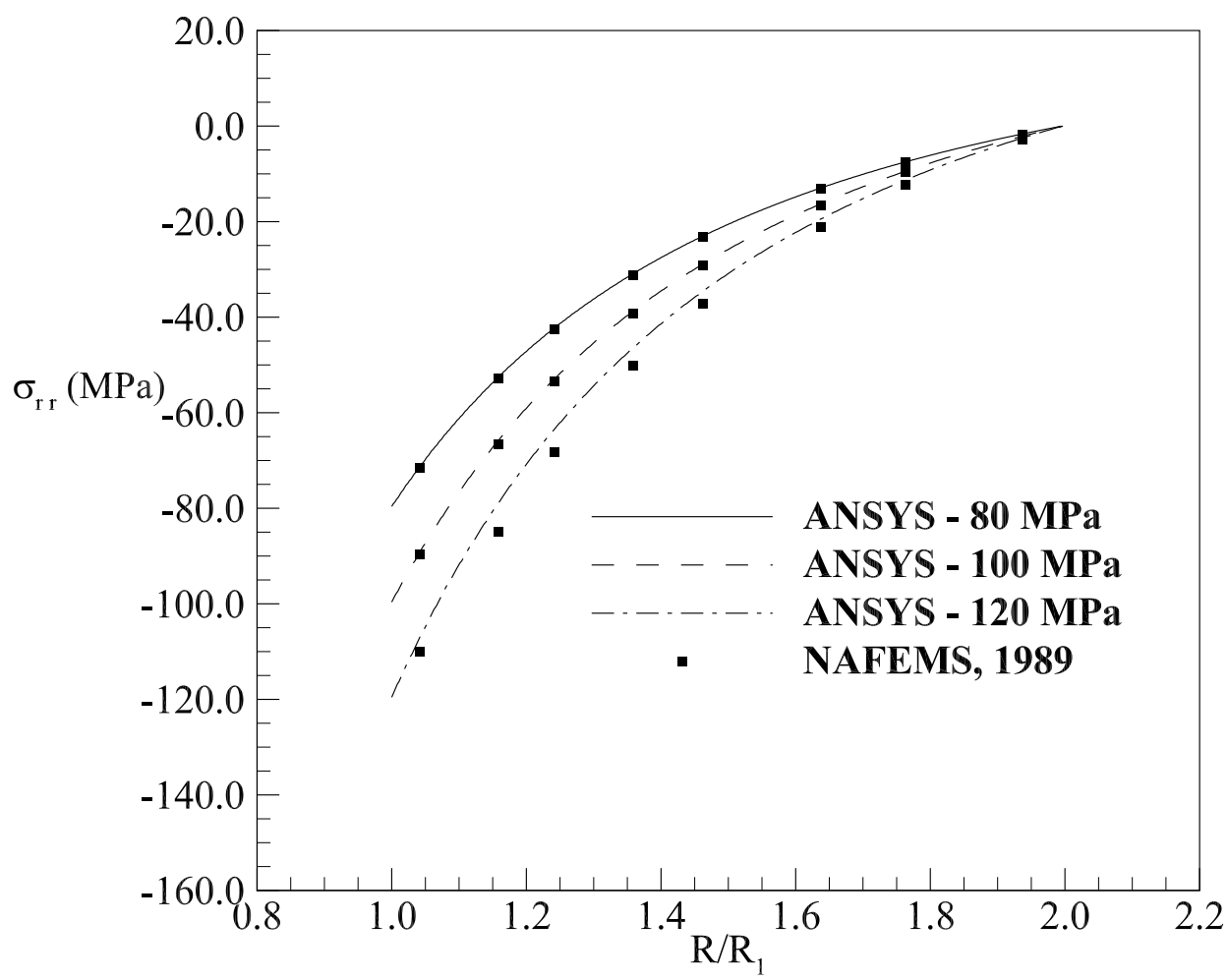

Figure C.3: Comparison of radial distribution of radial stress between the ANSYS solution and the reference solution.

\section{C.2 Circular Beam in Bending}

\section{C.2.1 Computational Model}

Fig. C.4 presents the computational model used for the circular beam in bending benchmark. The geometric and mechanical properties of the beam are the same as those used by NAFEMS (1993) as a recommended test for finite element codes, and are listed in Table C.4. A symmetry boundary condition is applied on the beam face at $x=0 \mathrm{~mm}$, while a moment, $M$ about the $\mathrm{y}$-axis is applied to the opposite face of the cylinder at $x=-50 \mathrm{~mm}$. All the faces and edges of the beam are constrained from moving or deforming in the $\mathrm{y}$ or $\mathrm{x}$ directions and from rotating about the $\mathrm{x}$ or $\mathrm{z}$ axes. The computational grid consists of approximately 20000 uniformly distributed tetrahedral elements, the details of which are presented in Table C.3 . 


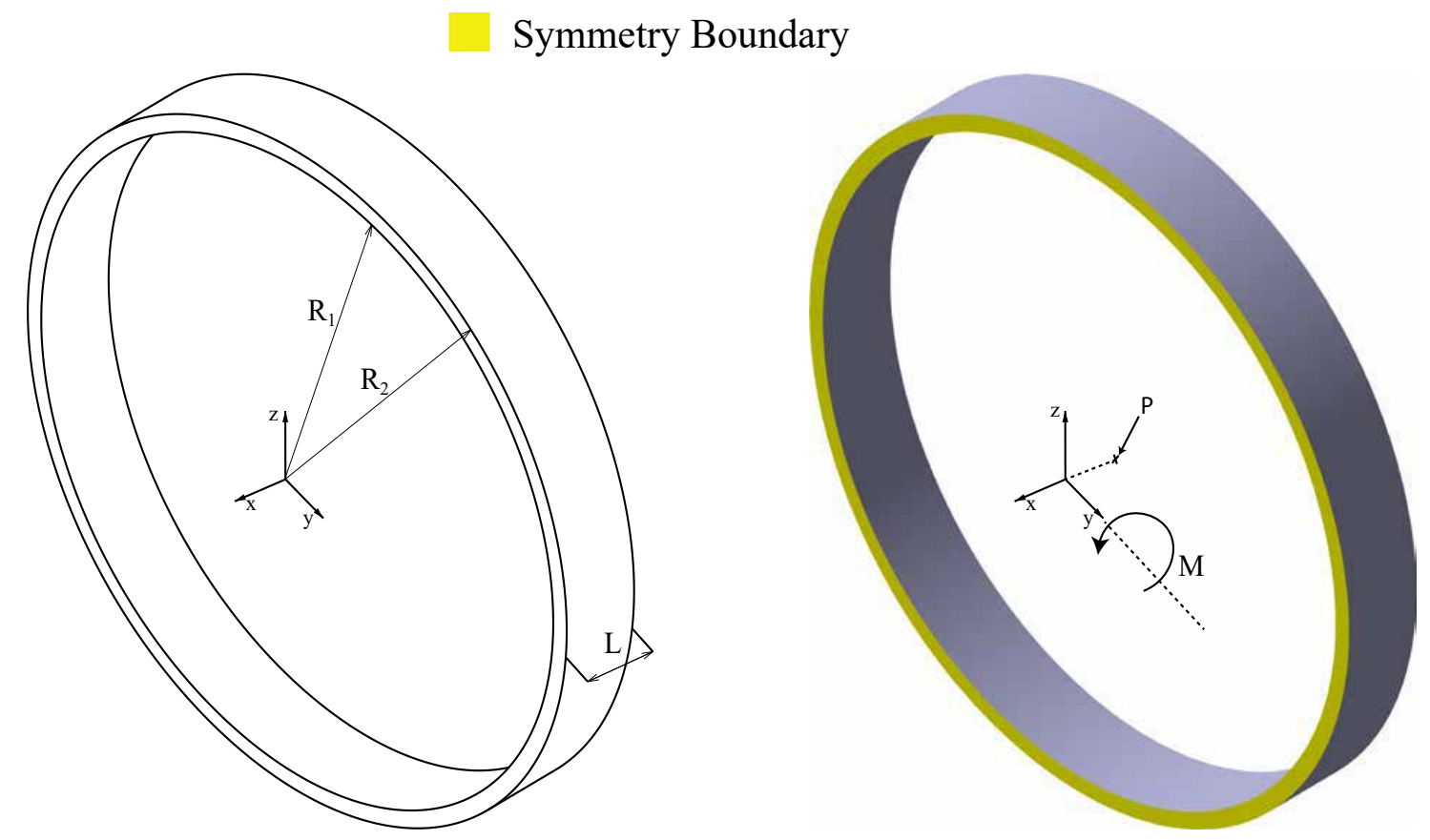

Figure C.4: Computational model of the circular beam.

Table C.3: Parameters of the computational grid.

\begin{tabular}{ccc}
\hline & $\begin{array}{c}\text { Element size }(\mathrm{mm}) \\
\min / \max \end{array}$ & $\begin{array}{c}\text { Spatial growth rate } \\
\min / \max \end{array}$ \\
\hline Circular Beam & $2.0 / 2.0$ & $0 \% / 0 \%$ \\
\hline
\end{tabular}

Table C.4: Geometric and mechanical properties of the circular beam.

\begin{tabular}{lc}
\hline Inner radius, $R_{1}$ & $390 \mathrm{~mm}$ \\
Outer radius, $R_{2}$ & $400 \mathrm{~mm}$ \\
Length, $L$ & $50 \mathrm{~mm}$ \\
Young's modulus & $193 \mathrm{GPa}$ \\
Poisson's ratio & 0.26 \\
\hline
\end{tabular}




\section{C.2.2 Results}

Fig. C.5 presents the moment-rotation curve for point P (Fig. C.4). The point is fixed in space, located along the $\mathrm{x}$-axis at $x=-100 \mathrm{~mm}$. Three moment magnitudes were applied to the beam and compared to the moment-rotation curve in NAFEMS (1993). The ANSYS results agree favorably with those of the reference solution (NAFEMS, 1993).

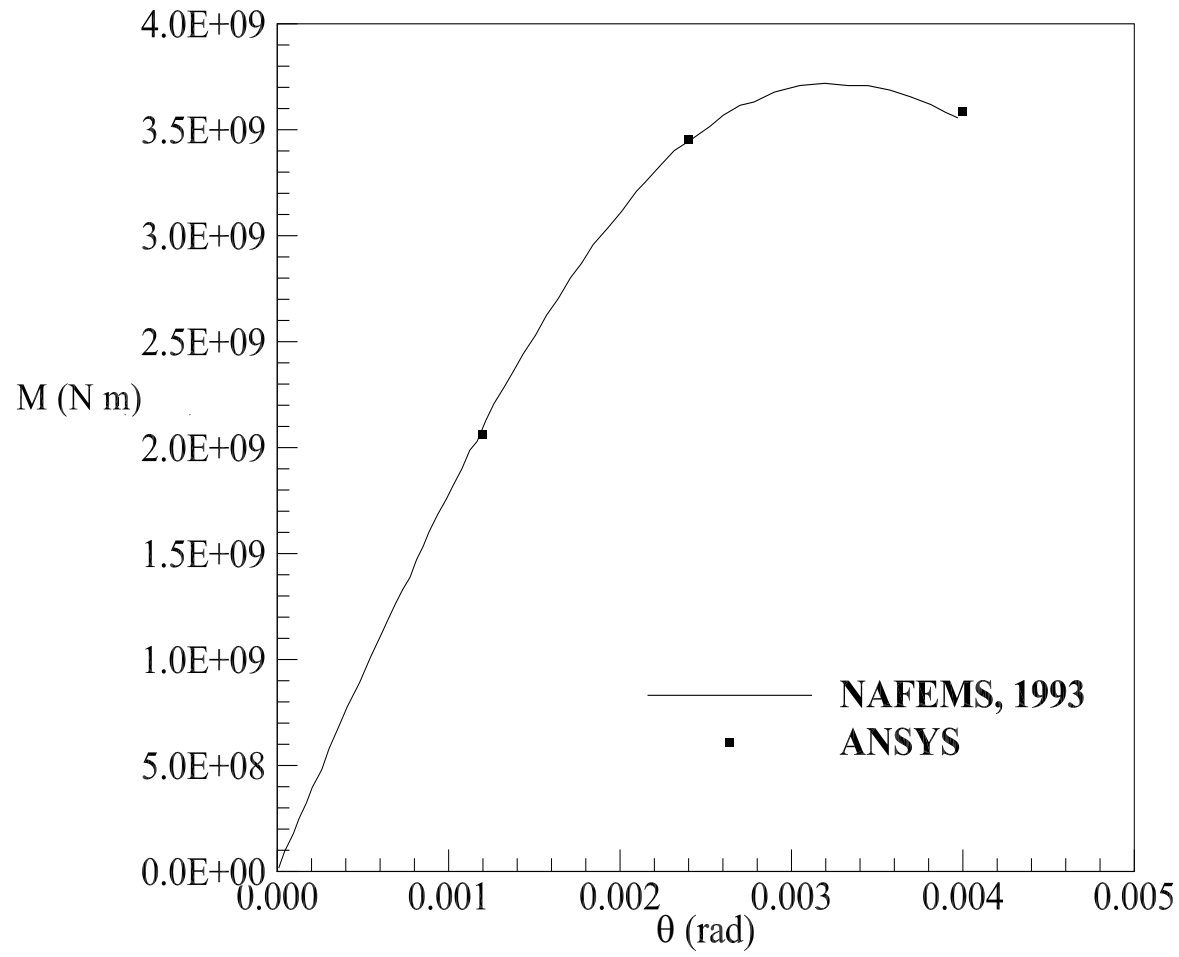

Figure C.5: Comparison of moment-rotation curves between ANSYS and reference solution. 


\section{Appendix D}

\section{Aerodynamic Performance Prediction Algorithm}

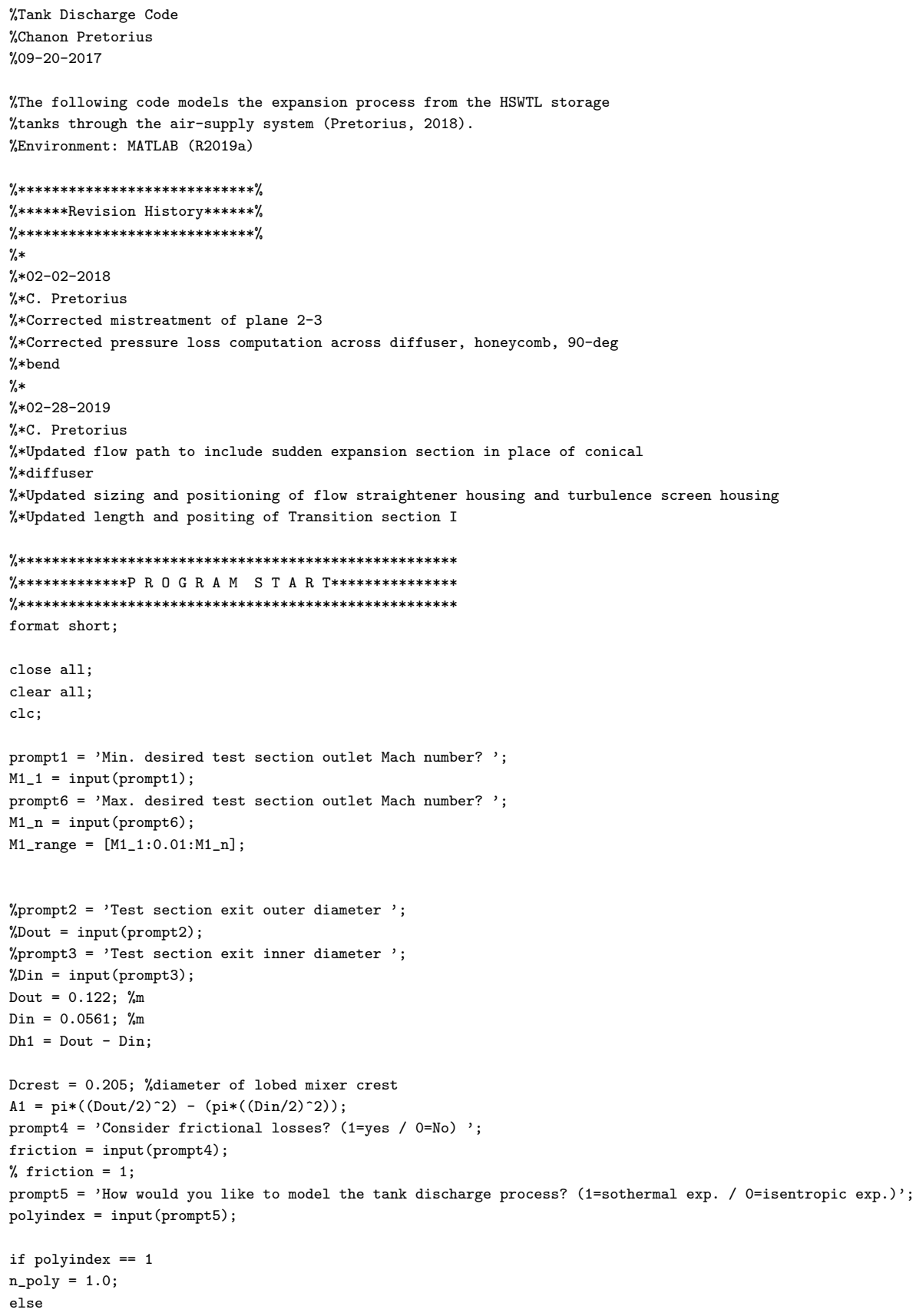




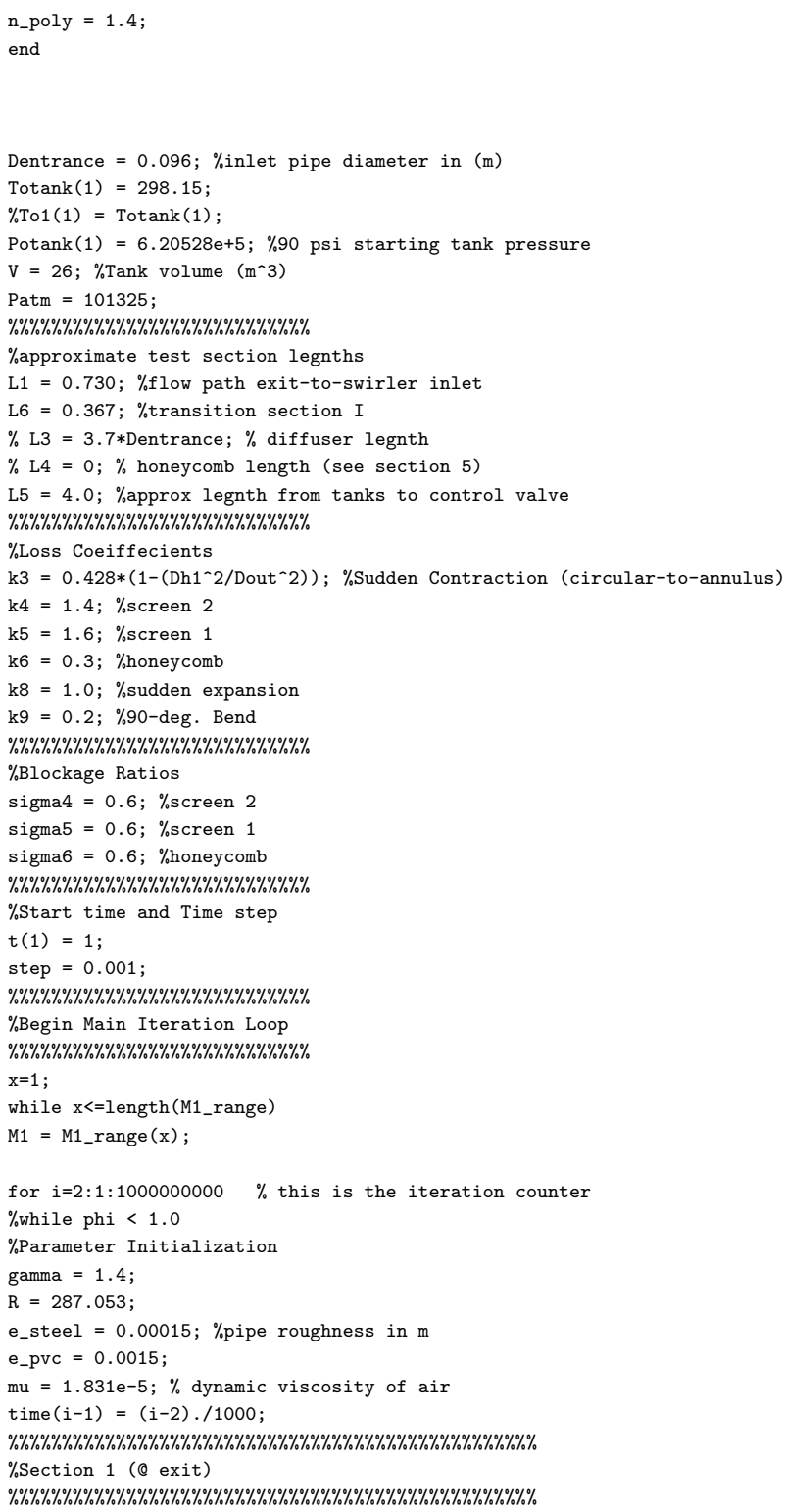




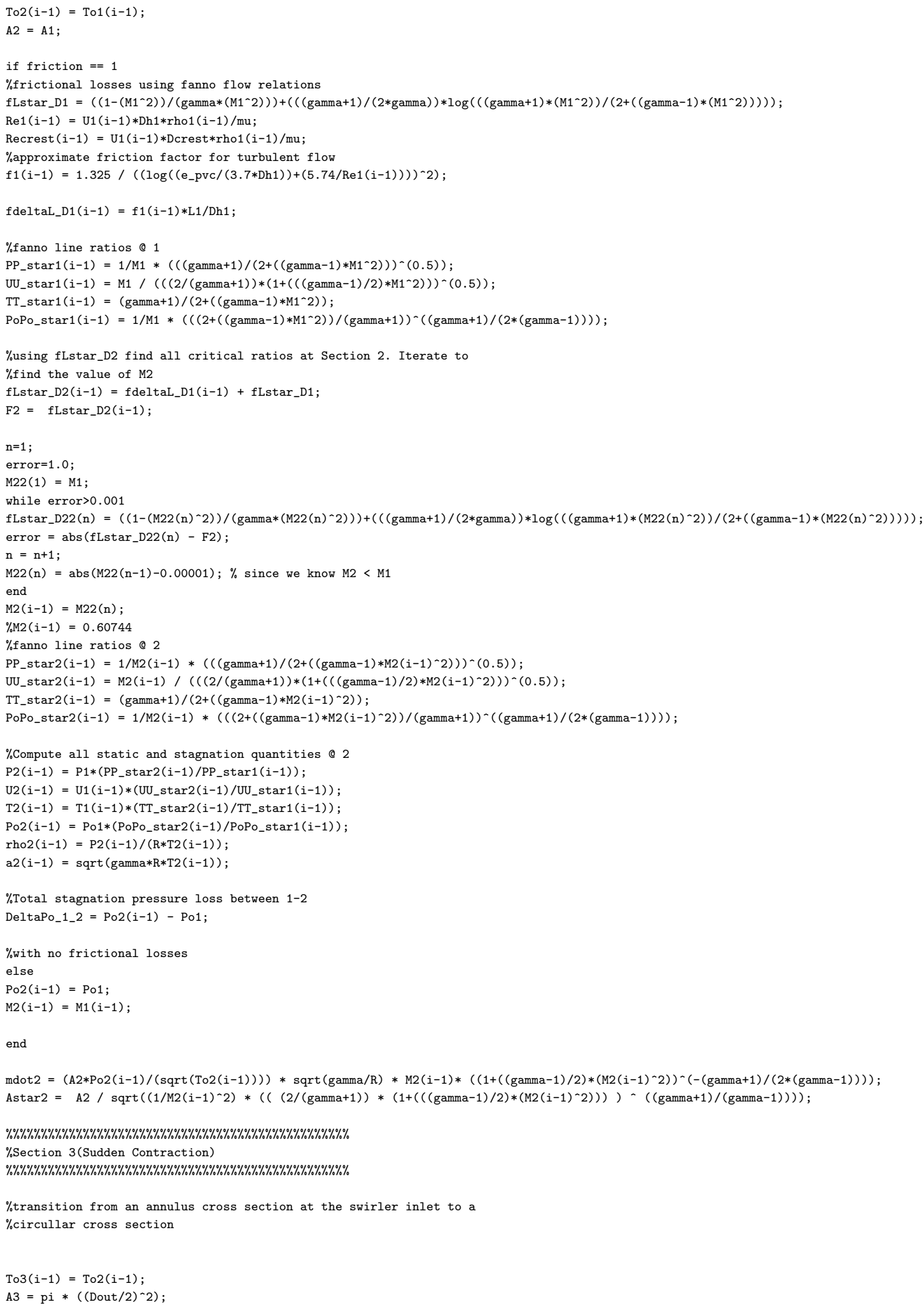




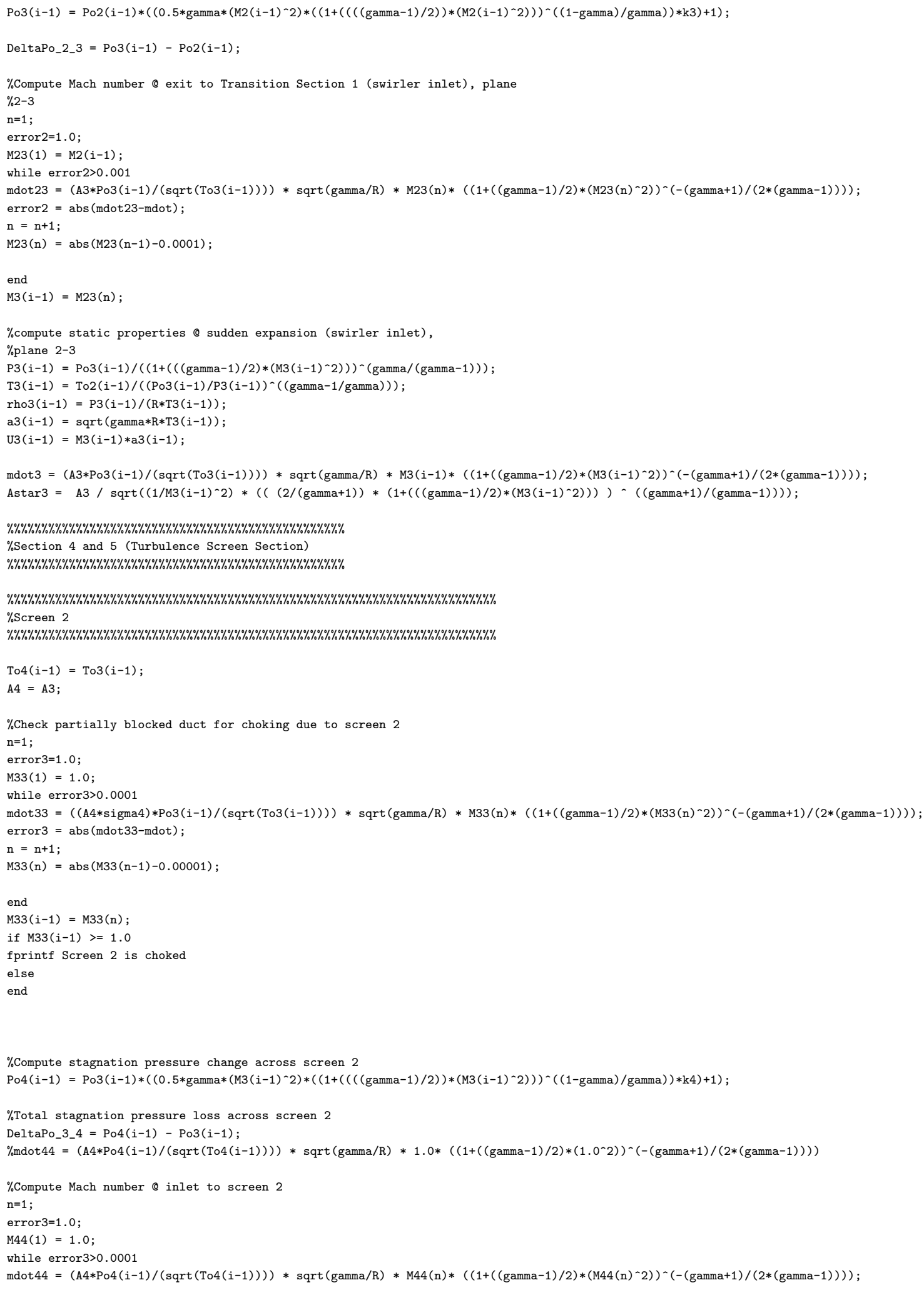




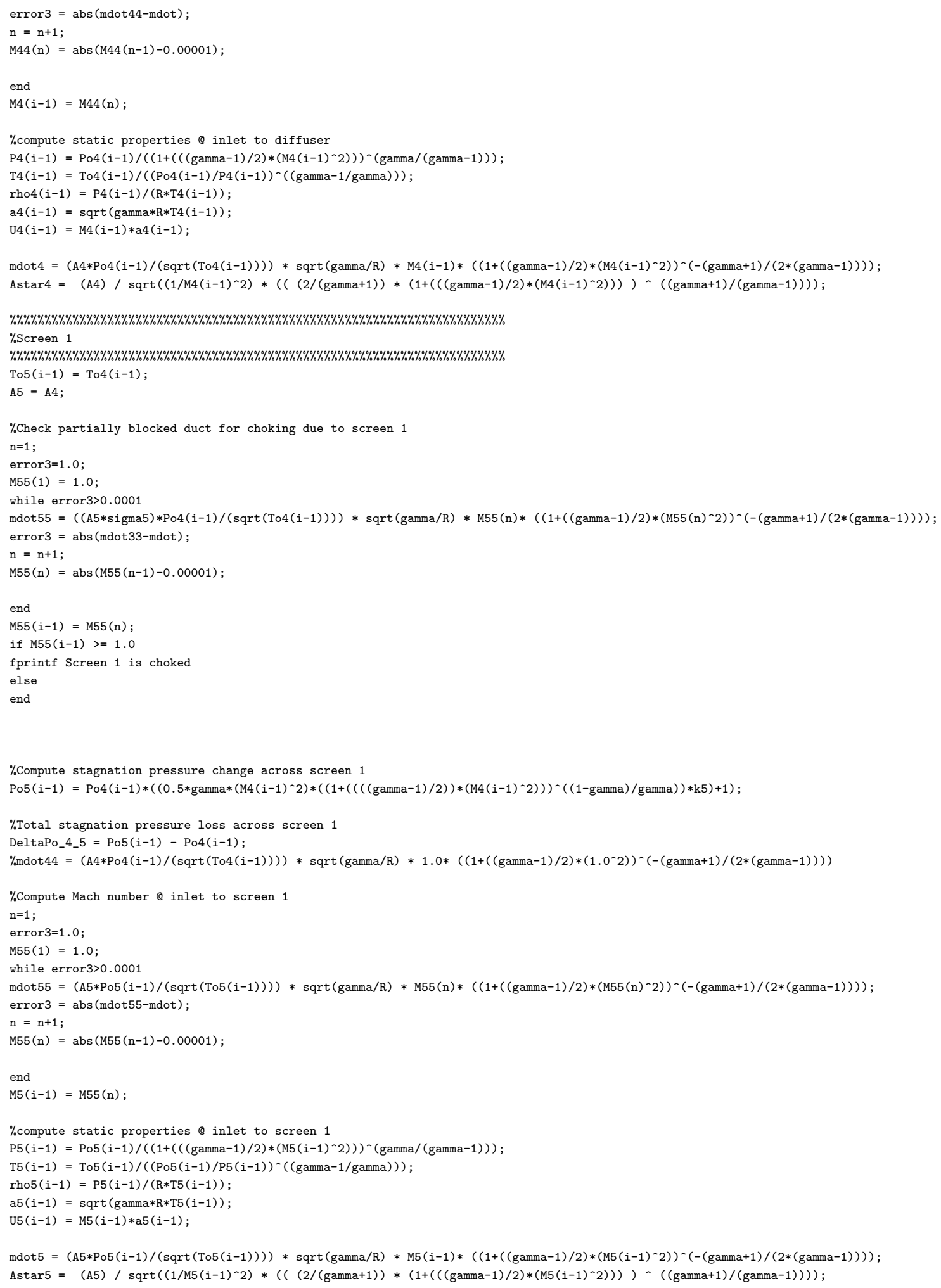




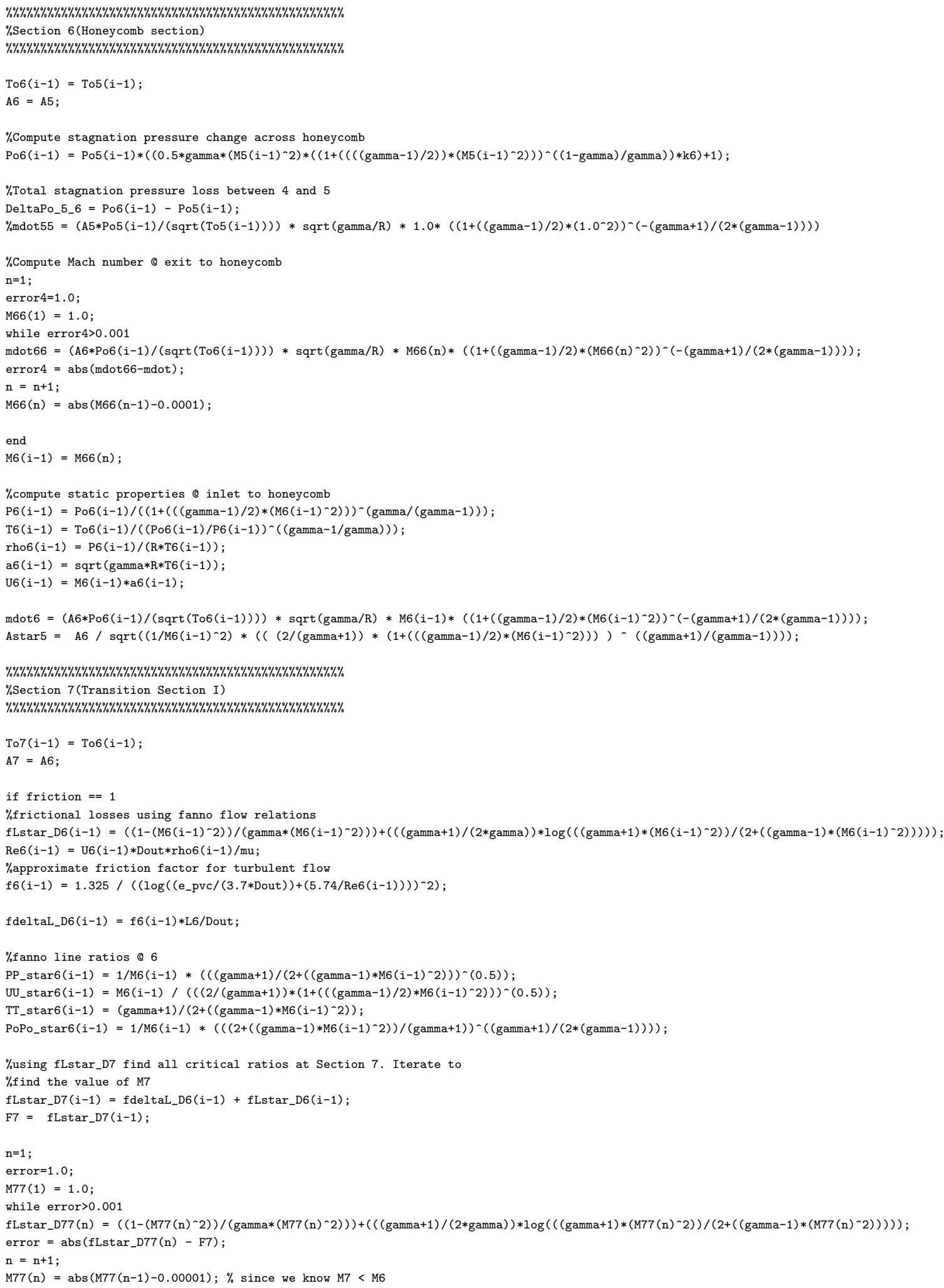


$M 7(i-1)=M 77(n)$

$\% \mathrm{M} 2(\mathrm{i}-1)=0.60744$

$\%$ fanno line ratios $Q 2$

PP_star7 $(i-1)=1 / \mathrm{M} 7(i-1) *((($ gamma+1) $) /(2+(($ gamma-1) $* \mathrm{M} 7(i-1) \wedge 2)))-(0.5))$;

UU_star7 $(i-1)=M 7(i-1) /(((2 /($ gamma +1$)) *(1+((($ gamma-1 $) / 2) * M 7(i-1) \wedge 2))) \sim(0.5))$

TT_star7 $(i-1)=($ gamma +1$) /(2+(($ gamma-1) $* \mathrm{M} 7(i-1))-2))$;

PoPo_star7 $(i-1)=1 / M 7(i-1) *(((2+(($ gamma-1) $) * M 7(i-1) \sim 2)) /($ gamma +1$)) \sim(($ gamma+1 $) /(2 *($ gamma-1 $))))$;

$\%$ Compute all static and stagnation quantities \& 7

$\mathrm{P} 7(i-1)=\mathrm{P} 6(i-1) *\left(\mathrm{PP}_{-} \operatorname{star} 7(\mathrm{i}-1) / \mathrm{PP}_{-} \operatorname{star} 6(i-1)\right) ;$

$\mathrm{U} 7(\mathrm{i}-1)=\mathrm{U} 6(\mathrm{i}-1) *\left(\mathrm{UU}_{-} \mathrm{s} \operatorname{tar} 7(\mathrm{i}-1) / \mathrm{UU}_{-} \mathrm{star} 6(\mathrm{i}-1)\right) ;$

$\mathrm{T} 7(\mathrm{i}-1)=\mathrm{T} 6(\mathrm{i}-1) *\left(\mathrm{TT}_{-} \mathrm{star} 7(\mathrm{i}-1) / \mathrm{TT}_{-} \mathrm{star6}(\mathrm{i}-1)\right) ;$

Po7 $(i-1)=$ Po6 (i-1) $*($ PoPo_star7(i-1)/PoPo_star6(i-1));

$\operatorname{rho7}(i-1)=\mathrm{P} 7(i-1) /(\mathrm{R} * \mathrm{~T} 7(\mathrm{i}-1))$;

$\mathrm{a} 7(i-1)=\operatorname{sqrt}(\operatorname{gamma} * \mathrm{R} * \mathrm{T7}(i-1))$;

\% Total stagnation pressure loss between 6-7

DeltaPo_6_7 = Po7(i-1) - Po6(i-1);

\%with no frictional losses

else

Po7 $(i-1)=\operatorname{Po6}(i-1)$;

$M 7(i-1)=M 6(i-1)$

end

$\operatorname{mdot} 7=(\mathrm{A} 7 * \operatorname{Po} 7(\mathrm{i}-1) /(\operatorname{sgrt}(\mathrm{To} 7(\mathrm{i}-1)))) * \operatorname{sqrt}(\operatorname{gamma} / \mathrm{R}) * \mathrm{M7}(\mathrm{i}-1) *((1+((\operatorname{gamma}-1) / 2) *(\mathrm{M} 7(\mathrm{i}-1) \sim 2)) \sim(-(\operatorname{gamma}+1) /(2 *(\operatorname{gamma}-1)))) ;$ Astar7 $=A 7 / \operatorname{sqrt}((1 / \operatorname{M7}(i-1)-2) *(((2 /($ gamma +1$)) *(1+((($ gamma-1) $) / 2) *(M 7(i-1) \wedge 2))))-(($ gamma +1$) /(\operatorname{gamma}-1)))) ;$

$\% \% \% \% \% \% \% \% \% \% \% \% \% \% \% \% \% \% \% \% \% \% \% \% \% \% \% \% \% \% \% \% \% \% \% \% \% \% \% \% \% \% \% \% \% \% \% \% \%$

$\%$ Section 8 (Sudden Expansion)

$\% \% \% \% \% \% \% \% \% \% \% \% \% \% \% \% \% \% \% \% \% \% \% \% \% \% \% \% \% \%$

$\operatorname{To8}(i-1)=\operatorname{To7}(i-1)$

A8 $=$ pi * ((Dentrance/2) 2$)$;

$\operatorname{Po} 8(\mathrm{i}-1)=\operatorname{Po}(\mathrm{i}-1) *((0.5 *$ gamma* $(M 7(\mathrm{i}-1) \wedge 2) *((1+(((($ gamma-1) $) / 2)) *(\mathrm{M} 7(\mathrm{i}-1) \wedge 2))) \wedge((1-$ gamma $) /$ gamma $)) * \mathrm{k} 8)+1) ;$

DeltaPo_7_8 = Po8(i-1) - Po7(i-1);

\%Compute Mach number @ entrance to sudden expansion

$\mathrm{n}=1$;

error $2=1.0$;

$\operatorname{M8}(1)=1.0 ;$

while error $2>0.001$

$\operatorname{mdot} 88=(\operatorname{A8} *$ Po8 $(i-1) /(\operatorname{sqrt}(\operatorname{To} 8(i-1)))) * \operatorname{sqrt}(\operatorname{gamma} / \mathrm{R}) * \operatorname{M88}(\mathrm{n}) *((1+((\operatorname{gamma}-1) / 2) *(\operatorname{M8} 8(\mathrm{n}) \sim 2)) \sim(-(\operatorname{gamma}+1) /(2 *(\operatorname{gamma}-1))))$

error 2 = abs (mdot88-mdot)

$\mathrm{n}=\mathrm{n}+1$;

$\operatorname{M8}(n)=\operatorname{abs}(\operatorname{M8}(n-1)-0.0001) ;$

end

M8 $(i-1)=\operatorname{M88}(n)$

$\%$ compute static properties 08

$\mathrm{P} 8(\mathrm{i}-1)=\mathrm{Po} 8(\mathrm{i}-1) /\left(\left(1+\left(\left((\text { gamma-1) } / 2) *(\mathrm{M} 8(\mathrm{i}-1))^{-} 2\right)\right)\right)^{-}(\right.$gamma $/($gamma-1) $)) ;$

$\mathrm{T} 8(\mathrm{i}-1)=\mathrm{T} 08(\mathrm{i}-1) /((\mathrm{Po} 8(\mathrm{i}-1) / \mathrm{P} 8(\mathrm{i}-1)) \sim(($ gamma-1/gamma $))) ;$

rho8 $(i-1)=P 8(i-1) /(R * T 8(i-1))$;

$\mathrm{a} 8(\mathrm{i}-1)=\operatorname{sqrt}(\operatorname{gamma} * \mathrm{R} * \mathrm{~T} 8(\mathrm{i}-1))$;

$\mathrm{U} 8(\mathrm{i}-1)=\mathrm{M} 8(\mathrm{i}-1) * \mathrm{a} 8(\mathrm{i}-1)$;

$\operatorname{mdot} 8=($ A8*Po8 $(i-1) /(\operatorname{sqrt}(\operatorname{To} 8(i-1)))) * \operatorname{sqrt}(\operatorname{gamma} / \mathrm{R}) * \operatorname{M8}(\mathrm{i}-1) *((1+((\operatorname{gamma}-1) / 2) *(\operatorname{M} 8(\mathrm{i}-1) \sim 2)) \sim(-(\operatorname{gamma}+1) /(2 *(\operatorname{gamma}-1))))$ Astar8 $=A 8 / \operatorname{sqrt}\left(\left(1 /\right.\right.$ M8 $\left.(i-1)^{-} 2\right) *\left(\left((2 /(\right.\right.$ gamma +1$\left.\left.\left.)) *\left(1+\left(((\operatorname{gamma}-1) / 2) *\left(M 8(i-1)^{-}-2\right)\right)\right)\right)-((\operatorname{gamma}+1) /(\operatorname{gamma}-1))\right)\right) ;$

$\% \% \% \% \% \% \% \% \% \% \% \% \% \% \% \% \% \% \% \% \% \% \% \% \% \% \% \% \% \% \% \% \% \% \% \% \% \% \% \% \% \% \% \% \% \% \% \% \% \%$

$\%$ Section 9 (Across 90 degree bend)

\%\%\%\%\%\%\%\%\%\%\%\%\%\%\%\%\%\%\%\%\%\%\%\%\%\%\%\%\%\%\%\%\%\%\%\%\%\%\%\%\%\%\%\%\%\%\%\%\%\%

$\operatorname{To9}(i-1)=\operatorname{To8}(i-1)$ 
$\%$ Compute stagnation pressure change across 90 -deg bend

Po9 $(i-1)=\operatorname{Po8}(i-1) *((0.5 *$ gamma $*(M 8(i-1)-2) *((1+(((($ gamma-1) $) / 2)) *($ M8 $(i-1) \sim 2))) \sim((1-$ gamma $) /$ gamma $)) * \mathrm{k} 9)+1)$;

$\%$ Total stagnation pressure loss between 5 and 6

DeltaPo_8_9 = Po9(i-1) - Po8(i-1);

$\%$ Compute Mach number $@$ exit to bend

$\mathrm{n}=1$;

error5=1.0;

M99(1) $=1.0$;

while error $5>0.001$

$\operatorname{mdot} 99=(\operatorname{A9} * \operatorname{Po9}(i-1) /(\operatorname{sqrt}(\operatorname{To9}(i-1)))) * \operatorname{sqrt}(\operatorname{gamma} / \mathrm{R}) * \operatorname{M99}(\mathrm{n}) *((1+((\operatorname{gamma}-1) / 2) *(\operatorname{M99}(\mathrm{n}) \sim 2)) \sim(-(\operatorname{gamma}+1) /(2 *(\operatorname{gamma}-1)))) ;$

error5 = abs (mdot99-mdot);

$\mathrm{n}=\mathrm{n}+1$;

$\operatorname{M99}(n)=\operatorname{abs}(\operatorname{M99}(n-1)-0.00001) ;$

end

M9 $(i-1)=\operatorname{M99}(n)$;

$\%$ compute static properties $@$ exit to diffuser

P9 $(i-1)=\operatorname{Po9}(i-1) /\left((1+(((\text { gamma-1 }) / 2) *(\text { M9 }(i-1) \wedge 2)))^{\wedge}(\right.$ gamma $/($ gamma-1) $))$;

$\operatorname{T9}(i-1)=\operatorname{To9}(i-1) /((\operatorname{Pog}(i-1) / P 9(i-1)) \sim(($ gamma-1/gamma $))) ;$

$\operatorname{rho} 9(i-1)=P 9(i-1) /(R * T 9(i-1))$;

a9 $(i-1)=\operatorname{sqrt}($ gamma $* \mathrm{R} * \mathrm{~T} 9(i-1))$;

U9 $(i-1)=$ M9 $(i-1) * a 9(i-1)$;

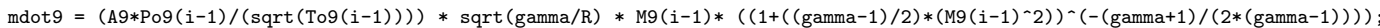

Astar9 $=A 9 / \operatorname{sqrt}((1 /$ M9 $(i-1)-2) *(((2 /($ gamma +1$)) *(1+((($ gamma-1) $) / 2) *($ M9 $(i-1)-2))))-(($ gamma +1$) /($ gamma-1) $)))$;

$\% \% \% \% \% \% \% \% \% \% \% \% \% \% \% \% \% \% \% \% \% \% \% \% \% \% \% \% \% \% \% \% \% \% \% \% \% \% \% \% \% \% \% \% \% \% \% \%$

$\%$ Section 10 (Across control valve)

$\% \% \% \% \% \% \% \% \% \% \% \% \% \% \% \% \% \% \% \% \% \% \% \% \% \% \% \% \% \% \% \% \% \% \% \% \% \% \% \% \% \% \% \% \% \% \% \% \% \% \% \%$

$\%$ assume that stagnation pressure upstream of valve is equal to tank pressure

Po10 (i-1) = Potank $(i-1)$

$\%$ assume that the valve is choked, so velocity just upstream is $M=1.0$

$\mathrm{M} 10(\mathrm{i}-1)=1.0$;

\% Po_10 $(\mathrm{i}-1)=\operatorname{Po9}(\mathrm{i}-1) *\left(\left(0.5 *\right.\right.$ gamma $\left.*\left(\mathrm{M} 9(\mathrm{i}-1)^{\sim} 2\right) *\left(\left(1+\left((((\text { gamma-1) }) / 2)) *\left(\mathrm{M} 9(\mathrm{i}-1)^{\wedge} 2\right)\right)\right)^{\wedge}(((1-\operatorname{gamma}) / \operatorname{gamma})) * \mathrm{k} 10\right)+1\right) ;$

To10 $(i-1)=\operatorname{To9}(i-1)$;

$\mathrm{T} 10(i-1)=\mathrm{To} 10(\mathrm{i}-1) /((1+((($ gamma-1) $/ 2) * \mathrm{M} 10(\mathrm{i}-1) \sim 2))) ;$

$\mathrm{P} 10(\mathrm{i}-1)=\mathrm{Po10}(\mathrm{i}-1) /((\mathrm{To1})(\mathrm{i}-1) / \mathrm{T} 10(\mathrm{i}-1)) \sim($ gamma $/($ gamma-1) $))) ;$

rho10 $(i-1)=\mathrm{P} 10(i-1) /(\mathrm{R} * \mathrm{~T} 10(i-1))$;

\%assuming that stagnation pressure upstream of valve is the same as the

$\%$ tank pressure

$\left.\% \operatorname{Avalve}(i-1)=\operatorname{mdot} /\left((\operatorname{Po} 10(i-1) / \operatorname{sqrt}(\operatorname{To} 10(i-1))) *(\operatorname{sqrt}(\operatorname{gamma} / \mathrm{R})) * M 10(i-1) *\left(\left(1+\left(((\operatorname{gamma}-1) / 2) *\left(\operatorname{M10}(i-1)^{\wedge} 2\right)\right)\right)\right)^{\wedge}((-\operatorname{gamma}+1) /(2 *(\operatorname{gamma}-1)))\right)\right) ; \% \mathrm{~m}^{\wedge} 2$

$\left.\operatorname{Avalve}(i-1)=\operatorname{mdot} /\left((\operatorname{Po1} 0(i-1) /(\operatorname{sqrt}(\operatorname{To} 10(i-1)))) * \operatorname{sqrt}(\operatorname{gamma} / \mathrm{R}) * \operatorname{M10}(\mathrm{i}-1) *\left(\left(1+\left(((\operatorname{gamma}-1) / 2) *\left((\operatorname{M10}(\mathrm{i}-1))^{-} 2\right)\right)\right)\right)^{-}(-(\operatorname{gamma}+1) /(2 *(\operatorname{gamma}-1)))\right)\right) ;$

phi $=$ Avalve $(i-1) / A 9 ;$

flag1 $=$ phi; \%phi is the percentage that the valve is opened. Codse stops when phi $=1.0$. Keeep flag on output to see real time progresssion of valve opening.

$\%$ keeping flag1 active slows down computation, but provides feedback that

$\%$ code is progressing in time

$\%$ end

$\% \operatorname{mdot} 10=(\operatorname{Avalve}(\mathrm{i}-1) * \operatorname{Po} 10(\mathrm{i}-1) /(\operatorname{sqrt}($ To10 $(i-1)))) * \operatorname{sqrt}(\operatorname{gamma} / \mathrm{R}) * \mathrm{M} 10(\mathrm{i}-1) *((1+((\operatorname{gamma}-1) / 2) *(\mathrm{M} 10(\mathrm{i}-1) \wedge-2)) \sim(-(\operatorname{gamma}+1) /(2 *(\operatorname{gamma}-1)))) ;$

$\operatorname{mdot} 10=(\operatorname{Avalve}(i-1) * \operatorname{Po} 10(i-1) /(\operatorname{sqrt}(\operatorname{To} 10(i-1)))) * \operatorname{sqrt}(\operatorname{gamma} / \mathrm{R}) *$ M10 $(\mathrm{i}-1) *((1+((\operatorname{gamma}-1) / 2) *(\operatorname{M10}(\mathrm{i}-1) \wedge 2)) \sim(-(\operatorname{gamma}+1) /(2 *(\operatorname{gamma}-1)))) ;$

$\% \% \% \% \% \% \% \% \% \% \% \% \% \% \% \% \% \% \% \% \% \% \% \% \% \% \% \% \% \% \% \% \% \% \% \% \% \% \% \%$

$\%$ Storage Tanks

$\% \% \% \% \% \% \% \% \% \% \% \% \% \% \% \% \% \% \% \% \% \% \% \% \% \% \% \% \% \% \% \% \% \% \% \% \% \% \% \%$

$\%$ If valve is fully open, stop run 


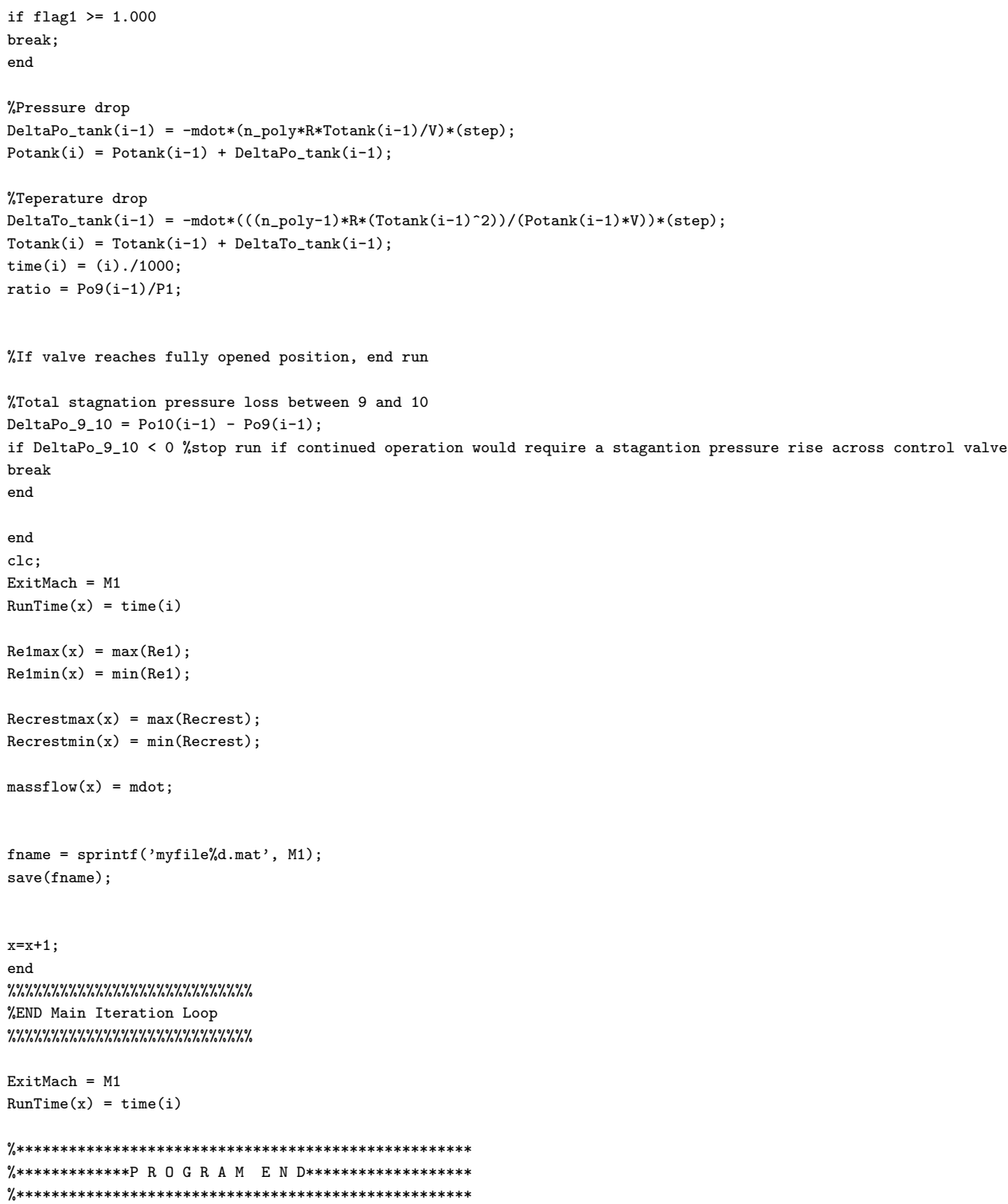




\section{Appendix E}

\section{Technical Drawings for the Rectilinear Test Section}

This page left intentionally blank. 


\begin{tabular}{|l|l|l|l|}
\hline Part & Designation & Material & Quantity \\
\hline 0 & Existing Steel Fange & Steel & - \\
\hline 1 & $7 / 8$ UNC Bolt & Steel & 8 \\
\hline 2 & Wedge & Aluminum & 2 \\
\hline 3 & M6 Machine Screw & Steel & 12 \\
\hline 4 & Lobed Tail & Steel & 1 \\
\hline 5 & Hub Tail & Aluminum & 1 \\
\hline
\end{tabular}
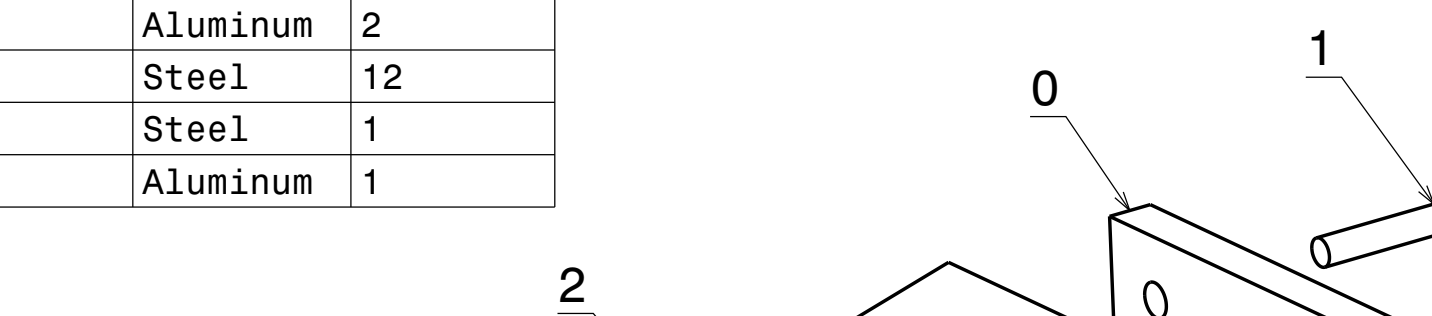

(2)
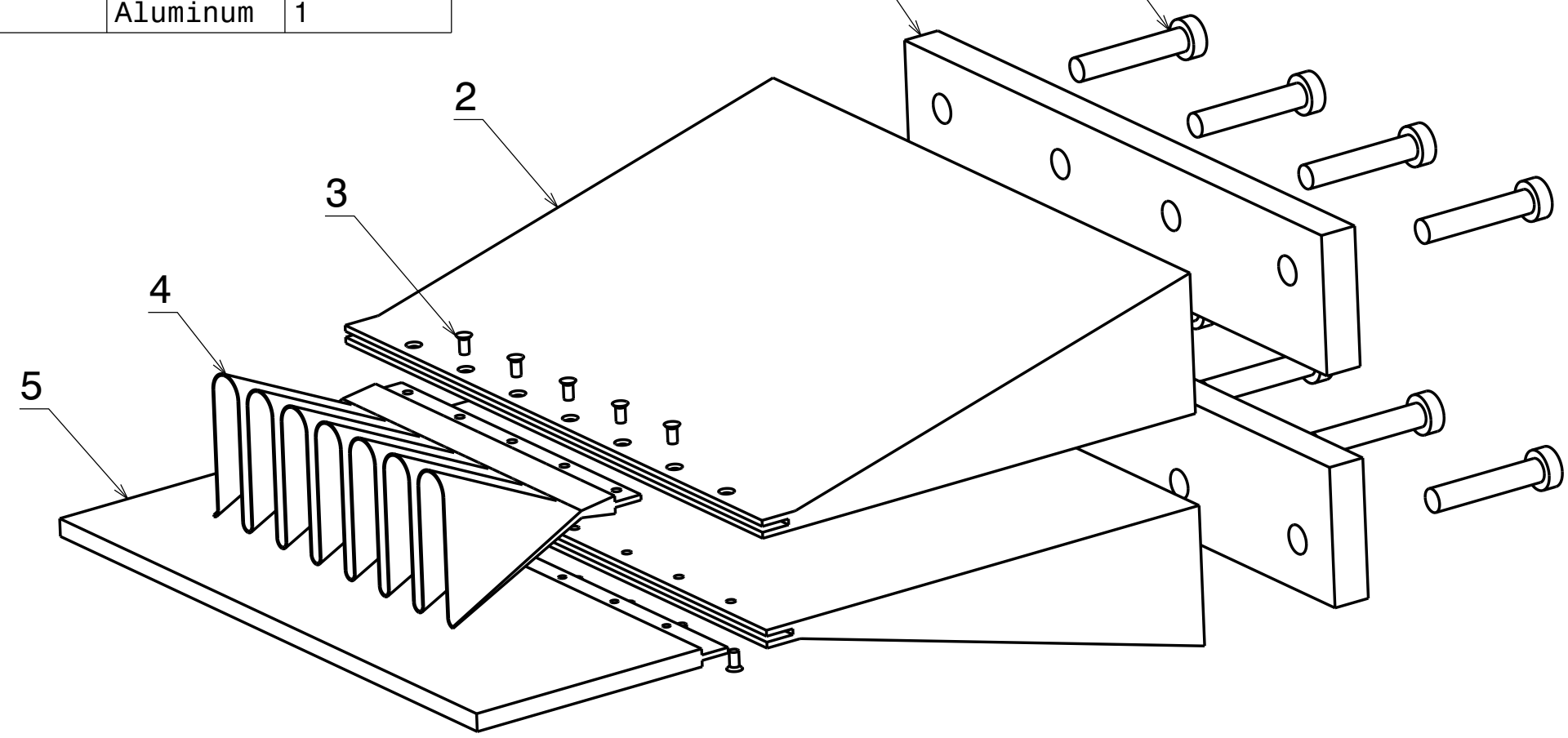

3

N

\begin{tabular}{|c|c|c|c|c|c|c|c|}
\hline \multirow{4}{*}{\multicolumn{2}{|c|}{$\begin{array}{l}\text { DESIGNED BY: } \\
\text { Chanon Pretorius } \\
\text { EMAIL: } \\
\text { chanonpretorius@ecmail.carleton.ca } \\
\text { PHONE: } 613-462-5243 \\
\quad 613\end{array}$}} & \multirow{3}{*}{\multicolumn{4}{|c|}{$\begin{array}{c}\text { Rectilinear Test Section Assembly: } \\
\text { Exploded View }\end{array}$}} & $H$ & - \\
\hline & & & & & & G & - \\
\hline & & & & & & $\mathrm{F}$ & - \\
\hline & & \multirow{2}{*}{\multicolumn{4}{|c|}{ CARLETON UNIVERSITY }} & $\mathrm{E}$ & - \\
\hline A4 & $\uplus \$$ & & & & & D & - \\
\hline \multirow{2}{*}{$1: 5$} & \multirow{2}{*}{ inches } & TOLERANCES & DRAWING NUMBER & MATERIAL & SHEET & C & $2019-08-31$ \\
\hline & & $\begin{array}{l}+/ .0 .005 \text { in unless } \\
\text { otherwise specified }\end{array}$ & 1 & $\begin{array}{l}\text { See proceeding } \\
\text { drawings }\end{array}$ & $1 / 1$ & $B$ & 2019-08-27 \\
\hline
\end{tabular}

\begin{tabular}{|l|l|l|l|l|l|l|l|}
\hline &
\end{tabular} 


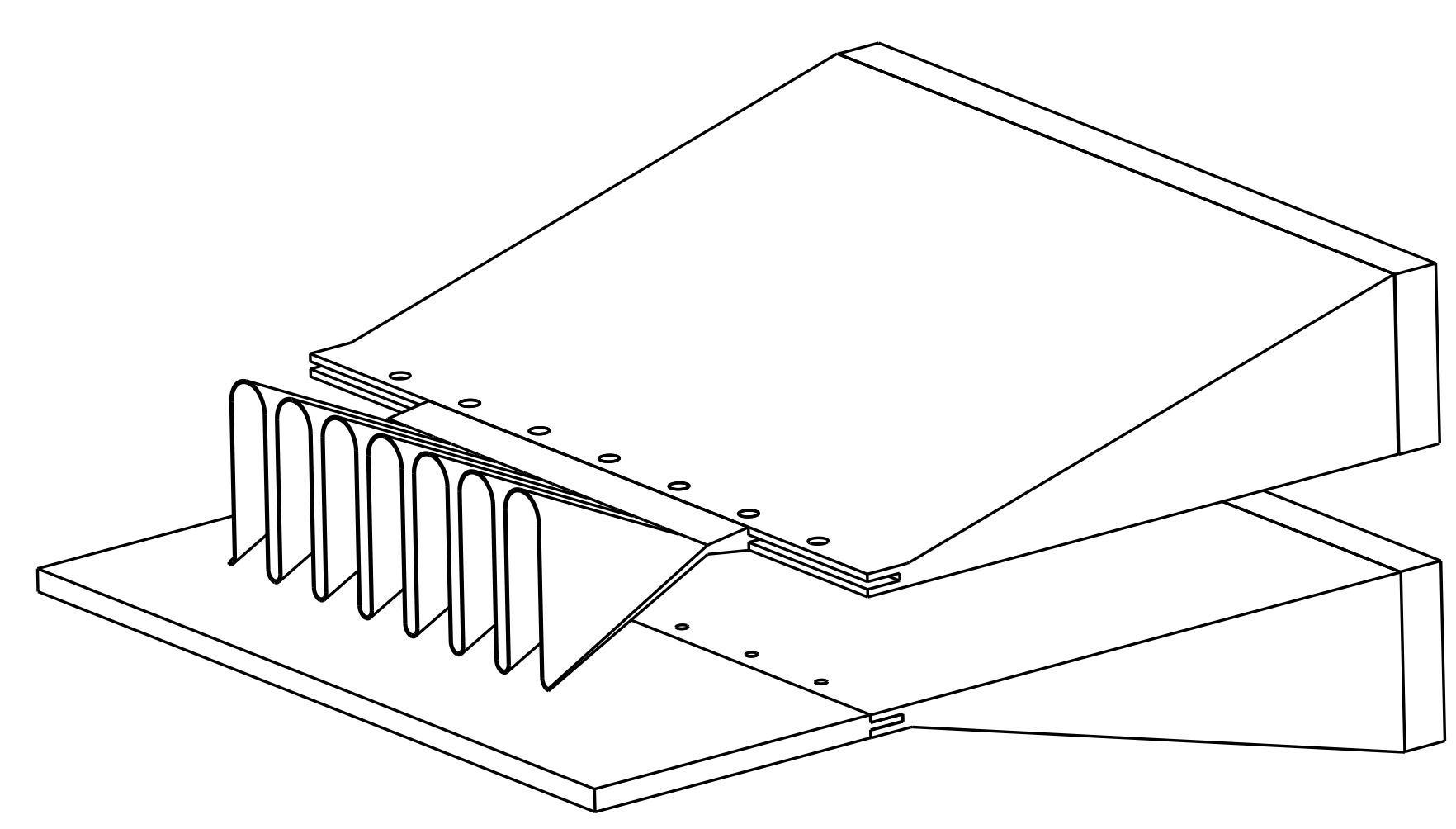

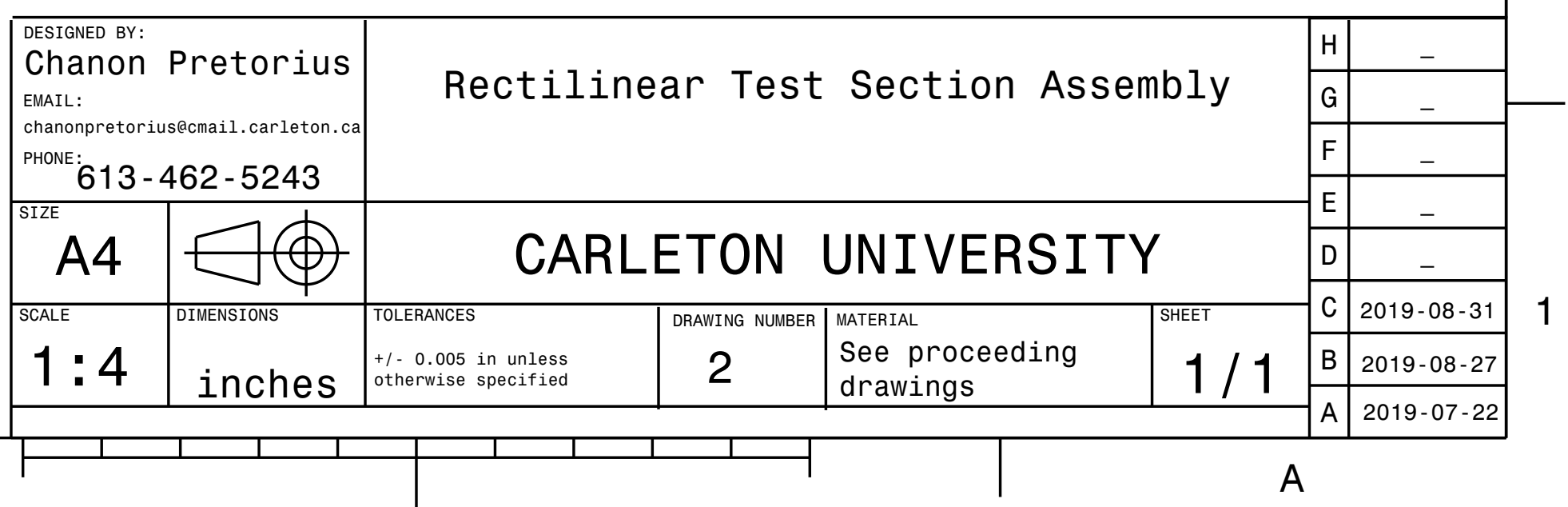


口

0

๓

$\varangle$

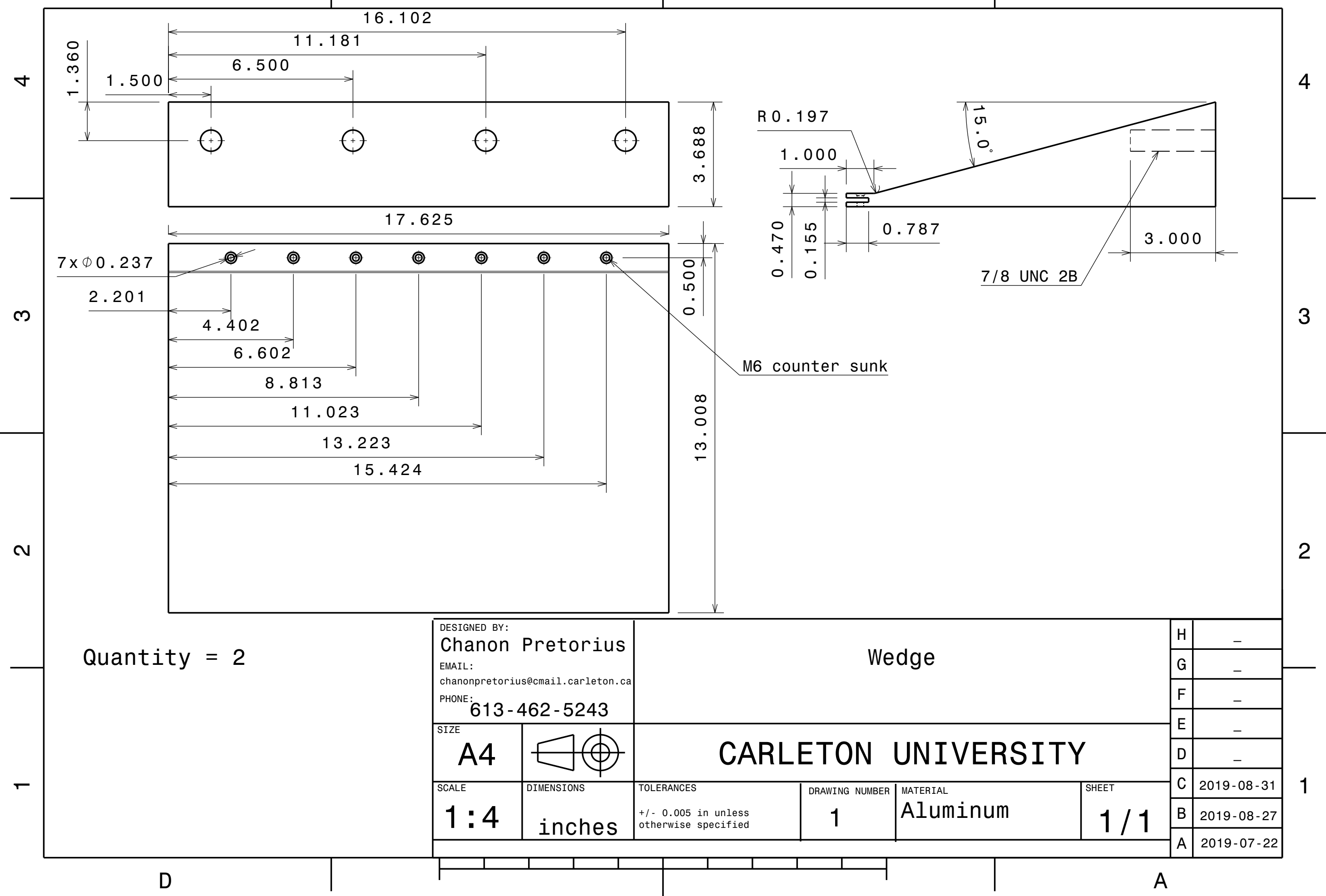



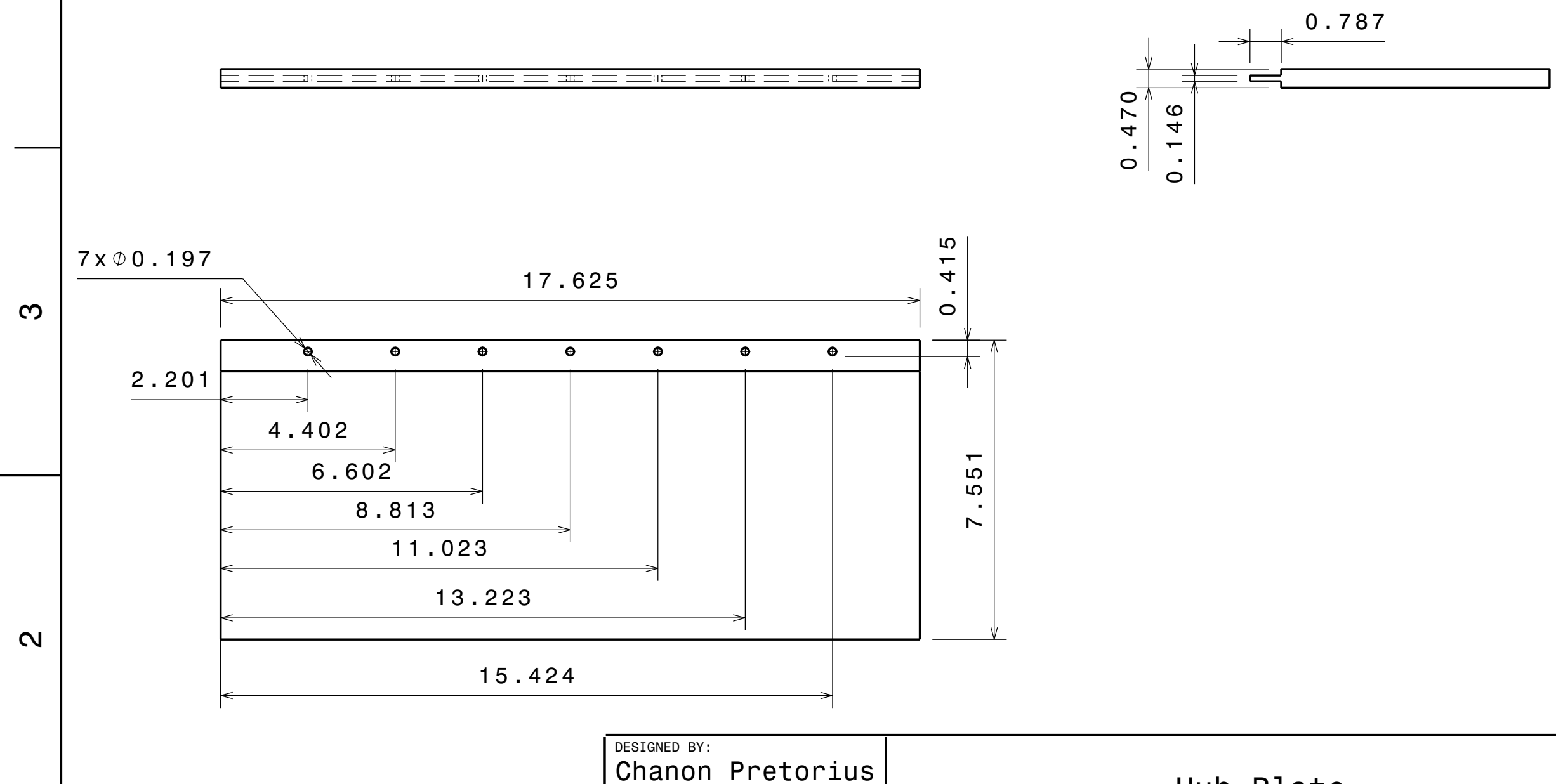

EMAIL:
chanonpretorius@cmail.carleton.ca

Hub Plate

PHONE: 613 - $462-5243$

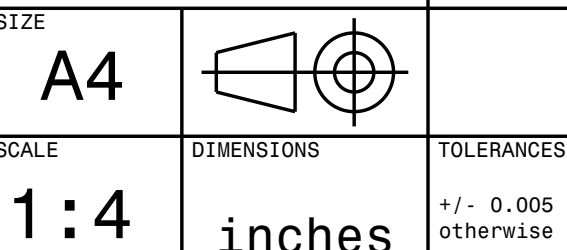

CARLETON UNIVERSITY

\begin{tabular}{|c|l|l} 
DRAWING NUMBER & MATERIAL \\
4 & Aluminum
\end{tabular}

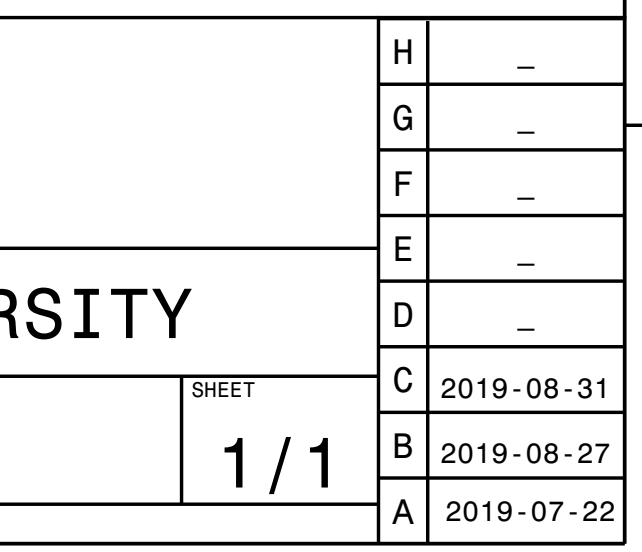




\section{R 0.552}

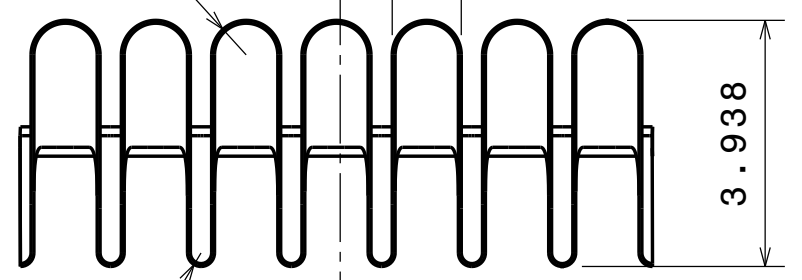

R 0.216

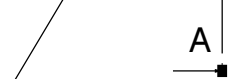

$m$

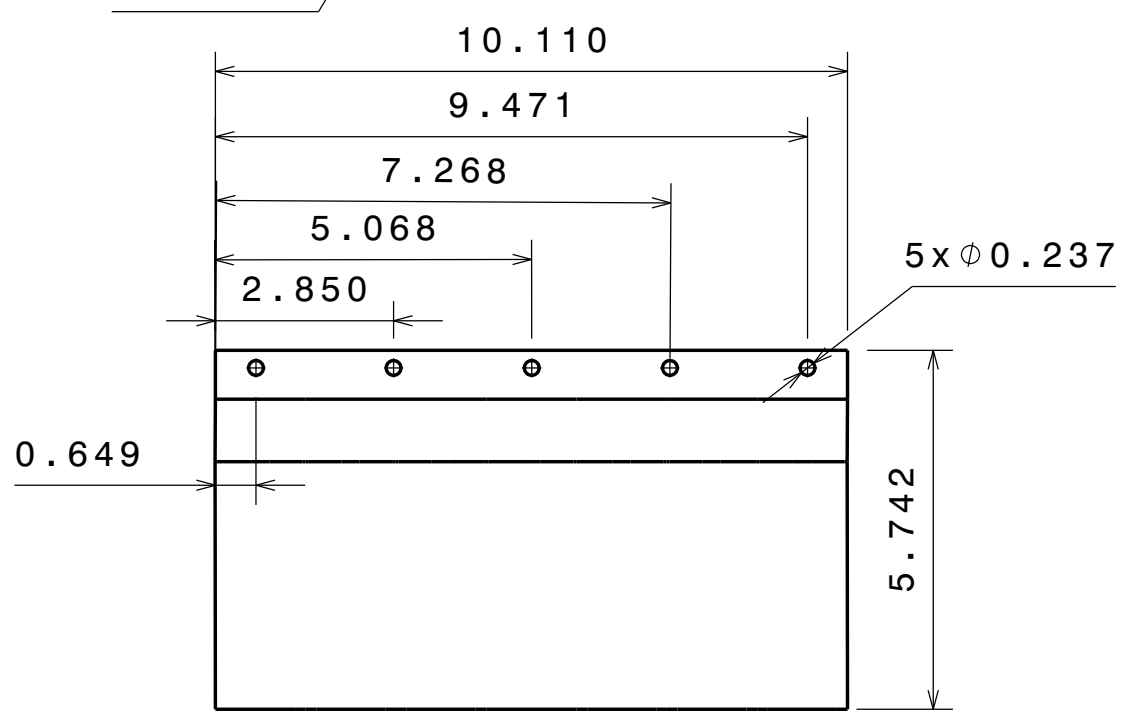

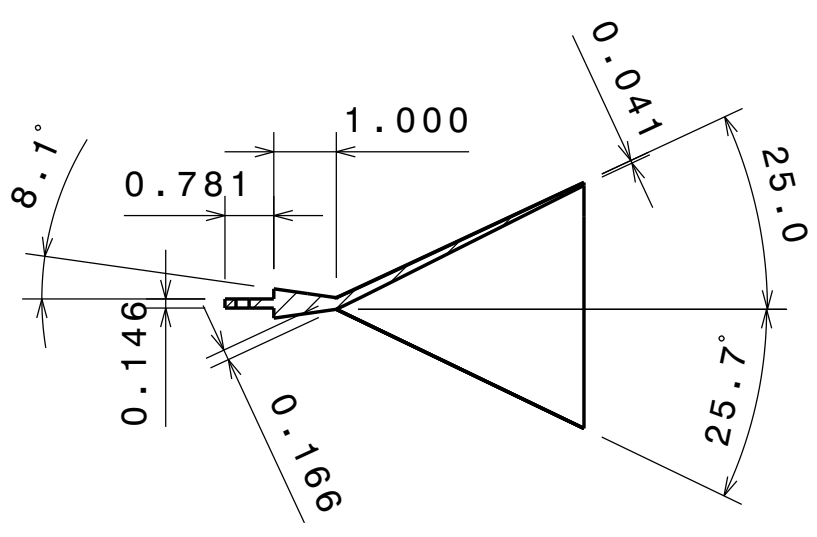

4

3

\begin{tabular}{|c|c|c|c|c|c|c|c|}
\hline \multirow{3}{*}{\multicolumn{2}{|c|}{$\begin{array}{l}\text { DESIGNED BY: } \\
\text { Chanon Pretorius } \\
\text { EMAIL: } \\
\text { chanonpretoriusecmail.carleton.ca } \\
\text { PHONE: } \\
\quad 613-462-5243 \\
\end{array}$}} & \multirow{3}{*}{\multicolumn{4}{|c|}{ Lobed tail }} & $\mathrm{H}$ & - \\
\hline & & & & & & $G$ & - \\
\hline & & & & & & $\mathrm{F}$ & - \\
\hline \multirow{2}{*}{ A4 } & \multirow[b]{2}{*}{ 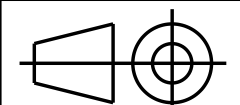 } & \multirow{2}{*}{\multicolumn{4}{|c|}{ CARLETON UNIVERSITY }} & $\mathrm{E}$ & - \\
\hline & & & & & & D & - \\
\hline \multirow{2}{*}{ 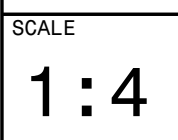 } & \multirow{2}{*}{$\begin{array}{l}\text { DIMENSIONS } \\
\text { inches }\end{array}$} & TOLERANCES & DRAWING NUMBER & \begin{tabular}{|l|l|} 
MATERIAL \\
\end{tabular} & \multirow{3}{*}{$\begin{array}{r}\text { SHEET } \\
1 / 1\end{array}$} & C & 2019-08-31 \\
\hline & & $\begin{array}{l}+1-0.005 \text { in unless } \\
\text { otherwise specified }\end{array}$ & 5 & Steel & & B & $2019-08-27$ \\
\hline \multicolumn{2}{|c|}{ 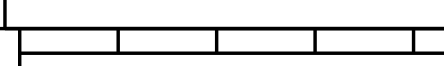 } & $I$ & 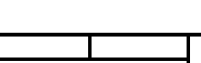 & & & A & $2019-07-22$ \\
\hline
\end{tabular}




\section{Appendix F}

\section{Validation of the Numerical Grid Used to Resolve the Expansion/Compression Wave Pattern of an \\ Under-expanded Jet}

\section{F.1 Computational Model}

The validation study presented in this appendix focuses on the sudden expansion of an under-expanded jet into a stagnant environment. In this flow, the series of expansion and compression waves and their mutual interactions constitute the most challenging flow features for a CFD algorithm. These waves may be incorrectly positioned and/or artificially smeared if the discretization scheme or spatial resolution results in excessive numerical (artificial) diffusion. The same may result from a turbulence model producing excessive amount of turbulent diffusion (eddy viscosity). The validation study presented herein aims to establish the suitability of the formal orders of discretization chosen for the spatial derivatives, the fineness of the chosen spatial grid, and the accuracy of the shear-stress-transport turbulence closure.

Fig. F.1 presents the computational domain and boundary conditions. Fig. F.2 presents a streamwise cross-sectional view of the computational grid where approximately 100 elements are placed across the orifice diameter of the jet. Table F.1 presents the values of the flow-field parameters at initialization. All quantities are normalized by the jet-exit diameter, $D_{j}=2.54 \mathrm{~mm}$, the inflow stagnation temperature, $T_{o_{i n}}=298 \mathrm{~K}$, the outflow static pressure, $P_{\text {out }}=100 \mathrm{kPa}$, and the reference velocity, $V_{r e f}=340 \mathrm{~m} / \mathrm{s}$, where $V_{\text {ref }}$ is equal to the speed of sound $(M=1.0)$ in air. 


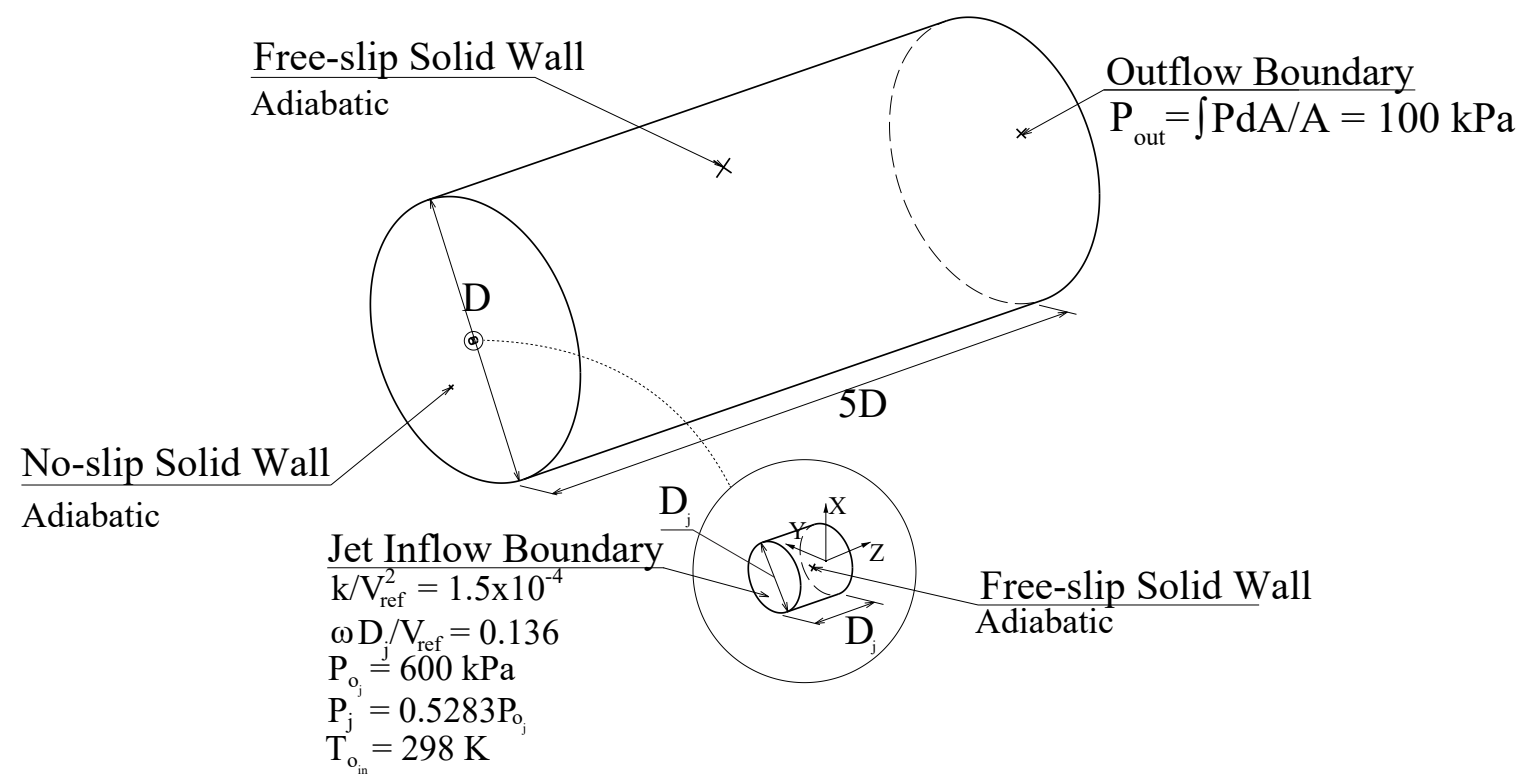

Figure F.1: Computational domain and boundary conditions.

Table F.1: Initial values of the normalized flow-field parameters in the domain.

\begin{tabular}{lc}
\hline$V_{x} / V_{\text {ref }}$ & 0.0 \\
$V_{y} / V_{\text {ref }}$ & 0.0 \\
$V_{z} / V_{\text {ref }}$ & 0.0 \\
$T_{o} / T_{\text {oin }_{\text {in }}}$ & 1.0 \\
$P / P_{\text {out }}$ & 1.0 \\
$k / V_{\text {ref }}^{2}$ & 0 \\
$\omega \Delta R / V_{\text {ref }}$ & 0 \\
\hline
\end{tabular}




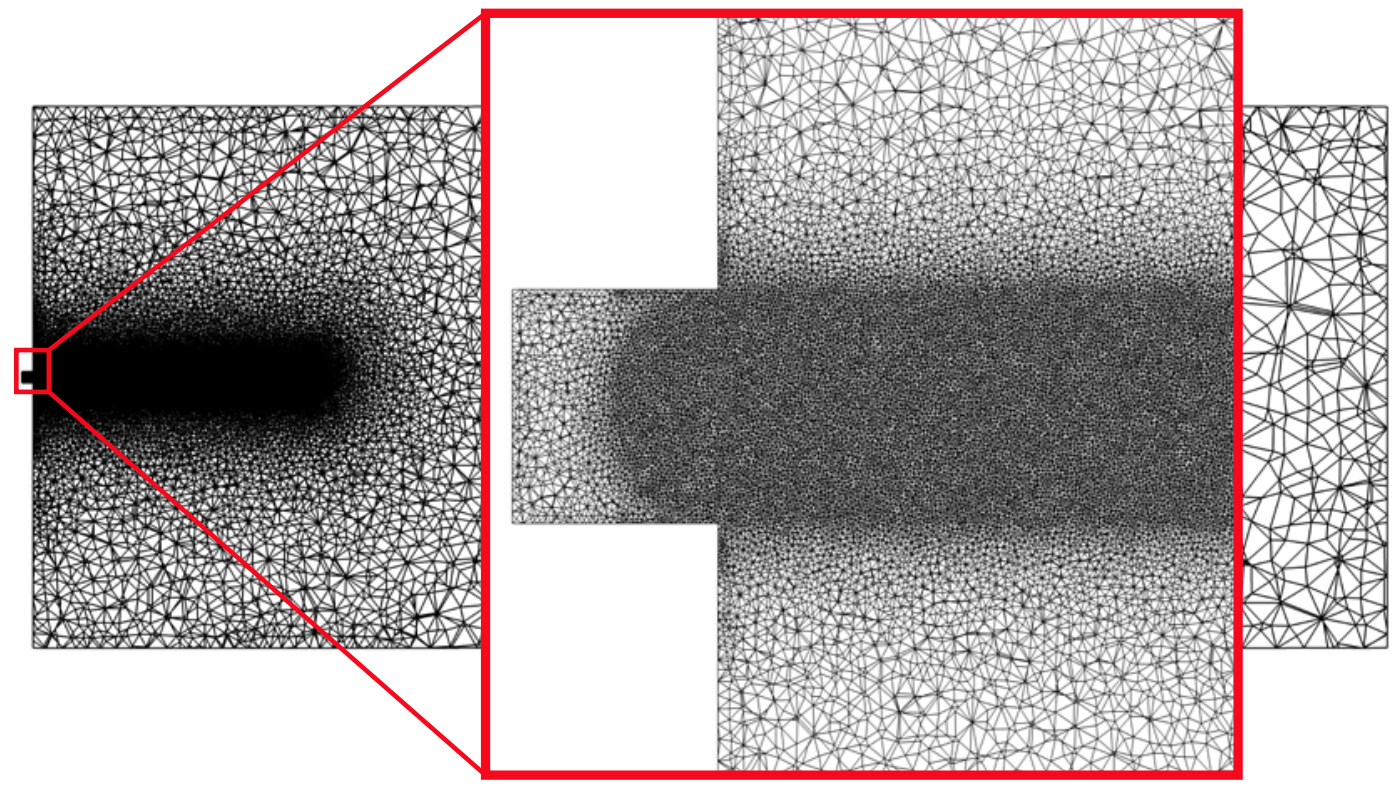

Figure F.2: Streamwise cross-section of the computational grid.

\section{F.2 Computational Results}

Fig. F.3 presents a numerical Schlieren image showing the density gradient magnitude of the under-expanded jet. Fig. F.3 (a) presents a qualitative comparison of the various features of the jet with an experimental Schlieren image of an under-expanded jet from the literature (Xiao et al., 2019). Fig. F.3 (b) shows the computed jet with relevant quantities measured. One of these quantities is the Prandtl-Meyer expansion angle, $\omega$. Table F.2 present a comparison of the Prandtl-Meyer expansion angle of the jet as computed by the simulation and predicted by the Prandtl-Meyer expansion theory, where $\omega$ is defined as:

$$
\omega=\sqrt{\frac{\gamma+1}{\gamma-1}} \arctan \sqrt{\left(M^{2}-1\right) \frac{\gamma-1}{\gamma+1}}-\arctan \sqrt{M^{2}-1}
$$

where $M=1.82$ is the mach number of the flow downstream of the expansion fan as computed by the simulation. The seconded quantity measured in Fig. F.3 is the distance of the Mach disk from the jet-exit normalized by the orifice diameter, $X_{m} / D_{j}$. Table F.2 presents a comparison of $X_{m} / D_{j}$ as computed by the simulation and predicted by the empirical correlation of Crist et al. (1966): 


$$
\frac{X_{m}}{D_{j}}=\sqrt{\frac{P_{o_{j}}}{2.4 P_{b}}}
$$

where $X_{m}$ is the distance of the Mach disk from the jet-exit, and $P_{b}=P_{\text {out }}$ is the back pressure into which the jet discharges. The favorable agreement of the results in Table F.2 confirms that the spatial resolution of the computational grid is indeed sufficient to accurately resolve the expansion and compression wave patterns in an under-expanded jet.
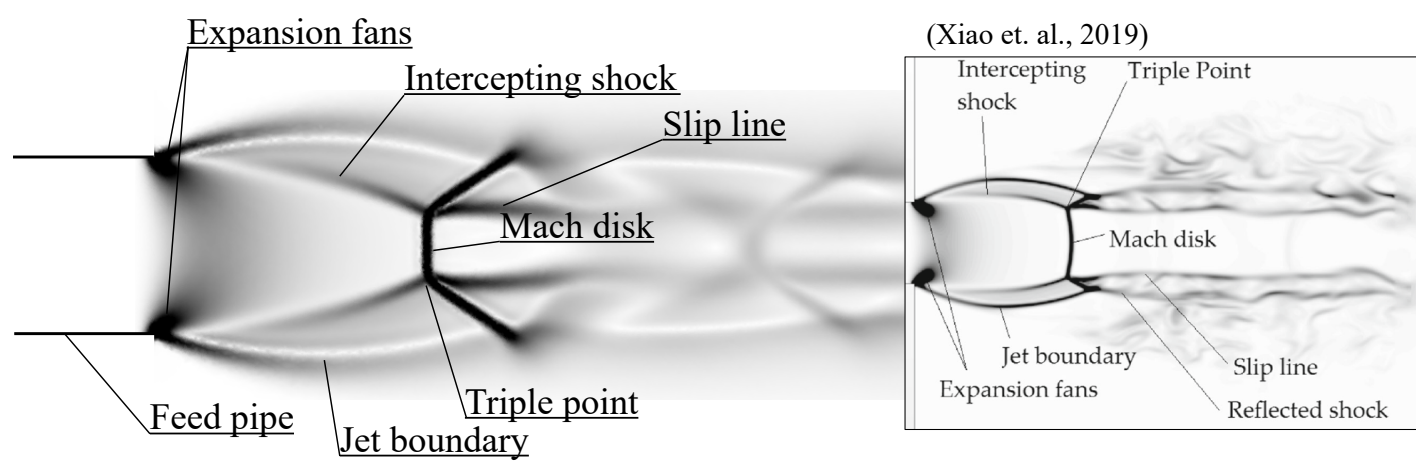

(a)

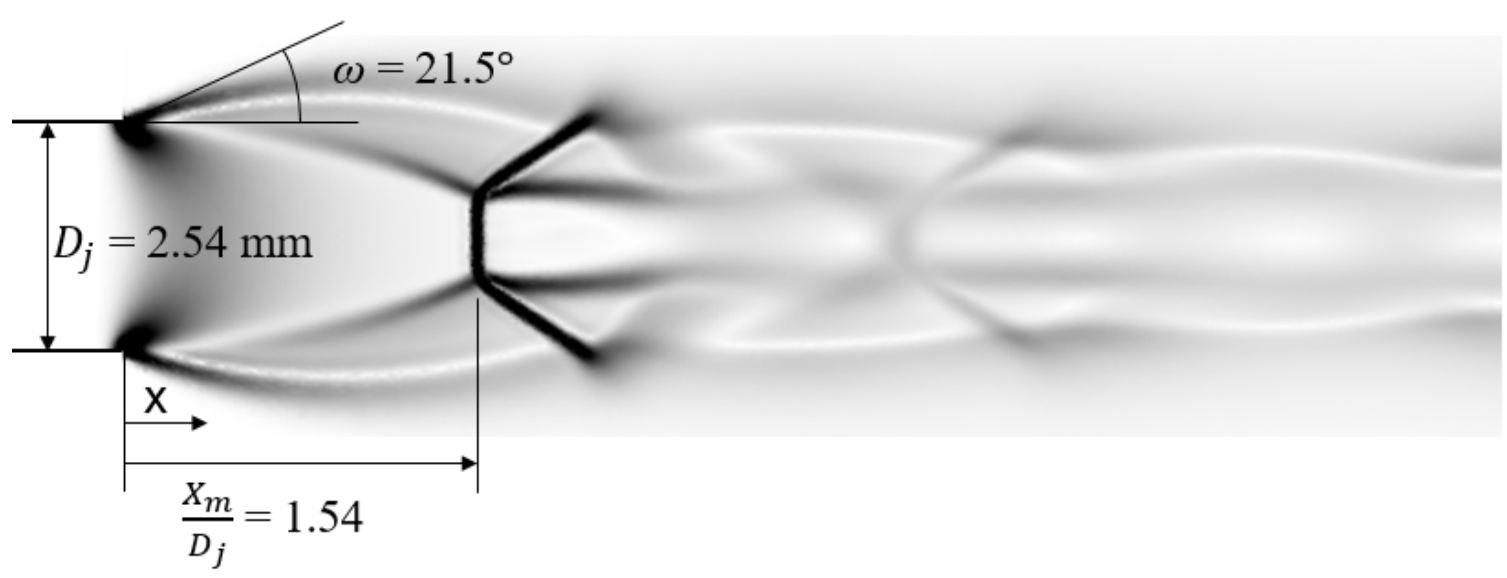

(b)

Figure F.3: (a) Density gradient-magnitude in the computed under-expanded jet showing the expansion/compression wave pattern as compared to an experimental Schlieren image (Xiao et al., 2019) and (b) Measurements of the expansion angle $(\omega)$ and Mach disk location $\left(X_{m} / D_{j}\right)$ in the computed under-expanded jet. 
Table F.2: Comparison of computational results to empirical and analytical predictions.

\begin{tabular}{c|ccc}
\hline & Simulation & $\begin{array}{c}\text { Analytical Solution } \\
\text { (Prandtl-Meyer Theory) }\end{array}$ & $\begin{array}{c}\text { Empirical Correlation } \\
\text { (Crist et al., 1966) }\end{array}$ \\
\hline$\omega$ & $21.5^{\circ}$ & $21.3^{\circ}$ & - \\
$\frac{X_{m}}{D_{j}}$ & 1.54 & - & 1.54 \\
\hline
\end{tabular}




\section{Appendix G}

\section{Seed Injection Port Design - Additional Figures}

This page left intentionally blank. 

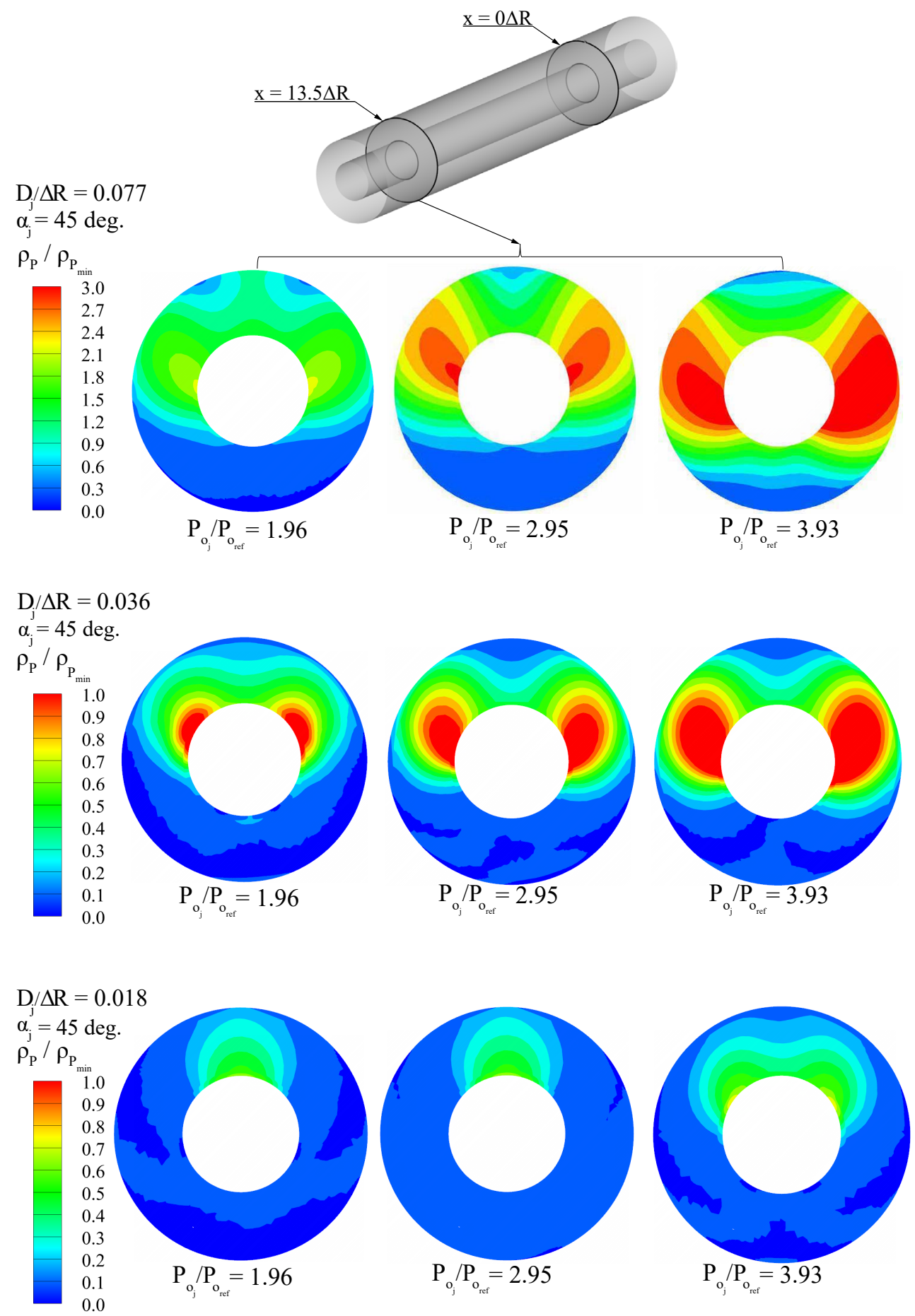

Figure G.1: Seed-particle density distribution in the $x=13.5 \Delta R$ plane as a function of $D_{j} / \Delta R$ and $P_{o_{j}} / P_{o_{r e f}}$ for $\alpha_{j}=45 \mathrm{deg}$. 

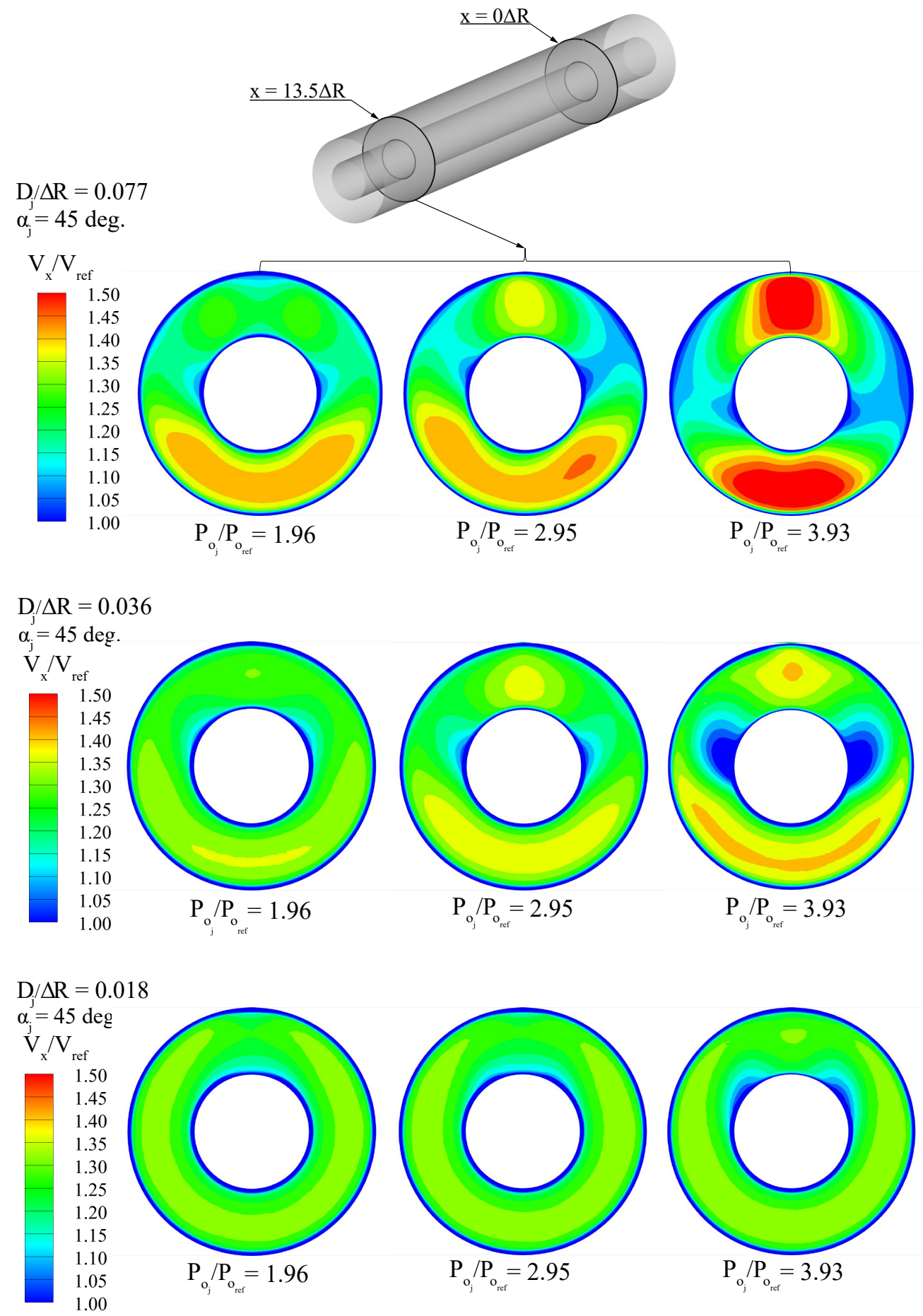

Figure G.2: Axial velocity distribution in the $x=13.5 \Delta R$ plane as a function of $D_{j} / \Delta R$ and $P_{o_{j}} / P_{o_{r e f}}$ for $\alpha_{j}=45 \mathrm{deg}$. 

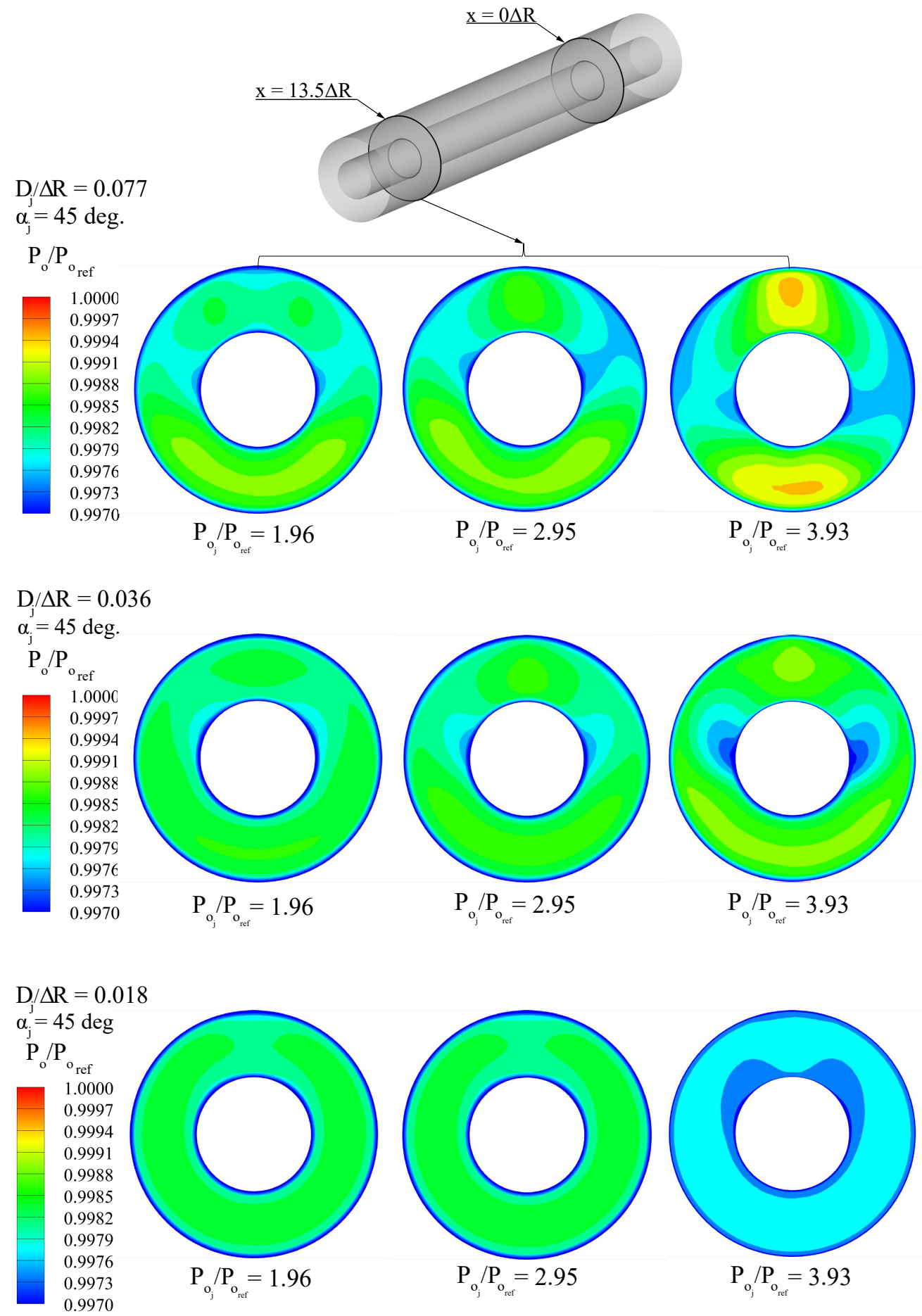

Figure G.3: Stagnation pressure distribution in the $x=13.5 \Delta R$ plane as a function of $D_{j} / \Delta R$ and $P_{o_{j}} / P_{o_{r e f}}$ for $\alpha_{j}=45 \mathrm{deg}$. 

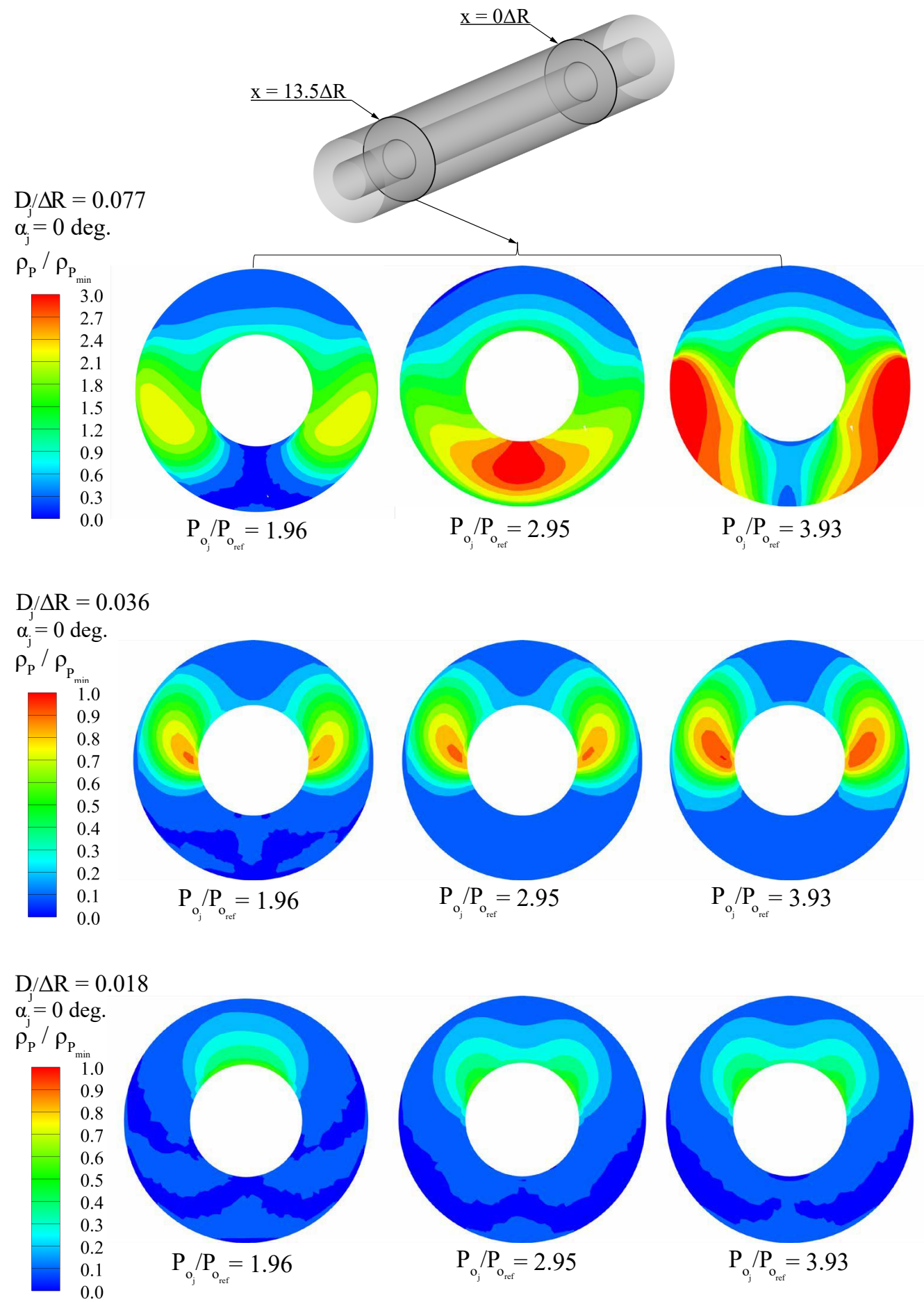

Figure G.4: Seed-particle density distribution in the $x=13.5 \Delta R$ plane as a function of $D_{j} / \Delta R$ and $P_{o_{j}} / P_{o_{r e f}}$ for $\alpha_{j}=0$ deg. 

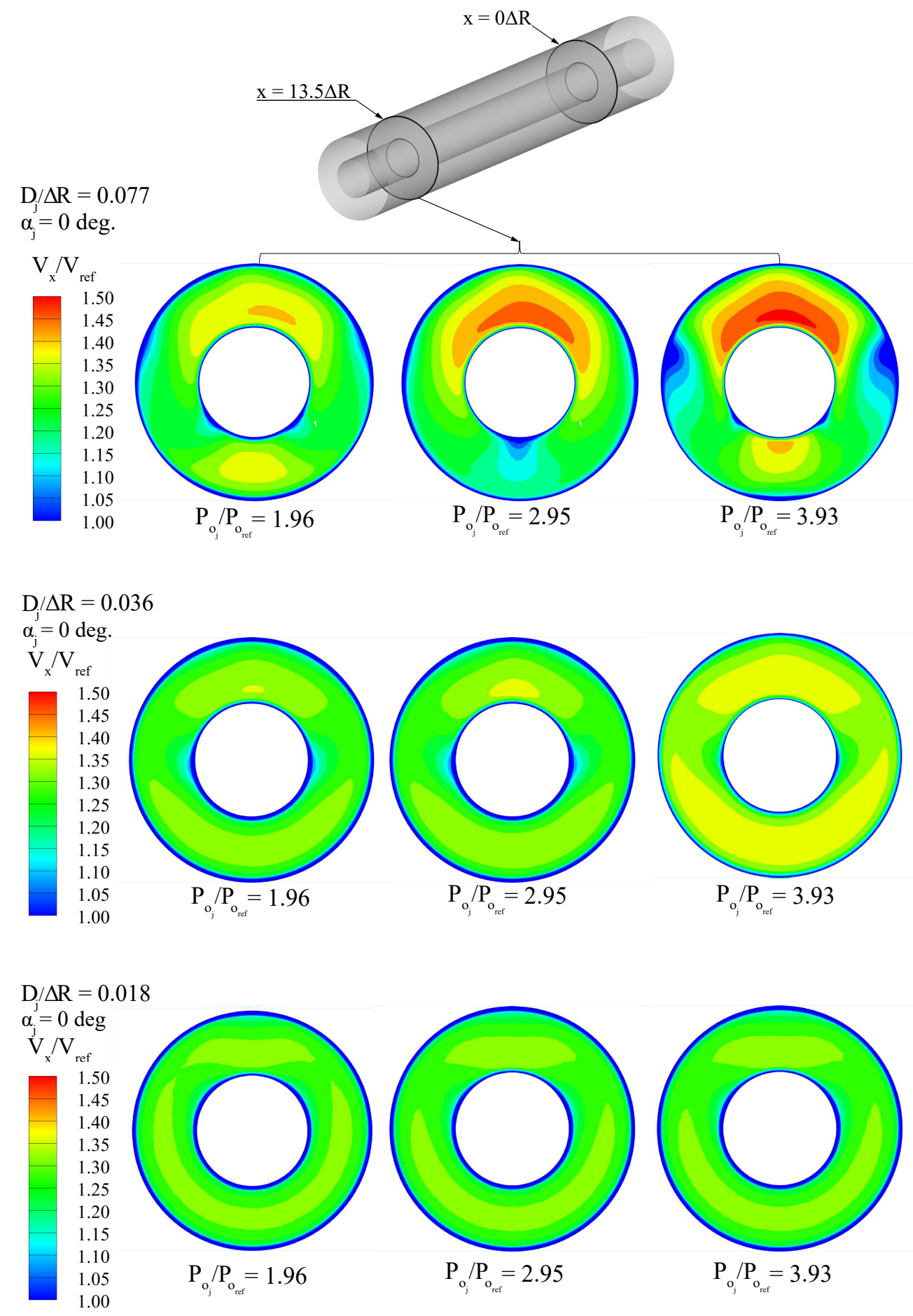

Figure G.5: Axial velocity distribution in the $x=13.5 \Delta R$ plane as a function of $D_{j} / \Delta R$ and $P_{o_{j}} / P_{o_{r e f}}$, and $\alpha_{j}=0$ deg. 

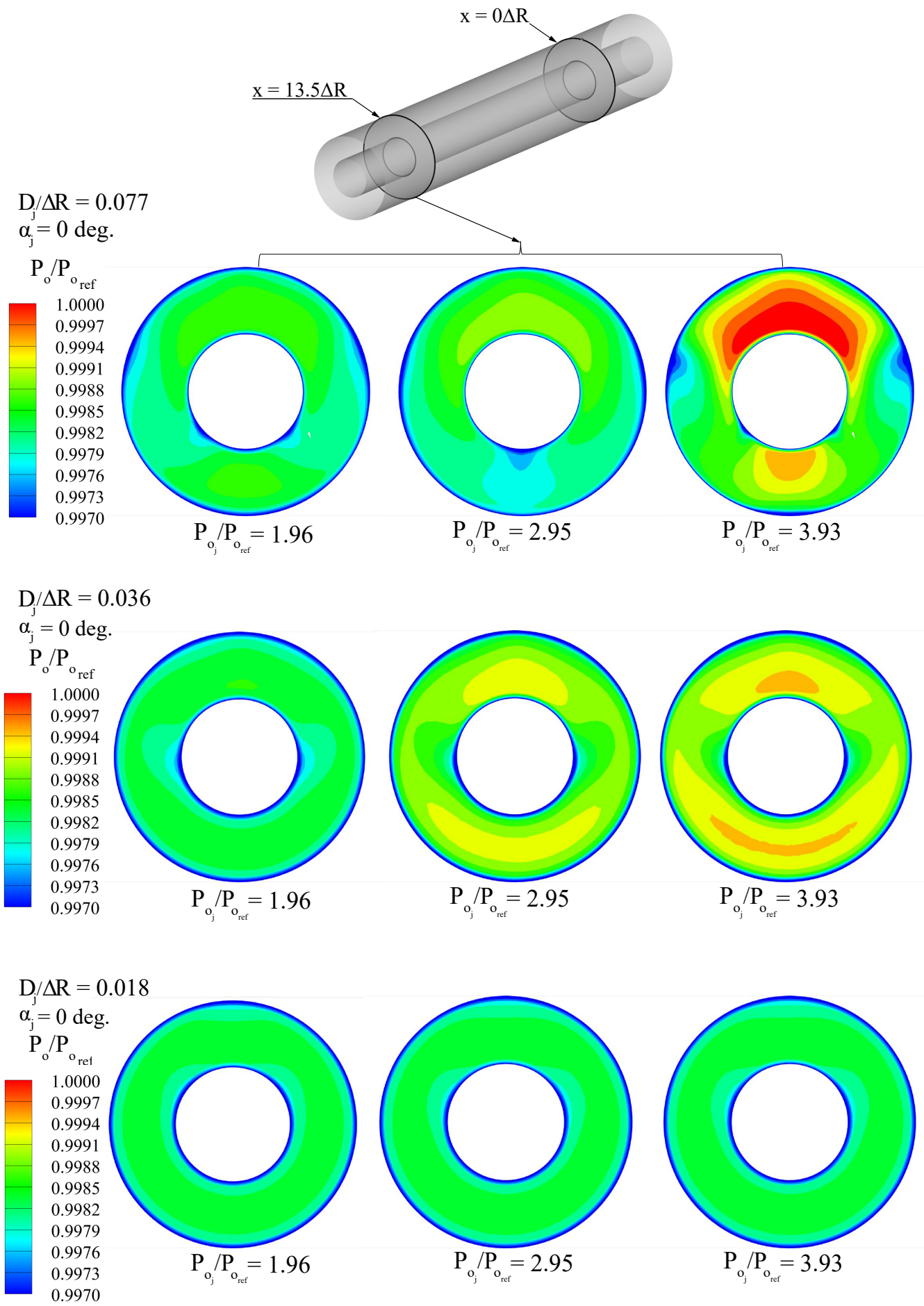

Figure G.6: Stagnation pressure distribution in the $x=13.5 \Delta R$ plane as a function of $D_{j} / \Delta R$ and $P_{o_{j}} / P_{o_{\text {ref }}}$ for $\alpha_{j}=0 \mathrm{deg}$. 


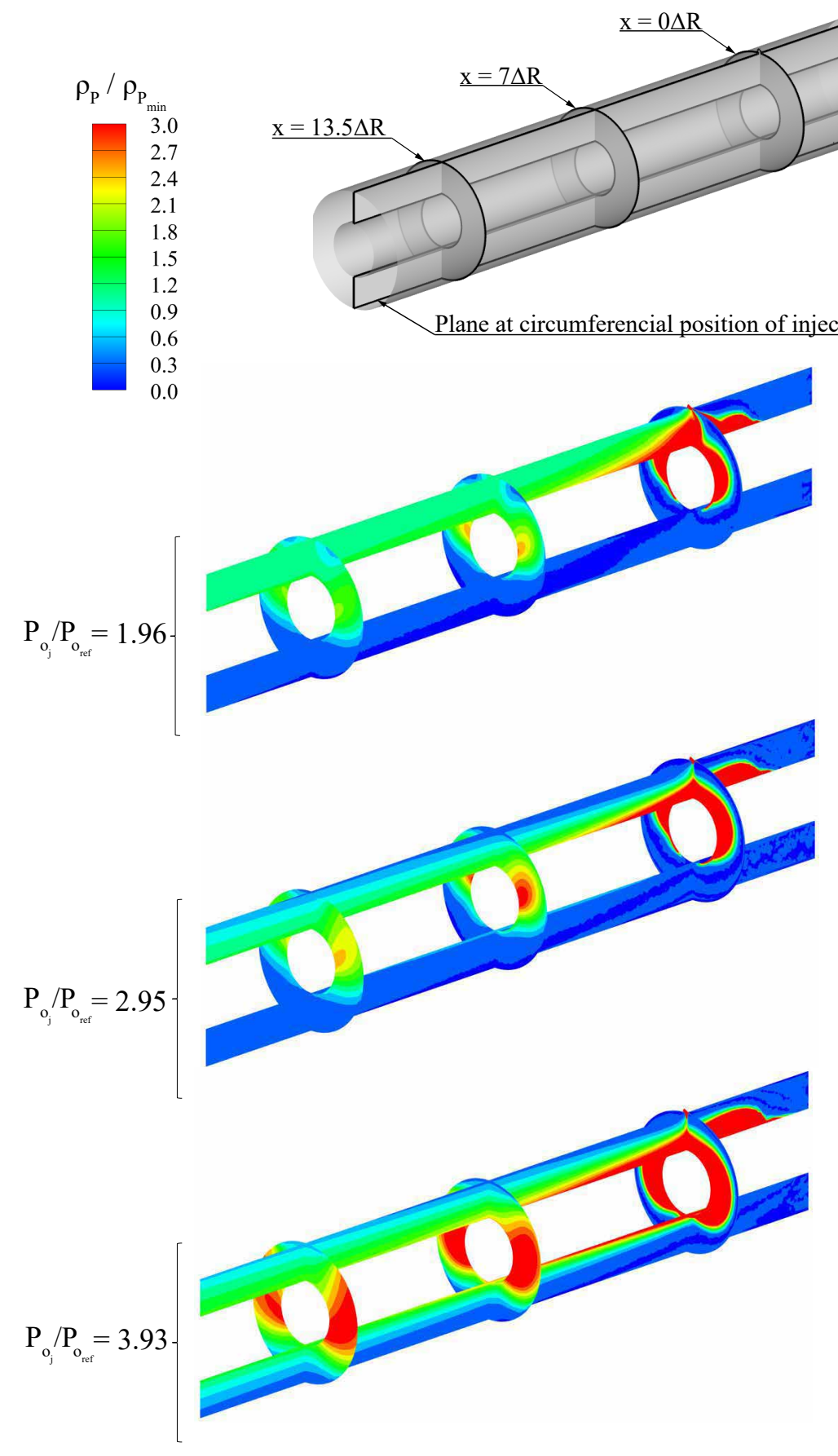

Figure G.7: Streamwise seed-particle density distributions in the $y=0$ plane as a function of $P_{o_{j}} / P_{o_{r e f}}$ for $D_{j} / \Delta R=0.077$ and $\alpha_{j}=45 \mathrm{deg}$. 


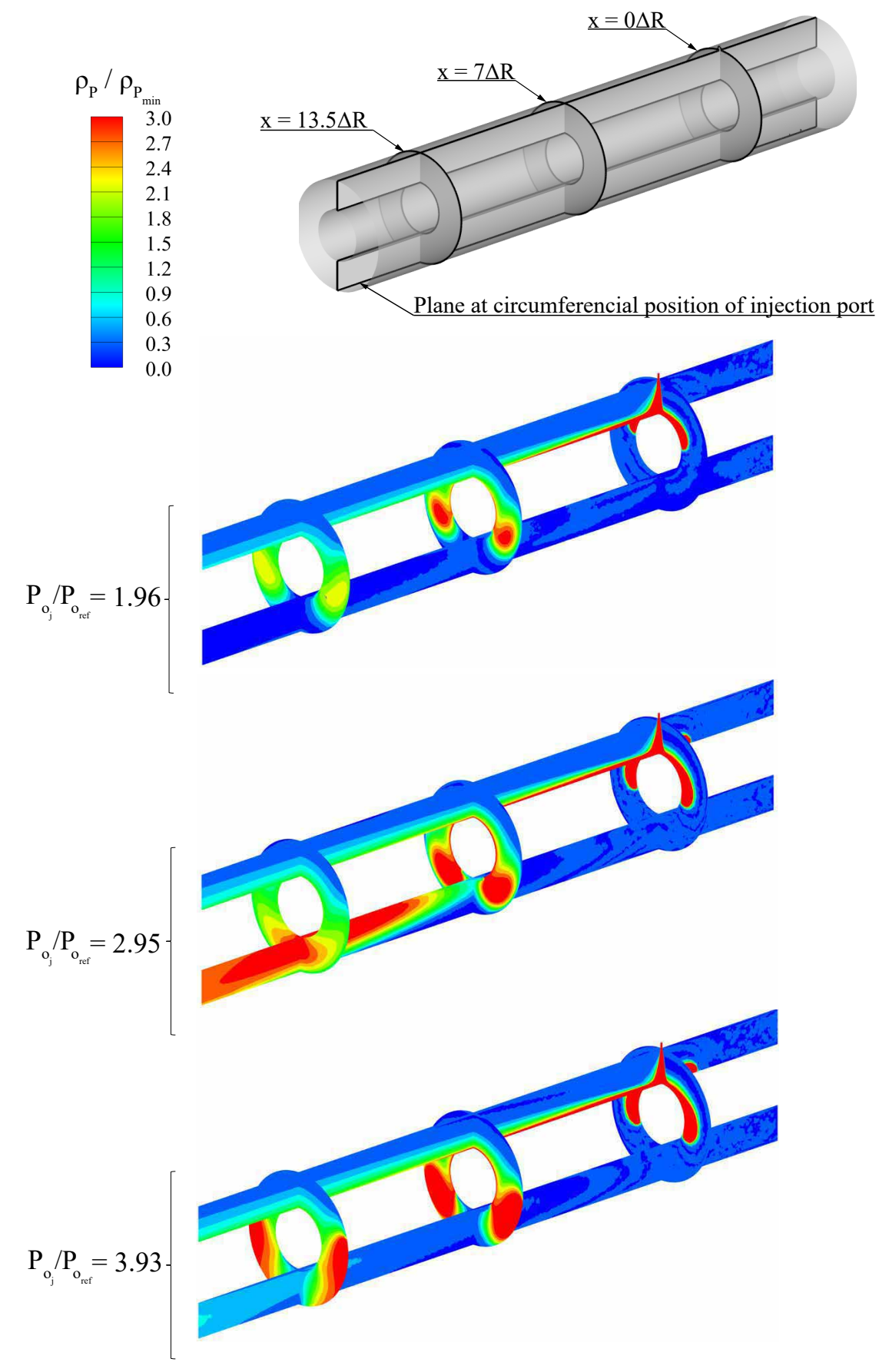

Figure G.8: Streamwise seed-particle density distributions as a function of $P_{o_{j}} / P_{o_{r e f}}$ for $D_{j} / \Delta R=0.077$ and $\alpha_{j}=0$ deg. 


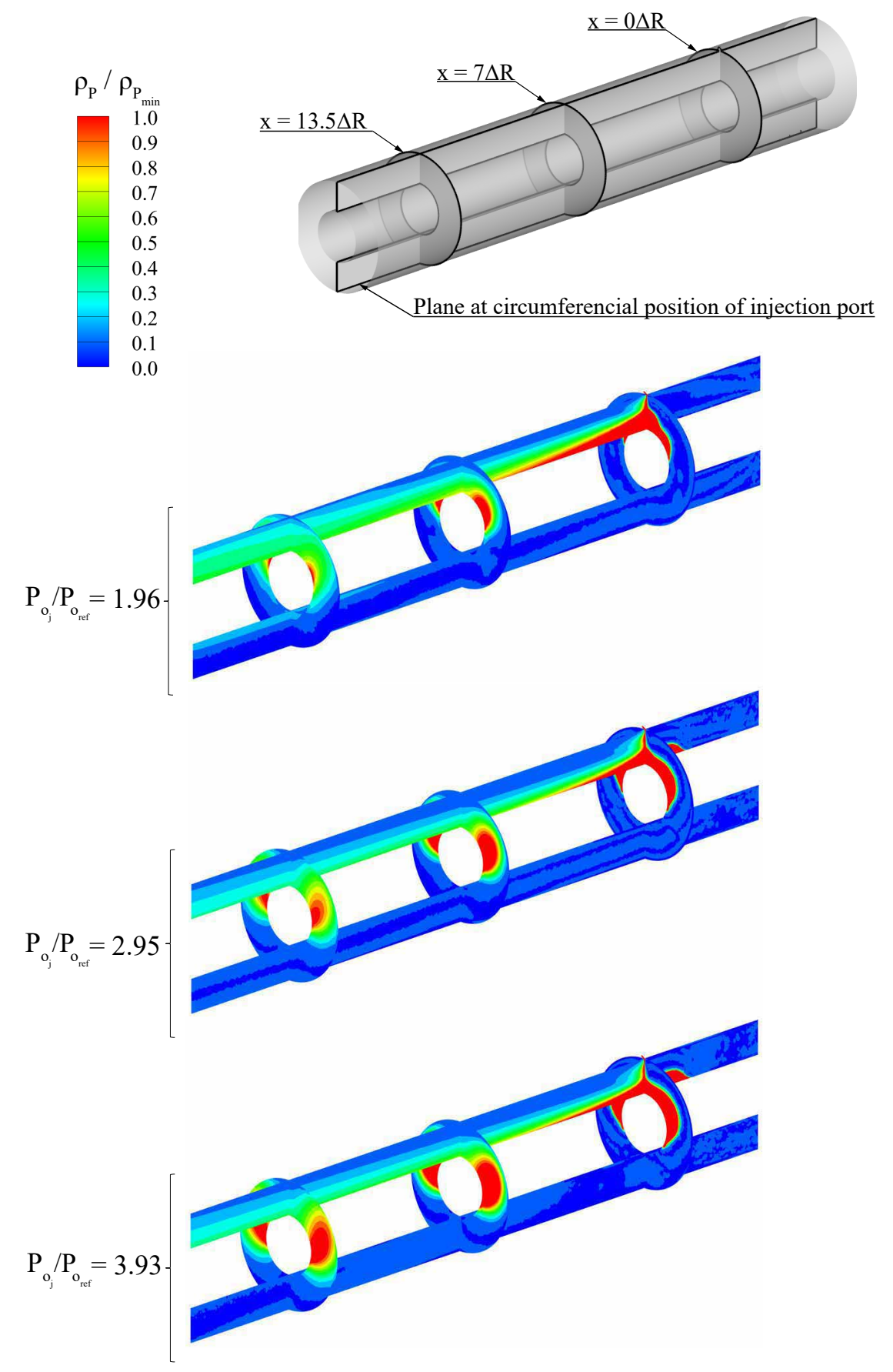

Figure G.9: Streamwise seed-particle density distributions as a function of $P_{o_{j}} / P_{o_{r e f}}$ for $D_{j} / \Delta R=0.036$ and $\alpha_{j}=45 \mathrm{deg}$. 


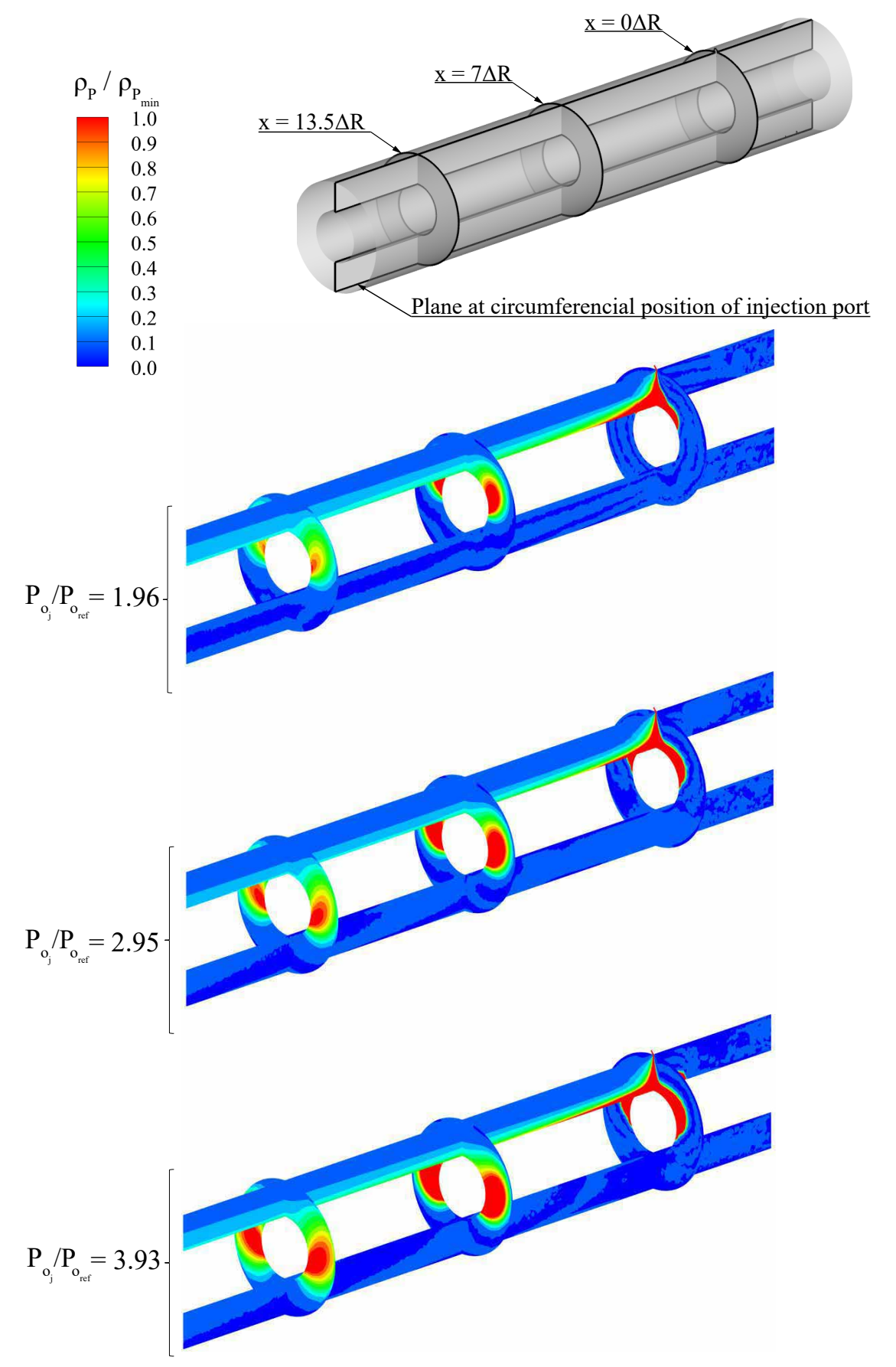

Figure G.10: Streamwise seed-particle density distributions as a function of $P_{o_{j}} / P_{o_{r e f}}$ for $D_{j} / \Delta R=0.036$ and $\alpha_{j}=30 \mathrm{deg}$. 


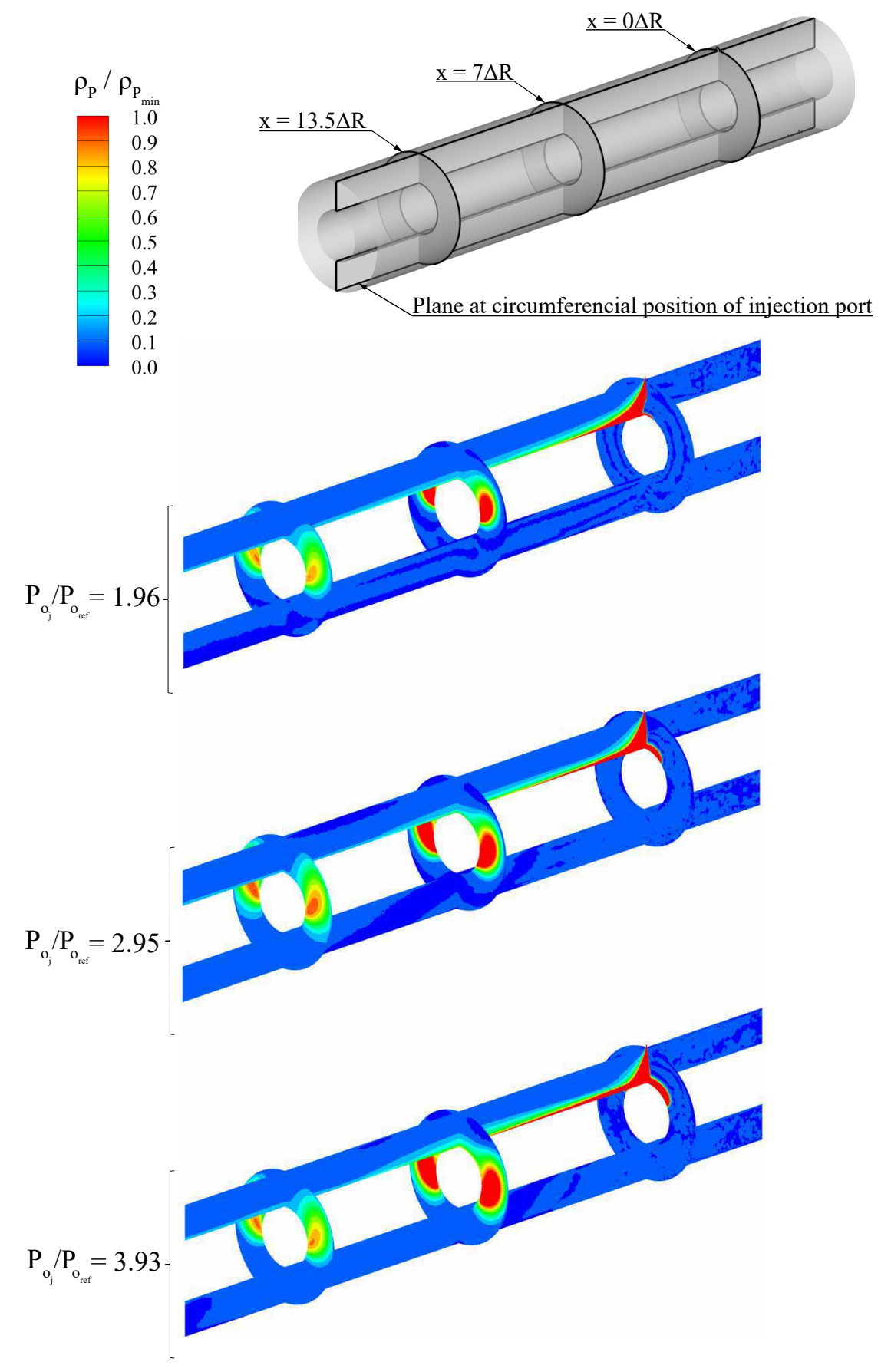

Figure G.11: Streamwise seed-particle density distributions as a function of $P_{o_{j}} / P_{o_{r e f}}$ for $D_{j} / \Delta R=0.036$ and $\alpha_{j}=0$ deg. 


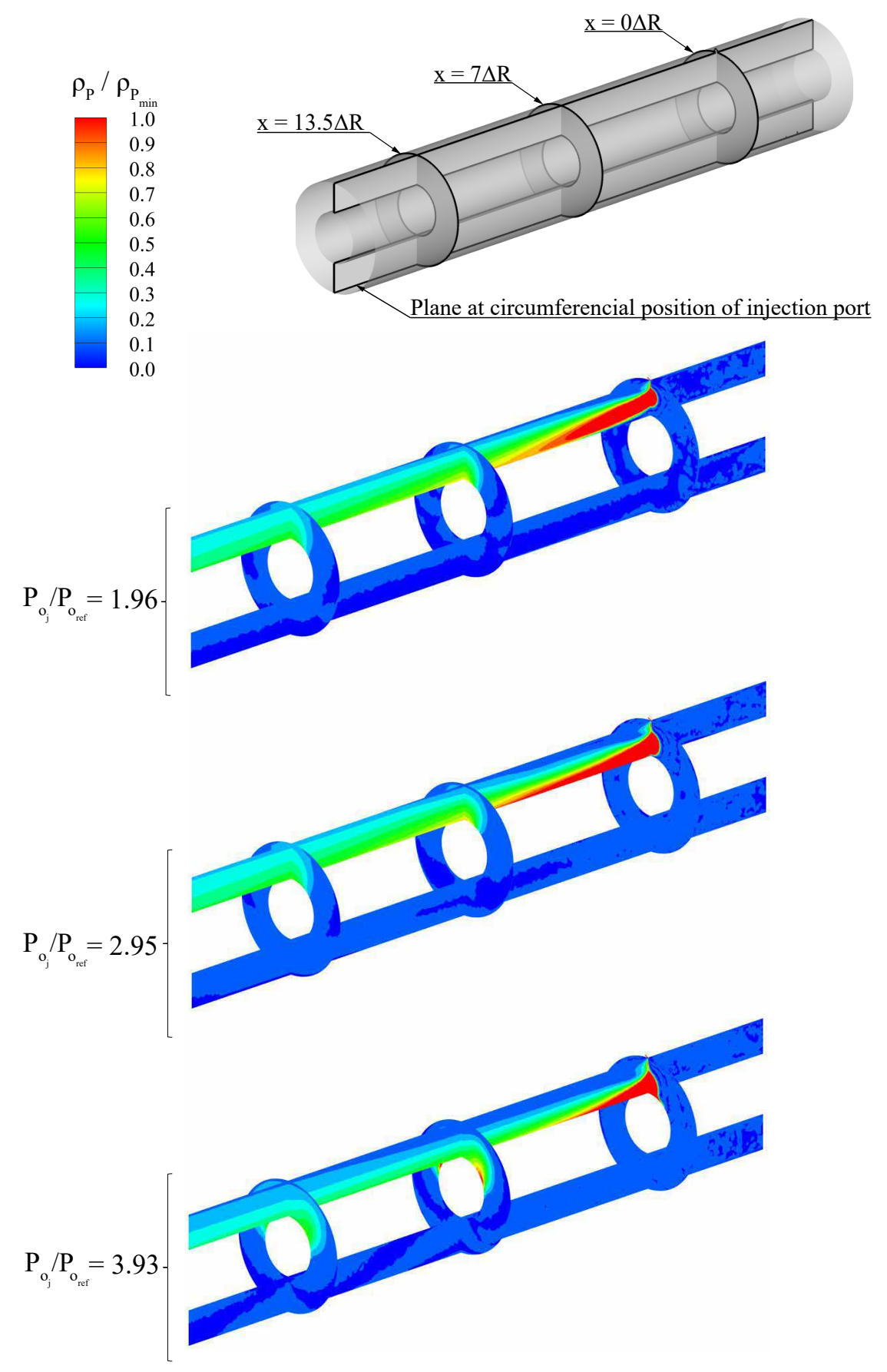

Figure G.12: Streamwise seed-particle density distributions as a function of $P_{o_{j}} / P_{o_{r e f}}$ for $D_{j} / \Delta R=0.018$ and $\alpha_{j}=45 \mathrm{deg}$. 


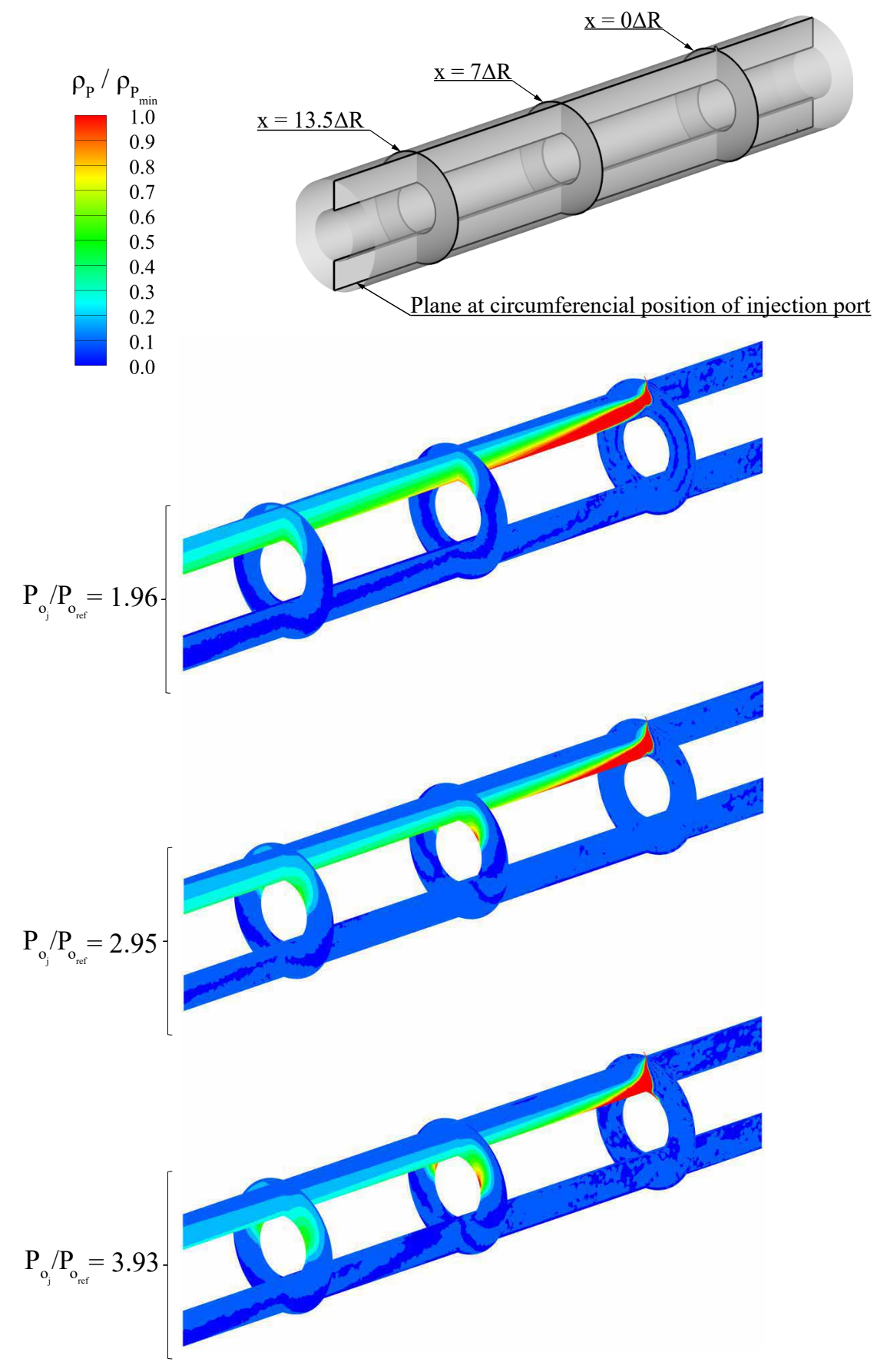

Figure G.13: Streamwise seed-particle density distributions as a function of $P_{o_{j}} / P_{o_{r e f}}$ for $D_{j} / \Delta R=0.018$ and $\alpha_{j}=30$ deg. 


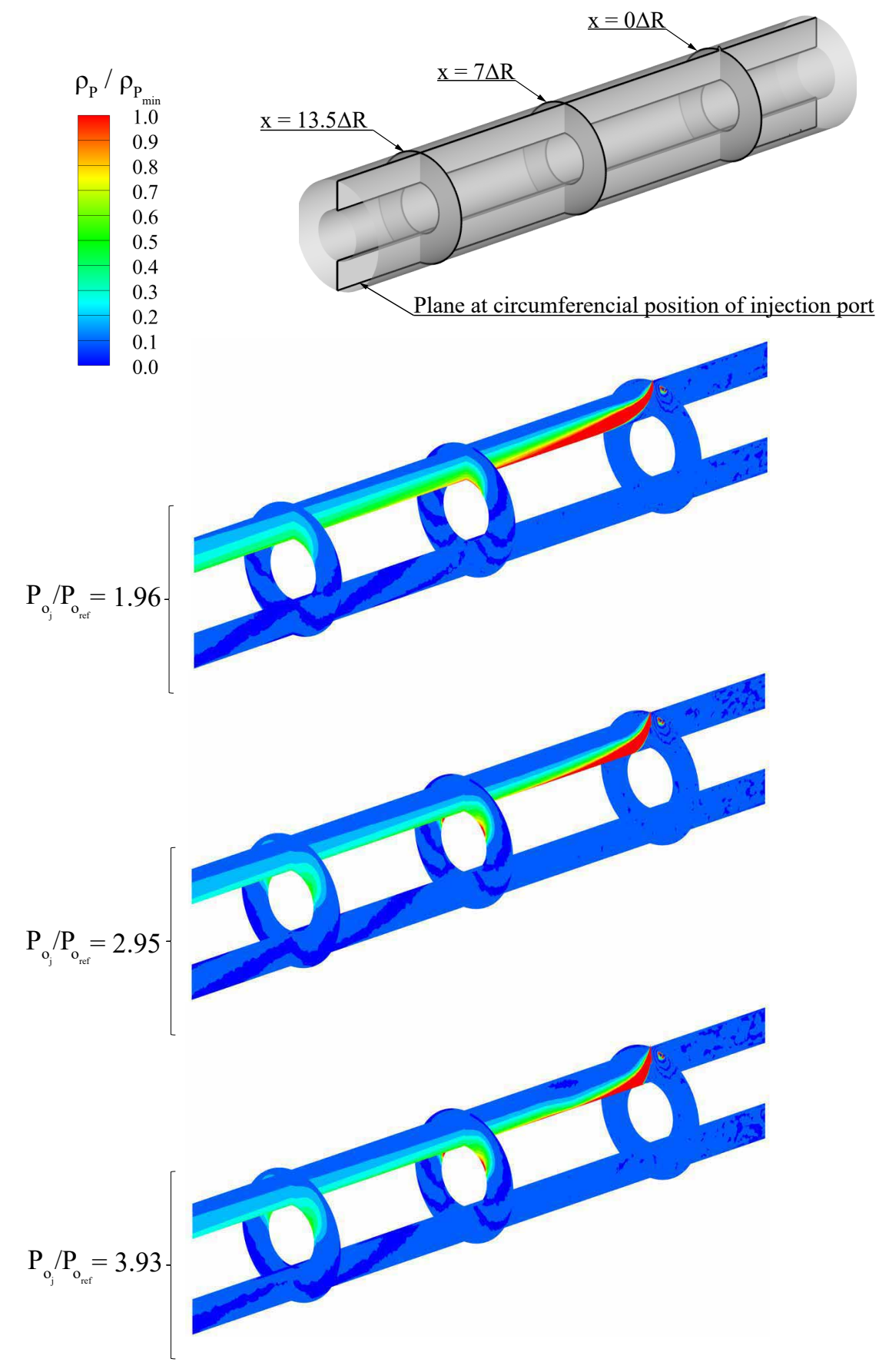

Figure G.14: Streamwise seed-particle density distributions as a function of $P_{o_{j}} / P_{o_{r e f}}$ for $D_{j} / \Delta R=0.018$ and $\alpha_{j}=0$ deg. 